\title{
Small Punch Testing to Estimate Mechanical Properties of Additively Manufactured Ti-6Al-4V
}

Enrico Lucon

Jake Benzing Nik Hrabe

This publication is available free of charge from: https://doi.org/10.6028/NIST.TN.2096 


\title{
Small Punch Testing to Estimate Mechanical Properties of Additively Manufactured Ti-6Al-4V
}

\author{
Enrico Lucon \\ Jake Benzing \\ Nik Hrabe \\ Applied Chemicals and Materials Division \\ Material Measurement Laboratory
}

This publication is available free of charge from:

https://doi.org/10.6028/NIST.TN.2096

June 2020

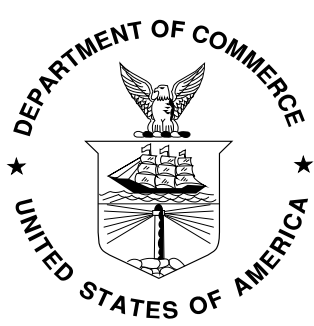

U.S. Department of Commerce

Wilbur L. Ross, Jr., Secretary

National Institute of Standards and Technology Walter Copan, NIST Director and Undersecretary of Commerce for Standards and Technology 
Certain commercial entities, equipment, or materials may be identified in this document in order to describe an experimental procedure or concept adequately. Such identification is not intended to imply recommendation or endorsement by the National Institute of Standards and Technology, nor is it intended to imply that the entities, materials, or equipment are necessarily the best available for the purpose.

National Institute of Standards and Technology Technical Note 2096

Natl. Inst. Stand. Technol. Tech. Note 2096, 277 pages (June 2020) CODEN: NTNOEF

This publication is available free of charge from: https://doi.org/10.6028/NIST.TN.2096 


\begin{abstract}
Small Punch (SP) testing is a methodology that uses small disk-shaped specimens, generally 8 $\mathrm{mm}$ in diameter and $0.5 \mathrm{~mm}$ thick, to estimate mechanical properties of metallic materials, such as tensile properties, fracture toughness, and ductile-to-brittle transition temperature. Empirical correlations are typically used to infer conventional mechanical properties from characteristic forces and displacements obtained from the test record. Most of the available literature relates to SP testing of steels, while relatively little information is available for other metallic materials. At NIST in Boulder, Colorado, SP tests were conducted on additively manufactured (AM) Ti-6Al-4V with different processing parameters and heat treatment conditions. The shape of force/punch displacement curves appeared different than typically reported for conventionally manufactured steels, and correlations with tensile parameters were generally weaker than those published for steel samples. We are led to conclude that the application of the SP technique, characterized by a biaxial loading mode, to materials with high anisotropy, such as current batches of AM Ti-6Al-4V, may be somewhat problematic and therefore of limited applicability. Finally, the use of actuator displacement instead of punch displacement in test analyses appeared to cause a generalized worsening of the correlations.
\end{abstract}

\title{
Key words
}

additive manufacturing, empirical correlations, fracture toughness, Small Punch, tensile properties, Ti-6Al-4V. 


\section{Table of Contents}

1. Introduction ....................................................................................................................... 12

2. Experimental setup for SP testing.................................................................... 14

3. Material and conditions investigated .................................................................... 16

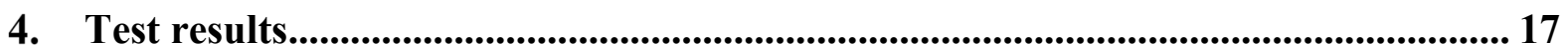

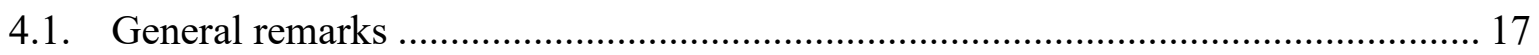

4.2. Preliminary tests (standard HIP, scan length $=56 \mathrm{~mm}$, supported) ....................... 19

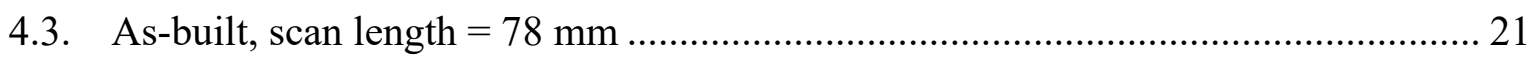

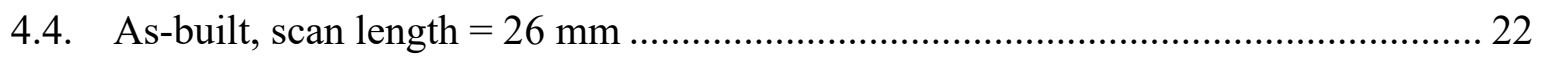

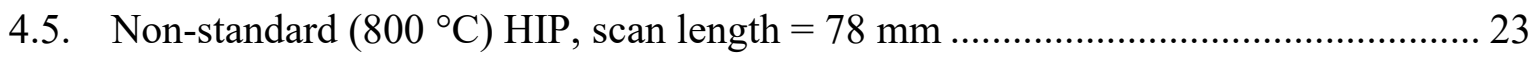

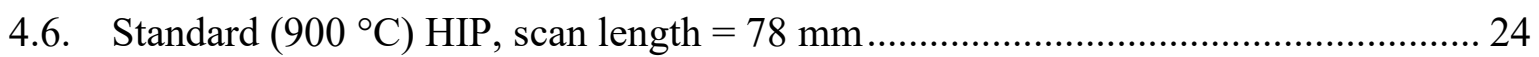

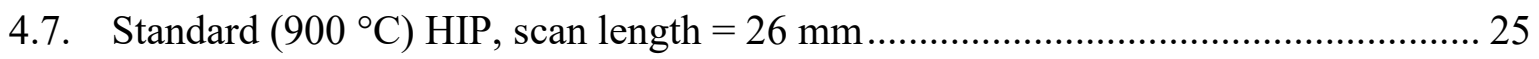

4.8. Non-standard $\left(1050^{\circ} \mathrm{C}\right)+$ additional $\left(800^{\circ} \mathrm{C}\right) \mathrm{HIP}$, scan length $=78 \mathrm{~mm}$.............. 26

4.9. Comparison between different conditions (non-supported specimens) ………........ 27

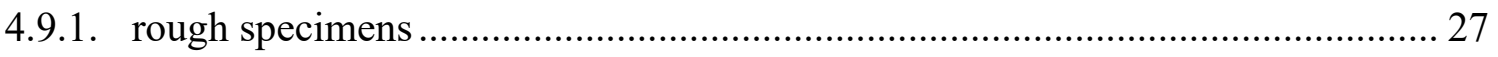

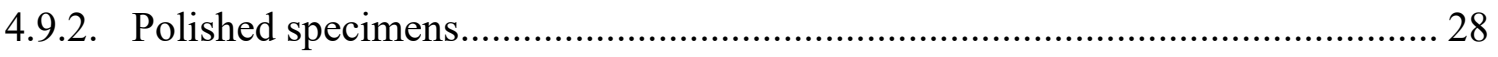

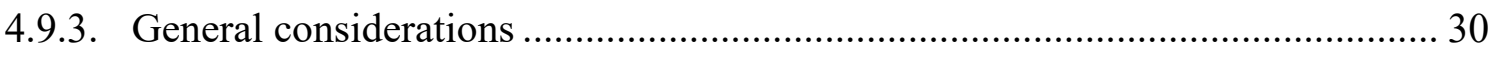

5. Correlations with tensile properties.................................................................................. 31

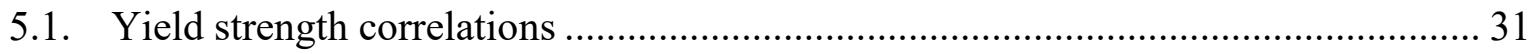

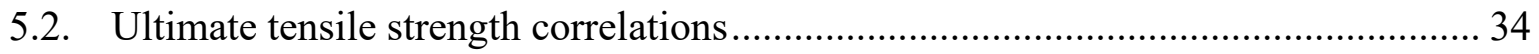

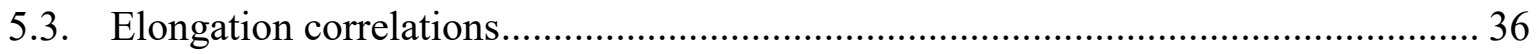

6. Correlations with fracture toughness .........................................................................39

7. Use of actuator displacement in the analyses .................................................................. 40

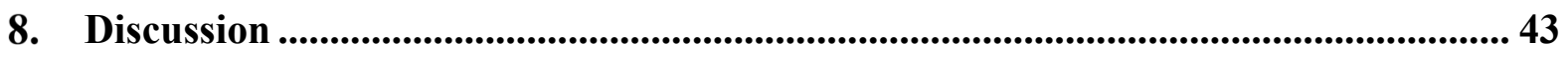

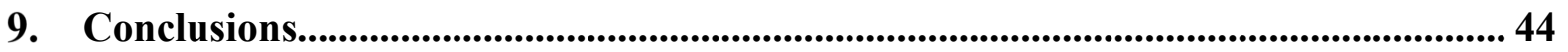

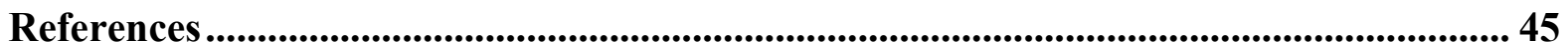

\section{List of Figures}

Figure 1 - Schematic representation of the SP test method. ……….................................... 12

Figure 2 - Typical form of a SP force-deflection diagram for steel, showing five distinct

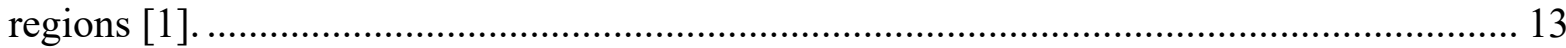
Figure 3 - SP testing fixture used at NIST, shown disassembled (left) and assembled (right).

Figure 4 - SP testing fixture mounted on the test machine with the extensometer for punch displacement measurement .......................................................................................... 15

Figure 5 - Dimensions and tolerances of the SP specimens. ............................................ 16 
Figure 6 - Force-punch displacement curve for a SP test on AM Ti64 (as-built, scan length = $78 \mathrm{~mm})$.

Figure 7 - Determination of two alternative values for the elastic-plastic transition force, $F_{\mathrm{e}, \text { proj }}$ and $F_{\mathrm{e} \text {,int. }}$. The green and red lines are the two linear fits, while the blue squares are experimental force-displacement points.

Figure 8 - Values of elastic-plastic transition forces and maximum forces as a function of estimated maximum strain rate.

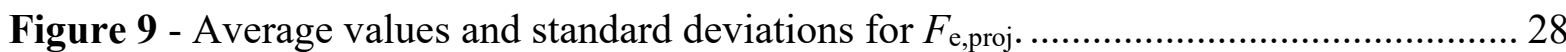

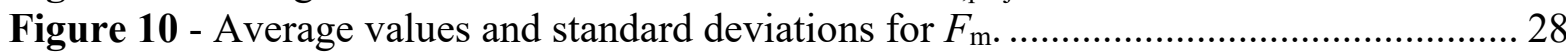

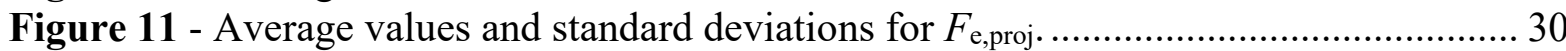

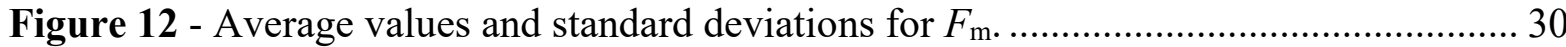

Figure 13 - Correlations between $F_{\text {e,proj }} / h_{0}^{2}$ and yield strength for rough and polished specimens of AM Ti64

Figure 14 - Correlations between $F_{\mathrm{e}, \text { int }} / h_{0}^{2}$ and yield strength for rough and polished specimens of AM Ti64 33

Figure 15 - Correlations between $F_{0.1 \mathrm{~mm}, \mathrm{off}} / h_{0}^{2}$ and yield strength for rough and polished specimens of AM Ti64

Figure 16 - Correlations between $F_{\mathrm{m}} / h_{0}{ }^{2}$ and ultimate tensile strength for rough and polished specimens of AM Ti64 35

Figure 17 - Correlations between $F_{\mathrm{m}} /\left(h_{0} u_{\mathrm{m}}\right)$ and ultimate tensile strength for rough and polished specimens of AM Ti64.

Figure 18 - Correlations between $F_{\text {infl }} /\left(h_{0} u_{\mathrm{m}}\right)$ and ultimate tensile strength for rough and polished specimens of AM Ti64.

Figure 19 - Correlations between $F_{0.65 \mathrm{~mm}} / h_{0}^{2}$ and ultimate tensile strength for rough and polished specimens of AM Ti64. 36

Figure 20 - Correlations between $\left(u_{\mathrm{f}}-h_{0}\right)$ and total elongation for rough and polished specimens of AM Ti64

Figure 21 - Correlations between $u_{\mathrm{m}} / h_{0}$ and uniform elongation for rough and polished specimens of AM Ti64 38

Figure 22 - Correlations between $F_{\mathrm{m}} / h_{0}^{2}$ and ultimate tensile strength for rough and polished specimens of AM Ti64. 39

Figure 23 - Empirical correlations between $J_{\mathrm{Q}}$ and $E_{\mathrm{SP}} /\left(h_{0} u_{\mathrm{f}}\right)$ for rough and polished SP specimens of AM Ti64

Figure 24 - Mean characteristic force values obtained for rough specimens using extensometer and actuator displacements, with $\pm 1 \sigma$ error bands.

Figure 25 - Mean characteristic displacement values obtained for rough specimens using extensometer and actuator displacements, with $\pm 1 \sigma$ error bands.

Figure 26 - Mean characteristic energy values obtained for rough specimens using extensometer and actuator displacements, with $\pm 1 \sigma$ error bands. 42

Figure 25 - Force-displacement curve for a SP test on ODS 14YWT steel [37] 44

\section{List of Tables}

Table 1 - Test matrix (for material condition codes, see bullet list in Section 3 above)....... 17

Table 2 - Tensile properties for the different material conditions. 
Table 3 - Test results for supported specimens with standard HIP and scan length $=56 \mathrm{~mm}$ (linear elastic slopes and forces).......................................................................... 20

Table 4 - Test results for supported specimens with standard HIP and scan length $=56 \mathrm{~mm}$ (displacements and energies).

Table 5 - Test results for rough as-built specimens with scan length $=78 \mathrm{~mm}$ (linear elastic slopes and forces).

Table 6 - Test results for rough as-built specimens with scan length $=78 \mathrm{~mm}$ (displacements and energies).

Table 7 - Test results for polished as-built specimens with scan length $=78 \mathrm{~mm}$ (linear elastic slopes and forces).

Table 8 - Test results for polished as-built specimens with scan length $=78 \mathrm{~mm}$

(displacements and energies).

Table 9 - Test results for rough as-built specimens with scan length $=26 \mathrm{~mm}$ (linear elastic slopes and forces).

Table 10 - Test results for rough as-built specimens with scan length $=26 \mathrm{~mm}$ (displacements and energies).

Table 11 - Test results for polished as-built specimens with scan length $=26 \mathrm{~mm}$ (linear elastic slopes and forces).

Table 12 - Test results for polished as-built specimens with scan length $=26 \mathrm{~mm}$ (displacements and energies).

Table 13 - Test results for rough specimens with non-standard HIP and scan length $=26 \mathrm{~mm}$ (linear elastic slopes and forces).

Table 14 - Test results for rough specimens with non-standard HIP and scan length $=26 \mathrm{~mm}$ (displacements and energies).

Table 15 - Test results for polished specimens with non-standard HIP and scan length $=26$ $\mathrm{mm}$ (linear elastic slopes and forces).

Table 16 - Test results for polished specimens with non-standard HIP and scan length = 26 $\mathrm{mm}$ (displacements and energies).

Table 17 - Test results for rough specimens with standard HIP and scan length $=78 \mathrm{~mm}$ (linear elastic slopes and forces).

Table 18 - Test results for rough specimens with standard HIP and scan length $=78 \mathrm{~mm}$ (displacements and energies).

Table 19 - Test results for polished specimens with standard HIP and scan length $=78 \mathrm{~mm}$ (linear elastic slopes and forces).

Table 20 - Test results for polished specimens with standard HIP and scan length $=78 \mathrm{~mm}$ (displacements and energies).

Table 21 - Test results for rough specimens with standard HIP and scan length $=26 \mathrm{~mm}$ (linear elastic slopes and forces).

Table 22 - Test results for rough specimens with standard HIP and scan length $=26 \mathrm{~mm}$ (displacements and energies).

Table 23 - Test results for polished specimens with standard HIP and scan length $=26 \mathrm{~mm}$ (linear elastic slopes and forces).

Table 24 - Test results for polished specimens with standard HIP and scan length $=26 \mathrm{~mm}$ (displacements and energies).

Table 25 - Test results for rough specimens with standard + additional HIP and scan length = $78 \mathrm{~mm}$ (linear elastic slopes and forces)....... 26 
Table 26 - Test results for rough specimens with standard + additional HIP and scan length =

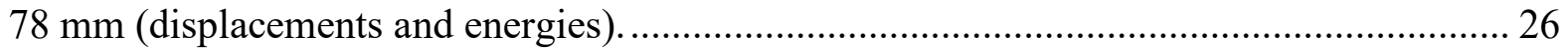

Table 27 - Test results for polished specimens with standard + additional HIP and scan

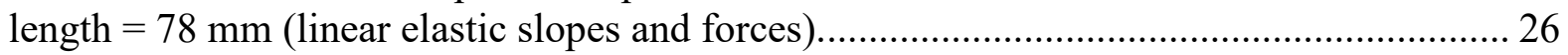

Table 28 - Test results for polished specimens with standard + additional HIP and scan

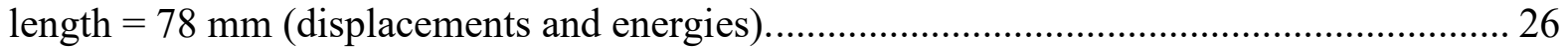

Table 29 - Average values and standard deviations for rough specimens in the different conditions (elastic slopes and forces).

Table 30 - Average values and standard deviations for rough specimens in the different conditions (displacements and energies).

Table 31 - Average values and standard deviations for polished specimens in the different conditions (elastic slopes and forces).

Table 32 - Average values and standard deviations for polished specimens in the different conditions (displacements and energies).

Table 33 - Summary of yield strength correlations obtained for rough specimens of AM Ti64.

Table 34 - Summary of yield strength correlations obtained for polished specimens of AM Ti64

Table 35 - Summary of ultimate tensile strength correlations obtained for rough specimens of AM Ti64.

Table 36 - Summary of ultimate tensile strength correlations obtained for polished specimens of AM Ti64.

Table 37 - Summary of total elongation correlations obtained for rough specimens of AM

Ti64

Table 38 - Summary of total elongation correlations obtained for polished specimens of AM

Ti64.

Table 39 - Uniform elongation correlation obtained for rough specimens of AM Ti64....... 38

Table 40 - Uniform elongation correlation obtained for polished specimens of AM Ti64. .. 38

Table 41 - Elastic-plastic fracture toughness measured on four AM Ti64 conditions [14]... 39

Table 42 - Correlation coefficients for extensometer-based and actuator-based correlations for rough specimens. In the last column, red arrows indicate a decrease in $r$ going from extensometer to actuator (two red arrows = substantial decrease), green arrows an increase, and black horizontal arrows a minor variation.

\section{List of Annexes}

ANNEX 1 SP Test Results for Standard HIP, scan length $=56 \mathrm{~mm}$, supported (rough specimens)

ANNEX 2 SP Test Results for As built, scan length $=78 \mathrm{~mm}$, non-supported (rough specimens)

ANNEX 3 SP Test Results for As built, scan length $=78 \mathrm{~mm}$, non-supported (polished specimens)

ANNEX 4 SP Test Results for As built, scan length $=26 \mathrm{~mm}$, non-supported (rough specimens) 
ANNEX 4 SP Test Results for As built, scan length $=26 \mathrm{~mm}$, non-supported (rough specimens)

ANNEX 5 SP Test Results for As built, scan length $=26 \mathrm{~mm}$, non-supported (polished specimens)

ANNEX 6 SP Test Results for $800{ }^{\circ} \mathrm{C} \mathrm{HIP}$, scan length $=78 \mathrm{~mm}$, non-supported (rough specimens)

ANNEX 7 SP Test Results for $800{ }^{\circ} \mathrm{C}$ HIP, scan length $=78 \mathrm{~mm}$, non-supported (polished specimens)

ANNEX 8 SP Test Results for $900{ }^{\circ} \mathrm{C} \mathrm{HIP}$, scan length $=78 \mathrm{~mm}$, non-supported (rough specimens)

ANNEX 9 SP Test Results for $900{ }^{\circ} \mathrm{C}$ HIP, scan length $=78 \mathrm{~mm}$, non-supported (polished specimens)

ANNEX 10 SP Test Results for $900{ }^{\circ} \mathrm{C} \mathrm{HIP}$, scan length $=26 \mathrm{~mm}$, non-supported (rough specimens)

ANNEX 11 SP Test Results for $900{ }^{\circ} \mathrm{C} \mathrm{HIP}$, scan length $=26 \mathrm{~mm}$, non-supported (polished specimens)

ANNEX 12 SP Test Results for $1050{ }^{\circ} \mathrm{C}+800{ }^{\circ} \mathrm{C}$ HIP, scan length $=78 \mathrm{~mm}$, non-supported (rough specimens)

ANNEX 13 SP Test Results for $1050{ }^{\circ} \mathrm{C}+800^{\circ} \mathrm{C}$ HIP, scan length $=78 \mathrm{~mm}$, non-supported (polished specimens)

ANNEX 14 Correlations obtained for AM Ti64 between yield strength and SP parameters

ANNEX 15 Correlations obtained for AM Ti64 between ultimate tensile strength and SP parameters

ANNEX 16 Correlations obtained for AM Ti64 between total/uniform elongation and SP parameters 


\section{Glossary}

\begin{tabular}{|c|c|}
\hline $\mathrm{AM}$ & Additive Manufacturing / Additively Manufactured \\
\hline ASTM & American Society for Testing and Materials \\
\hline CEN & European Committee for Standardization \\
\hline EBM & Electron-Beam Melting, aka Electron-Beam Additive Manufacturing \\
\hline EDM & Electro-Discharge Machining \\
\hline$D$ & In SP testing, specimen diameter (mm) \\
\hline $\mathrm{d} v / \mathrm{d} t$ & In SP testing, punch displacement rate $(\mathrm{mm} / \mathrm{s})$ \\
\hline$E_{\mathrm{m}}$ & In SP testing, total energy calculated up to $u_{\mathrm{m}}(\mathrm{J})$ \\
\hline$E_{\mathrm{PL}}$ & In SP testing, plastic energy calculated up to $u_{\mathrm{m}}(\mathrm{J})$ \\
\hline$E_{\mathrm{SP}}$ & In SP testing, fracture energy calculated up to $u_{\mathrm{f}}(\mathrm{J})$ \\
\hline$\varepsilon_{\mathrm{f}}$ & In SP testing, effective fracture strain \\
\hline$\dot{\varepsilon}_{S P}^{\max }$ & In SP testing, estimated maximum strain rate $(1 / \mathrm{s})$ \\
\hline$\varepsilon_{\mathrm{t}}$ & In tensile testing, total elongation $(\%)$ \\
\hline$\varepsilon_{\mathrm{u}}$ & In tensile testing, uniform elongation $(\%)$ \\
\hline$F$ & Force $(\mathrm{N})$ \\
\hline$F_{\mathrm{e}}$ & In SP testing, elastic-plastic transition force $(\mathrm{N})$ \\
\hline$F_{\mathrm{h} 0 / 10, \text { off }}$ & $\begin{array}{l}\text { In SP testing, force at the intersection between the test record and a line parallel to } \\
\text { the slope of the initial linear region with an offset of } 0.1 \cdot h_{0}(\mathrm{~N})\end{array}$ \\
\hline$F_{\text {infl }}$ & In SP testing, force at the inflection point of the curve $\left(\mathrm{d}^{2} F / \mathrm{d} u^{2}=0\right)(\mathrm{N})$ \\
\hline$F_{\mathrm{m}}$ & In $\mathrm{SP}$ testing, maximum force $(\mathrm{N})$ \\
\hline$F_{0.1 \mathrm{~mm}, \mathrm{off}}$ & $\begin{array}{l}\text { In SP testing, force at the intersection between the test record and a line parallel to } \\
\text { the slope of the initial linear region with an offset of } 0.1 \mathrm{~mm}(\mathrm{~N})\end{array}$ \\
\hline$F_{0.1 \mathrm{~mm}}$ & In SP testing, force corresponding to a displacement value of $0.1 \mathrm{~mm}(\mathrm{~N})$ \\
\hline$F_{0.48 \mathrm{~mm}}$ & In SP testing, force corresponding to a displacement value of $0.48 \mathrm{~mm}(\mathrm{~N})$ \\
\hline$F_{0.5 \mathrm{~mm}}$ & In SP testing, force corresponding to a displacement value of $0.5 \mathrm{~mm}(\mathrm{~N})$ \\
\hline$F_{0.645 \mathrm{~mm}}$ & In SP testing, force corresponding to a displacement value of $0.645 \mathrm{~mm}(\mathrm{~N})$ \\
\hline$F_{0.65 \mathrm{~mm}}$ & In SP testing, force corresponding to a displacement value of $0.65 \mathrm{~mm}(\mathrm{~N})$ \\
\hline$F_{0.9 \mathrm{~mm}}$ & In SP testing, force corresponding to a displacement value of $0.9 \mathrm{~mm}(\mathrm{~N})$ \\
\hline$f(v)$ & In SP testing, bilinear function used to determine $F_{\mathrm{e}}[10,11]$. \\
\hline$h_{0}$ & In SP testing, initial specimen thickness (mm) \\
\hline ISO & International Standardization Organization \\
\hline$K$ & In SP testing, curvature parameter according to [30] \\
\hline$K V$ & Charpy absorbed energy $(\mathrm{J})$ \\
\hline$r$ & Pearson correlation coefficient \\
\hline$R a$ & Surface roughness $(\mu \mathrm{m})$ \\
\hline$R_{\mathrm{m}}$ & In tensile testing, tensile strength $(\mathrm{MPa})$ \\
\hline
\end{tabular}


$R_{\mathrm{p} 02} \quad$ In tensile testing, yield strength (MPa)

Slope $_{\text {ini }} \quad$ In SP testing, slope of the initial elastic region of the curve $(\mathrm{N} / \mathrm{mm})$

SP Small Punch

$t_{\text {calc }} \quad$ Calculated value of the $t$-test statistic

$t_{\text {critical }} \quad$ Critical value of the $t$-test statistic (if $t_{\text {calc }}>t_{\text {critical }}$, means are statistically different)

$u \quad$ Specimen deflection $(\mathrm{mm})$

$v \quad$ Punch displacement $(\mathrm{mm})$

$v_{\mathrm{f}} \quad$ In SP testing, punch displacement corresponding to a $20 \%$ force drop with respect to maximum force $(\mathrm{mm})$

$v_{\mathrm{m}} \quad$ In SP testing, punch displacement at maximum force $(\mathrm{mm})$

$v_{1 \mathrm{p}} \quad$ In SP testing, punch displacement at the occurrence of the first significant pop-in $(\mathrm{mm})$. 


\section{Introduction}

In the field of experimental techniques based on sub-size or miniaturized specimens, destructive testing of very small disk-shaped specimens allows characterizing the mechanical properties of service-exposed plant components or structures with a minimal amount of material extracted from the component [1]. Moreover, a considerable number of disk specimens can be extracted from machining leftovers or already tested conventional specimens.

The Small Punch (SP) test, also known as the Disk Bend test, was developed in the mid-1980s [2,3] through the use of tiny disks of $3 \mathrm{~mm}$ diameter and $0.25 \mathrm{~mm}$ thickness, centrally loaded by a spherical ball or hemispherical punch, and expanded into a larger lower die. The test system was a module that could be placed between the loading platens of a tensile machine and subsequently loaded in compression [3]. The outcome is a bulge in the disk rather than a shear cut, as in a similar methodology called the Shear Punch test [4]. Although disks of these dimensions are still used for SP testing, nowadays the most popular specimen geometry is a round disk with a diameter of $8 \mathrm{~mm}$ and a thickness of $0.5 \mathrm{~mm}$, which is the geometry used in this study. The use of square specimens $(10 \mathrm{~mm} \times 10 \mathrm{~mm})$ has also been reported [5] but is not as common.

A schematic representation of the SP test method is shown in Fig. 1.
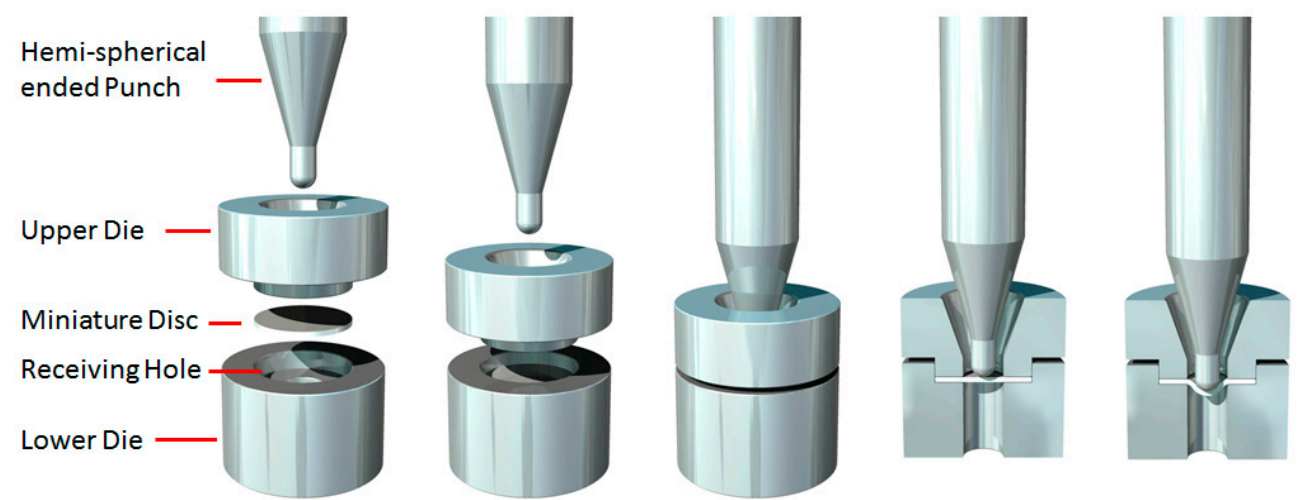

Figure 1 - Schematic representation of the SP test method.

The general form of a SP force/deflection test record for a steel specimen is shown in Fig. 2 [1]. Five distinct regions can be identified:

1. Elastic region,

2. Departure from linearity (elastic-plastic transition),

3. Local bending, transitioning to a membrane stress regime,

4. Membrane stress regime, and

5. Final failure region.

The general form of the test record suggests that yield stress may be associated with the change in slope between regions 1 and 2, while the ultimate tensile stress may be related to the maximum force, and ductility to maximum deflection. However, in the case of steels showing low ductility, the membrane stress regime and final failure region may be virtually absent or minimized. 


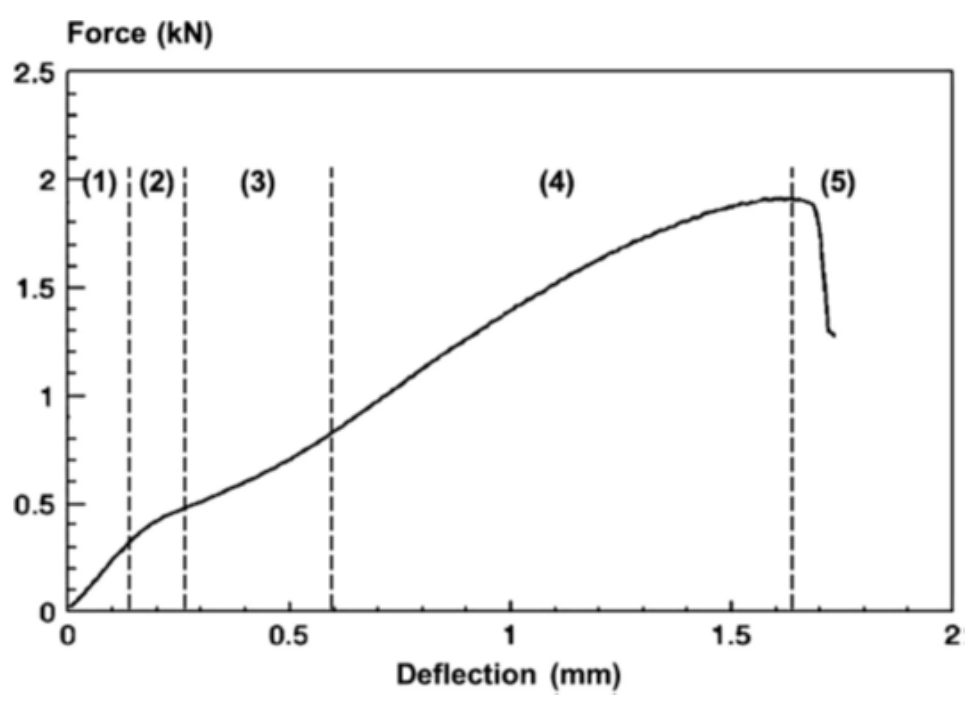

Figure 2 - Typical form of a SP force-deflection diagram for steel, showing five distinct regions [1].

Characteristic values of force, displacement, and energy (calculated by integrating force and displacement) are identified on the test record. These values are generally fed into empirical relationships to obtain estimates of specific mechanical parameters, such as tensile properties, ductile-to-brittle transition temperature, fracture toughness, for the material under investigation. Numerous empirical correlations are available in the literature, and have been developed by comparing characteristic parameters from SP tests with tensile properties, transition temperature data, and fracture toughness values measured by means of conventional tests.

In most cases, correlations appear to be strongly dependent on the material (or the class of material) under investigation, and cannot be expected to be applicable to other materials or material conditions [5].

However, alternative approaches of a more analytical nature have also been proposed. Several authors have matched force-displacement curves from SP tests, up to the point of observed crack initiation, to a database of curves corresponding to a range of stress-strain constitutive behaviors. The model used in this case is a Ramberg-Osgood model with a possible modification to accommodate the discontinuous yield observed in several low-alloy steels [6]. Other analytical methods have also been proposed, involving the use of Neural Networks and Finite Element simulations [7-9]. The approach used in this report for the analysis of SP test results, however, is strictly of a correlative nature.

Even though researchers all over the world have been performing SP tests since the 1980s, an official test standard issued by an internationally recognized standardization body (ASTM or ISO) has yet to be developed.

The currently available document that most closely resembles a test standard is a European CEN ${ }^{1}$ Workshop Agreement, CWA 15627 (Small Punch Test Method for Metallic Materials), issued in 2007 [10]. At the time of writing, a Draft ASTM Test Method for Small Punch Testing of Metallic Materials [11], modeled after CWA 15627, is being developed inside the ASTM E10.02 Sub-Committee (Behavior and Use of Nuclear Materials), and is in the final balloting stages.

${ }^{1}$ CEN: Comité Européen de Normalisation (European Committee for Standardization). 
The development of SP testing at NIST in Boulder, $\mathrm{CO}$, and the validation of the relevant experimental and analytical procedures was covered in a recently published NIST Internal Report [12]. In this report, we present the results of SP testing conducted with the objective of deriving estimates of mechanical properties (tensile and fracture toughness) for additively manufactured (AM) Ti-6Al-4V in various conditions. Previously published investigations focused on the measurement of Charpy impact toughness [13] and room temperature elastic-plastic fracture toughness [14] of the same material under various manufacturing conditions.

Additive Manufacturing (AM), previously referred to as $3 \mathrm{D}$ printing, is a process in which material is joined or solidified under computer control to create a threedimensional object, with material being fused (such as liquid molecules or powder grains being fused together), typically layer by layer. In the 1990s, 3D printing was considered only suitable to produce functional or aesthetical prototypes. Nowadays, the precision, repeatability, and material range have increased to the point that $3 \mathrm{D}$ printing, or $\mathrm{AM}$, is considered an industrial production technology. twofold:

The objective of the NIST Additive Manufacturing Fatigue and Fracture Project is

- Develop appropriate measurement science for fatigue and fracture behavior of additively manufactured metals, to underpin a rapid qualification framework.

- Determine the effect of processing (including post-processing) and structure (e.g. internal defects, external defects, residual stress, crystallographic texture, grain size, and chemistry) on fatigue and fracture properties of additively manufactured metals.

Ti-6Al-4V (hereinafter referred to as Ti64) is the most widely used titanium alloy, featuring good machinability and excellent mechanical properties. It offers the best all-round performance for a variety of weight reduction applications in aerospace, automotive, and marine equipment. Its high strength, low weight, and outstanding corrosion resistance has led to a wide range of successful applications that demand high levels of reliable performance in surgery and medicine, aerospace, automotive, chemical plants, power generation, oil and gas extraction, sports, and other major industries.

\section{Experimental setup for SP testing}

The fixture developed at NIST for SP testing, following the recommendations in refs. $[10,11]$, consists of an upper and a lower die, a rod (100 mm long, $2.5 \mathrm{~mm}$ diameter), and a ball (2.5 mm diameter). The combination of the rod and ball constitute the punch, which is driven through the specimen, held between the upper and lower dies. The fixture is shown in Fig. 3 in both disassembled (left) and assembled (right) form.

The fixture was mounted on a universal electro-mechanical test machine, equipped with a $5 \mathrm{kN}$ capacity load cell and an extensometer ${ }^{2}$. The extensometer was attached to one of the columns of the machine in order to measure the relative displacement between the machine actuator and the machine frame, in close proximity to the punch. Fig. 4 shows the fixture mounted on the test machine and the positioning of the extensometer with respect to the

${ }^{2}$ Load cell, machine actuator, and extensometer are regularly calibrated in accordance with ASTM E4 and E83. Based on calibrations current at the time of testing, maximum errors were found to be less than $0.7 \%$ for force values, less than $1.5 \%$ for actuator displacements, and less than $2 \%$ for extensometer displacement values. 
machine actuator. Unlike the extensometer signal, actuator displacement includes the compliance of the whole test system.

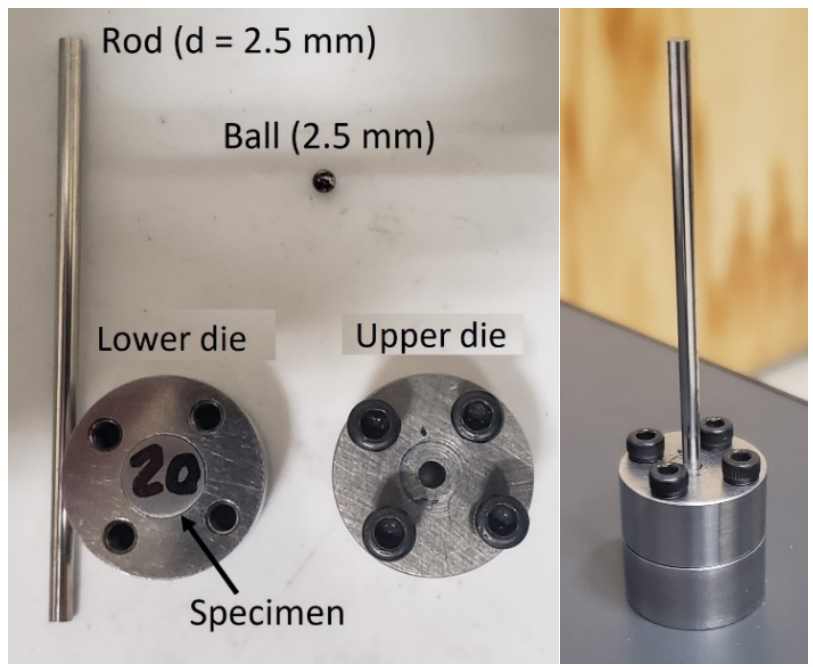

Figure 3 - SP testing fixture used at NIST, shown disassembled (left) and assembled (right).

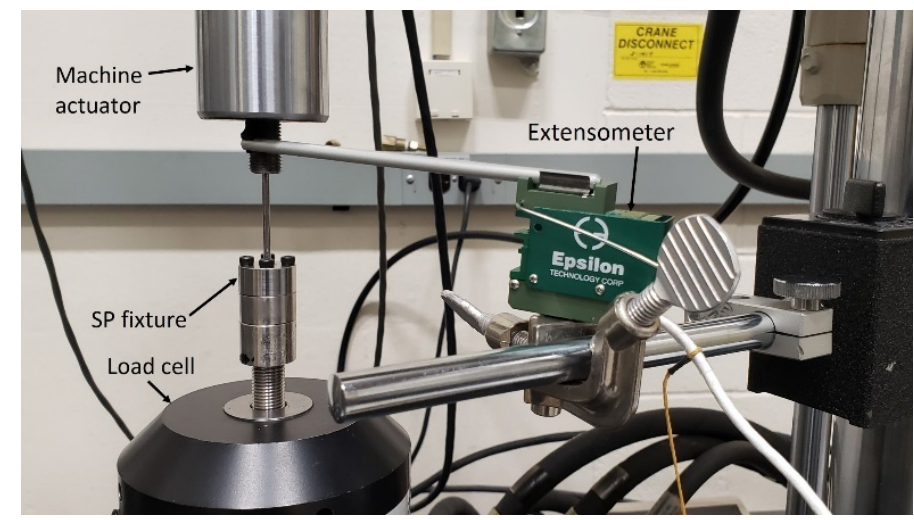

Figure 4 - SP testing fixture mounted on the test machine with the extensometer for punch displacement measurement.

All tests (except for a preliminary series described in Sec. 4.2) were performed at room temperature $\left(21^{\circ} \mathrm{C} \pm 2{ }^{\circ} \mathrm{C}\right)$ in actuator displacement control, at rates between $0.001 \mathrm{~mm} / \mathrm{s}$ and $0.003 \mathrm{~mm} / \mathrm{s}$. Force, actuator displacement, and punch displacement (extensometer) data were recorded at a sampling frequency of $1 \mathrm{~Hz}$. To account for the compliance of the test system on punch displacement, actuator and extensometer displacements were recorded without a specimen in place, and then subtracted from displacements measured during the tests. ${ }^{3}$

\footnotetext{
${ }^{3}$ In a few SP tests, punch displacement is larger than actuator displacement, contrary to expectations. The reason for this is unclear, and the most likely explanation is related to the different positioning of the extensometer from one test to the other. The use of a clip-on-gage, always located in the same spot, instead of an extensometer should improve the consistency of the displacement signals in future tests.
} 


\section{Material and conditions investigated}

The AM Ti64 parts used in this study were fabricated using an EBM powder bed fusion Arcam $^{4}$ A1 machine (software version 3.2.132,60 kV, $50 \mu \mathrm{m}$ layer thickness) and standard Arcam Ti64 gas-atomized powder (70 $\mu \mathrm{m}$ average diameter).

The following conditions (AM processing parameters), for which conventional tensile and fracture toughness test results had been previously measured [14,15], were investigated:

a. As-built condition (i.e., no heat treatment).

b. Non-standard Ti64 HIP $\left(800{ }^{\circ} \mathrm{C}, 100 \mathrm{MPa}, 2 \mathrm{~h}\right.$, Ar environment, standard heating and cooling rates ${ }^{5}$ ).

c. Standard Ti64 HIP $\left(900^{\circ} \mathrm{C}, 100 \mathrm{MPa}, 2 \mathrm{~h}\right.$, Ar environment, standard heating and cooling rates $\left.^{4}\right)$.

d. Non-standard Ti64 HIP $\left(1050{ }^{\circ} \mathrm{C}, 100 \mathrm{MPa}, 2 \mathrm{~h}\right.$, rapid cooling ${ }^{6}$ in Ar) with an additional HIP $\left(800{ }^{\circ} \mathrm{C}, 30 \mathrm{MPa}, 2 \mathrm{~h}\right.$, slow cooling ${ }^{4}$ in $\mathrm{Ar}-$ meant for martensite tempering).

e. Scan lengths ${ }^{7}$ of:

$\mathrm{e} 1.78 \mathrm{~mm}$ and

e2. $26 \mathrm{~mm}$.

In order to assess the applicability of SP testing to AM Ti64, a preliminary series of 10 tests was performed on supported ${ }^{8}$ specimens, all in standard HIP condition $\left(900{ }^{\circ} \mathrm{C}\right)$, corresponding to a different scan length $(52 \mathrm{~mm})$. An additional objective of these preliminary tests was to investigate the influence of different actuator displacement rates on the test results.

All SP disks were machined from AM Ti64 blocks by electrical discharge machining $(\mathrm{EDM})$ in accordance with the drawing in Fig. 5.

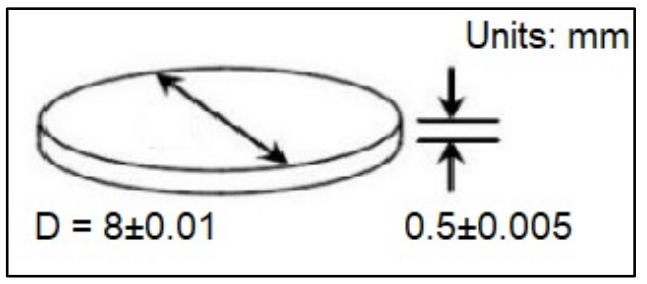

Figure 5 - Dimensions and tolerances of the SP specimens.

After machining, some of the specimens were polished to the surface finish required by the ASTM Draft [10], Ra $\leq 0.25 \mu \mathrm{m}$, by means of abrasive paper with an abrasive grit size designation P400 followed by fine grinding (P1200). As a result, polished disks had a thickness ranging from $0.43 \mathrm{~mm}$ to $0.48 \mathrm{~mm}$. The rest of the specimens (rough disks) had surface roughness in the range $\mathrm{Ra}=3 \mu \mathrm{m}$ to $4 \mu \mathrm{m}$. This allowed us to investigate the influence of surface finish on SP test results.

\footnotetext{
${ }^{4}$ Certain commercial software, equipment, instruments or materials are identified in this paper to adequately specify the experimental procedure. Such identification is not intended to imply recommendation or endorsement by the National Institute of Standards and Technology, nor is it intended to imply that the equipment or materials identified are necessarily the best available for the purpose.

${ }^{5} 12{ }^{\circ} \mathrm{C} /$ minute.

${ }^{6} 1600{ }^{\circ} \mathrm{C} /$ minute.

${ }^{7}$ Scan length is a manufacturer-specific parameter that corresponds to the distance the electron beam travels on a single track before turning around to begin the next track. It has been shown to determine energy density and affect texture [16].

${ }^{8}$ Specimens directly attached to the build plate were identified as "non-supported", while "supported" specimens (such as the ones tested in the preliminary phase) were connected to the build plate by means of standard thin wafer supports.
} 
The overall test matrix, excluding the preliminary study, is presented in Table 1 . The total number of tests performed was 55 (36 on rough specimens and 19 on polished specimens). All specimens were extracted from non-supported parts, and were tested at room temperature $\left(21^{\circ} \mathrm{C} \pm 2{ }^{\circ} \mathrm{C}\right)$.

Table 1 - Test matrix (for material condition codes, see bullet list in Section 3 above).

\begin{tabular}{|c|c|c|}
\hline \multirow{2}{*}{$\begin{array}{c}\text { Material } \\
\text { condition }\end{array}$} & \multicolumn{2}{c|}{ Number of tests performed } \\
\cline { 2 - 3 } aough disks & Polished disks \\
\hline a,e1 & 6 & 3 \\
\hline a,e2 & 6 & 3 \\
\hline b,e1 & 6 & 4 \\
\hline c,e1 & 6 & 2 \\
\hline c,e2 & 6 & 4 \\
\hline d,e1 & 6 & 3 \\
\hline
\end{tabular}

The main tensile properties for the different conditions investigated, obtained from conventional tensile tests [15] and used for the correlations with SP test results, are listed in Table 2.

Table 2 - Tensile properties for the different material conditions.

\begin{tabular}{|c|c|c|c|c|}
\hline $\begin{array}{l}\text { Material } \\
\text { condition }\end{array}$ & $\begin{array}{c}\mathbf{R}_{\mathrm{p} 02} \\
(\mathrm{MPa})\end{array}$ & $\begin{array}{c}\mathbf{R}_{\mathbf{m}} \\
(\mathbf{M P a})\end{array}$ & $\begin{array}{c}\mathcal{E}_{\mathrm{u}} \\
(\%)\end{array}$ & $\begin{array}{c}8 \mathrm{f} \\
(\%)\end{array}$ \\
\hline a,e1 & 879 & 981 & 9.5 & 27.8 \\
\hline $\mathrm{a}, \mathrm{e} 2$ & 875 & 972 & 9.1 & 25.5 \\
\hline b,e1 & 864 & 969 & 10.1 & 31.7 \\
\hline c,e1 & 838 & 951 & 10.0 & 31.0 \\
\hline c,e2 & 799 & 918 & 10.0 & 31.8 \\
\hline d,e1 & 885 & 985 & 7.9 & 18.8 \\
\hline
\end{tabular}

\section{Test results}

\subsection{General remarks}

An example of force/punch displacement curve for condition $\{b, e 1\}$ is shown in Fig. 6. Depending on material condition, one or more force drops were observed before, at, and/or after maximum force.

The SP curves obtained in this study are qualitatively similar to those reported in the literature for other investigations on EBM AM Ti64 [17-20], and differ significantly from conventional SP test records for steel specimens, as in the example illustrated in Fig. 2. However, the five regions shown in Fig. 1 can still be reasonably identified, see Fig. 6, and the analysis of the test can be conducted in accordance with Refs. [10,11]. 


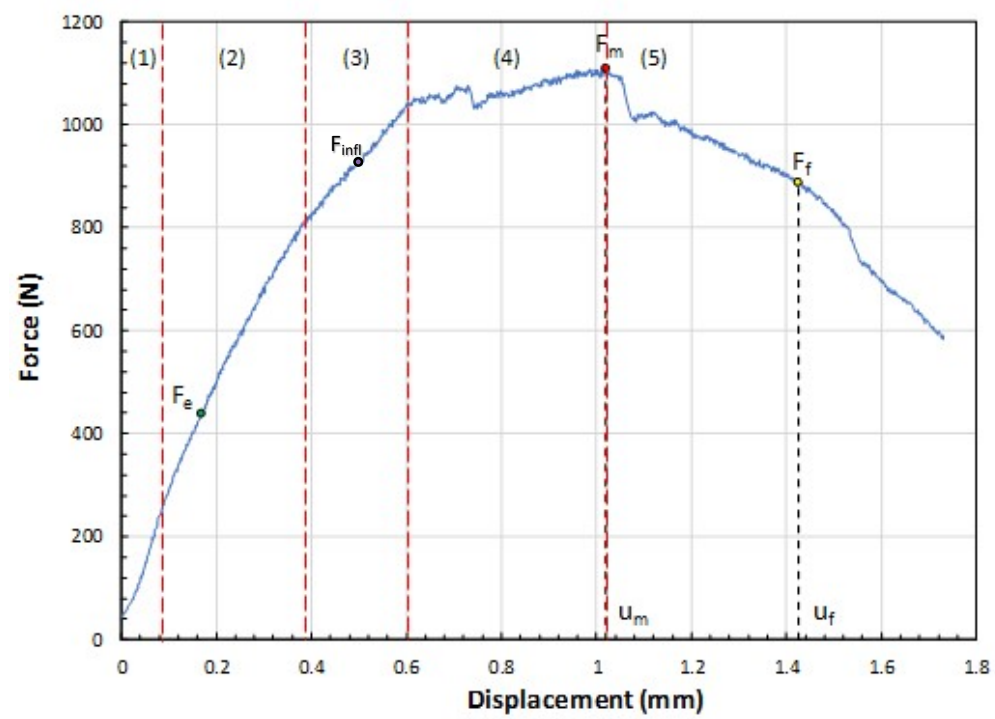

Figure 6 - Force-punch displacement curve for a SP test on AM Ti64 (as-built, scan length $=78 \mathrm{~mm})$.

From the analysis of each individual force-punch displacement curve the following force values, to be used for correlation with tensile properties, were extracted:

- $\quad$ elastic-plastic transition force, $F_{\mathrm{e}}$ (Fig. 6)

- $\quad$ maximum force, $F_{\mathrm{m}}$ (Fig. 6)

- force at the intersection between the test record and a line parallel to the slope of the initial linear region with an offset of $0.1 \cdot h_{0}, F_{\mathrm{h} 0 / 10 \text {,off }}$

- force at the inflection point of the curve $\left(\mathrm{d}^{2} F / \mathrm{d} u^{2}=0\right), F_{\text {infl }}$ (Fig. 6)

- force at the intersection between the test record and a line parallel to the slope of the initial linear region with an offset of $0.1 \mathrm{~mm}, F_{0.1 \mathrm{~mm} \text {,off }}$

- force corresponding to a displacement value of $0 . \mathrm{XXX} \mathrm{mm}, F_{0 . \mathrm{XXXmm}}$ (where $0 . \mathrm{XXX}=$ $0.48,0.645$, or 0.65 ).

Note that, in accordance with Refs. [10,11], the elastic-plastic transition force, $F_{\mathrm{e}}$, is obtained as the projection on the test record of the intersection between two linear fits. However, one of the yield strength correlations that have been proposed in the literature directly uses the force corresponding to the intersection of the regression lines. Therefore, in this investigation, the two values are labelled respectively $F_{\text {e,proj }}$ and $F_{\text {e,int }}$ (see Fig. 7 for an illustration of the two values).

The following punch displacement values, corresponding to forces bearing the same subscript, will also recorded: $u_{\mathrm{e}}, u_{\mathrm{m}}$ (Fig. 6), $u_{\mathrm{h} 0 / 10, \mathrm{off}}, u_{\mathrm{infl}}, u_{0.1 \mathrm{~mm}, \mathrm{off}}$, and $u_{\mathrm{e} 1.5}$.

Finally, three energy values were also calculated for each test:

- total energy calculated up to $u_{\mathrm{m}}, E_{\mathrm{m}}$;

- $\quad$ plastic energy calculated up to $u_{\mathrm{m}}, E_{\mathrm{SP}}$;

- fracture energy calculated up to $u_{\mathrm{f}}, E_{\mathrm{SP}}$.

Additionally, the slope of the initial elastic part of the test record, Slope $e_{\text {ini, }}$ as well as forces and displacements corresponding to test end ( $F_{\mathrm{f}}$ and $u_{\mathrm{f}}$, Fig. 6$)$, were also reported. 


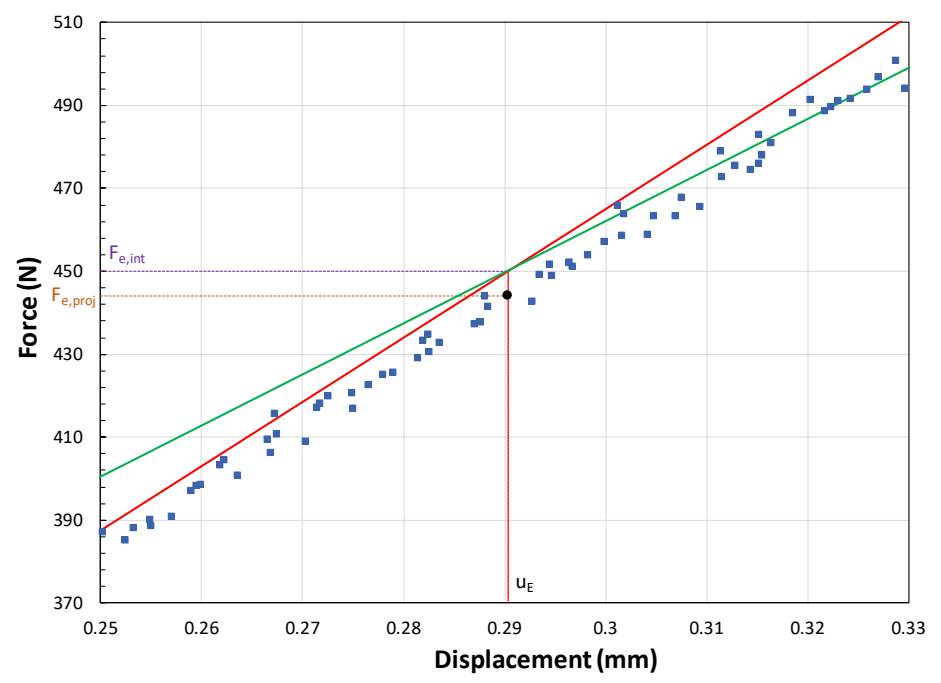

Figure 7 - Determination of two alternative values for the elastic-plastic transition force, $F_{\text {e,proj }}$ and $F_{\mathrm{e} \text {,int. }}$ The green and red lines are the two linear fits, while the blue squares are experimental force-displacement points.

All the analyses were performed by means of a macro-enabled spreadsheet, which has been described in [12]. This same report also provides specific details on the determination of the individual characteristic values of force, displacement, and energy.

\subsection{Preliminary tests (standard HIP, scan length $=56 \mathrm{~mm}$, supported)}

Ten SP specimens (all rough) were tested in the preliminary series, with test speeds (actuator displacement rates) ranging between $0.0007 \mathrm{~mm} / \mathrm{s}$ and $0.0084 \mathrm{~mm} / \mathrm{s}$. Note that the most commonly used test rate in the literature is $0.0015 \mathrm{~mm} / \mathrm{s}$. The applied displacement rates correspond to a range of maximum estimated strain rate $\dot{\varepsilon}_{\max }=7.35 \times 10^{-4} \mathrm{~s}^{-1}$ to 8.38 $\times 10^{-3} \mathrm{~s}^{-1}[10,11]$, i.e., more than an order of magnitude.

The results obtained are presented, with average values and standard deviations (absolute and relative ${ }^{9}$ ), in Table 3 (linear elastic slopes and characteristic forces) and Table 4 (characteristic displacements and energies). Data reported in the Tables show that forces and displacements corresponding to the elastic-plastic transition exhibit the largest standard deviations.

\footnotetext{
${ }^{9}$ The relative standard deviation, expressed in \%, is obtained by dividing the absolute standard deviation by the average value. This is also called coefficient of variation $(\mathrm{CV})$.
} 
Table 3 - Test results for supported specimens with standard HIP and scan length $=56 \mathrm{~mm}$ (linear elastic slopes and forces).

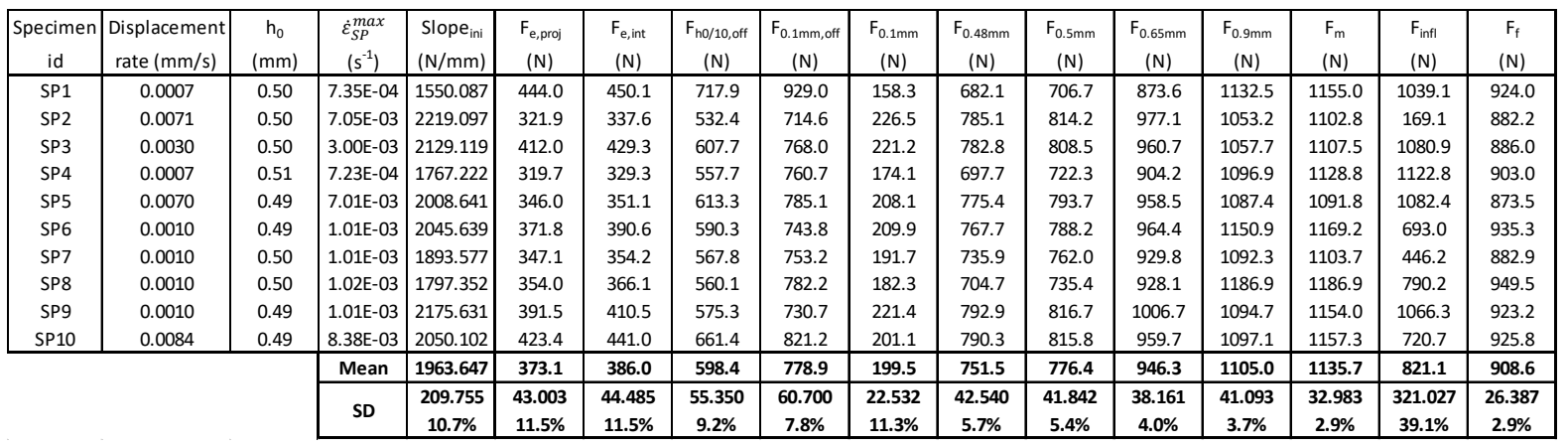

Table 4 - Test results for supported specimens with standard HIP and scan length $=56 \mathrm{~mm}$ (displacements and energies).

\begin{tabular}{|c|c|c|c|c|c|c|c|c|c|c|c|c|c|}
\hline $\begin{array}{c}\text { Specimen } \\
\text { id }\end{array}$ & $\begin{array}{c}\text { Displacement } \\
\text { rate }(\mathrm{mm} / \mathrm{s})\end{array}$ & $\begin{array}{c}\mathrm{h}_{0} \\
(\mathrm{~mm})\end{array}$ & $\begin{array}{c}\dot{\varepsilon}_{S P}^{\max } \\
\left(\mathrm{s}^{-1}\right)\end{array}$ & $\begin{array}{c}\mathrm{u}_{\mathrm{e}} \\
(\mathrm{mm})\end{array}$ & $\begin{array}{c}\mathrm{u}_{\mathrm{ho} / 10, \text { off }}(\mathrm{mm}) \\
\mathrm{u}_{0.1 \mathrm{~mm}, \text { off }} \\
(\mathrm{mm})\end{array}$ & $\begin{array}{c}\mathrm{u}_{\mathrm{e} 1.5} \\
(\mathrm{~mm})\end{array}$ & $\begin{array}{c}\mathrm{u}_{\mathrm{m}} \\
(\mathrm{mm})\end{array}$ & $\begin{array}{c}\mathrm{u}_{\text {infl }} \\
(\mathrm{mm})\end{array}$ & $\begin{array}{c}\mathrm{u}_{\mathrm{f}} \\
(\mathrm{mm})\end{array}$ & $\begin{array}{c}\mathrm{E}_{\mathrm{SP}} \\
(\mathrm{J})\end{array}$ & $\begin{array}{c}\mathrm{E}_{\mathrm{m}} \\
(\mathrm{J})\end{array}$ & $\begin{array}{c}\mathrm{E}_{\mathrm{PL}} \\
(\mathrm{J})\end{array}$ \\
\hline SP1 & 0.0007 & 0.50 & $7.35 \mathrm{E}-04$ & 0.290 & 0.513 & 0.701 & 0.074 & 1.076 & 1.300 & 1.475 & 1.18 & 0.76 & 0.33 \\
SP2 & 0.0071 & 0.50 & $7.05 \mathrm{E}-03$ & 0.152 & 0.291 & 0.423 & 0.366 & 1.053 & 2.299 & 1.500 & 1.25 & 0.79 & 0.52 \\
SP3 & 0.0030 & 0.50 & $3.00 \mathrm{E}-03$ & 0.202 & 0.336 & 0.463 & 0.042 & 1.034 & 0.991 & 1.529 & 1.28 & 0.77 & 0.48 \\
SP4 & 0.0007 & 0.51 & $7.23 \mathrm{E}-04$ & 0.186 & 0.366 & 0.532 & 0.097 & 1.102 & 0.963 & & & 0.80 & 0.44 \\
SP5 & 0.0070 & 0.49 & $7.01 \mathrm{E}-03$ & 0.175 & 0.356 & 0.491 & 0.067 & 0.886 & 0.878 & & & 0.60 & 0.30 \\
SP6 & 0.0010 & 0.49 & $1.01 \mathrm{E}-03$ & 0.191 & 0.339 & 0.464 & 0.081 & 0.881 & 1.734 & 1.505 & 1.28 & 0.61 & 0.27 \\
SP7 & 0.0010 & 0.50 & $1.01 \mathrm{E}-03$ & 0.187 & 0.351 & 0.498 & 0.086 & 0.894 & 0.257 & 1.675 & 1.38 & 0.59 & 0.27 \\
SP8 & 0.0010 & 0.50 & $1.02 \mathrm{E}-03$ & 0.204 & 0.362 & 0.535 & 0.111 & 0.899 & 1.729 & 1.478 & 1.20 & 0.59 & 0.20 \\
SP9 & 0.0010 & 0.49 & $1.01 \mathrm{E}-03$ & 0.189 & 0.315 & 0.436 & 0.086 & 1.132 & 1.428 & 1.576 & 1.37 & 0.90 & 0.59 \\
SP10 & 0.0084 & 0.49 & $8.38 \mathrm{E}-03$ & 0.215 & 0.373 & 0.502 & 0.076 & 1.121 & 0.419 & 1.560 & 1.34 & 0.87 & 0.55 \\
\hline
\end{tabular}

Test and analysis details for each of the tests performed on this group of specimens are provided in Annex 1.

The influence of estimated maximum strain rate on elastic-plastic transition forces $\left(F_{\text {e,proj }}\right)$ and maximum forces $\left(F_{\mathrm{m}}\right)$ is illustrated in Fig. 8. For both force values, the slopes of the linear fits are statistically not different from zero based on ANOVA tests, indicating that the influence of displacement rate within the investigated range $(0.0007 \mathrm{~mm} / \mathrm{s}$ to $0.0084 \mathrm{~mm} / \mathrm{s})$ is negligible.

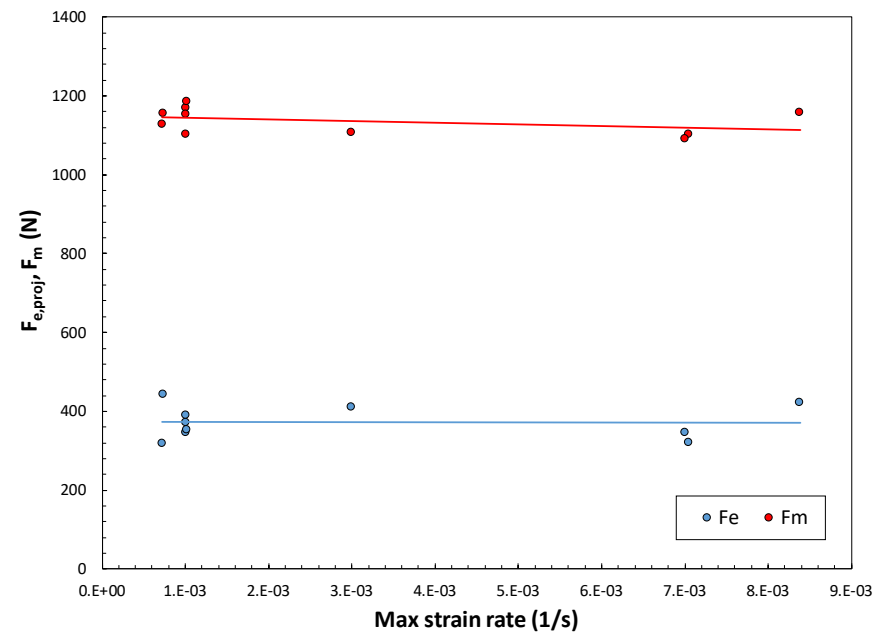

Figure 8 - Values of elastic-plastic transition forces and maximum forces as a function of estimated maximum strain rate. 


\subsection{As-built, scan length $=78 \mathrm{~mm}$}

Test results for the rough specimens is presented in Tables 5 and 6, which describe elastic slope/forces and displacements/energies, respectively. Likewise, Tables 7 and 8 describe the equivalent measurements for the polished specimens.

Table 5 - Test results for rough as-built specimens with scan length $=78 \mathrm{~mm}$ (linear elastic slopes and forces).

\begin{tabular}{|c|c|c|c|c|c|c|c|c|c|c|c|c|c|c|c|c|}
\hline $\begin{array}{c}\text { Specimen } \\
\text { id }\end{array}$ & $\begin{array}{c}\begin{array}{c}\text { Displacement } \\
\text { rate }(\mathrm{mm} / \mathrm{s})\end{array} \\
\end{array}$ & $\begin{array}{c}\mathrm{h}_{0} \\
(\mathrm{~mm})\end{array}$ & $\begin{array}{l}\dot{\varepsilon}_{S P}^{\max } \\
\left(\mathrm{s}^{-1}\right)\end{array}$ & $\begin{array}{l}\text { Slope }_{\mathrm{el}} \\
(\mathrm{N} / \mathrm{mm}) \\
\end{array}$ & $\begin{array}{l}F_{e, p r o j} \\
(N)\end{array}$ & $\begin{array}{l}F_{e, i n t} \\
(N)\end{array}$ & $\begin{array}{l}\mathrm{F}_{\mathrm{h} 0 / 10, \text { off }} \\
\text { (N) }\end{array}$ & $\begin{array}{c}F_{0.1 \mathrm{~mm}, \text { off }} \\
(\mathrm{N})\end{array}$ & $\begin{array}{l}\mathrm{F}_{0.1 \mathrm{~mm}} \\
(\mathrm{~N})\end{array}$ & $\begin{array}{l}\mathrm{F}_{0.48 \mathrm{~mm}} \\
(\mathrm{~N})\end{array}$ & $\begin{array}{l}\mathrm{F}_{0.5 \mathrm{~mm}} \\
(\mathrm{~N})\end{array}$ & $\begin{array}{l}\mathrm{F}_{0.65 \mathrm{~mm}} \\
(\mathrm{~N})\end{array}$ & $\begin{array}{l}\mathrm{F}_{0.9 \mathrm{~mm}} \\
(\mathrm{~N})\end{array}$ & $\begin{array}{l}F_{m} \\
(N)\end{array}$ & $\begin{array}{l}F_{\text {infl }} \\
(\mathrm{N}) \\
\end{array}$ & $\begin{array}{l}F_{f} \\
(N)\end{array}$ \\
\hline SP1 & 0.0009 & 0.51 & 9.34E-04 & 2098.05 & 352.6 & 361.0 & 621.7 & 808.2 & 216.0 & 807.1 & 831.2 & 995.7 & 1057.2 & 1070.8 & 645.2 & 856.7 \\
\hline SP2 & 0.0010 & 0.50 & 9.27E-04 & 2693.16 & 394.4 & 404.7 & 638.2 & 841.7 & 271.0 & 930.2 & 944.1 & 996.4 & 1047.2 & 1065.2 & 1038.6 & 852.2 \\
\hline SP4 & 0.0010 & 0.48 & $9.64 \mathrm{E}-04$ & 3070.90 & 511.2 & 549.7 & 674.5 & 767.8 & 333.2 & 884.0 & 901.6 & 986.5 & 1103.1 & 1109.4 & 709.8 & 887.5 \\
\hline SP5 & 0.0010 & 0.49 & $9.64 \mathrm{E}-04$ & 2960.92 & 396.4 & 424.7 & 571.1 & 732.9 & 299.7 & 879.4 & 899.0 & 973.8 & 1088.7 & 1098.1 & 635.9 & 878.4 \\
\hline SP6 & 0.0010 & 0.49 & 9.69E-04 & 2769.78 & 507.7 & 526.7 & 703.1 & 851.9 & 288.5 & 919.3 & 951.0 & 1009.7 & 1024.5 & 1050.7 & 740.5 & 840.6 \\
\hline SP7 & 0.0011 & 0.51 & \begin{tabular}{|l|} 
\\
\end{tabular} & 2772.76 & 438.4 & 470.2 & 653.3 & 822.8 & 288.2 & 915.7 & 927.0 & 1059.5 & 1083.8 & 1109.3 & 703.4 & 887.4 \\
\hline & & & Mean & 2727.6 & 433.4 & 456.2 & 643.7 & 804.2 & 282.8 & 889.3 & 909.0 & 1003.6 & 1067.4 & 1083.9 & 745.6 & 867.1 \\
\hline & & & SD & $\begin{array}{c}338.711 \\
12.4 \%\end{array}$ & $\begin{array}{l}64.851 \\
15.0 \%\end{array}$ & $\begin{array}{c}72.952 \\
16.0 \%\end{array}$ & $\begin{array}{c}45.500 \\
7.1 \%\end{array}$ & $\begin{array}{c}45.717 \\
5.7 \%\end{array}$ & $\begin{array}{l}38.718 \\
13.7 \%\end{array}$ & $\begin{array}{c}45.085 \\
5.1 \%\end{array}$ & $\begin{array}{c}43.665 \\
4.8 \%\end{array}$ & $\begin{array}{c}29.862 \\
3.0 \%\end{array}$ & $\begin{array}{c}29.503 \\
2.8 \%\end{array}$ & $\begin{array}{c}24.972 \\
2.3 \%\end{array}$ & $\begin{array}{c}149.028 \\
20.0 \%\end{array}$ & $\begin{array}{c}19.978 \\
2.3 \%\end{array}$ \\
\hline
\end{tabular}

Table 6 - Test results for rough as-built specimens with scan length $=78 \mathrm{~mm}$ (displacements and energies).

\begin{tabular}{|c|c|c|c|c|c|c|c|c|c|c|c|c|c|}
\hline $\begin{array}{c}\text { Specimen } \\
\text { id }\end{array}$ & $\begin{array}{c}\text { Displacement } \\
\text { rate }(\mathrm{mm} / \mathrm{s})\end{array}$ & $\begin{array}{c}\mathrm{h}_{0} \\
(\mathrm{~mm})\end{array}$ & $\begin{array}{c}\dot{\varepsilon}_{S P}^{\max } \\
\left(\mathrm{s}^{-1}\right)\end{array}$ & $\begin{array}{c}\mathrm{u}_{\mathrm{e}} \\
(\mathrm{mm})\end{array}$ & $\begin{array}{c}\mathrm{u}_{\mathrm{h} / 10, \text { off }}(\mathrm{mm}) \\
\left(\mathrm{u}_{0.1 \mathrm{~mm}, \text { off }}\right. \\
(\mathrm{mm})\end{array}$ & $\begin{array}{c}\mathrm{u}_{\mathrm{e} 1.5} \\
(\mathrm{~mm})\end{array}$ & $\begin{array}{c}\mathrm{u}_{\mathrm{m}} \\
(\mathrm{mm})\end{array}$ & $\begin{array}{c}\mathrm{u}_{\text {infl }} \\
(\mathrm{mm})\end{array}$ & $\begin{array}{c}\mathrm{u}_{\mathrm{f}} \\
(\mathrm{mm})\end{array}$ & $\begin{array}{c}\mathrm{E}_{\mathrm{SP}} \\
(\mathrm{J})\end{array}$ & $\begin{array}{c}\mathrm{E}_{\mathrm{m}} \\
(\mathrm{J})\end{array}$ & $\begin{array}{c}\mathrm{E}_{\mathrm{PL}} \\
(\mathrm{J})\end{array}$ \\
\hline SP1 & 0.0009 & 0.51 & $9.34 \mathrm{E}-04$ & 0.172 & 0.346 & 0.487 & 0.074 & 0.938 & 1.568 & 1.297 & 1.02 & 0.67 & 0.39 \\
SP2 & 0.0010 & 0.50 & $9.27 \mathrm{E}-04$ & 0.150 & 0.287 & 0.413 & 0.070 & 0.866 & 0.902 & 1.345 & 1.11 & 0.65 & 0.44 \\
SP4 & 0.0010 & 0.48 & $9.64 \mathrm{E}-04$ & 0.179 & 0.271 & 0.351 & 0.317 & 0.900 & 1.639 & 1.405 & 1.19 & 0.69 & 0.49 \\
SP5 & 0.0010 & 0.49 & $9.64 \mathrm{E}-04$ & 0.143 & 0.243 & 0.348 & 0.085 & 0.864 & 1.656 & 1.373 & 1.16 & 0.64 & 0.44 \\
SP6 & 0.0010 & 0.49 & $9.69 \mathrm{E}-04$ & 0.190 & 0.304 & 0.408 & 0.525 & 0.977 & 1.608 & 1.479 & 1.26 & 0.77 & 0.57 \\
SP7 & 0.0011 & 0.51 & $1.01 \mathrm{E}-03$ & 0.170 & 0.286 & 0.398 & 0.063 & 1.019 & 1.592 & 1.425 & 1.22 & 0.83 & 0.60 \\
\hline
\end{tabular}

Table 7 - Test results for polished as-built specimens with scan length $=78 \mathrm{~mm}$ (linear elastic slopes and forces).

\begin{tabular}{|c|c|c|c|c|c|c|c|c|c|c|c|c|c|c|c|c|}
\hline $\begin{array}{c}\text { Specimen } \\
\text { id }\end{array}$ & $\begin{array}{l}\text { Displacement } \\
\text { rate }(\mathrm{mm} / \mathrm{s})\end{array}$ & $\begin{array}{c}\mathrm{h}_{0} \\
(\mathrm{~mm})\end{array}$ & $\begin{array}{l}\dot{\varepsilon}_{S P}^{\max } \\
\left(\mathrm{s}^{-1}\right)\end{array}$ & $\begin{array}{l}\text { Slope }_{\text {ini }} \\
(\mathrm{N} / \mathrm{mm})\end{array}$ & $\begin{array}{l}F_{e, p r o j} \\
(N)\end{array}$ & $\begin{array}{l}F_{e, \text { int }} \\
(\mathrm{N})\end{array}$ & $\begin{array}{c}\mathrm{F}_{\mathrm{h} 0 / 10, \text { off }} \\
\text { (N) }\end{array}$ & $\begin{array}{l}\mathrm{F}_{0.1 \mathrm{~mm} \text {, off }} \\
\text { (N) }\end{array}$ & $\begin{array}{l}F_{0.1 \mathrm{~mm}} \\
(\mathrm{~N})\end{array}$ & $\begin{array}{c}\mathrm{F}_{0.48 \mathrm{~mm}} \\
(\mathrm{~N})\end{array}$ & $\begin{array}{l}F_{0.5 \mathrm{~mm}} \\
(\mathrm{~N})\end{array}$ & $\begin{array}{l}\mathrm{F}_{0.65 \mathrm{~mm}} \\
(\mathrm{~N})\end{array}$ & $\begin{array}{c}\mathrm{F}_{0.9 \mathrm{~mm}} \\
(\mathrm{~N})\end{array}$ & $\begin{array}{l}F_{m} \\
(N)\end{array}$ & $\begin{array}{l}F_{\text {infl }} \\
(\mathrm{N})\end{array}$ & $\begin{array}{r}F_{f} \\
(N) \\
\end{array}$ \\
\hline SP8 & 0.0014 & 0.45 & $1.44 \mathrm{E}-03$ & 3519.91 & 387.3 & 421.6 & 521.6 & 694.8 & 336.9 & 903.1 & 921.2 & 911.2 & 972.6 & 975.4 & 505.2 & 780.3 \\
\hline SP9 & 14 & 0.45 & 1.44E-03 & 3278.01 & 453.6 & 495.0 & 577.5 & 721.5 & 334.5 & 868.3 & 870.6 & 939.4 & 1006.8 & 1010.7 & 970.1 & 808.5 \\
\hline SP10 & 0.0014 & 0.45 & 1. $43 \mathrm{E}-03$ & 2907.81 & 390.0 & 419.9 & 532.1 & 698.4 & 298.2 & 845.6 & 852.7 & 865.9 & 937.9 & 955.3 & 487.2 & 764.3 \\
\hline & & & Mean & 3235.2 & 410.3 & 445.5 & 543.7 & 704.9 & 323.2 & 872.3 & 891.4 & 906.9 & 972.5 & 980.5 & 654.1 & 784.4 \\
\hline & & & SD & $\begin{array}{c}308.286 \\
9.5 \%\end{array}$ & $\begin{array}{c}37.534 \\
9.1 \%\end{array}$ & $\begin{array}{c}42.895 \\
9.6 \%\end{array}$ & $\begin{array}{c}29.705 \\
5.5 \%\end{array}$ & $\begin{array}{c}14.482 \\
2.1 \%\end{array}$ & $\begin{array}{c}21.680 \\
6.7 \%\end{array}$ & $\begin{array}{c}28.996 \\
3.3 \%\end{array}$ & $\begin{array}{c}35.128 \\
3.9 \%\end{array}$ & $\begin{array}{c}30.395 \\
3.4 \%\end{array}$ & $\begin{array}{c}28.143 \\
2.9 \%\end{array}$ & $\begin{array}{c}28.013 \\
2.9 \%\end{array}$ & $\begin{array}{c}273.751 \\
41.8 \%\end{array}$ & $\begin{array}{c}22.411 \\
2.9 \%\end{array}$ \\
\hline
\end{tabular}

Table 8 - Test results for polished as-built specimens with scan length $=78 \mathrm{~mm}$ (displacements and energies).

\begin{tabular}{|c|c|c|c|c|c|c|c|c|c|c|c|c|c|}
\hline $\begin{array}{c}\text { Specimen } \\
\text { id }\end{array}$ & \begin{tabular}{|c|}
$\begin{array}{c}\text { Displacement } \\
\text { rate }(\mathrm{mm} / \mathrm{s})\end{array}$ \\
\end{tabular} & $\begin{array}{c}\mathrm{h}_{0} \\
(\mathrm{~mm})\end{array}$ & $\begin{array}{l}\dot{\varepsilon}_{S P}^{\max } \\
\left(\mathrm{s}^{-1}\right) \\
\end{array}$ & $\begin{array}{c}\mathrm{u}_{\mathrm{e}} \\
(\mathrm{mm})\end{array}$ & $\begin{array}{c}u_{\text {ho/ } 10, \text { off }} \\
(\mathrm{mm})\end{array}$ & $\begin{array}{c}u_{0.1 \mathrm{~mm}, \text { off }} \\
(\mathrm{mm})\end{array}$ & $\begin{array}{r}\mathrm{u}_{\mathrm{e} 1.5} \\
(\mathrm{~mm})\end{array}$ & $\begin{array}{r}\mathrm{u}_{\mathrm{m}} \\
(\mathrm{mm})\end{array}$ & $\begin{array}{r}u_{\text {infl }} \\
(\mathrm{mm}) \\
\end{array}$ & $\begin{array}{c}\mathrm{u}_{\mathrm{f}} \\
(\mathrm{mm})\end{array}$ & $\begin{array}{l}E_{S P} \\
(J) \\
\end{array}$ & $\begin{array}{l}E_{m} \\
(J)\end{array}$ & $\begin{array}{l}E_{P L} \\
(J)\end{array}$ \\
\hline SP8 & 0.0014 & 0.45 & $1.44 \mathrm{E}-03$ & 0.120 & 0.194 & 0.298 & 0.328 & 0.861 & 1.624 & 1.250 & 0.98 & 0.63 & 0.49 \\
\hline SP9 & 0.0014 & 0.45 & $1.44 \mathrm{E}-03$ & 0.151 & 0.221 & 0.321 & 0.311 & 0.893 & 1.010 & 1.271 & 1.01 & 0.66 & 0.50 \\
\hline SP10 & 0.0014 & 0.45 & $1.43 \mathrm{E}-03$ & 0.144 & 0.228 & 0.340 & 0.399 & 0.976 & 1.611 & 1.351 & 1.03 & 0.70 & 0.54 \\
\hline & & & Mean & 0.138 & 0.214 & 0.320 & 0.346 & 0.910 & 1.415 & 1.291 & 1.006 & 0.661 & 0.512 \\
\hline & & & \multirow{2}{*}{ SD } & 0.016 & 0.018 & 0.021 & 0.047 & 0.059 & 0.351 & 0.053 & 0.026 & 0.036 & 0.027 \\
\hline & & & & $11.9 \%$ & $8.5 \%$ & $6.7 \%$ & $13.5 \%$ & $6.5 \%$ & $24.8 \%$ & $4.1 \%$ & $2.6 \%$ & $5.5 \%$ & $5.3 \%$ \\
\hline
\end{tabular}

Test and analysis details for each of the tests performed on this group of specimens are provided in Annex 2 (rough) and Annex 3 (polished). 


\subsection{As-built, scan length $=26 \mathrm{~mm}$}

Test results for the rough specimens is presented in Tables 9 and 10, which describe elastic slope/forces and displacements/energies, respectively. Likewise, Tables 11 and 12 describe the equivalent measurements for the polished specimens.

Table 9 - Test results for rough as-built specimens with scan length $=26 \mathrm{~mm}$ (linear elastic slopes and forces).

\begin{tabular}{|c|c|c|c|c|c|c|c|c|c|c|c|c|c|c|c|c|}
\hline $\begin{array}{c}\text { Specimen } \\
\text { id }\end{array}$ & $\begin{array}{c}\text { Displacement } \\
\text { rate }(\mathrm{mm} / \mathrm{s})\end{array}$ & $\begin{array}{c}\mathrm{h}_{0} \\
(\mathrm{~mm})\end{array}$ & $\begin{array}{l}\dot{\varepsilon}_{S P}^{\max } \\
\left(\mathrm{s}^{-1}\right) \\
\end{array}$ & $\begin{array}{r}\text { Slope }_{\text {el }} \\
(\mathrm{N} / \mathrm{mm})\end{array}$ & $\begin{array}{l}\mathrm{F}_{\mathrm{e}, \mathrm{proj}} \\
(\mathrm{N})\end{array}$ & $\begin{array}{l}F_{e, \text { int }} \\
\text { (N) }\end{array}$ & $\begin{array}{c}\mathrm{F}_{\mathrm{h} 0 / 10, \text { off }} \\
(\mathrm{N})\end{array}$ & $\begin{array}{c}F_{0.1 \mathrm{~mm}, \text { off }} \\
(\mathrm{N})\end{array}$ & $\begin{array}{l}\mathrm{F}_{0.1 \mathrm{~mm}} \\
(\mathrm{~N})\end{array}$ & $\begin{array}{l}F_{0.48 m m} \\
(\mathrm{~N})\end{array}$ & $\begin{array}{l}\mathrm{F}_{0.5 \mathrm{~mm}} \\
(\mathrm{~N})\end{array}$ & $\begin{array}{l}\mathrm{F}_{0.65 \mathrm{~mm}} \\
(\mathrm{~N})\end{array}$ & $\begin{array}{l}\mathrm{F}_{0.9 \mathrm{~mm}} \\
(\mathrm{~N})\end{array}$ & $\begin{array}{l}F_{m} \\
(N) \\
\end{array}$ & $\begin{array}{l}F_{\text {infl }} \\
(\mathrm{N})\end{array}$ & $\begin{array}{l}F_{f} \\
(N)\end{array}$ \\
\hline SP1 & 0.0009 & 0.51 & $1.03 \mathrm{E}-03$ & 1876.53 & 398.1 & 408.4 & 643.2 & 787.6 & 188.5 & 745.2 & 770.4 & 920.4 & 1005.9 & 1093.5 & 1000.8 & 874.8 \\
\hline SP2 & 0.0010 & 0.50 & $1.04 \mathrm{E}-03$ & 1860.63 & 449.1 & 457.0 & 673.4 & 831.5 & 186.6 & 758.0 & 777.1 & 939.1 & 1002.7 & 1158.1 & 630.8 & 926.5 \\
\hline SP4 & 0.0010 & 0.50 & $1.04 \mathrm{E}-03$ & 1666.44 & 452.2 & 456.5 & 780.4 & 974.2 & 163.0 & 736.5 & 763.6 & 947.0 & 1060.2 & 1071.7 & 349.1 & 857.3 \\
\hline SP5 & 0.0010 & 0.49 & $1.04 \mathrm{E}-03$ & 2016.45 & 383.2 & 393.2 & 612.4 & 785.9 & 198.4 & 774.2 & 799.9 & 921.8 & 1061.4 & 1107.0 & 740.2 & 885.6 \\
\hline SP6 & 0.0010 & 0.49 & $1.03 \mathrm{E}-03$ & 1745.38 & 401.5 & 408.2 & 675.0 & 872.4 & 174.9 & 738.2 & 762.2 & 922.1 & 1003.1 & 1090.5 & 715.8 & 872.4 \\
\hline SP7 & 0.0011 & 0.49 & $1.04 \mathrm{E}-03$ & 1752.85 & 364.6 & 370.9 & 637.4 & 832.6 & 172.4 & 725.1 & 742.0 & 915.6 & 1055.6 & 1088.0 & 634.4 & 870.4 \\
\hline & & & Mean & 1819.7 & 408.1 & 415.7 & 670.3 & 847.4 & 180.6 & 746.2 & 769.2 & 927.7 & 1031.5 & 1101.5 & 678.5 & 881.2 \\
\hline & & & SD & $\begin{array}{c}124.177 \\
6.8 \% \\
\end{array}$ & $\begin{array}{c}35.413 \\
8.7 \% \\
\end{array}$ & $\begin{array}{c}34.645 \\
8.3 \% \\
\end{array}$ & $\begin{array}{c}58.835 \\
8.8 \% \\
\end{array}$ & $\begin{array}{c}70.053 \\
8.3 \% \\
\end{array}$ & $\begin{array}{c}12.844 \\
7.1 \% \\
\end{array}$ & $\begin{array}{c}17.464 \\
2.3 \% \\
\end{array}$ & $\begin{array}{c}19.085 \\
2.5 \% \\
\end{array}$ & $\begin{array}{c}12.365 \\
1.3 \% \\
\end{array}$ & $\begin{array}{c}30.302 \\
2.9 \%\end{array}$ & $\begin{array}{c}29.973 \\
2.7 \% \\
\end{array}$ & $\begin{array}{c}210.609 \\
31.0 \%\end{array}$ & $\begin{array}{c}23.978 \\
2.7 \% \\
\end{array}$ \\
\hline
\end{tabular}

Table 10 - Test results for rough as-built specimens with scan length $=26 \mathrm{~mm}$ (displacements and energies).

\begin{tabular}{|c|c|c|c|c|c|c|c|c|c|c|c|c|c|}
\hline $\begin{array}{c}\text { Specimen } \\
\text { id }\end{array}$ & $\begin{array}{c}\text { Displacement } \\
\text { rate }(\mathrm{mm} / \mathrm{s})\end{array}$ & $\begin{array}{c}\mathrm{h}_{0} \\
(\mathrm{~mm})\end{array}$ & $\begin{array}{c}\dot{\varepsilon}_{S P}^{\max } \\
\left(\mathrm{s}^{-1}\right)\end{array}$ & $\begin{array}{c}\mathrm{u}_{\mathrm{e}} \\
(\mathrm{mm})\end{array}$ & $\begin{array}{c}\mathrm{u}_{\mathrm{h} 0 / 10, \text { off }}(\mathrm{mm}) \\
\left(\mathrm{u}_{0.1 \mathrm{~mm}, \text { off }}\right. \\
(\mathrm{mm})\end{array}$ & $\begin{array}{c}\mathrm{u}_{\mathrm{e} 1.5} \\
(\mathrm{~mm})\end{array}$ & $\begin{array}{c}\mathrm{u}_{\mathrm{m}} \\
(\mathrm{mm})\end{array}$ & $\begin{array}{c}\mathrm{u}_{\text {infl }} \\
(\mathrm{mm})\end{array}$ & $\begin{array}{c}\mathrm{u}_{\mathrm{f}} \\
(\mathrm{mm})\end{array}$ & $\begin{array}{c}\mathrm{E}_{\mathrm{SP}} \\
(\mathrm{J})\end{array}$ & $\begin{array}{c}\mathrm{E}_{\mathrm{m}} \\
(\mathrm{J})\end{array}$ & $\begin{array}{c}\mathrm{E}_{\mathrm{PL}} \\
(\mathrm{J})\end{array}$ \\
\hline SP1 & 0.0009 & 0.51 & $1.03 \mathrm{E}-03$ & 0.218 & 0.393 & 0.520 & 0.100 & 1.142 & 0.774 & 1.585 & 1.28 & 0.84 & 0.52 \\
SP2 & 0.0010 & 0.50 & $1.04 \mathrm{E}-03$ & 0.246 & 0.412 & 0.547 & 0.084 & 1.150 & 1.799 & 1.463 & 1.21 & 0.88 & 0.51 \\
SP4 & 0.0010 & 0.50 & $1.04 \mathrm{E}-03$ & 0.274 & 0.519 & 0.685 & 0.105 & 0.987 & 1.765 & 1.395 & 1.09 & 0.68 & 0.33 \\
SP5 & 0.0010 & 0.49 & $1.04 \mathrm{E}-03$ & 0.195 & 0.355 & 0.490 & 0.083 & 1.152 & 0.452 & 1.481 & 1.20 & 0.88 & 0.57 \\
SP6 & 0.0010 & 0.49 & $1.03 \mathrm{E}-03$ & 0.234 & 0.437 & 0.601 & 0.079 & 1.058 & 1.752 & 1.558 & 1.25 & 0.75 & 0.41 \\
SP7 & 0.0011 & 0.49 & $1.04 \mathrm{E}-03$ & 0.212 & 0.414 & 0.576 & 0.093 & 1.101 & 1.794 & 1.524 & 1.21 & 0.79 & 0.45 \\
\hline
\end{tabular}

Table 11 - Test results for polished as-built specimens with scan length $=26 \mathrm{~mm}$ (linear elastic slopes and forces).

\begin{tabular}{|c|c|c|c|c|c|c|c|c|c|c|c|c|c|c|c|c|c|c|}
\hline $\begin{array}{c}\text { Specimen } \\
\text { id }\end{array}$ & $\begin{array}{c}\text { Displacement } \\
\text { rate }(\mathrm{mm} / \mathrm{s})\end{array}$ & $\begin{array}{c}\mathrm{h}_{0} \\
(\mathrm{~mm})\end{array}$ & $\begin{array}{l}\dot{\varepsilon}_{S P}^{\max } \\
\left(s^{-1}\right)\end{array}$ & $\begin{array}{l}\text { Slope }_{\text {ini }} \\
(\mathrm{N} / \mathrm{mm})\end{array}$ & $\begin{array}{l}F_{e, p r o j} \\
\text { (N) }\end{array}$ & $\begin{array}{l}\mathrm{F}_{\mathrm{e}, \text { int }} \\
\text { (N) }\end{array}$ & $\begin{array}{c}\mathrm{F}_{\mathrm{h} / 10, \text { off }} \\
\text { (N) }\end{array}$ & $\begin{array}{l}F_{0.1 \mathrm{~mm}, \text { off }} \\
\text { (N) }\end{array}$ & $\begin{array}{l}\mathrm{F}_{0.1 \mathrm{~mm}} \\
\text { (N) }\end{array}$ & $\begin{array}{c}\mathrm{F}_{0.48 \mathrm{~mm}} \\
\text { (N) }\end{array}$ & $\begin{array}{l}\mathrm{F}_{0.5 \mathrm{~mm}} \\
\text { (N) }\end{array}$ & $\begin{array}{l}\mathrm{F}_{0.65 \mathrm{~mm}} \\
\text { (N) }\end{array}$ & $\begin{array}{l}\mathrm{F}_{0.9 \mathrm{~mm}} \\
\text { (N) }\end{array}$ & $\begin{array}{l}\mathrm{F}_{\mathrm{ept}} \\
\text { (N) }\end{array}$ & $\begin{array}{l}\mathrm{F}_{\mathrm{e} 1.5} \\
\text { (N) }\end{array}$ & $\begin{array}{l}F_{m} \\
\text { (N) }\end{array}$ & $\begin{array}{l}F_{\text {infl }} \\
\text { (N) }\end{array}$ & $\begin{array}{l}F_{f} \\
(N)\end{array}$ \\
\hline SP8 & 0.0014 & 0.45 & \begin{tabular}{|l}
$1.44 \mathrm{E}-03$ \\
\end{tabular} & 3010.25 & $\begin{array}{l}418.2 \\
\end{array}$ & 454.3 & 558.7 & 705.8 & 310.4 & 853.6 & 875.2 & 956.3 & $\begin{array}{l}934.8 \\
\end{array}$ & 81.4 & 760.3 & 968.0 & 515.4 & 774.4 \\
\hline SP9 & 0.0014 & 0.44 & $1.44 \mathrm{E}-03$ & 2889.12 & 428.5 & 466.5 & 552.1 & 694.6 & 295.4 & 818.5 & 818.5 & 897.2 & 926.0 & 702.3 & 732.7 & 956.8 & 487.4 & 765.4 \\
\hline SP10 & 0.0014 & 0.45 & $1.45 \mathrm{E}-03$ & 3283.40 & 363.6 & 404.3 & 522.6 & 688.9 & 317.7 & 880.6 & 898.1 & 949.1 & 962.4 & 430.5 & 658.3 & 981.3 & 76.1 & 785.1 \\
\hline & & & Mean & 3060.9 & 403.4 & $\begin{array}{l}441.7 \\
\end{array}$ & 544.4 & 696.4 & 307.8 & 850.9 & $\begin{array}{l}866.8 \\
\end{array}$ & 939.7 & 939.5 & 404.8 & 717.1 & $\begin{array}{l}968.7 \\
\end{array}$ & 359.6 & 775.0 \\
\hline & & & SD & 201.966 & 34.849 & 32.945 & 19.237 & 8.628 & 11.394 & 31.135 & 33.928 & 28.546 & 15.826 & 311.251 & 52.720 & 12.301 & 245.929 & 9.841 \\
\hline
\end{tabular}

Table 12 - Test results for polished as-built specimens with scan length $=26 \mathrm{~mm}$ (displacements and energies).

\begin{tabular}{|c|c|c|c|c|c|c|c|c|c|c|c|c|c|}
\hline $\begin{array}{c}\text { Specimen } \\
\text { id }\end{array}$ & $\begin{array}{c}\text { Displacement } \\
\text { rate }(\mathrm{mm} / \mathrm{s})\end{array}$ & $\begin{array}{c}\mathrm{h}_{0} \\
(\mathrm{~mm})\end{array}$ & $\begin{array}{c}\dot{\varepsilon}_{S P}^{\max } \\
\left(\mathrm{s}^{-1}\right)\end{array}$ & $\begin{array}{c}\mathrm{u}_{\mathrm{e}} \\
(\mathrm{mm})\end{array}$ & $\begin{array}{c}\mathrm{u}_{\text {ho/10,off }} \\
(\mathrm{mm})\end{array}$ & $\begin{array}{c}\mathrm{u}_{0.1 \mathrm{~mm}, \text { off }}(\mathrm{mm}) \\
(\mathrm{mm})\end{array}$ & $\begin{array}{c}\mathrm{u}_{\mathrm{e} 1.5} \\
(\mathrm{~mm})\end{array}$ & $\begin{array}{c}\mathrm{u}_{\mathrm{m}} \\
(\mathrm{mm})\end{array}$ & $\begin{array}{c}\mathrm{u}_{\text {infl }} \\
(\mathrm{mm})\end{array}$ & $\begin{array}{c}\mathrm{u}_{\mathrm{f}} \\
(\mathrm{mm})\end{array}$ & $\begin{array}{c}E_{\mathrm{SP}} \\
(\mathrm{J})\end{array}$ & $\begin{array}{c}E_{\mathrm{m}} \\
(\mathrm{J})\end{array}$ & $\begin{array}{c}E_{\text {PL }} \\
(\mathrm{J})\end{array}$ \\
\hline SP8 & 0.0014 & 0.45 & $1.44 \mathrm{E}-03$ & 0.151 & 0.231 & 0.335 & 0.379 & 0.680 & 1.661 & 1.245 & 0.95 & 0.44 & 0.28 \\
SP9 & 0.0014 & 0.44 & $1.44 \mathrm{E}-03$ & 0.161 & 0.236 & 0.341 & 0.372 & 0.844 & 1.631 & 1.232 & 0.91 & 0.57 & 0.42 \\
SP10 & 0.0014 & 0.45 & $1.45 E-03$ & 0.123 & 0.205 & 0.310 & 0.294 & 0.818 & 0.018 & 1.219 & 0.94 & 0.58 & 0.43 \\
\hline
\end{tabular}

Test and analysis details for each of the tests performed on this group of specimens are provided in Annex 4 (rough) and Annex 5 (polished). 


\subsection{Non-standard $\left(800^{\circ} \mathrm{C}\right) \mathrm{HIP}$, scan length $=78 \mathrm{~mm}$}

Test results for the rough specimens is presented in Tables 13 and 14, which describe elastic slope/forces and displacements/energies, respectively. Likewise, Tables 15 and 16 describe the equivalent measurements for the polished specimens.

Table 13 - Test results for rough specimens with non-standard HIP and scan length $=26 \mathrm{~mm}$ (linear elastic slopes and forces).

\begin{tabular}{|c|c|c|c|c|c|c|c|c|c|c|c|c|c|c|c|c|}
\hline $\begin{array}{c}\text { Specimen } \\
\text { id }\end{array}$ & $\begin{array}{c}\text { Displacement } \\
\text { rate }(\mathrm{mm} / \mathrm{s})\end{array}$ & $\begin{array}{c}\mathrm{h}_{0} \\
(\mathrm{~mm})\end{array}$ & $\begin{array}{l}\dot{\varepsilon}_{S P}^{\max } \\
\left(\mathrm{s}^{-1}\right)\end{array}$ & $\begin{array}{l}\text { Slope }_{\text {el }} \\
(\mathrm{N} / \mathrm{mm})\end{array}$ & $\begin{array}{l}F_{e, p r o j} \\
(\mathrm{~N})\end{array}$ & $\begin{array}{l}\mathrm{F}_{\mathrm{e}, \mathrm{int}} \\
(\mathrm{N})\end{array}$ & $\begin{array}{c}\mathrm{F}_{\mathrm{h} 0 / 10, \text { off }} \\
(\mathrm{N})\end{array}$ & $\begin{array}{c}F_{0.1 \mathrm{~mm}, \text { off }} \\
(\mathrm{N})\end{array}$ & $\begin{array}{l}\mathrm{F}_{0.1 \mathrm{~mm}} \\
(\mathrm{~N})\end{array}$ & $\begin{array}{l}\mathrm{F}_{0.48 \mathrm{~mm}} \\
(\mathrm{~N})\end{array}$ & $\begin{array}{l}\mathrm{F}_{0.5 \mathrm{~mm}} \\
(\mathrm{~N})\end{array}$ & $\begin{array}{c}F_{0.65 \mathrm{~mm}} \\
(\mathrm{~N})\end{array}$ & $\begin{array}{l}\mathrm{F}_{0.9 \mathrm{~mm}} \\
(\mathrm{~N})\end{array}$ & $\begin{array}{l}F_{m} \\
(N) \\
\end{array}$ & $\begin{array}{l}F_{\text {infl }} \\
(\mathrm{N})\end{array}$ & $\begin{array}{l}F_{f} \\
(N)\end{array}$ \\
\hline SP1 & 0.0009 & 0.49 & $1.00 \mathrm{E}-03$ & 1958.20 & 443.2 & 455.3 & 691.4 & 868.9 & 202.7 & 790.4 & 815.5 & 959.6 & 1032.8 & 1082.5 & 468.1 & 866.0 \\
\hline SP2 & 0.0010 & 0.50 & $1.01 \mathrm{E}-03$ & 2210.27 & 445.7 & 460.3 & 630.4 & 761.0 & 228.5 & 803.0 & 820.2 & 938.4 & 1020.1 & 1068.2 & 628.6 & 854.6 \\
\hline SP4 & 0.0010 & 0.49 & $1.01 \mathrm{E}-03$ & 1801.57 & 487.1 & 492.4 & 663.9 & 828.7 & 179.3 & 737.4 & 765.0 & 915.6 & 1126.4 & 1157.1 & 590.9 & 925.7 \\
\hline SP5 & 0.0010 & 0.50 & $1.02 \mathrm{E}-03$ & 1639.54 & 428.9 & 438.4 & 677.5 & 817.2 & 164.1 & 692.9 & 714.6 & 879.5 & 1054.7 & 1106.8 & 577.5 & 885.4 \\
\hline SP6 & 0.0010 & 0.51 & $1.04 \mathrm{E}-03$ & 1754.55 & 436.8 & 447.0 & 658.3 & 845.0 & 173.6 & 722.7 & 747.9 & 927.5 & 1056.0 & 1070.9 & 565.7 & 856.7 \\
\hline SP7 & 0.0011 & 0.49 & $1.02 \mathrm{E}-03$ & 1754.55 & 472.8 & 447.0 & 781.3 & 904.7 & 194.5 & 763.6 & 786.6 & 930.1 & 1093.5 & 1131.1 & 960.9 & 904.9 \\
\hline & & & Mean & 1853.1 & 452.4 & 456.7 & 683.8 & 837.6 & 190.5 & 751.7 & 774.9 & 925.1 & 1063.9 & 1102.8 & 632.0 & 882.2 \\
\hline & & & SD & $\begin{array}{c}203.128 \\
11.0 \%\end{array}$ & $\begin{array}{c}22.560 \\
5.0 \%\end{array}$ & $\begin{array}{c}19.048 \\
4.2 \%\end{array}$ & $\begin{array}{c}51.957 \\
7.6 \%\end{array}$ & $\begin{array}{c}48.816 \\
5.8 \%\end{array}$ & $\begin{array}{c}23.321 \\
12.2 \%\end{array}$ & $\begin{array}{c}41.878 \\
5.6 \%\end{array}$ & $\begin{array}{c}40.725 \\
5.3 \%\end{array}$ & $\begin{array}{c}26.722 \\
2.9 \%\end{array}$ & $\begin{array}{c}39.555 \\
3.7 \%\end{array}$ & $\begin{array}{c}35.741 \\
3.2 \%\end{array}$ & $\begin{array}{c}169.768 \\
26.9 \%\end{array}$ & $\begin{array}{c}28.593 \\
3.2 \%\end{array}$ \\
\hline
\end{tabular}

Table 14 - Test results for rough specimens with non-standard HIP and scan length $=26 \mathrm{~mm}$ (displacements and energies).

\begin{tabular}{|c|c|c|c|c|c|c|c|c|c|c|c|c|c|}
\hline $\begin{array}{c}\text { Specimen } \\
\text { id }\end{array}$ & $\begin{array}{c}\text { Displacement } \\
\text { rate }(\mathrm{mm} / \mathrm{s})\end{array}$ & $\begin{array}{c}\mathrm{h}_{0} \\
(\mathrm{~mm})\end{array}$ & $\begin{array}{c}\dot{\varepsilon}_{S P}^{\max } \\
\left(\mathrm{s}^{-1}\right)\end{array}$ & $\begin{array}{c}\mathrm{u}_{\mathrm{e}} \\
(\mathrm{mm})\end{array}$ & $\begin{array}{c}\mathrm{u}_{\mathrm{ho} / 10, \text { off }} \\
(\mathrm{mm})\end{array}$ & $\begin{array}{c}\mathrm{u}_{0.1 \mathrm{~mm}, \text { off }} \\
(\mathrm{mm})\end{array}$ & $\begin{array}{c}\mathrm{u}_{\mathrm{e} 1.5} \\
(\mathrm{~mm})\end{array}$ & $\begin{array}{c}\mathrm{u}_{\mathrm{m}} \\
(\mathrm{mm})\end{array}$ & $\begin{array}{c}\mathrm{u}_{\text {infl }} \\
(\mathrm{mm})\end{array}$ & $\begin{array}{c}\mathrm{u}_{\mathrm{f}} \\
(\mathrm{mm})\end{array}$ & $\begin{array}{c}\mathrm{E}_{\mathrm{SP}} \\
(\mathrm{J})\end{array}$ & $\begin{array}{c}\mathrm{E}_{\mathrm{m}} \\
(\mathrm{J})\end{array}$ & $\begin{array}{c}\mathrm{E}_{\mathrm{PL}} \\
(\mathrm{J})\end{array}$ \\
\hline SP1 & 0.0009 & 0.49 & $1.00 \mathrm{E}-03$ & 0.232 & 0.404 & 0.544 & -0.025 & 0.822 & 1.734 & 1.335 & 1.05 & 0.54 & 0.24 \\
SP2 & 0.0010 & 0.50 & $1.01 \mathrm{E}-03$ & 0.208 & 0.336 & 0.445 & 0.060 & 1.074 & 0.332 & 1.533 & 1.25 & 0.80 & 0.54 \\
SP4 & 0.0010 & 0.49 & $1.01 \mathrm{E}-03$ & 0.273 & 0.419 & 0.560 & 0.119 & 0.946 & 1.731 & 1.458 & 1.20 & 0.65 & 0.28 \\
SP5 & 0.0010 & 0.50 & $1.02 \mathrm{E}-03$ & 0.267 & 0.464 & 0.598 & 0.086 & 1.038 & 1.766 & 1.371 & 1.05 & 0.71 & 0.34 \\
SP6 & 0.0010 & 0.51 & $1.04 \mathrm{E}-03$ & 0.255 & 0.426 & 0.582 & 0.108 & 0.917 & 1.782 & 1.413 & 1.09 & 0.60 & 0.27 \\
SP7 & 0.0011 & 0.49 & $1.02 \mathrm{E}-03$ & 0.255 & 0.495 & 0.616 & 0.091 & 1.115 & 1.422 & 1.476 & 1.22 & 0.84 & 0.48 \\
\hline
\end{tabular}

Table 15 - Test results for polished specimens with non-standard HIP and scan length $=26$ $\mathrm{mm}$ (linear elastic slopes and forces).

\begin{tabular}{|c|c|c|c|c|c|c|c|c|c|c|c|c|c|c|c|c|}
\hline $\begin{array}{c}\text { Specimen } \\
\text { id }\end{array}$ & $\begin{array}{c}\text { Displacement } \\
\text { rate }(\mathrm{mm} / \mathrm{s})\end{array}$ & $\begin{array}{c}\mathrm{h}_{0} \\
(\mathrm{~mm}) \\
\end{array}$ & $\begin{array}{c}\dot{\varepsilon}_{S P}^{\max } \\
\left(\mathrm{s}^{-1}\right) \\
\end{array}$ & $\begin{array}{r}\text { Slope }_{\text {ini }} \\
(\mathrm{N} / \mathrm{mm})\end{array}$ & $\begin{array}{l}\mathrm{F}_{\mathrm{e}, \text { proj }} \\
(\mathrm{N})\end{array}$ & $\begin{array}{l}\mathrm{F}_{\mathrm{e}, \text { int }} \\
(\mathrm{N})\end{array}$ & $\begin{array}{c}\mathrm{F}_{\mathrm{h} 0 / 10, \text { off }} \\
\text { (N) }\end{array}$ & $\begin{array}{l}F_{0.1 \mathrm{~mm}, \text { off }} \\
(\mathrm{N})\end{array}$ & $\begin{array}{l}\mathrm{F}_{0.1 \mathrm{~mm}} \\
(\mathrm{~N})\end{array}$ & $\begin{array}{l}\mathrm{F}_{0.48 \mathrm{~mm}} \\
(\mathrm{~N})\end{array}$ & $\begin{array}{l}\mathrm{F}_{0.5 \mathrm{~mm}} \\
(\mathrm{~N}) \\
\end{array}$ & $\begin{array}{c}\mathrm{F}_{0.65 \mathrm{~mm}} \\
(\mathrm{~N})\end{array}$ & $\begin{array}{l}\mathrm{F}_{0.9 \mathrm{~mm}} \\
(\mathrm{~N}) \\
\end{array}$ & $\begin{array}{l}\mathrm{F}_{\mathrm{m}} \\
(\mathrm{N}) \\
\end{array}$ & $\begin{array}{l}F_{\text {infl }} \\
(\mathrm{N}) \\
\end{array}$ & $\begin{array}{l}F_{f} \\
(N)\end{array}$ \\
\hline SP7 & 0.0014 & 0.45 & $1.44 \mathrm{E}-03$ & 3521.07 & 431.3 & 473.5 & 559.8 & 698.0 & 348.9 & 874.7 & 890.4 & 922.1 & 1005.9 & 1013.5 & 988.7 & 810.8 \\
\hline SP8 & 0.0014 & 0.44 & $1.44 \mathrm{E}-03$ & 2983.80 & 381.2 & 413.1 & 513.8 & 661.1 & 298.0 & 832.4 & 850.4 & 961.4 & 918.9 & 963.9 & 592.8 & 771.1 \\
\hline SP9 & 0.0014 & 0.44 & $1.43 \mathrm{E}-03$ & 3115.09 & 393.2 & 426.2 & 528.3 & 656.9 & 317.5 & 833.0 & 853.6 & 967.8 & 916.7 & 971.8 & 623.1 & 777.4 \\
\hline SP10 & 0.0014 & 0.44 & 1.43E-03 & 3258.38 & 348.2 & 381.0 & 484.1 & 630.0 & 314.7 & 854.8 & 874.9 & 917.2 & 989.1 & 996.4 & 686.5 & 797.1 \\
\hline & & & Mean & 3219.6 & 388.5 & 423.4 & 521.5 & 661.5 & 319.8 & 848.7 & 871.9 & 938.1 & 967.3 & 986.4 & 722.8 & 789.1 \\
\hline & & & SD & $\begin{array}{c}230.154 \\
7.1 \%\end{array}$ & $\begin{array}{c}34.326 \\
8.8 \%\end{array}$ & $\begin{array}{c}38.373 \\
9.1 \%\end{array}$ & $\begin{array}{c}31.487 \\
6.0 \%\end{array}$ & $\begin{array}{c}27.941 \\
4.2 \%\end{array}$ & $\begin{array}{c}21.276 \\
6.7 \%\end{array}$ & $\begin{array}{c}20.176 \\
2.4 \%\end{array}$ & $\begin{array}{c}19.307 \\
2.2 \%\end{array}$ & $\begin{array}{c}24.342 \\
2.6 \%\end{array}$ & $\begin{array}{c}45.744 \\
4.7 \%\end{array}$ & $\begin{array}{c}22.768 \\
2.3 \%\end{array}$ & $\begin{array}{c}181.546 \\
25.1 \%\end{array}$ & $\begin{array}{c}18.215 \\
2.3 \%\end{array}$ \\
\hline
\end{tabular}

Table 16 - Test results for polished specimens with non-standard HIP and scan length $=26$ $\mathrm{mm}$ (displacements and energies).

\begin{tabular}{|c|c|c|c|c|c|c|c|c|c|c|c|c|c|}
\hline \begin{tabular}{|c|} 
Specimen \\
id
\end{tabular} & \begin{tabular}{|l|} 
Displacement \\
rate $(\mathrm{mm} / \mathrm{s})$
\end{tabular} & $\begin{array}{c}\mathrm{h}_{0} \\
(\mathrm{~mm})\end{array}$ & $\begin{array}{l}\dot{\varepsilon}_{S P}^{\max } \\
\left(\mathrm{s}^{-1}\right)\end{array}$ & $\begin{array}{c}\mathrm{u}_{\mathrm{e}} \\
(\mathrm{mm})\end{array}$ & $\begin{array}{c}\mathrm{u}_{\mathrm{hO} / 10, \text { off }} \\
(\mathrm{mm})\end{array}$ & $\begin{array}{c}\mathrm{u}_{0.1 \mathrm{~mm}, \text { off }} \\
(\mathrm{mm})\end{array}$ & $\begin{array}{l}\mathrm{u}_{\mathrm{e} 1.5} \\
(\mathrm{~mm})\end{array}$ & $\begin{array}{c}\mathrm{u}_{\mathrm{m}} \\
(\mathrm{mm})\end{array}$ & $\begin{array}{c}\mathrm{u}_{\text {infl }} \\
(\mathrm{mm})\end{array}$ & $\begin{array}{c}\mathrm{u}_{\mathrm{f}} \\
(\mathrm{mm})\end{array}$ & $\begin{array}{l}E_{S P} \\
(J)\end{array}$ & $\begin{array}{l}E_{m} \\
(J)\end{array}$ & $\begin{array}{l}E_{P L} \\
\text { (J) }\end{array}$ \\
\hline SP7 & 0.0014 & 0.45 & $1.44 \mathrm{E}-03$ & 0.134 & 0.205 & 0.299 & 0.219 & 0.885 & 1.026 & 1.278 & 1.03 & 0.66 & 0.51 \\
\hline SP8 & 0.0014 & 0.44 & 1. $44 \mathrm{E}-03$ & 0.138 & 0.217 & 0.322 & 0.334 & 0.654 & 1.666 & 1.404 & 1.05 & 0.40 & 0.25 \\
\hline SP9 & 0.0014 & 0.44 & $1.43 \mathrm{E}-03$ & 0.137 & 0.214 & 0.311 & 0.381 & 0.691 & 1.662 & 1.475 & 1.14 & 0.44 & 0.29 \\
\hline SP10 & 0.0014 & 0.44 & 1. $43 \mathrm{E}-03$ & 0.117 & 0.193 & 0.294 & 0.265 & 0.900 & 1.416 & 1.306 & 1.02 & 0.64 & 0.49 \\
\hline & & & Mean & 0.132 & 0.207 & 0.306 & 0.300 & 0.782 & 1.442 & 1.366 & 1.061 & 0.538 & 0.387 \\
\hline & & & \multirow{2}{*}{ SD } & 0.010 & 0.011 & 0.012 & 0.072 & 0.128 & 0.301 & 0.090 & 0.055 & 0.133 & 0.136 \\
\hline & & & & $7.6 \%$ & $5.2 \%$ & $4.1 \%$ & $24.1 \%$ & $16.4 \%$ & $20.9 \%$ & $6.6 \%$ & $5.1 \%$ & $24.8 \%$ & $35.3 \%$ \\
\hline
\end{tabular}

Test and analysis details for each of the tests performed on this group of specimens are provided in Annex 6 (rough) and Annex 7 (polished). 


\subsection{Standard $\left(900^{\circ} \mathrm{C}\right) \mathrm{HIP}$, scan length $=78 \mathrm{~mm}$}

Six rough specimens and four polished specimens were tested. Results are shown in Table 17 (elastic slopes and forces) and Table 18 (displacements and energies) for rough specimens, and Table 19 (elastic slopes and forces) and Table 20 (displacements and energies) for polished specimens.

Table 17 - Test results for rough specimens with standard HIP and scan length $=78 \mathrm{~mm}$ (linear elastic slopes and forces).

\begin{tabular}{|c|c|c|c|c|c|c|c|c|c|c|c|c|c|c|c|c|}
\hline $\begin{array}{c}\text { Specimen } \\
\text { id }\end{array}$ & $\begin{array}{c}\begin{array}{c}\text { Displacement } \\
\text { rate }(\mathrm{mm} / \mathrm{s})\end{array} \\
\end{array}$ & $\begin{array}{c}\mathrm{h}_{0} \\
(\mathrm{~mm})\end{array}$ & $\begin{array}{l}\dot{\varepsilon}_{S P}^{\max } \\
\left(\mathrm{s}^{-1}\right)\end{array}$ & $\begin{array}{l}\text { Slope }_{\text {el }} \\
(\mathrm{N} / \mathrm{mm})\end{array}$ & $\begin{array}{l}\mathrm{F}_{\mathrm{e}, \mathrm{proj}} \\
(\mathrm{N})\end{array}$ & $\begin{array}{l}F_{e, \text { int }} \\
\text { (N) }\end{array}$ & $\begin{array}{c}\mathrm{F}_{\mathrm{h} 0 / 10, \text { off }} \\
(\mathrm{N})\end{array}$ & $\begin{array}{l}F_{0.1 \mathrm{~mm}, \text { off }} \\
\text { (N) }\end{array}$ & $\begin{array}{l}\mathrm{F}_{0.1 \mathrm{~mm}} \\
(\mathrm{~N})\end{array}$ & $\begin{array}{c}\mathrm{F}_{0.48 \mathrm{~mm}} \\
(\mathrm{~N})\end{array}$ & $\begin{array}{l}\mathrm{F}_{0.5 \mathrm{~mm}} \\
(\mathrm{~N})\end{array}$ & $\begin{array}{l}\mathrm{F}_{0.65 \mathrm{~mm}} \\
(\mathrm{~N})\end{array}$ & $\begin{array}{l}\mathrm{F}_{0.9 \mathrm{~mm}} \\
(\mathrm{~N})\end{array}$ & $\begin{array}{l}F_{m} \\
(N)\end{array}$ & $\begin{array}{l}F_{\text {infl }} \\
(\mathrm{N})\end{array}$ & $\begin{array}{l}F_{f} \\
(N)\end{array}$ \\
\hline SP2 & 0.0009 & 0.51 & $1.02 \mathrm{E}-03$ & 2099.36 & 401.3 & 417.4 & 583.9 & 720.0 & 207.5 & 762.7 & 784.5 & 918.6 & 1071.1 & 1123.6 & 650.9 & 898.9 \\
\hline SP3 & 0.0010 & 0.50 & $1.01 \mathrm{E}-03$ & 1839.49 & 415.5 & 420.7 & 609.6 & 766.2 & 189.0 & 729.5 & 748.0 & 917.2 & 1037.6 & 1095.6 & 731.2 & 876.5 \\
\hline SP4 & 0.0010 & 0.55 & $1.00 \mathrm{E}-03$ & 825.63 & 345.3 & 344.0 & 1011.2 & 1004.9 & 82.4 & 391.9 & 410.4 & 521.4 & 743.9 & 1022.4 & 5.0 & 817.9 \\
\hline SP5 & 0.0010 & 0.51 & $1.01 \mathrm{E}-03$ & 1987.01 & 342.7 & 350.8 & 539.8 & 723.3 & 201.3 & 748.8 & 773.6 & 958.5 & 1047.8 & 1085.0 & 577.3 & 868.0 \\
\hline SP7 & 0.0010 & 0.51 & $1.02 \mathrm{E}-03$ & 2057.23 & 447.6 & 457.9 & 662.2 & 792.1 & 211.6 & 791.3 & 810.9 & 946.7 & 1090.2 & 1112.3 & 515.1 & 889.9 \\
\hline SP8 & 0.0011 & 0.52 & $1.01 \mathrm{E}-03$ & 2064.56 & 358.3 & 371.4 & 595.4 & 781.4 & 209.2 & 787.7 & 809.0 & 983.9 & 1042.8 & 1067.5 & 486.6 & 854.0 \\
\hline & & & Mean & 1812.2 & 385.1 & 393.7 & 667.0 & 798.0 & 183.5 & 702.0 & 722.7 & 874.4 & 1005.6 & 1084.4 & 494.3 & 867.5 \\
\hline & & & SD & $\begin{array}{c}492.099 \\
27.2 \%\end{array}$ & $\begin{array}{l}42.872 \\
11.1 \%\end{array}$ & $\begin{array}{l}45.216 \\
11.5 \%\end{array}$ & \begin{tabular}{|l|}
173.226 \\
$26.0 \%$
\end{tabular} & $\begin{array}{c}105.646 \\
13.2 \%\end{array}$ & $\begin{array}{l}50.196 \\
27.4 \%\end{array}$ & $\begin{array}{l}153.698 \\
21.9 \%\end{array}$ & $\begin{array}{c}154.800 \\
21.4 \%\end{array}$ & $\begin{array}{c}174.744 \\
20.0 \%\end{array}$ & \begin{tabular}{c|}
129.710 \\
$12.9 \%$
\end{tabular} & $\begin{array}{c}36.274 \\
3.3 \%\end{array}$ & \begin{tabular}{|l|}
255.897 \\
$51.8 \%$
\end{tabular} & $\begin{array}{c}29.019 \\
3.3 \%\end{array}$ \\
\hline
\end{tabular}

Table 18 - Test results for rough specimens with standard HIP and scan length $=78 \mathrm{~mm}$ (displacements and energies).

\begin{tabular}{|c|c|c|c|c|c|c|c|c|c|c|c|c|c|}
\hline $\begin{array}{c}\text { Specimen } \\
\text { id } \\
\end{array}$ & \begin{tabular}{|c|}
$\begin{array}{c}\text { Displacement } \\
\text { rate }(\mathrm{mm} / \mathrm{s})\end{array}$ \\
\end{tabular} & $\begin{array}{c}\mathrm{h}_{0} \\
(\mathrm{~mm})\end{array}$ & $\begin{array}{l}\dot{\varepsilon}_{S P}^{\max } \\
\left(s^{-1}\right)\end{array}$ & $\begin{array}{c}\mathrm{u}_{\mathrm{e}} \\
(\mathrm{mm})\end{array}$ & $\begin{array}{c}u_{\mathrm{h} 0 / 10, \text { off }} \\
(\mathrm{mm})\end{array}$ & $\begin{array}{c}\mathrm{u}_{0.1 \mathrm{~mm}, \text { off }} \\
(\mathrm{mm})\end{array}$ & $\begin{array}{r}u_{\mathrm{e} 1.5} \\
(\mathrm{~mm})\end{array}$ & $\begin{array}{c}\mathrm{u}_{\mathrm{m}} \\
(\mathrm{mm})\end{array}$ & $\begin{array}{c}\mathrm{u}_{\mathrm{infl}} \\
(\mathrm{mm})\end{array}$ & $\begin{array}{c}\mathrm{u}_{\mathrm{f}} \\
(\mathrm{mm})\end{array}$ & $\begin{array}{l}E_{S P} \\
(J)\end{array}$ & $\begin{array}{l}E_{m} \\
(J)\end{array}$ & $\begin{array}{l}E_{P L} \\
(J)\end{array}$ \\
\hline SP2 & 0.0009 & 0.51 & $1.02 \mathrm{E}-03$ & 0.199 & 0.328 & 0.444 & 0.064 & 1.091 & 1.748 & 1.450 & 1.18 & 0.81 & 0.51 \\
\hline SP3 & 0.0010 & 0.50 & $1.01 \mathrm{E}-03$ & 0.229 & 0.383 & 0.517 & 0.082 & 1.094 & 0.483 & 1.567 & 1.28 & 0.79 & 0.47 \\
\hline SP4 & 0.0010 & 0.55 & $1.00 \mathrm{E}-03$ & 0.417 & 1.276 & 1.320 & 0.176 & 1.267 & -0.107 & 1.653 & 1.02 & 0.66 & 0.03 \\
\hline SP5 & 0.0010 & 0.51 & $1.01 \mathrm{E}-03$ & 0.177 & 0.322 & 0.465 & 0.064 & 0.837 & 1.742 & 1.463 & 1.17 & 0.54 & 0.24 \\
\hline SP7 & 0.0010 & 0.51 & $1.02 \mathrm{E}-03$ & 0.223 & 0.373 & 0.485 & 0.066 & 0.870 & 0.268 & 1.525 & 1.25 & 0.59 & 0.29 \\
\hline SP8 & 0.0011 & 0.52 & $1.01 \mathrm{E}-03$ & 0.180 & 0.339 & 0.479 & 0.066 & 0.950 & 1.747 & 1.337 & 1.05 & 0.67 & 0.39 \\
\hline & & & Mean & 0.237 & 0.504 & 0.618 & 0.086 & 1.018 & 0.980 & 1.499 & 1.158 & 0.678 & 0.322 \\
\hline & & & \multirow[t]{2}{*}{ SD } & 0.090 & 0.379 & 0.345 & 0.044 & 0.163 & 0.860 & 0.109 & 0.104 & 0.108 & 0.176 \\
\hline & & & & $38.1 \%$ & $75.3 \%$ & $55.7 \%$ & $51.4 \%$ & $16.0 \%$ & $87.7 \%$ & $7.2 \%$ & $9.0 \%$ & $16.0 \%$ & $54.7 \%$ \\
\hline
\end{tabular}

Table 19 - Test results for polished specimens with standard HIP and scan length $=78 \mathrm{~mm}$ (linear elastic slopes and forces).

\begin{tabular}{|c|c|c|c|c|c|c|c|c|c|c|c|c|c|c|c|c|}
\hline $\begin{array}{c}\text { Specimen } \\
\text { id }\end{array}$ & \begin{tabular}{|c} 
Displacement \\
rate $(\mathrm{mm} / \mathrm{s})$
\end{tabular} & $\begin{array}{c}\mathrm{h}_{0} \\
(\mathrm{~mm})\end{array}$ & $\begin{array}{l}\dot{\varepsilon}_{S P}^{\max } \\
\left(\mathrm{s}^{-1}\right) \\
\end{array}$ & $\begin{array}{r}S^{S^{\prime} o p e_{\text {ini }}} \\
(\mathrm{N} / \mathrm{mm})\end{array}$ & $\begin{array}{l}F_{e, \text { proj }} \\
\text { (N) }\end{array}$ & $\begin{array}{l}\mathrm{F}_{\mathrm{e}, \mathrm{int}} \\
(\mathrm{N})\end{array}$ & $\begin{array}{c}F_{\text {ho/ } 10, \text { off }} \\
(\mathrm{N})\end{array}$ & $\begin{array}{c}F_{0.1 \mathrm{~mm}, \text { off }} \\
(\mathrm{N})\end{array}$ & $\begin{array}{c}F_{0.1 \mathrm{~mm}} \\
(\mathrm{~N})\end{array}$ & $\begin{array}{c}\mathrm{F}_{0.48 \mathrm{~mm}} \\
(\mathrm{~N})\end{array}$ & $\begin{array}{l}\mathrm{F}_{0.5 \mathrm{~mm}} \\
(\mathrm{~N})\end{array}$ & $\begin{array}{c}\mathrm{F}_{0.65 \mathrm{~mm}} \\
(\mathrm{~N})\end{array}$ & $\begin{array}{l}\mathrm{F}_{0.9 \mathrm{~mm}} \\
(\mathrm{~N})\end{array}$ & $\begin{array}{l}F_{m} \\
(N) \\
\end{array}$ & $\begin{array}{l}F_{\text {infl }} \\
(\mathrm{N}) \\
\end{array}$ & $\begin{array}{r}F_{f} \\
(N) \\
\end{array}$ \\
\hline SP9 & 0.0014 & 0.45 & $1.43 \mathrm{E}-03$ & 2910.50 & 414.0 & 438.5 & 568.5 & 715.2 & 301.3 & 877.3 & 898.4 & 938.6 & 1015.8 & 1031.9 & 622.9 & 825.5 \\
\hline SP10 & 0.0014 & 0.46 & 1.44E-03 & 3178.87 & 357.8 & 385.1 & 496.9 & 656.5 & 311.3 & 869.2 & 881.3 & 971.0 & 1074.2 & 1081.8 & 573.1 & 865.4 \\
\hline & & & Mean & 3044.7 & 385.9 & 411.8 & 532.7 & 685.9 & 306.3 & 873.2 & \begin{tabular}{|l|}
889.8 \\
\end{tabular} & 954.8 & 1045.0 & 1056.8 & 598.0 & 845.5 \\
\hline & & & SD & $\begin{array}{c}189.766 \\
6.2 \%\end{array}$ & $\begin{array}{l}39.775 \\
10.3 \%\end{array}$ & $\begin{array}{c}37.792 \\
9.2 \%\end{array}$ & $\begin{array}{c}50.614 \\
9.5 \%\end{array}$ & $\begin{array}{c}41.524 \\
6.1 \%\end{array}$ & $\begin{array}{l}7.047 \\
2.3 \%\end{array}$ & $\begin{array}{l}5.735 \\
0.7 \%\end{array}$ & $\begin{array}{c}12.130 \\
1.4 \%\end{array}$ & $\begin{array}{c}22.911 \\
2.4 \%\end{array}$ & $\begin{array}{c}41.296 \\
4.0 \%\end{array}$ & $\begin{array}{c}35.293 \\
3.3 \%\end{array}$ & $\begin{array}{c}35.269 \\
5.9 \%\end{array}$ & $\begin{array}{c}28.234 \\
3.3 \%\end{array}$ \\
\hline
\end{tabular}

Table 20 - Test results for polished specimens with standard HIP and scan length $=78 \mathrm{~mm}$ (displacements and energies).

\begin{tabular}{|c|c|c|c|c|c|c|c|c|c|c|c|c|c|}
\hline $\begin{array}{c}\text { Specimen } \\
\text { id }\end{array}$ & $\begin{array}{c}\text { Displacement } \\
\text { rate }(\mathrm{mm} / \mathrm{s})\end{array}$ & $\begin{array}{c}\mathrm{h}_{0} \\
(\mathrm{~mm})\end{array}$ & $\begin{array}{c}\dot{\varepsilon}_{S P}^{\max } \\
\left(\mathrm{s}^{-1}\right)\end{array}$ & $\begin{array}{c}\mathrm{u}_{\mathrm{e}} \\
(\mathrm{mm})\end{array}$ & $\begin{array}{c}\mathrm{u}_{\mathrm{ho} / 10, \text { off }} \\
(\mathrm{mm})\end{array}$ & $\begin{array}{c}\mathrm{u}_{0.1 \mathrm{~mm}, \text { off }} \\
(\mathrm{mm})\end{array}$ & $\begin{array}{c}\mathrm{u}_{\mathrm{e} 1.5} \\
(\mathrm{~mm})\end{array}$ & $\begin{array}{c}\mathrm{u}_{\mathrm{m}} \\
(\mathrm{mm})\end{array}$ & $\begin{array}{c}\mathrm{u}_{\text {infl }} \\
(\mathrm{mm})\end{array}$ & $\begin{array}{c}\mathrm{u}_{\mathrm{f}} \\
(\mathrm{mm})\end{array}$ & $\begin{array}{c}\mathrm{E}_{\mathrm{SP}} \\
(\mathrm{J})\end{array}$ & $\begin{array}{c}\mathrm{E}_{\mathrm{m}} \\
(\mathrm{J})\end{array}$ & $\begin{array}{c}\mathrm{E}_{\mathrm{PL}} \\
(\mathrm{J})\end{array}$ \\
\hline SP9 & 0.0014 & 0.45 & $1.43 \mathrm{E}-03$ & 0.151 & 0.241 & 0.347 & 0.391 & 0.955 & 1.663 & 1.448 & 1.18 & 0.71 & 0.53 \\
SP10 & 0.0014 & 0.46 & $1.44 \mathrm{E}-03$ & 0.121 & 0.203 & 0.307 & 0.354 & 0.891 & 1.676 & 1.306 & 1.07 & 0.66 & 0.47 \\
\hline
\end{tabular}

Test and analysis details for each of the tests performed on this group of specimens are provided in Annex 8 (rough) and Annex 9 (polished). 


\subsection{Standard $\left(900^{\circ} \mathrm{C}\right) \mathrm{HIP}$, scan length $=26 \mathrm{~mm}$}

Test results for the rough specimens is presented in Tables 21 and 22, which describe elastic slope/forces and displacements/energies, respectively. Likewise, Tables 23 and 24 describe the equivalent measurements for the polished specimens.

Table 21 - Test results for rough specimens with standard HIP and scan length $=26 \mathrm{~mm}$ (linear elastic slopes and forces).

\begin{tabular}{|c|c|c|c|c|c|c|c|c|c|c|c|c|c|c|c|c|}
\hline $\begin{array}{c}\text { Specimen } \\
\text { id }\end{array}$ & $\begin{array}{c}\text { Displacement } \\
\text { rate }(\mathrm{mm} / \mathrm{s})\end{array}$ & $\begin{array}{c}\mathrm{h}_{0} \\
(\mathrm{~mm})\end{array}$ & $\begin{array}{l}\dot{\varepsilon}_{S P}^{\max } \\
\left(\mathrm{s}^{-1}\right) \\
\end{array}$ & 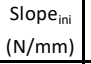 & $\begin{array}{l}\mathrm{F}_{\mathrm{e}, \mathrm{proj}} \\
(\mathrm{N})\end{array}$ & $\begin{array}{l}F_{e, i n t} \\
(N)\end{array}$ & $\begin{array}{c}\mathrm{F}_{\mathrm{h} 0 / 10, \text { off }} \\
(\mathrm{N})\end{array}$ & $\begin{array}{c}F_{0.1 \mathrm{~mm}, \text { off }} \\
(\mathrm{N})\end{array}$ & $\begin{array}{l}\mathrm{F}_{0.1 \mathrm{~mm}} \\
(\mathrm{~N})\end{array}$ & $\begin{array}{l}F_{0.48 m m} \\
(\mathrm{~N})\end{array}$ & $\begin{array}{l}\mathrm{F}_{0.5 \mathrm{~mm}} \\
(\mathrm{~N})\end{array}$ & $\begin{array}{l}\mathrm{F}_{0.65 \mathrm{~mm}} \\
(\mathrm{~N})\end{array}$ & $\begin{array}{l}\mathrm{F}_{0.9 \mathrm{~mm}} \\
(\mathrm{~N})\end{array}$ & $\begin{array}{l}F_{m} \\
(N) \\
\end{array}$ & $\begin{array}{l}F_{\text {infl }} \\
(\mathrm{N})\end{array}$ & $\begin{array}{l}F_{f} \\
(N)\end{array}$ \\
\hline SP1 & 0.0011 & 0.49 & $1.10 \mathrm{E}-03$ & 1685.886 & 343.4 & 356.2 & 595.2 & 759.3 & 176.4 & 682.5 & 710.3 & 880.1 & 1035.2 & 1121.8 & 584.1 & 897.5 \\
\hline SP2 & 0.0012 & 0.49 & $1.17 \mathrm{E}-03$ & 1482.609 & 412.2 & 420.5 & 668.2 & 920.9 & 146.5 & 641.9 & 668.0 & 844.5 & 1062.3 & 1085.3 & & 868.2 \\
\hline SP3 & 0.0011 & 0.50 & $1.14 \mathrm{E}-03$ & 1615.912 & 371.8 & 383.3 & 584.3 & 743.4 & 157.2 & 663.6 & 691.1 & 838.0 & 1046.7 & 1097.0 & 1083.1 & 877.6 \\
\hline SP4 & 0.0012 & 0.49 & $1.16 \mathrm{E}-03$ & 1821.886 & 369.8 & 379.0 & 580.9 & 724.9 & 187.2 & 704.5 & 724.9 & 860.5 & 1049.7 & 1094.2 & 688.8 & 875.4 \\
\hline SP5 & 0.0012 & 0.48 & 1.17E-03 & 1641.543 & 381.6 & 390.5 & 573.8 & 715.8 & 162.4 & 662.9 & 679.5 & 828.5 & 1026.5 & 1071.5 & 46.1 & 857.2 \\
\hline SP6 & 0.0012 & 0.50 & $1.15 \mathrm{E}-03$ & 1952.197 & 410.2 & 423.9 & 590.8 & 712.0 & 200.5 & 728.9 & 753.7 & 902.6 & 1027.1 & 1057.0 & 632.8 & 845.6 \\
\hline & & & Mean & 1700.006 & 381.5 & 392.2 & 598.9 & 762.7 & 171.7 & 680.7 & 704.6 & 859.0 & 1041.3 & 1087.8 & 607.0 & 870.2 \\
\hline & & & SD & $\begin{array}{c}165.170 \\
9.7 \%\end{array}$ & $\begin{array}{c}26.249 \\
6.9 \% \\
\end{array}$ & $\begin{array}{c}25.916 \\
6.6 \%\end{array}$ & $\begin{array}{c}34.762 \\
5.8 \% \\
\end{array}$ & $\begin{array}{l}79.509 \\
10.4 \% \\
\end{array}$ & $\begin{array}{l}20.112 \\
11.7 \% \\
\end{array}$ & $\begin{array}{c}31.655 \\
4.7 \% \\
\end{array}$ & $\begin{array}{c}31.632 \\
4.5 \% \\
\end{array}$ & $\begin{array}{c}28.027 \\
3.3 \% \\
\end{array}$ & $\begin{array}{c}14.132 \\
1.4 \% \\
\end{array}$ & $\begin{array}{c}22.394 \\
2.1 \% \\
\end{array}$ & $\begin{array}{c}370.515 \\
61.0 \% \\
\end{array}$ & $\begin{array}{c}17.915 \\
2.1 \% \\
\end{array}$ \\
\hline
\end{tabular}

Table 22 - Test results for rough specimens with standard HIP and scan length $=26 \mathrm{~mm}$ (displacements and energies).

\begin{tabular}{|c|c|c|c|c|c|c|c|c|c|c|c|c|c|}
\hline $\begin{array}{c}\text { Specimen } \\
\text { id }\end{array}$ & $\begin{array}{c}\text { Displacement } \\
\text { rate }(\mathrm{mm} / \mathrm{s})\end{array}$ & $\begin{array}{c}\mathrm{h}_{0} \\
(\mathrm{~mm})\end{array}$ & $\begin{array}{c}\dot{\varepsilon}_{S P}^{\max } \\
\left(\mathrm{s}^{-1}\right)\end{array}$ & $\begin{array}{c}\mathrm{u}_{\mathrm{e}} \\
(\mathrm{mm})\end{array}$ & $\begin{array}{c}\mathrm{u}_{\mathrm{h} / 10, \text { off }} \\
(\mathrm{mm})\end{array}$ & $\begin{array}{c}\mathrm{u}_{0.1 \mathrm{~mm}, \text { off }} \\
(\mathrm{mm})\end{array}$ & $\begin{array}{c}\mathrm{u}_{\mathrm{e} 1.5} \\
(\mathrm{~mm})\end{array}$ & $\begin{array}{c}\mathrm{u}_{\mathrm{m}} \\
(\mathrm{mm})\end{array}$ & $\begin{array}{c}\mathrm{u}_{\text {infl }} \\
(\mathrm{mm})\end{array}$ & $\begin{array}{c}\mathrm{u}_{\mathrm{f}} \\
(\mathrm{mm})\end{array}$ & $\begin{array}{c}\mathrm{E}_{\mathrm{SP}} \\
(\mathrm{J})\end{array}$ & $\begin{array}{c}\mathrm{E}_{\mathrm{m}} \\
(\mathrm{J})\end{array}$ & $\begin{array}{c}\mathrm{E}_{\mathrm{PL}} \\
(\mathrm{J})\end{array}$ \\
\hline SP1 & 0.0011 & 0.49 & $1.10 \mathrm{E}-03$ & 0.211 & 0.403 & 0.552 & 0.042 & 1.201 & 0.389 & 1.491 & 1.18 & 0.89 & 0.51 \\
$\mathrm{SP} 2$ & 0.0012 & 0.49 & $1.17 \mathrm{E}-03$ & 0.284 & 0.501 & 0.721 & 0.057 & 0.924 & & 1.799 & 1.44 & 0.56 & 0.16 \\
$\mathrm{SP3}$ & 0.0011 & 0.50 & $1.14 \mathrm{E}-03$ & 0.237 & 0.412 & 0.561 & 0.082 & 1.213 & 1.131 & 1.726 & 1.39 & 0.88 & 0.50 \\
SP4 & 0.0012 & 0.49 & $1.16 \mathrm{E}-03$ & 0.208 & 0.369 & 0.498 & 0.114 & 1.238 & 2.005 & 1.800 & 1.49 & 0.92 & 0.60 \\
SP5 & 0.0012 & 0.48 & $1.17 \mathrm{E}-03$ & 0.238 & 0.400 & 0.536 & 0.078 & 1.146 & 0.014 & 1.733 & 1.38 & 0.80 & 0.45 \\
SP6 & 0.0012 & 0.50 & $1.15 \mathrm{E}-03$ & 0.217 & 0.353 & 0.465 & 0.559 & 0.948 & 1.994 & 1.708 & 1.38 & 0.63 & 0.34 \\
\hline
\end{tabular}

Table 23 - Test results for polished specimens with standard HIP and scan length $=26 \mathrm{~mm}$ (linear elastic slopes and forces).

\begin{tabular}{|c|c|c|c|c|c|c|c|c|c|c|c|c|c|c|c|c|}
\hline $\begin{array}{c}\text { Specimen } \\
\text { id } \\
\end{array}$ & \begin{tabular}{|l} 
Displacement \\
rate $(\mathrm{mm} / \mathrm{s})$
\end{tabular} & $\begin{array}{c}\mathrm{h}_{0} \\
(\mathrm{~mm}) \\
\end{array}$ & $\begin{array}{l}\dot{\varepsilon}_{S P}^{\max } \\
\left(\mathrm{s}^{-1}\right) \\
\end{array}$ & $\begin{array}{r}S^{S l o p e}{ }_{\text {ini }} \\
(\mathrm{N} / \mathrm{mm})\end{array}$ & $\begin{array}{c}\mathrm{F}_{\mathrm{e}, \text { proj }} \\
(\mathrm{N})\end{array}$ & $\begin{array}{l}\mathrm{F}_{\mathrm{e}, \text { int }} \\
(\mathrm{N})\end{array}$ & $\begin{array}{c}F_{\text {ho/ } 10, \text { off }} \\
(\mathrm{N})\end{array}$ & \begin{tabular}{|c|}
$F_{0.1 \mathrm{~mm}, \text { off }}$ \\
$(\mathrm{N})$
\end{tabular} & $\begin{array}{c}\mathrm{F}_{0.1 \mathrm{~mm}} \\
(\mathrm{~N})\end{array}$ & $\begin{array}{c}\mathrm{F}_{0.48 \mathrm{~mm}} \\
(\mathrm{~N})\end{array}$ & $\begin{array}{c}\mathrm{F}_{0.5 \mathrm{~mm}} \\
(\mathrm{~N})\end{array}$ & $\begin{array}{c}\mathrm{F}_{0.65 \mathrm{~mm}} \\
(\mathrm{~N})\end{array}$ & $\begin{array}{c}\mathrm{F}_{0.9 \mathrm{~mm}} \\
(\mathrm{~N})\end{array}$ & $\begin{array}{l}\mathrm{F}_{\mathrm{m}} \\
(\mathrm{N}) \\
\end{array}$ & $\begin{array}{l}F_{\text {infl }} \\
(\mathrm{N}) \\
\end{array}$ & $\begin{array}{l}F_{f} \\
(N)\end{array}$ \\
\hline SP7 & 0.0014 & 0.45 & $1.44 \mathrm{E}-03$ & 3201.67 & 373.5 & 397.2 & 522.2 & 653.3 & 309.9 & 870.1 & 882.4 & 965.2 & 918.4 & 992.2 & 593.6 & 793.7 \\
\hline SP8 & 0.0014 & 0.46 & $1.43 E-03$ & 3288.00 & 372.2 & 402.0 & 515.6 & 660.5 & 324.6 & 882.8 & 902.5 & 1026.6 & 1079.7 & 1091.8 & 559.0 & 873.4 \\
\hline SP9 & 0.0015 & 0.45 & $1.46 \mathrm{E}-03$ & 2989.78 & 373.8 & 406.2 & 531.7 & 690.7 & 300.6 & 882.8 & 915.7 & 961.6 & 969.8 & 995.9 & 509.4 & 796.7 \\
\hline SP10 & 0.0014 & 0.45 & 1.44E-03 & 2849.28 & 434.7 & 467.2 & 595.3 & 748.0 & 293.0 & 872.8 & 878.4 & 921.6 & 1033.5 & 1040.8 & 967.1 & 832.7 \\
\hline & & & Mean & 2919.5 & 404.3 & 436.7 & 563.5 & 719.3 & 296.8 & 877.8 & 894.8 & 968.8 & 1000.3 & 1018.4 & 738.3 & 814.7 \\
\hline & & & SD & $\begin{array}{c}99.345 \\
3.4 \%\end{array}$ & $\begin{array}{c}43.068 \\
10.7 \%\end{array}$ & $\begin{array}{c}43.075 \\
9.9 \%\end{array}$ & $\begin{array}{c}44.949 \\
8.0 \%\end{array}$ & $\begin{array}{c}40.531 \\
5.6 \%\end{array}$ & $\begin{array}{l}5.429 \\
1.8 \%\end{array}$ & $\begin{array}{l}7.055 \\
0.8 \%\end{array}$ & $\begin{array}{c}17.491 \\
2.0 \%\end{array}$ & $\begin{array}{c}43.314 \\
4.5 \%\end{array}$ & $\begin{array}{c}70.800 \\
7.1 \%\end{array}$ & $\begin{array}{c}31.765 \\
3.1 \%\end{array}$ & $\begin{array}{c}323.589 \\
43.8 \%\end{array}$ & $\begin{array}{c}25.412 \\
3.1 \%\end{array}$ \\
\hline
\end{tabular}

Table 24 - Test results for polished specimens with standard HIP and scan length $=26 \mathrm{~mm}$ (displacements and energies).

\begin{tabular}{|c|c|c|c|c|c|c|c|c|c|c|c|c|c|}
\hline $\begin{array}{c}\text { Specimen } \\
\text { id }\end{array}$ & $\begin{array}{c}\text { Displacement } \\
\text { rate }(\mathrm{mm} / \mathrm{s})\end{array}$ & $\begin{array}{c}\mathrm{h}_{0} \\
(\mathrm{~mm})\end{array}$ & $\begin{array}{c}\dot{\varepsilon}_{S P}^{\max } \\
\left(\mathrm{s}^{-1}\right)\end{array}$ & $\begin{array}{c}\mathrm{u}_{\mathrm{e}} \\
(\mathrm{mm})\end{array}$ & $\begin{array}{c}\mathrm{u}_{\mathrm{ho} / 10, \text { off }} \\
(\mathrm{mm})\end{array}$ & $\begin{array}{c}\mathrm{u}_{0.1 \mathrm{~mm}, \text { off }} \\
(\mathrm{mm})\end{array}$ & $\begin{array}{c}\mathrm{u}_{\mathrm{e} 1.5} \\
(\mathrm{~mm})\end{array}$ & $\begin{array}{c}\mathrm{u}_{\mathrm{m}} \\
(\mathrm{mm})\end{array}$ & $\begin{array}{c}\mathrm{u}_{\text {infl }} \\
(\mathrm{mm})\end{array}$ & $\begin{array}{c}\mathrm{u}_{\mathrm{f}} \\
(\mathrm{mm})\end{array}$ & $\begin{array}{c}\mathrm{E}_{\mathrm{SP}} \\
(\mathrm{J})\end{array}$ & $\begin{array}{c}\mathrm{E}_{\mathrm{m}} \\
(\mathrm{J})\end{array}$ & $\begin{array}{c}\mathrm{E}_{\mathrm{PL}} \\
(\mathrm{J})\end{array}$ \\
\hline SP7 & 0.0014 & 0.45 & $1.44 \mathrm{E}-03$ & 0.124 & 0.209 & 0.305 & 0.269 & 0.618 & 1.667 & 1.369 & 1.05 & 0.38 & 0.23 \\
SP8 & 0.0014 & 0.46 & $1.43 \mathrm{E}-03$ & 0.122 & 0.203 & 0.302 & 0.357 & 0.832 & 1.487 & 1.273 & 1.06 & 0.62 & 0.43 \\
SP9 & 0.0015 & 0.45 & $1.46 \mathrm{E}-03$ & 0.136 & 0.223 & 0.333 & 0.380 & 0.558 & 1.648 & 1.323 & 1.04 & 0.32 & 0.16 \\
SP10 & 0.0014 & 0.45 & $1.44 \mathrm{E}-03$ & 0.164 & 0.254 & 0.364 & 0.457 & 0.899 & 0.707 & 1.356 & 1.09 & 0.65 & 0.46 \\
\hline
\end{tabular}

Test and analysis details for each of the tests performed on this group of specimens are provided in Annex 10 (rough) and Annex 11 (polished). 


\subsection{Non-standard $\left(1050^{\circ} \mathrm{C}\right)+$ additional $\left(800^{\circ} \mathrm{C}\right) \mathrm{HIP}$, scan length $=78 \mathrm{~mm}$}

Test results for the rough specimens is presented in Tables 25 and 26, which describe elastic slope/forces and displacements/energies, respectively. Likewise, Tables 27 and 28 describe the equivalent measurements for the polished specimens.

Table 25 - Test results for rough specimens with standard + additional HIP and scan length = $78 \mathrm{~mm}$ (linear elastic slopes and forces).

\begin{tabular}{|c|c|c|c|c|c|c|c|c|c|c|c|c|c|c|c|c|}
\hline $\begin{array}{c}\text { Specimen } \\
\text { id }\end{array}$ & \begin{tabular}{|c|} 
Displacement \\
rate $(\mathrm{mm} / \mathrm{s})$
\end{tabular} & $\begin{array}{c}\mathrm{h}_{0} \\
(\mathrm{~mm})\end{array}$ & $\begin{array}{l}\dot{\varepsilon}_{S P}^{\max } \\
\left(s^{-1}\right)\end{array}$ & \begin{tabular}{|c|} 
Slope $_{\text {ini }}$ \\
$(\mathrm{N} / \mathrm{mm})$
\end{tabular} & $\begin{array}{l}\mathrm{F}_{\mathrm{e}, \mathrm{proj}} \\
(\mathrm{N})\end{array}$ & $\begin{array}{l}\mathrm{F}_{\mathrm{e}, \mathrm{int}} \\
\text { (N) }\end{array}$ & $\begin{array}{c}F_{\mathrm{ho} / 10, \text { off }} \\
\text { (N) }\end{array}$ & \begin{tabular}{|c|}
$F_{0.1 \mathrm{~mm}, \text { off }}$ \\
$(\mathrm{N})$
\end{tabular} & $\begin{array}{l}\mathrm{F}_{0.1 \mathrm{~mm}} \\
(\mathrm{~N})\end{array}$ & $\begin{array}{c}\mathrm{F}_{0.48 \mathrm{~mm}} \\
(\mathrm{~N})\end{array}$ & $\begin{array}{l}\mathrm{F}_{0.5 \mathrm{~mm}} \\
(\mathrm{~N})\end{array}$ & $\begin{array}{c}\mathrm{F}_{0.65 \mathrm{~mm}} \\
(\mathrm{~N})\end{array}$ & $\begin{array}{c}\mathrm{F}_{0.9 \mathrm{~mm}} \\
(\mathrm{~N})\end{array}$ & $\begin{array}{l}F_{m} \\
\text { (N) }\end{array}$ & $\begin{array}{l}\mathrm{F}_{\text {infl }} \\
\text { (N) }\end{array}$ & $\begin{array}{l}F_{f} \\
(N)\end{array}$ \\
\hline SP1 & 0.0010 & 0.47 & $1.04 \mathrm{E}-03$ & 2139.702 & 407.3 & 420.1 & 576.8 & 703.8 & 218.3 & 768.0 & 791.8 & 932.3 & 1126.7 & 1192.9 & 702.3 & 954.3 \\
\hline SP2 & 0.0011 & 0.48 & $1.07 \mathrm{E}-03$ & 2011.628 & 365.1 & 377.5 & 570.1 & 732.7 & 207.9 & 748.0 & 779.8 & 931.9 & 1138.0 & 1194.5 & 1189.5 & 955.6 \\
\hline SP4 & 0.0011 & 0.49 & $1.07 \mathrm{E}-03$ & 1844.336 & 456.7 & 465.1 & 646.7 & 771.7 & 182.3 & 731.7 & 753.7 & 910.0 & 1138.2 & 1230.4 & 714.9 & 984.3 \\
\hline SP5 & 0.0011 & 0.49 & $1.08 \mathrm{E}-03$ & 1930.462 & 475.7 & 485.1 & 644.0 & 765.3 & 196.2 & 748.9 & 772.8 & 928.4 & 1162.1 & 1225.3 & 61.4 & 980.2 \\
\hline SP6 & 0.0011 & 0.48 & $1.11 \mathrm{E}-03$ & |1918.267 & 414.5 & 430.1 & 594.2 & 713.8 & 193.2 & 724.3 & 747.6 & 901.9 & 1106.7 & 1156.0 & 901.5 & 924.8 \\
\hline SP7 & 0.0011 & 0.47 & $1.04 \mathrm{E}-03$ & 1874.916 & 380.2 & 393.6 & 604.0 & 742.3 & 191.4 & 732.4 & 752.2 & 912.0 & 1122.9 & 1196.6 & 678.8 & 957.2 \\
\hline & & & Mean & 1953.218 & 416.6 & 428.6 & 606.0 & $\begin{array}{l}738.3 \\
\end{array}$ & 198.2 & 742.2 & 766.3 & 919.4 & 1132.4 & 1199.3 & 708.1 & 959.4 \\
\hline & & & SD & \begin{tabular}{|c|}
107.623 \\
$5.5 \%$
\end{tabular} & $\begin{array}{l}42.811 \\
10.3 \%\end{array}$ & $\begin{array}{c}41.099 \\
9.6 \%\end{array}$ & $\begin{array}{c}32.814 \\
5.4 \%\end{array}$ & $\begin{array}{c}27.146 \\
3.7 \%\end{array}$ & $\begin{array}{c}12.817 \\
6.5 \%\end{array}$ & $\begin{array}{c}15.961 \\
2.2 \%\end{array}$ & $\begin{array}{c}17.816 \\
2.3 \%\end{array}$ & $\begin{array}{c}13.055 \\
1.4 \%\end{array}$ & $\begin{array}{c}18.590 \\
1.6 \%\end{array}$ & $\begin{array}{c}26.767 \\
2.2 \%\end{array}$ & $\begin{array}{c}371.000 \\
52.4 \%\end{array}$ & $\begin{array}{c}21.413 \\
2.2 \%\end{array}$ \\
\hline
\end{tabular}

Table 26 - Test results for rough specimens with standard + additional HIP and scan length $=$ $78 \mathrm{~mm}$ (displacements and energies).

\begin{tabular}{|c|c|c|c|c|c|c|c|c|c|c|c|c|c|}
\hline $\begin{array}{c}\text { Specimen } \\
\text { id }\end{array}$ & $\begin{array}{c}\text { Displacement } \\
\text { rate }(\mathrm{mm} / \mathrm{s})\end{array}$ & $\begin{array}{c}\mathrm{h}_{0} \\
(\mathrm{~mm})\end{array}$ & $\begin{array}{l}\dot{\varepsilon}_{S P}^{\max } \\
\left(\mathrm{s}^{-1}\right)\end{array}$ & $\begin{array}{c}\mathrm{u}_{\mathrm{e}} \\
(\mathrm{mm})\end{array}$ & $\begin{array}{c}u_{\mathrm{h} 0 / 10, \text { off }} \\
(\mathrm{mm})\end{array}$ & $\begin{array}{c}\mathrm{u}_{0.1 \mathrm{~mm}, \text { off }} \\
(\mathrm{mm})\end{array}$ & $\begin{array}{c}\mathrm{u}_{\mathrm{e} 1.5} \\
(\mathrm{~mm})\end{array}$ & $\begin{array}{c}\mathrm{u}_{\mathrm{m}} \\
(\mathrm{mm})\end{array}$ & $\begin{array}{c}\mathrm{u}_{\text {infl }} \\
(\mathrm{mm})\end{array}$ & $\begin{array}{c}\mathrm{u}_{\mathrm{f}} \\
(\mathrm{mm})\end{array}$ & $\begin{array}{l}E_{S P} \\
(J)\end{array}$ & $\begin{array}{l}E_{m} \\
(J)\end{array}$ & $\begin{array}{l}E_{P L} \\
(J)\end{array}$ \\
\hline SP1 & 0.0010 & 0.47 & $1.04 \mathrm{E}-03$ & 0.196 & 0.320 & 0.430 & 0.104 & 1.066 & 1.760 & 1.498 & 1.29 & 0.81 & 0.48 \\
\hline SP2 & 0.0011 & 0.48 & $1.07 \mathrm{E}-03$ & 0.188 & 0.335 & 0.465 & 0.072 & 1.071 & 1.077 & 1.458 & 1.23 & 0.80 & 0.45 \\
\hline SP4 & 0.0011 & 0.49 & $1.07 \mathrm{E}-03$ & 0.252 & 0.401 & 0.519 & 0.096 & 1.065 & 1.831 & 1.560 & 1.34 & 0.79 & 0.38 \\
\hline SP5 & 0.0011 & 0.49 & $1.08 \mathrm{E}-03$ & 0.251 & 0.385 & 0.496 & 0.066 & 1.005 & 0.027 & 1.524 & 1.32 & 0.74 & 0.35 \\
\hline SP6 & 0.0011 & 0.48 & $1.11 \mathrm{E}-03$ & 0.224 & 0.360 & 0.472 & 0.075 & 1.002 & 0.649 & 1.628 & 1.36 & 0.71 & 0.36 \\
\hline SP7 & 0.0011 & 0.47 & $1.04 \mathrm{E}-03$ & 0.210 & 0.372 & 0.496 & 0.082 & 1.023 & 1.843 & 1.412 & 1.14 & 0.73 & 0.35 \\
\hline & & & Mean & 0.220 & 0.362 & 0.480 & 0.083 & 1.039 & 1.198 & 1.513 & 1.28 & 0.76 & 0.39 \\
\hline & & & SD & $\begin{array}{l}0.027 \\
12.4 \%\end{array}$ & $\begin{array}{l}0.031 \\
8.5 \%\end{array}$ & $\begin{array}{l}0.031 \\
6.5 \%\end{array}$ & $\begin{array}{l}0.015 \\
17.7 \%\end{array}$ & $\begin{array}{l}0.032 \\
3.1 \%\end{array}$ & $\begin{array}{l}0.751 \\
62.7 \%\end{array}$ & $\begin{array}{l}0.076 \\
5.0 \%\end{array}$ & $\begin{array}{l}0.083 \\
6.5 \%\end{array}$ & $\begin{array}{l}0.043 \\
5.7 \%\end{array}$ & $\begin{array}{l}0.055 \\
14.1 \%\end{array}$ \\
\hline
\end{tabular}

Table 27 - Test results for polished specimens with standard + additional HIP and scan length $=78 \mathrm{~mm}$ (linear elastic slopes and forces).

\begin{tabular}{|c|c|c|c|c|c|c|c|c|c|c|c|c|c|c|c|c|}
\hline $\begin{array}{c}\text { Specimen } \\
\text { id }\end{array}$ & $\begin{array}{l}\text { Displacement } \\
\text { rate }(\mathrm{mm} / \mathrm{s})\end{array}$ & $\begin{array}{c}\mathrm{h}_{0} \\
(\mathrm{~mm})\end{array}$ & $\begin{array}{l}\dot{\varepsilon}_{S P}^{\max } \\
\left(\mathrm{s}^{-1}\right)\end{array}$ & $\begin{array}{l}\text { Slope }_{\text {ini }} \\
(\mathrm{N} / \mathrm{mm})\end{array}$ & $\begin{array}{c}\mathrm{F}_{\mathrm{e}, \text { proj }} \\
(\mathrm{N})\end{array}$ & $\begin{array}{l}F_{e, \text { int }} \\
(N)\end{array}$ & $\begin{array}{c}\mathrm{F}_{\mathrm{hO} / 10, \text { off }} \\
(\mathrm{N})\end{array}$ & $\begin{array}{l}F_{0.1 \mathrm{~mm}, \text { off }} \\
(\mathrm{N})\end{array}$ & $\begin{array}{c}\mathrm{F}_{0.1 \mathrm{~mm}} \\
(\mathrm{~N})\end{array}$ & $\begin{array}{c}F_{0.48 m m} \\
(N)\end{array}$ & $\begin{array}{l}\mathrm{F}_{0.5 \mathrm{~mm}} \\
(\mathrm{~N})\end{array}$ & $\begin{array}{c}\mathrm{F}_{0.65 \mathrm{~mm}} \\
(\mathrm{~N})\end{array}$ & $\begin{array}{l}\mathrm{F}_{0.9 \mathrm{~mm}} \\
(\mathrm{~N})\end{array}$ & $\begin{array}{l}F_{m} \\
(N)\end{array}$ & $\begin{array}{l}F_{\text {infl }} \\
(N)\end{array}$ & $\begin{array}{l}F_{f} \\
(N)\end{array}$ \\
\hline SP8 & 0.0014 & 0.43 & $1.43 \mathrm{E}-03$ & 2957.72 & 367.2 & 392.6 & 490.5 & 657.8 & 295.8 & 833.8 & 859.5 & 975.0 & 999.1 & 1023.0 & 849.4 & 818.4 \\
\hline SP9 & 015 & 0.46 & 47E-03 & 3214.86 & 418.1 & 453.3 & 576.1 & 713.0 & 321.2 & 908.7 & 920.9 & 1039.7 & 1045.0 & 1064.1 & 545.6 & 851.3 \\
\hline SP10 & 0.0015 & 0.44 & $1.46 \mathrm{E}-03$ & 3294.63 & 403.9 & 436.2 & 549.7 & 691.2 & 325.1 & 904.6 & 926.6 & 1069.5 & 1024.2 & 1096.7 & 895.1 & 877.4 \\
\hline & & & Mean & 3254.7 & 411.0 & 444.7 & 562.9 & 702.1 & 323.1 & 906.7 & 902.3 & 1028.1 & 1022.8 & 1080.4 & 720.4 & 864.3 \\
\hline & & & SD & $\begin{array}{c}56.403 \\
1.7 \%\end{array}$ & $\begin{array}{c}10.033 \\
2.4 \%\end{array}$ & $\begin{array}{c}12.074 \\
2.7 \%\end{array}$ & $\begin{array}{c}18.685 \\
3.3 \%\end{array}$ & $\begin{array}{c}15.372 \\
2.2 \%\end{array}$ & $\begin{array}{l}2.758 \\
0.9 \%\end{array}$ & $\begin{array}{l}2.886 \\
0.3 \%\end{array}$ & $\begin{array}{c}37.219 \\
4.1 \%\end{array}$ & $\begin{array}{c}48.302 \\
4.7 \%\end{array}$ & $\begin{array}{c}22.945 \\
2.2 \%\end{array}$ & $\begin{array}{c}23.017 \\
2.1 \%\end{array}$ & $\begin{array}{c}247.087 \\
34.3 \%\end{array}$ & $\begin{array}{c}18.413 \\
2.1 \%\end{array}$ \\
\hline
\end{tabular}

Table 28 - Test results for polished specimens with standard + additional HIP and scan length $=78 \mathrm{~mm}$ (displacements and energies).

\begin{tabular}{|c|c|c|c|c|c|c|c|c|c|c|c|c|c|}
\hline $\begin{array}{c}\text { Specimen } \\
\text { id }\end{array}$ & $\begin{array}{c}\text { Displacement } \\
\text { rate }(\mathrm{mm} / \mathrm{s})\end{array}$ & $\begin{array}{c}\mathrm{h}_{0} \\
(\mathrm{~mm})\end{array}$ & $\begin{array}{l}\dot{\varepsilon}_{S P}^{\max } \\
\left(\mathrm{s}^{-1}\right)\end{array}$ & $\begin{array}{c}\mathrm{u}_{\mathrm{e}} \\
(\mathrm{mm})\end{array}$ & $\begin{array}{c}u_{\mathrm{h} / 10, \text { off }} \\
(\mathrm{mm})\end{array}$ & $\begin{array}{c}\mathrm{u}_{0.1 \mathrm{~mm}, \text { off }} \\
(\mathrm{mm})\end{array}$ & $\begin{array}{r}\mathrm{u}_{\mathrm{e} 1.5} \\
(\mathrm{~mm})\end{array}$ & $\begin{array}{r}\mathrm{u}_{\mathrm{m}} \\
(\mathrm{mm})\end{array}$ & $\begin{array}{r}u_{\text {infl }} \\
(\mathrm{mm})\end{array}$ & $\begin{array}{c}\mathrm{u}_{\mathrm{f}} \\
(\mathrm{mm})\end{array}$ & $\begin{array}{l}E_{S p} \\
(J)\end{array}$ & $\begin{array}{l}E_{m} \\
(J)\end{array}$ & $\begin{array}{l}E_{P L} \\
(J)\end{array}$ \\
\hline SP8 & 0.0014 & 0.43 & $1.43 \mathrm{E}-03$ & 0.133 & 0.209 & 0.323 & 0.386 & 0.761 & 1.174 & 1.260 & 0.97 & 0.51 & 0.33 \\
\hline SP9 & 0.0015 & 0.46 & 1.47E-03 & 0.141 & 0.226 & 0.322 & 0.342 & 0.719 & 1.614 & 1.242 & 1.02 & 0.51 & 0.33 \\
\hline SP10 & 0.0015 & 0.44 & $1.46 \mathrm{E}-03$ & 0.132 & 0.211 & 0.311 & 0.282 & 0.693 & 0.470 & 1.109 & 0.89 & 0.48 & 0.30 \\
\hline & & & Mean & 0.137 & 0.219 & 0.316 & 0.312 & 0.706 & 1.042 & 1.176 & 0.958 & 0.494 & 0.315 \\
\hline & & & SD & 0.006 & 0.011 & 0.008 & 0.043 & 0.019 & 0.809 & 0.094 & 0.089 & 0.019 & 0.023 \\
\hline
\end{tabular}

Test and analysis details for each of the tests performed on this group of specimens are provided in Annex 12 (rough) and Annex 13 (polished). 


\subsection{Comparison between different conditions (non-supported specimens)}

\subsection{1. rough specimens}

Average values and standard deviations (absolute and relative) for the various characteristic parameters of the different conditions examined (excluding the preliminary tests) are compared in Table 29 (elastic slopes and forces) and Table 30 (displacements and energies).

Table 29 - Average values and standard deviations for rough specimens in the different conditions (elastic slopes and forces).

\begin{tabular}{|c|c|c|c|c|c|c|c|c|c|c|c|c|c|c|c|}
\hline $\begin{array}{c}\text { Material } \\
\text { condition }\end{array}$ & $\begin{array}{c}\text { Scan } \\
\text { length }(\mathrm{mm}) \\
\end{array}$ & $\begin{array}{c}\begin{array}{c}\text { Displacement } \\
\text { rate }(\mathrm{mm} / \mathrm{s})\end{array} \\
\end{array}$ & $\begin{array}{r}\text { Slope }_{\text {ini }} \\
(\mathrm{N} / \mathrm{mm}) \\
\end{array}$ & $\begin{array}{l}F_{e, \text { proj }} \\
(\mathrm{N})\end{array}$ & $\begin{array}{l}F_{e, \text { int }} \\
(\mathrm{N})\end{array}$ & $\begin{array}{c}\mathrm{F}_{\mathrm{h} 0 / 10, \text { off }} \\
(\mathrm{N})\end{array}$ & $\begin{array}{c}F_{0.1 \mathrm{~mm}, \text { off }} \\
(\mathrm{N})\end{array}$ & $\begin{array}{l}F_{0.1 \mathrm{~mm}} \\
(\mathrm{~N})\end{array}$ & $\begin{array}{c}\mathrm{F}_{0.48 \mathrm{~mm}} \\
(\mathrm{~N})\end{array}$ & $\begin{array}{l}\mathrm{F}_{0.5 \mathrm{~mm}} \\
(\mathrm{~N})\end{array}$ & $\begin{array}{c}\mathrm{F}_{0.65 \mathrm{~mm}} \\
(\mathrm{~N})\end{array}$ & $\begin{array}{l}\mathrm{F}_{0.9 \mathrm{~mm}} \\
(\mathrm{~N})\end{array}$ & $\begin{array}{r}F_{m} \\
(N) \\
\end{array}$ & $\begin{array}{l}F_{\text {infl }} \\
(\mathrm{N})\end{array}$ & $\begin{array}{r}F_{f} \\
(N) \\
\end{array}$ \\
\hline \multirow{6}{*}{ As-Built } & \multirow{3}{*}{78} & \multirow{3}{*}{0.001} & 2727.595 & 433.4 & 456.2 & 643.7 & 804.2 & 282.8 & 889.3 & 909.0 & 1003.6 & 1067.4 & 1083.9 & 745.6 & 867.1 \\
\hline & & & 338.711 & 64.9 & 73.0 & 45.5 & 45.7 & 38.7 & 45.1 & 43.7 & 29.9 & 29.5 & 25.0 & 149.0 & 20.0 \\
\hline & & & $12.4 \%$ & $15.0 \%$ & $16.0 \%$ & $7.1 \%$ & $5.7 \%$ & $13.7 \%$ & $5.1 \%$ & $4.8 \%$ & $3.0 \%$ & $2.8 \%$ & $2.3 \%$ & $20.0 \%$ & $2.3 \%$ \\
\hline & \multirow{3}{*}{26} & \multirow{3}{*}{0.001} & 1819.712 & 408.1 & 415.7 & 670.3 & 847.4 & 180.6 & 746.2 & 769.2 & 927.7 & 1031.5 & 1101.5 & 678.5 & 881.2 \\
\hline & & & 124.177 & 35.4 & 34.6 & 58.8 & 70.1 & 12.8 & 17.5 & 19.1 & 12.4 & 30.3 & 30.0 & 210.6 & 24.0 \\
\hline & & & $6.8 \%$ & $8.7 \%$ & $8.3 \%$ & $8.8 \%$ & $8.3 \%$ & $7.1 \%$ & $2.3 \%$ & $2.5 \%$ & $1.3 \%$ & $2.9 \%$ & $2.7 \%$ & $31.0 \%$ & $2.7 \%$ \\
\hline \multirow{3}{*}{$\begin{array}{l}\text { HIP } \\
800^{\circ} \mathrm{C}\end{array}$} & \multirow{3}{*}{78} & \multirow{3}{*}{0.001} & 1853.112 & 452.4 & 456.7 & 683.8 & 837.6 & 190.5 & 751.7 & 774.9 & 925.1 & 1063.9 & 1102.8 & 632.0 & 882.2 \\
\hline & & & 203.128 & 22.6 & 19.0 & 52.0 & 48.8 & 23.3 & 41.9 & 40.7 & 26.7 & 39.6 & 35.7 & 169.8 & 28.6 \\
\hline & & & $11.0 \%$ & $5.0 \%$ & $4.2 \%$ & $7.6 \%$ & $5.8 \%$ & $12.2 \%$ & $5.6 \%$ & $5.3 \%$ & $2.9 \%$ & $3.7 \%$ & $3.2 \%$ & $26.9 \%$ & $3.2 \%$ \\
\hline \multirow{6}{*}{$\begin{array}{l}\text { HIP } \\
900^{\circ} \mathrm{C}\end{array}$} & \multirow{3}{*}{78} & \multirow{3}{*}{0.001} & 1812.213 & 385.1 & 393.7 & 667.0 & 798.0 & 183.5 & 702.0 & 722.7 & 874.4 & 1005.6 & 1084.4 & 494.3 & 867.5 \\
\hline & & & 492.099 & 42.9 & 45.2 & 173.2 & 105.6 & 50.2 & 153.7 & 154.8 & 174.7 & 129.7 & 36.3 & 255.9 & 29.0 \\
\hline & & & $27.2 \%$ & $11.1 \%$ & $11.5 \%$ & $26.0 \%$ & $13.2 \%$ & $27.4 \%$ & $21.9 \%$ & $21.4 \%$ & $20.0 \%$ & $12.9 \%$ & $3.3 \%$ & $51.8 \%$ & $3.3 \%$ \\
\hline & \multirow{3}{*}{26} & \multirow{3}{*}{0.001} & 1700.006 & 381.5 & 392.2 & 598.9 & 762.7 & 171.7 & 680.7 & 704.6 & 859.0 & 1041.3 & 1087.8 & 607.0 & 870.2 \\
\hline & & & 165.170 & 26.2 & 25.9 & 34.8 & 79.5 & 20.1 & 31.7 & 31.6 & 28.0 & 14.1 & 22.4 & 370.5 & 17.9 \\
\hline & & & $9.7 \%$ & $6.9 \%$ & $6.6 \%$ & $5.8 \%$ & $10.4 \%$ & $11.7 \%$ & $4.7 \%$ & $4.5 \%$ & $3.3 \%$ & $1.4 \%$ & $2.1 \%$ & $61.0 \%$ & $2.1 \%$ \\
\hline HIP & \multirow{3}{*}{78} & \multirow{3}{*}{0.001} & 1953.218 & 416.6 & 428.6 & 606.0 & 738.3 & 198.2 & 742.2 & 766.3 & 919.4 & 1132.4 & 1199.3 & 708.1 & 959.4 \\
\hline $1050^{\circ} \mathrm{C}+$ & & & 107.623 & 42.8 & 41.1 & 32.8 & 27.1 & 12.8 & 16.0 & 17.8 & 13.1 & 18.6 & 26.8 & 371.0 & 21.4 \\
\hline $800^{\circ} \mathrm{C}$ & & & $5.5 \%$ & $10.3 \%$ & $9.6 \%$ & $5.4 \%$ & $3.7 \%$ & $6.5 \%$ & $2.2 \%$ & $2.3 \%$ & $1.4 \%$ & $1.6 \%$ & $2.2 \%$ & $52.4 \%$ & $2.2 \%$ \\
\hline
\end{tabular}

Table 30 - Average values and standard deviations for rough specimens in the different conditions (displacements and energies).

\begin{tabular}{|c|c|c|c|c|c|c|c|c|c|c|c|c|}
\hline $\begin{array}{c}\text { Material } \\
\text { condition }\end{array}$ & $\begin{array}{c}\text { Scan } \\
\text { length }(\mathrm{mm}) \\
\end{array}$ & \begin{tabular}{|c|c|}
$\begin{array}{c}\text { Displacement } \\
\text { rate }(\mathrm{mm} / \mathrm{s})\end{array}$ \\
\end{tabular} & $\begin{array}{c}\mathrm{u}_{\mathrm{e}} \\
(\mathrm{mm})\end{array}$ & $\begin{array}{c}u_{\text {ho/ } 10, \text { off }} \\
(\mathrm{mm})\end{array}$ & $\begin{array}{c}u_{0.1 \mathrm{~mm}, \text { off }} \\
(\mathrm{mm})\end{array}$ & $\begin{array}{r}\mathrm{u}_{\mathrm{e} 1.5} \\
(\mathrm{~mm})\end{array}$ & $\begin{array}{c}\mathrm{u}_{\mathrm{m}} \\
(\mathrm{mm})\end{array}$ & $\begin{array}{r}\mathrm{u}_{\text {infl }} \\
(\mathrm{mm})\end{array}$ & $\begin{array}{c}\mathrm{u}_{\mathrm{f}} \\
(\mathrm{mm})\end{array}$ & $\begin{array}{l}E_{S P} \\
(J) \\
\end{array}$ & $\begin{array}{l}E_{m} \\
(J) \\
\end{array}$ & $\begin{array}{l}E_{P L} \\
(J) \\
\end{array}$ \\
\hline \multirow{6}{*}{ As-Built } & \multirow{3}{*}{78} & \multirow{3}{*}{0.001} & 0.167 & 0.290 & 0.401 & 0.189 & 0.928 & 1.494 & 1.387 & 1.16 & 0.71 & 0.49 \\
\hline & & & 0.018 & 0.035 & 0.051 & 0.192 & 0.062 & 0.292 & 0.064 & 0.09 & 0.07 & 0.08 \\
\hline & & & $1.8 \%$ & $3.5 \%$ & $5.1 \%$ & $19.2 \%$ & $6.7 \%$ & $29.2 \%$ & $4.6 \%$ & $7.4 \%$ & $10.5 \%$ & $17.0 \%$ \\
\hline & \multirow{3}{*}{26} & \multirow{3}{*}{0.001} & 0.230 & 0.422 & 0.570 & 0.091 & 1.098 & 1.389 & 1.501 & 1.21 & 0.80 & 0.47 \\
\hline & & & 0.028 & 0.055 & 0.069 & 0.010 & 0.066 & 0.610 & 0.069 & 0.07 & 0.08 & 0.09 \\
\hline & & & $12.2 \%$ & $13.0 \%$ & $12.1 \%$ & $11.6 \%$ & $6.0 \%$ & $43.9 \%$ & $4.6 \%$ & $5.5 \%$ & $9.8 \%$ & $18.7 \%$ \\
\hline \multirow{3}{*}{$\begin{array}{l}\text { HIP } \\
800^{\circ} \mathrm{C}\end{array}$} & \multirow{3}{*}{78} & \multirow{3}{*}{0.001} & 0.248 & 0.424 & 0.558 & 0.073 & 0.985 & 1.461 & 1.431 & 1.14 & 0.69 & 0.36 \\
\hline & & & 0.024 & 0.055 & 0.061 & 0.052 & 0.110 & 0.569 & 0.073 & 0.09 & 0.12 & 0.12 \\
\hline & & & $9.7 \%$ & $12.9 \%$ & $10.9 \%$ & $71.5 \%$ & $11.2 \%$ & $39.0 \%$ & $5.1 \%$ & $7.8 \%$ & $17.1 \%$ & $34.6 \%$ \\
\hline \multirow{6}{*}{$\begin{array}{l}\text { HIP } \\
900^{\circ} \mathrm{C}\end{array}$} & \multirow{3}{*}{78} & \multirow{3}{*}{0.001} & 0.237 & 0.504 & 0.618 & 0.086 & 1.018 & 0.980 & 1.499 & 1.16 & 0.68 & 0.32 \\
\hline & & & 0.090 & 0.379 & 0.345 & 0.044 & 0.163 & 0.860 & 0.109 & 0.10 & 0.11 & 0.18 \\
\hline & & & $38.1 \%$ & $75.3 \%$ & $55.7 \%$ & $51.4 \%$ & $16.0 \%$ & $87.7 \%$ & $7.2 \%$ & $9.0 \%$ & $16.0 \%$ & $54.7 \%$ \\
\hline & \multirow{3}{*}{26} & \multirow{3}{*}{0.001} & 0.233 & 0.406 & 0.555 & 0.155 & 1.112 & 1.107 & 1.709 & 1.38 & 0.78 & 0.43 \\
\hline & & & 0.028 & 0.052 & 0.089 & 0.199 & 0.140 & 0.909 & 0.114 & 0.10 & 0.15 & 0.15 \\
\hline & & & $12.1 \%$ & $12.7 \%$ & $16.0 \%$ & $128.1 \%$ & $12.6 \%$ & $82.1 \%$ & $6.6 \%$ & $7.6 \%$ & $19.3 \%$ & $36.0 \%$ \\
\hline HIP & \multirow{3}{*}{78} & \multirow{3}{*}{0.001} & 0.220 & 0.362 & 0.480 & 0.083 & 1.039 & 1.198 & 1.513 & 1.28 & 0.76 & 0.39 \\
\hline $1050^{\circ} \mathrm{C}+$ & & & 0.027 & 0.031 & 0.031 & 0.015 & 0.032 & 0.751 & 0.076 & 0.08 & 0.04 & 0.06 \\
\hline $800^{\circ} \mathrm{C}$ & & & $12.4 \%$ & $8.5 \%$ & $6.5 \%$ & $17.7 \%$ & $3.1 \%$ & $62.7 \%$ & $5.0 \%$ & $6.5 \%$ & $5.7 \%$ & $14.1 \%$ \\
\hline
\end{tabular}

Comparisons in terms of $F_{\text {e,proj }}$ and $F_{\mathrm{m}}$ are illustrated in Fig. 9 and Fig. 10, respectively. Considering that $F_{\mathrm{e} \text {,proj }}$ is directly proportional to the yield strength and $F_{\mathrm{m}}$ is directly proportional to the ultimate tensile strength, the condition which exhibited the best combination of tensile properties, in terms of high values and low standard deviations, corresponds to the non-standard HIP treatment $\left(800^{\circ} \mathrm{C}\right)$ with scan length $=78 \mathrm{~mm}$. 
A decrease in scan length (from $78 \mathrm{~mm}$ to $26 \mathrm{~mm}$ ) appears to have a minor influence on both lower yield strength and ultimate tensile strength, as can be observed for the as-built and standard HIP conditions.

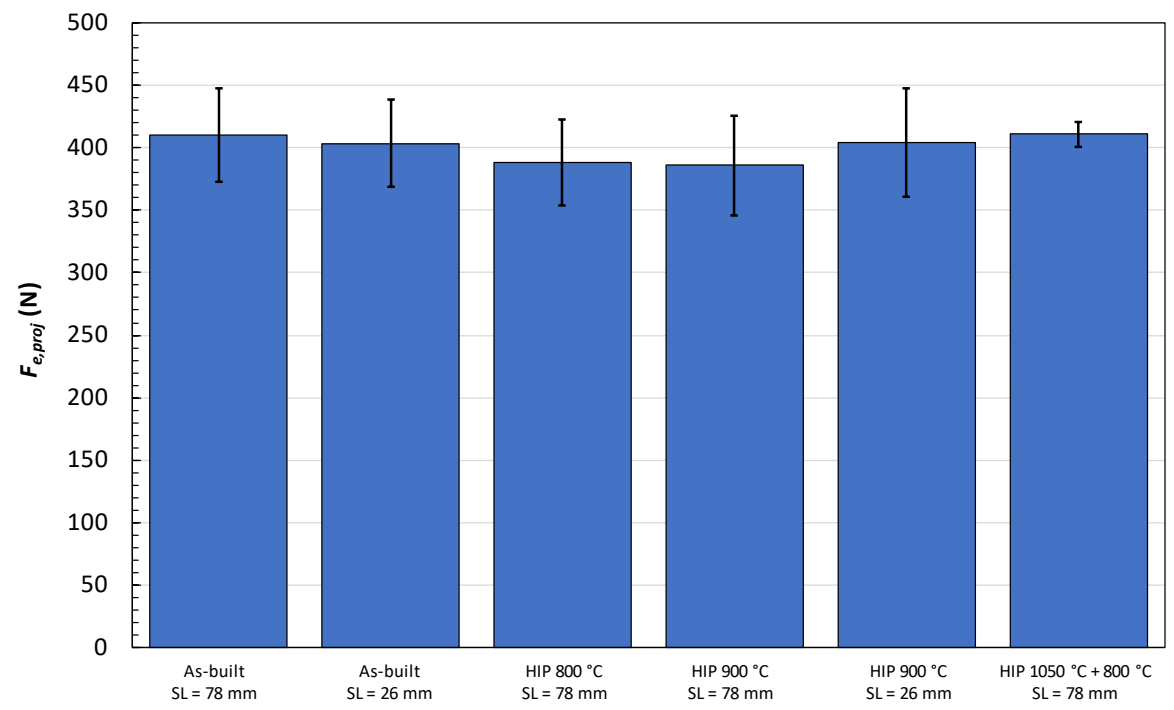

Figure 9 - Average values and standard deviations for $F_{\text {e,proj. }}$.

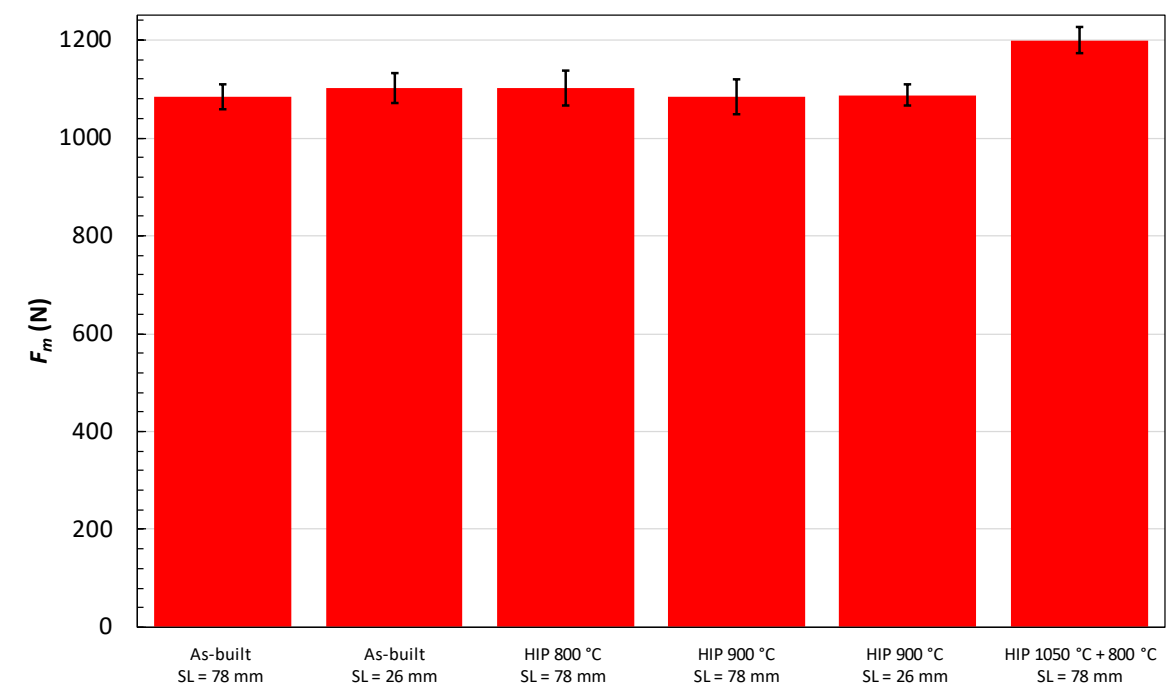

Figure 10 - Average values and standard deviations for $F_{\mathrm{m}}$.

\subsubsection{Polished specimens}

Average values and standard deviations (absolute and relative) for the various characteristic parameters of the different conditions examined (excluding the preliminary tests) are compared in Table 31 (elastic slopes and forces) and Table 32 (displacements and energies). 
Table 31 - Average values and standard deviations for polished specimens in the different conditions (elastic slopes and forces).

\begin{tabular}{|c|c|c|c|c|c|c|c|c|c|c|c|c|c|c|c|c|}
\hline $\begin{array}{c}\text { Material } \\
\text { condition }\end{array}$ & $\begin{array}{c}\text { Scan } \\
\text { length }\end{array}$ & \begin{tabular}{|c|} 
Supported \\
$(\mathrm{Y} / \mathrm{N})$
\end{tabular} & $\begin{array}{c}\text { Displacement } \\
\text { rate }(\mathrm{mm} / \mathrm{s})\end{array}$ & $\begin{array}{r}\text { Slope }_{\text {ini }} \\
(\mathrm{N} / \mathrm{mm}) \\
\end{array}$ & $\begin{array}{l}\mathrm{F}_{\mathrm{e}, \mathrm{proj}} \\
(\mathrm{N})\end{array}$ & $\begin{array}{l}\mathrm{F}_{\mathrm{e}, \text { int }} \\
(\mathrm{N})\end{array}$ & $\begin{array}{c}\mathrm{F}_{\mathrm{h} 0 / 10, \text { off }} \\
(\mathrm{N})\end{array}$ & $\begin{array}{l}F_{0.1 \mathrm{~mm}, \text { off }} \\
(\mathrm{N})\end{array}$ & $\begin{array}{l}\mathrm{F}_{0.1 \mathrm{~mm}} \\
(\mathrm{~N})\end{array}$ & $\begin{array}{c}\mathrm{F}_{0.48 \mathrm{~mm}} \\
(\mathrm{~N})\end{array}$ & $\begin{array}{l}\mathrm{F}_{0.5 \mathrm{~mm}} \\
(\mathrm{~N})\end{array}$ & $\begin{array}{c}\mathrm{F}_{0.65 \mathrm{~mm}} \\
(\mathrm{~N})\end{array}$ & $\begin{array}{l}\mathrm{F}_{0.9 \mathrm{~mm}} \\
(\mathrm{~N})\end{array}$ & $\begin{array}{l}F_{m} \\
(N)\end{array}$ & $\begin{array}{l}F_{\text {infl }} \\
(\mathrm{N})\end{array}$ & $\begin{array}{c}F_{f} \\
(N)\end{array}$ \\
\hline \multirow{6}{*}{ As-Built } & \multirow{3}{*}{78} & \multirow{3}{*}{$\mathrm{N}$} & \multirow{3}{*}{0.0014} & 3235.245 & 410.3 & 445.5 & 543.7 & 704.9 & 323.2 & 872.3 & 891.4 & 906.9 & 972.5 & 980.5 & 654.1 & 784.4 \\
\hline & & & & 308.286 & 37.534 & 42.895 & 29.705 & 14.482 & 21.680 & 28.996 & 35.128 & 30.395 & 28.143 & 28.013 & 273.751 & 22.411 \\
\hline & & & & $9.5 \%$ & $9.1 \%$ & $9.6 \%$ & $5.5 \%$ & $2.1 \%$ & $6.7 \%$ & $3.3 \%$ & $3.9 \%$ & $3.4 \%$ & $2.9 \%$ & $2.9 \%$ & $41.8 \%$ & $2.9 \%$ \\
\hline & \multirow{3}{*}{26} & \multirow{3}{*}{$\mathrm{N}$} & \multirow{3}{*}{0.0014} & 3060.926 & 403.4 & 441.7 & 544.4 & 696.4 & 307.8 & 850.9 & 866.8 & 939.7 & 939.5 & 968.7 & 359.6 & 775.0 \\
\hline & & & & 201.966 & 34.849 & 32.945 & 19.237 & 8.628 & 11.394 & 31.135 & 33.928 & 28.546 & 15.826 & 12.301 & 245.929 & 9.841 \\
\hline & & & & $6.6 \%$ & $8.6 \%$ & $7.5 \%$ & $3.5 \%$ & $1.2 \%$ & $3.7 \%$ & $3.7 \%$ & $3.9 \%$ & $3.0 \%$ & $1.7 \%$ & $1.3 \%$ & $68.4 \%$ & $1.3 \%$ \\
\hline \multirow{3}{*}{$\begin{array}{c}\text { HIP } \\
800^{\circ} \mathrm{C}\end{array}$} & \multirow{3}{*}{78} & \multirow{3}{*}{$\mathrm{N}$} & \multirow{3}{*}{0.0014} & 3219.586 & 388.5 & 423.4 & 521.5 & 661.5 & 319.8 & 848.7 & 871.9 & 938.1 & 967.3 & 986.4 & 722.8 & 789.1 \\
\hline & & & & 230.154 & 34.326 & 38.373 & 31.487 & 27.941 & 21.276 & 20.176 & 19.307 & 24.342 & 45.744 & 22.768 & 181.546 & 18.215 \\
\hline & & & & $7.1 \%$ & $8.8 \%$ & $9.1 \%$ & $6.0 \%$ & $4.2 \%$ & $6.7 \%$ & $2.4 \%$ & $2.2 \%$ & $2.6 \%$ & $4.7 \%$ & $2.3 \%$ & $25.1 \%$ & $2.3 \%$ \\
\hline \multirow{6}{*}{$\begin{array}{l}\text { HIP } \\
900^{\circ} \mathrm{C}\end{array}$} & \multirow{3}{*}{78} & \multirow{3}{*}{$\mathrm{N}$} & \multirow{3}{*}{0.0014} & 3044.687 & 385.9 & 411.8 & 532.7 & 685.9 & 306.3 & 873.2 & 889.8 & 954.8 & 1045.0 & 1056.8 & 598.0 & 845.5 \\
\hline & & & & 189.766 & 39.775 & 37.792 & 50.614 & 41.524 & 7.047 & 5.735 & 12.130 & 22.911 & 41.296 & 35.293 & 35.269 & 28.234 \\
\hline & & & & $6.2 \%$ & $10.3 \%$ & $9.2 \%$ & $9.5 \%$ & $6.1 \%$ & $2.3 \%$ & $0.7 \%$ & $1.4 \%$ & $2.4 \%$ & $4.0 \%$ & $3.3 \%$ & $5.9 \%$ & $3.3 \%$ \\
\hline & \multirow{3}{*}{26} & \multirow{3}{*}{$\mathrm{N}$} & \multirow{3}{*}{0.0014} & 2919.529 & 404.3 & 436.7 & 563.5 & 719.3 & 296.8 & 877.8 & 894.8 & 968.8 & 1000.3 & 1018.4 & 738.3 & 814.7 \\
\hline & & & & 99.345 & 43.068 & 43.075 & 44.949 & 40.531 & 5.429 & 7.055 & 17.491 & 43.314 & 70.800 & 31.765 & 323.589 & 25.412 \\
\hline & & & & $3.4 \%$ & $10.7 \%$ & $9.9 \%$ & $8.0 \%$ & $5.6 \%$ & $1.8 \%$ & $0.8 \%$ & $2.0 \%$ & $4.5 \%$ & $7.1 \%$ & $3.1 \%$ & $43.8 \%$ & $3.1 \%$ \\
\hline \multirow{3}{*}{$\begin{array}{c}\text { HIP } \\
1050^{\circ} \mathrm{C}+ \\
900^{\circ} \mathrm{C}\end{array}$} & \multirow{3}{*}{78} & \multirow{3}{*}{$\mathrm{N}$} & \multirow{3}{*}{0.0015} & 3254.743 & 411.0 & 444.7 & 562.9 & 702.1 & 323.1 & 906.7 & 902.3 & 1028.1 & 1022.8 & 1080.4 & 720.4 & 864.3 \\
\hline & & & & 56.403 & 10.033 & 12.074 & 18.685 & 15.372 & 2.758 & 2.886 & 37.219 & 48.302 & 22.945 & 23.017 & 247.087 & 18.413 \\
\hline & & & & $1.7 \%$ & $2.4 \%$ & $2.7 \%$ & $3.3 \%$ & $2.2 \%$ & $0.9 \%$ & $0.3 \%$ & $4.1 \%$ & $4.7 \%$ & $2.2 \%$ & $2.1 \%$ & $34.3 \%$ & $2.1 \%$ \\
\hline
\end{tabular}

Table 32 - Average values and standard deviations for polished specimens in the different conditions (displacements and energies).

\begin{tabular}{|c|c|c|c|c|c|c|c|c|c|c|c|c|c|}
\hline $\begin{array}{c}\text { Material } \\
\text { condition }\end{array}$ & $\begin{array}{c}\text { Scan } \\
\text { length }\end{array}$ & \begin{tabular}{|c}
$\begin{array}{c}\text { Supported } \\
(\mathrm{Y} / \mathrm{N})\end{array}$ \\
\end{tabular} & \begin{tabular}{|c|} 
Displacement \\
rate $(\mathrm{mm} / \mathrm{s})$
\end{tabular} & $\begin{array}{r}\mathrm{u}_{\mathrm{e}} \\
(\mathrm{mm}) \\
\end{array}$ & $\begin{array}{c}u_{\text {ho/ } 10, \text { off }} \\
(\mathrm{mm})\end{array}$ & $\begin{array}{c}u_{0.1 \mathrm{~mm}, \text { off }} \\
(\mathrm{mm})\end{array}$ & $\begin{array}{r}\mathrm{u}_{\mathrm{e} 1.5} \\
(\mathrm{~mm}) \\
\end{array}$ & $\begin{array}{r}u_{m} \\
(\mathrm{~mm}) \\
\end{array}$ & $\begin{array}{c}\mathrm{u}_{\text {infl }} \\
(\mathrm{mm})\end{array}$ & $\begin{array}{c}\mathrm{u}_{\mathrm{f}} \\
(\mathrm{mm})\end{array}$ & $\begin{array}{l}E_{S P} \\
(J) \\
\end{array}$ & $\begin{array}{l}E_{m} \\
(J)\end{array}$ & $\begin{array}{l}E_{P L} \\
(J) \\
\end{array}$ \\
\hline \multirow{6}{*}{ As-Built } & \multirow{3}{*}{78} & \multirow{3}{*}{$\mathrm{N}$} & \multirow{3}{*}{0.0014} & 0.138 & 0.214 & 0.320 & 0.346 & 0.910 & 1.415 & 1.291 & 1.01 & 0.66 & 0.51 \\
\hline & & & & 0.016 & 0.018 & 0.021 & 0.047 & 0.059 & 0.351 & 0.053 & 0.026 & 0.036 & 0.027 \\
\hline & & & & $11.9 \%$ & $8.5 \%$ & $6.7 \%$ & $13.5 \%$ & $6.5 \%$ & $24.8 \%$ & $4.1 \%$ & $2.6 \%$ & $5.5 \%$ & $5.3 \%$ \\
\hline & \multirow{3}{*}{26} & \multirow{3}{*}{$\mathrm{N}$} & \multirow{3}{*}{0.0014} & 0.145 & 0.224 & 0.329 & 0.348 & 0.780 & 1.103 & 1.232 & 0.93 & 0.53 & 0.38 \\
\hline & & & & 0.020 & 0.017 & 0.016 & 0.047 & 0.088 & 0.940 & 0.013 & 0.021 & 0.080 & 0.082 \\
\hline & & & & $13.6 \%$ & $7.5 \%$ & $5.0 \%$ & $13.6 \%$ & $11.3 \%$ & $85.2 \%$ & $1.1 \%$ & $2.2 \%$ & $15.1 \%$ & $21.8 \%$ \\
\hline \multirow{3}{*}{$\begin{array}{l}\text { HIP } \\
800^{\circ} \mathrm{C}\end{array}$} & \multirow{3}{*}{78} & \multirow{3}{*}{$\mathrm{N}$} & \multirow{3}{*}{0.0014} & 0.132 & 0.207 & 0.306 & 0.300 & 0.782 & 1.442 & 1.366 & 1.06 & 0.54 & 0.39 \\
\hline & & & & 0.010 & 0.011 & 0.012 & 0.072 & 0.128 & 0.301 & 0.090 & 0.055 & 0.133 & 0.136 \\
\hline & & & & $7.6 \%$ & $5.2 \%$ & $4.1 \%$ & $24.1 \%$ & $16.4 \%$ & $20.9 \%$ & $6.6 \%$ & $5.1 \%$ & $24.8 \%$ & $35.3 \%$ \\
\hline \multirow{6}{*}{$\begin{array}{c}\text { HIP } \\
900^{\circ} \mathrm{C}\end{array}$} & \multirow{3}{*}{78} & \multirow{3}{*}{$\mathrm{N}$} & \multirow{3}{*}{0.0014} & 0.136 & 0.222 & 0.327 & 0.373 & 0.923 & 1.669 & 1.377 & 1.13 & 0.69 & 0.50 \\
\hline & & & & 0.021 & 0.026 & 0.028 & 0.026 & 0.045 & 0.010 & 0.100 & 0.079 & 0.038 & 0.039 \\
\hline & & & & $15.4 \%$ & $11.9 \%$ & $8.7 \%$ & $7.1 \%$ & $4.9 \%$ & $0.6 \%$ & $7.3 \%$ & $7.0 \%$ & $5.6 \%$ & $7.7 \%$ \\
\hline & \multirow{3}{*}{26} & \multirow{3}{*}{$\mathrm{N}$} & \multirow{3}{*}{0.0014} & 0.150 & 0.238 & 0.348 & 0.418 & 0.728 & 1.177 & 1.340 & 1.06 & 0.49 & 0.31 \\
\hline & & & & 0.020 & 0.022 & 0.022 & 0.054 & 0.242 & 0.665 & 0.023 & 0.034 & 0.232 & 0.215 \\
\hline & & & & $13.2 \%$ & $9.2 \%$ & $6.3 \%$ & $13.0 \%$ & $33.2 \%$ & $56.5 \%$ & $1.7 \%$ & $3.2 \%$ & $47.4 \%$ & $69.1 \%$ \\
\hline HIP & \multirow{3}{*}{78} & \multirow{3}{*}{$\mathrm{N}$} & \multirow{3}{*}{0.0015} & 0.137 & 0.219 & 0.316 & 0.312 & 0.706 & 1.042 & 1.176 & 0.96 & 0.49 & 0.31 \\
\hline $1050^{\circ} \mathrm{C}+$ & & & & 0.006 & 0.011 & 0.008 & 0.043 & 0.019 & 0.809 & 0.094 & 0.089 & 0.019 & 0.023 \\
\hline $900^{\circ} \mathrm{C}$ & & & & $4.4 \%$ & $4.9 \%$ & $2.5 \%$ & $13.8 \%$ & $2.6 \%$ & $77.6 \%$ & $8.0 \%$ & $9.3 \%$ & $3.8 \%$ & $7.4 \%$ \\
\hline
\end{tabular}

Comparisons for $F_{\text {e,proj }}$ and $F_{\mathrm{m}}$ are illustrated in Fig. 11 and Fig. 12, respectively. Unlike rough specimens, for polished specimen the condition which exhibited the best combination of tensile properties, in terms of high values and low standard deviations, corresponds to the non-standard multiple HIP treatment $\left(1050^{\circ} \mathrm{C}+800^{\circ} \mathrm{C}\right)$ with scan length $=78 \mathrm{~mm}$.

A univocal effect of scan length cannot be detected for polished specimens, as can be observed from the as-built and standard HIP conditions. 


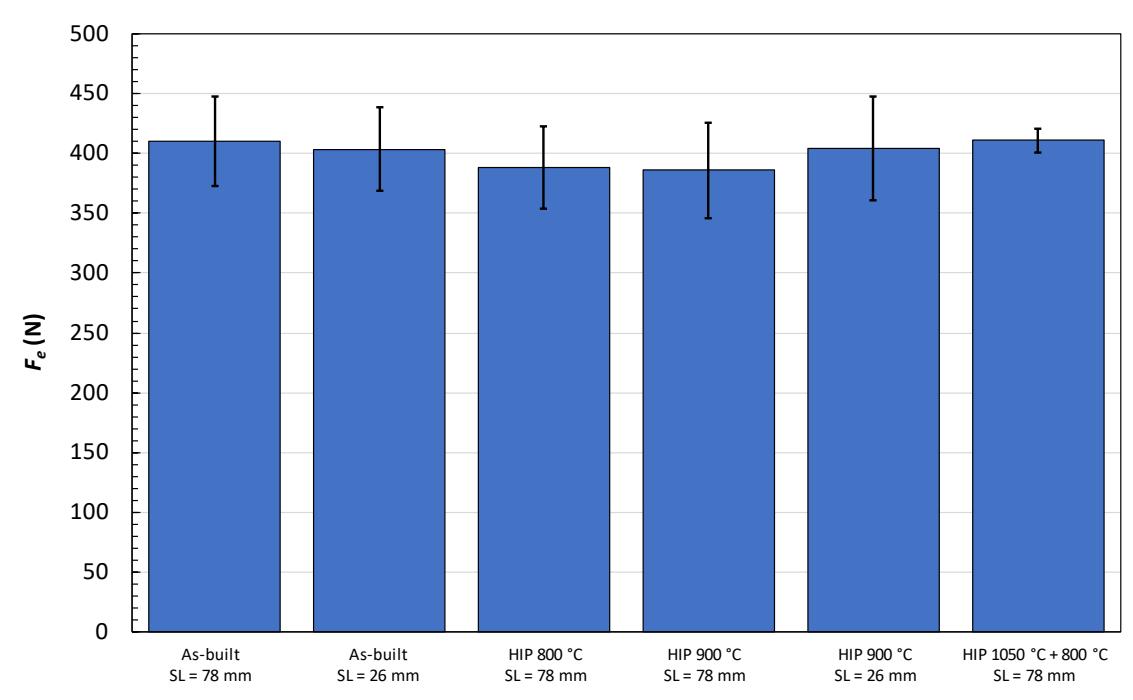

Figure 11 - Average values and standard deviations for $F_{\text {e,proj. }}$

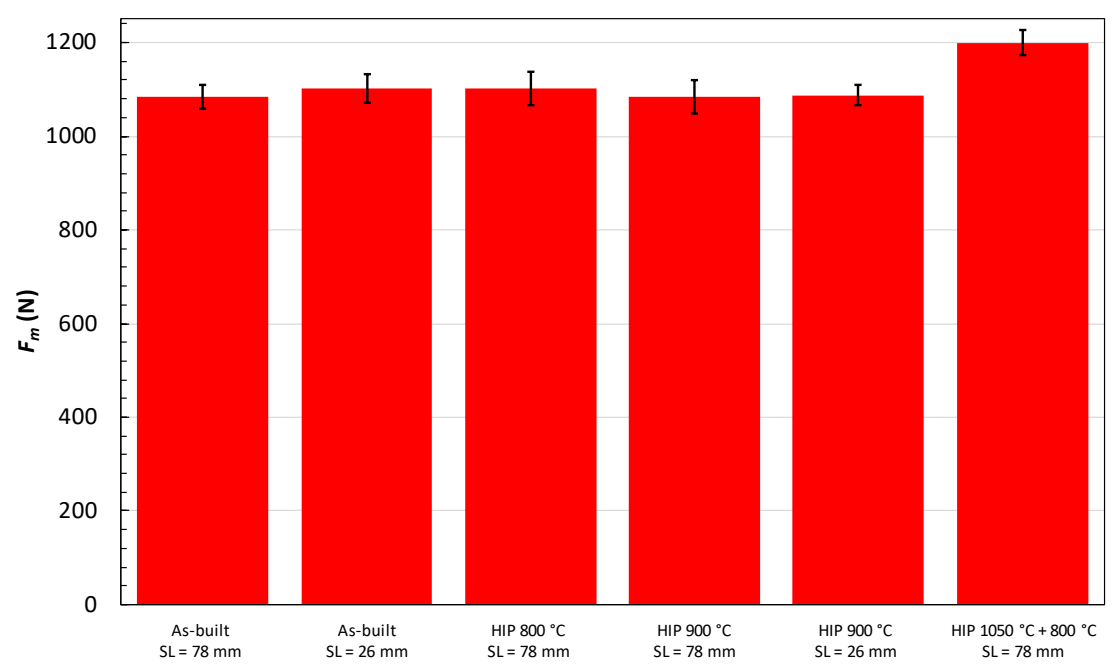

Figure 12 - Average values and standard deviations for $F_{\mathrm{m}}$.

\subsubsection{General considerations}

It must be stressed that average values in Tables 29 to 31 should not be compared directly between rough and polished specimens, as these latter had a lower thickness $(0.43 \mathrm{~mm}$ - $0.47 \mathrm{~mm})$ than the former $(0.5 \mathrm{~mm})$. However, standard deviations can be legitimately compared.

The SP parameter that exhibits the least variability $(1.3 \%-3.3 \%)$ is the maximum force $F_{\mathrm{m}}$, whereas elastic-plastic transition forces $F_{\text {e,proj }}$ and $F_{\text {e,int, which are calculated from }}$ the intersection of two linear fits, yielded relative standard deviations in the range $2.4 \%-$ $15.0 \%$. Interestingly, displacements at maximum force, $u_{\mathrm{m}}$, generally showed larger scatter than displacements corresponding to a $20 \%$ force drop from the maximum, $u_{\mathrm{f}}$. In terms of calculated energies, the most consistent parameter is the fracture energy, $E_{\mathrm{SP}}$.

No obvious effect of surface finish (rough vs. polished) could be detected on the variability of SP parameters. 


\section{Correlations with tensile properties}

Many empirical correlations between normalized SP parameters and conventional tensile properties have been proposed in the literature, almost exclusively for steels. For most of these correlations, the accuracy of the predicted strength values (yield and ultimate) was reported to be in the order of $\pm 25 \mathrm{MPa}$. Specifically:

- $\quad$ Yield strength has been mostly correlated with $F_{\mathrm{e}} / h_{0}^{2}$ [21-27]. Alternative correlations for $R_{\mathrm{p} 02}$ have been proposed with:

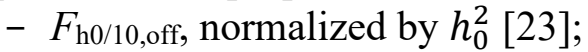

- $F_{0.1 \mathrm{~mm}, \mathrm{off}}$, normalized by $h_{0}^{2}[22,24]$;

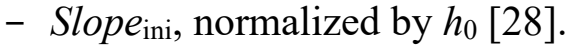

- Tensile strength has been mostly correlated with $F_{\mathrm{m}}$, normalized either by $h_{0}^{2}[23,25,27]$ or $h_{0} \cdot u_{\mathrm{m}}[21-27,29]$. Other force values that have been correlated with $R_{\mathrm{m}}$ are:

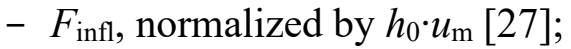

- $F_{0.48 \mathrm{~mm}}[27], F_{0.645 \mathrm{~mm}}[25]$, and $F_{0.65 \mathrm{~mm}}$ [28], all normalized by $h_{0}^{2}$.

- Total elongation has been correlated with $u_{\mathrm{m}}[23,31], u_{\mathrm{m}} / h_{0}[23,32]$, and $\frac{u_{f}-h_{0}}{h_{0}}[33]$.

Almost every correlation was proposed in the generic linear form:

$$
Y=\alpha_{1} X+\alpha_{2}
$$

where $Y$ is the tensile property of interest, $X$ is the normalized SP parameter, and $\alpha_{1}, \alpha_{2}$ are slope and intercept, respectively, of a least-squares linear regression between $X$ and $Y$.

As far as energy values are concerned, in the literature they are only used to construct a transition curve by performing tests at different temperatures. The flexural point of the energy/temperature curve is then empirically correlated to the ductile-to-brittle transition temperature established from Charpy tests [34,35].

In previous work by the authors [12], correlations for three investigated steels (A533B cl. 1, 4340 low-energy, and 4340 high-energy) were derived and compared with those proposed by other authors. A similar approach was taken for this investigation.

The strength of the established correlations is hereinafter defined on the basis of the Pearson correlation coefficient, $r$ :

- strong $(r>0.70)$ - color: green,

- moderate $(0.70 \leq r<0.50)$ - color: yellow,

- weak $(0.50 \leq r<0.30)$ - color: pink, or

- absent/no correlation $(r \leq 0.30)$ - color: red.

\subsection{Yield strength correlations}

A summary of the yield strength correlations obtained for rough specimens is provided in Table 33, while the same information is shown in Table 34 for polished specimens. The Tables present the values of the linear coefficients (slope and intercept), as well as the values of the correlation coefficient $r$. The yield properties to be correlated with SP parameters were measured in [15]. 
Table 33 - Summary of yield strength correlations obtained for rough specimens of AM Ti64.

\begin{tabular}{|c|c|c|c|c|c|c|c|c|c|}
\hline & Slope & 0.1462 & 0.1190 & 0.0264 & 0.1853 & 0.0987 & 0.0737 & 0.0227 \\
\hline & & Intercept & 610.82 & 657.04 & 754.16 & 372.04 & 536.89 & 786.89 & 766.22 \\
\hline & & $r$ & 0.6661 & 0.5574 & 0.5597 & 0.6946 & 0.5079 & 0.5553 & 0.5338 \\
\hline & & Tensile & & SP & SP & SP & SP & SP & SP \\
\hline \multirow{2}{*}{$\begin{array}{c}\text { Material } \\
\text { condition }\end{array}$} & \multirow{2}{*}{$\begin{array}{c}\text { Scan length } \\
(\mathrm{mm})\end{array}$} & property & parameter & parameter & parameter & parameter & parameter & parameter & parameter \\
\hline & & $0.2 \%$ YS & $\mathrm{F}_{\mathrm{e}, \mathrm{proj}} / \mathrm{h}_{0}{ }^{2}$ & $\mathrm{~F}_{\mathrm{e}, \mathrm{int}} / \mathrm{h}_{0}{ }^{2}$ & $F_{e, p r o j} /\left(u_{e} h_{0}\right)$ & $\mathrm{F}_{\mathrm{h} 0 / 10, \text { off }} / \mathrm{h}_{0}{ }^{2}$ & $\mathrm{~F}_{0.1 \mathrm{~mm}, \mathrm{off}} / \mathrm{h}_{0}{ }^{2}$ & $\mathbf{R}_{\mathrm{p} 0.2, \text { Hähner }}$ & Slope $_{\text {in }} / \mathrm{h}_{0}$ \\
\hline \multirow{2}{*}{ As-Built } & 78 & 879 & 1767.1 & 1860.5 & 5229.1 & 2614.9 & 3261.8 & 1329.3 & 5504.99 \\
\hline & 26 & 875 & 1653.8 & 1684.5 & 3596.0 & 2717.5 & 3437.7 & 1097.1 & 3664.67 \\
\hline HIP $800^{\circ} \mathrm{C}$ & 78 & 864 & 1837.0 & 1853.6 & 3694.5 & 2777.0 & 3400.1 & 986.2 & 3732.27 \\
\hline \multirow{2}{*}{$\operatorname{HIP} 900^{\circ} \mathrm{C}$} & 78 & 838 & 1451.6 & 1484.6 & 3446.2 & 2474.9 & 2978.5 & 881.4 & 3532.78 \\
\hline & 26 & 799 & 1578.6 & 1565.1 & 3361.1 & 2478.7 & 3157.5 & 668.5 & 3456.76 \\
\hline HIP $1050+800^{\circ} \mathrm{C}$ & 78 & 885 & 1805.4 & 1622.8 & 3955.3 & 2629.1 & 3204.4 & 720.6 & 4072.12 \\
\hline
\end{tabular}

Table 34 - Summary of yield strength correlations obtained for polished specimens of AM Ti64.

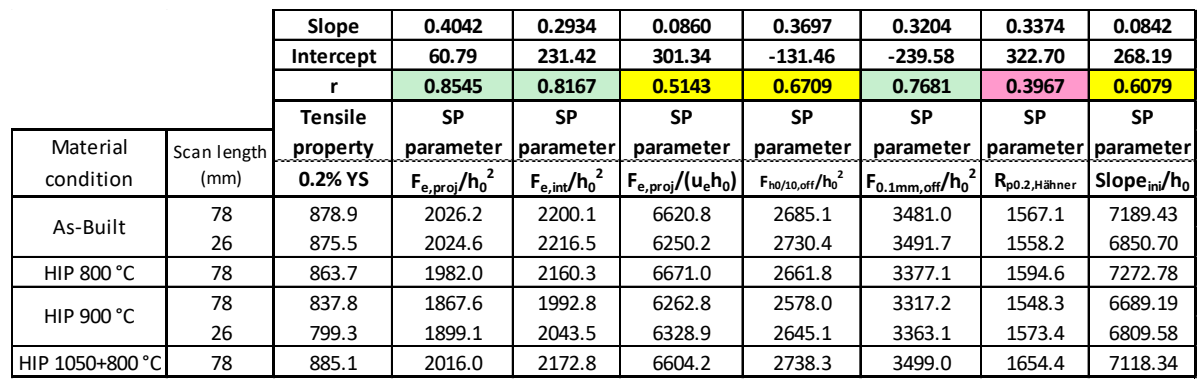

All the correlations established for rough specimens were moderate $(0.70 \leq r<0.50)$, while for polished specimens three of the correlations were found to be strong $(r>0.70)$ and one weak $(0.50 \leq r<0.30)$. The three strong correlations obtained are depicted in Figs. 13 to 15 , which also include data and linear fits for rough specimens.

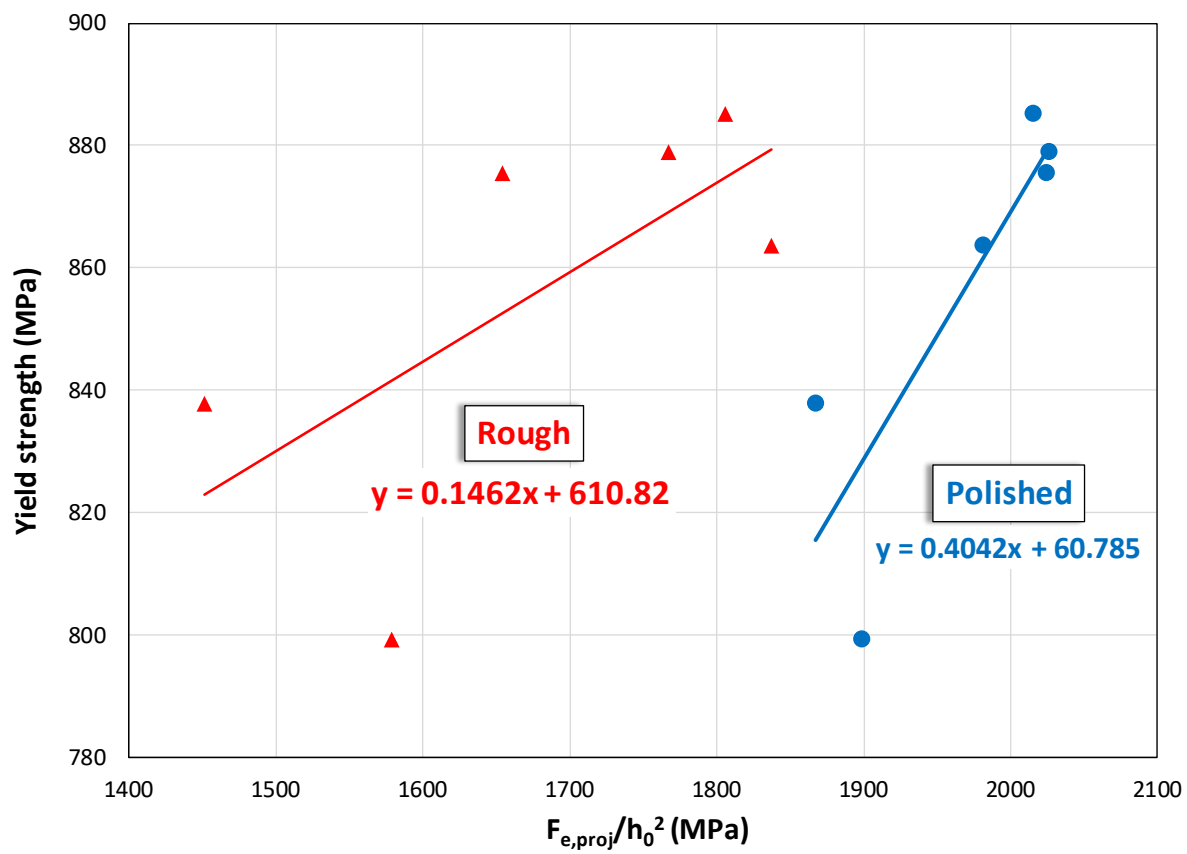

Figure 13 - Correlations between $F_{\text {e,proj }} / h_{0}^{2}$ and yield strength for rough and polished specimens of AM Ti64. 


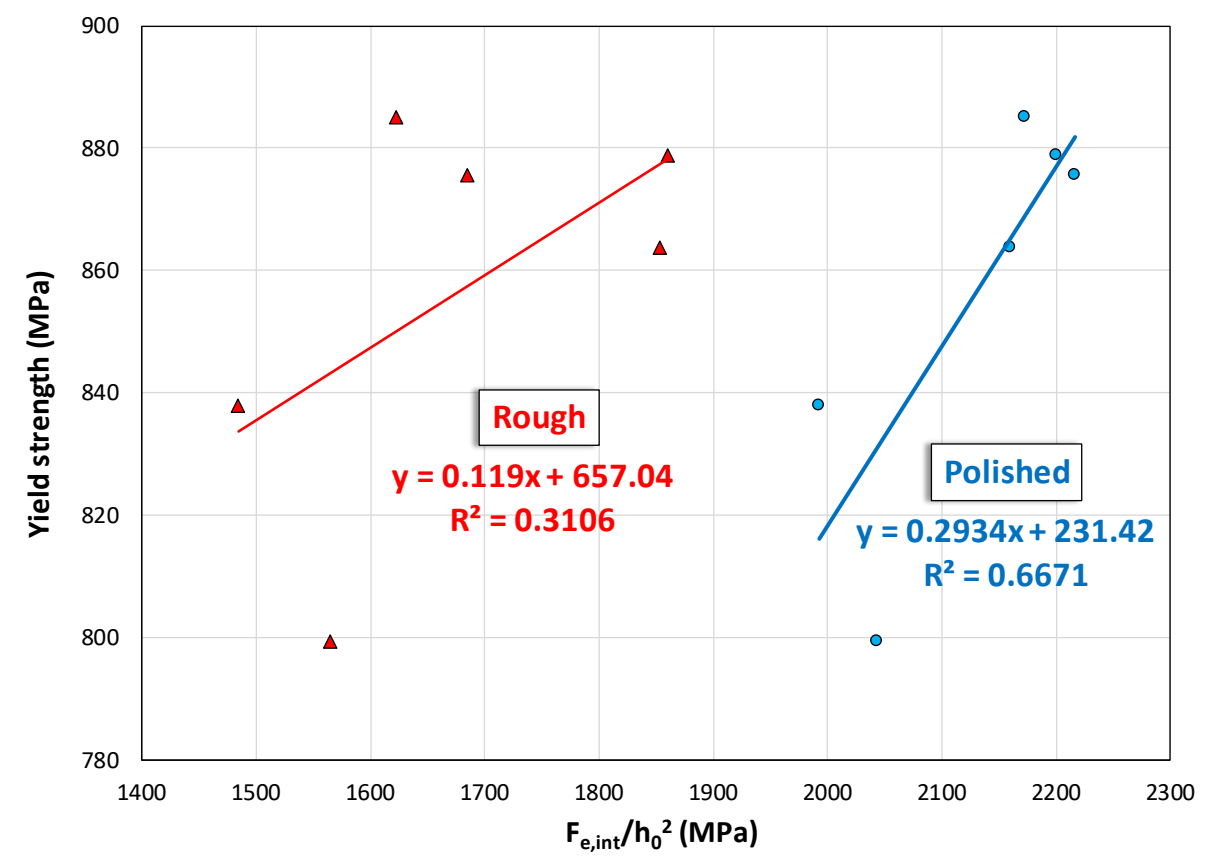

Figure 14 - Correlations between $F_{\mathrm{e}, \text { int }} / h_{0}^{2}$ and yield strength for rough and polished specimens of AM Ti64.

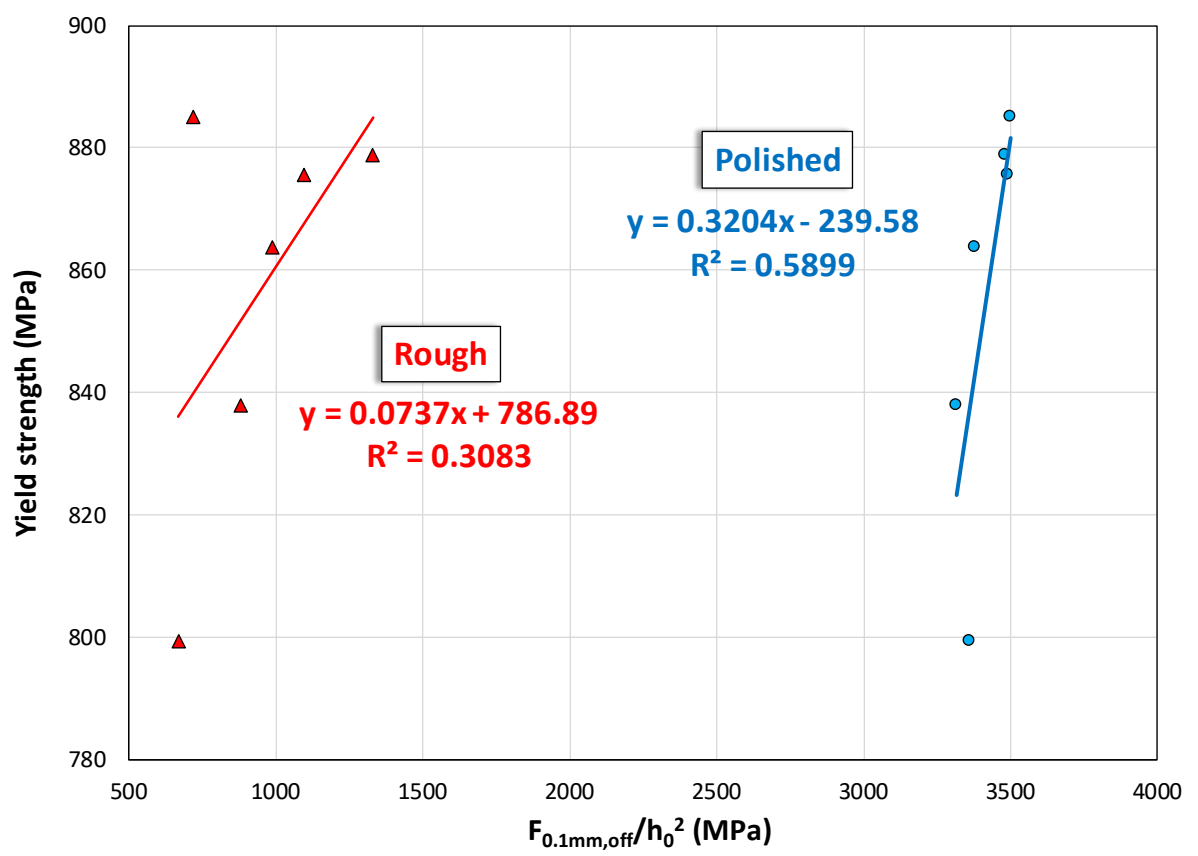

Figure 15 - Correlations between $F_{0.1 \mathrm{~mm}, \text { off }} / h_{0}^{2}$ and yield strength for rough and polished specimens of AM Ti64.

All the yield strength correlations, obtained for both rough and polished specimens, are documented in Annex 14. 


\subsection{Ultimate tensile strength correlations}

A summary of the ultimate tensile strength correlations obtained for rough specimens is provided in Table 35, while the same information is shown in Table 36 for polished specimens. The Tables present the values of the linear coefficients (slope and intercept), as well as the values of the correlation coefficient $r$. The ultimate tensile values to be correlated with SP parameters were measured in [15].

Table 35 - Summary of ultimate tensile strength correlations obtained for rough specimens of AM Ti64.

\begin{tabular}{|c|c|c|c|c|c|c|c|}
\hline & Slope & 0.0273 & 0.1074 & 0.0817 & 0.0530 & 0.0654 \\
\hline & & Intercept & 839.28 & 726.27 & 861.04 & 800.01 & 717.99 \\
\hline & & $r$ & 0.4040 & 0.7386 & 0.8852 & 0.7036 & 0.7312 \\
\hline & & Tensile & SP & SP & SP & SP & SP \\
\hline \multirow{2}{*}{$\begin{array}{c}\text { Material } \\
\text { condition }\end{array}$} & \multirow{2}{*}{$\begin{array}{c}\text { Scan length } \\
(\mathrm{mm})\end{array}$} & property & parameter & parameter & parameter & parameter & parameter \\
\hline & & UTS & $\mathrm{F}_{\mathrm{m}} / \mathrm{h}_{0}{ }^{2}$ & $F_{m} /\left(h_{0} u_{m}\right)$ & $\mathrm{F}_{\text {inf }} /\left(\mathrm{h}_{0} \mathrm{u}_{\mathrm{m}}\right)$ & $\mathrm{F}_{0.48 \mathrm{~mm}} / \mathrm{h}_{0}{ }^{2}$ & $\mathrm{~F}_{0.65 \mathrm{~mm}} / \mathrm{h}_{0}{ }^{2}$ \\
\hline \multirow{2}{*}{ As-Built } & 78 & 981 & 4401.2 & 2364.3 & 1631.7 & 3612.1 & 4071.6 \\
\hline & 26 & 972 & 4467.9 & 2024.4 & 1232.0 & 3027.0 & 3762.7 \\
\hline HIP $800^{\circ} \mathrm{C}$ & 78 & 969 & 4476.0 & 2277.9 & 1281.6 & 3050.9 & 3753.5 \\
\hline \multirow{2}{*}{$\operatorname{HIP} 900^{\circ} \mathrm{C}$} & 78 & 951 & 4079.7 & 2116.3 & 1001.8 & 2663.4 & 3314.6 \\
\hline & 26 & 918 & 4502.7 & 2015.7 & 889.0 & 2816.2 & 3554.2 \\
\hline $\mathrm{HIP} 1050+800^{\circ} \mathrm{C}$ & 78 & 985 & 5207.7 & 2407.2 & 1416.1 & 3224.8 & 3994.1 \\
\hline
\end{tabular}

Table 36 - Summary of ultimate tensile strength correlations obtained for polished specimens of AM Ti64.

\begin{tabular}{|c|c|c|c|c|c|c|c|}
\hline & Slope & 0.0129 & -0.0167 & 0.0004 & 0.1362 & 0.0242 \\
\hline & & Intercept & 897.43 & 1010.27 & 961.83 & 374.43 & 847.22 \\
\hline & & $r$ & 0.1066 & -0.2458 & 0.0081 & 0.5135 & 0.2539 \\
\hline & & Tensile & SP & SP & SP & SP & SP \\
\hline \multirow{2}{*}{$\begin{array}{c}\text { Material } \\
\text { condition }\end{array}$} & \multirow{2}{*}{$\begin{array}{c}\text { Scan length } \\
(\mathrm{mm})\end{array}$} & property & parameter & parameter & parameter & parameter & parameter \\
\hline & & UTS & $\mathrm{F}_{\mathrm{m}} / \mathrm{h}_{0}{ }^{2}$ & $F_{m} /\left(h_{0} u_{m}\right)$ & $F_{\text {inft }} /\left(h_{0} u_{m}\right)$ & $\mathrm{F}_{0.48 \mathrm{~mm}} / \mathrm{h}_{0}{ }^{2}$ & $\mathrm{~F}_{0.65 \mathrm{~mm}} / \mathrm{h}_{0}{ }^{2}$ \\
\hline \multirow{2}{*}{ As-Built } & 78 & 980.9 & 4841.8 & 2402.0 & 1608.7 & 4307.8 & 4471.7 \\
\hline & 26 & 971.7 & 4856.1 & 2802.6 & 1068.0 & 4263.9 & 4681.2 \\
\hline $\mathrm{HIP} 800^{\circ} \mathrm{C}$ & 78 & 969.1 & 5037.5 & 2902.2 & 2081.8 & 4334.3 & 4814.0 \\
\hline \multirow{2}{*}{ HIP $900^{\circ} \mathrm{C}$} & 78 & 950.6 & 5104.0 & 2520.4 & 1424.0 & 4219.9 & 4612.1 \\
\hline & 26 & 917.8 & 5029.3 & 3240.3 & 2003.7 & 4284.6 & 4729.5 \\
\hline HIP $1050+800^{\circ} \mathrm{C}$ & 78 & 985.3 & 5408.8 & 3313.0 & 2393.5 & 4492.1 & 5237.1 \\
\hline
\end{tabular}

Interestingly, the results point in a different direction with respect to what we obtained for the yield strength. Correlations are generally much stronger for rough specimens (four strong and one weak) than for polished specimens (four non-existent and one moderate).

The empirical relationships established between $R_{\mathrm{m}}$ and $F_{\mathrm{m}} / h_{0}^{2}, F_{\mathrm{m}} /\left(h_{0} u_{\mathrm{m}}\right), F_{\text {infl }} /\left(h_{0} u_{\mathrm{m}}\right)$, and $F_{0.65 \mathrm{~mm}} / h_{0}^{2}$ are shown in Figs. 16-19 for both groups of specimens.

All the ultimate tensile strength correlations, obtained for both rough and polished specimens, are documented in Annex 15. 


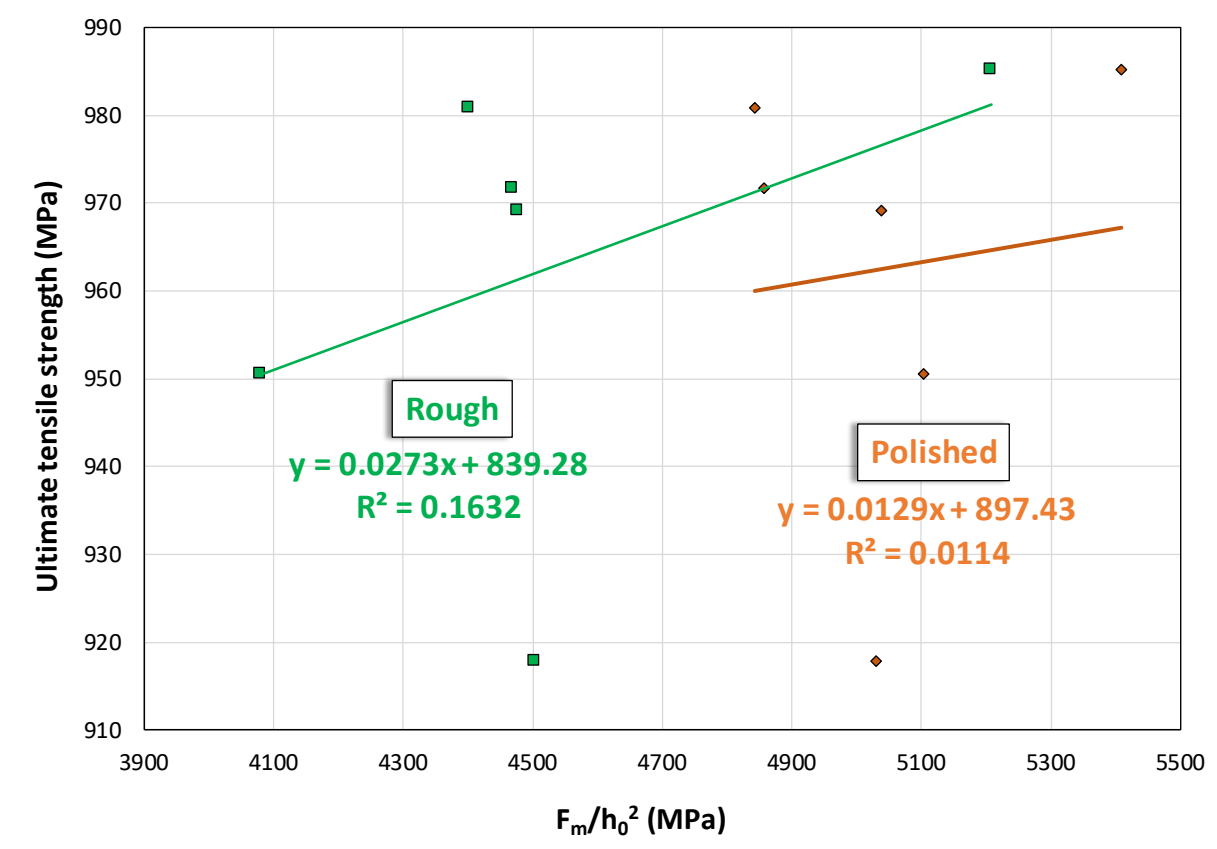

Figure 16 - Correlations between $F_{\mathrm{m}} / h_{0}^{2}$ and ultimate tensile strength for rough and polished specimens of AM Ti64.

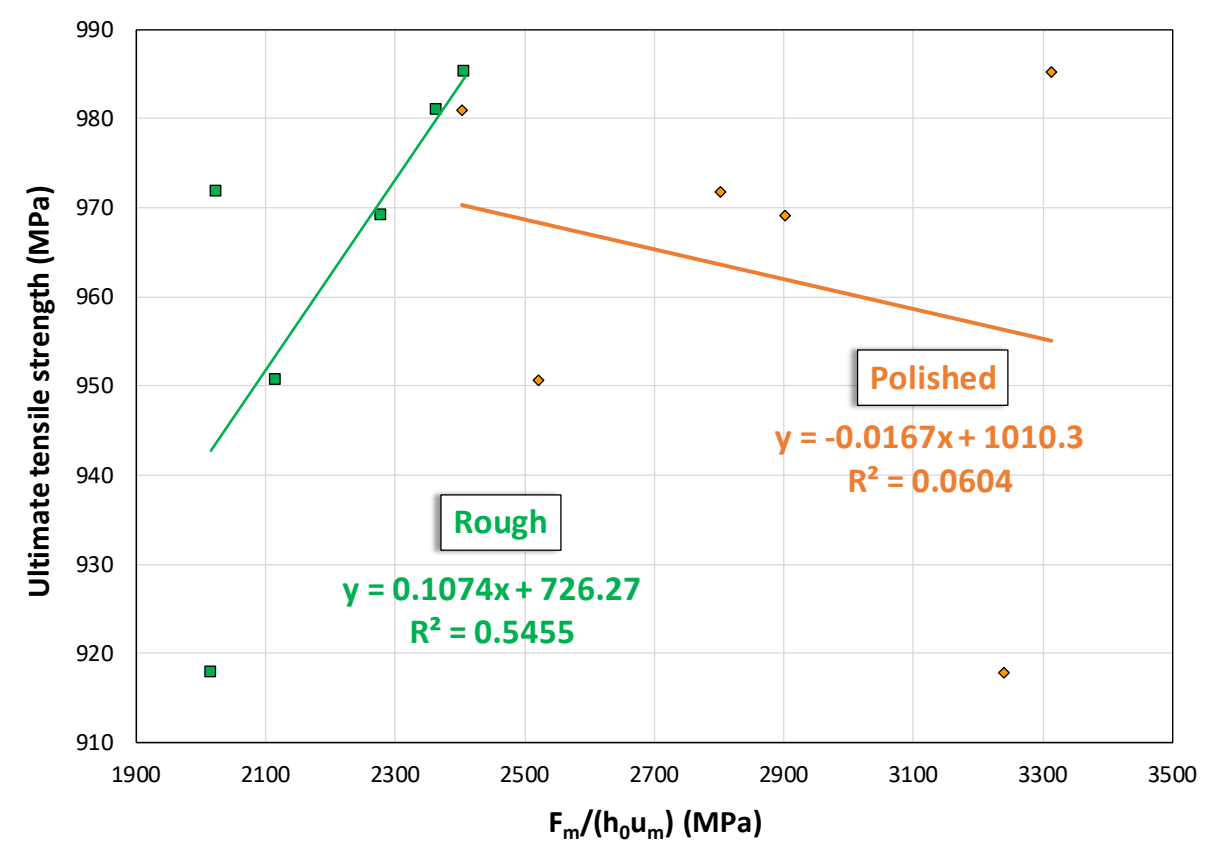

Figure 17 - Correlations between $F_{\mathrm{m}} /\left(h_{0} u_{\mathrm{m}}\right)$ and ultimate tensile strength for rough and polished specimens of AM Ti64. 


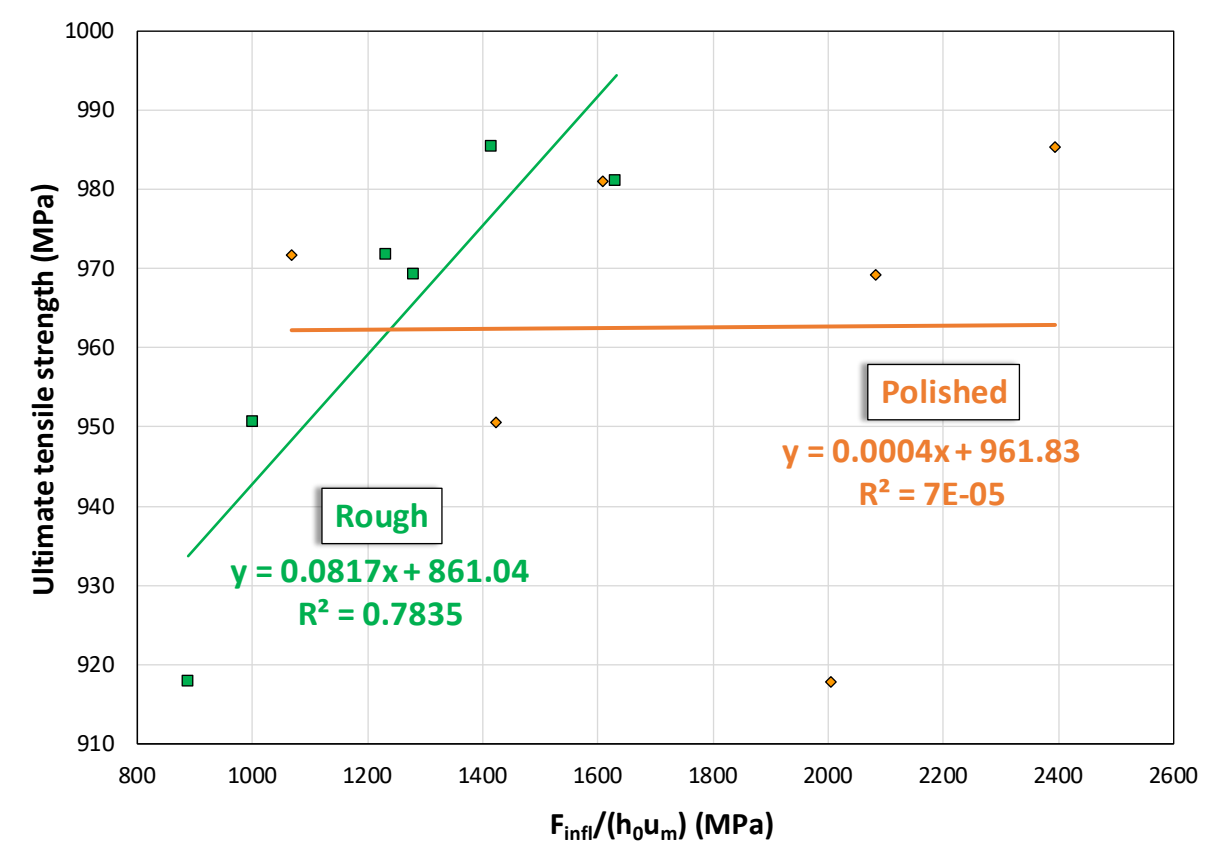

Figure 18 - Correlations between $F_{\text {infl }} /\left(h_{0} u_{\mathrm{m}}\right)$ and ultimate tensile strength for rough and polished specimens of AM Ti64.

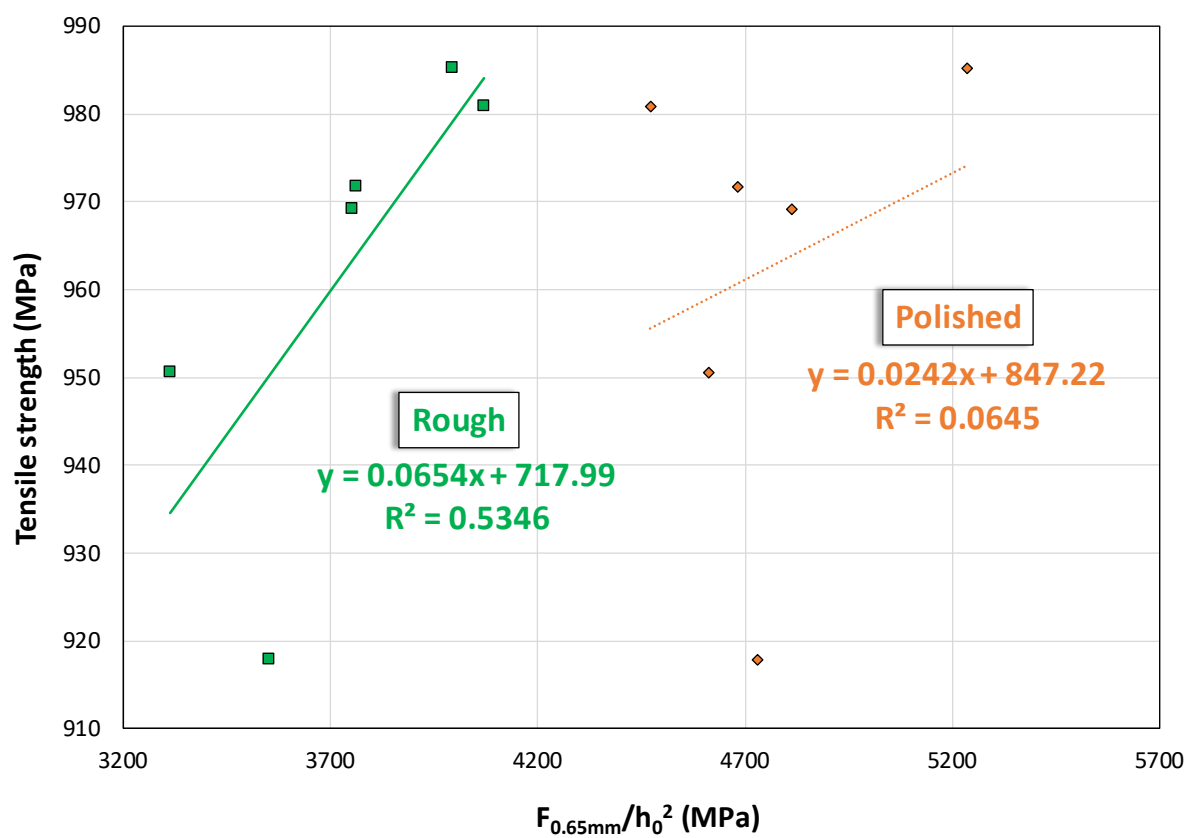

Figure 19 - Correlations between $F_{0.65 \mathrm{~mm}} / h_{0}^{2}$ and ultimate tensile strength for rough and polished specimens of AM Ti64.

\subsection{Elongation correlations}

A summary of the correlations with total elongation from tensile tests obtained for rough specimens is provided in Table 37, while the same information is shown in Table 38 for 
polished specimens. The Tables present the values of the linear coefficients (slope and intercept), as well as the values of the correlation coefficient $r$. The total elongation values to be correlated with SP parameters were measured in [15].

Table 37 - Summary of total elongation correlations obtained for rough specimens of AM Ti64.

\begin{tabular}{|c|c|c|c|c|}
\hline & Slope & -0.0861 & -0.0089 \\
\hline & & Intercept & 0.46 & 0.30 \\
\hline & & $r$ & -0.2681 & -0.0436 \\
\hline & & Tensile & SP & SP \\
\hline \multirow{2}{*}{$\begin{array}{c}\text { Material } \\
\text { condition }\end{array}$} & \multirow{2}{*}{$\begin{array}{c}\text { Scan length } \\
(\mathrm{mm})\end{array}$} & property & parameter & parameter \\
\hline & & $\mathrm{TE}$ & $u_{m} / h_{0}$ & $\left(u_{f}-h_{0}\right) / h_{0}$ \\
\hline \multirow{2}{*}{ As-Built } & 78 & $27.8 \%$ & 1.867 & 1.796 \\
\hline & 26 & $25.5 \%$ & 2.211 & 2.023 \\
\hline $\mathrm{HIP} 800^{\circ} \mathrm{C}$ & 78 & $31.7 \%$ & 1.984 & 1.882 \\
\hline \multirow{2}{*}{$\operatorname{HIP} 900^{\circ} \mathrm{C}$} & 78 & $31.0 \%$ & 1.968 & 1.902 \\
\hline & 26 & $31.8 \%$ & 2.262 & 2.477 \\
\hline HIP $1050+800^{\circ} \mathrm{C}$ & 78 & $18.8 \%$ & 2.165 & 2.152 \\
\hline
\end{tabular}

Table 38 - Summary of total elongation correlations obtained for polished specimens of AM Ti64.

\begin{tabular}{|c|c|c|c|c|}
\hline & Slope & 0.0832 & 0.3047 \\
\hline & & Intercept & 0.13 & -0.30 \\
\hline & & $r$ & 0.3036 & 0.8838 \\
\hline & & Tensile & SP & SP \\
\hline \multirow{2}{*}{$\begin{array}{l}\text { Material } \\
\text { condition }\end{array}$} & \multirow{2}{*}{$\begin{array}{c}\text { Scan length } \\
(\mathrm{mm})\end{array}$} & property & parameter & parameter \\
\hline & & $\mathrm{TE}$ & $u_{m} / h_{0}$ & $\left(u_{f}-h_{0}\right) / h_{0}$ \\
\hline \multirow{2}{*}{ As-Built } & 78 & $27.8 \%$ & 2.023 & 1.868 \\
\hline & 26 & $25.5 \%$ & 1.749 & 1.759 \\
\hline $\mathrm{HIP} 800^{\circ} \mathrm{C}$ & 78 & $31.7 \%$ & 1.767 & 2.088 \\
\hline \multirow{2}{*}{$\operatorname{HIP} 900^{\circ} \mathrm{C}$} & 78 & $31.0 \%$ & 2.029 & 2.029 \\
\hline & 26 & $31.8 \%$ & 1.605 & 1.941 \\
\hline HIP $1050+800^{\circ} \mathrm{C}$ & 78 & $18.8 \%$ & 1.636 & 1.717 \\
\hline
\end{tabular}

For rough specimens, negative (and therefore non-physical) correlations were found between $\varepsilon_{\mathrm{t}}$ and both $u_{\mathrm{m}} / h_{0}$ and $\frac{u_{\mathrm{f}}-h_{0}}{h_{0}}$ (Fig. 20). In the case of polished specimens, however, both correlations were positive, and the correlation with $\frac{u_{\mathrm{f}}-h_{0}}{h_{0}}$ (Fig. 20) was quite strong.

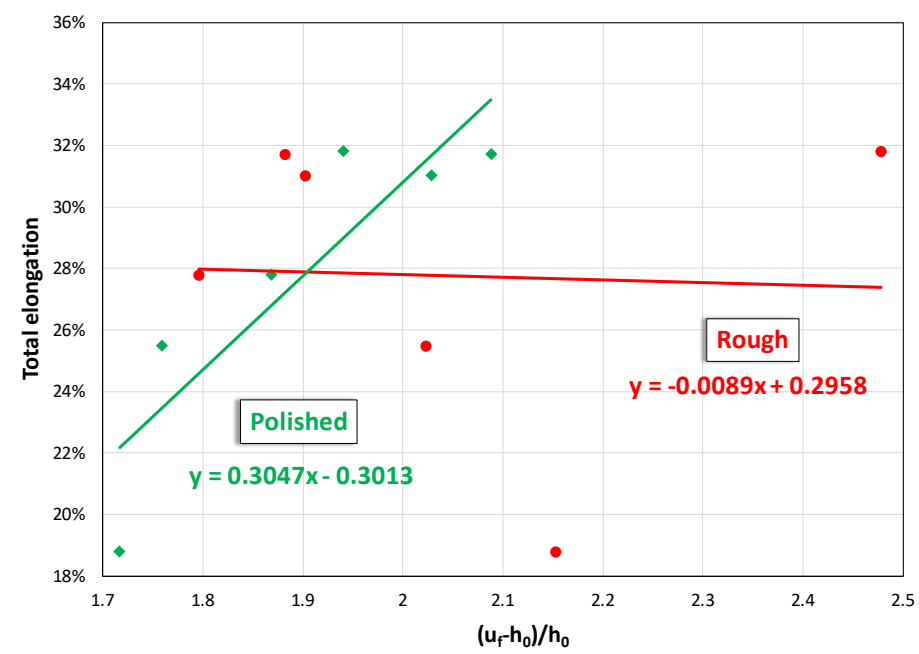

Figure 20 - Correlations between $\left(u_{\mathrm{f}}-h_{0}\right)$ and total elongation for rough and polished specimens of AM Ti64. 
We also attempted to correlate uniform elongation, $\varepsilon_{\mathrm{u}}$, to the normalized punch displacement at maximum force, $u_{\mathrm{m}} / h_{0}$ (Tables 39-40 and Fig. 21). Once again, a non-physical negative relationship was found for rough specimens, whereas a weak $(r=0.39)$ positive correlation was obtained for polished specimens.

Table 39 - Uniform elongation correlation obtained for rough specimens of AM Ti64.

\begin{tabular}{|c|c|c|c|}
\hline & Slope & -0.0179 \\
\hline & & Intercept & 0.13 \\
\hline & & $r$ & -0.3420 \\
\hline & & Tensile & SP \\
\hline \multirow{2}{*}{$\begin{array}{c}\text { Material } \\
\text { condition }\end{array}$} & \multirow{2}{*}{$\begin{array}{c}\text { Scan length } \\
(\mathrm{mm})\end{array}$} & property & parameter \\
\hline & & UE & $u_{m} / h_{0}$ \\
\hline \multirow{2}{*}{ As-Built } & 78 & $9.5 \%$ & 1.867 \\
\hline & 26 & $9.1 \%$ & 2.211 \\
\hline HIP $800^{\circ} \mathrm{C}$ & 78 & $10.1 \%$ & 1.984 \\
\hline \multirow{2}{*}{ HIP $900^{\circ} \mathrm{C}$} & 78 & $10.0 \%$ & 1.968 \\
\hline & 26 & $10.0 \%$ & 2.262 \\
\hline HIP $1050+800^{\circ} \mathrm{C}$ & 78 & $7.9 \%$ & 2.165 \\
\hline
\end{tabular}

Table 40 - Uniform elongation correlation obtained for polished specimens of AM Ti64.

\begin{tabular}{|c|c|c|c|}
\hline & Slope & 0.0173 \\
\hline & & Intercept & 0.06 \\
\hline & & $r$ & 0.3870 \\
\hline & & Tensile & \\
\hline \multirow{2}{*}{$\begin{array}{l}\text { Material } \\
\text { condition }\end{array}$} & \multirow{2}{*}{$\begin{array}{c}\text { Scan length } \\
(\mathrm{mm})\end{array}$} & property & parameter \\
\hline & & UE & $\mathrm{u}_{\mathrm{m}} / \mathrm{h}_{0}$ \\
\hline \multirow{2}{*}{ As-Built } & 78 & $9.5 \%$ & 2.023 \\
\hline & 26 & $9.1 \%$ & 1.749 \\
\hline $\mathrm{HIP} 800^{\circ} \mathrm{C}$ & 78 & $10.1 \%$ & 1.767 \\
\hline \multirow{2}{*}{$\operatorname{HIP} 900^{\circ} \mathrm{C}$} & 78 & $10.0 \%$ & 2.029 \\
\hline & 26 & $10.0 \%$ & 1.605 \\
\hline HIP $1050+800^{\circ} \mathrm{C}$ & 78 & $7.9 \%$ & 1.636 \\
\hline
\end{tabular}

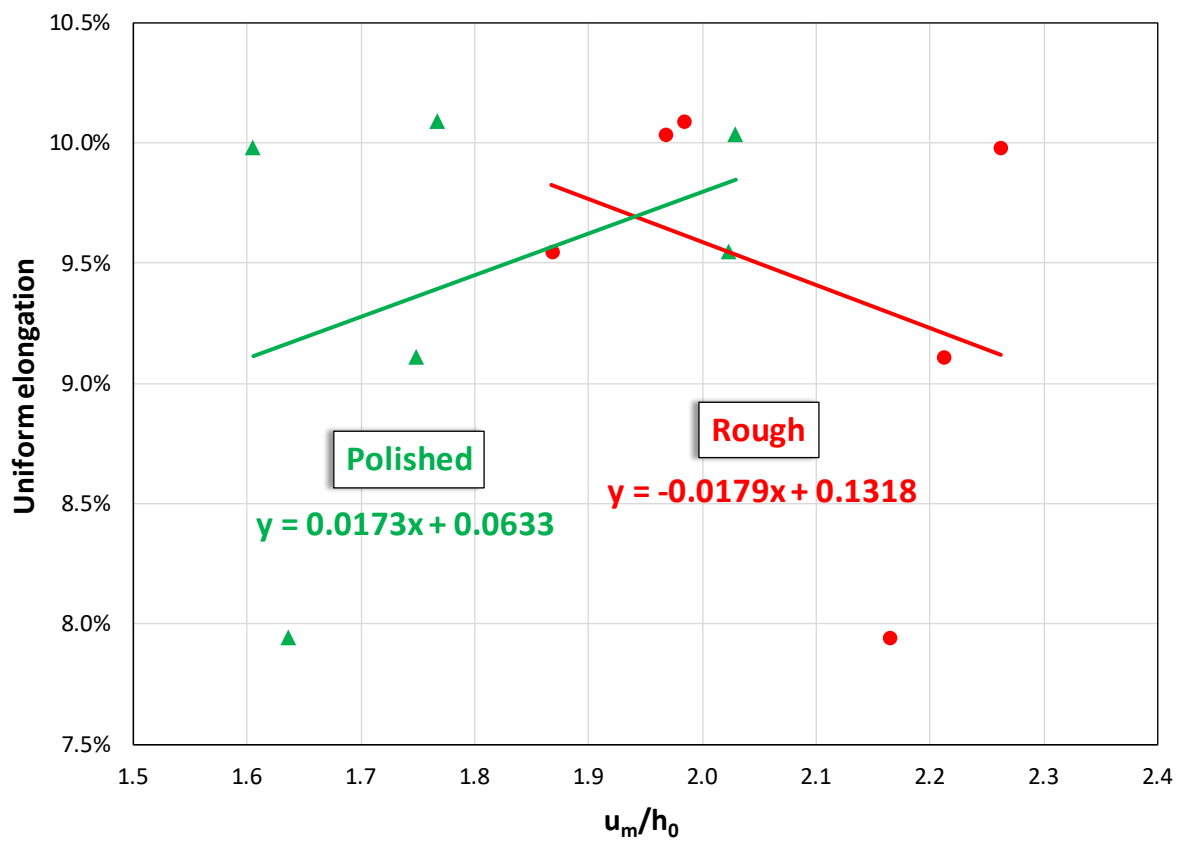

Figure 21 - Correlations between $u_{\mathrm{m}} / h_{0}$ and uniform elongation for rough and polished specimens of AM Ti64. 
All the total and uniform elongation correlations, obtained for both rough and polished specimens, are documented in Annex 16.

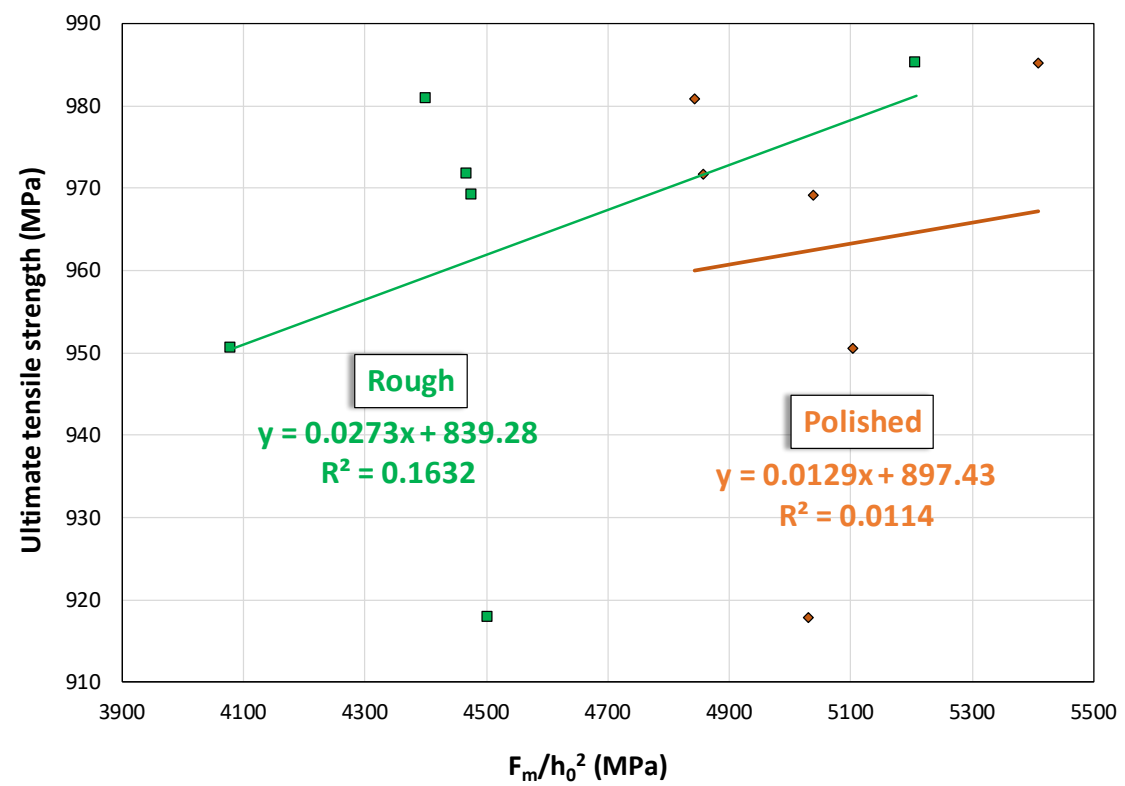

Figure 22 - Correlations between $F_{\mathrm{m}} / h_{0}^{2}$ and ultimate tensile strength for rough and polished specimens of AM Ti64.

\section{Correlations with fracture toughness}

In the literature, fracture toughness has been generally estimated from SP test results by means of analytical approaches involving finite element calculations [37,38]. Most of the few published empirical correlations were established between critical $J$-integral $\left(J_{\text {Ic }}, J_{\mathrm{Q}}\right)$ and effective (or biaxial) fracture strain [10,23,38,39], calculated as:

$$
\varepsilon_{\mathrm{f}}=\ln \left(\frac{h_{0}}{h_{\mathrm{f}}}\right)
$$

However, at the time of writing, $h_{\mathrm{f}}$ values were not available for the specimens tested.

The room temperature elastic-plastic fracture toughness of four of the AM Ti64 investigated conditions (as-built and non-standard $900{ }^{\circ} \mathrm{C}$ HIP, two scan lengths) was measured on fatigue precracked Charpy-type specimens in a previous investigation [14], by means of the Elastic Compliance single-specimen methodology, in terms of size-sensitive critical value of $J$-integral, $J_{\mathrm{Q}}$ (Table 41).

Table 41 - Elastic-plastic fracture toughness measured on four AM Ti64 conditions [14].

\begin{tabular}{|cc|}
\hline $\begin{array}{c}\text { Material } \\
\text { condition }\end{array}$ & $\begin{array}{c}\boldsymbol{J}_{\mathbf{Q}} \\
\left(\mathbf{k N} / \mathbf{m}^{2}\right)\end{array}$ \\
\hline $\mathbf{a , e 1}$ & 121.14 \\
\hline $\mathbf{a , e 2}$ & 93.23 \\
\hline $\mathbf{c , e 1}$ & 157.67 \\
\hline $\mathbf{c , e 2}$ & 151.61 \\
\hline
\end{tabular}


In this study, we attempted novel correlations between $J_{\mathrm{Q}}$ and the following normalized SP energy values: $\frac{E_{\mathrm{SP}}}{h_{0} u_{\mathrm{f}}}, \frac{E_{\mathrm{m}}}{h_{0} u_{\mathrm{m}}}$, and $\frac{E_{\mathrm{PL}}}{h_{0} u_{\mathrm{m}, \mathrm{pl}}}$. In each case, SP energy was normalized by the product of the initial thickness by the corresponding displacement value. For $E_{\mathrm{PL}}$, the plastic component of $u_{\mathrm{m}}$ was calculated as:

$$
u_{\mathrm{m}, \mathrm{pl}}=u_{\mathrm{m}}-\frac{F_{\mathrm{m}}}{\text { Slope }_{\mathrm{ini}}} .
$$

For both rough and polished specimens, moderately strong correlations were found between $J_{\mathrm{Q}}$ and the normalized fracture energy $\frac{E_{S P}}{h_{0} u_{f}}(r=0.60$ and 0.62 , respectively, Fig. 17). The remaining correlations obtained were negative and therefore unacceptable for rough specimens, while in the case of polished specimens a weak correlation was obtained for the plastic energy, $\frac{E_{P L}}{h_{0} u_{m, p l}}(r=0.43)$, and no correlation was observed with the normalized total energy, $\frac{E_{m}}{h_{0} u_{m}}(r=0.18)$.

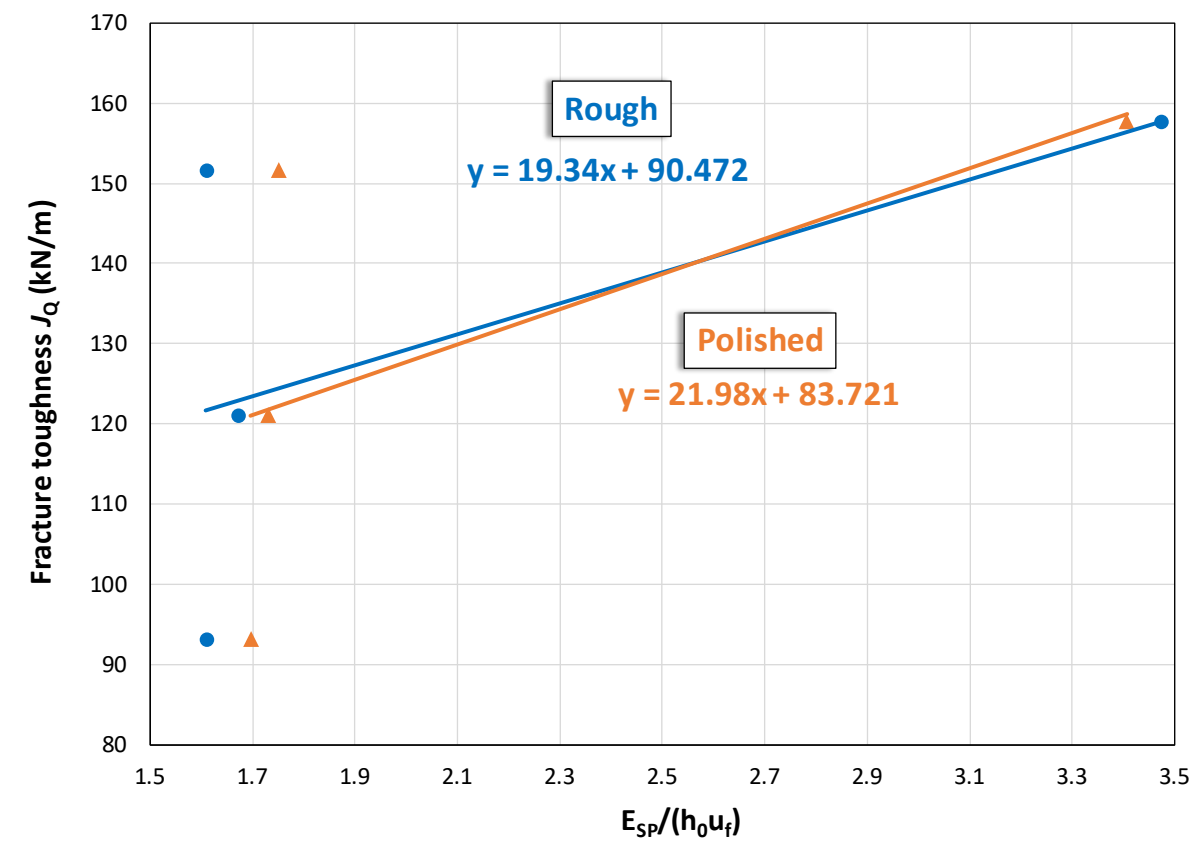

Figure 23 - Empirical correlations between $J_{\mathrm{Q}}$ and $E_{\mathrm{SP}} /\left(h_{0} u_{\mathrm{f}}\right)$ for rough and polished SP specimens of AM Ti64.

\section{Use of actuator displacement in the analyses}

Two displacement signals were recorded during SP testing: the signal of the extensometer, which corresponds to punch displacement, and the displacement of the machine actuator. Several authors have also reported the use of another displacement (specimen deflection), measured by a transducer (LVDT) positioned below the specimen being tested.

All the analyses of the tests performed on rough specimens were repeated using actuator displacement as a surrogate of punch displacement. Comparisons between average values and standard deviations for selected characteristic force, displacement, and energy 
values obtained using extensometer and actuator displacements are illustrated in Fig. 24 ( $F_{\text {e,proj }}$ and $\left.F_{0.1 \mathrm{~mm}, \mathrm{off}}\right)$, Fig. 25 ( $u_{\mathrm{e}}, u_{0.1 \mathrm{~mm}, \mathrm{off}}, u_{\mathrm{m}}$, and $\left.u_{\mathrm{f}}\right)$, and Fig. $26\left(E_{\mathrm{SP}}, E_{\mathrm{m}}\right.$, and $\left.E_{\mathrm{PL}}\right)$.

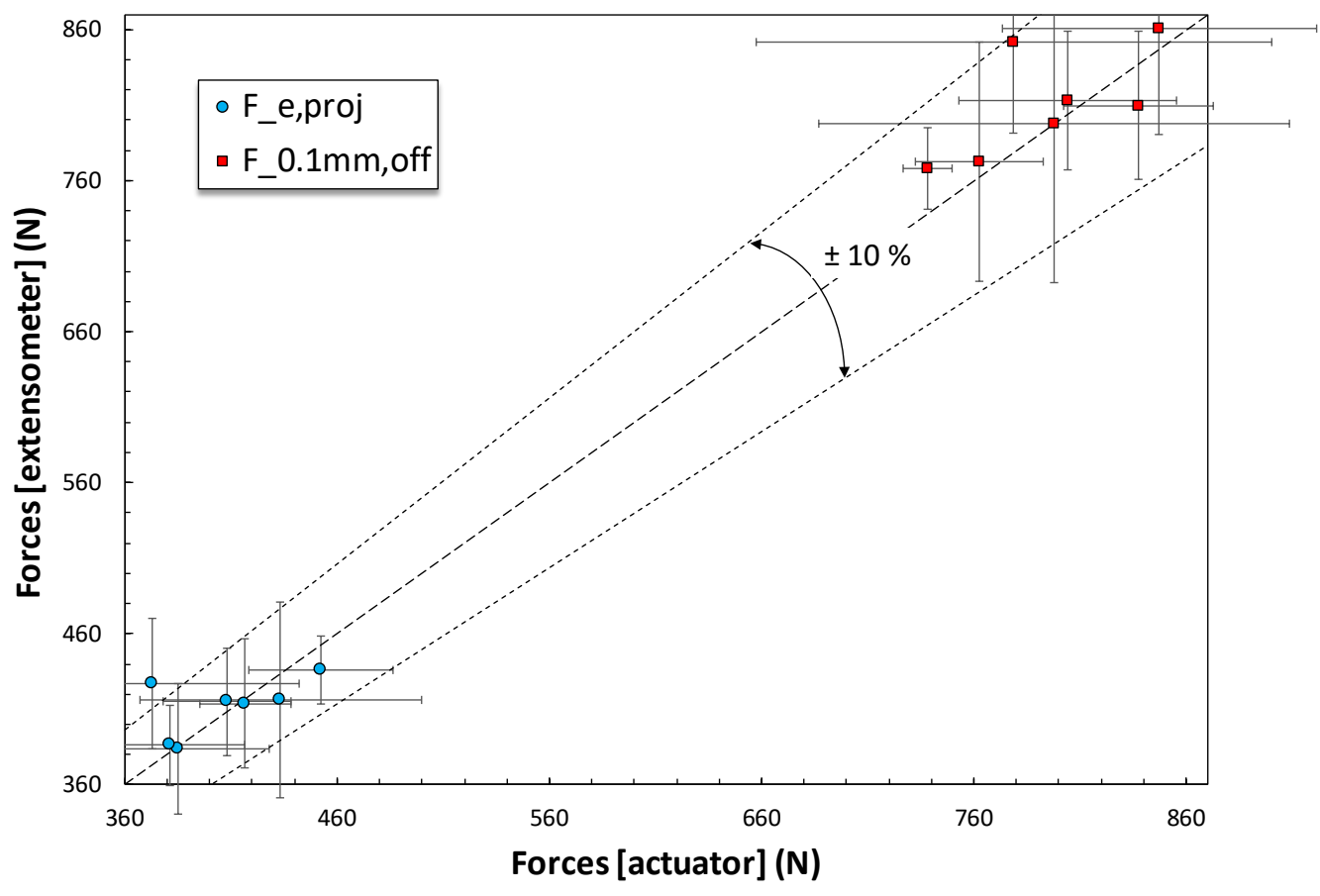

Figure 24 - Mean characteristic force values obtained for rough specimens using extensometer and actuator displacements, with $\pm 1 \sigma$ error bands.

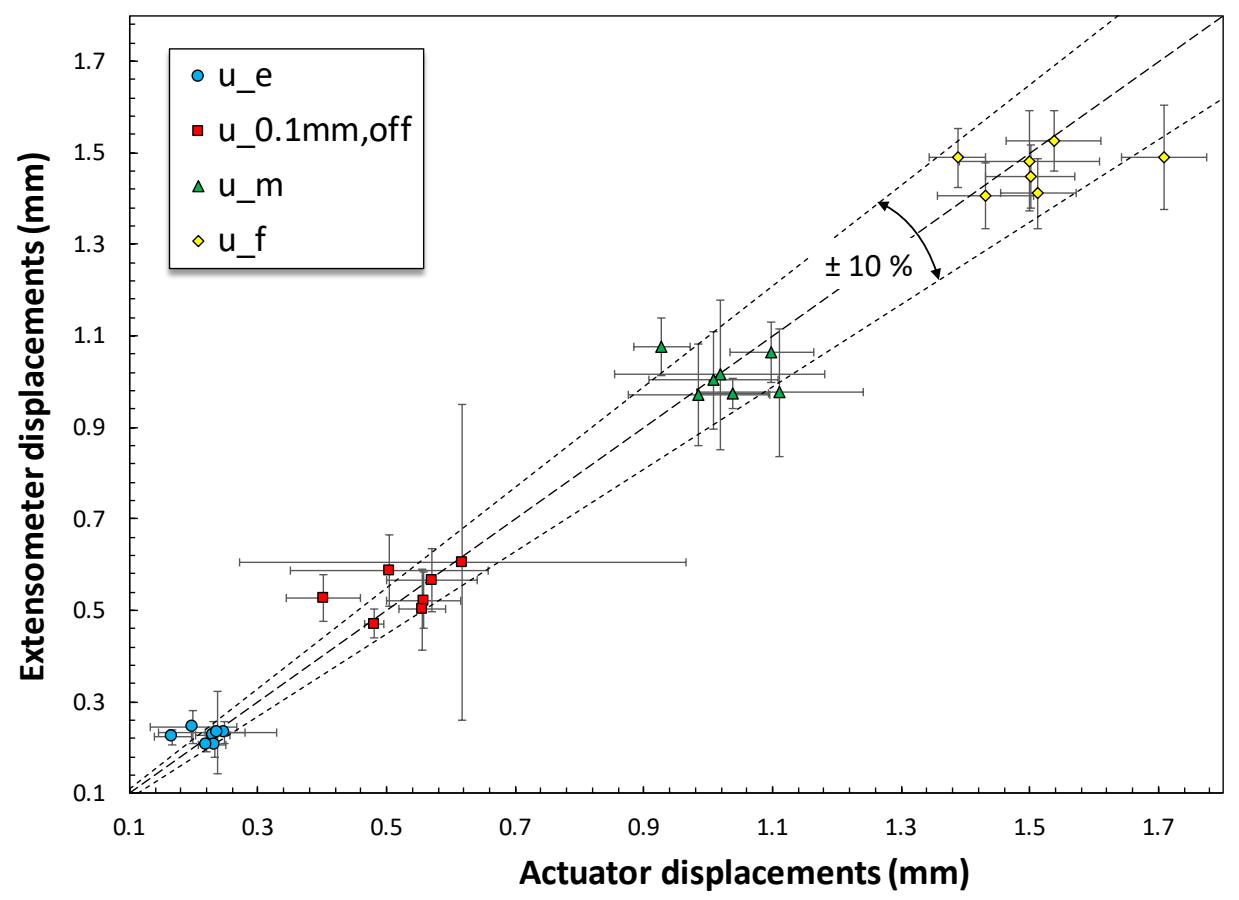

Figure 25 - Mean characteristic displacement values obtained for rough specimens using extensometer and actuator displacements, with $\pm 1 \sigma$ error bands. 


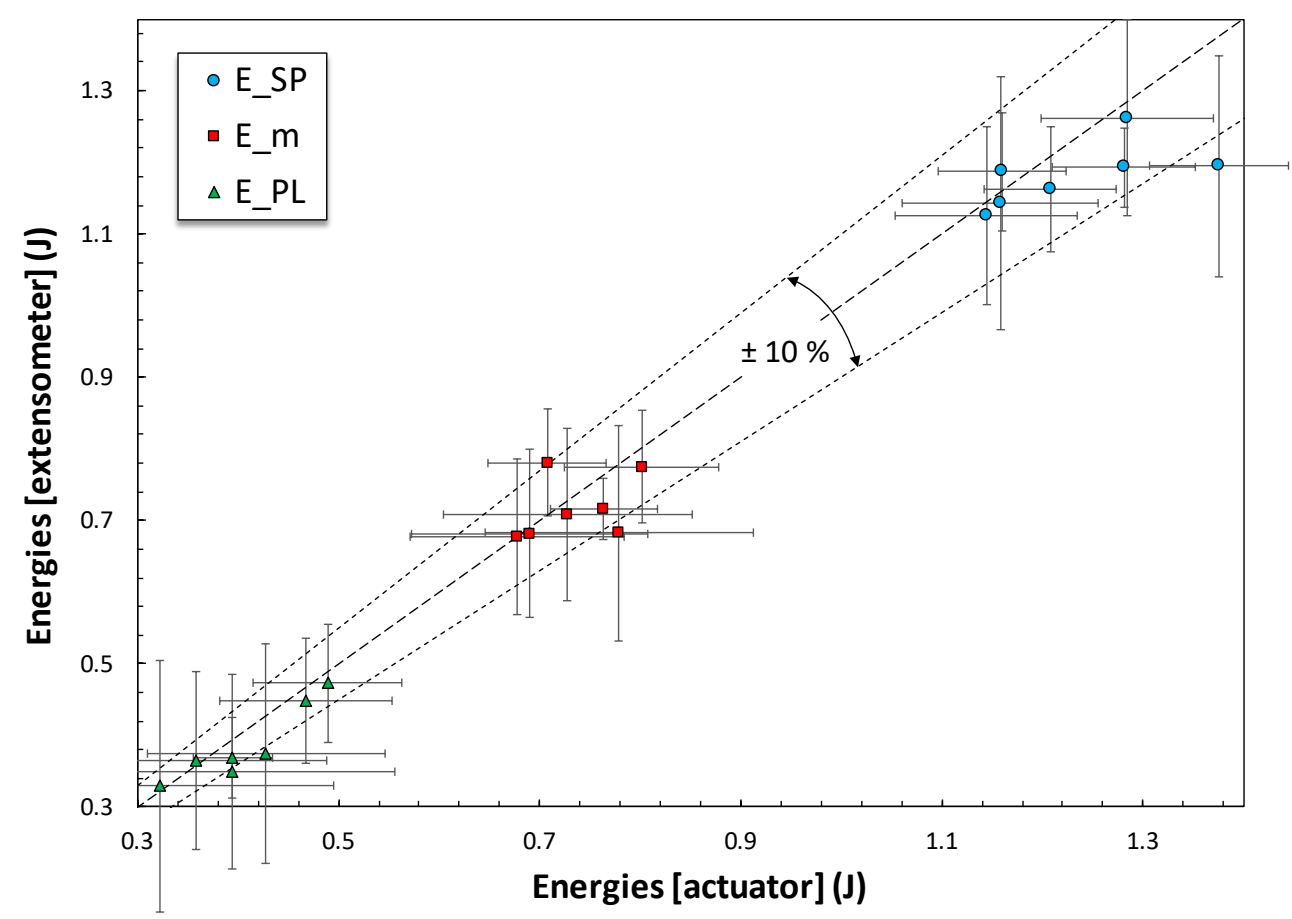

Figure 26 - Mean characteristic energy values obtained for rough specimens using extensometer and actuator displacements, with $\pm 1 \sigma$ error bands.

The data shown in Figs. 24-26 show a relatively modest influence of the choice of displacement signal on the characteristic parameters obtained from SP tests, also considering the relatively large standard deviations. Two qualitative remarks can nonetheless be formulated:

- Most of the average values are within $\pm 10 \%$ of each other.

- In case of force values (Fig. 24), a specific trend is not clearly visible. For displacements (Fig. 25) and energies (Fig. 26), it can be observed that actuator-based values tend to be generally higher than extensometer-based values.

The most practical information, however, is the effect of using actuator displacements to derive correlations with tensile properties, in a hypothetical situation where no external displacement transducer is available. Such an effect is illustrated in Table 42, which compares values of Pearson's correlation coefficient, $r$, from selected empirical correlations established by using SP extensometer-based and actuator-based parameters for rough specimens.

Note that we will not report details on specific actuator-based empirical correlations here, as the reference procedure this document uses is based on measuring punch displacement by means of an extensometer. 
Table 42 - Correlation coefficients for extensometer-based and actuator-based correlations for rough specimens. In the last column, red arrows indicate a decrease in $r$ going from extensometer to actuator (two red arrows = substantial decrease), green arrows an increase, and black horizontal arrows a minor variation.

\begin{tabular}{|c|c|c|c|c|}
\hline \multirow[b]{2}{*}{ Y-variable } & \multirow[b]{2}{*}{ X-variable } & \multicolumn{2}{|c|}{ Correlation coefficients, $r$} & \multirow[b]{2}{*}{ Change } \\
\hline & & Extensometer & Actuator & \\
\hline \multirow{4}{*}{$\begin{array}{c}\text { Yield } \\
\text { strength }\end{array}$} & $\mathrm{F}_{\mathrm{e}, \mathrm{proj}} / \mathrm{h}_{0}{ }^{2}$ & 0.6661 & 0.5619 & $\Downarrow$ \\
\hline & $\mathrm{F}_{\mathrm{e}, \mathrm{proj}} /\left(\mathrm{u}_{\mathrm{e}} \mathrm{h}_{0}\right)$ & 0.5597 & 0.4458 & $\Downarrow$ \\
\hline & $\mathrm{F}_{0.1 \mathrm{~mm}, \mathrm{off}} / \mathrm{h}_{0}^{2}$ & 0.1262 & 0.4257 & $\Uparrow$ \\
\hline & Slope $_{\mathrm{e}} / \mathrm{h}_{0}$ & 0.5338 & 0.3972 & $\Downarrow$ \\
\hline \multirow{2}{*}{$\begin{array}{c}\text { Ultimate } \\
\text { tensile } \\
\text { strength }\end{array}$} & $\mathrm{F}_{\mathrm{m}} / \mathrm{h}_{0}^{2}$ & \multicolumn{3}{|c|}{0.4040} \\
\hline & $\mathrm{F}_{\mathrm{m}} /\left(\mathrm{u}_{\mathrm{m}} \mathrm{h}_{0}\right)$ & 0.7386 & 0.0608 & ШШ \\
\hline UE & $\mathrm{u}_{\mathrm{m}} / \mathrm{h}_{0}$ & -0.3420 & -0.3580 & $\Leftrightarrow$ \\
\hline \multirow{2}{*}{ TE } & $\mathrm{u}_{\mathrm{f}} / \mathrm{h}_{0}$ & -0.2681 & -0.1304 & $\Leftrightarrow$ \\
\hline & $\left(u_{\mathrm{f}}-\mathrm{h}_{0}\right) / \mathrm{h}_{0}$ & -0.0436 & 0.1975 & $\Uparrow$ \\
\hline
\end{tabular}

As can be seen in Table 42, the use of actuator displacement generally tends to weaken the strength of the strongest correlations that were established on the basis of extensometer displacement. Relative improvements are only observed for relationships that were already rather shaky, or even non-physical (negative values of $r$ ).

\section{Discussion}

Across the board, the quality of empirical correlations between tensile properties and SP parameters for AM Ti64 was found to be poorer than that reported for steels in the open literature. This is hardly surprising, considering that the Small Punch methodology was developed for application to high-quality materials in the power industry [1], and was found to be inaccurate for highly anisotropic materials, such as oxide dispersion strengthened (ODS) steels [40]. As an example, the force-displacement curve for a SP test on ODS 14YWT steel [40] shown in Figure 24, exhibits several force drops ("pop-in" type events), associated with the steel's susceptibility to secondary cracking, and has a different form than the classical SP curve for homogeneous steels (Figure 1). 


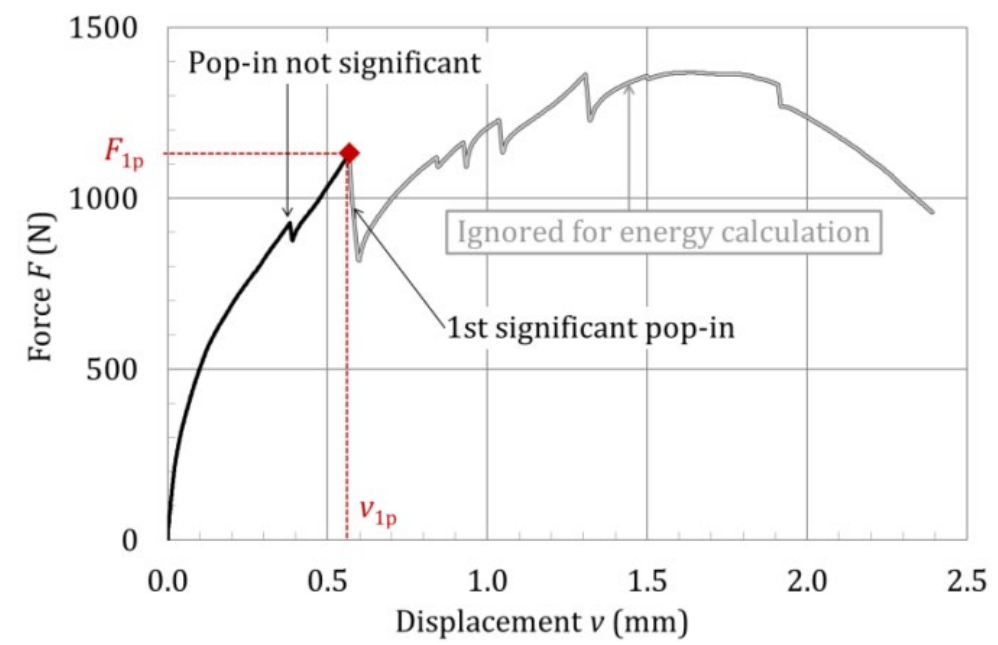

Figure 27 - Force-displacement curve for a SP test on ODS 14YWT steel [40].

It should also be noted that the range of tensile properties for the AM Ti64 investigated conditions, with respect to the property average value was relatively limited, particularly in the case of $R_{\mathrm{p} 02}$ and $R_{\mathrm{m}}(10.1 \%$ and $7 \%$, respectively). This makes it even more difficult to obtain strong correlations, particularly in the presence of significant experimental variability.

As far as the influence of specimen surface finish is concerned, we can generally state that polished specimens correlate slightly better with AM Ti64 tensile properties than rough specimens, which supports the inclusion of the $\mathrm{Ra}<0.25 \mu \mathrm{m}$ requirement in both the ASTM draft [11] and the CEN Workshop Agreement CWA15627:2007 [10]. Moreover, we observe that normalized force values are systematically larger for polished specimens.

Follow-up microstructural investigations are planned to provide a more complete interpretation of the SP results obtained in the different conditions, including measurements of final specimen thickness values, $h_{\mathrm{f}}$, on the fracture surface to be correlated with fracture toughness.

\section{Conclusions}

The overall quality of the empirical correlations that we obtained between tensile properties and SP test parameters for six conditions of AM Ti64 is not satisfactory. Most correlations were found to be non-existent or weak at best. This is not an unexpected conclusion, since the Small Punch methodology has been shown to have limited applicability for highly anisotropic materials, such as those currently produced via Additive Manufacturing. However, it's important to note that the AM community is actively working on decreasing the anisotropy of AM materials, and therefore these correlations could be revisited once significant progress has been made in this direction.

The available literature on the application of various SP testing techniques, including fatigue and creep, to various types of Ti64 (forged, cast, and EBM) [17-20, 41] demonstrates a significant level of interest in the material testing community. Published force-displacement curves of AM specimens [17-20] are similar to those we obtained in this study. Several references also show significantly lower tensile properties for AM Ti64 with respect to forged 
and cast material, as inferred from SP tests performed both at ambient and elevated temperatures $[17,19,20]$.

In our study, the strongest correlations (correlation coefficient $r>0.70^{10}$ ) for polished specimens were found between:

- Yield strength $\left(R_{\mathrm{p} 02}\right)$ and normalized SP elastic-plastic transition force $\left(F_{\mathrm{e}} / h_{0}^{2}\right)$, Eqs. $(2)$ and (3).

- Yield strength $\left(R_{\mathrm{p} 02}\right)$ and normalized SP force at $h_{0} / 10$ offset $\left(F_{h 0, \text { off }} / h_{0}^{2}\right)$ for rough specimens ( $\mathrm{Ra}>3 \mu \mathrm{m})$, Eq. (4).

- Yield strength $\left(R_{\mathrm{p} 02}\right)$ and normalized SP force at $0.1 \mathrm{~mm}$ offset $\left(F_{0.1 \mathrm{~mm}, \mathrm{off}} / h_{0}^{2}\right)$ for polished specimens $(\mathrm{Ra}<0.25 \mu \mathrm{m})$, Eq. (5).

- Total elongation $\left(\varepsilon_{\mathrm{t}}\right)$ and normalized SP displacement at fracture $\left(\frac{u_{\mathrm{f}}-h_{0}}{h_{0}}\right)$ for polished specimens, Eq. (8).

Correlations between fracture toughness and normalized SP energies were also attempted, but were not particularly successful, with a highest degree of correlation around $r$ $=0.60$ obtained between $J_{\mathrm{Q}}$ and normalized fracture energy.

The influence of surface roughness on SP characteristic parameters was found to be significant on specimen yielding and fracture, in that these phenomena tend to occur earlier in the test in the case of rougher specimens. Across the board, polished specimens $(\mathrm{Ra}<0.25 \mu \mathrm{m}$, in accordance with current normative or pre-normative documents) appear to yield better correlations.

Finally, the use of actuator displacement in the place of punch displacement (measured by an extensometer) in the analysis of SP tests did not have a particularly significant influence on characteristic parameters, but caused a generalized weakening of the empirical correlations with tensile properties.

\section{References}

[1] Lucon, E., 2014, "Testing of Small-Sized Specimens," Comprehensive Materials Processing, C. J. Van Tyne, ed., Elsevier Ltd., Vol. 1, pp. 135-163. http://dx.doi.org/10.1016/B978-0-08-096532-1.00110-2

[2] Harling, O. K., Lee, M., Sohn, D.-S., Kohse, G., and Lau, C. W., 1986, "The MIT Miniaturised Disc Bend Test," The Use of Small-Scale Specimens for Testing Irradiated Material, ASTM STP 888. W. R. Corwin, and G. E. Lucas, eds., ASTM, Philadelphia, PA, pp. 50-65.

[3] Manahan, M. P., Browning, A. E., Argon, A. S., and Harling, O. K., 1986, "Miniaturised Disc Bend Test Technique Development and Application," The Use of Small-Scale Specimens for Testing Irradiated Material, ASTM STP 888. W. R. Corwin, and G. E. Lucas, eds., ASTM, Philadelphia, PA, pp. 17-49.

[4] Hankin, G. L., Toloczko, M. B., Hamilton, M. L., Garner, F. A., and Faulkner, R. G., 1998, "Validation of the Shear Punch - Tensile Correlation Technique Using Irradiated Materials," Journal of Nuclear Materials, 258-263, pp. 1651-1656. http://dx.doi.org/10.1016/S0022-3115(98)00203-7

\footnotetext{
${ }^{10}$ To put this value into context, most published correlations for steels report correlation coefficients higher than 0.90 .
} 
[5] Bicego, V., Lucon, E., and Sampietri, C., 1998, "The 'Small Punch' Technique for Evaluating Quasi Non-Destructively the Mechanical Properties of Steels," In Fracture from Defects, ECF 12: Proceedings of the Twelfth European Conference on Fracture. M. W. Brown, E. R. de los Rios, K. J. Miller, eds., EMAS Publishing, London, Vol. 1, pp 1273-1278.

[6] Sainte-Catherine, C., Messier. J., Poussard, C., Rosinski, S., and Foulds, J., 2002, "Small Punch Test: EPRI-CEA Finite Element Simulation Benchmark and Inverse Method for the Estimation of Elastic Plastic Behavior," in Small Specimen Test Techniques: Fourth Volume, ASTM STP 1418, M. A. Sokolov, J. D. Landes, and G. E. Lucas, Eds., ASTM International, West Conshohocken, PA, pp. 350-370.

[7] Abendroth, M. and Kuna, M., 2003, "Determination of Deformation and Failure Properties of Ductile Materials by Means of the Small Punch Test and Neural Networks," Computational Materials Science, Vol 28, pp. 633-644. http://dx.doi.org/10.1016/j.commatsci.2003.08.031

[8] Abendroth, M. and Kuna, M., 2004, "Determination of Ductile Material Properties by Means of the Small Punch Test and Neural Networks," Advanced Engineering Materials, Vol 6, No. 7, 2004, pp. 536-540. http://dx.doi.org/10.1002/adem.200400405

[9] Kuna, M. and Abendroth, M., 2012, "Identification and Validation of Ductile Damage Parameters by the Small Punch Test," in Proceedings of the $2^{\text {nd }}$ International Conference SSTT, Determination of Mechanical Properties of Materials by Small Punch and Other Miniature Testing Techniques, Oct 2-4. 2012, Ostrava, Czech Republic, Ocelot Publ., ISBN 978-80-260-0079-2, pp. 4-18.

[10] CEN Workshop Agreement, CWA 15627:2007, "Small Punch Test Method for Metallic Materials," European Committee for Standardization, Brussels, Belgium.

[11] ASTM Subcommittee E10.02, 2019, "Standard Test Method for Small Punch Testing of Metallic Materials," Work Item WK61832, Version 01/29/19.

[12] Lucon, E., Benzing, J., and Hrabe, N., 2020, "Development and Validation of Small Punch Testing at NIST," NIST Internal Report 8303, Boulder, Colorado. https://doi.org/10.6028/NIST.IR.8303

[13] Lucon, E. and Hrabe, N., 2018, "Instrumented Impact Testing of Miniaturized Charpy Specimens of AM Ti-6Al-4V," Materials Performance and Characterization, Vol. 7, No. 1, pp. 126-138. https://doi.org/10.1520/MPC20170160

[14] Lucon, E., Benzing, J., and Hrabe, N., 2019, "Room Temperature Fracture Toughness Characterization of Additively Manufactured Ti-6Al-4V," NIST Technical Note 2065, Boulder, Colorado. https://doi.org/10.6028/NIST.TN.2065

[15] Benzing, J., Hrabe, N., Quinn, T., White, R., and Rentz, R., 2019, "Manipulation of microstructure and tensile properties of additively manufactured Ti-6Al-4V parts," presented at the $19^{\text {th }}$ International ASTM/ESIS Symposium on Fatigue and Fracture Mechanics ( $42^{\text {nd }}$ National Symposium on Fatigue and Fracture Mechanics), May 15-17, 2019, Denver, CO, USA.

[16] Hrabe, N., Gnäupel-Herold, T., and Quinn, T., 2017," Fatigue properties of a titanium alloy (Ti-6Al-4V) fabricated via electron beam melting (EBM): Effects of internal defects and residual stress," International Journal of Fatigue, Vol. 94, pp. 202-210.

[17] Lancaster, R., Davies, G., Illsley, H., Jeffs, S., and Baxter, G., 2016, "Structural Integrity of an Electron Beam Melted Titanium," Materials, 9, 470. http://dx.doi.org/10.3390/ma9060470 
[18] Lancaster, R. J., Illsey, H. W., Davies, G. R., Jeffs, S. P., and Baxter, G. J., 2016, "Modelling the small punch tensile behaviour of an aerospace alloy," Materials Science and Technology, 33:9, pp. 1065-1073 http://dx.doi.org/10.1080/02670836.2016.1230168

[19] Hurst, R. C., Lancaster, R. J., Jeffs, S. P., and Bache, M. R., 2016, "The contribution of small punch testing towards the development of materials for aero-engine applications," Theoretical and Applied Fracture Mechanics 86, pp. 69-77. http://dx.doi.org/10.1016/j.tafmec.2016.07.013

[20] Illsey, H., Lancaster, R., Hurst, R., Jeffs, S., and Baxter, G., 2017, "Mechanical Property Characterisation of Electron Beam Melted (EBM) via Small Punch Tensile Testing," Key Engineering Materials, Vol. 734, pp. 51-60.

[21] Ruan, Y., Spätig, P., and Victoria, M., 2002, "Assessment of mechanical properties of the martensitic steel EUROFER97 by means of punch tests," Journal of Nuclear Materials, Volumes 307-311, pp. 236-239. http://dx.doi.org/10.1016/S0022-3115(02)01194-7

[22] Matocha, K., 2012, "Determination of actual tensile and fracture characteristics of critical components of industrial plants under long term operation by SPT," Proceedings of the ASME 2012 Pressure Vessels \& Piping Conference, PVP2012, Toronto, Canada, July 1519, ASME Paper No. PVP2012-78553. http://dx.doi.org/10.1115/PVP2012-78553

[23] García, T.E., Rodríguez, C., Belzunce, F.J., and Suárez, C., 2014, "Estimation of the mechanical properties of metallic materials by means of the small punch test," J Alloys and Compounds, 582, pp. 708-717. http://dx.doi.org/10.1016/j.jallcom.2013.08.009

[24] Matocha, K., 2015, "Small-Punch Testing for Tensile and Fracture Behavior: Experiences and Way Forward," Small Specimen Test Techniques: $6^{\text {th }}$ Volume, ASTM STP 1576, M. A. Sokolov and E. Lucon, eds., West Conshohocken, PA, pp. 145-159. http://dx.doi.org/10.1520/STP157620140005

[25] Bruchhausen, M., Holmström, S., Simonovski, I., Austin, T., Lapetite, J.-M., Ripplinger, J.-M., and de Haan, F., 2016, "Recent developments in small punch testing: Tensile properties and DBTT," Theoretical and Applied Fracture Mechanics, 86, pp. 2-10. http://dx.doi.org/10.1016/j.tafmec.2016.09.012

[26] Altstadt, E., Ge, H.E., Kuksenko, V., Serrano, M., Houska, M., Lasan, M., Bruchhausen, M., Lapetite, and Dai, Y., 2016, "Critical evaluation of the small punch test as a screening procedure for mechanical properties," Journal of Nuclear Materials, 472, pp. 186-195. http://dx.doi.org/10.1016/j.jnucmat.2015.07.029

[27] Janča, A., Siegl, J., and Haušild, P., 2016, "Small punch test evaluation methods for material characterization," Journal of Nuclear Materials, 481, pp. 201-213. http://dx.doi.org/10.1016/j.jnucmat.2015.07.029

[28] Chica, J.C., Díez, P.M.B., and Calzada, M.P., 2018, "Development of an improved correlation method for the yield strength of steel alloys in the small punch test," Proceedings of $5^{\text {th }}$ International Small Sample Test Techniques, SSTT2018, Swansea University, UK, July 10-12, 2018, Ubiquity Proceedings. http://dx.doi.org/10.5334/uproc.12

[29] Kumar, K., Pooleery, A., Madhusoodanan, K., Singh, R.N., Chakravartty, J.K., Shriwastaw, R.S., Dutta, B.K., and Sinha, R.K., 2015, "Evaluation of ultimate tensile strength using Miniature Disk Bend Test," Journal of Nuclear Materials, 461, pp. 100111. http://dx.doi.org/10.1016/j.jnucmat.2015.02.029

[30] Altstadt, E., Houska, M., Simonovski, I., Bruchhausen, M., Holmström, S., and Lacalle, R., 2018, "On the estimation of ultimate tensile stress from small punch testing," 
International Journal of Mechanical Sciences, 136, pp. 85-93. http://dx.doi.org/10.1016/j.ijmecsci.2017.12.016

[31] Fleury, E. and Ha, J.S., 1998, "On the estimation of ultimate tensile stress from small punch testing," International Journal of Pressure Vessel and Piping, 75, pp. 699-706.

[32] Rodriguez, C., Garcia Cabezas, J., Cardenas, E., Belzunce, F. J., and Betegon, C., 2009, "Mechanical Properties Characterization of Heat-Affected Zone Using the Small Punch Test," Welding Research, Vol. 88, pp. 188-s-192-s.

[33] Yang, S., Ling, X., and Peng, D., 2018, "Elastic and plastic deformation behavior analysis in small punch test for mechanical properties evaluation," Journal of Central South University, 25(4), pp. 747-753. http://dx.doi.org/10.1007/s11771-018-3779-7

[34] Contreras, M.A., Rodríguez, C., Belzunce, F. J., and Betegón, C., 2008, "Use of the small punch test to determine the ductile-to-brittle transition temperature of structural steels," Fatigue \& Fracture of Engineering Materials \& Structures, 31, pp. 727-737. http://dx.doi.org/10.1111/j.1460-2695.2008.01259.x

[35] Matocha, K., 2015, "Small Punch Testing for Tensile and Fracture Behavior: Experiences and Way Forward," Small Specimen Test Techniques: $6^{\text {th }}$ Volume. STP 1576, Mikhail Sokolov and Enrico Lucon, Eds. ASTM International, West Conshohocken, PA, pp. 145159. http://dx.doi.org/10.1520/STP157620140005

[36] Foulds, J.R. and Jewett, C. W., 1991, "Miniature Specimen Test Technique for Estimating Toughness," Report GS-7526, Electric Power Research Institute, Palo Alto, CA.

[37] Foulds, J. R., Wu, M., Srivastav, S., and Jewett, C. W., 1997, "Fracture and Tensile Properties of ASTM Cross-Comparison Exercise A533B Steel by Small Punch Testing", Small Specimen Test Techniques, ASTM STP 1329, W. R. Corwin, S. T. Rosinski, and E. Van Walle, eds., ASTM, Philadelphia, PA, pp 557-574.

[38] Wang, Z.-X., Shi, H.-J., Lu J., Shi P., and Ma X.-F., 2008, "Small punch testing for assessing the fracture properties of the reactor vessel steel with different thicknesses," Nuclear Engineering and Design, 238, pp. 3186-3193. http://dx.doi.org/10.1016/j.nucengdes.2008.07.013

[39] Hurst, R. C. and Matocha, K., 2015, "A Renaissance in the Use of the Small Punch Testing Technique," Transactions, SMiRT-23, Manchester, United Kingdom, August 10-14, 2015, Division II. http://dx.doi.org/10.1115/PVP2015-45095

[40] Altstadt, E., Houska, M., and Das, A., 2018, "Effect of anisotropic microstructure of ODS steels on small punch test results," Proceedings of 5th International Small Sample Test Techniques, SSTT2018, Swansea University, UK, July 10-12, 2018, Ubiquity Proceedings. http://dx.doi.org/10.5334/uproc.4

[41] Lewis, D. T. S., Lancaster, R. J., Jeffs, S. P., Illsley, H. W., Davies, S. J., and Baxter, G. J., 2019, "Characterising the fatigue performance of additive materials using the small punch test," Materials Science \& Engineering A 754, pp. 719-727. https://doi.org/10.1016/j.msea.2019.03.115 


\section{ANNEX 1 \\ SP Test Results for \\ $900{ }^{\circ} \mathrm{C}$ HIP, scan length $=52 \mathrm{~mm}$, supported ("rough" specimens)}


Specimen ID: 900HIP_2_S_a_SP1

Material: AM Ti-64

Test speed: $0.000735 \mathrm{~mm} / \mathrm{s}$

Max strain rate: $7.35 \mathrm{E}-04 \mathrm{~s}^{-1}$

\begin{tabular}{|c|c|c|}
\hline \multicolumn{3}{|c|}{ TEST RESULTS } \\
\hline \multicolumn{3}{|c|}{ Force values } \\
\hline$F_{e, p r o j}=$ & 444.0 & $\mathbf{N}$ \\
\hline$F_{e, \text { int }}=$ & 450.1 & $\mathbf{N}$ \\
\hline$F_{h 0 / 10, \text { off }}=$ & 717.9 & $\mathbf{N}$ \\
\hline$F_{0.1 \mathrm{~mm}, \mathrm{off}}=$ & 929.0 & $\mathbf{N}$ \\
\hline$F_{0.1 \mathrm{~mm}}=$ & 158.3 & $\mathbf{N}$ \\
\hline$F_{0.48 \mathrm{~mm}}=$ & 682.1 & $\mathbf{N}$ \\
\hline$F_{0.5 \mathrm{~mm}}=$ & 706.7 & $\mathbf{N}$ \\
\hline$F_{0.65 \mathrm{~mm}}=$ & 873.6 & $\mathbf{N}$ \\
\hline$F_{0.9 \mathrm{~mm}}=$ & 1132.5 & $\mathbf{N}$ \\
\hline$F_{\text {ept }}=$ & 320.9 & $\mathbf{N}$ \\
\hline$F_{\mathrm{e} 1.5}=$ & 113.5 & $\mathbf{N}$ \\
\hline$F_{m}=$ & 1155.0 & $\mathbf{N}$ \\
\hline$F_{\text {infl }}=$ & 1039.1 & $\mathbf{N}$ \\
\hline$F_{f}=$ & 924.0 & $\mathbf{N}$ \\
\hline \multicolumn{3}{|c|}{ Displacement values } \\
\hline$u_{e}=$ & 0.290 & $\mathrm{~mm}$ \\
\hline$u_{h 0 / 10, \text { off }}=$ & 0.513 & $\mathrm{~mm}$ \\
\hline $\mathrm{u}_{0.1 \mathrm{~mm}, \mathrm{off}}=$ & 0.701 & $\mathrm{~mm}$ \\
\hline$u_{e 1.5}=$ & 0.074 & $\mathrm{~mm}$ \\
\hline$u_{m}=$ & 1.076 & $\mathrm{~mm}$ \\
\hline$u_{\text {infl }}=$ & 1.300 & $\mathrm{~mm}$ \\
\hline$u_{f}=$ & 1.475 & $\mathrm{~mm}$ \\
\hline \multicolumn{3}{|c|}{ Initial linear slope } \\
\hline Slope $_{\text {ini }}=$ & 1550.09 & $\mathrm{~N} / \mathrm{mm}$ \\
\hline \multicolumn{3}{|c|}{ Energy values } \\
\hline$E_{S P}=$ & 1.18 & J \\
\hline$E_{m}=$ & 0.76 & J \\
\hline$E_{P L}=$ & 0.33 & J \\
\hline
\end{tabular}




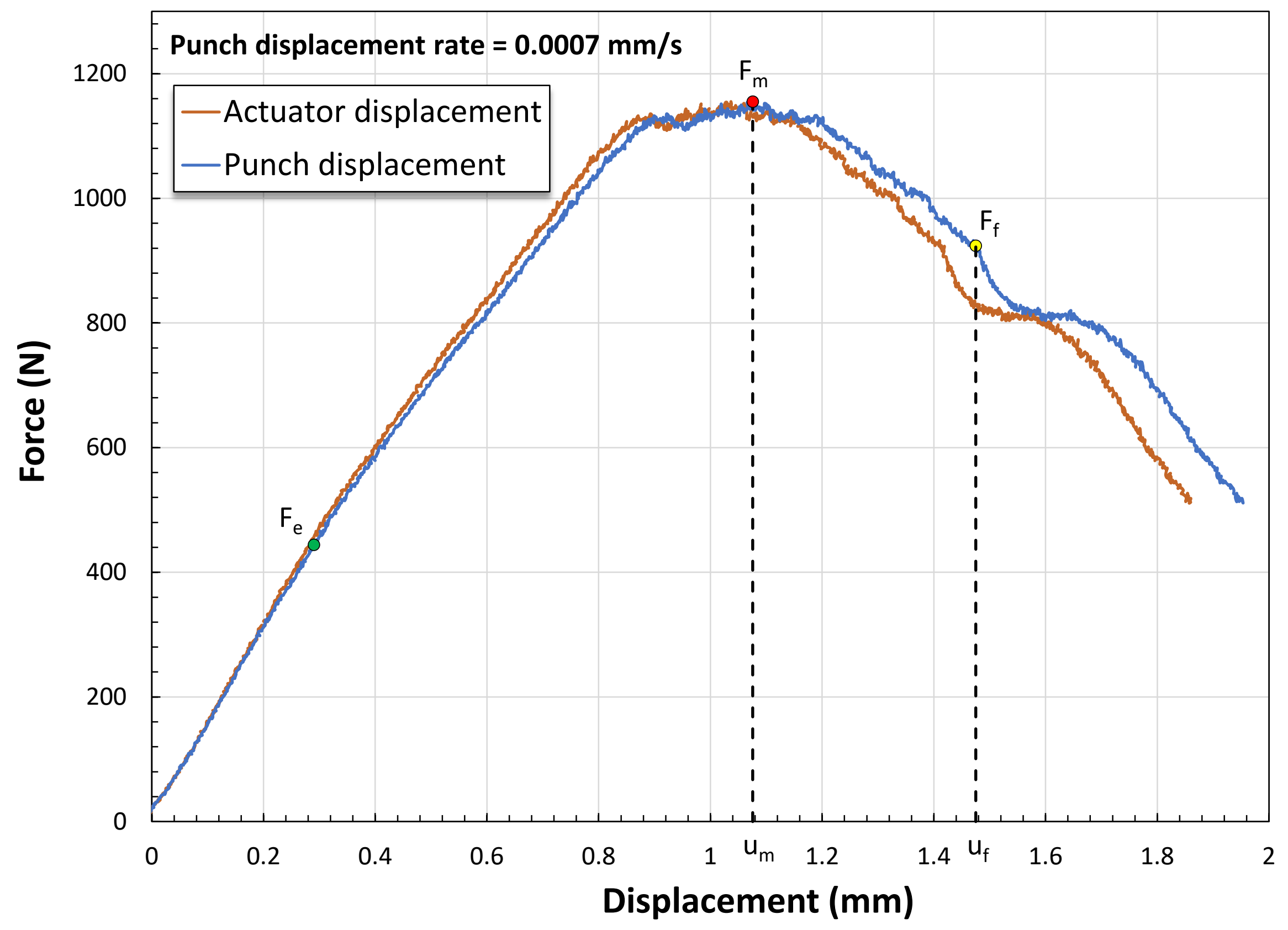




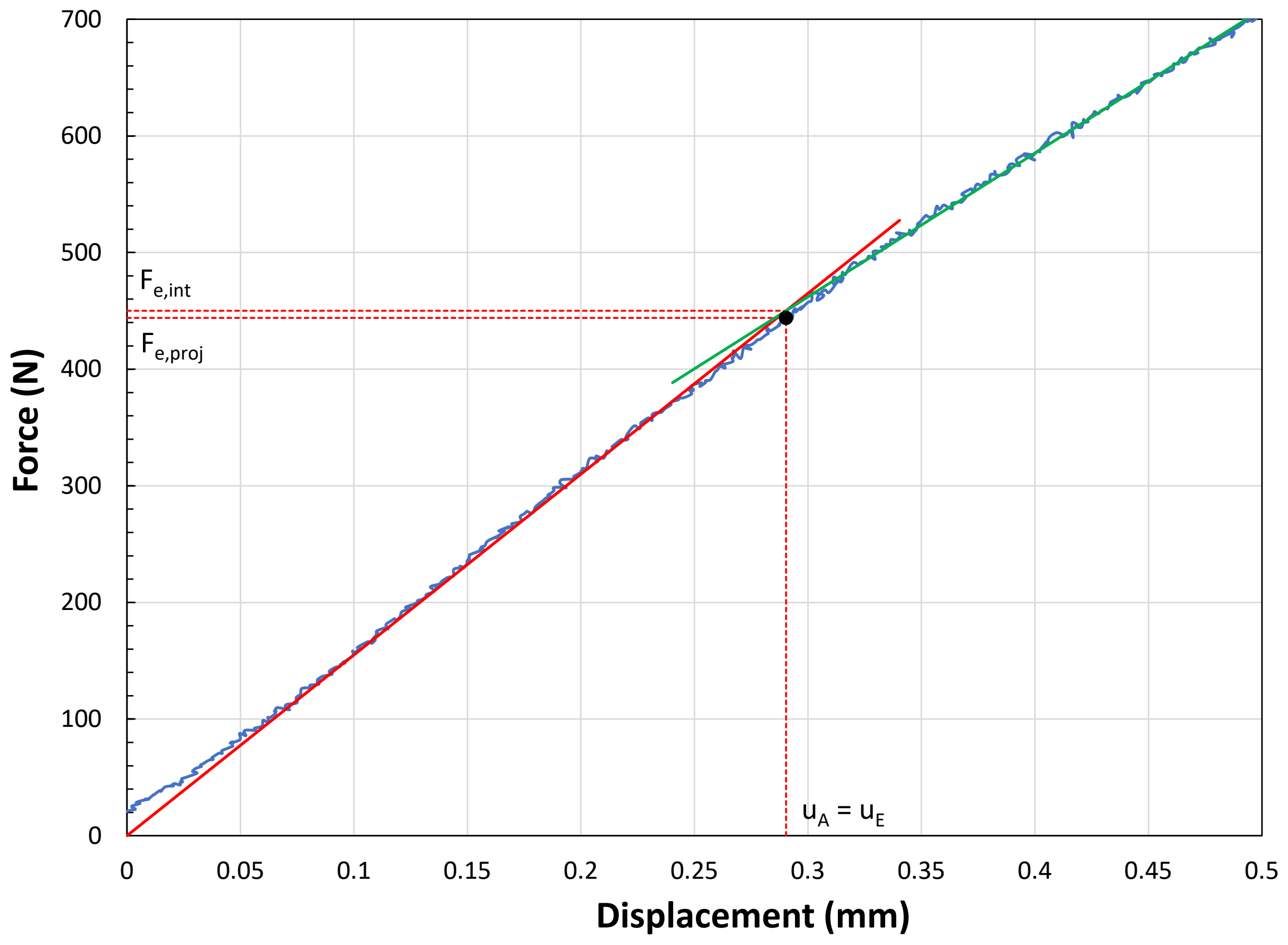


Specimen ID: 900HIP_2_S_a_SP2

Material: AM Ti-64

Test speed: $0.007052 \mathrm{~mm} / \mathrm{s}$

Max strain rate: $7.05 \mathrm{E}-03 \mathrm{~s}^{-1}$

\begin{tabular}{|c|c|c|}
\hline \multicolumn{3}{|c|}{ TEST RESULTS } \\
\hline \multicolumn{3}{|c|}{ Force values } \\
\hline$F_{e, p r o j}=$ & 321.9 & $\mathbf{N}$ \\
\hline$F_{e, \text { int }}=$ & 337.6 & $\mathbf{N}$ \\
\hline$F_{h 0 / 10, \text { off }}=$ & 532.4 & $\mathbf{N}$ \\
\hline$F_{0.1 \mathrm{~mm}, \mathrm{off}}=$ & 714.6 & $\mathbf{N}$ \\
\hline$F_{0.1 \mathrm{~mm}}=$ & 226.5 & $\mathbf{N}$ \\
\hline$F_{0.48 \mathrm{~mm}}=$ & 785.1 & $\mathbf{N}$ \\
\hline$F_{0.5 \mathrm{~mm}}=$ & 814.2 & $\mathbf{N}$ \\
\hline$F_{0.65 \mathrm{~mm}}=$ & 977.1 & $\mathbf{N}$ \\
\hline$F_{0.9 \mathrm{~mm}}=$ & 1053.2 & $\mathbf{N}$ \\
\hline$F_{\mathrm{ept}}=$ & 1069.2 & $\mathbf{N}$ \\
\hline$F_{\mathrm{e} 1.5}=$ & 635.6 & $\mathbf{N}$ \\
\hline $\mathbf{F}_{\mathrm{m}}=$ & 1102.8 & $\mathbf{N}$ \\
\hline$F_{\text {infl }}=$ & 169.1 & $\mathbf{N}$ \\
\hline$F_{f}=$ & 882.2 & $\mathbf{N}$ \\
\hline \multicolumn{3}{|c|}{ Displacement values } \\
\hline $\mathrm{u}_{\mathrm{e}}=$ & 0.152 & $\mathrm{~mm}$ \\
\hline$u_{h 0 / 10, \text { off }}=$ & 0.291 & $\mathrm{~mm}$ \\
\hline$u_{0.1 \mathrm{~mm}, \mathrm{off}}=$ & 0.423 & $\mathrm{~mm}$ \\
\hline$u_{\mathrm{e} 1.5}=$ & 0.366 & $\mathrm{~mm}$ \\
\hline$u_{m}=$ & 1.053 & $\mathrm{~mm}$ \\
\hline$u_{\text {infl }}=$ & 2.299 & $\mathrm{~mm}$ \\
\hline $\mathbf{u}_{\mathrm{f}}=$ & 1.500 & $\mathrm{~mm}$ \\
\hline \multicolumn{3}{|c|}{ Initial linear slope } \\
\hline Slope $_{\text {ini }}=$ & 2219.10 & $\mathrm{~N} / \mathrm{mm}$ \\
\hline \multicolumn{3}{|c|}{ Energy values } \\
\hline$E_{S P}=$ & 1.25 & J \\
\hline$E_{m}=$ & 0.79 & $\mathbf{J}$ \\
\hline$E_{P L}=$ & 0.52 & $J$ \\
\hline
\end{tabular}




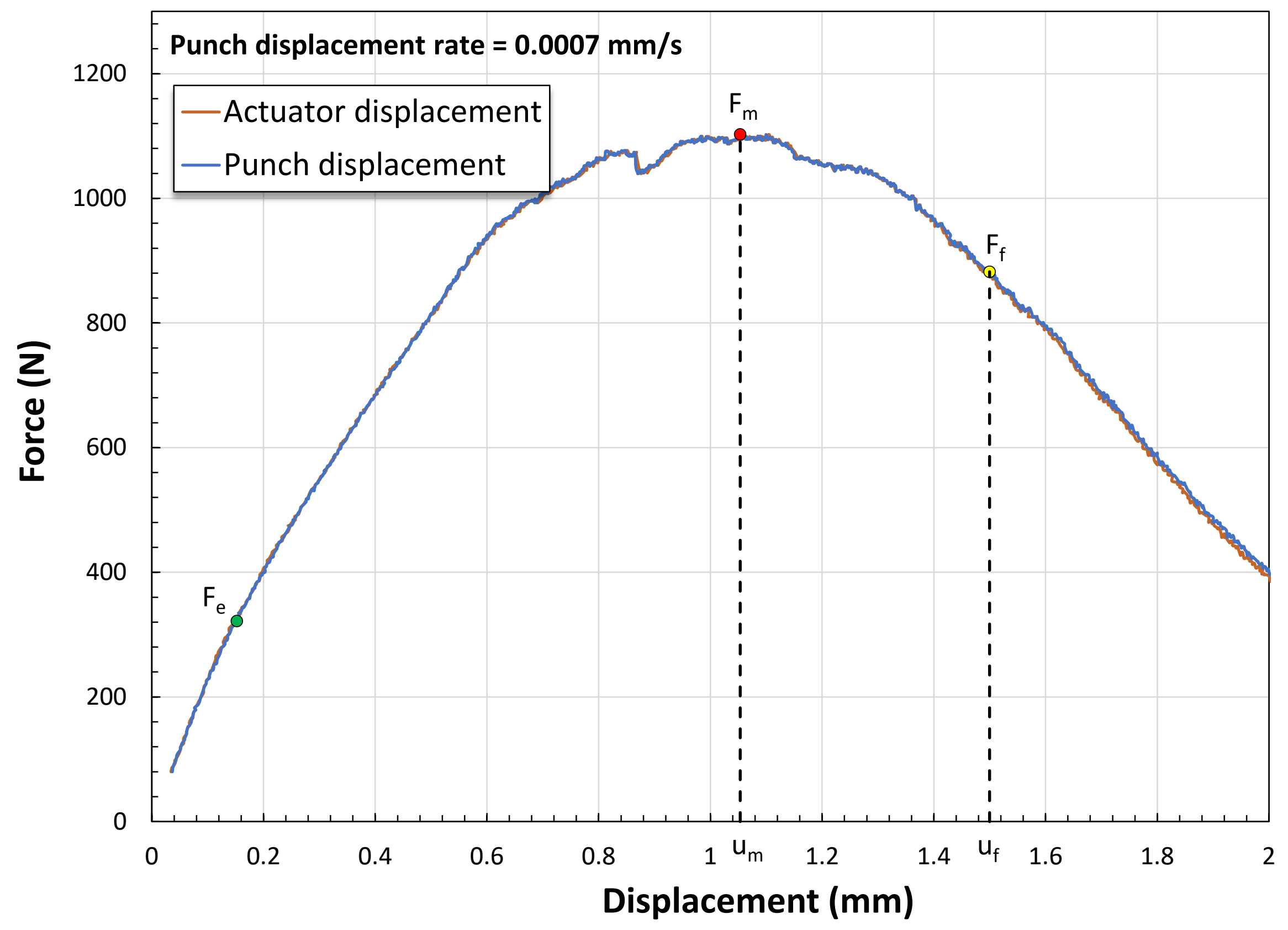




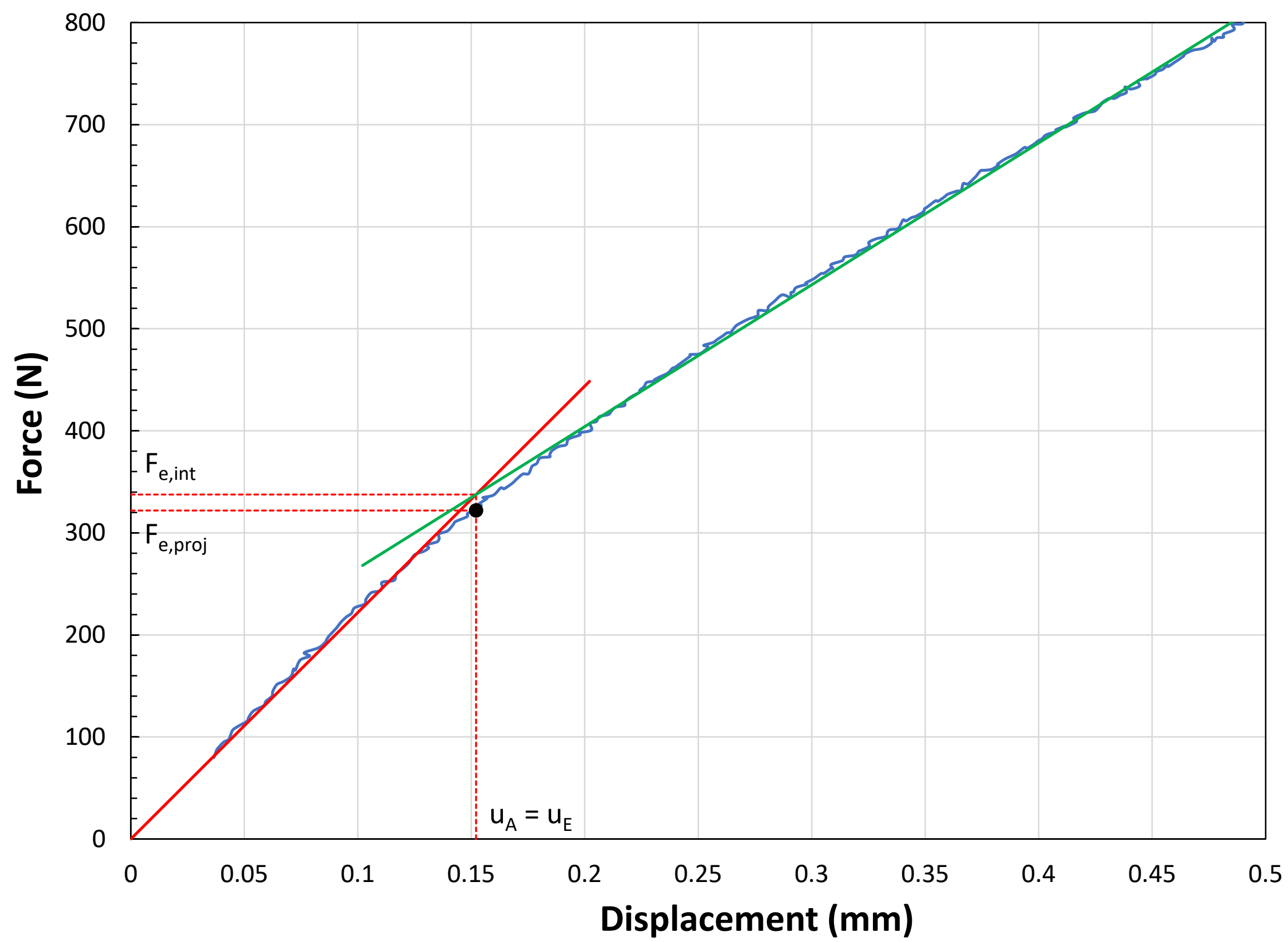


Specimen ID: 900HIP_2_S_a_SP3

Material: AM Ti-64

Test speed: $0.002996 \mathrm{~mm} / \mathrm{s}$

Max strain rate: $3.00 \mathrm{E}-03 \mathrm{~s}^{-1}$

\begin{tabular}{|c|c|c|}
\hline \multicolumn{3}{|c|}{ TEST RESULTS } \\
\hline \multicolumn{3}{|c|}{ Force values } \\
\hline$F_{e, p r o j}=$ & 412.0 & $\mathbf{N}$ \\
\hline$F_{e, \text { int }}=$ & 429.3 & $\mathbf{N}$ \\
\hline$F_{h 0 / 10, \text { off }}=$ & 607.7 & $\mathbf{N}$ \\
\hline$F_{0.1 \mathrm{~mm}, \mathrm{off}}=$ & 768.0 & $\mathbf{N}$ \\
\hline$F_{0.1 \mathrm{~mm}}=$ & 221.2 & $\mathbf{N}$ \\
\hline$F_{0.48 \mathrm{~mm}}=$ & 782.8 & $\mathbf{N}$ \\
\hline$F_{0.5 \mathrm{~mm}}=$ & 808.5 & $\mathbf{N}$ \\
\hline$F_{0.65 \mathrm{~mm}}=$ & 960.7 & $\mathbf{N}$ \\
\hline$F_{0.9 \mathrm{~mm}}=$ & 1057.7 & $\mathbf{N}$ \\
\hline$F_{\text {ept }}=$ & 856.8 & $\mathbf{N}$ \\
\hline$F_{e 1.5}=$ & 91.6 & $\mathbf{N}$ \\
\hline$F_{m}=$ & 1107.5 & $\mathbf{N}$ \\
\hline$F_{i n f l}=$ & 1080.9 & $\mathbf{N}$ \\
\hline$F_{f}=$ & 886.0 & $\mathbf{N}$ \\
\hline \multicolumn{3}{|c|}{ Displacement values } \\
\hline$u_{e}=$ & 0.202 & $\mathrm{~mm}$ \\
\hline$u_{h 0 / 10, \text { off }}=$ & 0.336 & $\mathrm{~mm}$ \\
\hline $\mathrm{u}_{0.1 \mathrm{~mm}, \mathrm{off}}=$ & 0.463 & $\mathrm{~mm}$ \\
\hline$u_{e 1.5}=$ & 0.042 & $\mathrm{~mm}$ \\
\hline$u_{m}=$ & 1.034 & $\mathrm{~mm}$ \\
\hline$u_{\text {infl }}=$ & 0.991 & $\mathrm{~mm}$ \\
\hline$u_{f}=$ & 1.529 & $\mathrm{~mm}$ \\
\hline \multicolumn{3}{|c|}{ Initial linear slope } \\
\hline Slope $_{\text {ini }}=$ & 2129.12 & $\mathrm{~N} / \mathrm{mm}$ \\
\hline \multicolumn{3}{|c|}{ Energy values } \\
\hline$E_{S P}=$ & 1.28 & J \\
\hline$E_{m}=$ & 0.77 & J \\
\hline$E_{P L}=$ & 0.48 & J \\
\hline
\end{tabular}




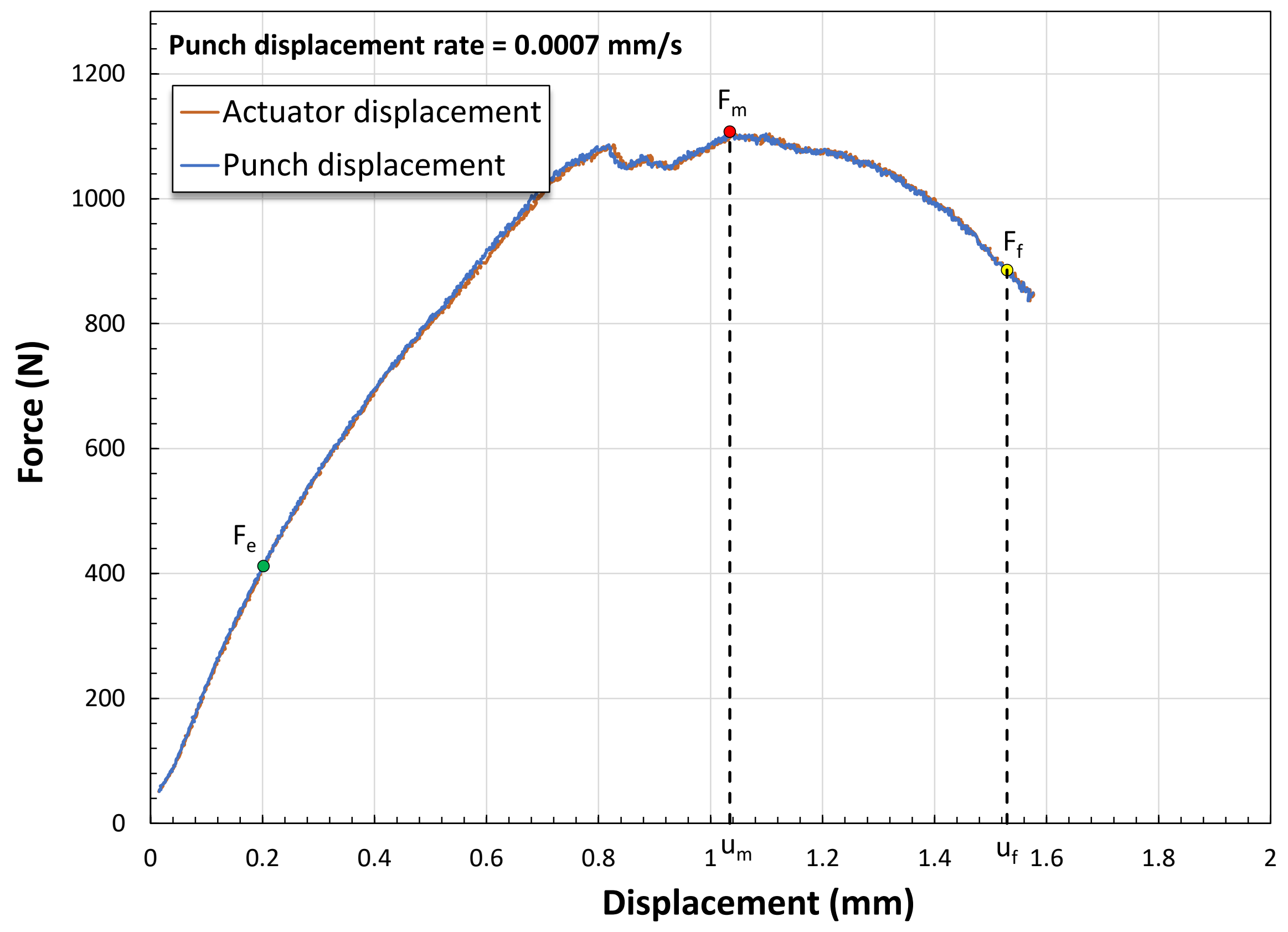




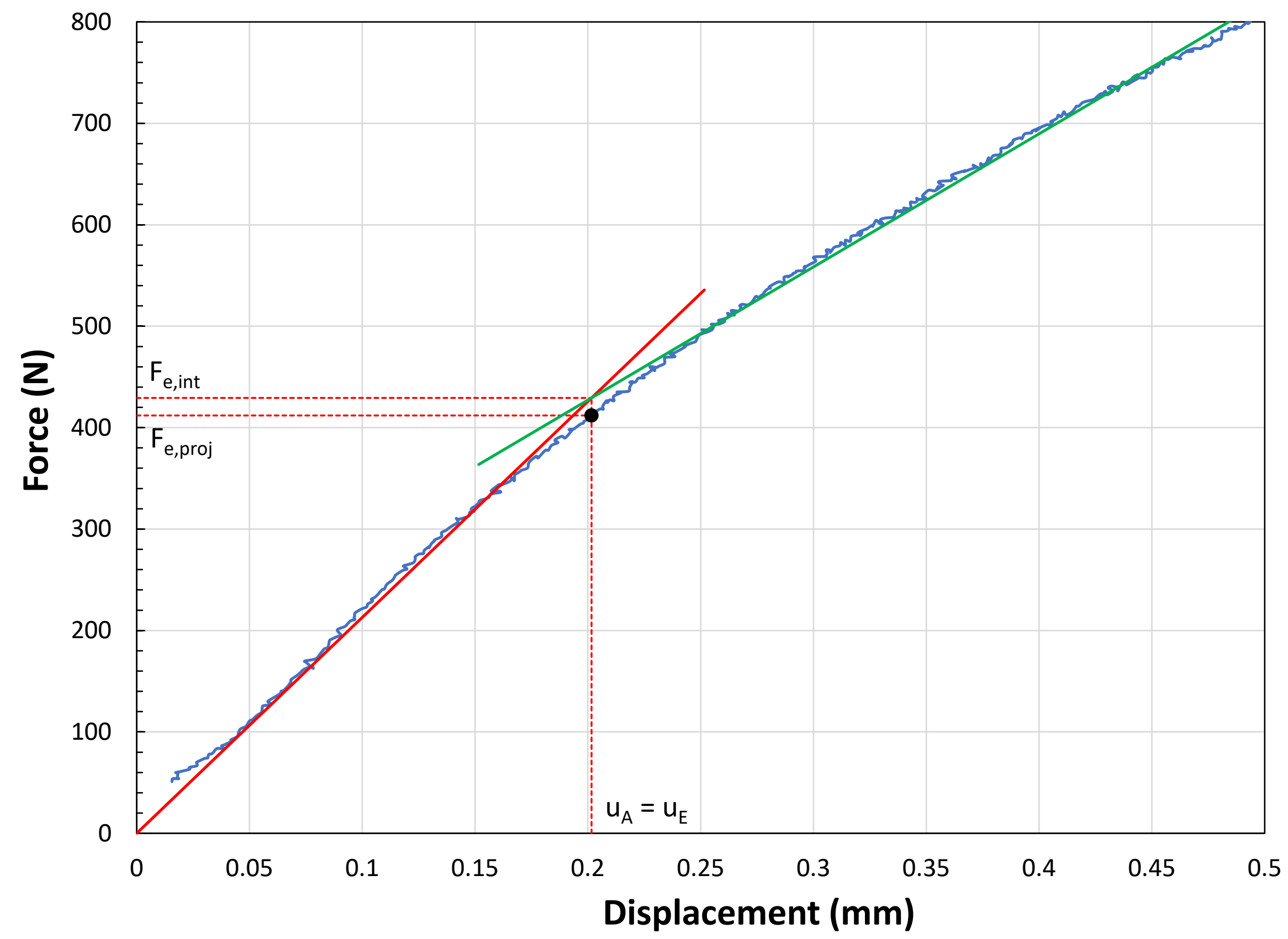


Specimen ID: 900HIP_2_S_a_SP4

Material: AM Ti-64

Test speed: $0.000723 \mathrm{~mm} / \mathrm{s}$

Max strain rate: $7.23 \mathrm{E}-04 \mathrm{~s}^{-1}$

\begin{tabular}{|c|c|c|}
\hline \multicolumn{3}{|c|}{ TEST RESULTS } \\
\hline \multicolumn{3}{|c|}{ Force values } \\
\hline$F_{e, p r o j}=$ & 319.7 & $\mathbf{N}$ \\
\hline$F_{e, \text { int }}=$ & 329.3 & $\mathbf{N}$ \\
\hline$F_{h 0 / 10, \text { off }}=$ & 557.7 & $\mathbf{N}$ \\
\hline$F_{0.1 \mathrm{~mm}, \mathrm{off}}=$ & 760.7 & $\mathbf{N}$ \\
\hline$F_{0.1 \mathrm{~mm}}=$ & 174.1 & $\mathbf{N}$ \\
\hline$F_{0.48 \mathrm{~mm}}=$ & 697.7 & $\mathbf{N}$ \\
\hline$F_{0.5 \mathrm{~mm}}=$ & 722.3 & $\mathbf{N}$ \\
\hline$F_{0.65 \mathrm{~mm}}=$ & 904.2 & $\mathbf{N}$ \\
\hline$F_{0.9 \mathrm{~mm}}=$ & 1096.9 & $\mathbf{N}$ \\
\hline$F_{\mathrm{ept}}=$ & 1117.1 & $\mathbf{N}$ \\
\hline$F_{\mathrm{e} 1.5}=$ & 172.4 & $\mathbf{N}$ \\
\hline $\mathbf{F}_{\mathrm{m}}=$ & 1128.8 & $\mathbf{N}$ \\
\hline$F_{\text {infl }}=$ & 1122.8 & $\mathbf{N}$ \\
\hline$F_{f}=$ & 903.0 & $\mathbf{N}$ \\
\hline \multicolumn{3}{|c|}{ Displacement values } \\
\hline $\mathrm{u}_{\mathrm{e}}=$ & 0.186 & $\mathrm{~mm}$ \\
\hline$u_{h 0 / 10, \text { off }}=$ & 0.366 & $\mathrm{~mm}$ \\
\hline$u_{0.1 \mathrm{~mm}, \mathrm{off}}=$ & 0.532 & $\mathrm{~mm}$ \\
\hline$u_{\mathrm{e} 1.5}=$ & 0.097 & $\mathrm{~mm}$ \\
\hline$u_{m}=$ & 1.102 & $\mathrm{~mm}$ \\
\hline$u_{\text {infl }}=$ & 0.963 & $\mathrm{~mm}$ \\
\hline$u_{f}=$ & & $\mathrm{mm}$ \\
\hline \multicolumn{3}{|c|}{ Initial linear slope } \\
\hline Slope $_{\text {ini }}=$ & 1767.22 & $\mathrm{~N} / \mathrm{mm}$ \\
\hline \multicolumn{3}{|c|}{ Energy values } \\
\hline$E_{S P}=$ & $\# \mathbf{N} / \mathbf{A}$ & J \\
\hline$E_{m}=$ & 0.80 & J \\
\hline$E_{P L}=$ & 0.44 & $\mathbf{J}$ \\
\hline
\end{tabular}




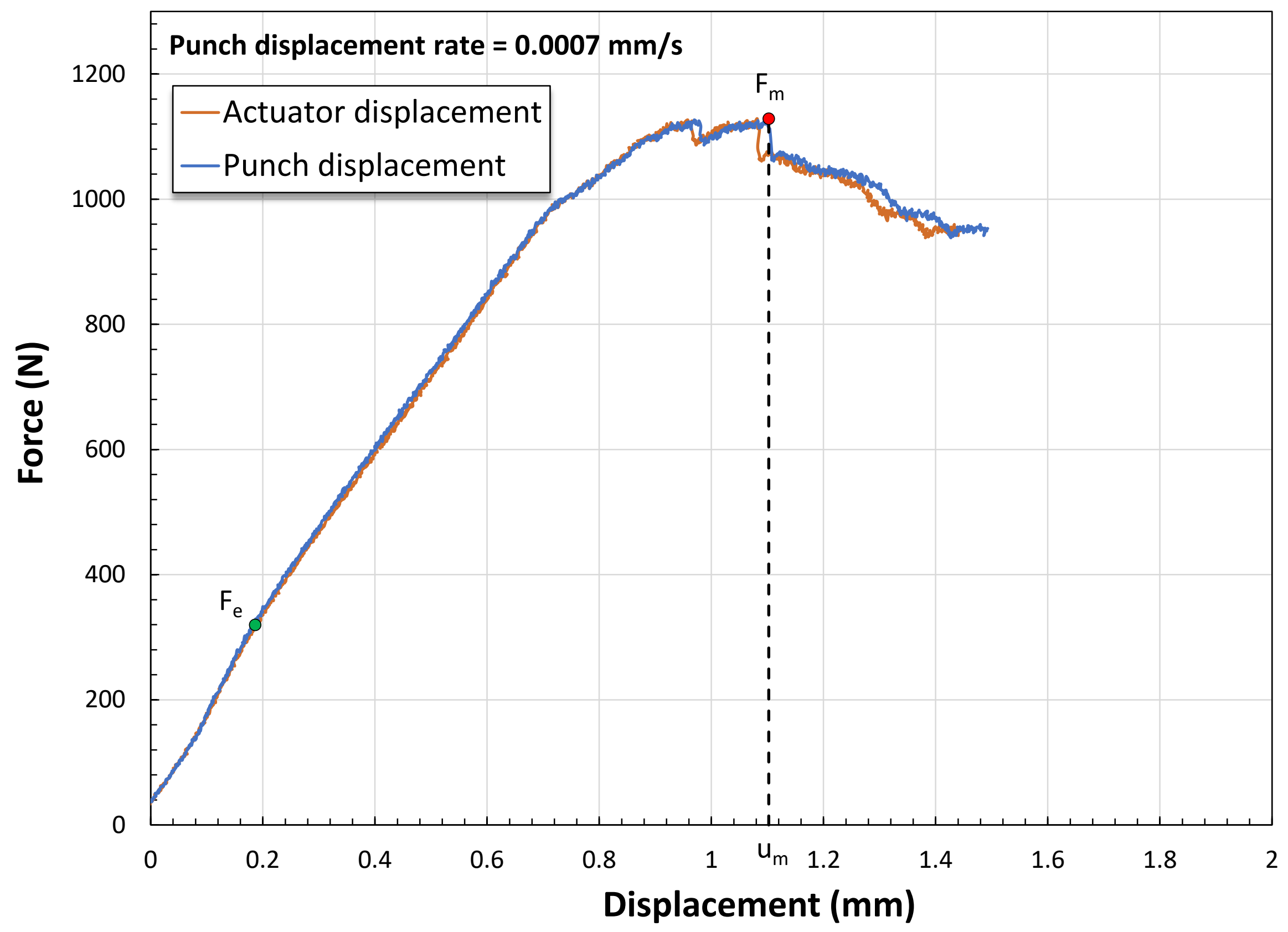




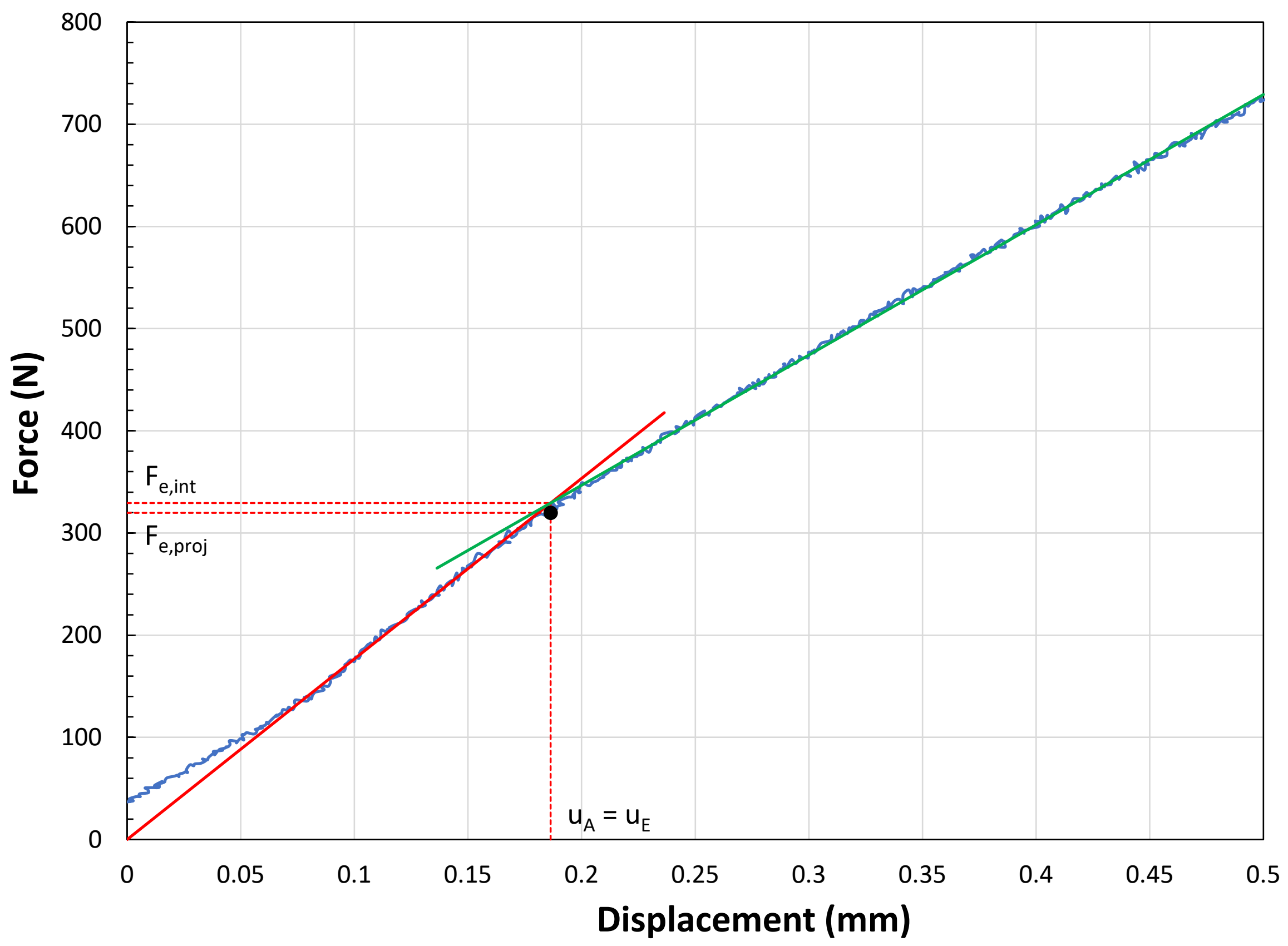


Specimen ID: 900HIP_2_S_a_SP5

Material: AM Ti-64

Test speed: $0.007006 \mathrm{~mm} / \mathrm{s}$

Max strain rate: $7.01 \mathrm{E}-03 \mathrm{~s}^{-1}$

\begin{tabular}{|c|c|c|}
\hline \multicolumn{3}{|c|}{ TEST RESULTS } \\
\hline \multicolumn{3}{|c|}{ Force values } \\
\hline$F_{e, p r o j}=$ & 346.0 & $\mathbf{N}$ \\
\hline$F_{e, \text { int }}=$ & 351.1 & $\mathbf{N}$ \\
\hline$F_{h 0 / 10, \text { off }}=$ & 613.3 & $\mathbf{N}$ \\
\hline$F_{0.1 \mathrm{~mm}, \mathrm{off}}=$ & 785.1 & $\mathbf{N}$ \\
\hline $\mathbf{F}_{0.1 \mathrm{~mm}}=$ & 208.1 & $\mathbf{N}$ \\
\hline$F_{0.48 \mathrm{~mm}}=$ & 775.4 & $\mathbf{N}$ \\
\hline$F_{0.5 \mathrm{~mm}}=$ & 793.7 & $\mathbf{N}$ \\
\hline$F_{0.65 \mathrm{~mm}}=$ & 958.5 & $\mathbf{N}$ \\
\hline$F_{0.9 \mathrm{~mm}}=$ & 1087.4 & $\mathbf{N}$ \\
\hline$F_{\text {ept }}=$ & 543.1 & $\mathbf{N}$ \\
\hline$F_{\mathrm{e} 1.5}=$ & 140.2 & $\mathbf{N}$ \\
\hline$F_{m}=$ & 1091.8 & $\mathbf{N}$ \\
\hline$F_{\text {infl }}=$ & 1082.4 & $\mathbf{N}$ \\
\hline$F_{f}=$ & 873.5 & $\mathbf{N}$ \\
\hline \multicolumn{3}{|c|}{ Displacement values } \\
\hline$u_{e}=$ & 0.175 & $\mathrm{~mm}$ \\
\hline$u_{h 0 / 10, \text { off }}=$ & 0.356 & $\mathrm{~mm}$ \\
\hline$u_{0.1 \mathrm{~mm}, \mathrm{off}}=$ & 0.491 & $\mathrm{~mm}$ \\
\hline $\mathrm{u}_{\mathrm{e} 1.5}=$ & 0.067 & $\mathrm{~mm}$ \\
\hline$u_{m}=$ & 0.886 & $\mathrm{~mm}$ \\
\hline$u_{\text {infl }}=$ & 0.878 & $\mathrm{~mm}$ \\
\hline$u_{f}=$ & & $\mathrm{mm}$ \\
\hline \multicolumn{3}{|c|}{ Initial linear slope } \\
\hline Slope $_{\text {ini }}=$ & 2008.64 & $\mathrm{~N} / \mathrm{mm}$ \\
\hline \multicolumn{3}{|c|}{ Energy values } \\
\hline$E_{S P}=$ & $\# \mathbf{N} / \mathrm{A}$ & J \\
\hline$E_{m}=$ & 0.60 & J \\
\hline$E_{P L}=$ & 0.30 & J \\
\hline
\end{tabular}




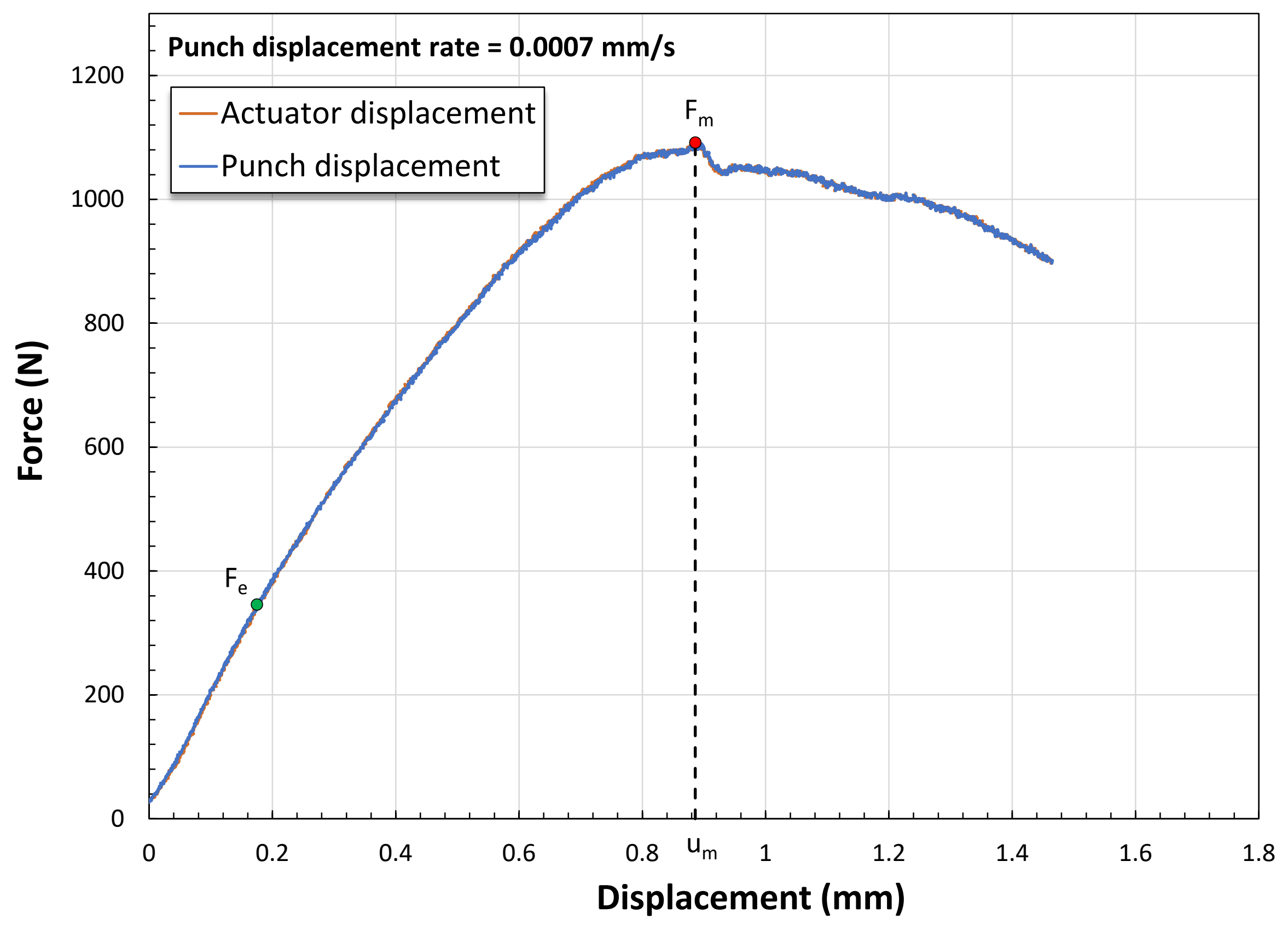




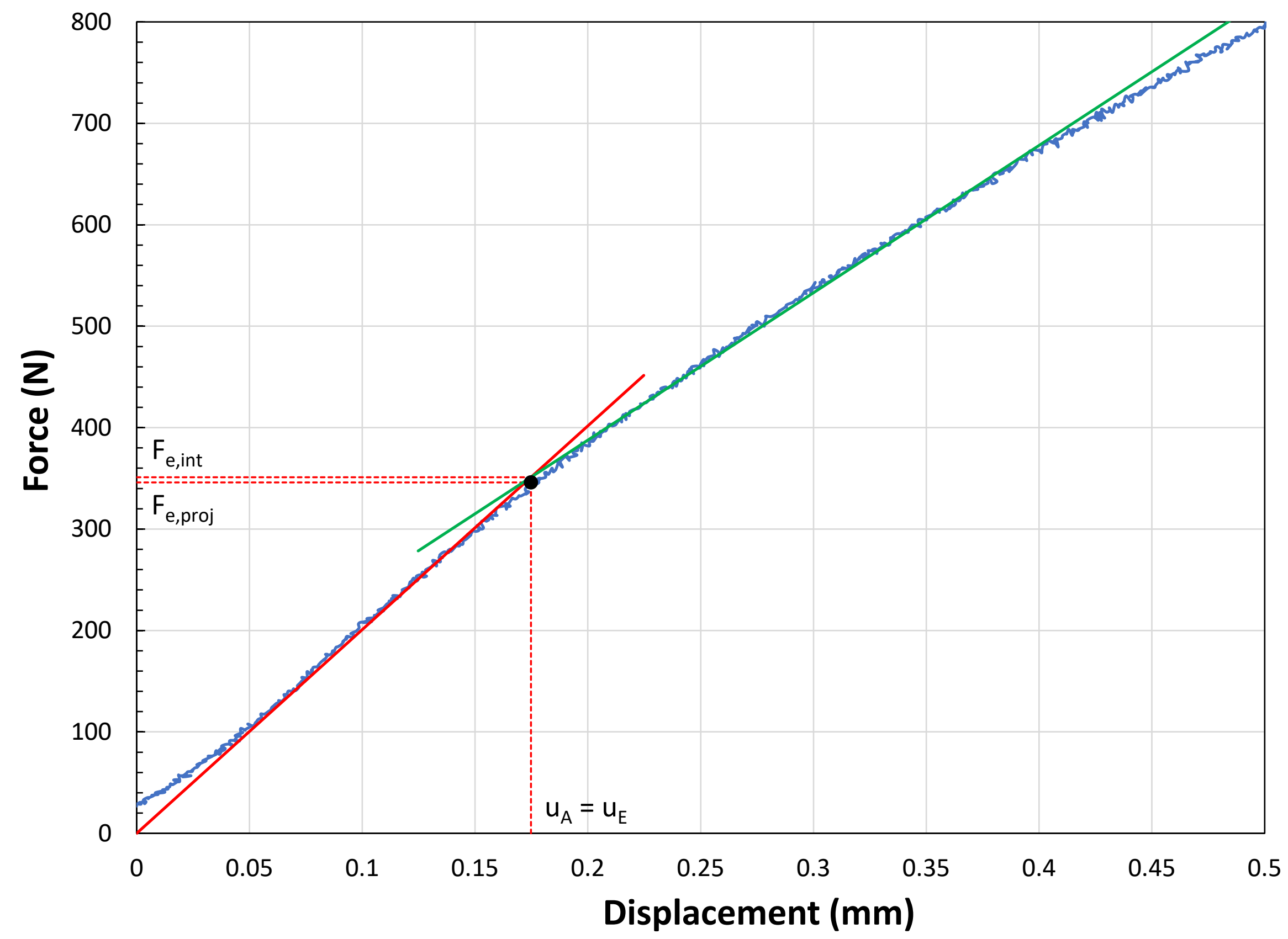


Specimen ID: 900HIP_2_S_a_SP6

Material: AM Ti-64

Test speed: $0.001014 \mathrm{~mm} / \mathrm{s}$

Max strain rate: $1.01 \mathrm{E}-03 \mathrm{~s}^{-1}$

\begin{tabular}{|c|c|c|}
\hline \multicolumn{3}{|c|}{ TEST RESULTS } \\
\hline \multicolumn{3}{|c|}{ Force values } \\
\hline$F_{e, p r o j}=$ & 371.8 & $\mathbf{N}$ \\
\hline$F_{e, \text { int }}=$ & 390.6 & $\mathbf{N}$ \\
\hline$F_{h 0 / 10, \text { off }}=$ & 590.3 & $\mathbf{N}$ \\
\hline$F_{0.1 \mathrm{~mm}, \mathrm{off}}=$ & 743.8 & $\mathbf{N}$ \\
\hline$F_{0.1 \mathrm{~mm}}=$ & 209.9 & $\mathbf{N}$ \\
\hline$F_{0.48 \mathrm{~mm}}=$ & 767.7 & $\mathbf{N}$ \\
\hline$F_{0.5 \mathrm{~mm}}=$ & 788.2 & $\mathbf{N}$ \\
\hline$F_{0.65 \mathrm{~mm}}=$ & 964.4 & $\mathbf{N}$ \\
\hline$F_{0.9 m m}=$ & 1150.9 & $\mathbf{N}$ \\
\hline$F_{\mathrm{ept}}=$ & 32.5 & $\mathbf{N}$ \\
\hline$F_{\mathrm{e} 1.5}=$ & 170.6 & $\mathbf{N}$ \\
\hline $\mathbf{F}_{\mathrm{m}}=$ & 1169.2 & $\mathbf{N}$ \\
\hline$F_{\text {infl }}=$ & 693.0 & $\mathbf{N}$ \\
\hline$F_{f}=$ & 935.3 & $\mathbf{N}$ \\
\hline \multicolumn{3}{|c|}{ Displacement values } \\
\hline$u_{e}=$ & 0.191 & $\mathrm{~mm}$ \\
\hline$u_{h 0 / 10, \text { off }}=$ & 0.339 & $\mathrm{~mm}$ \\
\hline$u_{0.1 \mathrm{~mm}, \mathrm{off}}=$ & 0.464 & $\mathrm{~mm}$ \\
\hline$u_{\mathrm{e} 1.5}=$ & 0.081 & $\mathrm{~mm}$ \\
\hline$u_{m}=$ & 0.881 & $\mathrm{~mm}$ \\
\hline$u_{\text {infl }}=$ & 1.734 & $\mathrm{~mm}$ \\
\hline$u_{f}=$ & 1.505 & $\mathrm{~mm}$ \\
\hline \multicolumn{3}{|c|}{ Initial linear slope } \\
\hline Slope $_{\text {ini }}=$ & 2045.64 & $\mathrm{~N} / \mathrm{mm}$ \\
\hline \multicolumn{3}{|c|}{ Energy values } \\
\hline$E_{S P}=$ & 1.28 & J \\
\hline$E_{m}=$ & 0.61 & J \\
\hline$E_{P L}=$ & 0.27 & $\mathbf{J}$ \\
\hline
\end{tabular}




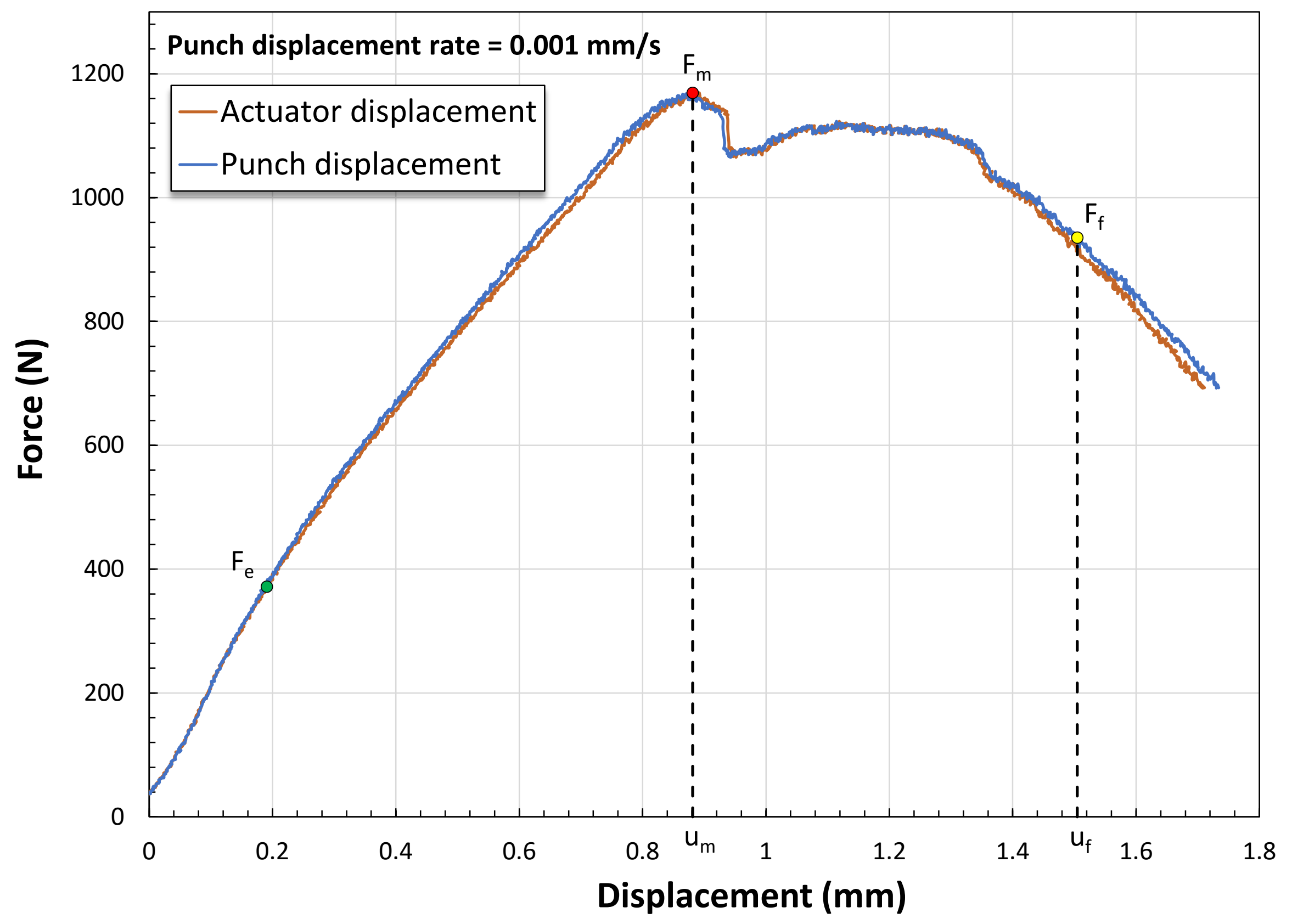




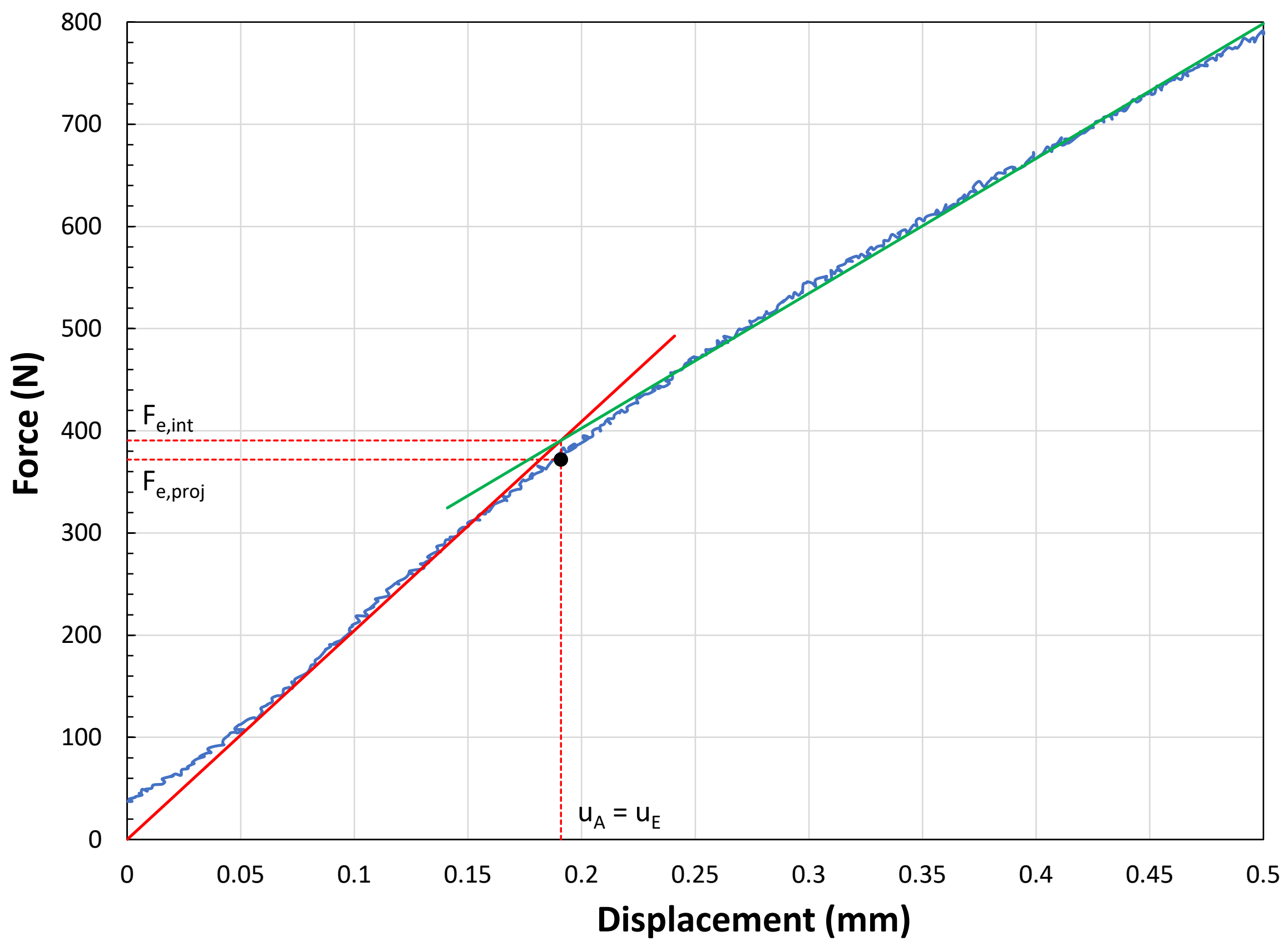


Specimen ID: 900HIP_2_S_a_SP7

Material: AM Ti-64

Test speed: $0.001006 \mathrm{~mm} / \mathrm{s}$

Max strain rate: $1.01 \mathrm{E}-03 \mathrm{~s}^{-1}$

\begin{tabular}{|c|c|c|}
\hline \multicolumn{3}{|c|}{ TEST RESULTS } \\
\hline \multicolumn{3}{|c|}{ Force values } \\
\hline$F_{e, p r o j}=$ & 347.1 & $\mathbf{N}$ \\
\hline$F_{e, \text { int }}=$ & 354.2 & $\mathbf{N}$ \\
\hline$F_{h 0 / 10, \text { off }}=$ & 567.8 & $\mathbf{N}$ \\
\hline$F_{0.1 \mathrm{~mm}, \mathrm{off}}=$ & 753.2 & $\mathbf{N}$ \\
\hline$F_{0.1 \mathrm{~mm}}=$ & 191.7 & $\mathbf{N}$ \\
\hline$F_{0.48 \mathrm{~mm}}=$ & 735.9 & $\mathbf{N}$ \\
\hline$F_{0.5 \mathrm{~mm}}=$ & 762.0 & $\mathbf{N}$ \\
\hline$F_{0.65 \mathrm{~mm}}=$ & 929.8 & $\mathbf{N}$ \\
\hline$F_{0.9 \mathrm{~mm}}=$ & 1092.3 & $\mathbf{N}$ \\
\hline$F_{\mathrm{ept}}=$ & 326.7 & $\mathbf{N}$ \\
\hline$F_{\mathrm{e} 1.5}=$ & 164.9 & $\mathbf{N}$ \\
\hline $\mathbf{F}_{\mathrm{m}}=$ & 1103.7 & $\mathbf{N}$ \\
\hline$F_{\text {infl }}=$ & 446.2 & $\mathbf{N}$ \\
\hline$F_{f}=$ & 882.9 & $\mathbf{N}$ \\
\hline \multicolumn{3}{|c|}{ Displacement values } \\
\hline$u_{e}=$ & 0.187 & $\mathrm{~mm}$ \\
\hline$u_{h 0 / 10, \text { off }}=$ & 0.351 & $\mathrm{~mm}$ \\
\hline$u_{0.1 \mathrm{~mm}, \mathrm{off}}=$ & 0.498 & $\mathrm{~mm}$ \\
\hline$u_{\mathrm{e} 1.5}=$ & 0.086 & $\mathrm{~mm}$ \\
\hline$u_{m}=$ & 0.894 & $\mathrm{~mm}$ \\
\hline$u_{\text {infl }}=$ & 0.257 & $\mathrm{~mm}$ \\
\hline$u_{f}=$ & 1.675 & $\mathrm{~mm}$ \\
\hline \multicolumn{3}{|c|}{ Initial linear slope } \\
\hline Slope $_{\text {ini }}=$ & 1893.58 & $\mathrm{~N} / \mathrm{mm}$ \\
\hline \multicolumn{3}{|c|}{ Energy values } \\
\hline$E_{S P}=$ & 1.38 & J \\
\hline$E_{m}=$ & 0.59 & J \\
\hline$E_{P L}=$ & 0.27 & $\mathbf{J}$ \\
\hline
\end{tabular}




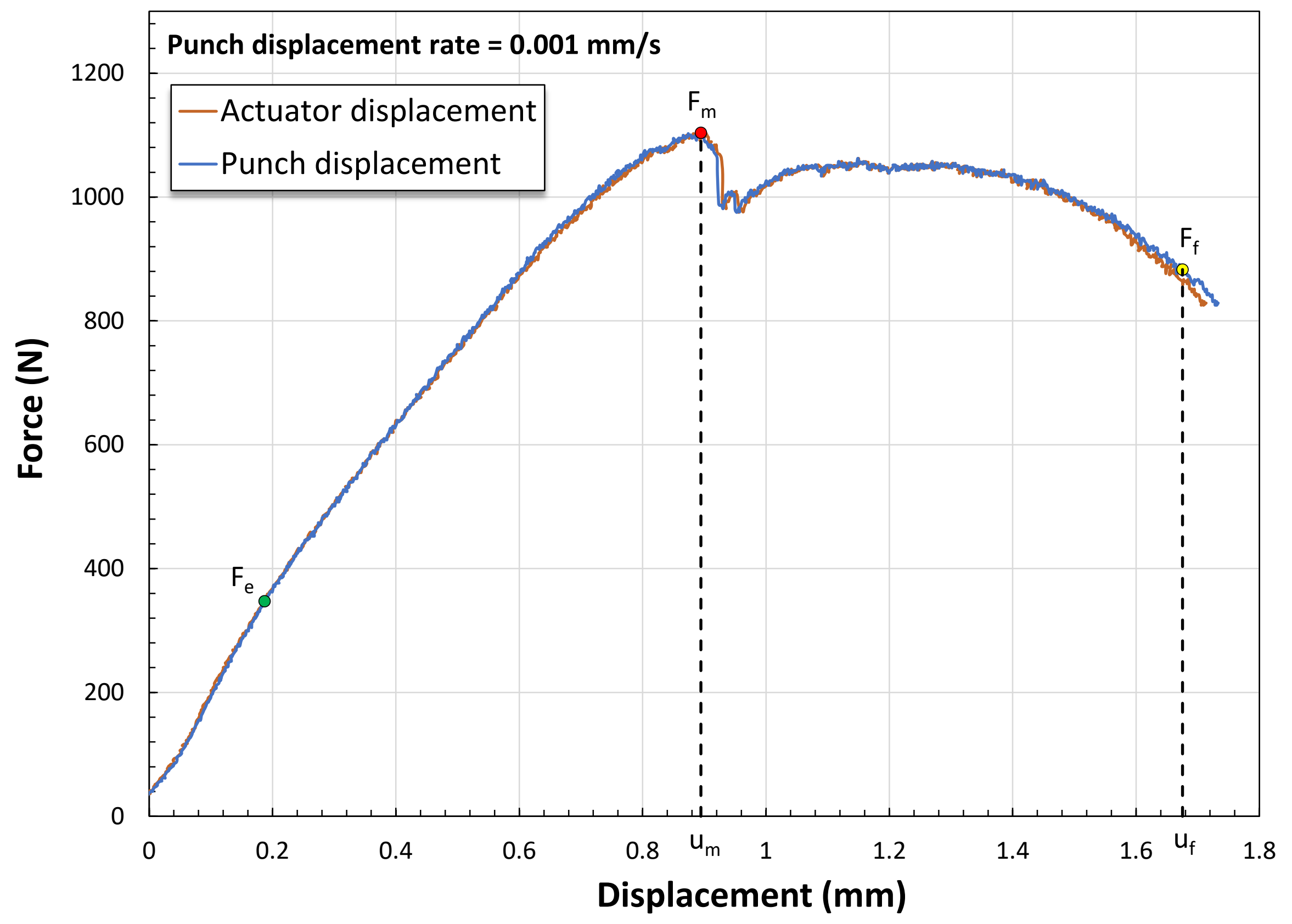




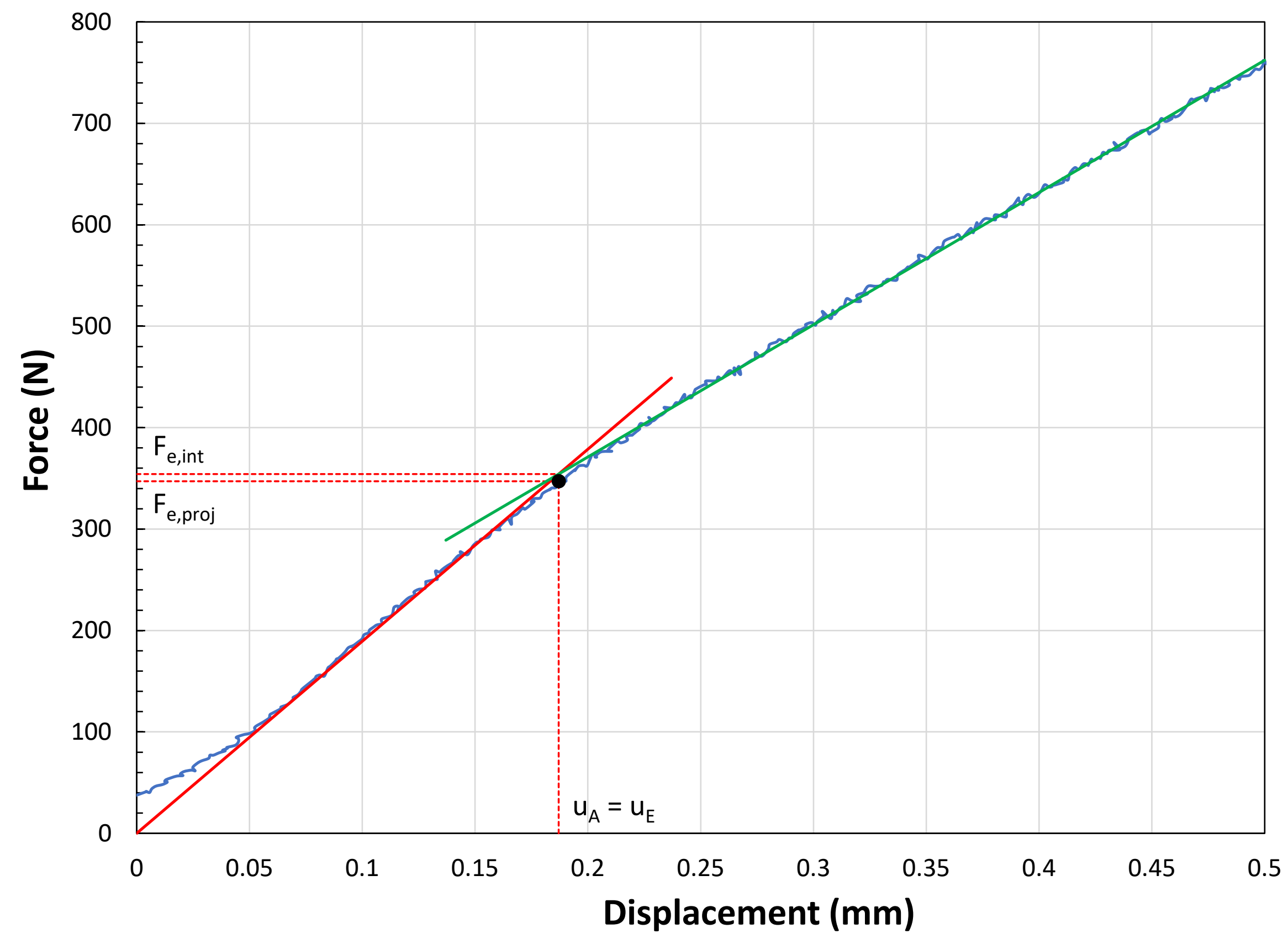


Specimen ID: 900HIP_2_S_a_SP8

Material: AM Ti-64

Test speed: $0.001025 \mathrm{~mm} / \mathrm{s}$

Max strain rate: $1.02 \mathrm{E}-03 \mathrm{~s}^{-1}$

\begin{tabular}{|c|c|c|}
\hline \multicolumn{3}{|c|}{ TEST RESULTS } \\
\hline \multicolumn{3}{|c|}{ Force values } \\
\hline$F_{e, p r o j}=$ & 354.0 & $\mathbf{N}$ \\
\hline$F_{e, \text { int }}=$ & 366.1 & $\mathbf{N}$ \\
\hline$F_{h 0 / 10, \text { off }}=$ & 560.1 & $\mathbf{N}$ \\
\hline$F_{0.1 \mathrm{~mm}, \mathrm{off}}=$ & 782.2 & $\mathbf{N}$ \\
\hline$F_{0.1 \mathrm{~mm}}=$ & 182.3 & $\mathbf{N}$ \\
\hline$F_{0.48 \mathrm{~mm}}=$ & 704.7 & $\mathbf{N}$ \\
\hline$F_{0.5 \mathrm{~mm}}=$ & 735.4 & $\mathbf{N}$ \\
\hline$F_{0.65 \mathrm{~mm}}=$ & 928.1 & $\mathbf{N}$ \\
\hline$F_{0.9 \mathrm{~mm}}=$ & 1186.9 & $\mathbf{N}$ \\
\hline$F_{\text {ept }}=$ & 855.9 & $\mathbf{N}$ \\
\hline$F_{\mathrm{e} 1.5}=$ & 199.5 & $\mathbf{N}$ \\
\hline$F_{m}=$ & 1186.9 & $\mathbf{N}$ \\
\hline$F_{\text {infl }}=$ & 790.2 & $\mathbf{N}$ \\
\hline$F_{f}=$ & 949.5 & $\mathbf{N}$ \\
\hline \multicolumn{3}{|c|}{ Displacement values } \\
\hline$u_{e}=$ & 0.204 & $\mathrm{~mm}$ \\
\hline$u_{h 0 / 10, \text { off }}=$ & 0.362 & $\mathrm{~mm}$ \\
\hline $\mathrm{u}_{0.1 \mathrm{~mm}, \mathrm{off}}=$ & 0.535 & $\mathrm{~mm}$ \\
\hline$u_{e 1.5}=$ & 0.111 & $\mathrm{~mm}$ \\
\hline$u_{m}=$ & 0.899 & $\mathrm{~mm}$ \\
\hline$u_{\text {infl }}=$ & 1.729 & $\mathrm{~mm}$ \\
\hline$u_{f}=$ & 1.478 & $\mathrm{~mm}$ \\
\hline \multicolumn{3}{|c|}{ Initial linear slope } \\
\hline Slope $_{\text {ini }}=$ & 1797.35 & $\mathrm{~N} / \mathrm{mm}$ \\
\hline \multicolumn{3}{|c|}{ Energy values } \\
\hline$E_{S P}=$ & 1.20 & J \\
\hline $\mathbf{E}_{\mathrm{m}}=$ & 0.59 & J \\
\hline$E_{P L}=$ & 0.20 & J \\
\hline
\end{tabular}




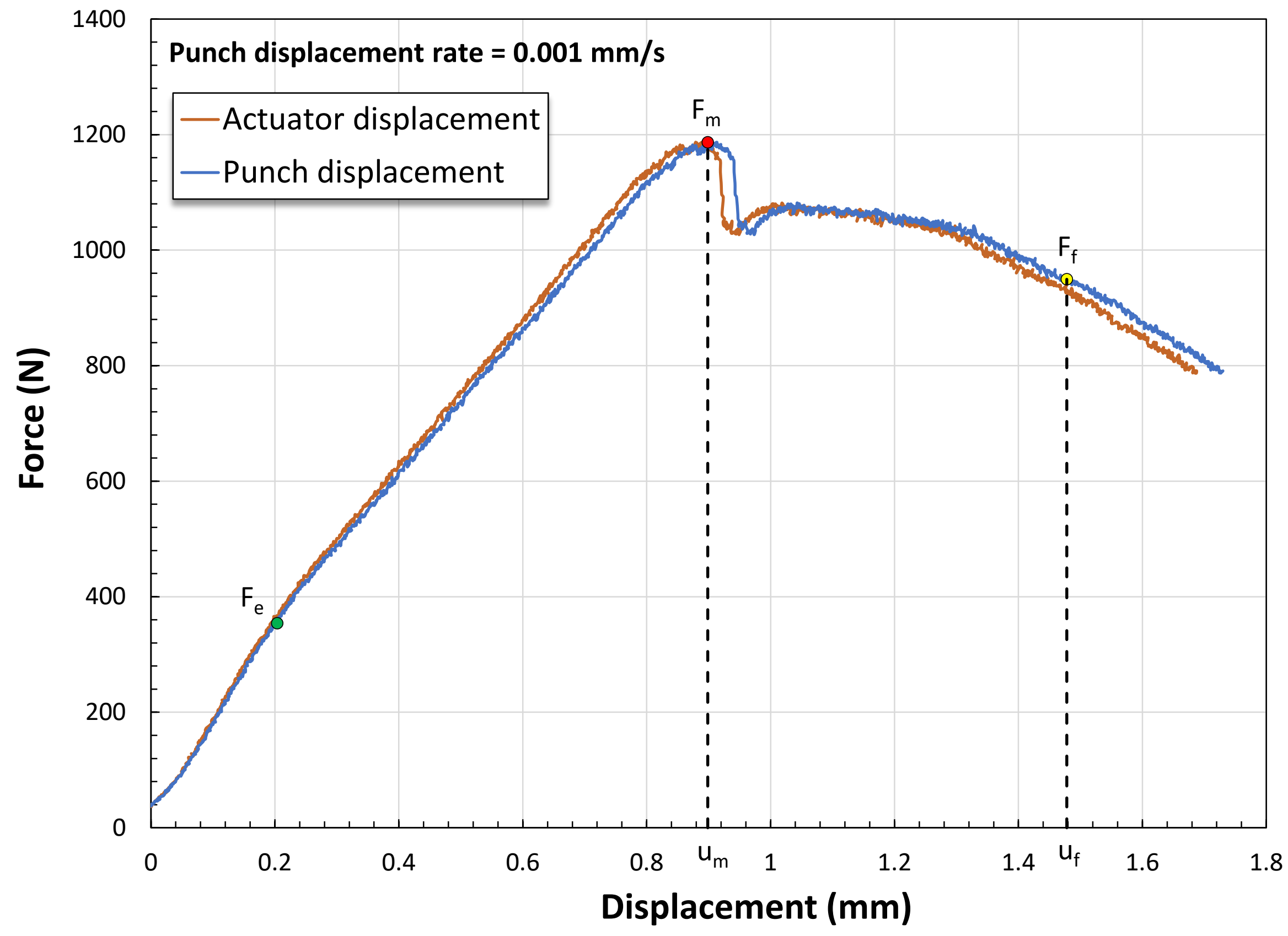




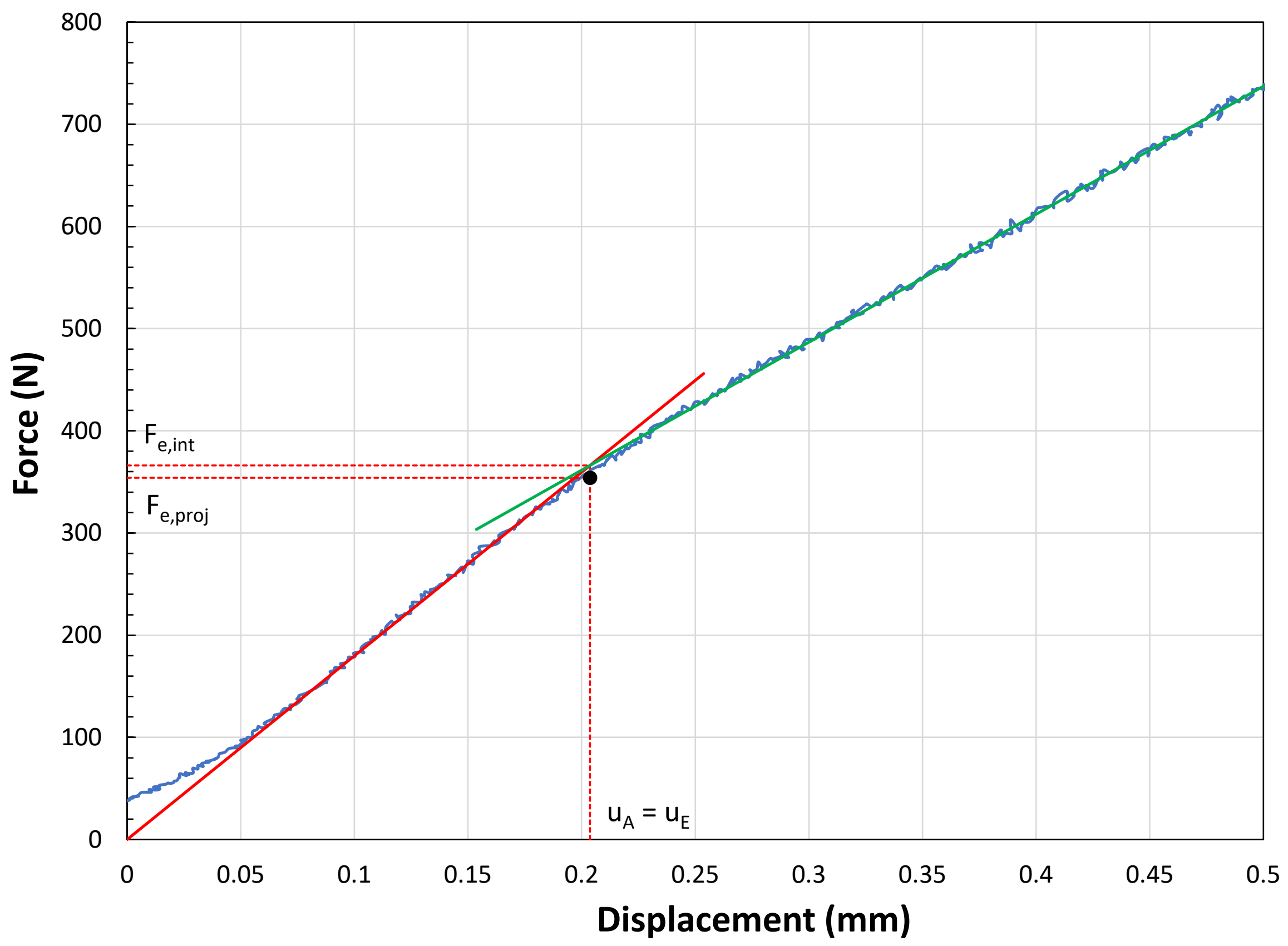


Specimen ID: 900HIP_2_S_a_SP9

Material: AM Ti-64

Test speed: $0.001009 \mathrm{~mm} / \mathrm{s}$

Max strain rate: $1.01 \mathrm{E}-03 \mathrm{~s}^{-1}$

\begin{tabular}{|c|c|c|}
\hline \multicolumn{3}{|c|}{ TEST RESULTS } \\
\hline \multicolumn{3}{|c|}{ Force values } \\
\hline$F_{e, p r o j}=$ & 391.5 & $\mathbf{N}$ \\
\hline$F_{e, \text { int }}=$ & 410.5 & $\mathbf{N}$ \\
\hline$F_{h 0 / 10, \text { off }}=$ & 575.3 & $\mathbf{N}$ \\
\hline$F_{0.1 \mathrm{~mm}, \mathrm{off}}=$ & 730.7 & $\mathbf{N}$ \\
\hline$F_{0.1 \mathrm{~mm}}=$ & 221.4 & $\mathbf{N}$ \\
\hline$F_{0.48 \mathrm{~mm}}=$ & 792.9 & $\mathbf{N}$ \\
\hline$F_{0.5 \mathrm{~mm}}=$ & 816.7 & $\mathbf{N}$ \\
\hline$F_{0.65 \mathrm{~mm}}=$ & 1006.7 & $\mathbf{N}$ \\
\hline$F_{0.9 \mathrm{~mm}}=$ & 1094.7 & $\mathbf{N}$ \\
\hline$F_{\text {ept }}=$ & 1120.6 & $\mathbf{N}$ \\
\hline$F_{e 1.5}=$ & 187.6 & $\mathbf{N}$ \\
\hline$F_{m}=$ & 1154.0 & $\mathbf{N}$ \\
\hline$F_{i n f l}=$ & 1066.3 & $\mathbf{N}$ \\
\hline$F_{f}=$ & 923.2 & $\mathbf{N}$ \\
\hline \multicolumn{3}{|c|}{ Displacement values } \\
\hline$u_{e}=$ & 0.189 & $\mathrm{~mm}$ \\
\hline$u_{h 0 / 10, \text { off }}=$ & 0.315 & $\mathrm{~mm}$ \\
\hline $\mathrm{u}_{0.1 \mathrm{~mm}, \mathrm{off}}=$ & 0.436 & $\mathrm{~mm}$ \\
\hline$u_{e 1.5}=$ & 0.086 & $\mathrm{~mm}$ \\
\hline$u_{m}=$ & 1.132 & $\mathrm{~mm}$ \\
\hline$u_{\text {infl }}=$ & 1.428 & $\mathrm{~mm}$ \\
\hline$u_{f}=$ & 1.576 & $\mathrm{~mm}$ \\
\hline \multicolumn{3}{|c|}{ Initial linear slope } \\
\hline Slope $_{\text {ini }}=$ & 2175.63 & $\mathrm{~N} / \mathrm{mm}$ \\
\hline \multicolumn{3}{|c|}{ Energy values } \\
\hline$E_{S P}=$ & 1.37 & J \\
\hline$E_{m}=$ & 0.90 & J \\
\hline$E_{P L}=$ & 0.59 & J \\
\hline
\end{tabular}




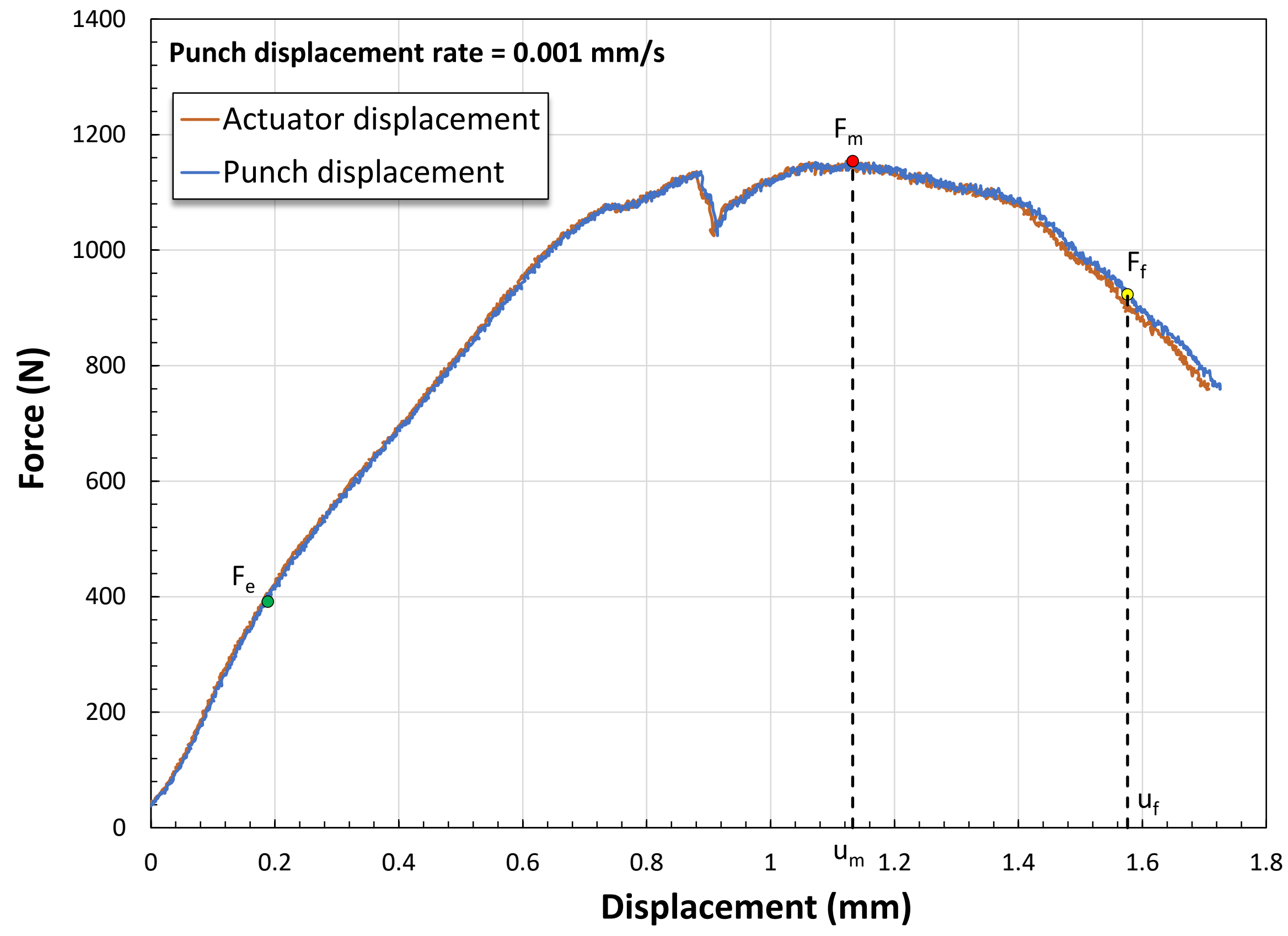




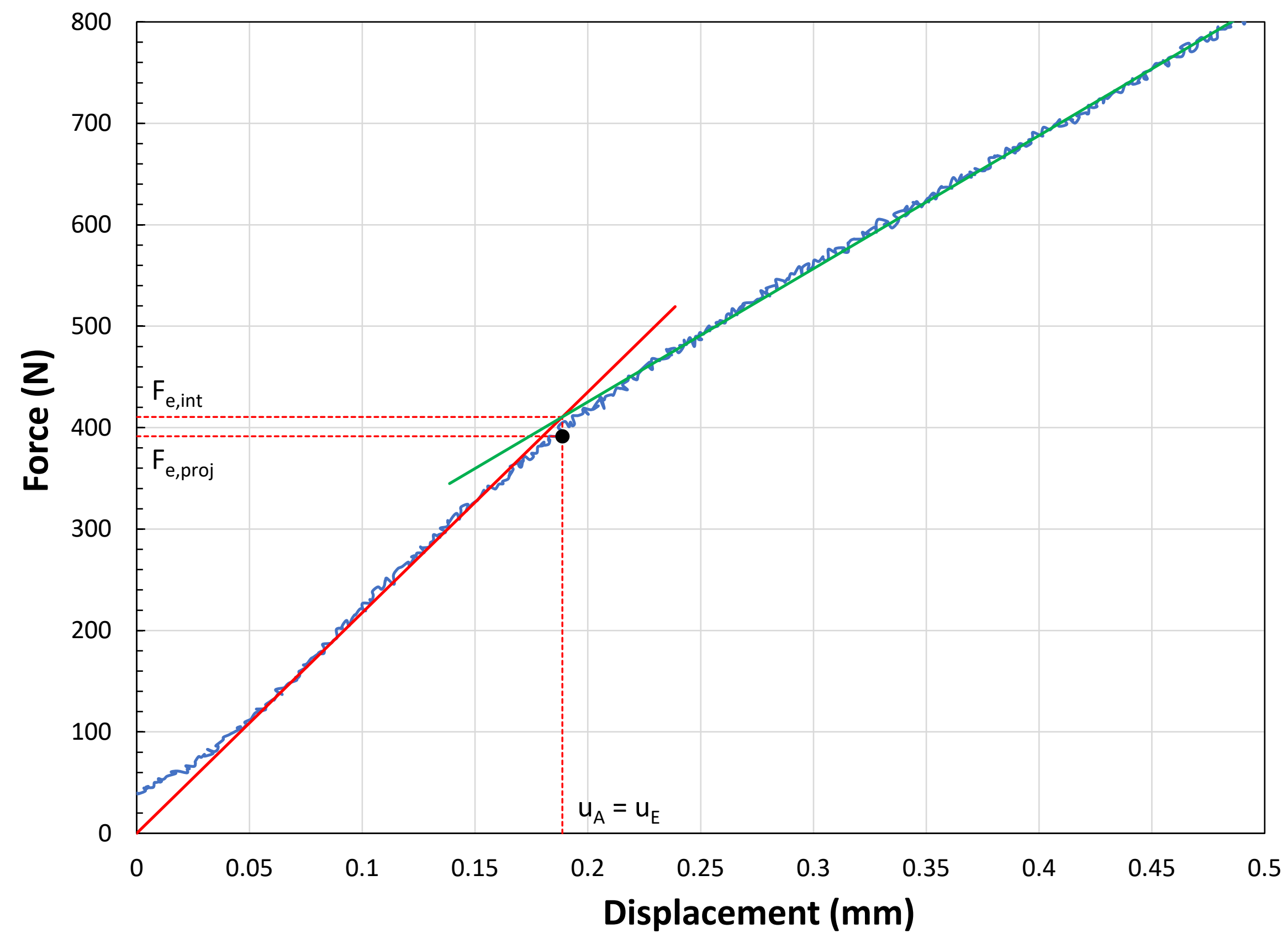


Specimen ID: 900HIP_2_S_a_SP10

Material: AM Ti-64

Test speed: $0.008383 \mathrm{~mm} / \mathrm{s}$

Max strain rate: $8.38 \mathrm{E}-03 \mathrm{~s}^{-1}$

\begin{tabular}{|c|c|c|}
\hline \multicolumn{3}{|c|}{ TEST RESULTS } \\
\hline \multicolumn{3}{|c|}{ Force values } \\
\hline$F_{e, p r o j}=$ & 423.4 & $\mathbf{N}$ \\
\hline $\mathbf{F}_{\mathrm{e}, \text { int }}=$ & 441.0 & $\mathbf{N}$ \\
\hline$F_{h 0 / 10, \text { off }}=$ & 661.4 & $\mathbf{N}$ \\
\hline$F_{0.1 \mathrm{~mm}, \text { off }}=$ & 821.2 & $\mathbf{N}$ \\
\hline$F_{0.1 \mathrm{~mm}}=$ & 201.1 & $\mathbf{N}$ \\
\hline$F_{0.48 \mathrm{~mm}}=$ & 790.3 & $\mathbf{N}$ \\
\hline $\mathrm{F}_{0.5 \mathrm{~mm}}=$ & 815.8 & $\mathbf{N}$ \\
\hline$F_{0.65 \mathrm{~mm}}=$ & 959.7 & $\mathbf{N}$ \\
\hline$F_{0.9 \mathrm{~mm}}=$ & 1097.1 & $\mathbf{N}$ \\
\hline$F_{\text {ept }}=$ & 977.1 & $\mathbf{N}$ \\
\hline$F_{\mathrm{e} 1.5}=$ & 164.5 & $\mathbf{N}$ \\
\hline$F_{m}=$ & 1157.3 & $\mathbf{N}$ \\
\hline$F_{\text {infl }}=$ & 720.7 & $\mathbf{N}$ \\
\hline$F_{f}=$ & 925.8 & $\mathbf{N}$ \\
\hline \multicolumn{3}{|c|}{ Displacement values } \\
\hline $\mathrm{u}_{\mathrm{e}}=$ & 0.215 & $\mathrm{~mm}$ \\
\hline$u_{h 0 / 10, \text { off }}=$ & 0.373 & $\mathrm{~mm}$ \\
\hline$u_{0.1 \mathrm{~mm}, \text { off }}=$ & 0.502 & $\mathrm{~mm}$ \\
\hline$u_{\mathrm{e} 1.5}=$ & 0.076 & $\mathrm{~mm}$ \\
\hline$u_{m}=$ & 1.121 & $\mathrm{~mm}$ \\
\hline$u_{i n f l}=$ & 0.419 & $\mathrm{~mm}$ \\
\hline$u_{f}=$ & 1.560 & $\mathrm{~mm}$ \\
\hline \multicolumn{3}{|c|}{ Initial linear slope } \\
\hline Slope $_{\text {ini }}=$ & 2050.10 & $\mathrm{~N} / \mathrm{mm}$ \\
\hline \multicolumn{3}{|c|}{ Energy values } \\
\hline$E_{S P}=$ & 1.34 & J \\
\hline$E_{m}=$ & 0.87 & J \\
\hline $\mathrm{E}_{\mathrm{PL}}=$ & 0.55 & J \\
\hline
\end{tabular}




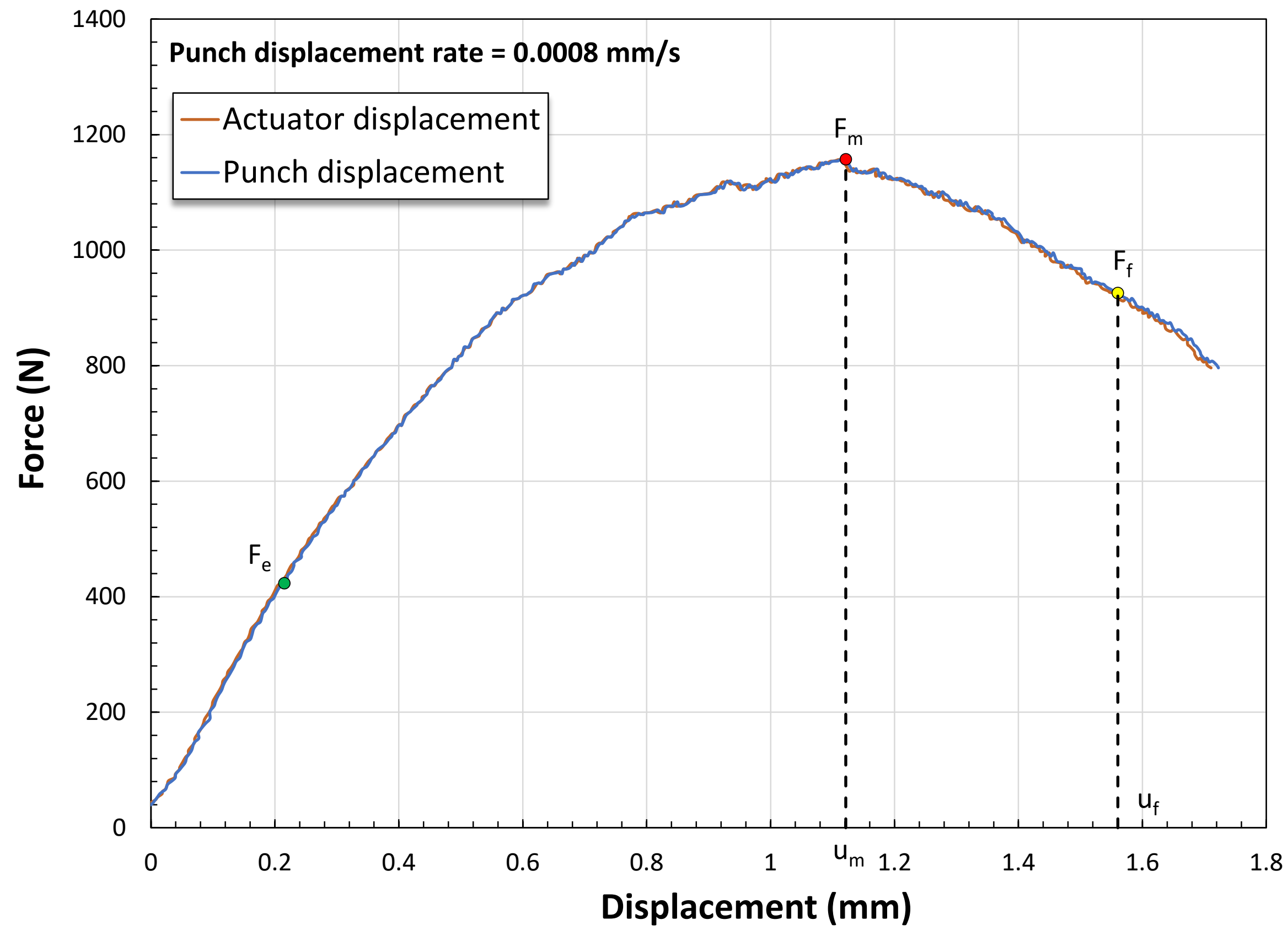




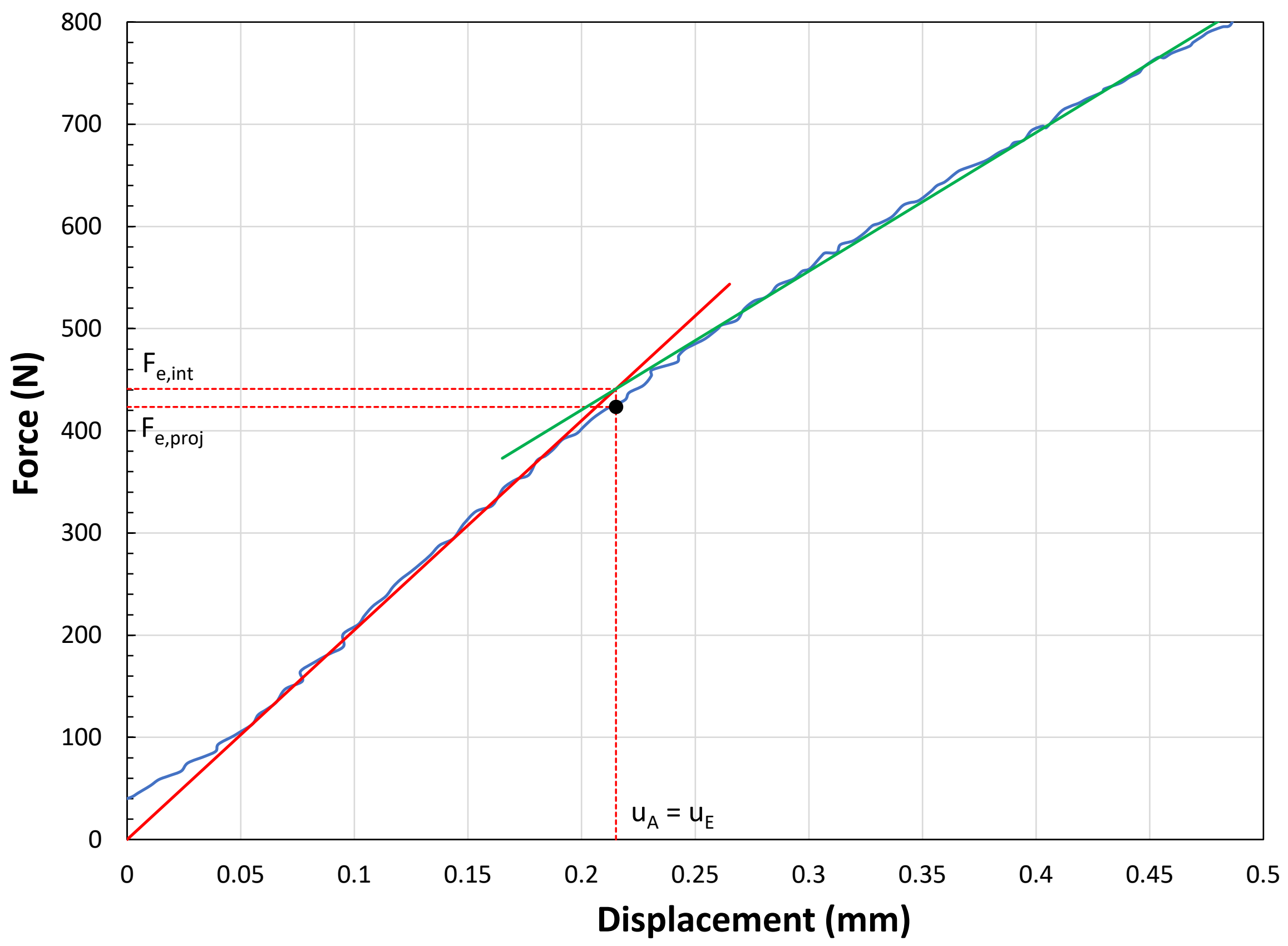




\section{ANNEX 2 \\ SP Test Results for \\ As built, scan length $=78 \mathrm{~mm}$, non-supported \\ ("rough" specimens)}


Specimen ID: AB_1_N_a_SP1

Material: AM Ti-64

Test speed: $0.000934 \mathrm{~mm} / \mathrm{s}$

Max strain rate: $9.34 \mathrm{E}-04 \mathrm{~s}^{-1}$

\begin{tabular}{|c|c|c|}
\hline \multicolumn{3}{|c|}{ TEST RESULTS } \\
\hline \multicolumn{3}{|c|}{ Force values } \\
\hline$F_{e, p r o j}=$ & 352.6 & $\mathbf{N}$ \\
\hline$F_{e, \text { int }}=$ & 361.0 & $\mathbf{N}$ \\
\hline$F_{h 0 / 10, \text { off }}=$ & 621.7 & $\mathbf{N}$ \\
\hline$F_{0.1 \mathrm{~mm}, \mathrm{off}}=$ & 808.2 & $\mathbf{N}$ \\
\hline$F_{0.1 \mathrm{~mm}}=$ & 216.0 & $\mathbf{N}$ \\
\hline$F_{0.48 \mathrm{~mm}}=$ & 807.1 & $\mathbf{N}$ \\
\hline$F_{0.5 \mathrm{~mm}}=$ & 831.2 & $\mathbf{N}$ \\
\hline$F_{0.65 \mathrm{~mm}}=$ & 995.7 & $\mathbf{N}$ \\
\hline$F_{0.9 \mathrm{~mm}}=$ & 1057.2 & $\mathbf{N}$ \\
\hline $\mathbf{F}_{\mathrm{ept}}=$ & 23.9 & $\mathbf{N}$ \\
\hline$F_{e 1.5}=$ & 155.8 & $\mathbf{N}$ \\
\hline $\mathbf{F}_{\mathrm{m}}=$ & 1070.8 & $\mathbf{N}$ \\
\hline$F_{\text {infl }}=$ & 645.2 & $\mathbf{N}$ \\
\hline$F_{f}=$ & 856.7 & $\mathbf{N}$ \\
\hline \multicolumn{3}{|c|}{ Displacement values } \\
\hline $\mathrm{u}_{\mathrm{e}}=$ & 0.172 & $\mathrm{~mm}$ \\
\hline$u_{h 0 / 10, \text { off }}=$ & 0.346 & $\mathrm{~mm}$ \\
\hline$u_{0.1 \mathrm{~mm}, \mathrm{off}}=$ & 0.487 & $\mathrm{~mm}$ \\
\hline$u_{\mathrm{e} 1.5}=$ & 0.074 & $\mathrm{~mm}$ \\
\hline$u_{m}=$ & 0.938 & $\mathrm{~mm}$ \\
\hline$u_{\text {infl }}=$ & 1.568 & $\mathrm{~mm}$ \\
\hline$u_{f}=$ & 1.297 & $\mathrm{~mm}$ \\
\hline \multicolumn{3}{|c|}{ Initial linear slope } \\
\hline Slope $_{\text {ini }}=$ & 2098.05 & $\mathrm{~N} / \mathrm{mm}$ \\
\hline \multicolumn{3}{|c|}{ Energy values } \\
\hline$E_{S P}=$ & 1.02 & J \\
\hline$E_{m}=$ & 0.67 & J \\
\hline$E_{P L}=$ & 0.39 & $\mathbf{J}$ \\
\hline
\end{tabular}




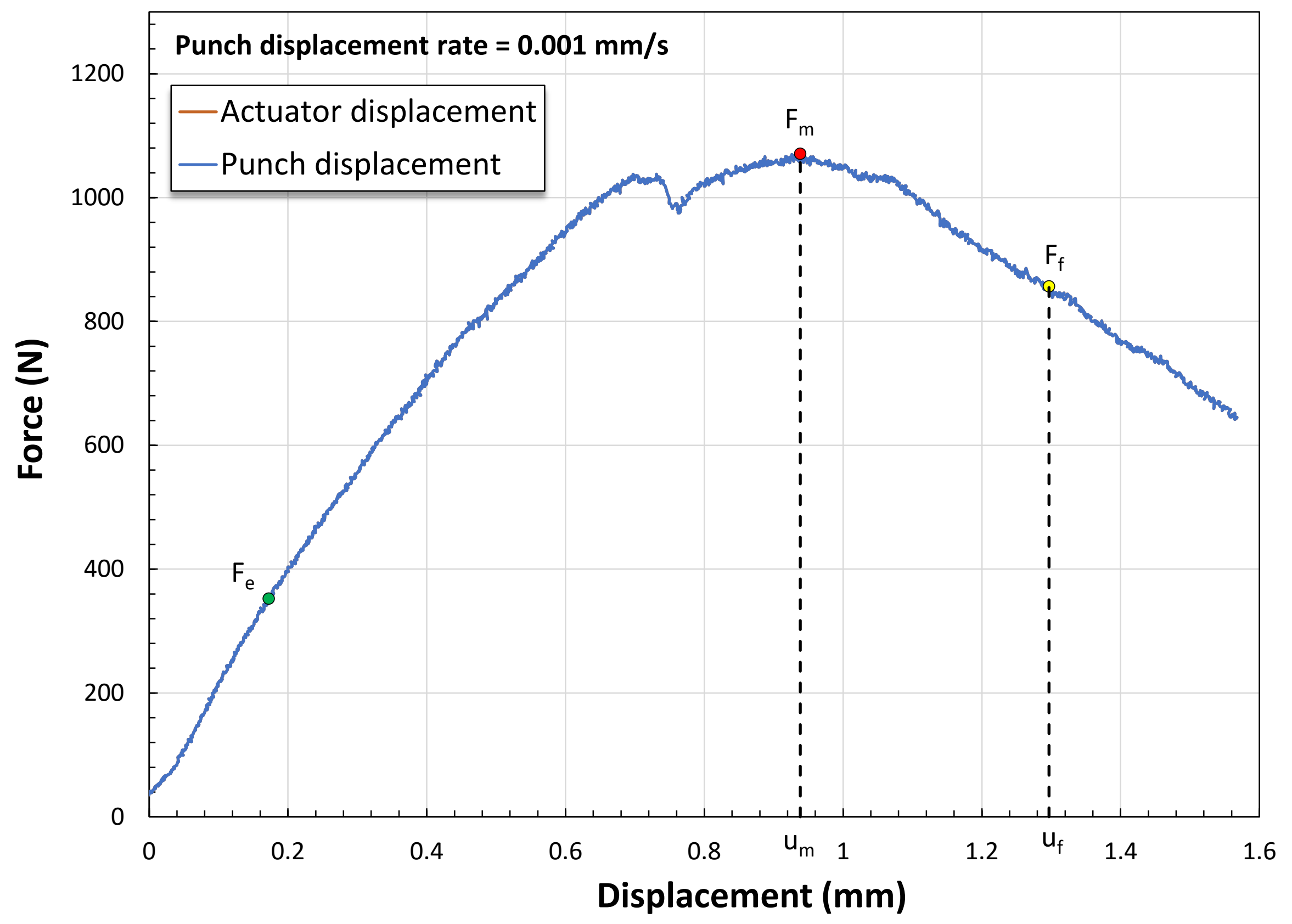




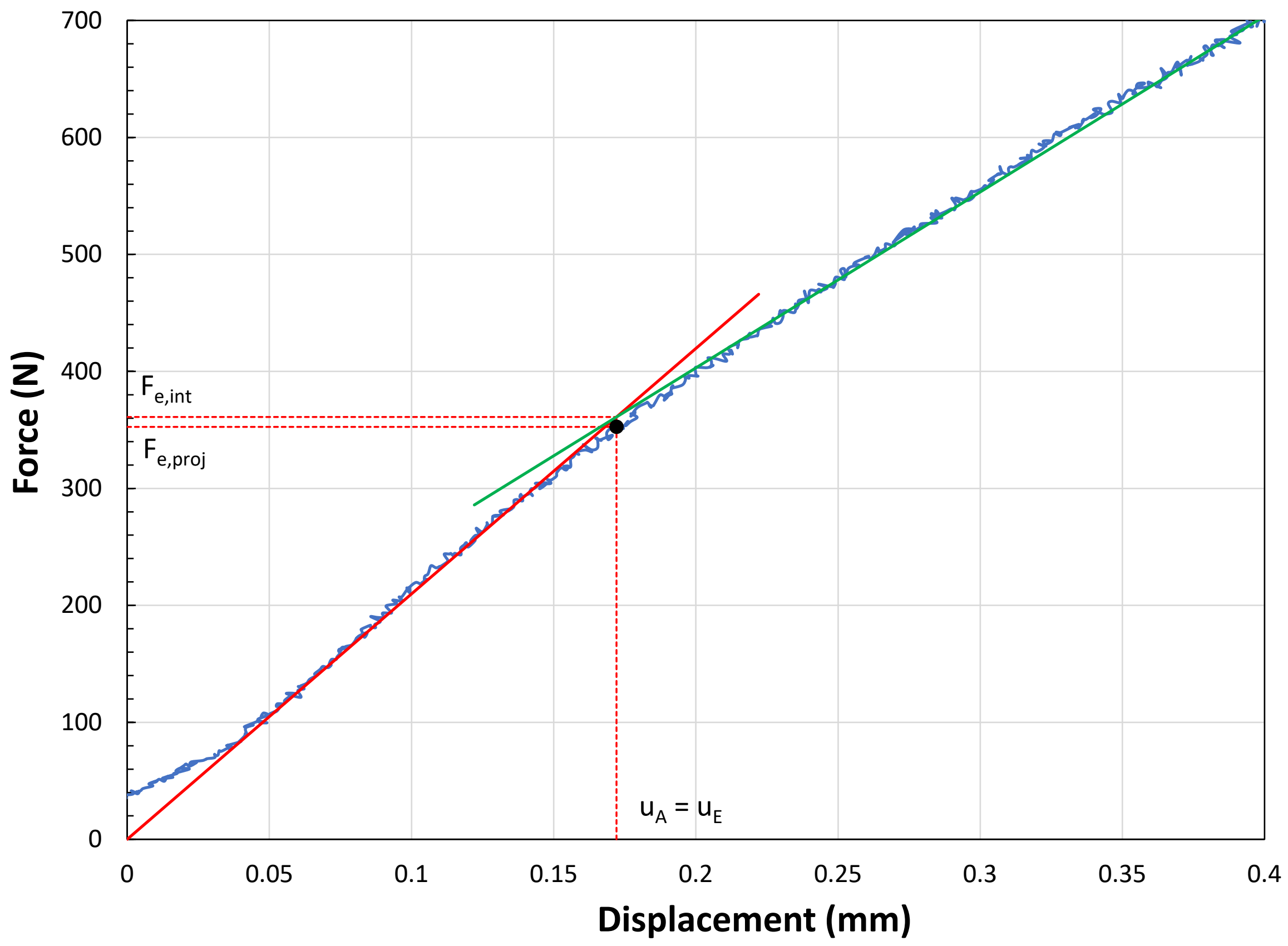


Specimen ID: AB_1_N_a_SP2

Material: AM Ti-64

Test speed: $0.000927 \mathrm{~mm} / \mathrm{s}$

Max strain rate: $9.27 \mathrm{E}-04 \mathrm{~s}^{-1}$

\begin{tabular}{|c|c|c|}
\hline \multicolumn{3}{|c|}{ TEST RESULTS } \\
\hline \multicolumn{3}{|c|}{ Force values } \\
\hline$F_{e, p r o j}=$ & 394.4 & $\mathbf{N}$ \\
\hline$F_{e, \text { int }}=$ & 404.7 & $\mathbf{N}$ \\
\hline$F_{h 0 / 10, \text { off }}=$ & 638.2 & $\mathbf{N}$ \\
\hline$F_{0.1 \mathrm{~mm}, \mathrm{off}}=$ & 841.7 & $\mathbf{N}$ \\
\hline$F_{0.1 \mathrm{~mm}}=$ & 271.0 & $\mathbf{N}$ \\
\hline$F_{0.48 \mathrm{~mm}}=$ & 930.2 & $\mathbf{N}$ \\
\hline$F_{0.5 \mathrm{~mm}}=$ & 944.1 & $\mathbf{N}$ \\
\hline$F_{0.65 \mathrm{~mm}}=$ & 996.4 & $\mathbf{N}$ \\
\hline$F_{0.9 \mathrm{~mm}}=$ & 1047.2 & $\mathbf{N}$ \\
\hline$F_{\mathrm{ept}}=$ & 704.5 & $\mathbf{N}$ \\
\hline$F_{\mathrm{e} 1.5}=$ & 190.1 & $\mathbf{N}$ \\
\hline $\mathbf{F}_{\mathrm{m}}=$ & 1065.2 & $\mathbf{N}$ \\
\hline$F_{\text {infl }}=$ & 1038.6 & $\mathbf{N}$ \\
\hline$F_{f}=$ & 852.2 & $\mathbf{N}$ \\
\hline \multicolumn{3}{|c|}{ Displacement values } \\
\hline$u_{e}=$ & 0.150 & $\mathrm{~mm}$ \\
\hline$u_{h 0 / 10, \text { off }}=$ & 0.287 & $\mathrm{~mm}$ \\
\hline$u_{0.1 \mathrm{~mm}, \mathrm{off}}=$ & 0.413 & $\mathrm{~mm}$ \\
\hline$u_{e 1.5}=$ & 0.070 & $\mathrm{~mm}$ \\
\hline$u_{m}=$ & 0.866 & $\mathrm{~mm}$ \\
\hline$u_{\text {infl }}=$ & 0.902 & $\mathrm{~mm}$ \\
\hline$u_{f}=$ & 1.345 & $\mathrm{~mm}$ \\
\hline \multicolumn{3}{|c|}{ Initial linear slope } \\
\hline Slope $_{\text {ini }}=$ & 2693.16 & $\mathrm{~N} / \mathrm{mm}$ \\
\hline \multicolumn{3}{|c|}{ Energy values } \\
\hline$E_{S P}=$ & 1.11 & J \\
\hline$E_{m}=$ & 0.65 & J \\
\hline$E_{P L}=$ & 0.44 & $\mathbf{J}$ \\
\hline
\end{tabular}




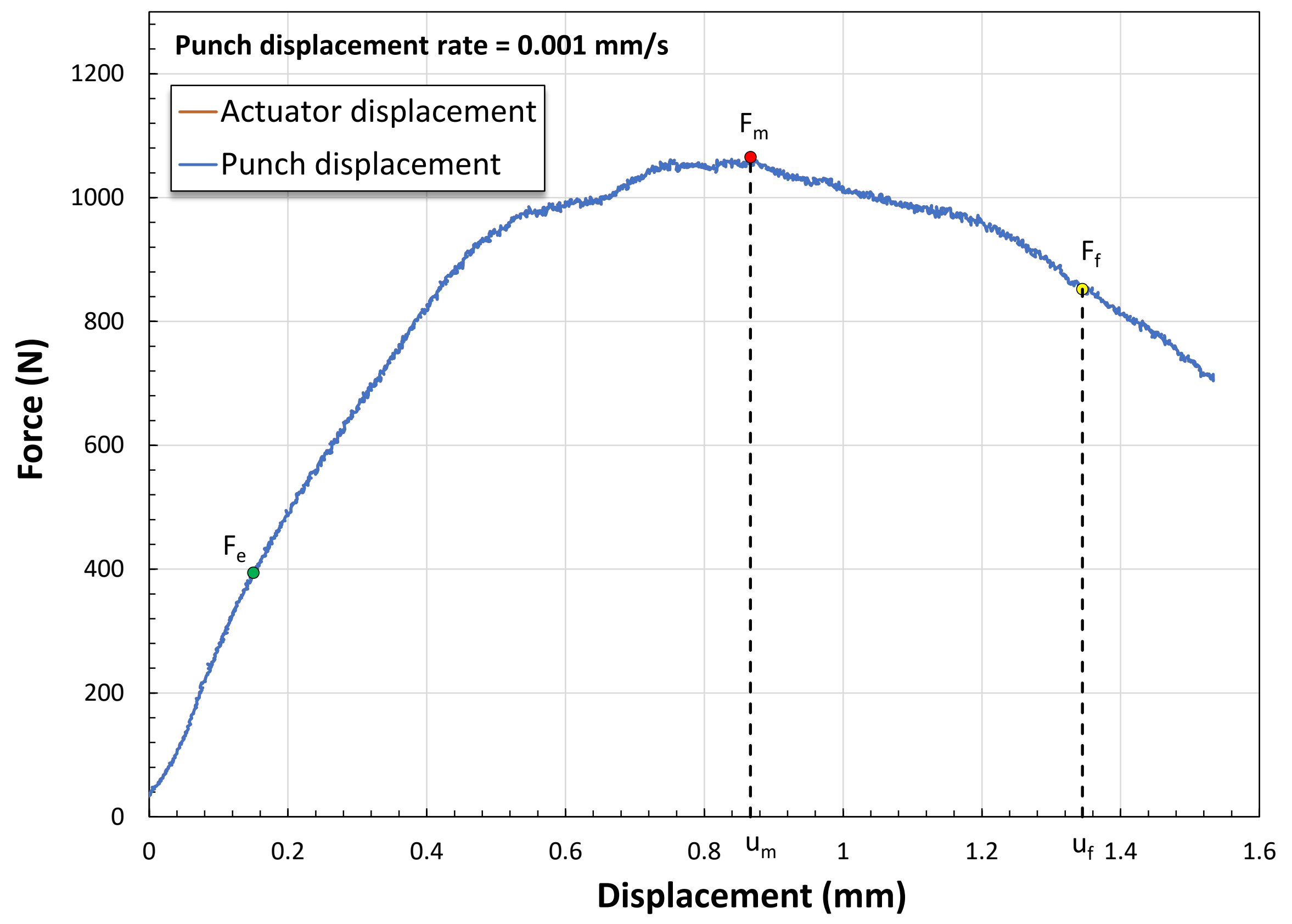




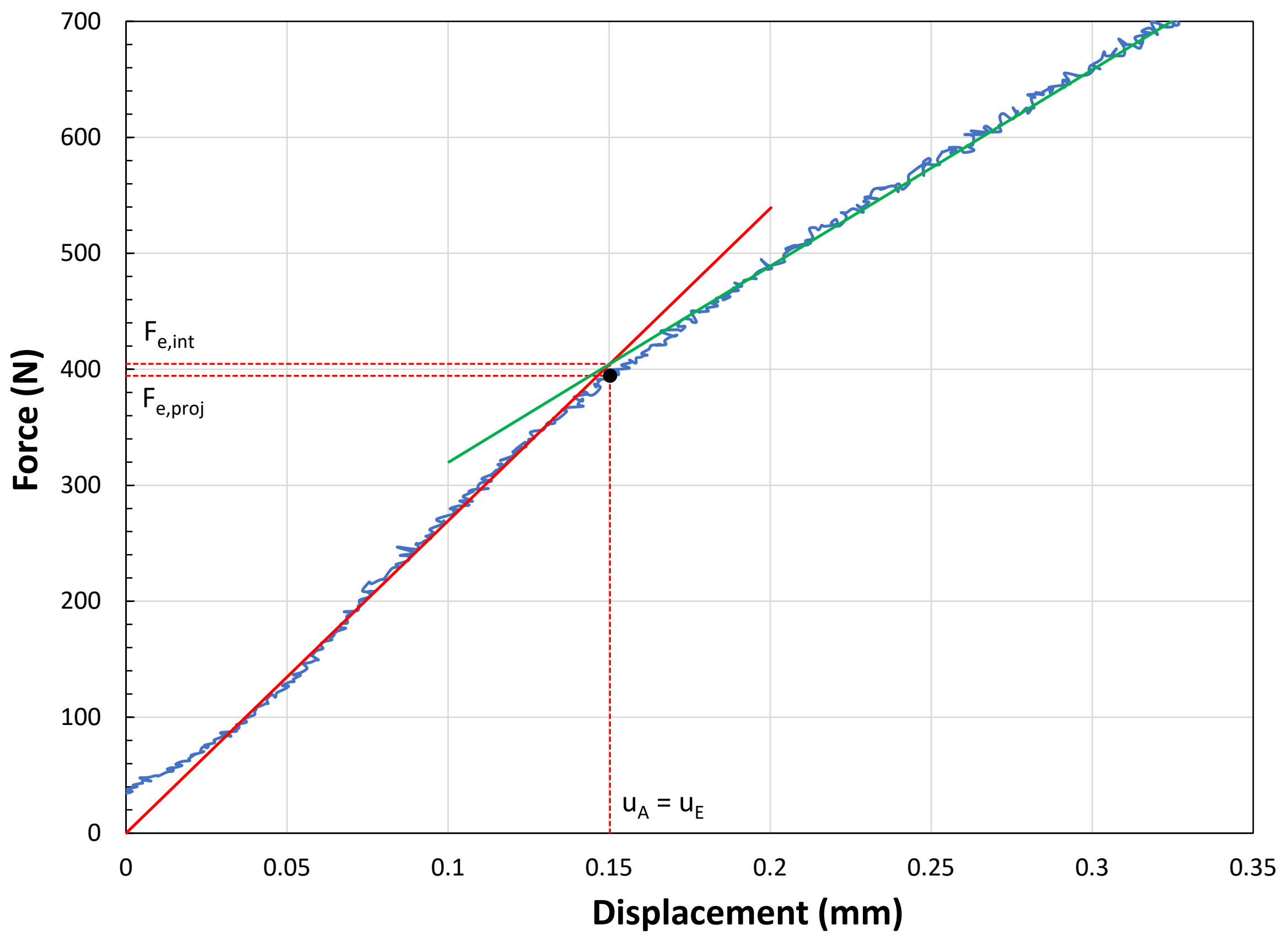


Specimen ID: AB_1_N_a_SP4

Material: AM Ti-64

Test speed: $0.000964 \mathrm{~mm} / \mathrm{s}$

Max strain rate: $9.64 \mathrm{E}-04 \mathrm{~s}^{-1}$

\begin{tabular}{|c|c|c|}
\hline \multicolumn{3}{|c|}{ TEST RESULTS } \\
\hline \multicolumn{3}{|c|}{ Force values } \\
\hline$F_{e, p r o j}=$ & 511.2 & $\mathbf{N}$ \\
\hline$F_{e, \text { int }}=$ & 549.7 & $\mathbf{N}$ \\
\hline$F_{h 0 / 10, \text { off }}=$ & 674.5 & $\mathbf{N}$ \\
\hline$F_{0.1 \mathrm{~mm}, \mathrm{off}}=$ & 767.8 & $\mathbf{N}$ \\
\hline$F_{0.1 \mathrm{~mm}}=$ & 333.2 & $\mathbf{N}$ \\
\hline$F_{0.48 \mathrm{~mm}}=$ & 884.0 & $\mathbf{N}$ \\
\hline$F_{0.5 \mathrm{~mm}}=$ & 901.6 & $\mathbf{N}$ \\
\hline$F_{0.65 \mathrm{~mm}}=$ & 986.5 & $\mathbf{N}$ \\
\hline$F_{0.9 m m}=$ & 1103.1 & $\mathbf{N}$ \\
\hline$F_{\mathrm{ept}}=$ & 792.6 & $\mathbf{N}$ \\
\hline$F_{\mathrm{e} 1.5}=$ & 732.4 & $\mathbf{N}$ \\
\hline $\mathbf{F}_{\mathrm{m}}=$ & 1109.4 & $\mathbf{N}$ \\
\hline$F_{\text {infl }}=$ & 709.8 & $\mathbf{N}$ \\
\hline$F_{f}=$ & 887.5 & $\mathbf{N}$ \\
\hline \multicolumn{3}{|c|}{ Displacement values } \\
\hline $\mathrm{u}_{\mathrm{e}}=$ & 0.179 & $\mathrm{~mm}$ \\
\hline$u_{h 0 / 10, \text { off }}=$ & 0.271 & $\mathrm{~mm}$ \\
\hline$u_{0.1 \mathrm{~mm}, \mathrm{off}}=$ & 0.351 & $\mathrm{~mm}$ \\
\hline$u_{\mathrm{e} 1.5}=$ & 0.317 & $\mathrm{~mm}$ \\
\hline$u_{m}=$ & 0.900 & $\mathrm{~mm}$ \\
\hline$u_{\text {infl }}=$ & 1.639 & $\mathrm{~mm}$ \\
\hline$u_{f}=$ & 1.405 & $\mathrm{~mm}$ \\
\hline \multicolumn{3}{|c|}{ Initial linear slope } \\
\hline Slope $_{\text {ini }}=$ & 3070.90 & $\mathrm{~N} / \mathrm{mm}$ \\
\hline \multicolumn{3}{|c|}{ Energy values } \\
\hline$E_{S P}=$ & 1.19 & J \\
\hline$E_{m}=$ & 0.69 & $\mathbf{J}$ \\
\hline$E_{P L}=$ & 0.49 & $J$ \\
\hline
\end{tabular}




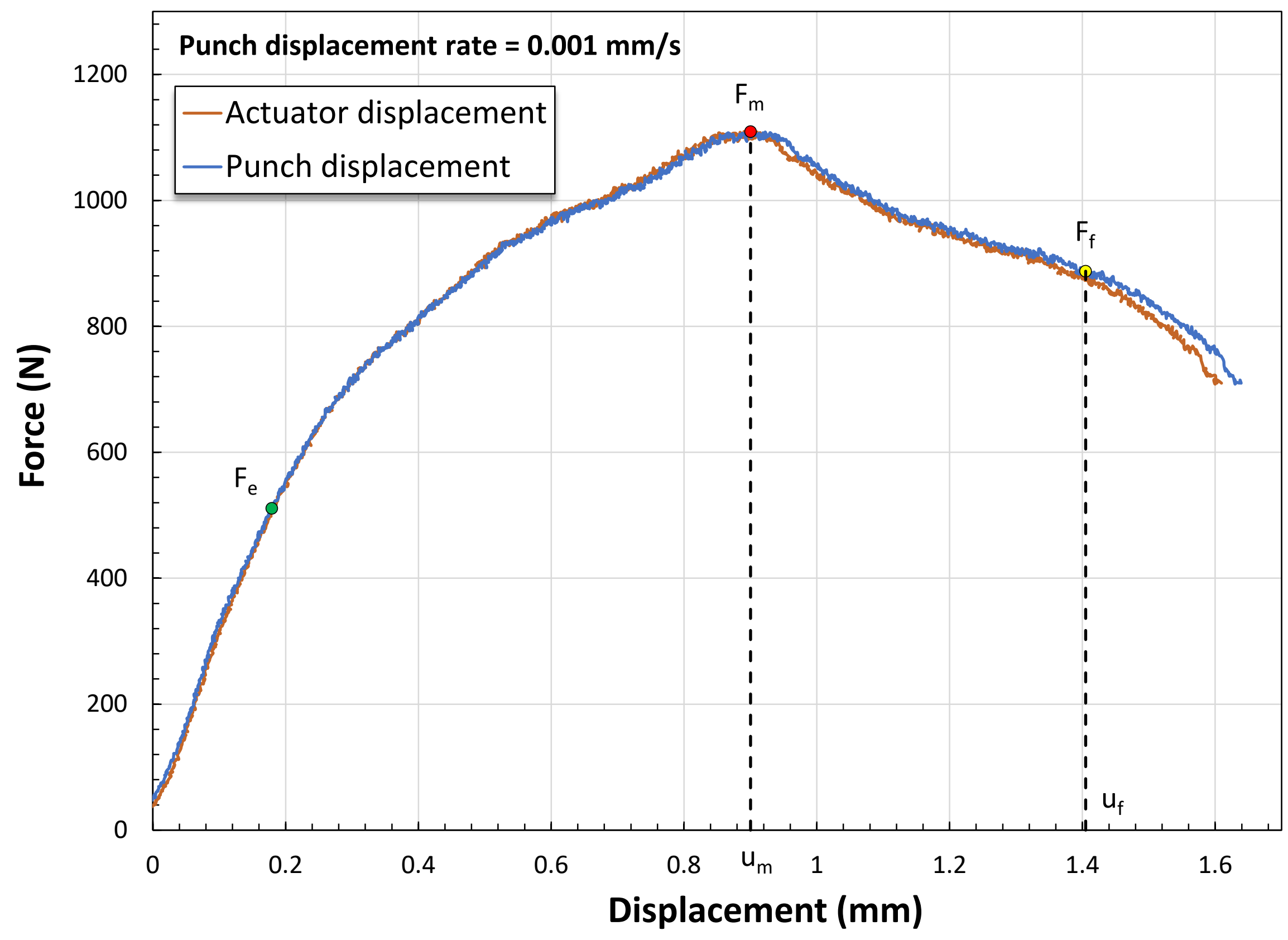




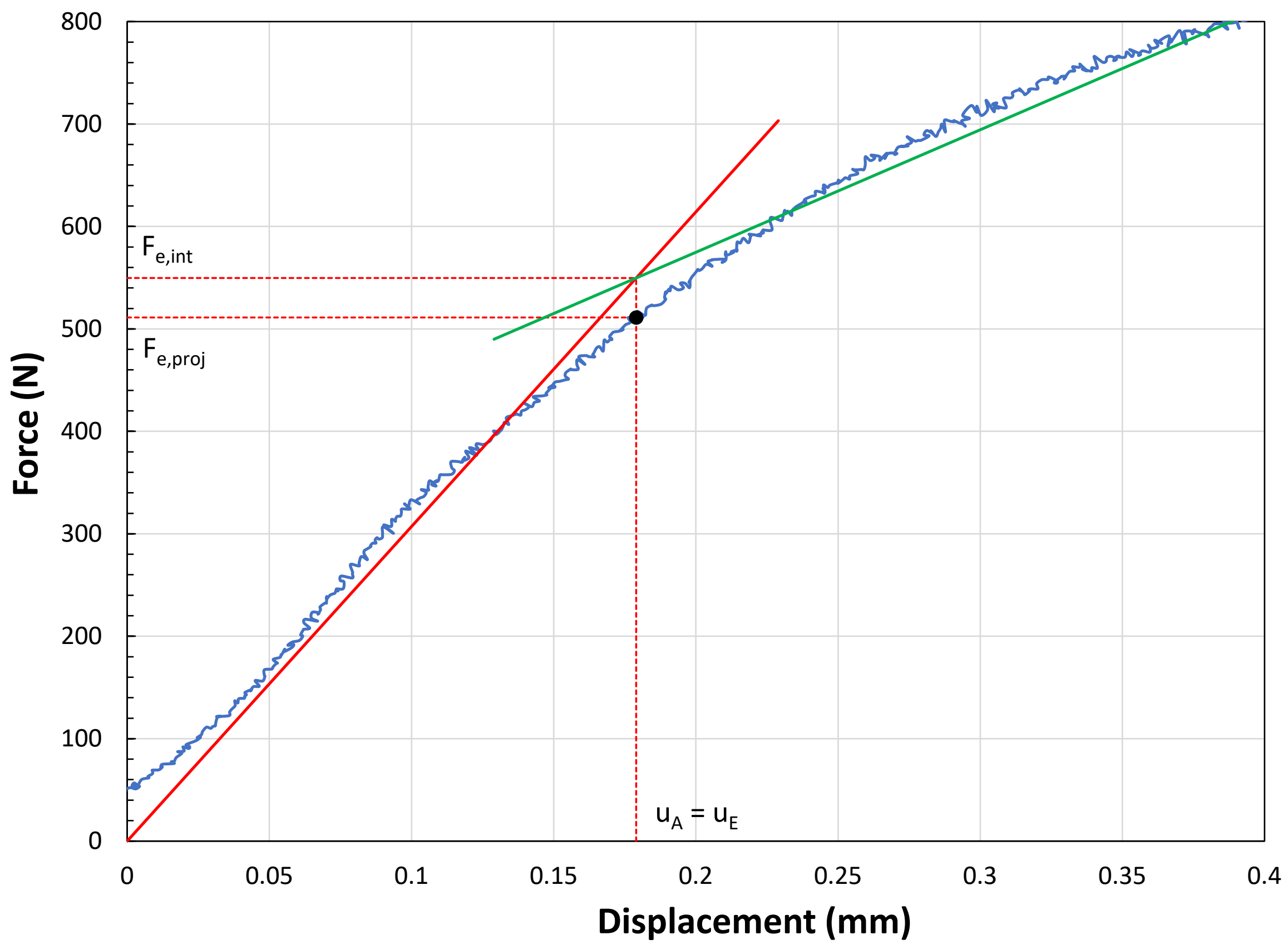


Specimen ID: AB_1_N_a_SP5

Material: AM Ti-64

Test speed: $0.000964 \mathrm{~mm} / \mathrm{s}$

Max strain rate: $9.64 \mathrm{E}-04 \mathrm{~s}^{-1}$

\begin{tabular}{|c|c|c|}
\hline \multicolumn{3}{|c|}{ TEST RESULTS } \\
\hline \multicolumn{3}{|c|}{ Force values } \\
\hline$F_{e, p r o j}=$ & 396.4 & $\mathbf{N}$ \\
\hline$F_{e, \text { int }}=$ & 424.7 & $\mathbf{N}$ \\
\hline$F_{h 0 / 10, \text { off }}=$ & 571.1 & $\mathbf{N}$ \\
\hline$F_{0.1 \mathrm{~mm}, \mathrm{off}}=$ & 732.9 & $\mathbf{N}$ \\
\hline$F_{0.1 \mathrm{~mm}}=$ & 299.7 & $\mathbf{N}$ \\
\hline$F_{0.48 \mathrm{~mm}}=$ & 879.4 & $\mathbf{N}$ \\
\hline$F_{0.5 \mathrm{~mm}}=$ & 899.0 & $\mathbf{N}$ \\
\hline$F_{0.65 \mathrm{~mm}}=$ & 973.8 & $\mathbf{N}$ \\
\hline$F_{0.9 \mathrm{~mm}}=$ & 1088.7 & $\mathbf{N}$ \\
\hline$F_{\mathrm{ept}}=$ & 887.5 & $\mathbf{N}$ \\
\hline$F_{\mathrm{e} 1.5}=$ & 261.9 & $\mathbf{N}$ \\
\hline $\mathbf{F}_{\mathrm{m}}=$ & 1098.1 & $\mathbf{N}$ \\
\hline$F_{\text {infl }}=$ & 635.9 & $\mathbf{N}$ \\
\hline$F_{f}=$ & 878.4 & $\mathbf{N}$ \\
\hline \multicolumn{3}{|c|}{ Displacement values } \\
\hline $\mathrm{u}_{\mathrm{e}}=$ & 0.143 & $\mathrm{~mm}$ \\
\hline$u_{h 0 / 10, \text { off }}=$ & 0.243 & $\mathrm{~mm}$ \\
\hline$u_{0.1 \mathrm{~mm}, \mathrm{off}}=$ & 0.348 & $\mathrm{~mm}$ \\
\hline$u_{\mathrm{e} 1.5}=$ & 0.085 & $\mathrm{~mm}$ \\
\hline$u_{m}=$ & 0.864 & $\mathrm{~mm}$ \\
\hline$u_{\text {infl }}=$ & 1.656 & $\mathrm{~mm}$ \\
\hline$u_{f}=$ & 1.373 & $\mathrm{~mm}$ \\
\hline \multicolumn{3}{|c|}{ Initial linear slope } \\
\hline Slope $_{\text {ini }}=$ & 2960.92 & $\mathrm{~N} / \mathrm{mm}$ \\
\hline \multicolumn{3}{|c|}{ Energy values } \\
\hline$E_{S P}=$ & 1.16 & J \\
\hline$E_{m}=$ & 0.64 & $\mathbf{J}$ \\
\hline$E_{P L}=$ & 0.44 & $\mathbf{J}$ \\
\hline
\end{tabular}




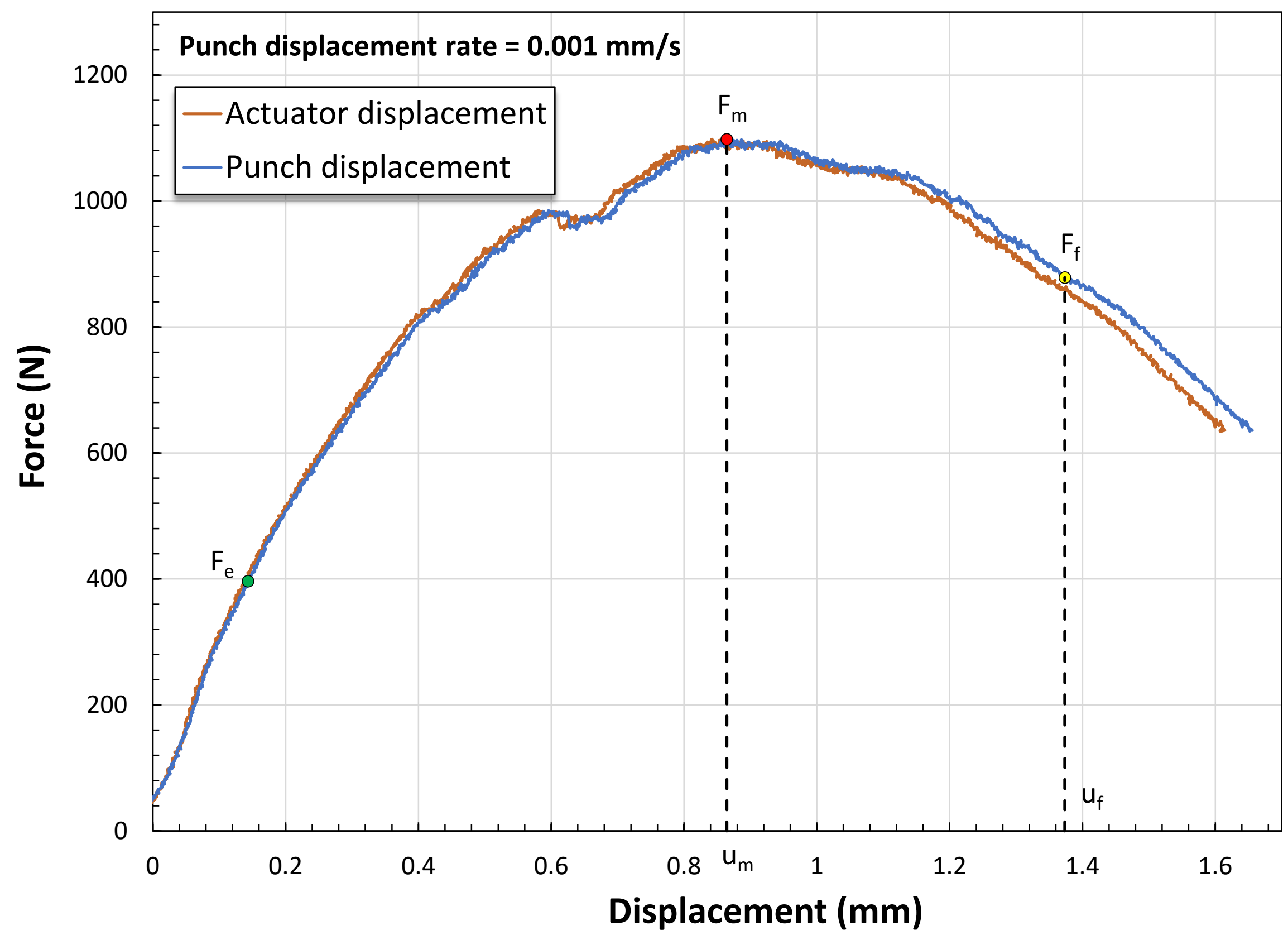




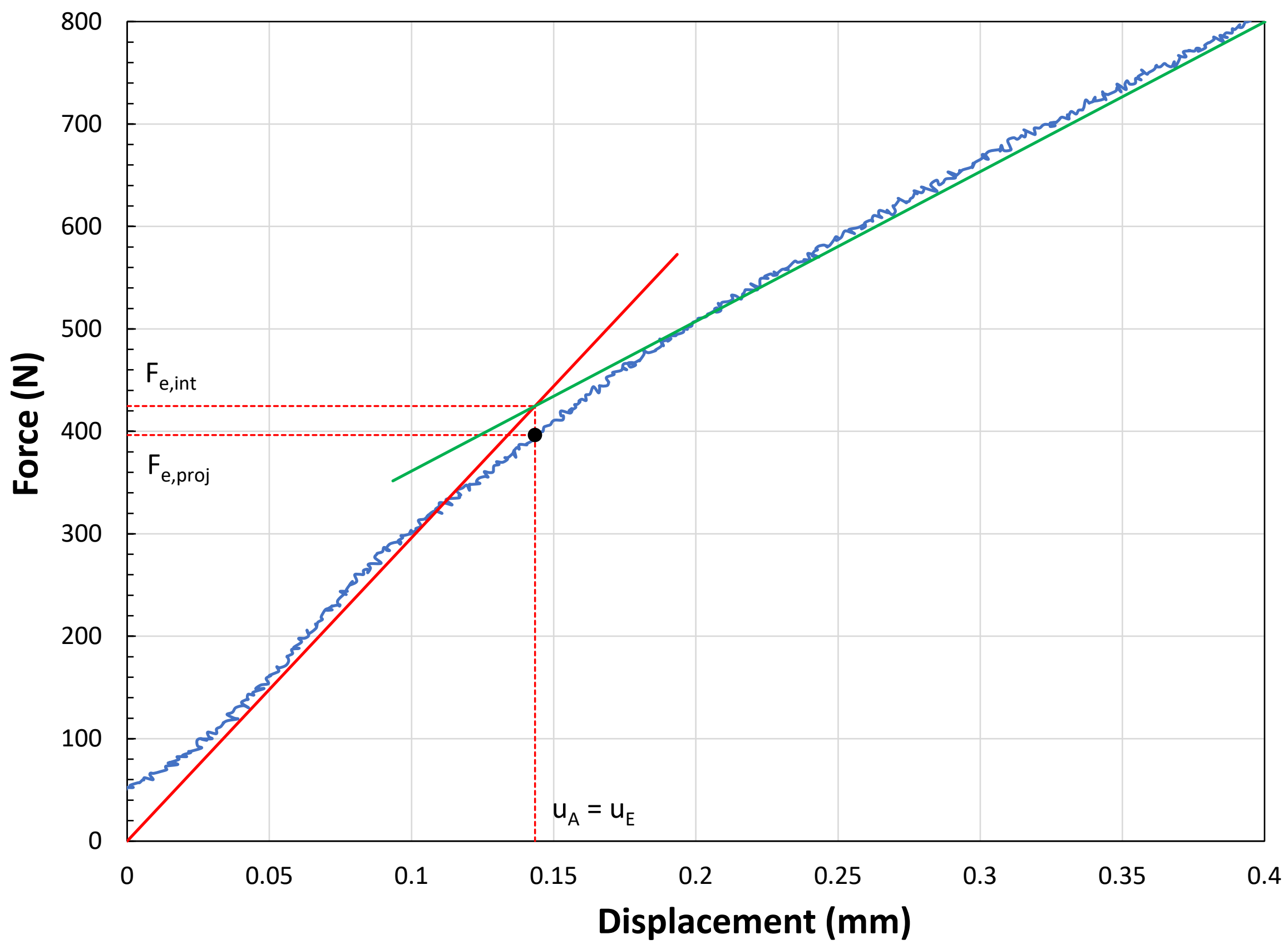


Specimen ID: AB_1_N_a_SP6

Material: AM Ti-64

Test speed: $0.000969 \mathrm{~mm} / \mathrm{s}$

Max strain rate: $9.69 \mathrm{E}-04 \mathrm{~s}^{-1}$

\begin{tabular}{|c|c|c|}
\hline \multicolumn{3}{|c|}{ TEST RESULTS } \\
\hline \multicolumn{3}{|c|}{ Force values } \\
\hline$F_{e, p r o j}=$ & 507.7 & $\mathbf{N}$ \\
\hline$F_{e, \text { int }}=$ & 526.7 & $\mathbf{N}$ \\
\hline$F_{h 0 / 10, \text { off }}=$ & 703.1 & $\mathbf{N}$ \\
\hline$F_{0.1 \mathrm{~mm}, \mathrm{off}}=$ & 851.9 & $\mathbf{N}$ \\
\hline$F_{0.1 \mathrm{~mm}}=$ & 288.5 & $\mathbf{N}$ \\
\hline$F_{0.48 \mathrm{~mm}}=$ & 919.3 & $\mathbf{N}$ \\
\hline$F_{0.5 \mathrm{~mm}}=$ & 951.0 & $\mathbf{N}$ \\
\hline$F_{0.65 \mathrm{~mm}}=$ & 1009.7 & $\mathbf{N}$ \\
\hline$F_{0.9 \mathrm{~mm}}=$ & 1024.5 & $\mathbf{N}$ \\
\hline$F_{\mathrm{ept}}=$ & 410.0 & $\mathbf{N}$ \\
\hline$F_{\mathrm{e} 1.5}=$ & 983.5 & $\mathbf{N}$ \\
\hline $\mathbf{F}_{\mathrm{m}}=$ & 1050.7 & $\mathbf{N}$ \\
\hline$F_{\text {infl }}=$ & 740.5 & $\mathbf{N}$ \\
\hline$F_{f}=$ & 840.6 & $\mathbf{N}$ \\
\hline \multicolumn{3}{|c|}{ Displacement values } \\
\hline $\mathrm{u}_{\mathrm{e}}=$ & 0.190 & $\mathrm{~mm}$ \\
\hline$u_{h 0 / 10, \text { off }}=$ & 0.304 & $\mathrm{~mm}$ \\
\hline$u_{0.1 \mathrm{~mm}, \mathrm{off}}=$ & 0.408 & $\mathrm{~mm}$ \\
\hline$u_{\mathrm{e} 1.5}=$ & 0.525 & $\mathrm{~mm}$ \\
\hline$u_{m}=$ & 0.977 & $\mathrm{~mm}$ \\
\hline$u_{\text {infl }}=$ & 1.608 & $\mathrm{~mm}$ \\
\hline$u_{f}=$ & 1.479 & $\mathrm{~mm}$ \\
\hline \multicolumn{3}{|c|}{ Initial linear slope } \\
\hline Slope $_{\text {ini }}=$ & 2769.78 & $\mathrm{~N} / \mathrm{mm}$ \\
\hline \multicolumn{3}{|c|}{ Energy values } \\
\hline$E_{S P}=$ & 1.26 & J \\
\hline$E_{m}=$ & 0.77 & $\mathbf{J}$ \\
\hline$E_{P L}=$ & 0.57 & $J$ \\
\hline
\end{tabular}




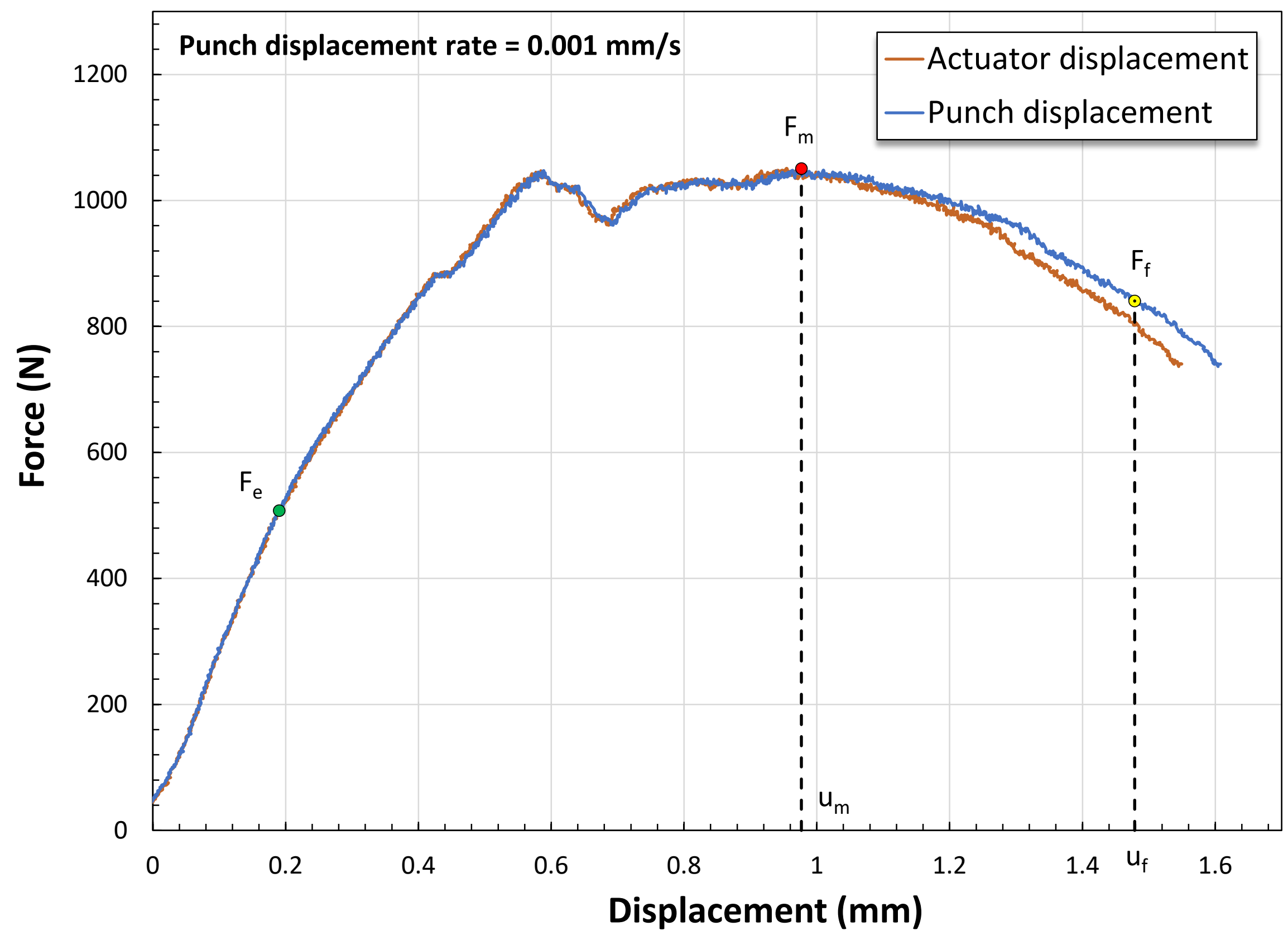




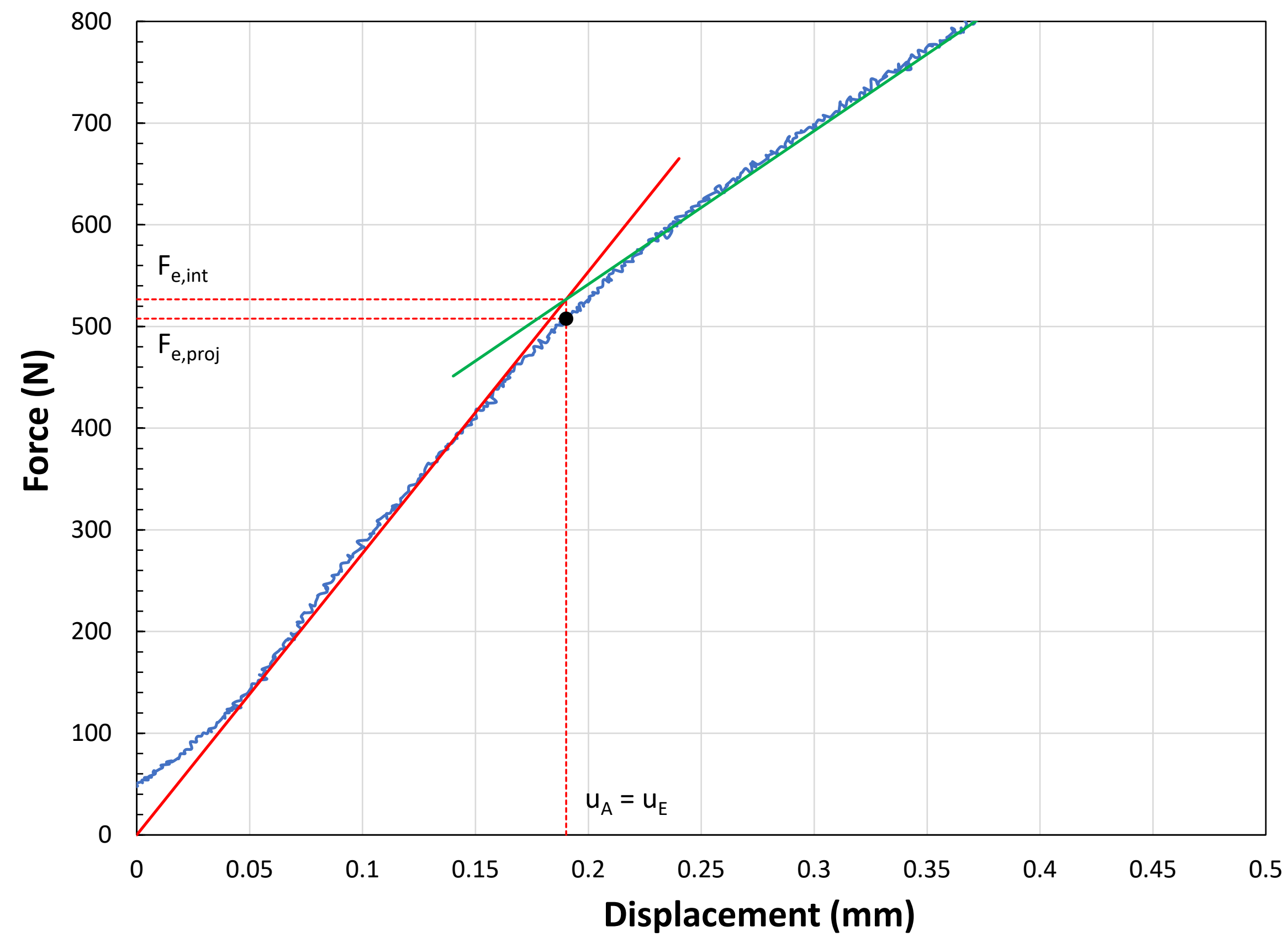


Specimen ID: AB_1_N_a_SP7

Material: AM Ti-64

Test speed: $0.001011 \mathrm{~mm} / \mathrm{s}$

Max strain rate: $1.01 \mathrm{E}-03 \mathrm{~s}^{-1}$

\begin{tabular}{|c|c|c|}
\hline \multicolumn{3}{|c|}{ TEST RESULTS } \\
\hline \multicolumn{3}{|c|}{ Force values } \\
\hline$F_{e, p r o j}=$ & 438.4 & $\mathbf{N}$ \\
\hline$F_{e, \text { int }}=$ & 470.2 & $\mathbf{N}$ \\
\hline$F_{h 0 / 10, \text { off }}=$ & 653.3 & $\mathbf{N}$ \\
\hline$F_{0.1 \mathrm{~mm}, \mathrm{off}}=$ & 822.8 & $\mathbf{N}$ \\
\hline$F_{0.1 \mathrm{~mm}}=$ & 288.2 & $\mathbf{N}$ \\
\hline$F_{0.48 \mathrm{~mm}}=$ & 915.7 & $\mathbf{N}$ \\
\hline$F_{0.5 \mathrm{~mm}}=$ & 927.0 & $\mathbf{N}$ \\
\hline$F_{0.65 \mathrm{~mm}}=$ & 1059.5 & $\mathbf{N}$ \\
\hline$F_{0.9 \mathrm{~mm}}=$ & 1083.8 & $\mathbf{N}$ \\
\hline$F_{\mathrm{ept}}=$ & 309.6 & $\mathbf{N}$ \\
\hline$F_{\mathrm{e} 1.5}=$ & 187.5 & $\mathbf{N}$ \\
\hline $\mathbf{F}_{\mathrm{m}}=$ & 1109.3 & $\mathbf{N}$ \\
\hline$F_{\text {infl }}=$ & 703.4 & $\mathbf{N}$ \\
\hline$F_{f}=$ & 887.4 & $\mathbf{N}$ \\
\hline \multicolumn{3}{|c|}{ Displacement values } \\
\hline$u_{e}=$ & 0.170 & $\mathrm{~mm}$ \\
\hline$u_{h 0 / 10, \text { off }}=$ & 0.286 & $\mathrm{~mm}$ \\
\hline$u_{0.1 \mathrm{~mm}, \mathrm{off}}=$ & 0.398 & $\mathrm{~mm}$ \\
\hline$u_{e 1.5}=$ & 0.063 & $\mathrm{~mm}$ \\
\hline$u_{m}=$ & 1.019 & $\mathrm{~mm}$ \\
\hline$u_{\text {infl }}=$ & 1.592 & $\mathrm{~mm}$ \\
\hline$u_{f}=$ & 1.425 & $\mathrm{~mm}$ \\
\hline \multicolumn{3}{|c|}{ Initial linear slope } \\
\hline Slope $_{\text {ini }}=$ & 2772.76 & $\mathrm{~N} / \mathrm{mm}$ \\
\hline \multicolumn{3}{|c|}{ Energy values } \\
\hline$E_{S P}=$ & 1.22 & J \\
\hline$E_{m}=$ & 0.83 & J \\
\hline$E_{P L}=$ & 0.60 & $J$ \\
\hline
\end{tabular}




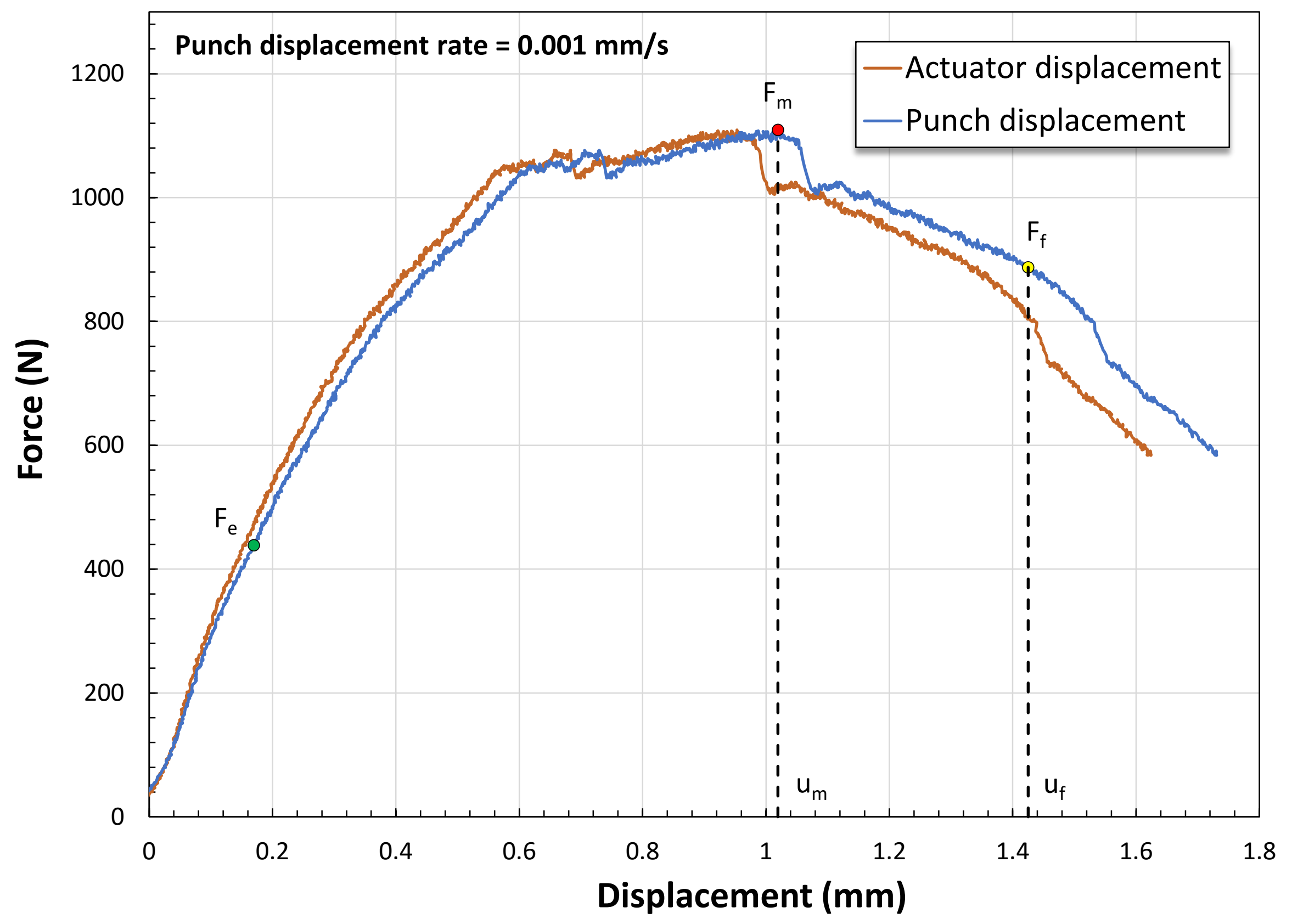




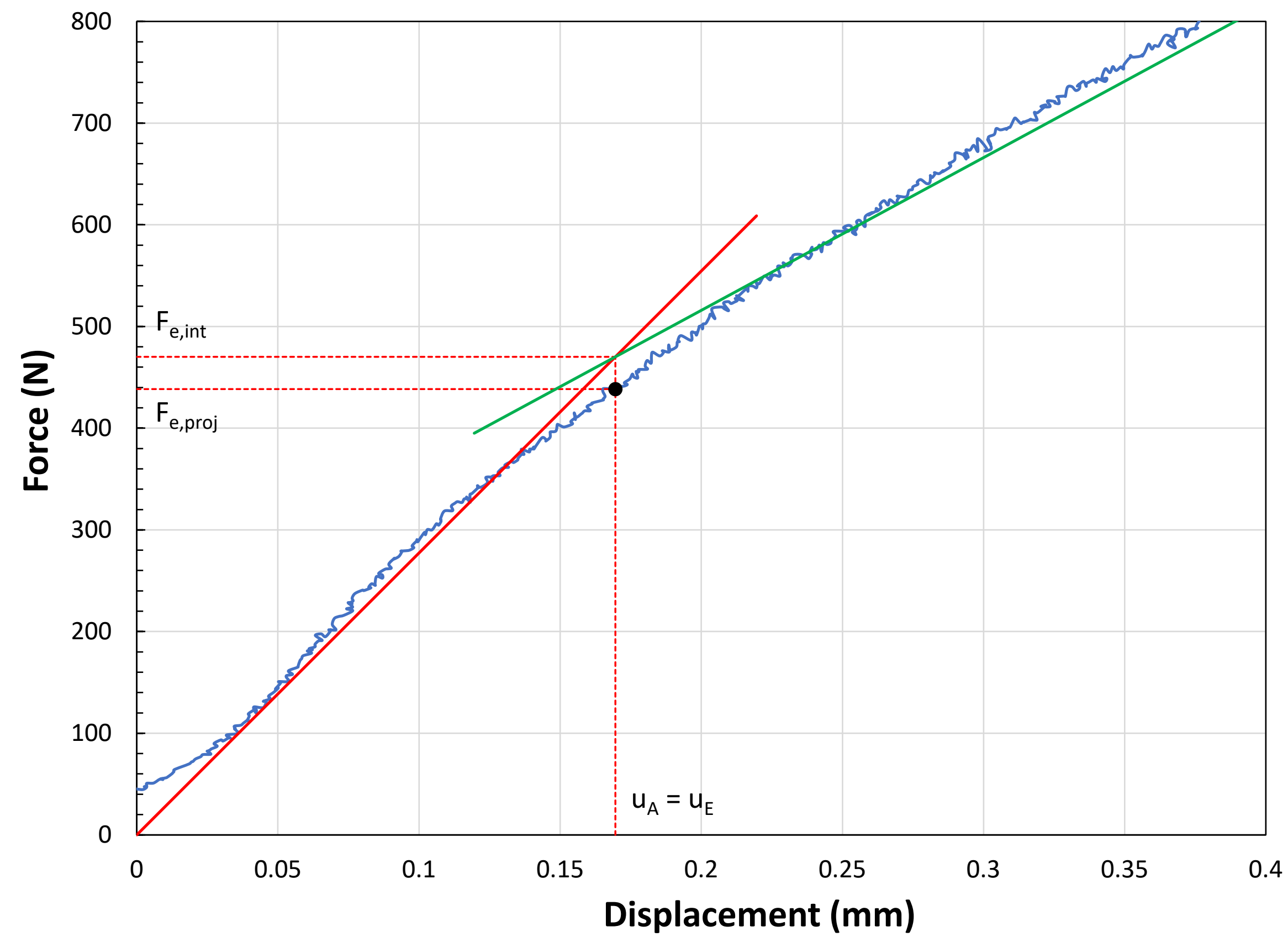




\section{ANNEX 3 \\ SP Test Results for \\ As built, scan length $=78 \mathrm{~mm}$, non-supported \\ (polished specimens)}


Specimen ID: AB_1_N_a_SP8 (polish

Material: AM Ti-64

Test speed: $0.001436 \mathrm{~mm} / \mathrm{s}$

Max strain rate: $1.44 \mathrm{E}-03 \mathrm{~s}^{-1}$

\begin{tabular}{|c|c|c|}
\hline \multicolumn{3}{|c|}{ TEST RESULTS } \\
\hline \multicolumn{3}{|c|}{ Force values } \\
\hline$F_{e, \text { proj }}=$ & 387.3 & $\mathbf{N}$ \\
\hline$F_{e, \text { int }}=$ & 421.6 & $\mathbf{N}$ \\
\hline$F_{h 0 / 10, \text { off }}=$ & 521.6 & $\mathbf{N}$ \\
\hline$F_{0.1 \mathrm{~mm}, \text { off }}=$ & 694.8 & $\mathbf{N}$ \\
\hline$F_{0.1 \mathrm{~mm}}=$ & 336.9 & $\mathbf{N}$ \\
\hline$F_{0.48 \mathrm{~mm}}=$ & 903.1 & $\mathbf{N}$ \\
\hline $\mathrm{F}_{0.5 \mathrm{~mm}}=$ & 921.2 & $\mathbf{N}$ \\
\hline$F_{0.65 \mathrm{~mm}}=$ & 911.2 & $\mathbf{N}$ \\
\hline$F_{0.9 \mathrm{~mm}}=$ & 972.6 & $\mathbf{N}$ \\
\hline$F_{\text {ept }}=$ & 783.2 & $\mathbf{N}$ \\
\hline $\mathrm{F}_{\mathrm{e} 1.5}=$ & 736.8 & $\mathbf{N}$ \\
\hline$F_{m}=$ & 975.4 & $\mathbf{N}$ \\
\hline$F_{\text {infl }}=$ & 505.2 & $\mathbf{N}$ \\
\hline$F_{f}=$ & 780.3 & $\mathbf{N}$ \\
\hline \multicolumn{3}{|c|}{ Displacement values } \\
\hline $\mathrm{u}_{\mathrm{e}}=$ & 0.120 & $\mathrm{~mm}$ \\
\hline$u_{h 0 / 10, \text { off }}=$ & 0.194 & $\mathrm{~mm}$ \\
\hline$u_{0.1 \mathrm{~mm}, \text { off }}=$ & 0.298 & $\mathrm{~mm}$ \\
\hline$u_{e 1.5}=$ & 0.328 & $\mathrm{~mm}$ \\
\hline$u_{m}=$ & 0.861 & $\mathrm{~mm}$ \\
\hline$u_{\text {infl }}=$ & 1.624 & $\mathrm{~mm}$ \\
\hline$u_{f}=$ & 1.250 & $\mathrm{~mm}$ \\
\hline \multicolumn{3}{|c|}{ Initial linear slope } \\
\hline Slope $_{\text {ini }}=$ & 3519.91 & $\mathrm{~N} / \mathrm{mm}$ \\
\hline \multicolumn{3}{|c|}{ Energy values } \\
\hline$E_{S P}=$ & 0.98 & J \\
\hline$E_{m}=$ & 0.63 & J \\
\hline $\mathrm{E}_{\mathrm{PL}}=$ & 0.49 & J \\
\hline
\end{tabular}




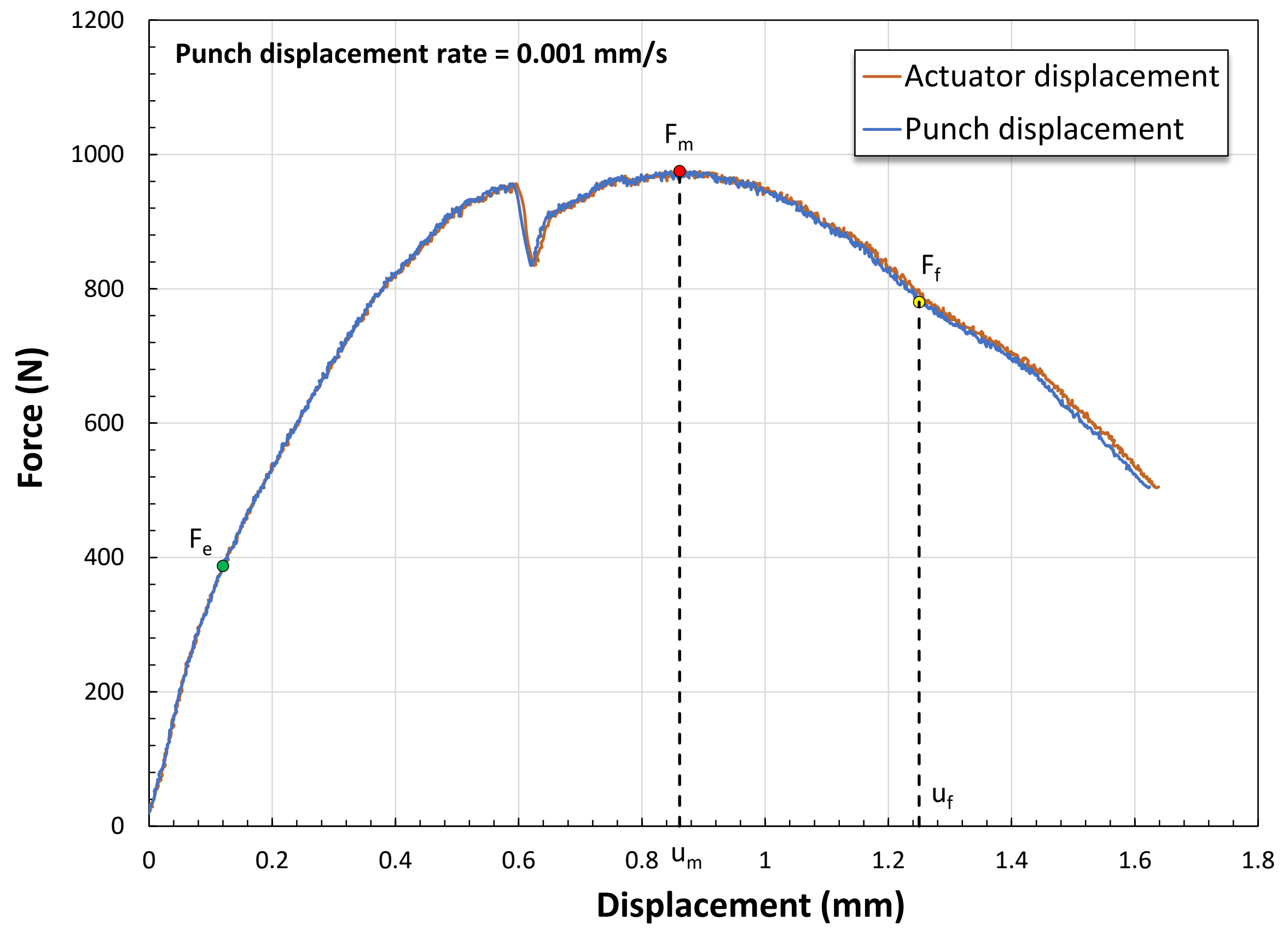




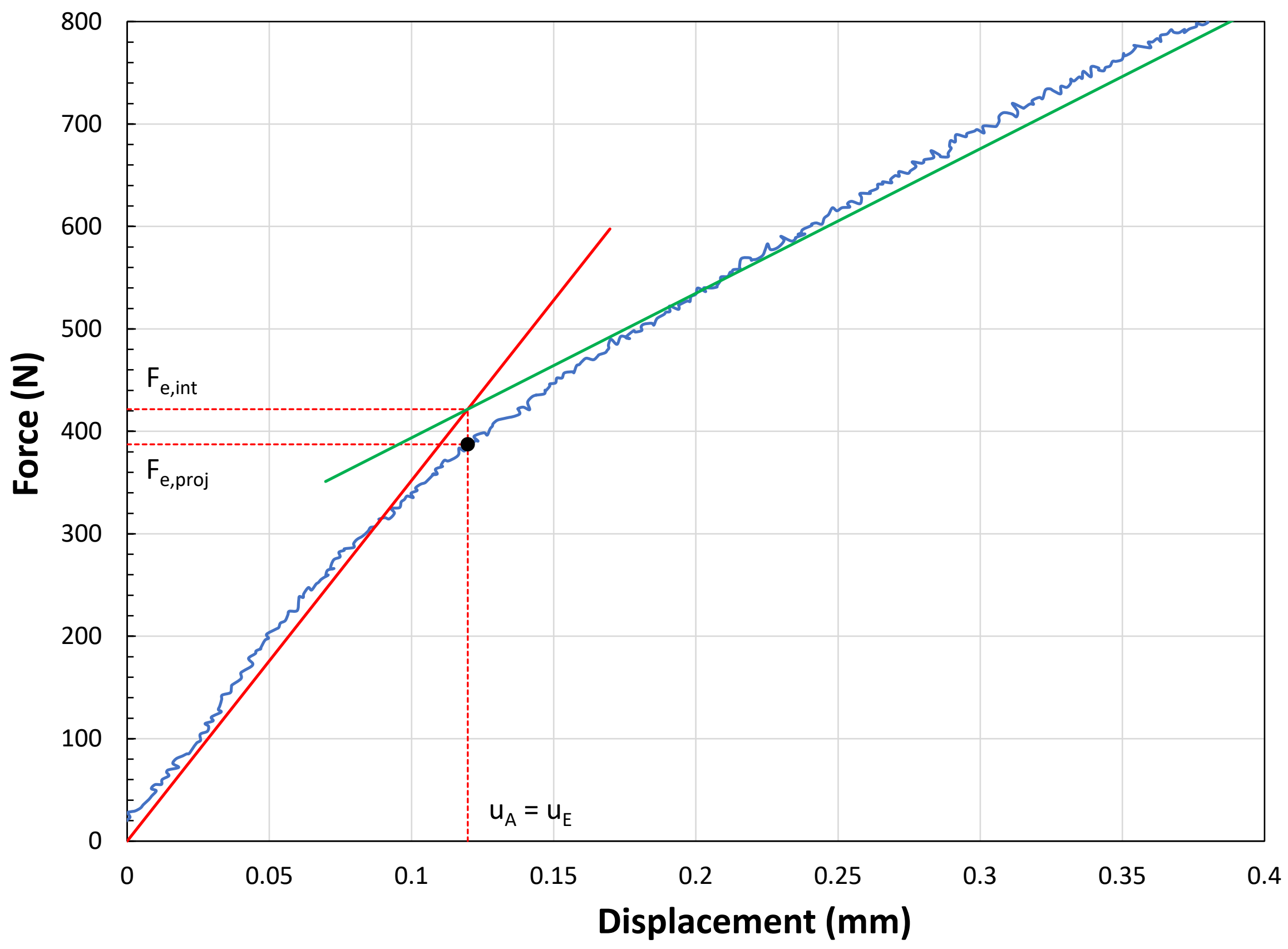


Specimen ID: AB_1_N_a_SP9 (polish

Material: AM Ti-64

Test speed: $0.001437 \mathrm{~mm} / \mathrm{s}$

Max strain rate: $1.44 \mathrm{E}-03 \mathrm{~s}^{-1}$

\begin{tabular}{|c|c|c|}
\hline \multicolumn{3}{|c|}{ TEST RESULTS } \\
\hline \multicolumn{3}{|c|}{ Force values } \\
\hline$F_{e, p r o j}=$ & 453.6 & $\mathbf{N}$ \\
\hline $\mathbf{F}_{\mathrm{e}, \text { int }}=$ & 495.0 & $\mathbf{N}$ \\
\hline$F_{h 0 / 10, \text { off }}=$ & 577.5 & $\mathbf{N}$ \\
\hline$F_{0.1 \mathrm{~mm}, \text { off }}=$ & 721.5 & $\mathbf{N}$ \\
\hline$F_{0.1 \mathrm{~mm}}=$ & 334.5 & $\mathbf{N}$ \\
\hline$F_{0.48 \mathrm{~mm}}=$ & 868.3 & $\mathbf{N}$ \\
\hline $\mathrm{F}_{0.5 \mathrm{~mm}}=$ & 870.6 & $\mathbf{N}$ \\
\hline$F_{0.65 \mathrm{~mm}}=$ & 939.4 & $\mathbf{N}$ \\
\hline$F_{0.9 \mathrm{~mm}}=$ & 1006.8 & $\mathbf{N}$ \\
\hline$F_{\text {ept }}=$ & 737.8 & $\mathbf{N}$ \\
\hline$F_{\mathrm{e} 1.5}=$ & 711.4 & $\mathbf{N}$ \\
\hline$F_{m}=$ & 1010.7 & $\mathbf{N}$ \\
\hline$F_{\text {infl }}=$ & 970.1 & $\mathbf{N}$ \\
\hline$F_{f}=$ & 808.5 & $\mathbf{N}$ \\
\hline \multicolumn{3}{|c|}{ Displacement values } \\
\hline $\mathrm{u}_{\mathrm{e}}=$ & 0.151 & $\mathrm{~mm}$ \\
\hline$u_{h 0 / 10, \text { off }}=$ & 0.221 & $\mathrm{~mm}$ \\
\hline$u_{0.1 \mathrm{~mm}, \text { off }}=$ & 0.321 & $\mathrm{~mm}$ \\
\hline$u_{\mathrm{e} 1.5}=$ & 0.311 & $\mathrm{~mm}$ \\
\hline$u_{m}=$ & 0.893 & $\mathrm{~mm}$ \\
\hline$u_{i n f l}=$ & 1.010 & $\mathrm{~mm}$ \\
\hline$u_{f}=$ & 1.271 & $\mathrm{~mm}$ \\
\hline \multicolumn{3}{|c|}{ Initial linear slope } \\
\hline Slope $_{\text {ini }}=$ & 3278.01 & $\mathrm{~N} / \mathrm{mm}$ \\
\hline \multicolumn{3}{|c|}{ Energy values } \\
\hline$E_{S P}=$ & 1.01 & J \\
\hline$E_{m}=$ & 0.66 & J \\
\hline$E_{p L}=$ & 0.50 & J \\
\hline
\end{tabular}




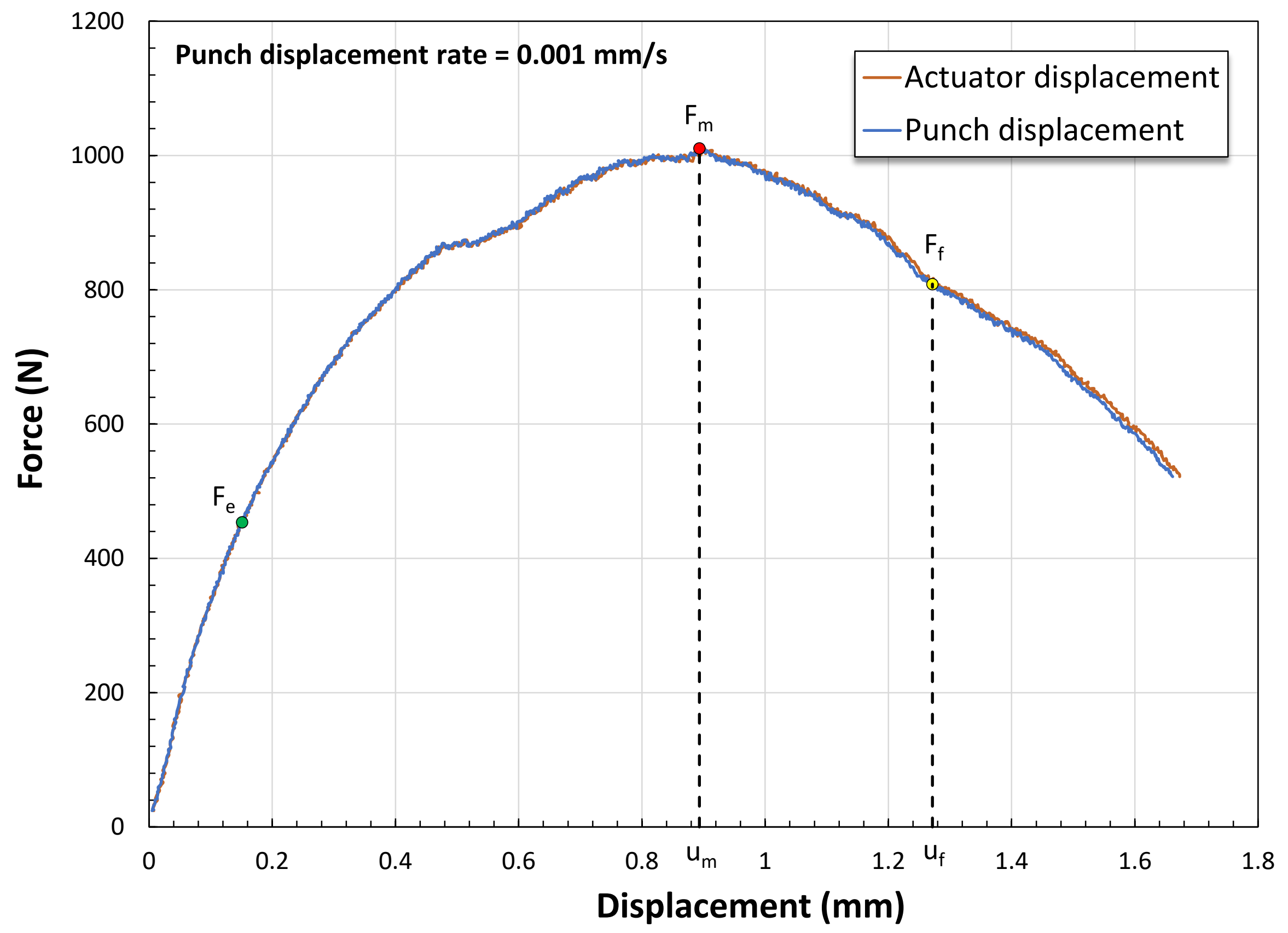




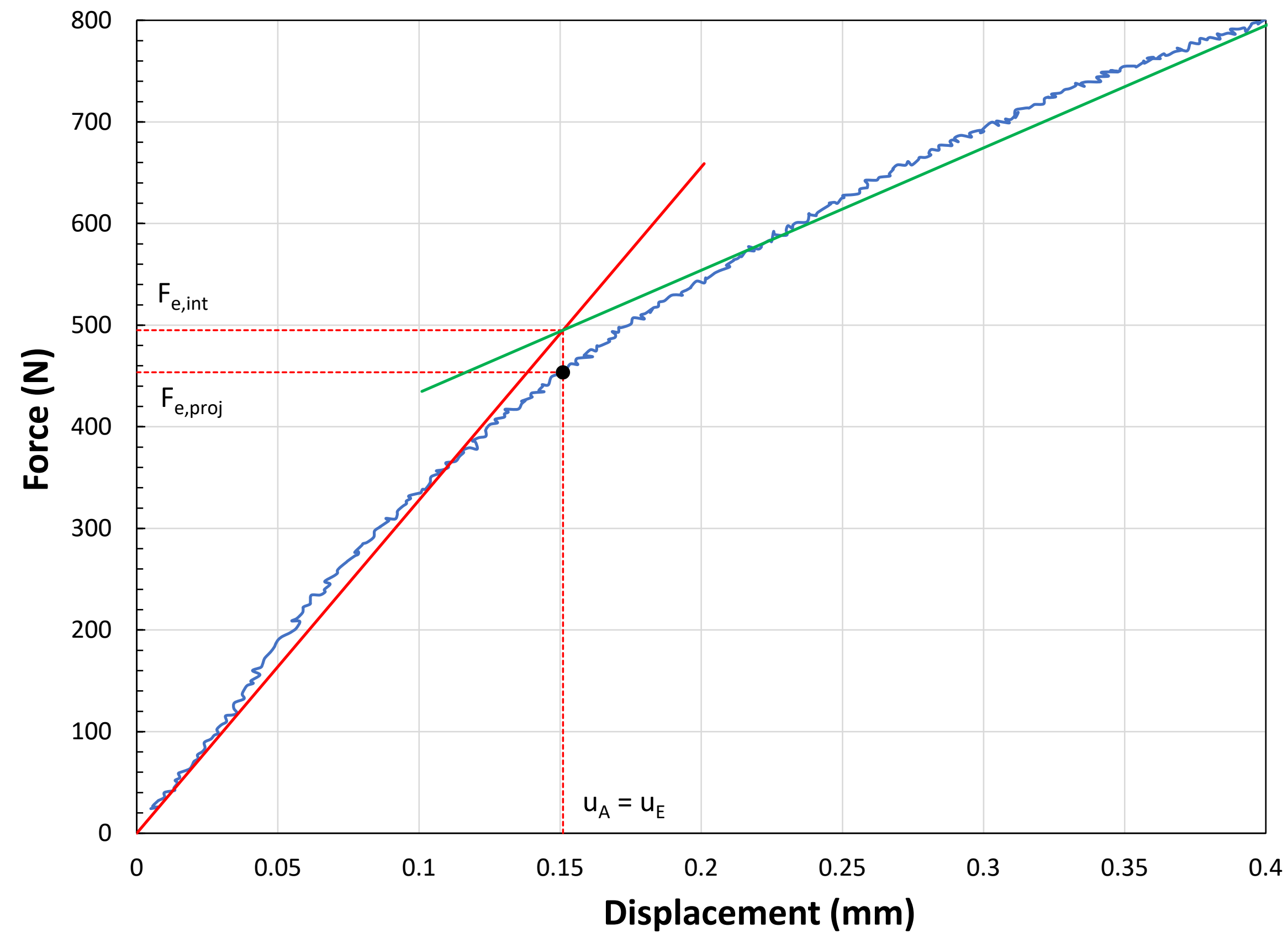


Specimen ID: AB_1_N_a_SP10 (polis

Material: AM Ti-64

Test speed: $0.00143 \mathrm{~mm} / \mathrm{s}$

Max strain rate: $1.43 \mathrm{E}-03 \mathrm{~s}^{-1}$

\begin{tabular}{|c|c|c|}
\hline \multicolumn{3}{|c|}{ TEST RESULTS } \\
\hline \multicolumn{3}{|c|}{ Force values } \\
\hline$F_{e, p r o j}=$ & 390.0 & $\mathbf{N}$ \\
\hline$F_{e, \text { int }}=$ & 419.9 & $\mathbf{N}$ \\
\hline$F_{h 0 / 10, \text { off }}=$ & 532.1 & $\mathbf{N}$ \\
\hline$F_{0.1 \mathrm{~mm}, \mathrm{off}}=$ & 698.4 & $\mathbf{N}$ \\
\hline$F_{0.1 \mathrm{~mm}}=$ & 298.2 & $\mathbf{N}$ \\
\hline$F_{0.48 \mathrm{~mm}}=$ & 845.6 & $\mathbf{N}$ \\
\hline$F_{0.5 \mathrm{~mm}}=$ & 852.7 & $\mathbf{N}$ \\
\hline$F_{0.65 \mathrm{~mm}}=$ & 865.9 & $\mathbf{N}$ \\
\hline$F_{0.9 m m}=$ & 937.9 & $\mathbf{N}$ \\
\hline$F_{\mathrm{ept}}=$ & 878.2 & $\mathbf{N}$ \\
\hline$F_{\mathrm{e} 1.5}=$ & 775.3 & $\mathbf{N}$ \\
\hline $\mathbf{F}_{\mathrm{m}}=$ & 955.3 & $\mathbf{N}$ \\
\hline$F_{\text {infl }}=$ & 487.2 & $\mathbf{N}$ \\
\hline$F_{f}=$ & 764.3 & $\mathbf{N}$ \\
\hline \multicolumn{3}{|c|}{ Displacement values } \\
\hline $\mathrm{u}_{\mathrm{e}}=$ & 0.144 & $\mathrm{~mm}$ \\
\hline$u_{h 0 / 10, \text { off }}=$ & 0.228 & $\mathrm{~mm}$ \\
\hline$u_{0.1 \mathrm{~mm}, \mathrm{off}}=$ & 0.340 & $\mathrm{~mm}$ \\
\hline$u_{\mathrm{e} 1.5}=$ & 0.399 & $\mathrm{~mm}$ \\
\hline$u_{m}=$ & 0.976 & $\mathrm{~mm}$ \\
\hline$u_{\text {infl }}=$ & 1.611 & $\mathrm{~mm}$ \\
\hline$u_{f}=$ & 1.351 & $\mathrm{~mm}$ \\
\hline \multicolumn{3}{|c|}{ Initial linear slope } \\
\hline Slope $_{\text {ini }}=$ & 2907.81 & $\mathrm{~N} / \mathrm{mm}$ \\
\hline \multicolumn{3}{|c|}{ Energy values } \\
\hline$E_{S P}=$ & 1.03 & J \\
\hline$E_{m}=$ & 0.70 & $\mathbf{J}$ \\
\hline$E_{P L}=$ & 0.54 & $\mathbf{J}$ \\
\hline
\end{tabular}




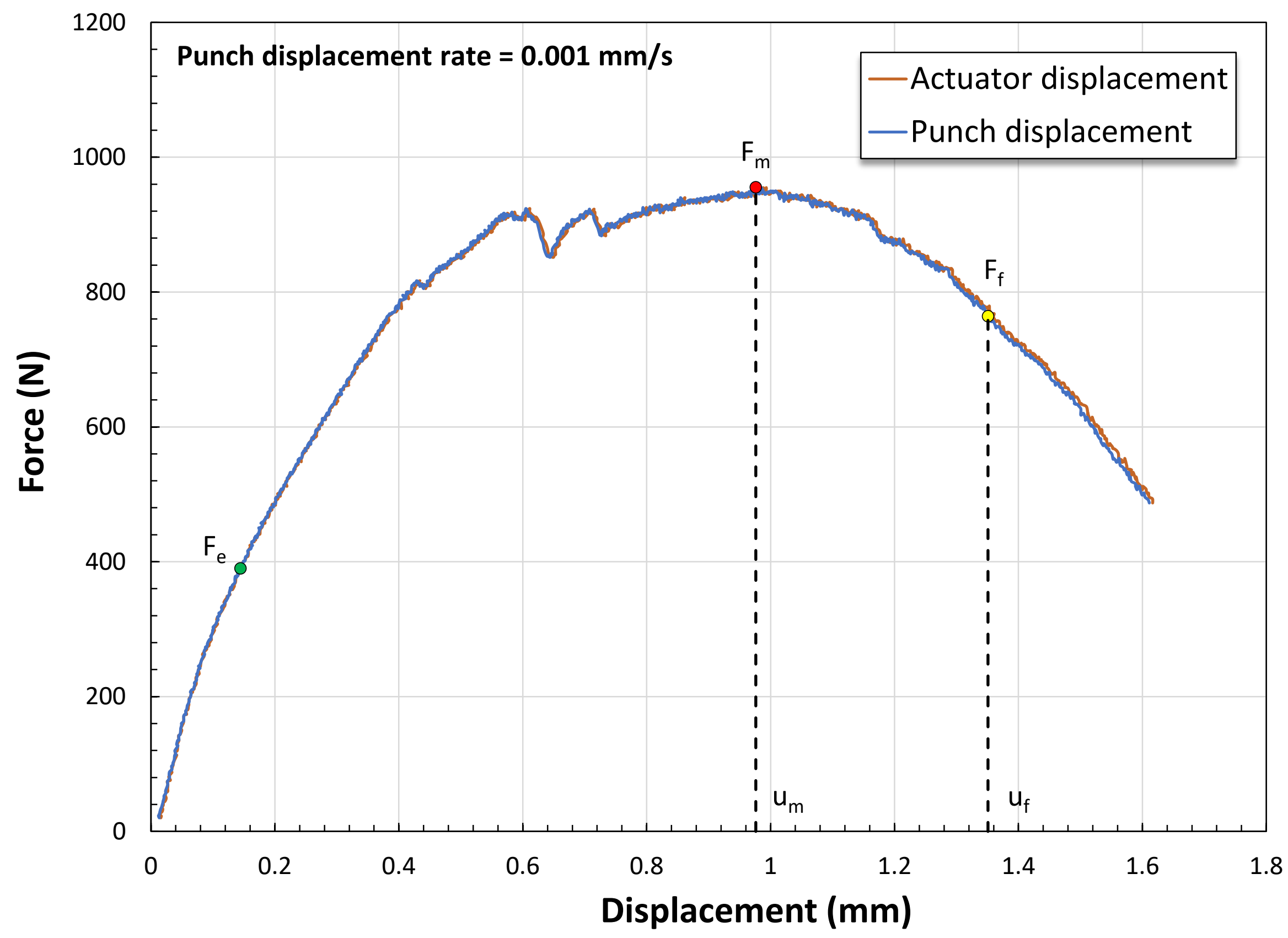




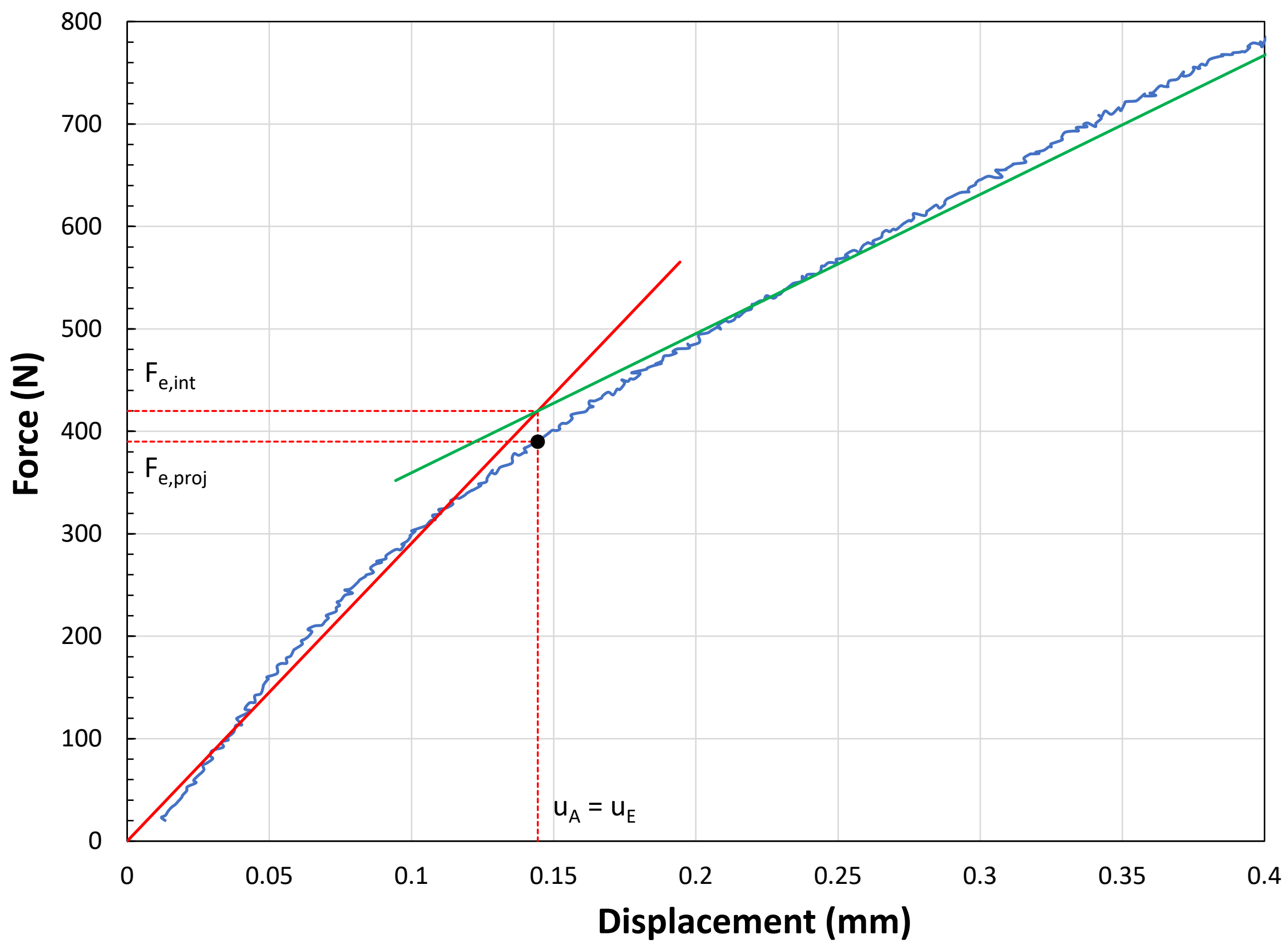




\section{ANNEX 4 \\ SP Test Results for \\ As built, scan length $=26 \mathrm{~mm}$, non-supported \\ ("rough" specimens)}


Specimen ID: AB_3_N_a_SP1

Material: AM Ti-64

Test speed: $0.001031 \mathrm{~mm} / \mathrm{s}$

Max strain rate: $1.03 \mathrm{E}-03 \mathrm{~s}^{-1}$

\begin{tabular}{|c|c|c|}
\hline \multicolumn{3}{|c|}{ TEST RESULTS } \\
\hline \multicolumn{3}{|c|}{ Force values } \\
\hline$F_{e, p r o j}=$ & 398.1 & $\mathbf{N}$ \\
\hline$F_{e, \text { int }}=$ & 408.4 & $\mathbf{N}$ \\
\hline$F_{h 0 / 10, \text { off }}=$ & 643.2 & $\mathbf{N}$ \\
\hline$F_{0.1 \mathrm{~mm}, \mathrm{off}}=$ & 787.6 & $\mathbf{N}$ \\
\hline$F_{0.1 \mathrm{~mm}}=$ & 188.5 & $\mathbf{N}$ \\
\hline$F_{0.48 \mathrm{~mm}}=$ & 745.2 & $\mathbf{N}$ \\
\hline$F_{0.5 \mathrm{~mm}}=$ & 770.4 & $\mathbf{N}$ \\
\hline$F_{0.65 \mathrm{~mm}}=$ & 920.4 & $\mathbf{N}$ \\
\hline$F_{0.9 \mathrm{~mm}}=$ & 1005.9 & $\mathbf{N}$ \\
\hline$F_{\mathrm{ept}}=$ & 117.8 & $\mathbf{N}$ \\
\hline$F_{\mathrm{e} 1.5}=$ & 192.6 & $\mathbf{N}$ \\
\hline $\mathbf{F}_{\mathrm{m}}=$ & 1093.5 & $\mathbf{N}$ \\
\hline$F_{\text {infl }}=$ & 1000.8 & $\mathbf{N}$ \\
\hline$F_{f}=$ & 874.8 & $\mathbf{N}$ \\
\hline \multicolumn{3}{|c|}{ Displacement values } \\
\hline $\mathrm{u}_{\mathrm{e}}=$ & 0.218 & $\mathrm{~mm}$ \\
\hline$u_{h 0 / 10, \text { off }}=$ & 0.393 & $\mathrm{~mm}$ \\
\hline$u_{0.1 \mathrm{~mm}, \mathrm{off}}=$ & 0.520 & $\mathrm{~mm}$ \\
\hline$u_{\mathrm{e} 1.5}=$ & 0.100 & $\mathrm{~mm}$ \\
\hline$u_{m}=$ & 1.142 & $\mathrm{~mm}$ \\
\hline$u_{\text {infl }}=$ & 0.774 & $\mathrm{~mm}$ \\
\hline$u_{f}=$ & 1.585 & $\mathrm{~mm}$ \\
\hline \multicolumn{3}{|c|}{ Initial linear slope } \\
\hline Slope $_{\text {ini }}=$ & 1876.53 & $\mathrm{~N} / \mathrm{mm}$ \\
\hline \multicolumn{3}{|c|}{ Energy values } \\
\hline$E_{S P}=$ & 1.28 & J \\
\hline$E_{m}=$ & 0.84 & J \\
\hline$E_{P L}=$ & 0.52 & $\mathbf{J}$ \\
\hline
\end{tabular}




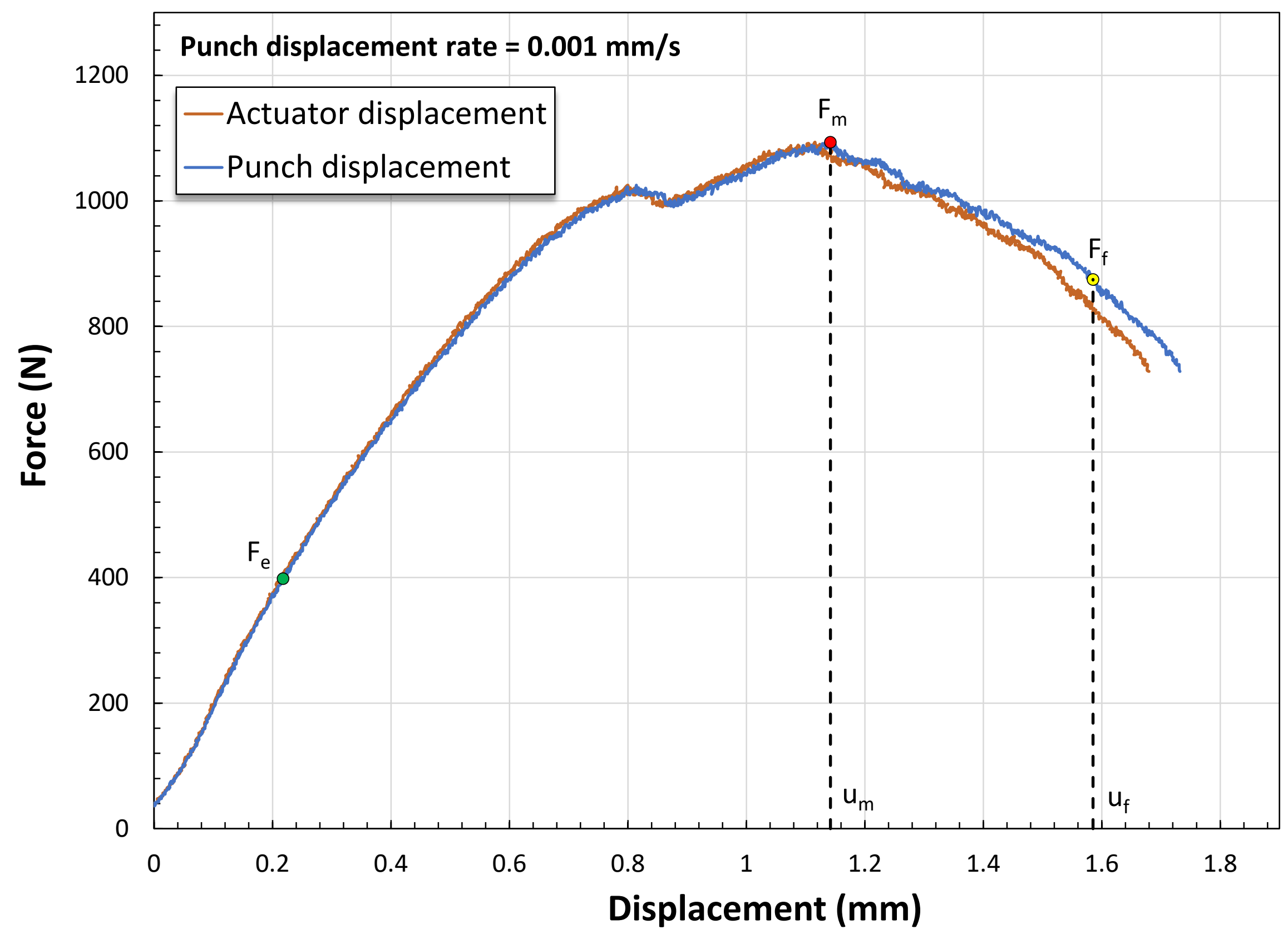




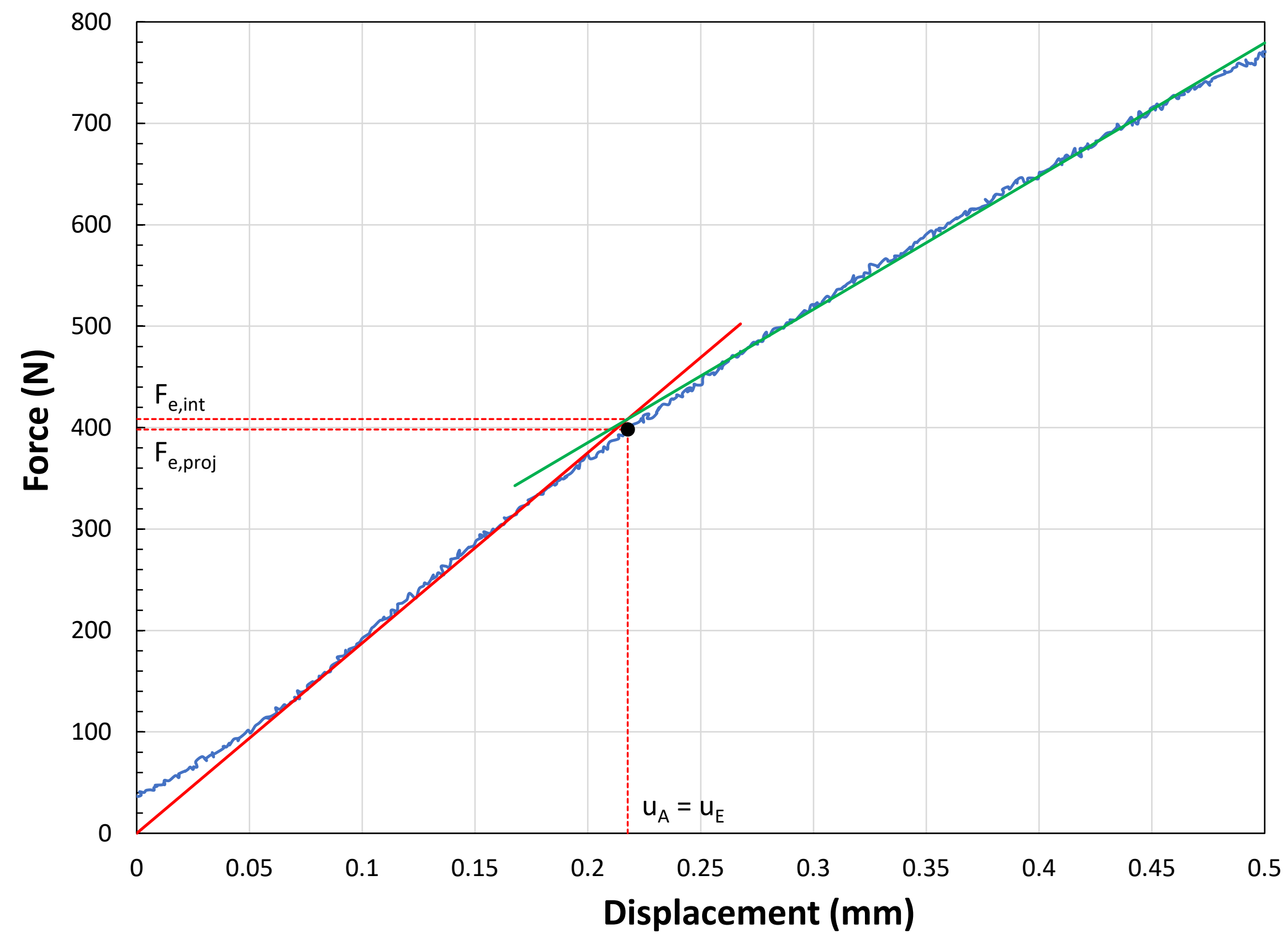


Specimen ID: AB_3_N_a_SP3

Material: AM Ti-64

Test speed: $0.00104 \mathrm{~mm} / \mathrm{s}$

Max strain rate: $1.04 \mathrm{E}-03 \mathrm{~s}^{-1}$

\begin{tabular}{|c|c|c|}
\hline \multicolumn{3}{|c|}{ TEST RESULTS } \\
\hline \multicolumn{3}{|c|}{ Force values } \\
\hline$F_{e, p r o j}=$ & 449.1 & $\mathbf{N}$ \\
\hline$F_{e, \text { int }}=$ & 457.0 & $\mathbf{N}$ \\
\hline$F_{h 0 / 10, \text { off }}=$ & 673.4 & $\mathbf{N}$ \\
\hline$F_{0.1 \mathrm{~mm}, \mathrm{off}}=$ & 831.5 & $\mathbf{N}$ \\
\hline$F_{0.1 \mathrm{~mm}}=$ & 186.6 & $\mathbf{N}$ \\
\hline$F_{0.48 \mathrm{~mm}}=$ & 758.0 & $\mathbf{N}$ \\
\hline$F_{0.5 \mathrm{~mm}}=$ & 777.1 & $\mathbf{N}$ \\
\hline$F_{0.65 \mathrm{~mm}}=$ & 939.1 & $\mathbf{N}$ \\
\hline$F_{0.9 \mathrm{~mm}}=$ & 1002.7 & $\mathbf{N}$ \\
\hline$F_{\mathrm{ept}}=$ & 609.0 & $\mathbf{N}$ \\
\hline$F_{\mathrm{e} 1.5}=$ & 156.4 & $\mathbf{N}$ \\
\hline $\mathbf{F}_{\mathrm{m}}=$ & 1158.1 & $\mathbf{N}$ \\
\hline$F_{\text {infl }}=$ & 630.8 & $\mathbf{N}$ \\
\hline$F_{f}=$ & 926.5 & $\mathbf{N}$ \\
\hline \multicolumn{3}{|c|}{ Displacement values } \\
\hline $\mathrm{u}_{\mathrm{e}}=$ & 0.246 & $\mathrm{~mm}$ \\
\hline$u_{h 0 / 10, \text { off }}=$ & 0.412 & $\mathrm{~mm}$ \\
\hline$u_{0.1 \mathrm{~mm}, \mathrm{off}}=$ & 0.547 & $\mathrm{~mm}$ \\
\hline$u_{\mathrm{e} 1.5}=$ & 0.084 & $\mathrm{~mm}$ \\
\hline$u_{m}=$ & 1.150 & $\mathrm{~mm}$ \\
\hline$u_{\text {infl }}=$ & 1.799 & $\mathrm{~mm}$ \\
\hline $\mathbf{u}_{\mathrm{f}}=$ & 1.463 & $\mathrm{~mm}$ \\
\hline \multicolumn{3}{|c|}{ Initial linear slope } \\
\hline Slope $_{\text {ini }}=$ & 1860.63 & $\mathrm{~N} / \mathrm{mm}$ \\
\hline \multicolumn{3}{|c|}{ Energy values } \\
\hline$E_{S P}=$ & 1.21 & J \\
\hline $\mathbf{E}_{m}=$ & 0.88 & $\mathbf{J}$ \\
\hline$E_{P L}=$ & 0.51 & $\mathbf{J}$ \\
\hline
\end{tabular}




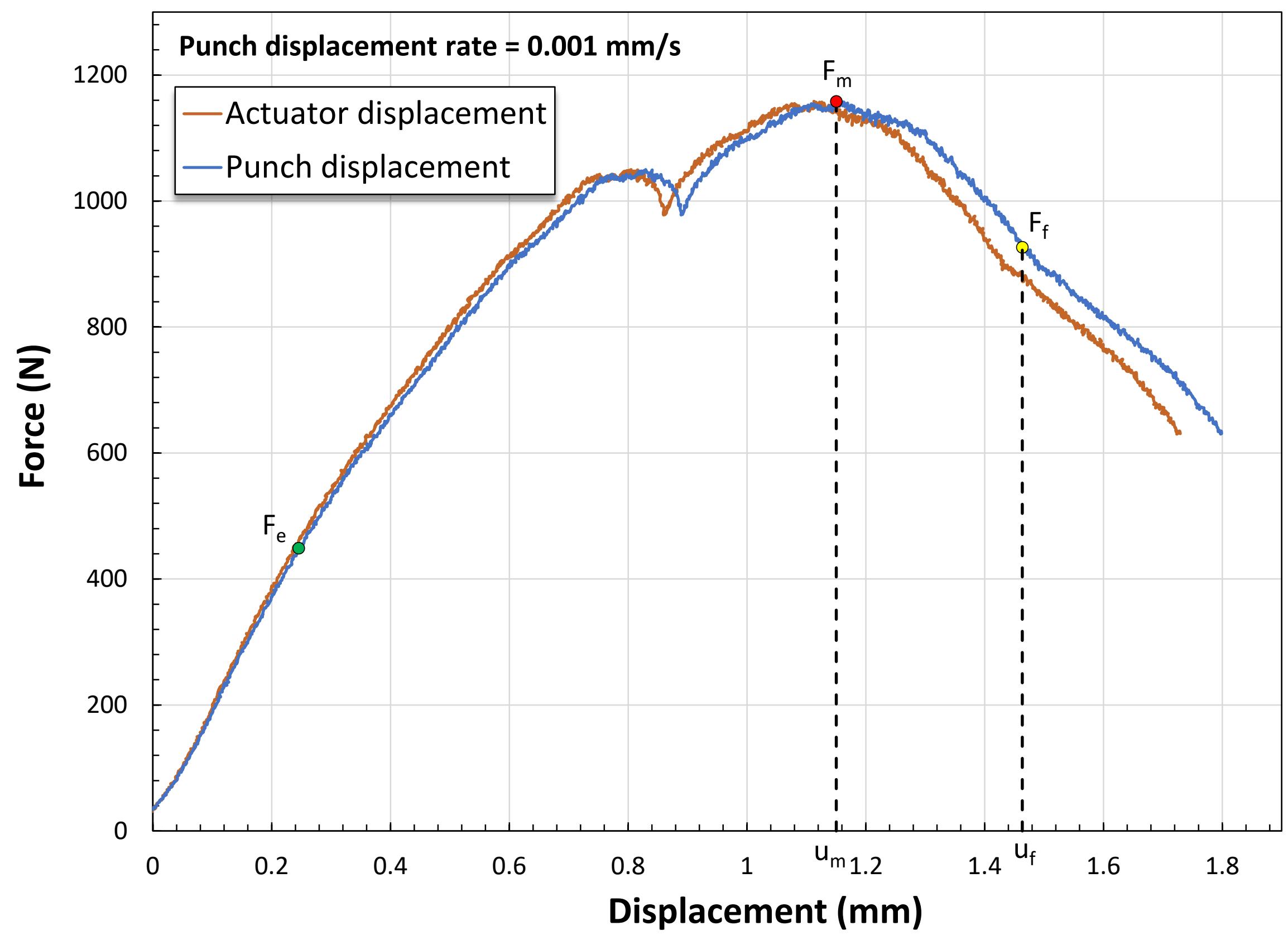




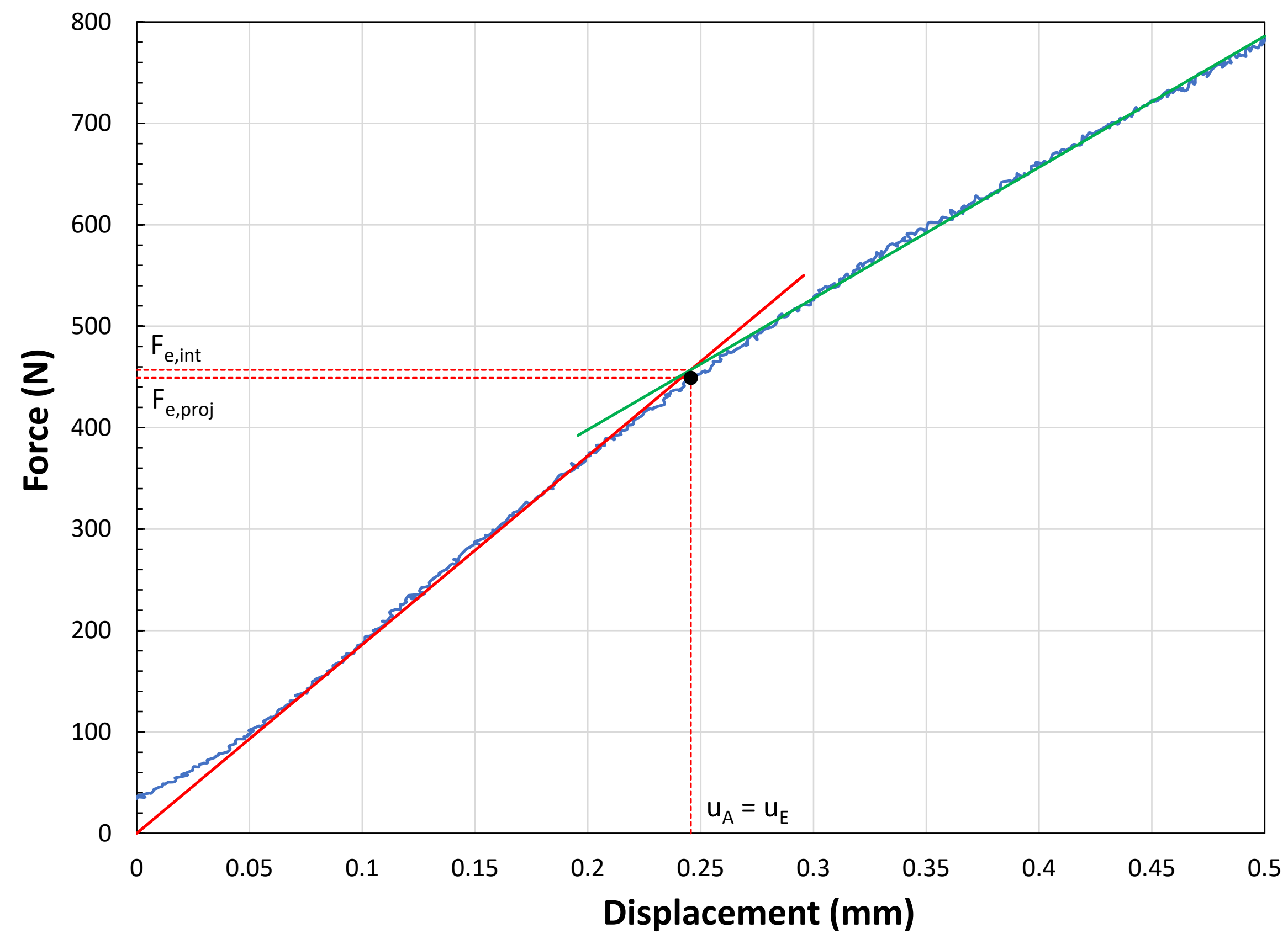


Specimen ID: AB_3_N_a_SP4

Material: AM Ti-64

Test speed: $0.001042 \mathrm{~mm} / \mathrm{s}$

Max strain rate: $1.04 \mathrm{E}-03 \mathrm{~s}^{-1}$

\begin{tabular}{|c|c|c|}
\hline \multicolumn{3}{|c|}{ TEST RESULTS } \\
\hline \multicolumn{3}{|c|}{ Force values } \\
\hline$F_{e, p r o j}=$ & 452.2 & $\mathbf{N}$ \\
\hline$F_{e, \text { int }}=$ & 456.5 & $\mathbf{N}$ \\
\hline$F_{h 0 / 10, \text { off }}=$ & 780.4 & $\mathbf{N}$ \\
\hline$F_{0.1 \mathrm{~mm}, \mathrm{off}}=$ & 974.2 & $\mathbf{N}$ \\
\hline$F_{0.1 \mathrm{~mm}}=$ & 163.0 & $\mathbf{N}$ \\
\hline$F_{0.48 \mathrm{~mm}}=$ & 736.5 & $\mathbf{N}$ \\
\hline$F_{0.5 \mathrm{~mm}}=$ & 763.6 & $\mathbf{N}$ \\
\hline$F_{0.65 \mathrm{~mm}}=$ & 947.0 & $\mathbf{N}$ \\
\hline$F_{0.9 \mathrm{~mm}}=$ & 1060.2 & $\mathbf{N}$ \\
\hline$F_{\mathrm{ept}}=$ & 942.2 & $\mathbf{N}$ \\
\hline$F_{\mathrm{e} 1.5}=$ & 174.1 & $\mathbf{N}$ \\
\hline $\mathbf{F}_{\mathrm{m}}=$ & 1071.7 & $\mathbf{N}$ \\
\hline$F_{\text {infl }}=$ & 349.1 & $\mathbf{N}$ \\
\hline$F_{f}=$ & 857.3 & $\mathbf{N}$ \\
\hline \multicolumn{3}{|c|}{ Displacement values } \\
\hline $\mathrm{u}_{\mathrm{e}}=$ & 0.274 & $\mathrm{~mm}$ \\
\hline$u_{h 0 / 10, \text { off }}=$ & 0.519 & $\mathrm{~mm}$ \\
\hline$u_{0.1 \mathrm{~mm}, \mathrm{off}}=$ & 0.685 & $\mathrm{~mm}$ \\
\hline$u_{\mathrm{e} 1.5}=$ & 0.105 & $\mathrm{~mm}$ \\
\hline$u_{m}=$ & 0.987 & $\mathrm{~mm}$ \\
\hline$u_{\text {infl }}=$ & 1.765 & $\mathrm{~mm}$ \\
\hline$u_{f}=$ & 1.395 & $\mathrm{~mm}$ \\
\hline \multicolumn{3}{|c|}{ Initial linear slope } \\
\hline Slope $_{\text {ini }}=$ & 1666.44 & $\mathrm{~N} / \mathrm{mm}$ \\
\hline \multicolumn{3}{|c|}{ Energy values } \\
\hline$E_{S P}=$ & 1.09 & J \\
\hline$E_{m}=$ & 0.68 & $\mathbf{J}$ \\
\hline$E_{P L}=$ & 0.33 & $\mathbf{J}$ \\
\hline
\end{tabular}




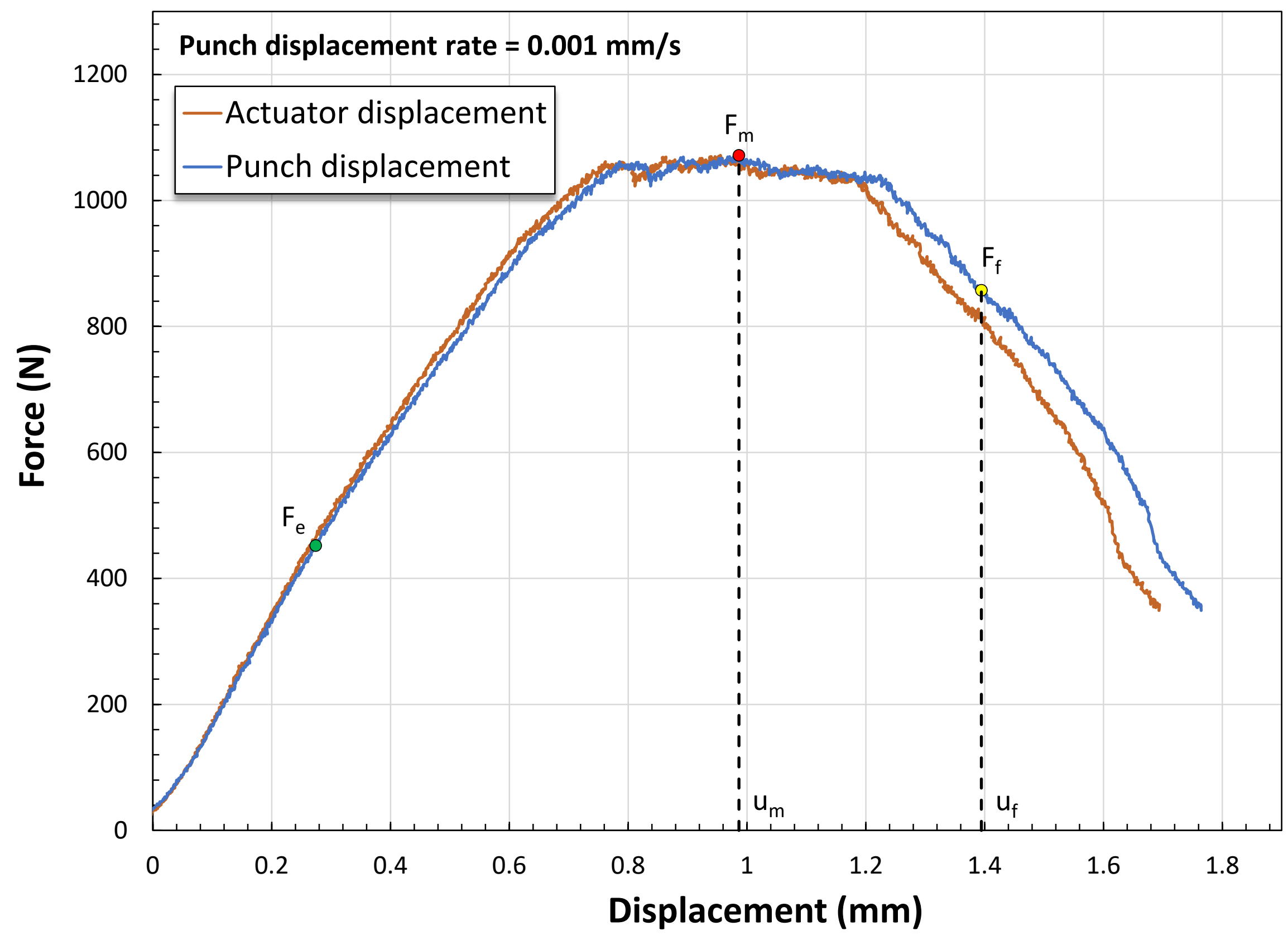




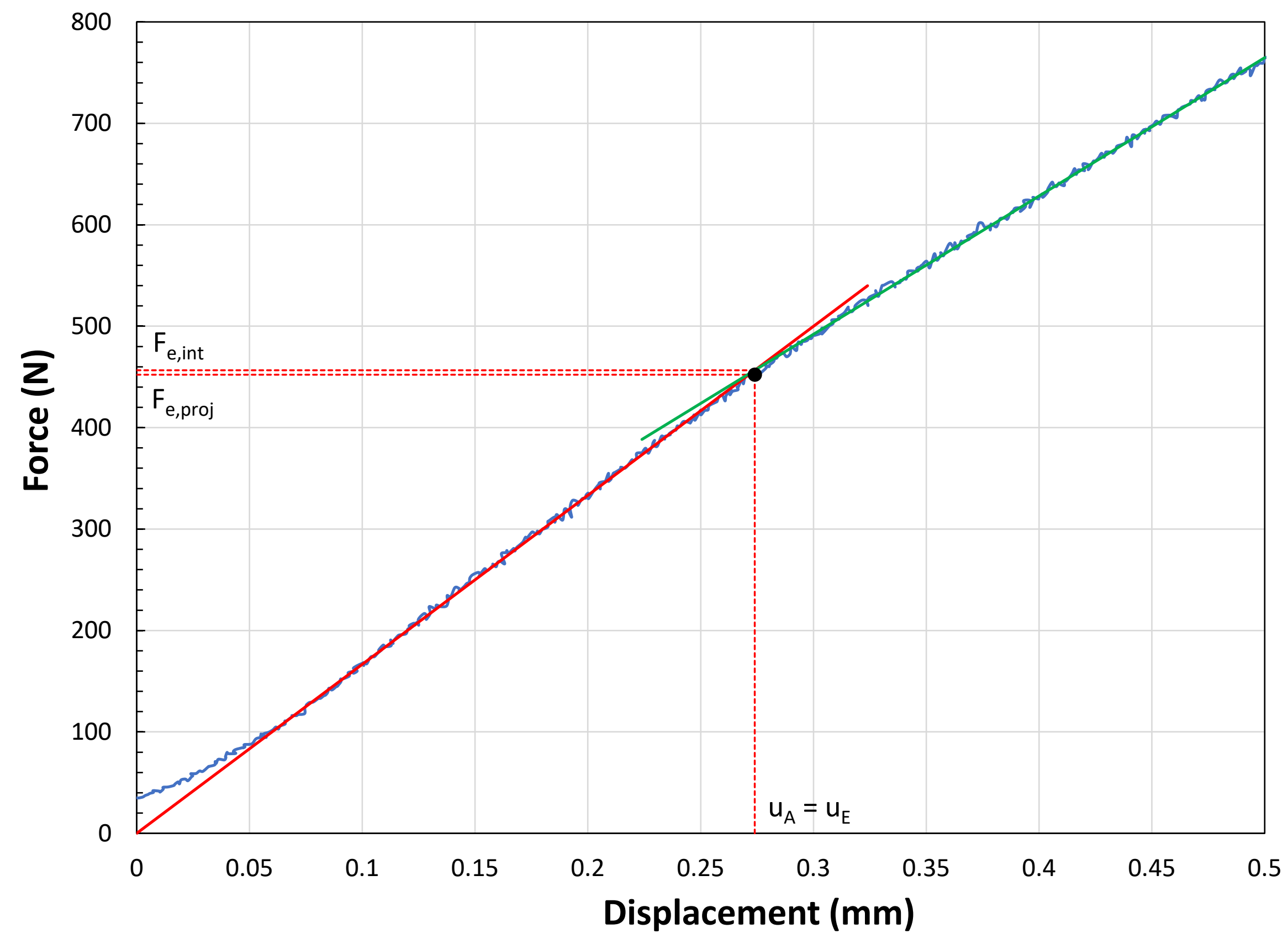


Specimen ID: AB_3_N_a_SP5

Material: AM Ti-64

Test speed: $0.00104 \mathrm{~mm} / \mathrm{s}$

Max strain rate: $1.04 \mathrm{E}-03 \mathrm{~s}^{-1}$

\begin{tabular}{|c|c|c|}
\hline \multicolumn{3}{|c|}{ TEST RESULTS } \\
\hline \multicolumn{3}{|c|}{ Force values } \\
\hline$F_{e, p r o j}=$ & 383.2 & $\mathbf{N}$ \\
\hline$F_{e, \text { int }}=$ & 393.2 & $\mathbf{N}$ \\
\hline$F_{h 0 / 10, \text { off }}=$ & 612.4 & $\mathbf{N}$ \\
\hline$F_{0.1 \mathrm{~mm}, \mathrm{off}}=$ & 785.9 & $\mathbf{N}$ \\
\hline$F_{0.1 \mathrm{~mm}}=$ & 198.4 & $\mathbf{N}$ \\
\hline$F_{0.48 \mathrm{~mm}}=$ & 774.2 & $\mathbf{N}$ \\
\hline$F_{0.5 \mathrm{~mm}}=$ & 799.9 & $\mathbf{N}$ \\
\hline$F_{0.65 \mathrm{~mm}}=$ & 921.8 & $\mathbf{N}$ \\
\hline$F_{0.9 \mathrm{~mm}}=$ & 1061.4 & $\mathbf{N}$ \\
\hline$F_{\mathrm{ept}}=$ & 613.7 & $\mathbf{N}$ \\
\hline$F_{\mathrm{e} 1.5}=$ & 163.9 & $\mathbf{N}$ \\
\hline $\mathbf{F}_{\mathrm{m}}=$ & 1107.0 & $\mathbf{N}$ \\
\hline$F_{\text {infl }}=$ & 740.2 & $\mathbf{N}$ \\
\hline$F_{f}=$ & 885.6 & $\mathbf{N}$ \\
\hline \multicolumn{3}{|c|}{ Displacement values } \\
\hline $\mathrm{u}_{\mathrm{e}}=$ & 0.195 & $\mathrm{~mm}$ \\
\hline$u_{h 0 / 10, \text { off }}=$ & 0.355 & $\mathrm{~mm}$ \\
\hline$u_{0.1 \mathrm{~mm}, \mathrm{off}}=$ & 0.490 & $\mathrm{~mm}$ \\
\hline$u_{\mathrm{e} 1.5}=$ & 0.083 & $\mathrm{~mm}$ \\
\hline$u_{m}=$ & 1.152 & $\mathrm{~mm}$ \\
\hline$u_{\text {infl }}=$ & 0.452 & $\mathrm{~mm}$ \\
\hline $\mathbf{u}_{\mathrm{f}}=$ & 1.481 & $\mathrm{~mm}$ \\
\hline \multicolumn{3}{|c|}{ Initial linear slope } \\
\hline Slope $_{\text {ini }}=$ & 2016.45 & $\mathrm{~N} / \mathrm{mm}$ \\
\hline \multicolumn{3}{|c|}{ Energy values } \\
\hline$E_{S P}=$ & 1.20 & J \\
\hline$E_{m}=$ & 0.88 & $\mathbf{J}$ \\
\hline$E_{P L}=$ & 0.57 & $J$ \\
\hline
\end{tabular}




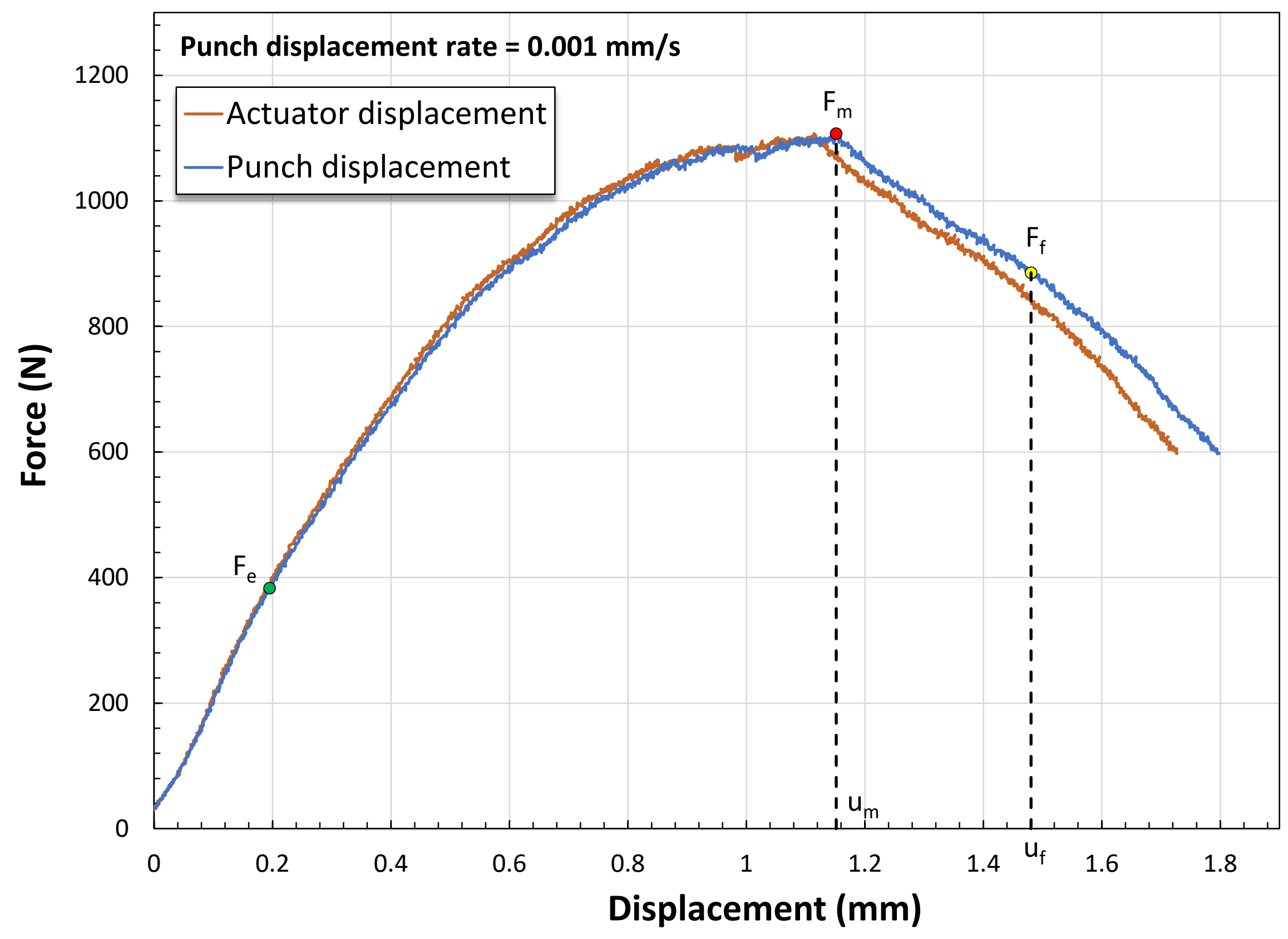




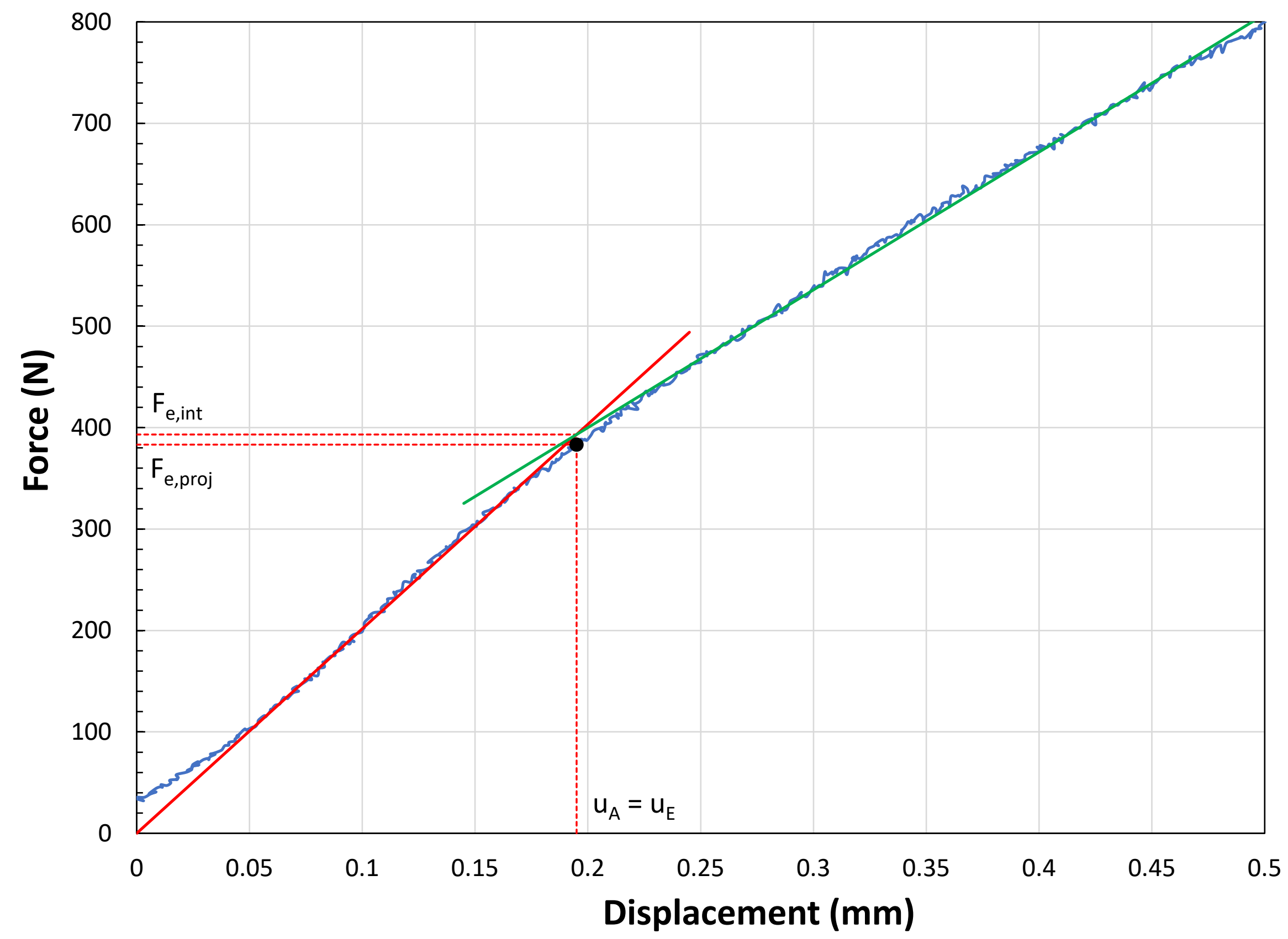


Specimen ID: AB_3_N_a_SP6

Material: AM Ti-64

Test speed: $0.001034 \mathrm{~mm} / \mathrm{s}$

Max strain rate: $1.03 \mathrm{E}-03 \mathrm{~s}^{-1}$

\begin{tabular}{|c|c|c|}
\hline \multicolumn{3}{|c|}{ TEST RESULTS } \\
\hline \multicolumn{3}{|c|}{ Force values } \\
\hline$F_{e, p r o j}=$ & 401.5 & $\mathbf{N}$ \\
\hline$F_{e, \text { int }}=$ & 408.2 & $\mathbf{N}$ \\
\hline$F_{h 0 / 10, \text { off }}=$ & 675.0 & $\mathbf{N}$ \\
\hline$F_{0.1 \mathrm{~mm}, \mathrm{off}}=$ & 872.4 & $\mathbf{N}$ \\
\hline$F_{0.1 \mathrm{~mm}}=$ & 174.9 & $\mathbf{N}$ \\
\hline$F_{0.48 \mathrm{~mm}}=$ & 738.2 & $\mathbf{N}$ \\
\hline$F_{0.5 \mathrm{~mm}}=$ & 762.2 & $\mathbf{N}$ \\
\hline$F_{0.65 \mathrm{~mm}}=$ & 922.1 & $\mathbf{N}$ \\
\hline$F_{0.9 \mathrm{~mm}}=$ & 1003.1 & $\mathbf{N}$ \\
\hline$F_{\mathrm{ept}}=$ & 756.4 & $\mathbf{N}$ \\
\hline$F_{\mathrm{e} 1.5}=$ & 140.7 & $\mathbf{N}$ \\
\hline $\mathbf{F}_{\mathrm{m}}=$ & 1090.5 & $\mathbf{N}$ \\
\hline$F_{\text {infl }}=$ & 715.8 & $\mathbf{N}$ \\
\hline$F_{f}=$ & 872.4 & $\mathbf{N}$ \\
\hline \multicolumn{3}{|c|}{ Displacement values } \\
\hline $\mathrm{u}_{\mathrm{e}}=$ & 0.234 & $\mathrm{~mm}$ \\
\hline$u_{h 0 / 10, \text { off }}=$ & 0.437 & $\mathrm{~mm}$ \\
\hline$u_{0.1 \mathrm{~mm}, \mathrm{off}}=$ & 0.601 & $\mathrm{~mm}$ \\
\hline$u_{\mathrm{e} 1.5}=$ & 0.079 & $\mathrm{~mm}$ \\
\hline$u_{m}=$ & 1.058 & $\mathrm{~mm}$ \\
\hline$u_{\text {infl }}=$ & 1.752 & $\mathrm{~mm}$ \\
\hline$u_{f}=$ & 1.558 & $\mathrm{~mm}$ \\
\hline \multicolumn{3}{|c|}{ Initial linear slope } \\
\hline Slope $_{\text {ini }}=$ & 1745.38 & $\mathrm{~N} / \mathrm{mm}$ \\
\hline \multicolumn{3}{|c|}{ Energy values } \\
\hline$E_{S P}=$ & 1.25 & J \\
\hline$E_{m}=$ & 0.75 & J \\
\hline$E_{P L}=$ & 0.41 & $\mathbf{J}$ \\
\hline
\end{tabular}




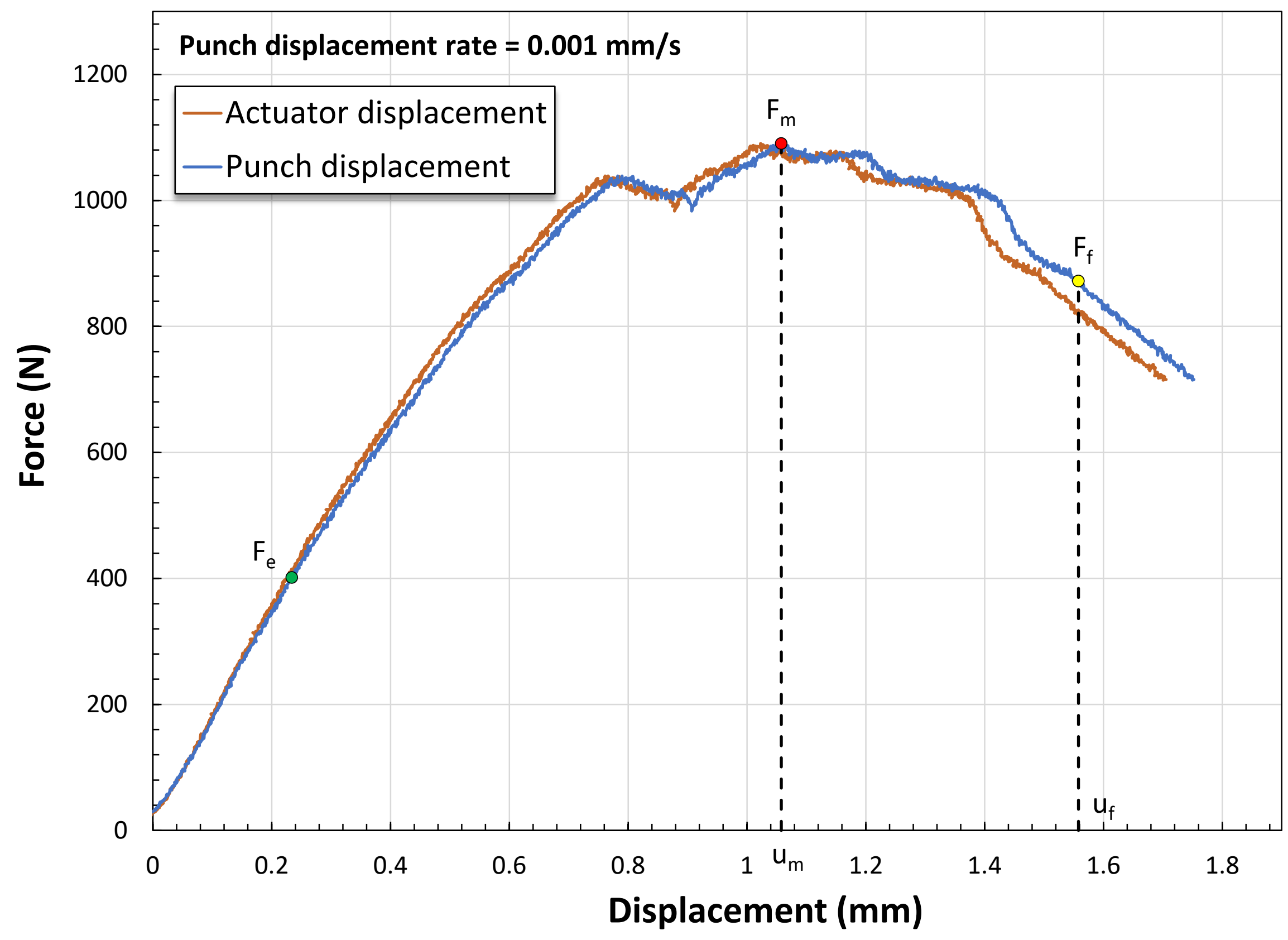




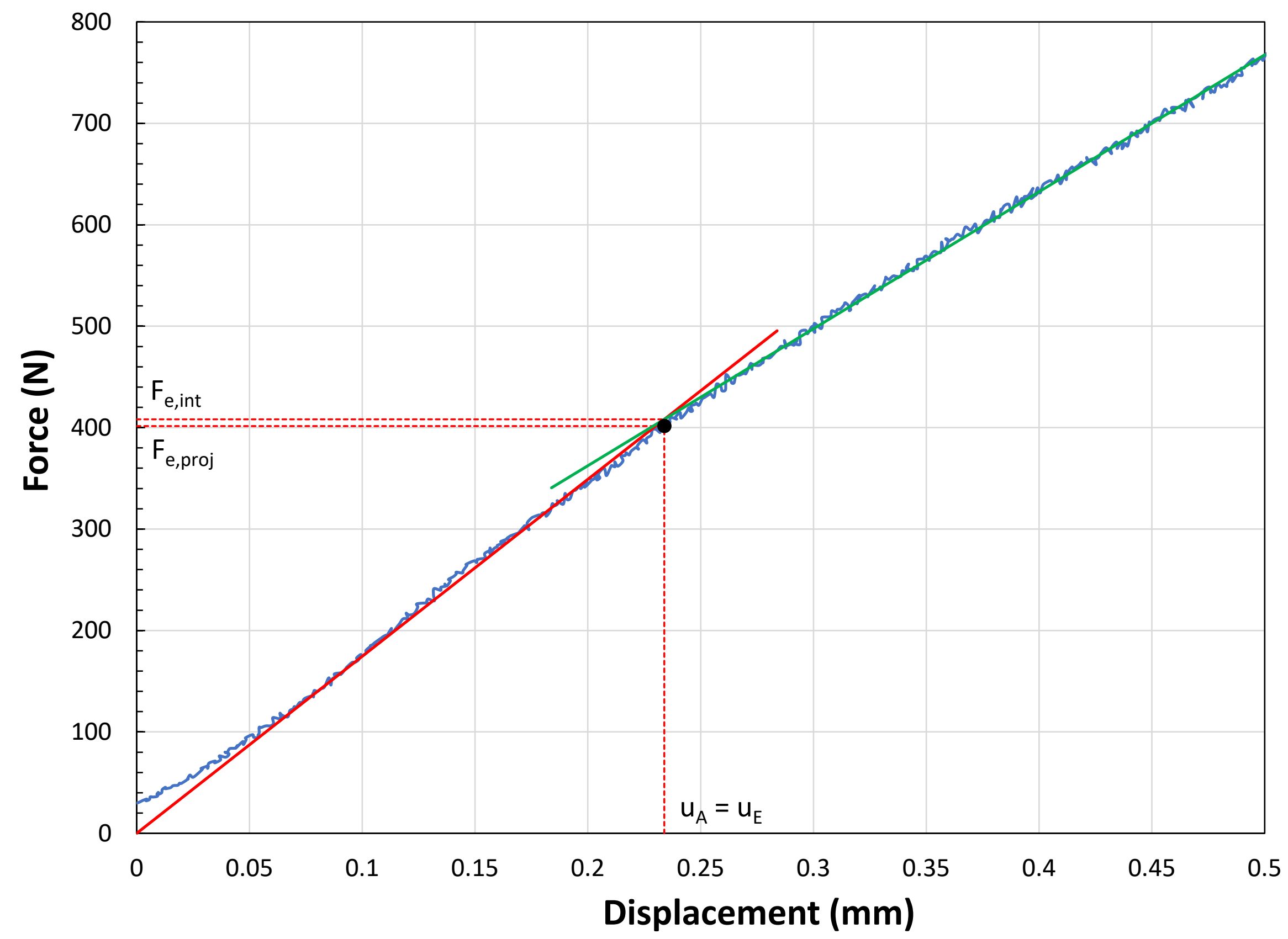


Specimen ID: AB_3_N_a_SP7

Material: AM Ti-64

Test speed: $0.001043 \mathrm{~mm} / \mathrm{s}$

Max strain rate: $1.04 \mathrm{E}-03 \mathrm{~s}^{-1}$

\begin{tabular}{|c|c|c|}
\hline \multicolumn{3}{|c|}{ TEST RESULTS } \\
\hline \multicolumn{3}{|c|}{ Force values } \\
\hline$F_{e, p r o j}=$ & 364.6 & $\mathbf{N}$ \\
\hline$F_{e, \text { int }}=$ & 370.9 & $\mathbf{N}$ \\
\hline$F_{h 0 / 10, \text { off }}=$ & 637.4 & $\mathbf{N}$ \\
\hline$F_{0.1 \mathrm{~mm}, \mathrm{off}}=$ & 832.6 & $\mathbf{N}$ \\
\hline$F_{0.1 \mathrm{~mm}}=$ & 172.4 & $\mathbf{N}$ \\
\hline$F_{0.48 \mathrm{~mm}}=$ & 725.1 & $\mathbf{N}$ \\
\hline$F_{0.5 \mathrm{~mm}}=$ & 742.0 & $\mathbf{N}$ \\
\hline$F_{0.65 \mathrm{~mm}}=$ & 915.6 & $\mathbf{N}$ \\
\hline$F_{0.9 \mathrm{~mm}}=$ & 1055.6 & $\mathbf{N}$ \\
\hline$F_{\mathrm{ept}}=$ & 241.5 & $\mathbf{N}$ \\
\hline$F_{\mathrm{e} 1.5}=$ & 164.6 & $\mathbf{N}$ \\
\hline $\mathbf{F}_{\mathrm{m}}=$ & 1088.0 & $\mathbf{N}$ \\
\hline$F_{\text {infl }}=$ & 634.4 & $\mathbf{N}$ \\
\hline$F_{f}=$ & 870.4 & $\mathbf{N}$ \\
\hline \multicolumn{3}{|c|}{ Displacement values } \\
\hline $\mathrm{u}_{\mathrm{e}}=$ & 0.212 & $\mathrm{~mm}$ \\
\hline$u_{h 0 / 10, \text { off }}=$ & 0.414 & $\mathrm{~mm}$ \\
\hline$u_{0.1 \mathrm{~mm}, \mathrm{off}}=$ & 0.576 & $\mathrm{~mm}$ \\
\hline$u_{\mathrm{e} 1.5}=$ & 0.093 & $\mathrm{~mm}$ \\
\hline$u_{m}=$ & 1.101 & $\mathrm{~mm}$ \\
\hline$u_{\text {infl }}=$ & 1.794 & $\mathrm{~mm}$ \\
\hline$u_{f}=$ & 1.524 & $\mathrm{~mm}$ \\
\hline \multicolumn{3}{|c|}{ Initial linear slope } \\
\hline Slope $_{\text {ini }}=$ & 1752.85 & $\mathrm{~N} / \mathrm{mm}$ \\
\hline \multicolumn{3}{|c|}{ Energy values } \\
\hline$E_{S P}=$ & 1.21 & J \\
\hline$E_{m}=$ & 0.79 & $\mathbf{J}$ \\
\hline$E_{P L}=$ & 0.45 & $\mathbf{J}$ \\
\hline
\end{tabular}




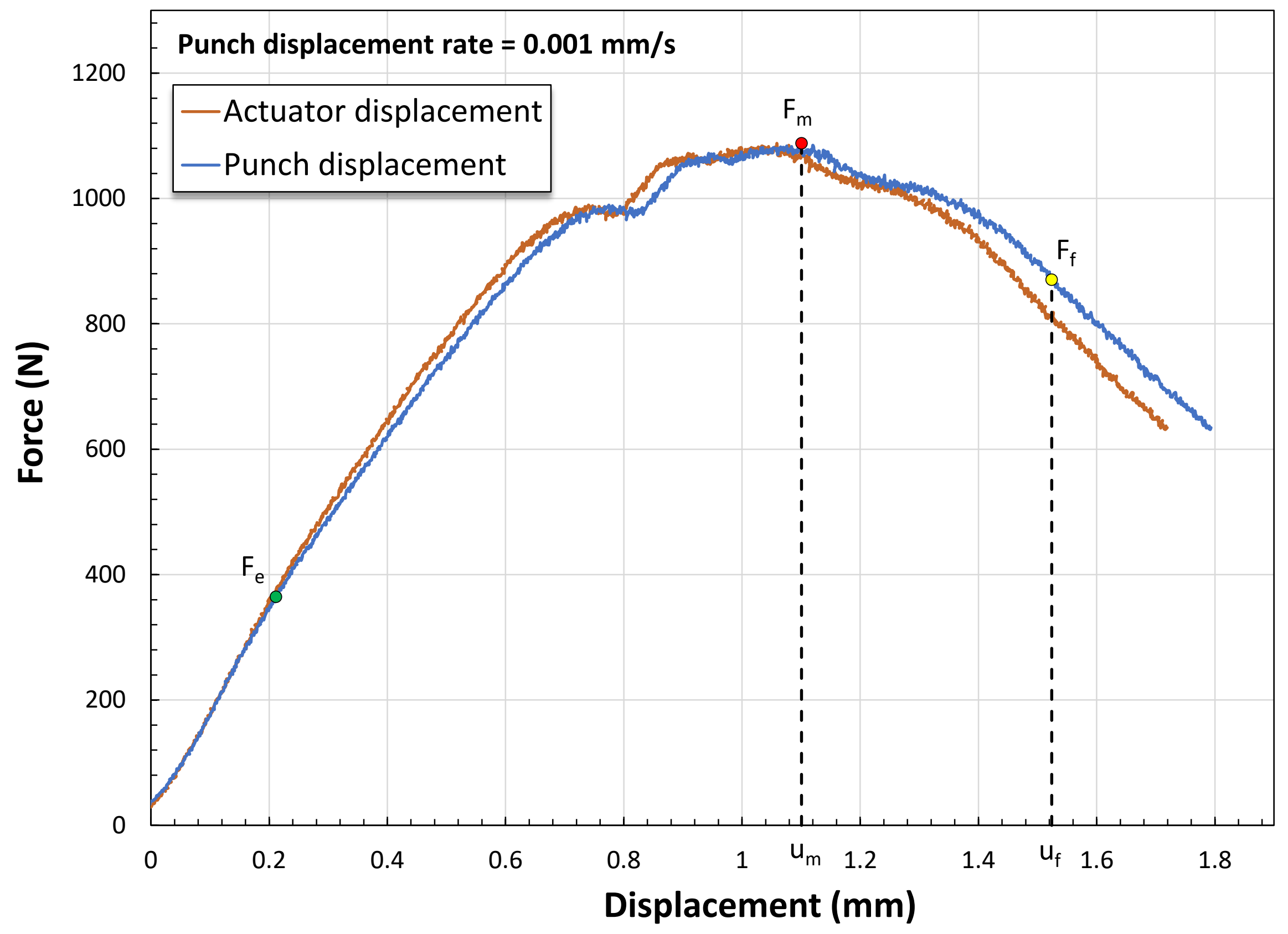




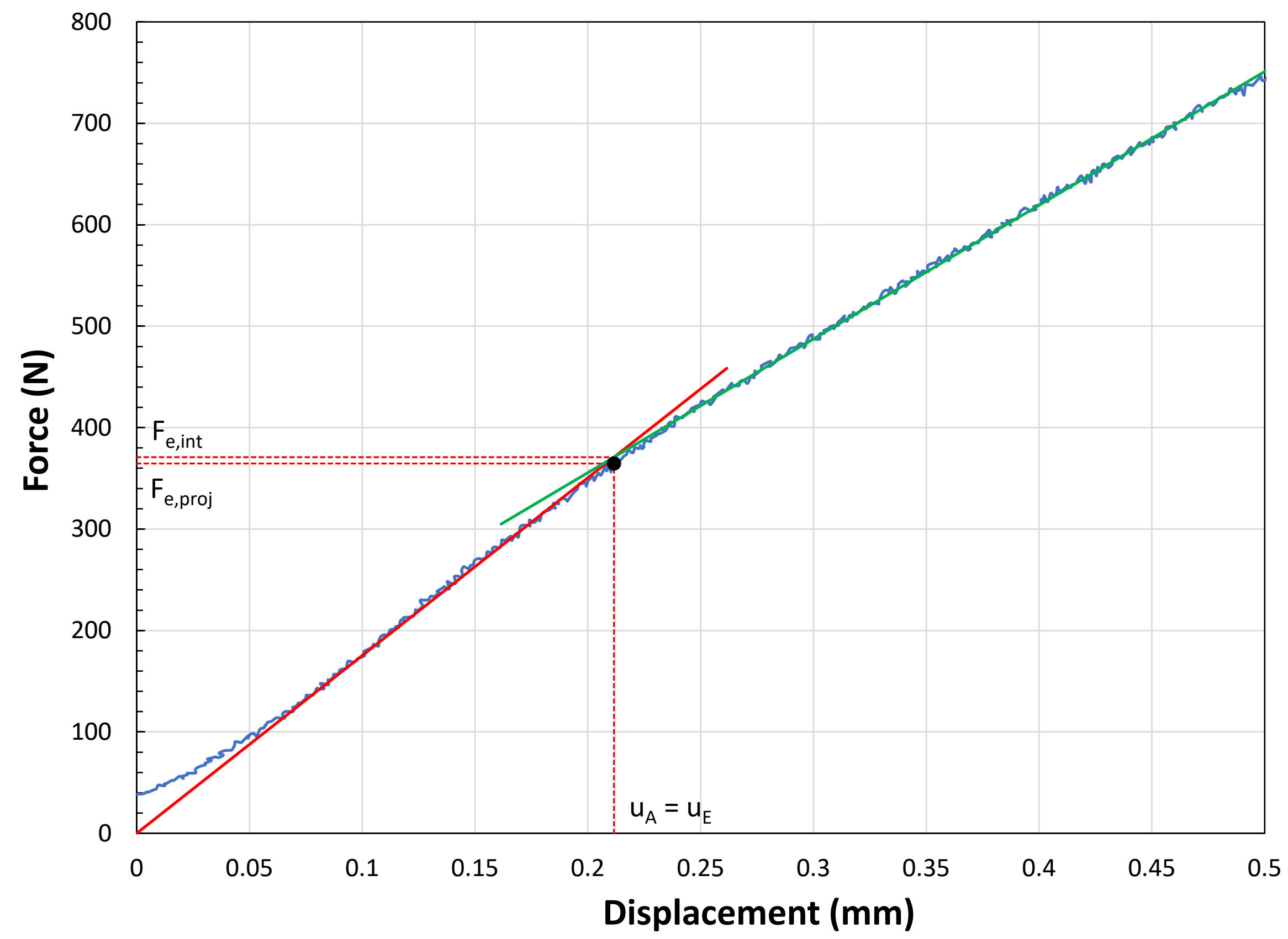




\section{ANNEX 5 \\ SP Test Results for \\ As built, scan length $=26 \mathrm{~mm}$, non-supported \\ (polished specimens)}


Specimen ID: AB_3_N_a_SP8 (polish

Material: AM Ti-64

Test speed: $0.001444 \mathrm{~mm} / \mathrm{s}$

Max strain rate: $1.44 \mathrm{E}-03 \mathrm{~s}^{-1}$

\begin{tabular}{|c|c|c|}
\hline \multicolumn{3}{|c|}{ TEST RESULTS } \\
\hline \multicolumn{3}{|c|}{ Force values } \\
\hline$F_{e, p r o j}=$ & 418.2 & $\mathbf{N}$ \\
\hline $\mathbf{F}_{\mathrm{e}, \text { int }}=$ & 454.3 & $\mathbf{N}$ \\
\hline$F_{h 0 / 10, \text { off }}=$ & 558.7 & $\mathbf{N}$ \\
\hline$F_{0.1 \mathrm{~mm}, \text { off }}=$ & 705.8 & $\mathbf{N}$ \\
\hline$F_{0.1 \mathrm{~mm}}=$ & 310.4 & $\mathbf{N}$ \\
\hline$F_{0.48 \mathrm{~mm}}=$ & 853.6 & $\mathbf{N}$ \\
\hline $\mathrm{F}_{0.5 \mathrm{~mm}}=$ & 875.2 & $\mathbf{N}$ \\
\hline$F_{0.65 \mathrm{~mm}}=$ & 956.3 & $\mathbf{N}$ \\
\hline$F_{0.9 \mathrm{~mm}}=$ & 934.8 & $\mathbf{N}$ \\
\hline$F_{\text {ept }}=$ & 81.4 & $\mathbf{N}$ \\
\hline$F_{\mathrm{e} 1.5}=$ & 760.3 & $\mathbf{N}$ \\
\hline$F_{m}=$ & 968.0 & $\mathbf{N}$ \\
\hline$F_{\text {infl }}=$ & 515.4 & $\mathbf{N}$ \\
\hline$F_{f}=$ & 774.4 & $\mathbf{N}$ \\
\hline \multicolumn{3}{|c|}{ Displacement values } \\
\hline $\mathrm{u}_{\mathrm{e}}=$ & 0.151 & $\mathrm{~mm}$ \\
\hline$u_{h 0 / 10, \text { off }}=$ & 0.231 & $\mathrm{~mm}$ \\
\hline$u_{0.1 \mathrm{~mm}, \text { off }}=$ & 0.335 & $\mathrm{~mm}$ \\
\hline$u_{\mathrm{e} 1.5}=$ & 0.379 & $\mathrm{~mm}$ \\
\hline$u_{m}=$ & 0.680 & $\mathrm{~mm}$ \\
\hline$u_{i n f l}=$ & 1.661 & $\mathrm{~mm}$ \\
\hline$u_{f}=$ & 1.245 & $\mathrm{~mm}$ \\
\hline \multicolumn{3}{|c|}{ Initial linear slope } \\
\hline Slope $_{\text {ini }}=$ & 3010.25 & $\mathrm{~N} / \mathrm{mm}$ \\
\hline \multicolumn{3}{|c|}{ Energy values } \\
\hline$E_{S P}=$ & 0.95 & J \\
\hline$E_{m}=$ & 0.44 & J \\
\hline $\mathrm{E}_{\mathrm{PL}}=$ & 0.28 & J \\
\hline
\end{tabular}




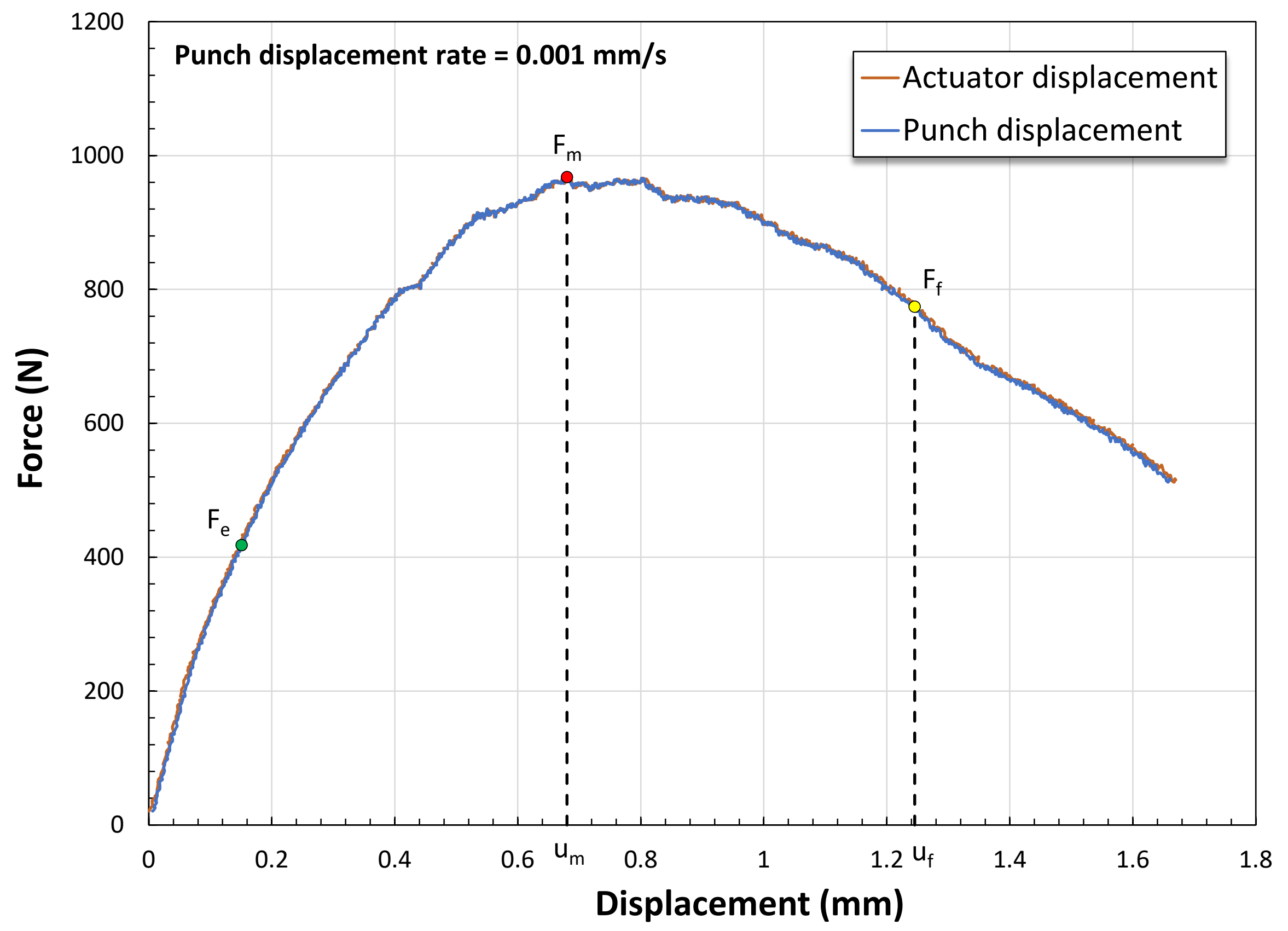




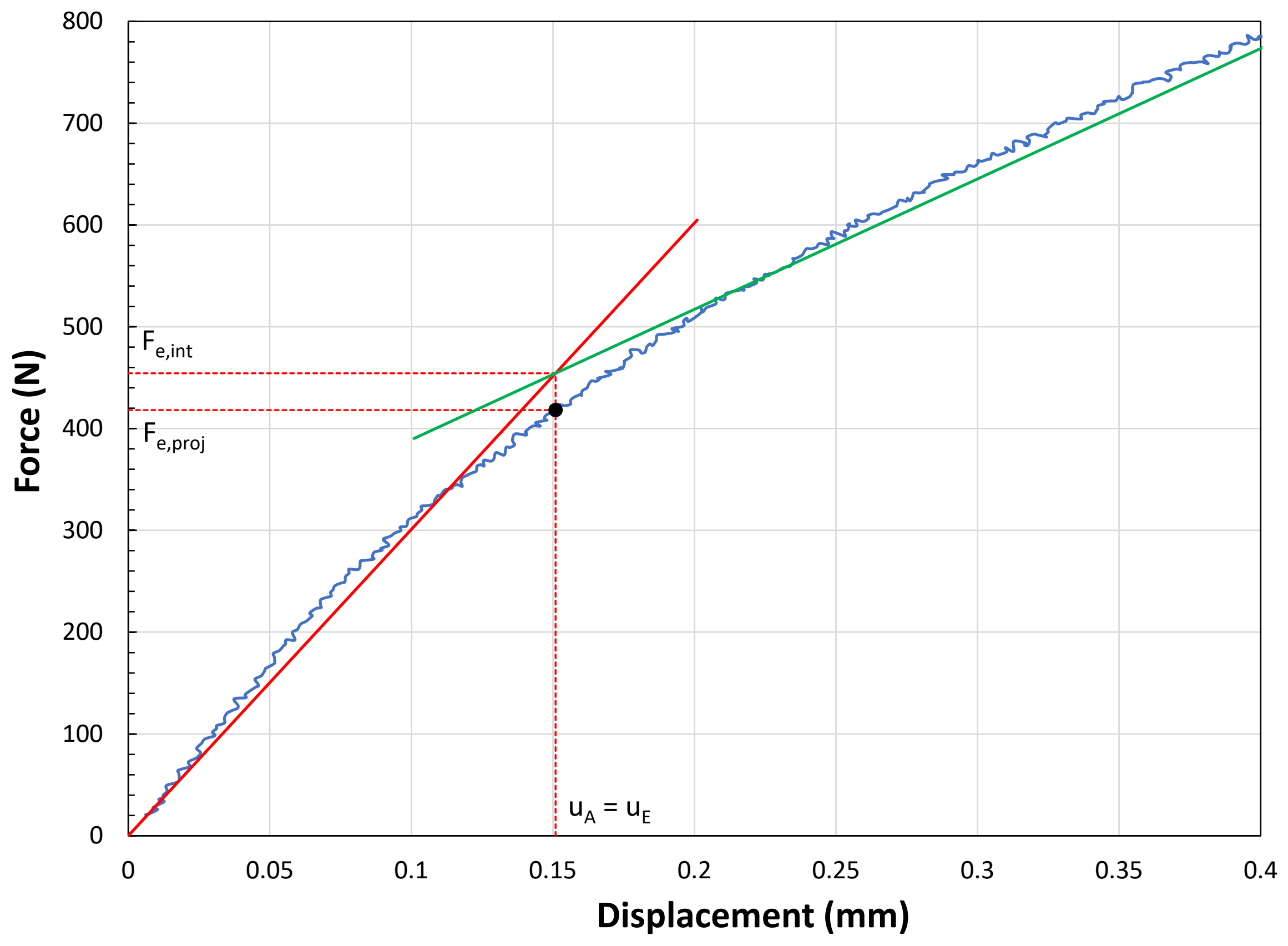


Specimen ID: AB_3_N_a_SP9 (polish

Material: AM Ti-64

Test speed: $0.001445 \mathrm{~mm} / \mathrm{s}$

Max strain rate: $1.44 \mathrm{E}-03 \mathrm{~s}^{-1}$

\begin{tabular}{|c|c|c|}
\hline \multicolumn{3}{|c|}{ TEST RESULTS } \\
\hline \multicolumn{3}{|c|}{ Force values } \\
\hline$F_{e, \text { proj }}=$ & 428.5 & $\mathbf{N}$ \\
\hline$F_{e, \text { int }}=$ & 466.5 & $\mathbf{N}$ \\
\hline$F_{h 0 / 10, \text { off }}=$ & 552.1 & $\mathbf{N}$ \\
\hline$F_{0.1 \mathrm{~mm}, \text { off }}=$ & 694.6 & $\mathbf{N}$ \\
\hline$F_{0.1 \mathrm{~mm}}=$ & 295.4 & $\mathbf{N}$ \\
\hline$F_{0.48 \mathrm{~mm}}=$ & 818.5 & $\mathbf{N}$ \\
\hline $\mathrm{F}_{0.5 \mathrm{~mm}}=$ & 818.5 & $\mathbf{N}$ \\
\hline$F_{0.65 \mathrm{~mm}}=$ & 897.2 & $\mathbf{N}$ \\
\hline$F_{0.9 \mathrm{~mm}}=$ & 926.0 & $\mathbf{N}$ \\
\hline$F_{\text {ept }}=$ & 702.3 & $\mathbf{N}$ \\
\hline $\mathrm{F}_{\mathrm{e} 1.5}=$ & 732.7 & $\mathbf{N}$ \\
\hline$F_{m}=$ & 956.8 & $\mathbf{N}$ \\
\hline$F_{\text {infl }}=$ & 487.4 & $\mathbf{N}$ \\
\hline$F_{f}=$ & 765.4 & $\mathbf{N}$ \\
\hline \multicolumn{3}{|c|}{ Displacement values } \\
\hline $\mathrm{u}_{\mathrm{e}}=$ & 0.161 & $\mathrm{~mm}$ \\
\hline$u_{h 0 / 10, \text { off }}=$ & 0.236 & $\mathrm{~mm}$ \\
\hline$u_{0.1 \mathrm{~mm}, \text { off }}=$ & 0.341 & $\mathrm{~mm}$ \\
\hline$u_{e 1.5}=$ & 0.372 & $\mathrm{~mm}$ \\
\hline$u_{m}=$ & 0.844 & $\mathrm{~mm}$ \\
\hline$u_{\text {infl }}=$ & 1.631 & $\mathrm{~mm}$ \\
\hline$u_{f}=$ & 1.232 & $\mathrm{~mm}$ \\
\hline \multicolumn{3}{|c|}{ Initial linear slope } \\
\hline Slope $_{\text {ini }}=$ & 2889.12 & $\mathrm{~N} / \mathrm{mm}$ \\
\hline \multicolumn{3}{|c|}{ Energy values } \\
\hline$E_{S P}=$ & 0.91 & J \\
\hline$E_{m}=$ & 0.57 & J \\
\hline $\mathrm{E}_{\mathrm{PL}}=$ & 0.42 & J \\
\hline
\end{tabular}




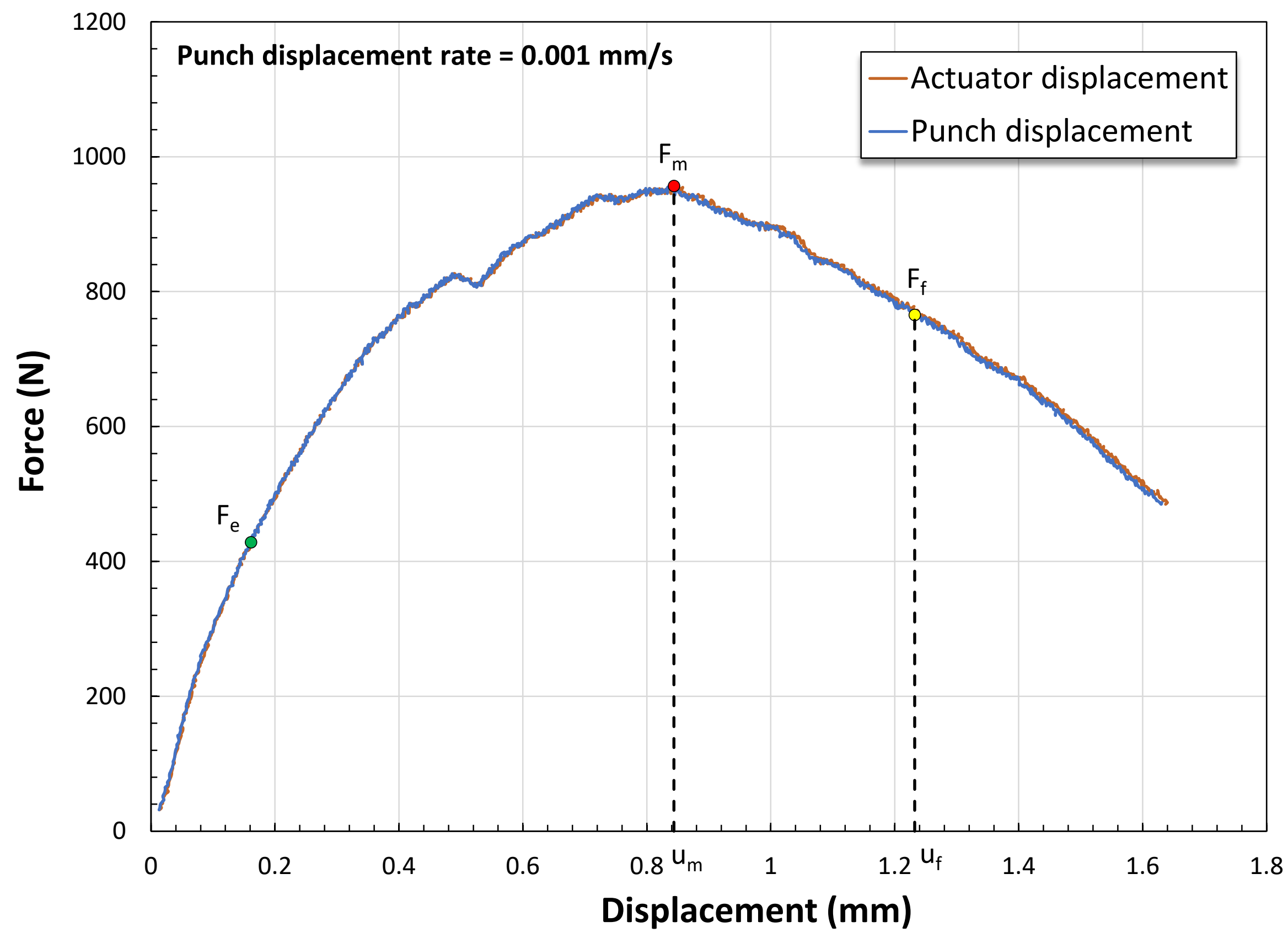




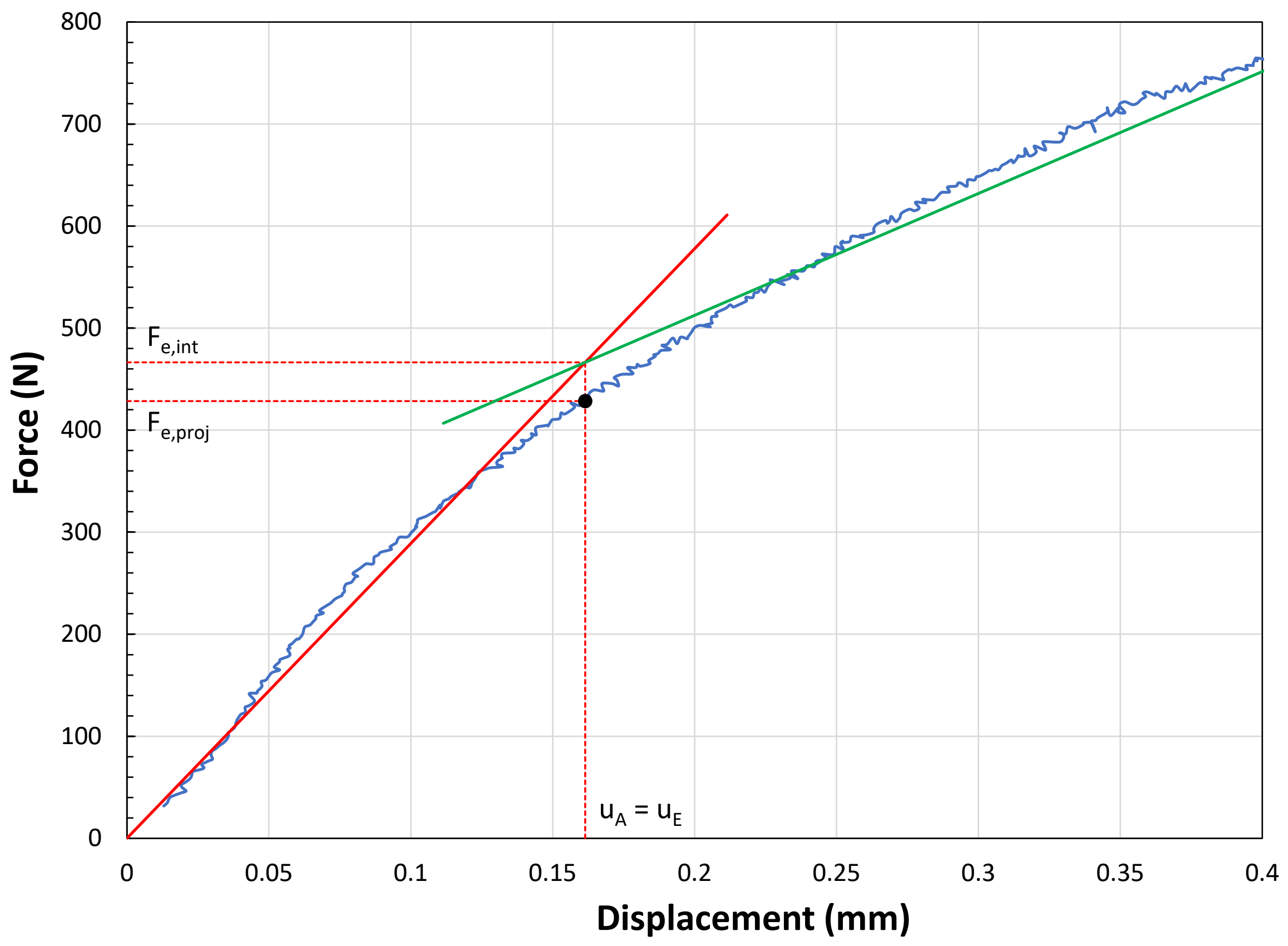


Specimen ID: AB_3_N_a_SP10 (polis

Material: AM Ti-64

Test speed: $0.001449 \mathrm{~mm} / \mathrm{s}$

Max strain rate: $1.45 \mathrm{E}-03 \mathrm{~s}^{-1}$

\begin{tabular}{|c|c|c|}
\hline \multicolumn{3}{|c|}{ TEST RESULTS } \\
\hline \multicolumn{3}{|c|}{ Force values } \\
\hline$F_{e, \text { proj }}=$ & 363.6 & $\mathbf{N}$ \\
\hline$F_{e, \text { int }}=$ & 404.3 & $\mathbf{N}$ \\
\hline$F_{h 0 / 10, \text { off }}=$ & 522.6 & $\mathbf{N}$ \\
\hline$F_{0.1 \mathrm{~mm}, \mathrm{off}}=$ & 688.9 & $\mathbf{N}$ \\
\hline$F_{0.1 \mathrm{~mm}}=$ & 317.7 & $\mathbf{N}$ \\
\hline$F_{0.48 \mathrm{~mm}}=$ & 880.6 & $\mathbf{N}$ \\
\hline $\mathrm{F}_{0.5 \mathrm{~mm}}=$ & 898.1 & $\mathbf{N}$ \\
\hline$F_{0.65 \mathrm{~mm}}=$ & 949.1 & $\mathbf{N}$ \\
\hline$F_{0.9 \mathrm{~mm}}=$ & 962.4 & $\mathbf{N}$ \\
\hline$F_{\text {ept }}=$ & 430.5 & $\mathbf{N}$ \\
\hline $\mathrm{F}_{\mathrm{e} 1.5}=$ & 658.3 & $\mathbf{N}$ \\
\hline$F_{m}=$ & 981.3 & $\mathbf{N}$ \\
\hline$F_{\text {infl }}=$ & 76.1 & $\mathbf{N}$ \\
\hline$F_{f}=$ & 785.1 & $\mathbf{N}$ \\
\hline \multicolumn{3}{|c|}{ Displacement values } \\
\hline $\mathrm{u}_{\mathrm{e}}=$ & 0.123 & $\mathrm{~mm}$ \\
\hline$u_{h 0 / 10, \text { off }}=$ & 0.205 & $\mathrm{~mm}$ \\
\hline $\mathrm{u}_{0.1 \mathrm{~mm}, \mathrm{off}}=$ & 0.310 & $\mathrm{~mm}$ \\
\hline$u_{e 1.5}=$ & 0.294 & $\mathrm{~mm}$ \\
\hline$u_{m}=$ & 0.818 & $\mathrm{~mm}$ \\
\hline$u_{\text {infl }}=$ & 0.018 & $\mathrm{~mm}$ \\
\hline$u_{f}=$ & 1.219 & $\mathrm{~mm}$ \\
\hline \multicolumn{3}{|c|}{ Initial linear slope } \\
\hline Slope $_{\text {ini }}=$ & 3283.40 & $\mathrm{~N} / \mathrm{mm}$ \\
\hline \multicolumn{3}{|c|}{ Energy values } \\
\hline$E_{S P}=$ & 0.94 & J \\
\hline$E_{m}=$ & 0.58 & J \\
\hline $\mathrm{E}_{\mathrm{PL}}=$ & 0.43 & J \\
\hline
\end{tabular}




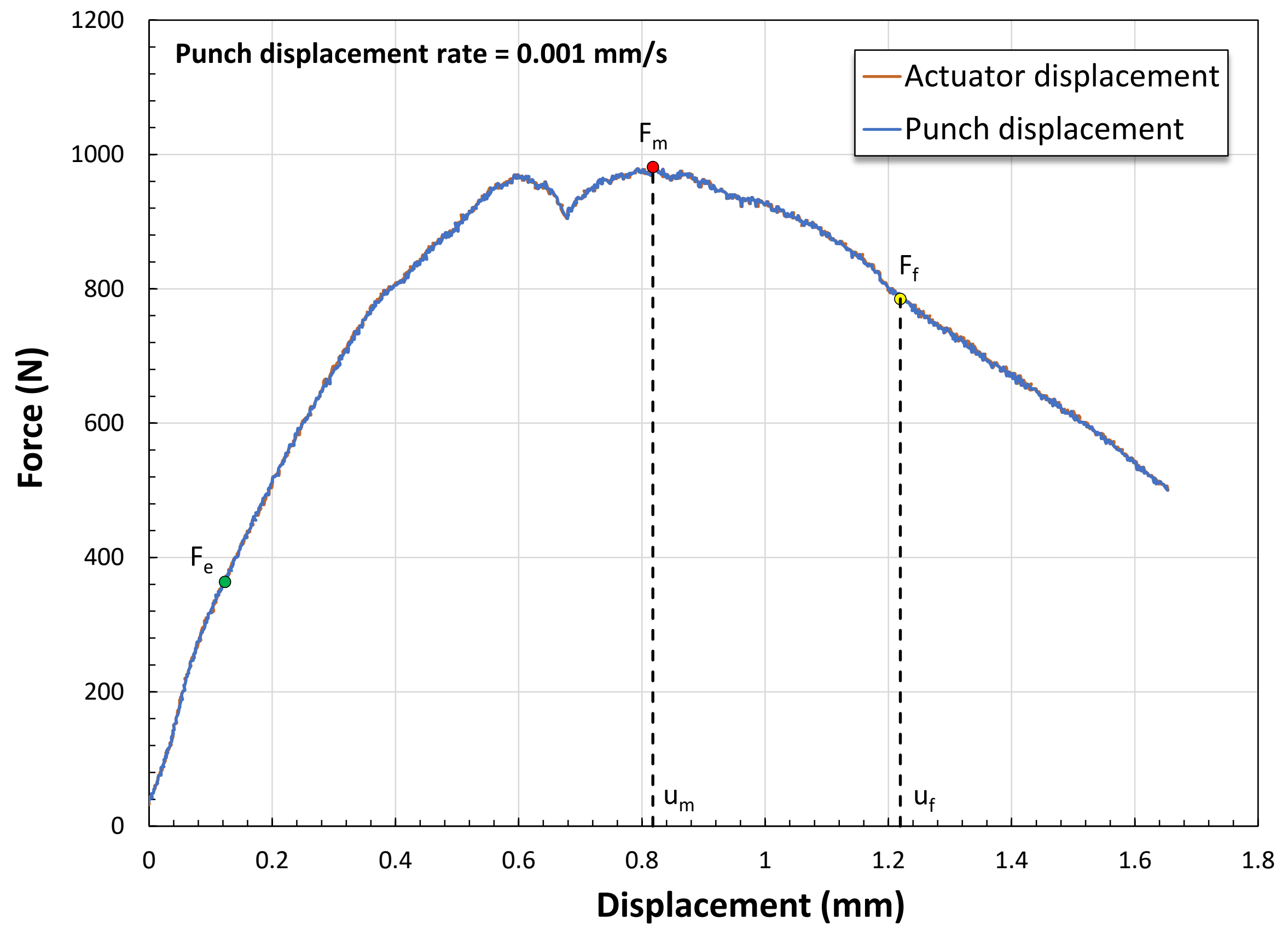




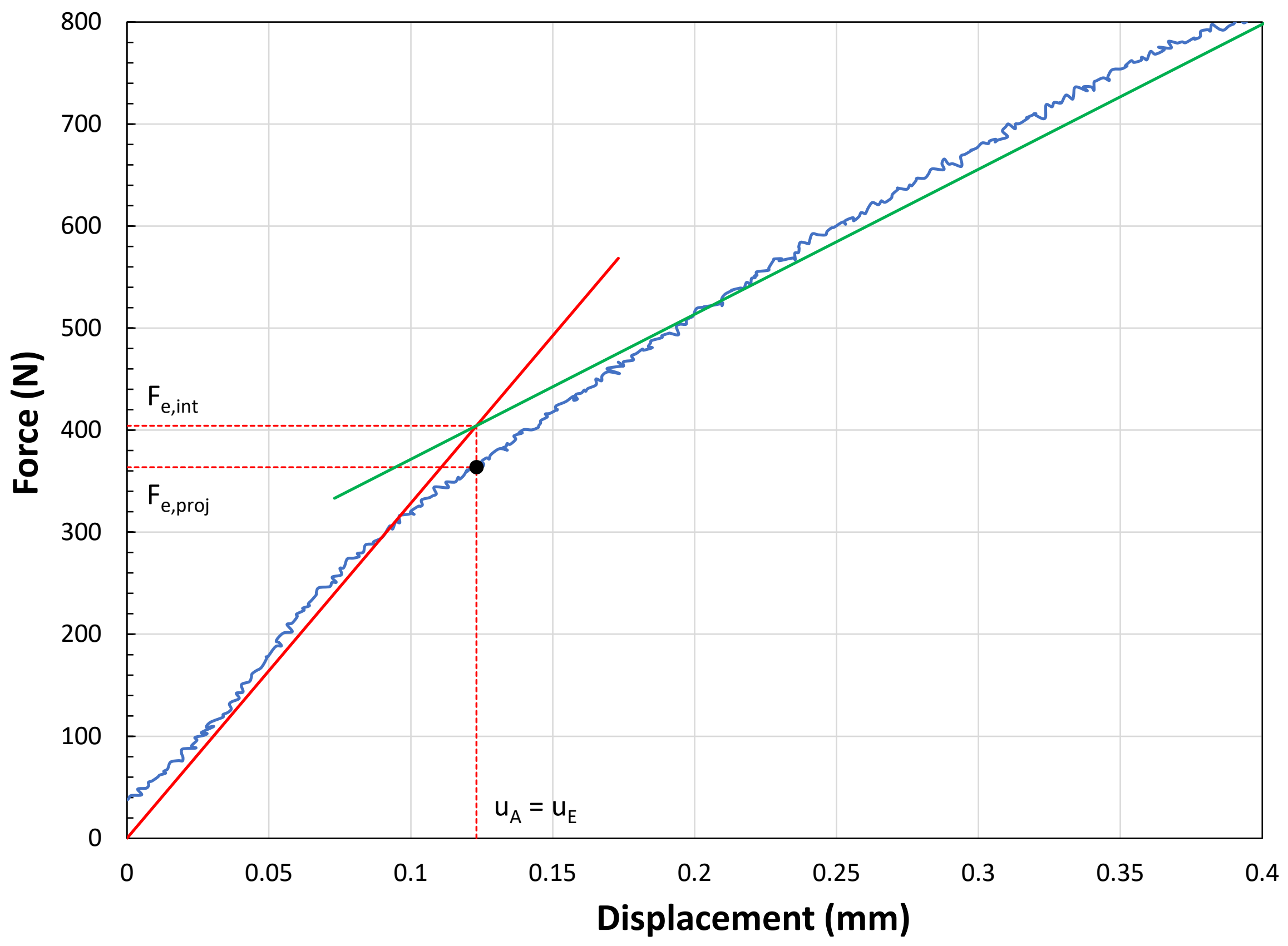




\section{ANNEX 6 \\ SP Test Results for \\ $800{ }^{\circ} \mathrm{C}$ HIP, scan length $=78 \mathrm{~mm}$, non-supported \\ ("rough" specimens)}


Specimen ID: 800HIP_1_N_a_SP1

Material: AM Ti-64

Test speed: $0.001004 \mathrm{~mm} / \mathrm{s}$

Max strain rate: $1.00 \mathrm{E}-03 \mathrm{~s}^{-1}$

\begin{tabular}{|c|c|c|}
\hline \multicolumn{3}{|c|}{ TEST RESULTS } \\
\hline \multicolumn{3}{|c|}{ Force values } \\
\hline$F_{e, p r o j}=$ & 443.2 & $\mathbf{N}$ \\
\hline$F_{e, \text { int }}=$ & 455.3 & $\mathbf{N}$ \\
\hline$F_{h 0 / 10, \text { off }}=$ & 691.4 & $\mathbf{N}$ \\
\hline$F_{0.1 \mathrm{~mm}, \mathrm{off}}=$ & 868.9 & $\mathbf{N}$ \\
\hline$F_{0.1 \mathrm{~mm}}=$ & 202.7 & $\mathbf{N}$ \\
\hline$F_{0.48 \mathrm{~mm}}=$ & 790.4 & $\mathbf{N}$ \\
\hline$F_{0.5 \mathrm{~mm}}=$ & 815.5 & $\mathbf{N}$ \\
\hline$F_{0.65 \mathrm{~mm}}=$ & 959.6 & $\mathbf{N}$ \\
\hline$F_{0.9 \mathrm{~mm}}=$ & 1032.8 & $\mathbf{N}$ \\
\hline$F_{\mathrm{ept}}=$ & 572.9 & $\mathbf{N}$ \\
\hline$F_{\mathrm{e} 1.5}=$ & 10.1 & $\mathbf{N}$ \\
\hline $\mathbf{F}_{\mathrm{m}}=$ & 1082.5 & $\mathbf{N}$ \\
\hline$F_{\text {infl }}=$ & 468.1 & $\mathbf{N}$ \\
\hline$F_{f}=$ & 866.0 & $\mathbf{N}$ \\
\hline \multicolumn{3}{|c|}{ Displacement values } \\
\hline $\mathrm{u}_{\mathrm{e}}=$ & 0.232 & $\mathrm{~mm}$ \\
\hline$u_{h 0 / 10, \text { off }}=$ & 0.404 & $\mathrm{~mm}$ \\
\hline$u_{0.1 \mathrm{~mm}, \mathrm{off}}=$ & 0.544 & $\mathrm{~mm}$ \\
\hline$u_{\mathrm{e} 1.5}=$ & -0.025 & $\mathrm{~mm}$ \\
\hline$u_{m}=$ & 0.822 & $\mathrm{~mm}$ \\
\hline$u_{\text {infl }}=$ & 1.734 & $\mathrm{~mm}$ \\
\hline$u_{f}=$ & 1.335 & $\mathrm{~mm}$ \\
\hline \multicolumn{3}{|c|}{ Initial linear slope } \\
\hline Slope $_{\text {ini }}=$ & 1958.20 & $\mathrm{~N} / \mathrm{mm}$ \\
\hline \multicolumn{3}{|c|}{ Energy values } \\
\hline$E_{S P}=$ & 1.05 & J \\
\hline$E_{m}=$ & 0.54 & $\mathbf{J}$ \\
\hline$E_{P L}=$ & 0.24 & $J$ \\
\hline
\end{tabular}




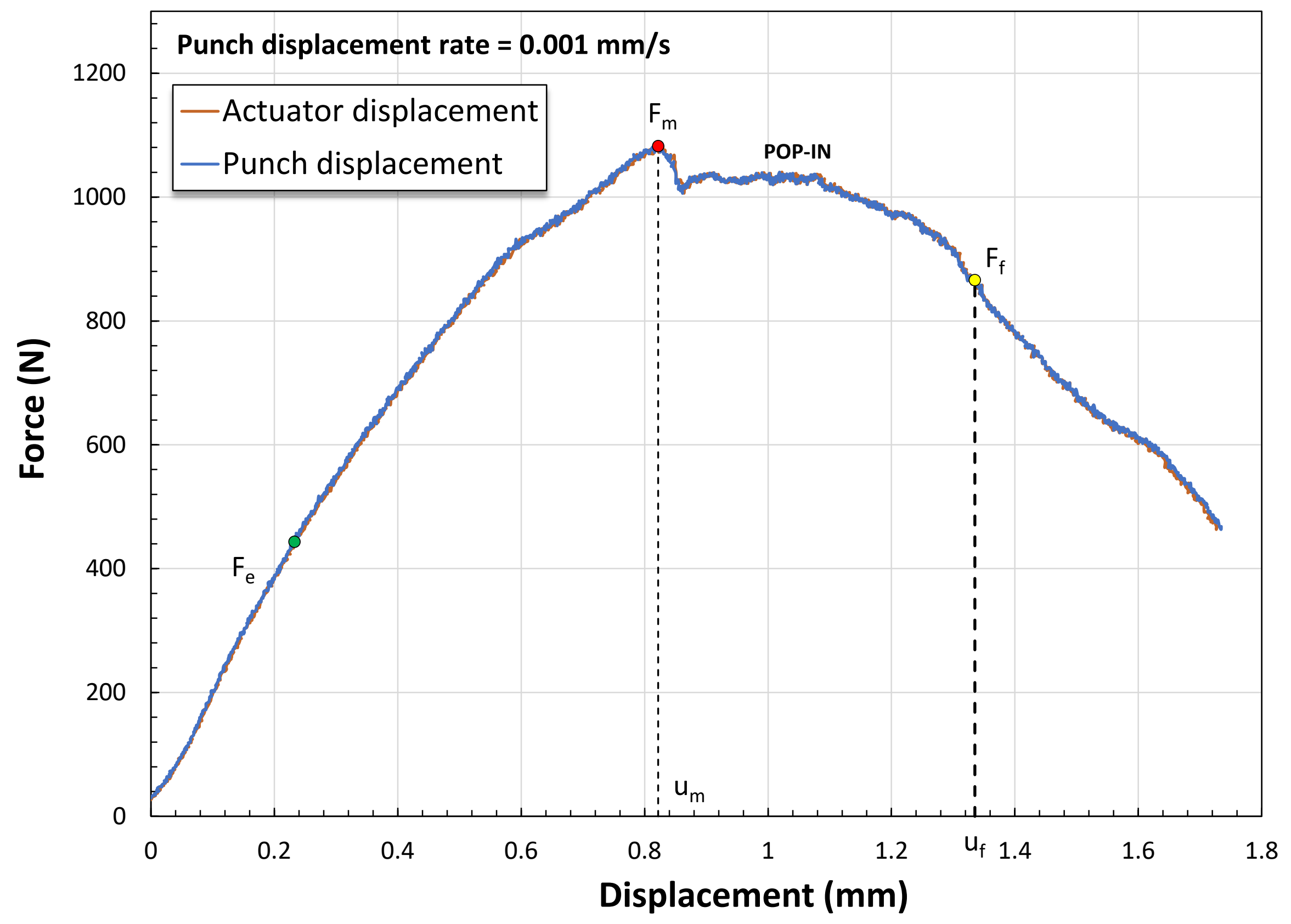




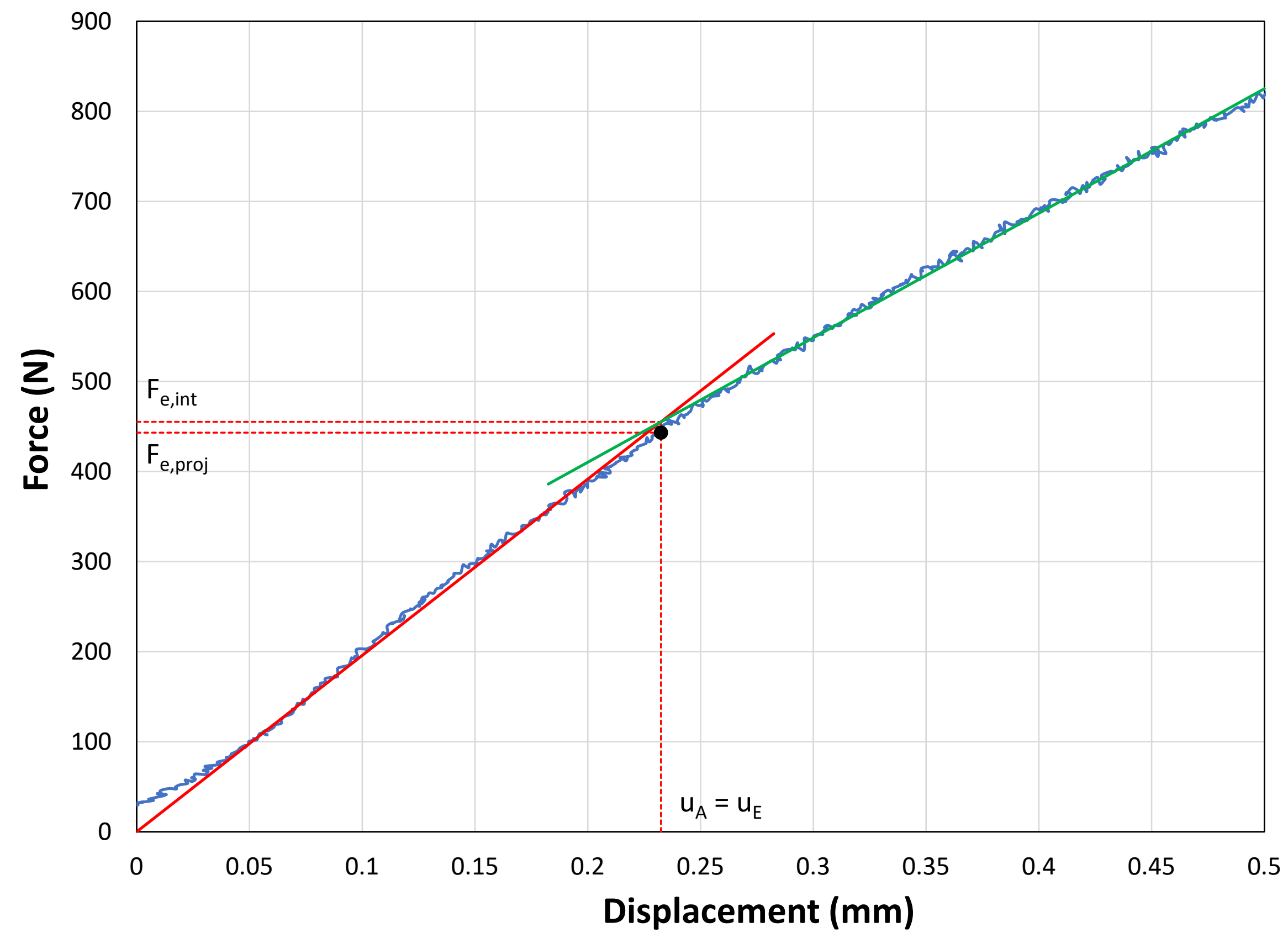


Specimen ID: 800HIP_1_N_a_SP2

Material: AM Ti-64

Test speed: $0.001005 \mathrm{~mm} / \mathrm{s}$

Max strain rate: $1.01 \mathrm{E}-03 \mathrm{~s}^{-1}$

\begin{tabular}{|c|c|c|}
\hline \multicolumn{3}{|c|}{ TEST RESULTS } \\
\hline \multicolumn{3}{|c|}{ Force values } \\
\hline$F_{e, p r o j}=$ & 445.7 & $\mathbf{N}$ \\
\hline$F_{e, \text { int }}=$ & 460.3 & $\mathbf{N}$ \\
\hline$F_{h 0 / 10, \text { off }}=$ & 630.4 & $\mathbf{N}$ \\
\hline$F_{0.1 \mathrm{~mm}, \mathrm{off}}=$ & 761.0 & $\mathbf{N}$ \\
\hline$F_{0.1 \mathrm{~mm}}=$ & 228.5 & $\mathbf{N}$ \\
\hline$F_{0.48 \mathrm{~mm}}=$ & 803.0 & $\mathbf{N}$ \\
\hline$F_{0.5 \mathrm{~mm}}=$ & 820.2 & $\mathbf{N}$ \\
\hline$F_{0.65 \mathrm{~mm}}=$ & 938.4 & $\mathbf{N}$ \\
\hline$F_{0.9 \mathrm{~mm}}=$ & 1020.1 & $\mathbf{N}$ \\
\hline $\mathbf{F}_{\mathrm{ept}}=$ & 966.0 & $\mathbf{N}$ \\
\hline$F_{\mathrm{e} 1.5}=$ & 136.8 & $\mathbf{N}$ \\
\hline $\mathbf{F}_{\mathrm{m}}=$ & 1068.2 & $\mathbf{N}$ \\
\hline$F_{\text {infl }}=$ & 628.6 & $\mathbf{N}$ \\
\hline$F_{f}=$ & 854.6 & $\mathbf{N}$ \\
\hline \multicolumn{3}{|c|}{ Displacement values } \\
\hline $\mathrm{u}_{\mathrm{e}}=$ & 0.208 & $\mathrm{~mm}$ \\
\hline$u_{h 0 / 10, \text { off }}=$ & 0.336 & $\mathrm{~mm}$ \\
\hline$u_{0.1 \mathrm{~mm}, \mathrm{off}}=$ & 0.445 & $\mathrm{~mm}$ \\
\hline$u_{\mathrm{e} 1.5}=$ & 0.060 & $\mathrm{~mm}$ \\
\hline$u_{m}=$ & 1.074 & $\mathrm{~mm}$ \\
\hline$u_{\text {infl }}=$ & 0.332 & $\mathrm{~mm}$ \\
\hline$u_{f}=$ & 1.533 & $\mathrm{~mm}$ \\
\hline \multicolumn{3}{|c|}{ Initial linear slope } \\
\hline Slope $_{\text {ini }}=$ & 2210.27 & $\mathrm{~N} / \mathrm{mm}$ \\
\hline \multicolumn{3}{|c|}{ Energy values } \\
\hline$E_{S P}=$ & 1.25 & J \\
\hline$E_{m}=$ & 0.80 & J \\
\hline$E_{P L}=$ & 0.54 & $\mathbf{J}$ \\
\hline
\end{tabular}




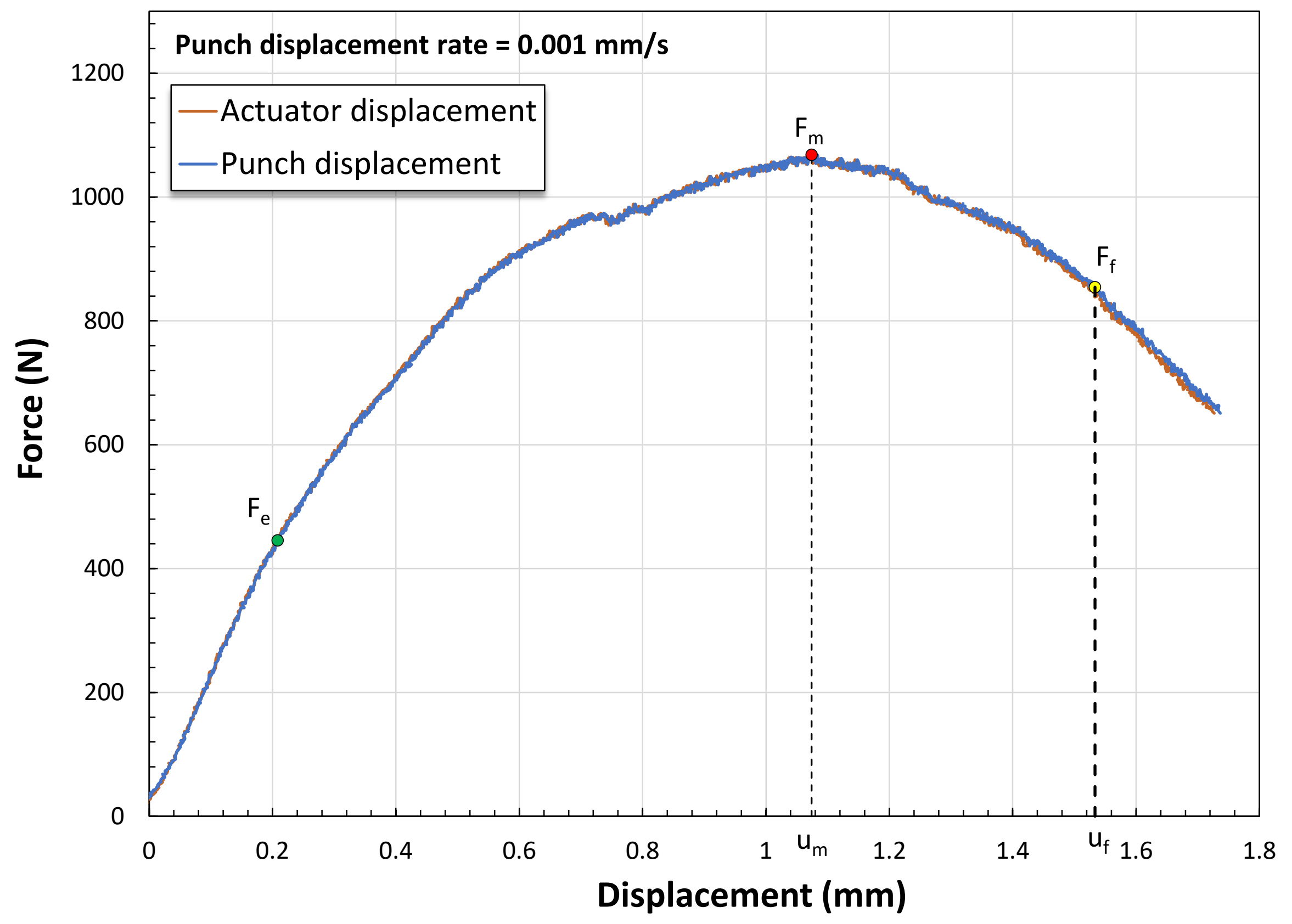




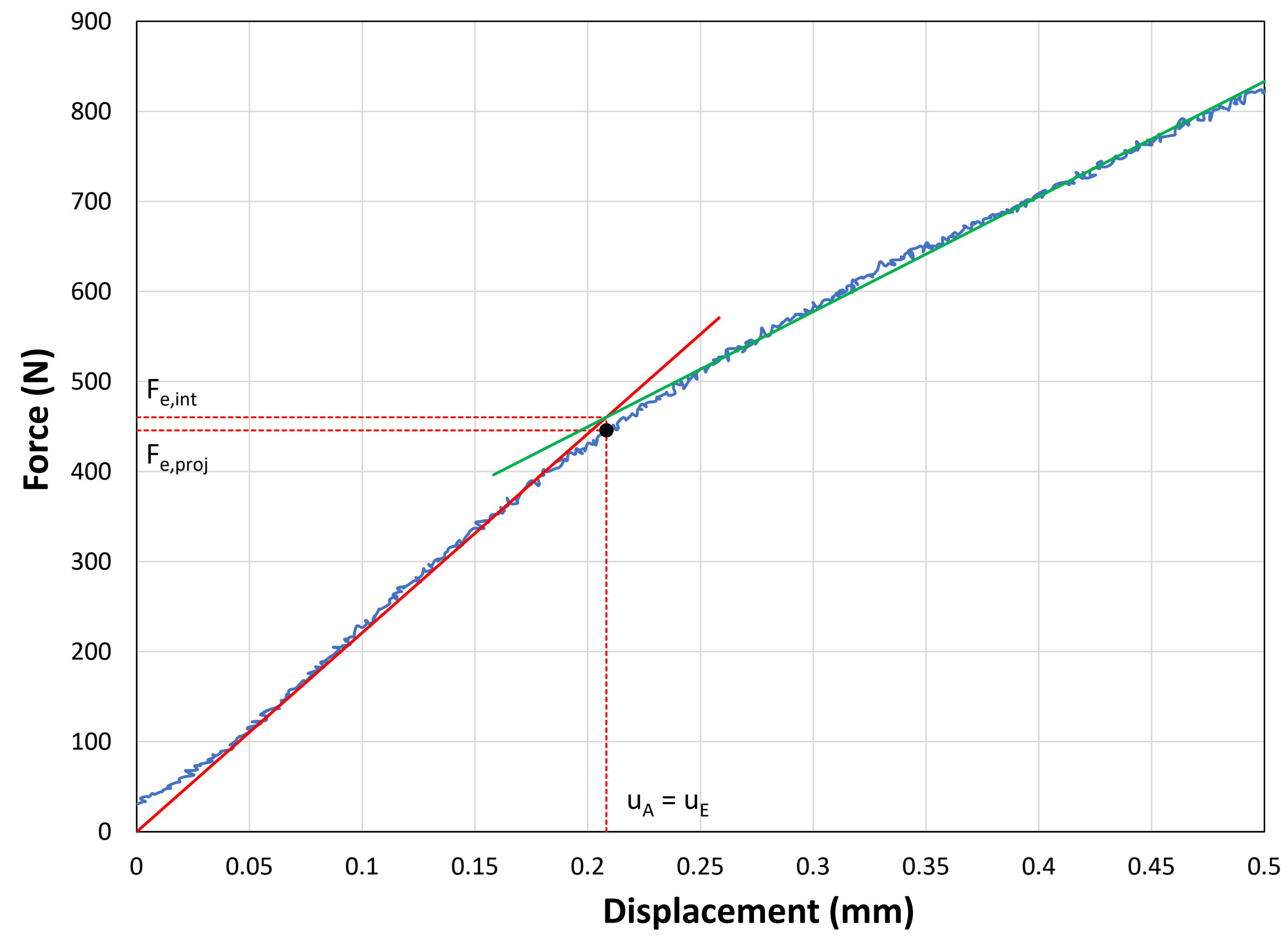


Specimen ID: 800HIP_1_N_a_SP3

Material: AM Ti-64

Test speed: $0.001012 \mathrm{~mm} / \mathrm{s}$

Max strain rate: $1.01 \mathrm{E}-03 \mathrm{~s}^{-1}$

\begin{tabular}{|c|c|c|}
\hline \multicolumn{3}{|c|}{ TEST RESULTS } \\
\hline \multicolumn{3}{|c|}{ Force values } \\
\hline$F_{e, p r o j}=$ & 487.1 & $\mathbf{N}$ \\
\hline$F_{e, \text { int }}=$ & 492.4 & $\mathbf{N}$ \\
\hline$F_{h 0 / 10, \text { off }}=$ & 663.9 & $\mathbf{N}$ \\
\hline$F_{0.1 \mathrm{~mm}, \mathrm{off}}=$ & 828.7 & $\mathbf{N}$ \\
\hline$F_{0.1 \mathrm{~mm}}=$ & 179.3 & $\mathbf{N}$ \\
\hline$F_{0.48 \mathrm{~mm}}=$ & 737.4 & $\mathbf{N}$ \\
\hline$F_{0.5 \mathrm{~mm}}=$ & 765.0 & $\mathbf{N}$ \\
\hline$F_{0.65 \mathrm{~mm}}=$ & 915.6 & $\mathbf{N}$ \\
\hline$F_{0.9 m m}=$ & 1126.4 & $\mathbf{N}$ \\
\hline$F_{\mathrm{ept}}=$ & 708.1 & $\mathbf{N}$ \\
\hline$F_{\mathrm{e} 1.5}=$ & 216.7 & $\mathbf{N}$ \\
\hline $\mathbf{F}_{\mathrm{m}}=$ & 1157.1 & $\mathbf{N}$ \\
\hline$F_{\text {infl }}=$ & 590.9 & $\mathbf{N}$ \\
\hline$F_{f}=$ & 925.7 & $\mathbf{N}$ \\
\hline \multicolumn{3}{|c|}{ Displacement values } \\
\hline$u_{e}=$ & 0.273 & $\mathrm{~mm}$ \\
\hline$u_{h 0 / 10, \text { off }}=$ & 0.419 & $\mathrm{~mm}$ \\
\hline$u_{0.1 \mathrm{~mm}, \mathrm{off}}=$ & 0.560 & $\mathrm{~mm}$ \\
\hline$u_{\mathrm{e} 1.5}=$ & 0.119 & $\mathrm{~mm}$ \\
\hline$u_{m}=$ & 0.946 & $\mathrm{~mm}$ \\
\hline$u_{\text {infl }}=$ & 1.731 & $\mathrm{~mm}$ \\
\hline$u_{f}=$ & 1.458 & $\mathrm{~mm}$ \\
\hline \multicolumn{3}{|c|}{ Initial linear slope } \\
\hline Slope $_{\text {ini }}=$ & 1801.57 & $\mathrm{~N} / \mathrm{mm}$ \\
\hline \multicolumn{3}{|c|}{ Energy values } \\
\hline$E_{S P}=$ & 1.20 & J \\
\hline$E_{m}=$ & 0.65 & J \\
\hline$E_{P L}=$ & 0.28 & $\mathbf{J}$ \\
\hline
\end{tabular}




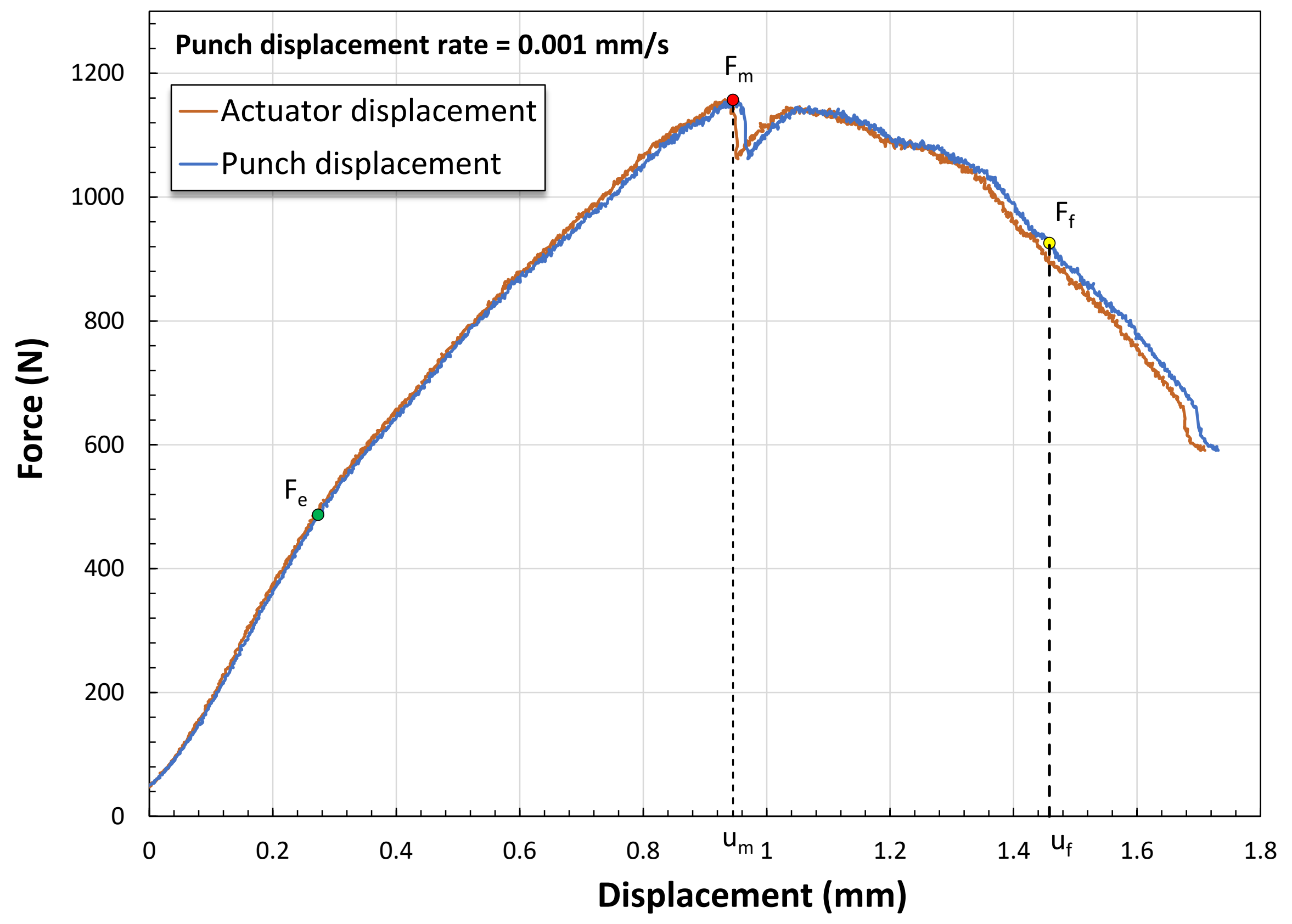




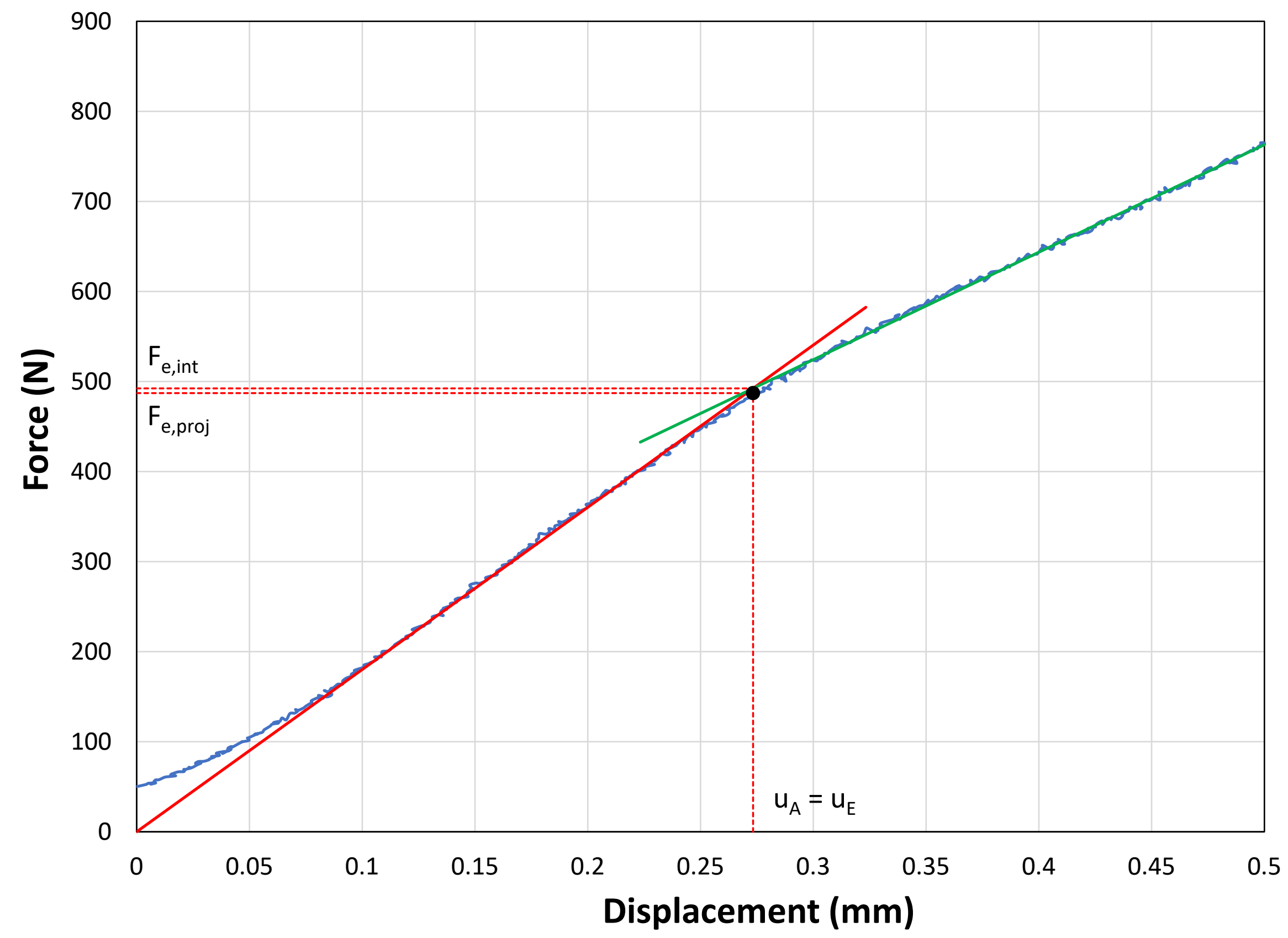


Specimen ID: 800HIP_1_N_a_SP4

Material: AM Ti-64

Test speed: $0.001023 \mathrm{~mm} / \mathrm{s}$

Max strain rate: $1.02 \mathrm{E}-03 \mathrm{~s}^{-1}$

\begin{tabular}{|c|c|c|}
\hline \multicolumn{3}{|c|}{ TEST RESULTS } \\
\hline \multicolumn{3}{|c|}{ Force values } \\
\hline$F_{e, p r o j}=$ & 428.9 & $\mathbf{N}$ \\
\hline$F_{e, \text { int }}=$ & 438.4 & $\mathbf{N}$ \\
\hline$F_{h 0 / 10, \text { off }}=$ & 677.5 & $\mathbf{N}$ \\
\hline$F_{0.1 \mathrm{~mm}, \mathrm{off}}=$ & 817.2 & $\mathbf{N}$ \\
\hline$F_{0.1 \mathrm{~mm}}=$ & 164.1 & $\mathbf{N}$ \\
\hline$F_{0.48 \mathrm{~mm}}=$ & 692.9 & $\mathbf{N}$ \\
\hline$F_{0.5 \mathrm{~mm}}=$ & 714.6 & $\mathbf{N}$ \\
\hline$F_{0.65 \mathrm{~mm}}=$ & 879.5 & $\mathbf{N}$ \\
\hline$F_{0.9 \mathrm{~mm}}=$ & 1054.7 & $\mathbf{N}$ \\
\hline$F_{\mathrm{ept}}=$ & 411.3 & $\mathbf{N}$ \\
\hline$F_{\mathrm{e} 1.5}=$ & 142.5 & $\mathbf{N}$ \\
\hline $\mathbf{F}_{\mathrm{m}}=$ & 1106.8 & $\mathbf{N}$ \\
\hline$F_{\text {infl }}=$ & 577.5 & $\mathbf{N}$ \\
\hline$F_{f}=$ & 885.4 & $\mathbf{N}$ \\
\hline \multicolumn{3}{|c|}{ Displacement values } \\
\hline $\mathrm{u}_{\mathrm{e}}=$ & 0.267 & $\mathrm{~mm}$ \\
\hline$u_{h 0 / 10, \text { off }}=$ & 0.464 & $\mathrm{~mm}$ \\
\hline$u_{0.1 \mathrm{~mm}, \mathrm{off}}=$ & 0.598 & $\mathrm{~mm}$ \\
\hline$u_{\mathrm{e} 1.5}=$ & 0.086 & $\mathrm{~mm}$ \\
\hline$u_{m}=$ & 1.038 & $\mathrm{~mm}$ \\
\hline$u_{\text {infl }}=$ & 1.766 & $\mathrm{~mm}$ \\
\hline$u_{f}=$ & 1.371 & $\mathrm{~mm}$ \\
\hline \multicolumn{3}{|c|}{ Initial linear slope } \\
\hline Slope $_{\text {ini }}=$ & 1639.54 & $\mathrm{~N} / \mathrm{mm}$ \\
\hline \multicolumn{3}{|c|}{ Energy values } \\
\hline$E_{S P}=$ & 1.05 & J \\
\hline$E_{m}=$ & 0.71 & $\mathbf{J}$ \\
\hline$E_{P L}=$ & 0.34 & $J$ \\
\hline
\end{tabular}




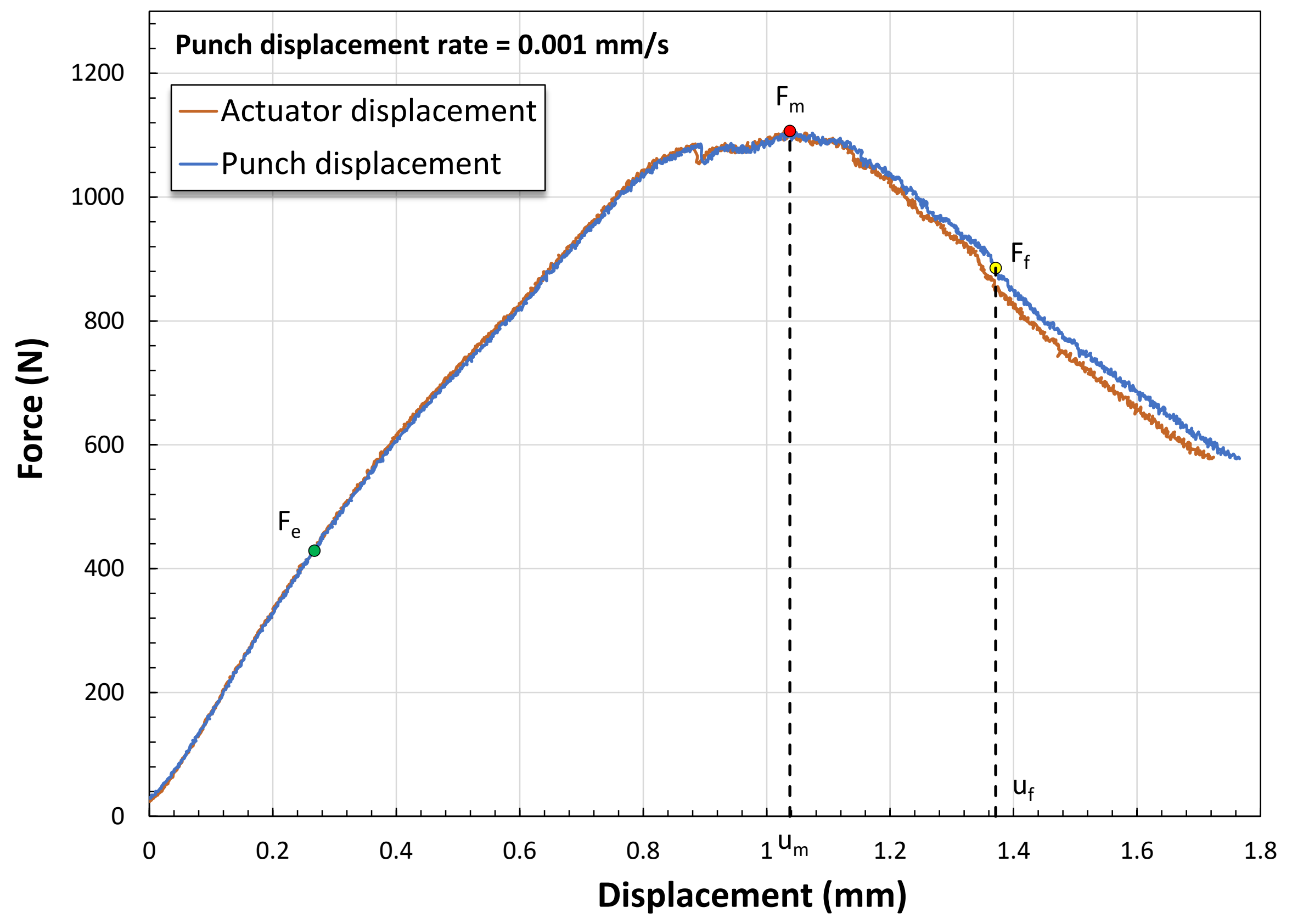




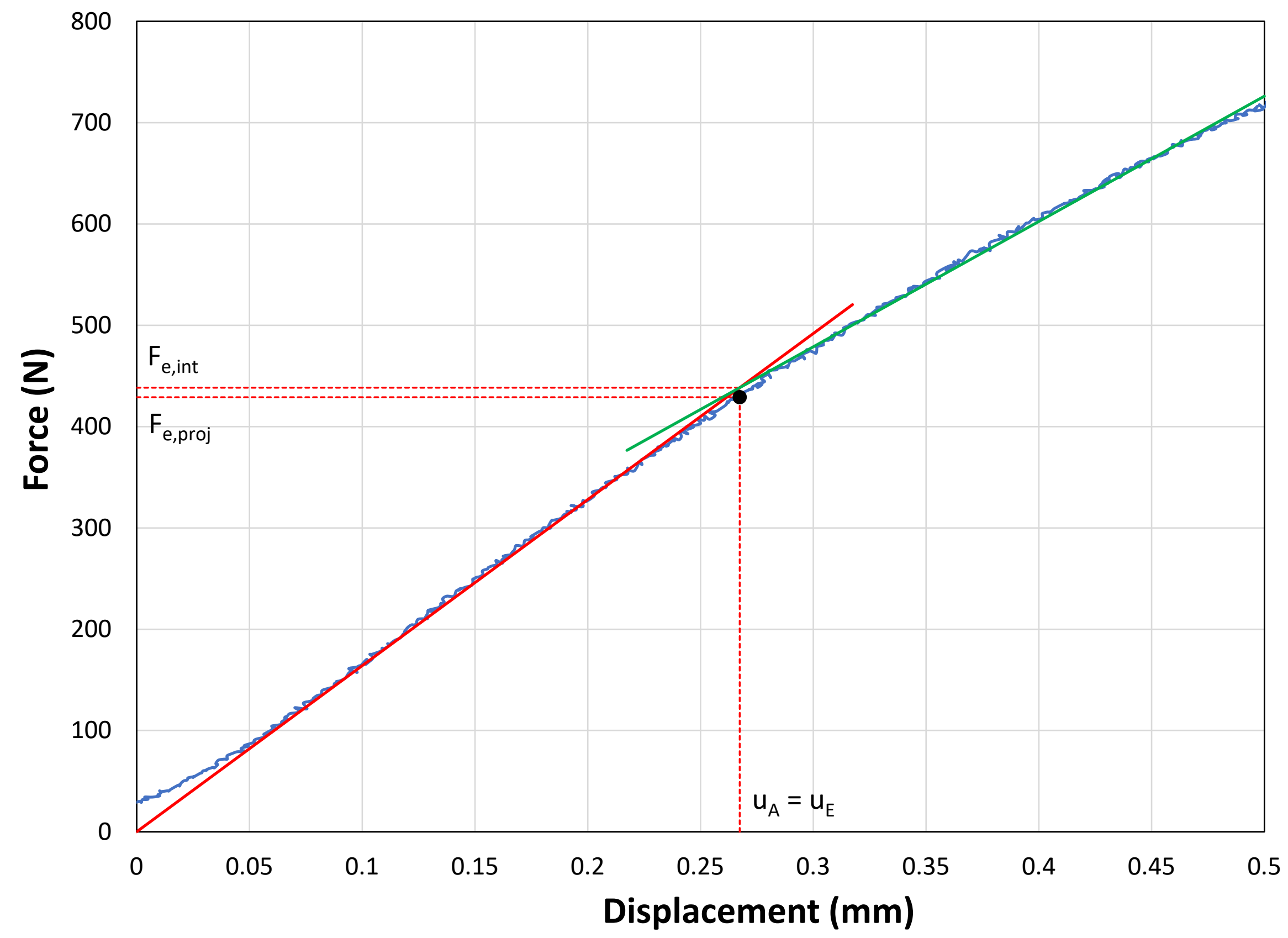


Specimen ID: 800HIP_1_N_a_SP5

Material: AM Ti-64

Test speed: $0.001036 \mathrm{~mm} / \mathrm{s}$

Max strain rate: $1.04 \mathrm{E}-03 \mathrm{~s}^{-1}$

\begin{tabular}{|c|c|c|}
\hline \multicolumn{3}{|c|}{ TEST RESULTS } \\
\hline \multicolumn{3}{|c|}{ Force values } \\
\hline$F_{e, p r o j}=$ & 436.8 & $\mathbf{N}$ \\
\hline$F_{e, \text { int }}=$ & 447.0 & $\mathbf{N}$ \\
\hline$F_{h 0 / 10, \text { off }}=$ & 658.3 & $\mathbf{N}$ \\
\hline$F_{0.1 \mathrm{~mm}, \mathrm{off}}=$ & 845.0 & $\mathbf{N}$ \\
\hline$F_{0.1 \mathrm{~mm}}=$ & 173.6 & $\mathbf{N}$ \\
\hline$F_{0.48 \mathrm{~mm}}=$ & 722.7 & $\mathbf{N}$ \\
\hline$F_{0.5 \mathrm{~mm}}=$ & 747.9 & $\mathbf{N}$ \\
\hline$F_{0.65 \mathrm{~mm}}=$ & 927.5 & $\mathbf{N}$ \\
\hline$F_{0.9 \mathrm{~mm}}=$ & 1056.0 & $\mathbf{N}$ \\
\hline$F_{\mathrm{ept}}=$ & 115.1 & $\mathbf{N}$ \\
\hline$F_{\mathrm{e} 1.5}=$ & 190.3 & $\mathbf{N}$ \\
\hline $\mathbf{F}_{\mathrm{m}}=$ & 1070.9 & $\mathbf{N}$ \\
\hline$F_{\text {infl }}=$ & 565.7 & $\mathbf{N}$ \\
\hline$F_{f}=$ & 856.7 & $\mathbf{N}$ \\
\hline \multicolumn{3}{|c|}{ Displacement values } \\
\hline $\mathrm{u}_{\mathrm{e}}=$ & 0.255 & $\mathrm{~mm}$ \\
\hline$u_{h 0 / 10, \text { off }}=$ & 0.426 & $\mathrm{~mm}$ \\
\hline$u_{0.1 \mathrm{~mm}, \mathrm{off}}=$ & 0.582 & $\mathrm{~mm}$ \\
\hline$u_{\mathrm{e} 1.5}=$ & 0.108 & $\mathrm{~mm}$ \\
\hline$u_{m}=$ & 0.917 & $\mathrm{~mm}$ \\
\hline$u_{\text {infl }}=$ & 1.782 & $\mathrm{~mm}$ \\
\hline$u_{f}=$ & 1.413 & $\mathrm{~mm}$ \\
\hline \multicolumn{3}{|c|}{ Initial linear slope } \\
\hline Slope $_{\text {ini }}=$ & 1754.55 & $\mathrm{~N} / \mathrm{mm}$ \\
\hline \multicolumn{3}{|c|}{ Energy values } \\
\hline$E_{S P}=$ & 1.09 & J \\
\hline$E_{m}=$ & 0.60 & J \\
\hline$E_{P L}=$ & 0.27 & $J$ \\
\hline
\end{tabular}




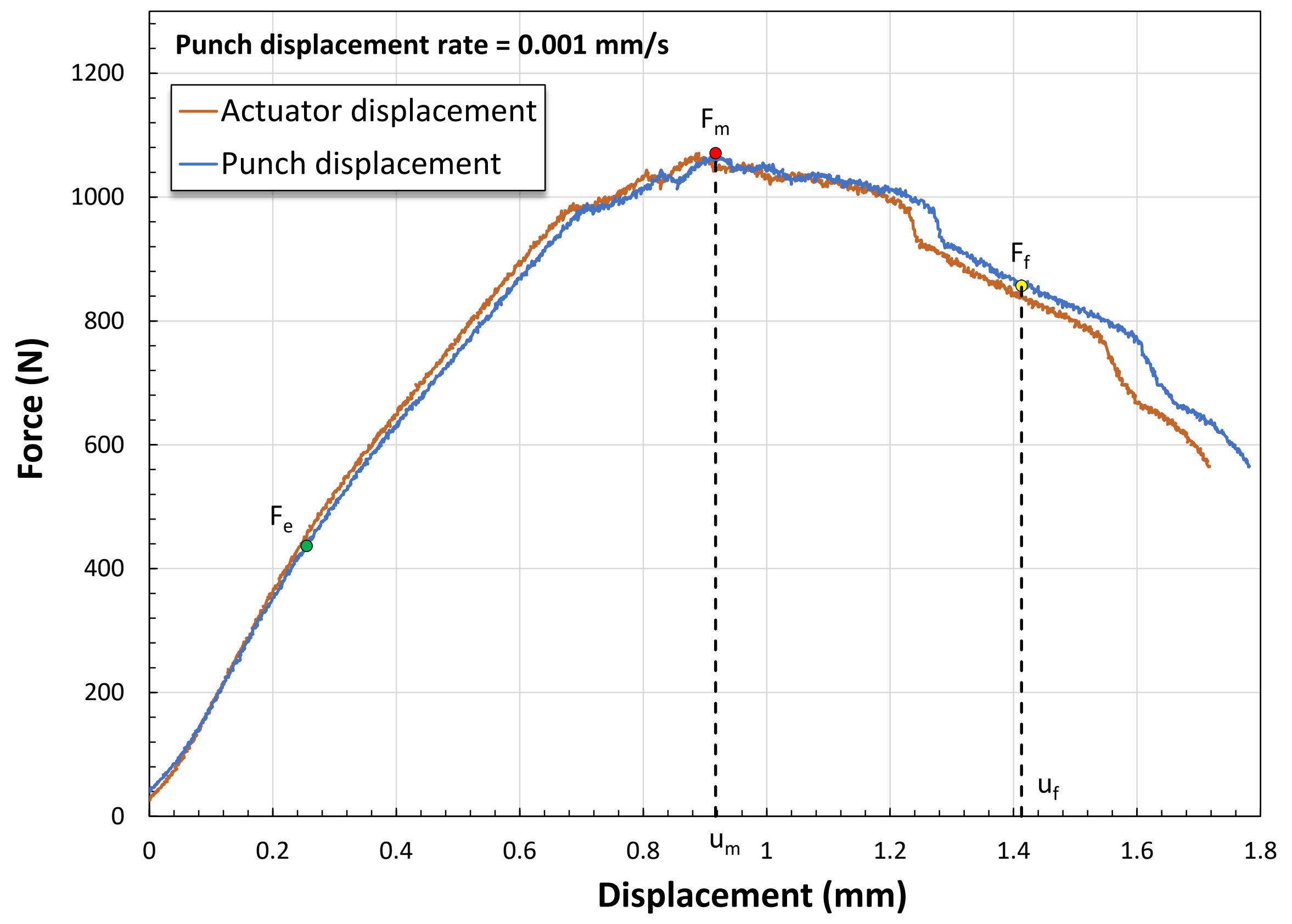




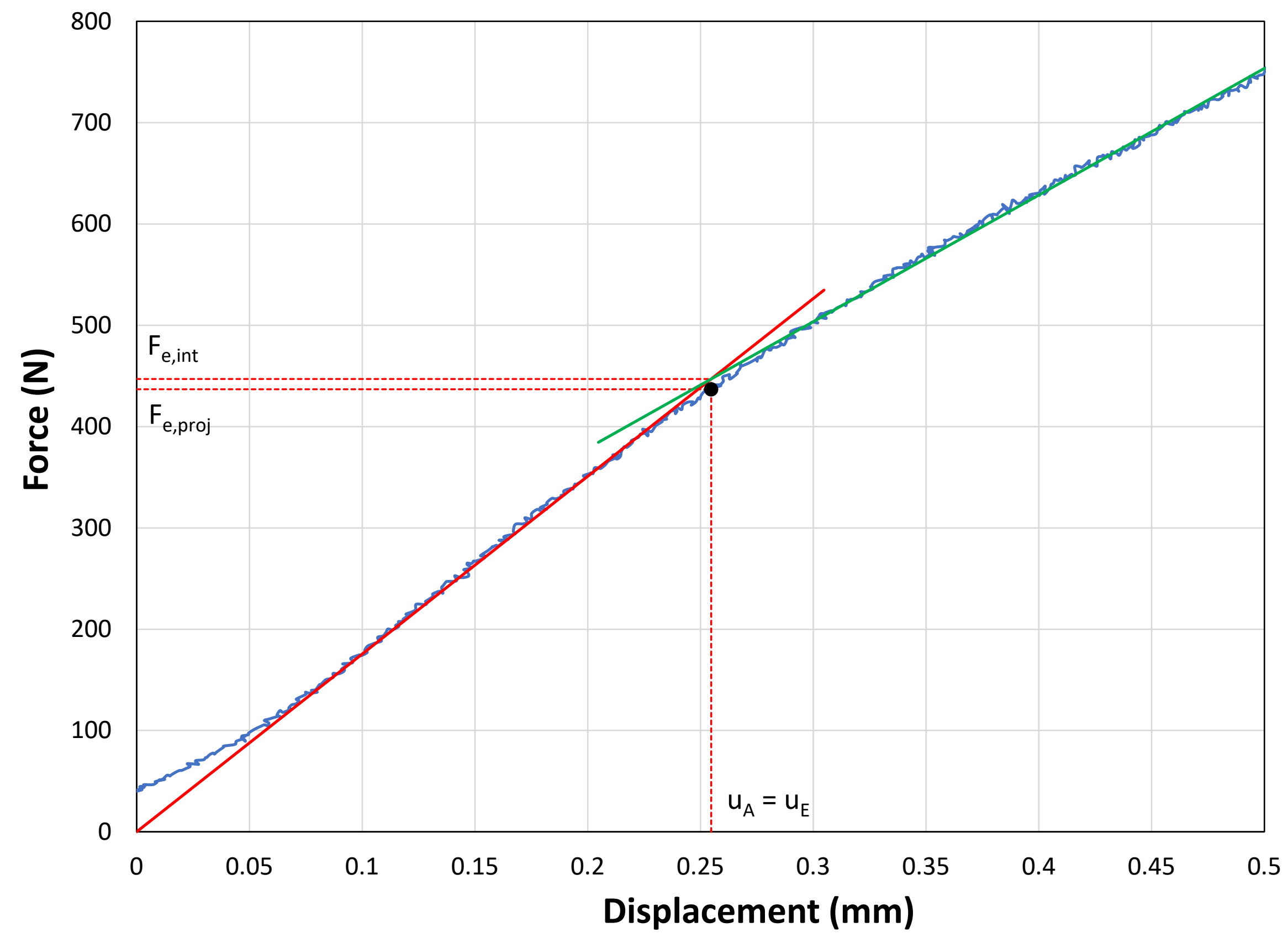


Specimen ID: 800HIP_1_N_a_SP6

Material: AM Ti-64

Test speed: $0.001022 \mathrm{~mm} / \mathrm{s}$

Max strain rate: $1.02 \mathrm{E}-03 \mathrm{~s}^{-1}$

\begin{tabular}{|c|c|c|}
\hline \multicolumn{3}{|c|}{ TEST RESULTS } \\
\hline \multicolumn{3}{|c|}{ Force values } \\
\hline$F_{e, p r o j}=$ & 472.8 & $\mathbf{N}$ \\
\hline$F_{e, \text { int }}=$ & 447.0 & $\mathbf{N}$ \\
\hline$F_{h 0 / 10, \text { off }}=$ & 781.3 & $\mathbf{N}$ \\
\hline$F_{0.1 \mathrm{~mm}, \mathrm{off}}=$ & 904.7 & $\mathbf{N}$ \\
\hline$F_{0.1 \mathrm{~mm}}=$ & 194.5 & $\mathbf{N}$ \\
\hline$F_{0.48 \mathrm{~mm}}=$ & 763.6 & $\mathbf{N}$ \\
\hline$F_{0.5 \mathrm{~mm}}=$ & 786.6 & $\mathbf{N}$ \\
\hline$F_{0.65 \mathrm{~mm}}=$ & 930.1 & $\mathbf{N}$ \\
\hline$F_{0.9 \mathrm{~mm}}=$ & 1093.5 & $\mathbf{N}$ \\
\hline$F_{\mathrm{ept}}=$ & 1122.7 & $\mathbf{N}$ \\
\hline$F_{\mathrm{e} 1.5}=$ & 180.0 & $\mathbf{N}$ \\
\hline $\mathbf{F}_{\mathrm{m}}=$ & 1131.1 & $\mathbf{N}$ \\
\hline$F_{\text {infl }}=$ & 960.9 & $\mathbf{N}$ \\
\hline$F_{f}=$ & 904.9 & $\mathbf{N}$ \\
\hline \multicolumn{3}{|c|}{ Displacement values } \\
\hline $\mathrm{u}_{\mathrm{e}}=$ & 0.255 & $\mathrm{~mm}$ \\
\hline$u_{h 0 / 10, \text { off }}=$ & 0.495 & $\mathrm{~mm}$ \\
\hline$u_{0.1 \mathrm{~mm}, \mathrm{off}}=$ & 0.616 & $\mathrm{~mm}$ \\
\hline$u_{\mathrm{e} 1.5}=$ & 0.091 & $\mathrm{~mm}$ \\
\hline$u_{m}=$ & 1.115 & $\mathrm{~mm}$ \\
\hline$u_{\text {infl }}=$ & 1.422 & $\mathrm{~mm}$ \\
\hline$u_{f}=$ & 1.476 & $\mathrm{~mm}$ \\
\hline \multicolumn{3}{|c|}{ Initial linear slope } \\
\hline Slope $_{\text {ini }}=$ & 1754.55 & $\mathrm{~N} / \mathrm{mm}$ \\
\hline \multicolumn{3}{|c|}{ Energy values } \\
\hline$E_{S P}=$ & 1.22 & J \\
\hline$E_{m}=$ & 0.84 & $\mathbf{J}$ \\
\hline$E_{P L}=$ & 0.48 & $J$ \\
\hline
\end{tabular}




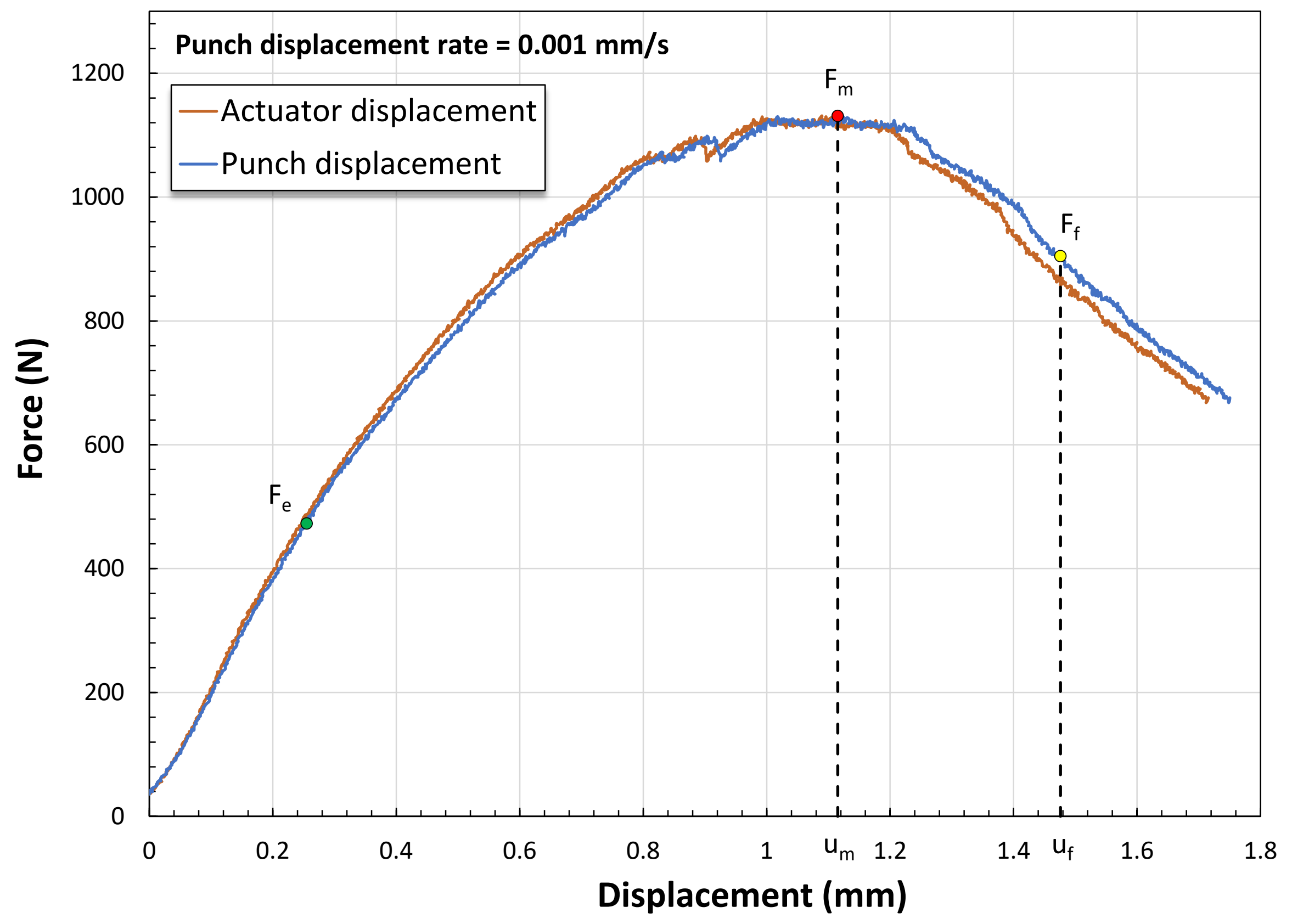




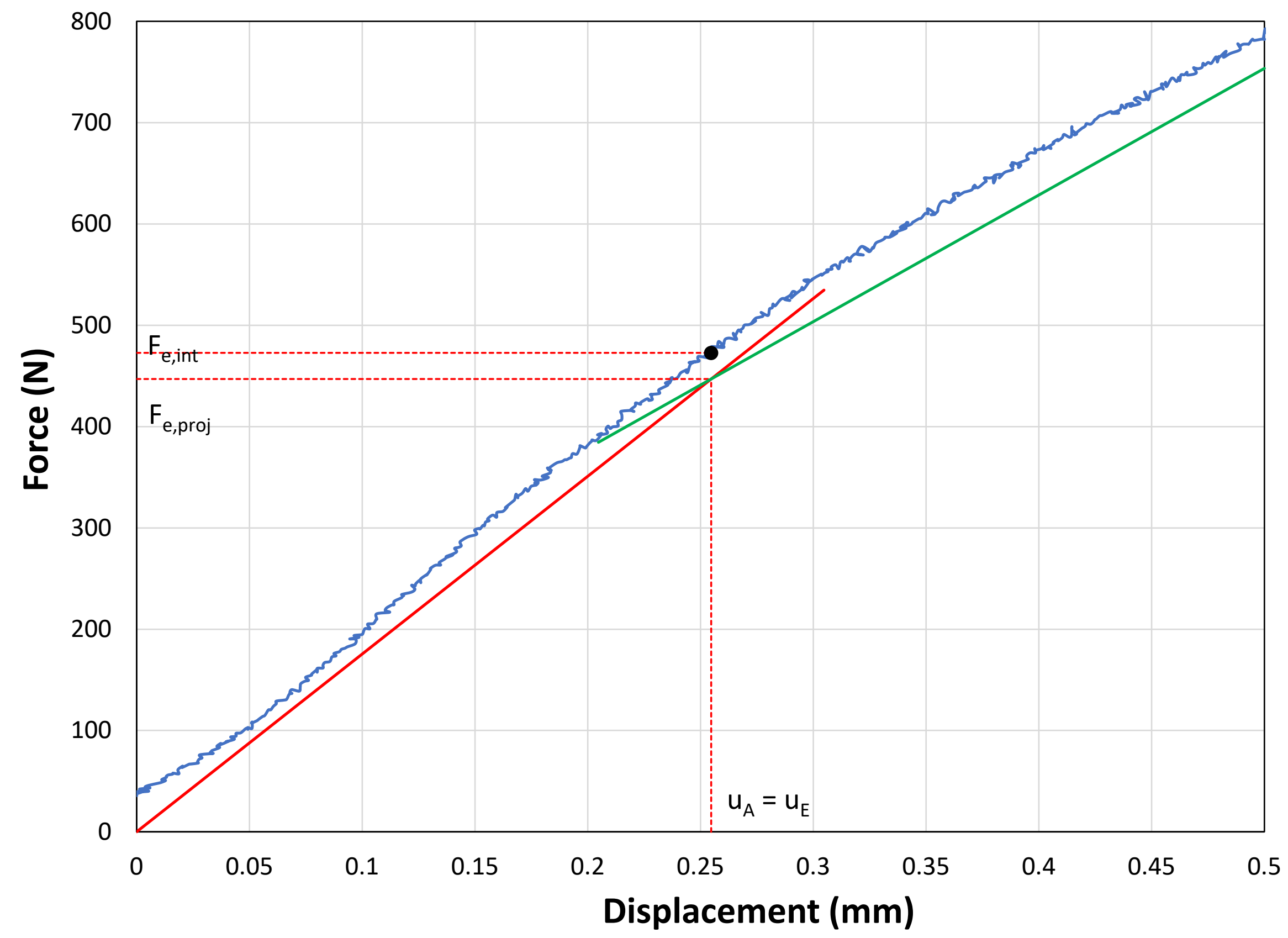




\section{ANNEX 7 \\ SP Test Results for \\ $800{ }^{\circ} \mathrm{C}$ HIP, scan length $=78 \mathrm{~mm}$, non-supported (polished specimens)}


Specimen ID: 800HIP_1_N_a_SP7 (p

Material: AM Ti-64

Test speed: $0.001441 \mathrm{~mm} / \mathrm{s}$

Max strain rate: $1.44 \mathrm{E}-03 \mathrm{~s}^{-1}$

\begin{tabular}{|c|c|c|}
\hline \multicolumn{3}{|c|}{ TEST RESULTS } \\
\hline \multicolumn{3}{|c|}{ Force values } \\
\hline$F_{e, p r o j}=$ & 431.3 & $\mathbf{N}$ \\
\hline $\mathbf{F}_{\mathrm{e}, \text { int }}=$ & 473.5 & $\mathbf{N}$ \\
\hline$F_{h 0 / 10, \text { off }}=$ & 559.8 & $\mathbf{N}$ \\
\hline$F_{0.1 \mathrm{~mm}, \text { off }}=$ & 698.0 & $\mathbf{N}$ \\
\hline$F_{0.1 \mathrm{~mm}}=$ & 348.9 & $\mathbf{N}$ \\
\hline$F_{0.48 m m}=$ & 874.7 & $\mathbf{N}$ \\
\hline $\mathrm{F}_{0.5 \mathrm{~mm}}=$ & 890.4 & $\mathbf{N}$ \\
\hline$F_{0.65 \mathrm{~mm}}=$ & 922.1 & $\mathbf{N}$ \\
\hline $\mathrm{F}_{0.9 \mathrm{~mm}}=$ & 1005.9 & $\mathbf{N}$ \\
\hline$F_{\text {ept }}=$ & 652.4 & $\mathbf{N}$ \\
\hline$F_{\mathrm{e} 1.5}=$ & 577.2 & $\mathbf{N}$ \\
\hline$F_{m}=$ & 1013.5 & $\mathbf{N}$ \\
\hline$F_{\text {infl }}=$ & 988.7 & $\mathbf{N}$ \\
\hline$F_{f}=$ & 810.8 & $\mathbf{N}$ \\
\hline \multicolumn{3}{|c|}{ Displacement values } \\
\hline $\mathrm{u}_{\mathrm{e}}=$ & 0.134 & $\mathrm{~mm}$ \\
\hline$u_{h 0 / 10, \text { off }}=$ & 0.205 & $\mathrm{~mm}$ \\
\hline$u_{0.1 \mathrm{~mm}, \text { off }}=$ & 0.299 & $\mathrm{~mm}$ \\
\hline$u_{\mathrm{e} 1.5}=$ & 0.219 & $\mathrm{~mm}$ \\
\hline$u_{m}=$ & 0.885 & $\mathrm{~mm}$ \\
\hline$u_{i n f l}=$ & 1.026 & $\mathrm{~mm}$ \\
\hline$u_{f}=$ & 1.278 & $\mathrm{~mm}$ \\
\hline \multicolumn{3}{|c|}{ Initial linear slope } \\
\hline Slope $_{\text {ini }}=$ & 3521.07 & $\mathrm{~N} / \mathrm{mm}$ \\
\hline \multicolumn{3}{|c|}{ Energy values } \\
\hline$E_{S P}=$ & 1.03 & J \\
\hline$E_{m}=$ & 0.66 & J \\
\hline$E_{p L}=$ & 0.51 & J \\
\hline
\end{tabular}




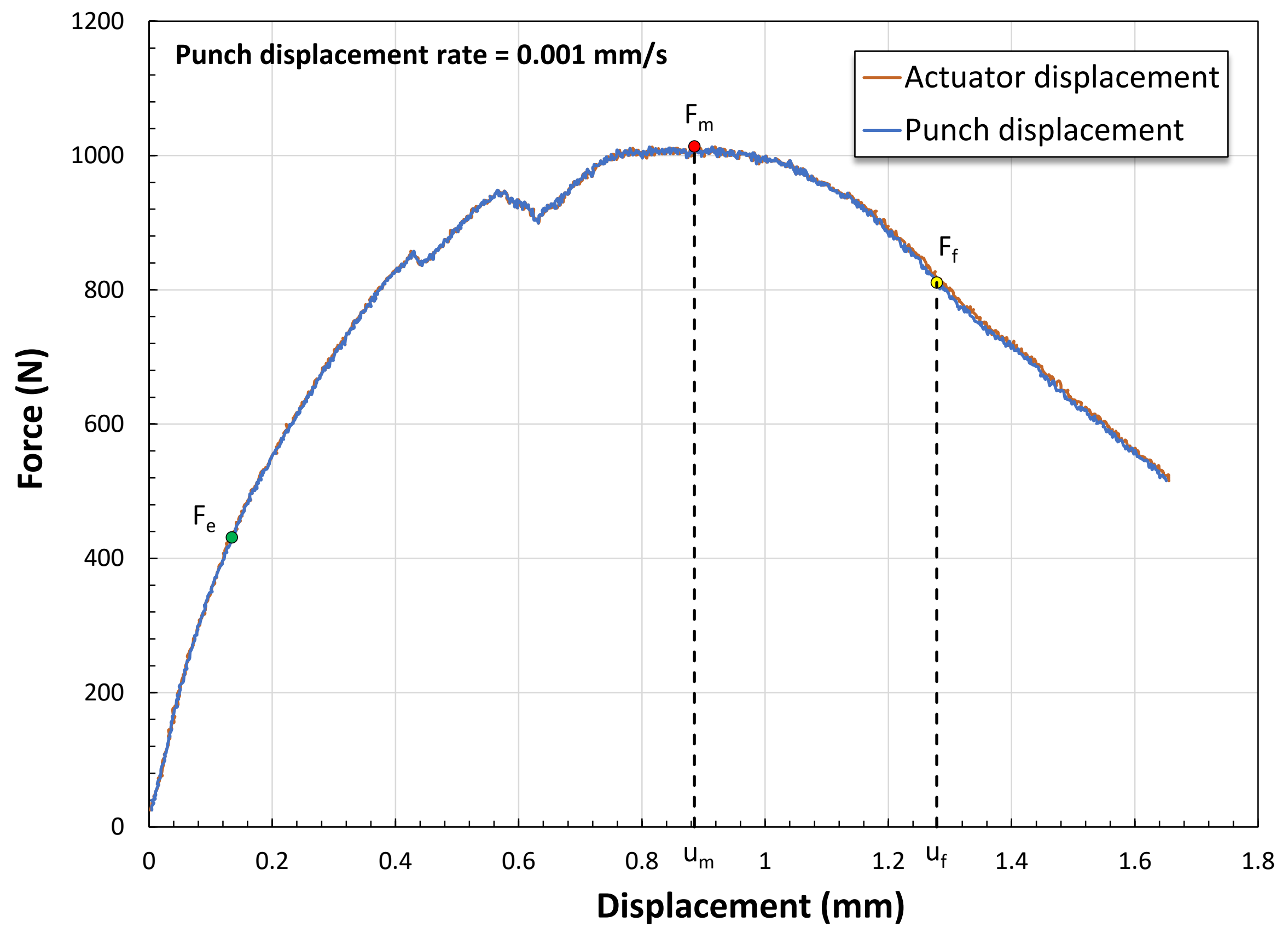




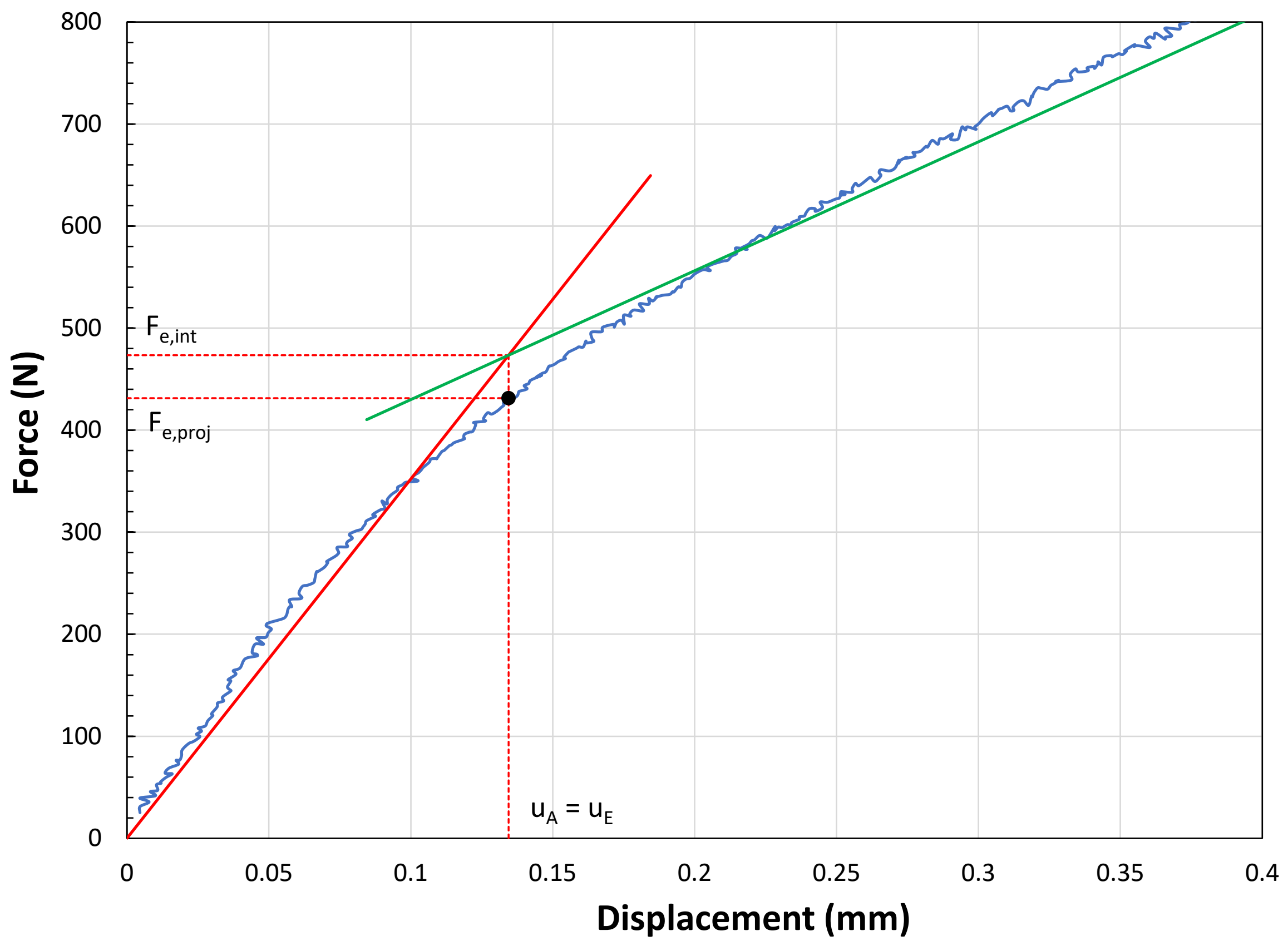


Specimen ID: 800HIP_1_N_a_SP8 (p

Material: AM Ti-64

Test speed: $0.001443 \mathrm{~mm} / \mathrm{s}$

Max strain rate: $1.44 \mathrm{E}-03 \mathrm{~s}^{-1}$

\begin{tabular}{|c|c|c|}
\hline \multicolumn{3}{|c|}{ TEST RESULTS } \\
\hline \multicolumn{3}{|c|}{ Force values } \\
\hline$F_{e, \text { proj }}=$ & 381.2 & $\mathbf{N}$ \\
\hline$F_{e, \text { int }}=$ & 413.1 & $\mathbf{N}$ \\
\hline$F_{h 0 / 10, \text { off }}=$ & 513.8 & $\mathbf{N}$ \\
\hline$F_{0.1 \mathrm{~mm}, \mathrm{off}}=$ & 661.1 & $\mathbf{N}$ \\
\hline$F_{0.1 \mathrm{~mm}}=$ & 298.0 & $\mathbf{N}$ \\
\hline$F_{0.48 \mathrm{~mm}}=$ & 832.4 & $\mathbf{N}$ \\
\hline $\mathrm{F}_{0.5 \mathrm{~mm}}=$ & 850.4 & $\mathbf{N}$ \\
\hline$F_{0.65 \mathrm{~mm}}=$ & 961.4 & $\mathbf{N}$ \\
\hline $\mathrm{F}_{0.9 \mathrm{~mm}}=$ & 918.9 & $\mathbf{N}$ \\
\hline$F_{\text {ept }}=$ & 722.6 & $\mathbf{N}$ \\
\hline $\mathrm{F}_{\mathrm{e} 1.5}=$ & 673.6 & $\mathbf{N}$ \\
\hline$F_{m}=$ & 963.9 & $\mathbf{N}$ \\
\hline$F_{\text {infl }}=$ & 592.8 & $\mathbf{N}$ \\
\hline$F_{f}=$ & 771.1 & $\mathbf{N}$ \\
\hline \multicolumn{3}{|c|}{ Displacement values } \\
\hline $\mathrm{u}_{\mathrm{e}}=$ & 0.138 & $\mathrm{~mm}$ \\
\hline$u_{h 0 / 10, \text { off }}=$ & 0.217 & $\mathrm{~mm}$ \\
\hline $\mathrm{u}_{0.1 \mathrm{~mm}, \mathrm{off}}=$ & 0.322 & $\mathrm{~mm}$ \\
\hline$u_{e 1.5}=$ & 0.334 & $\mathrm{~mm}$ \\
\hline$u_{m}=$ & 0.654 & $\mathrm{~mm}$ \\
\hline$u_{\text {infl }}=$ & 1.666 & $\mathrm{~mm}$ \\
\hline$u_{f}=$ & 1.404 & $\mathrm{~mm}$ \\
\hline \multicolumn{3}{|c|}{ Initial linear slope } \\
\hline Slope $_{\text {ini }}=$ & 2983.80 & $\mathrm{~N} / \mathrm{mm}$ \\
\hline \multicolumn{3}{|c|}{ Energy values } \\
\hline$E_{S P}=$ & 1.05 & J \\
\hline$E_{m}=$ & 0.40 & J \\
\hline $\mathrm{E}_{\mathrm{PL}}=$ & 0.25 & J \\
\hline
\end{tabular}




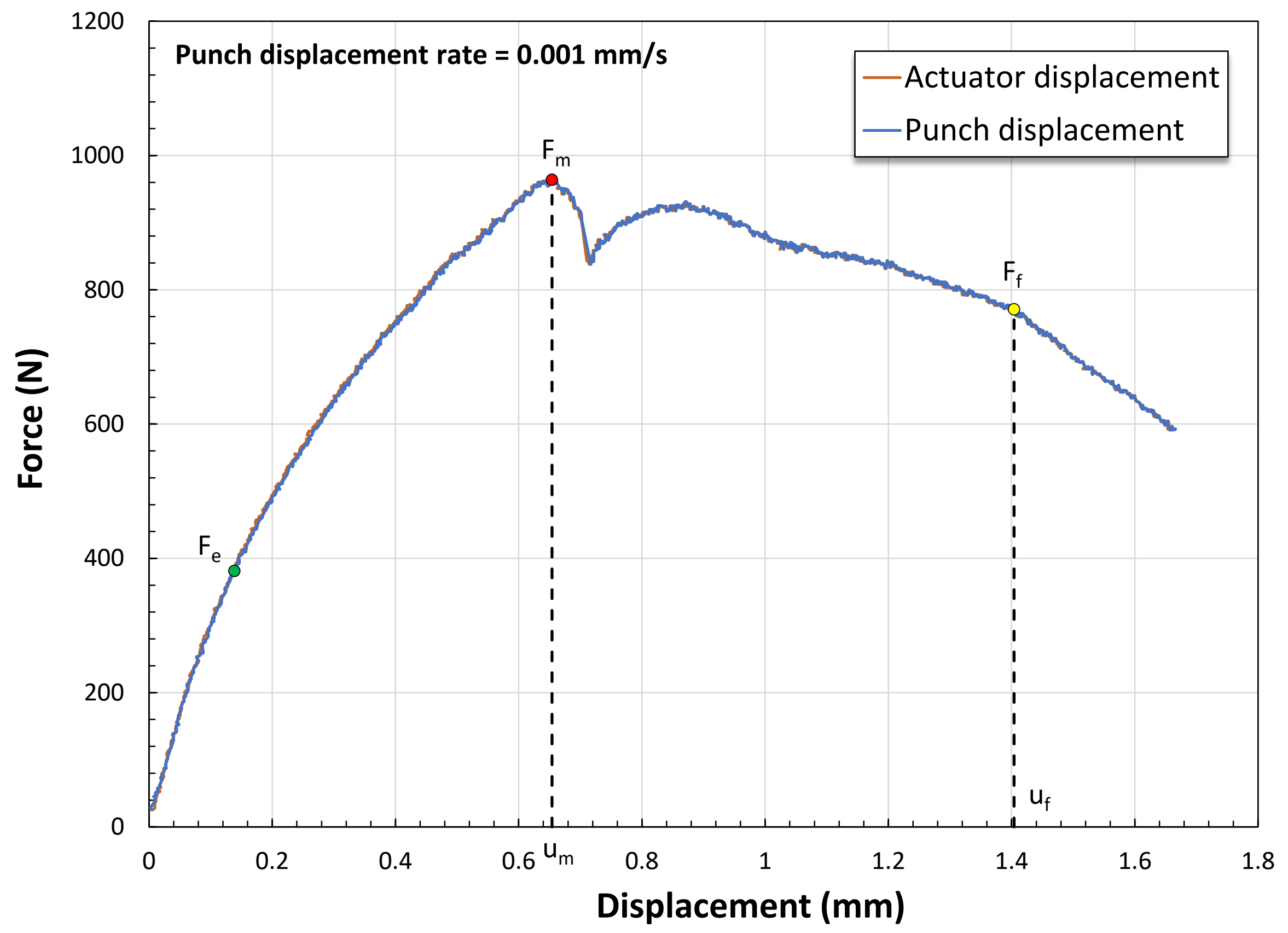




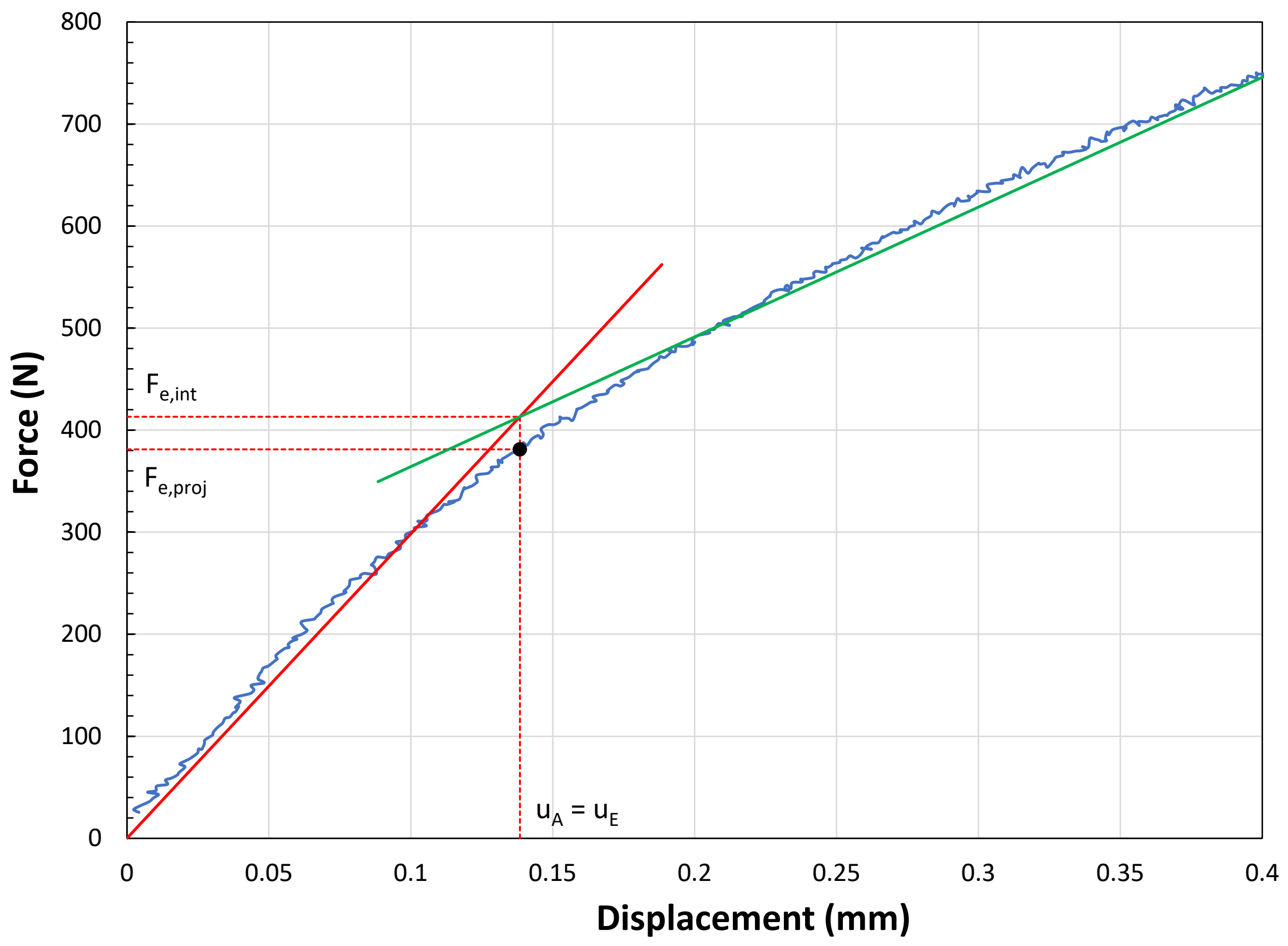


Specimen ID: 800HIP_1_N_a_SP9 (p

Material: AM Ti-64

Test speed: $0.001434 \mathrm{~mm} / \mathrm{s}$

Max strain rate: $1.43 \mathrm{E}-03 \mathrm{~s}^{-1}$

\begin{tabular}{|c|c|c|}
\hline \multicolumn{3}{|c|}{ TEST RESULTS } \\
\hline \multicolumn{3}{|c|}{ Force values } \\
\hline$F_{e, \text { proj }}=$ & 393.2 & $\mathbf{N}$ \\
\hline$F_{e, \text { int }}=$ & 426.2 & $\mathbf{N}$ \\
\hline$F_{h 0 / 10, \text { off }}=$ & 528.3 & $\mathbf{N}$ \\
\hline$F_{0.1 \mathrm{~mm}, \text { off }}=$ & 656.9 & $\mathbf{N}$ \\
\hline$F_{0.1 \mathrm{~mm}}=$ & 317.5 & $\mathbf{N}$ \\
\hline$F_{0.48 \mathrm{~mm}}=$ & 833.0 & $\mathbf{N}$ \\
\hline $\mathrm{F}_{0.5 \mathrm{~mm}}=$ & 853.6 & $\mathbf{N}$ \\
\hline$F_{0.65 \mathrm{~mm}}=$ & 967.8 & $\mathbf{N}$ \\
\hline$F_{0.9 \mathrm{~mm}}=$ & 916.7 & $\mathbf{N}$ \\
\hline$F_{\text {ept }}=$ & 613.3 & $\mathbf{N}$ \\
\hline $\mathrm{F}_{\mathrm{e} 1.5}=$ & 753.9 & $\mathbf{N}$ \\
\hline$F_{m}=$ & 971.8 & $\mathbf{N}$ \\
\hline$F_{\text {infl }}=$ & 623.1 & $\mathbf{N}$ \\
\hline$F_{f}=$ & 777.4 & $\mathbf{N}$ \\
\hline \multicolumn{3}{|c|}{ Displacement values } \\
\hline $\mathrm{u}_{\mathrm{e}}=$ & 0.137 & $\mathrm{~mm}$ \\
\hline$u_{h 0 / 10, \text { off }}=$ & 0.214 & $\mathrm{~mm}$ \\
\hline$u_{0.1 \mathrm{~mm}, \text { off }}=$ & 0.311 & $\mathrm{~mm}$ \\
\hline$u_{e 1.5}=$ & 0.381 & $\mathrm{~mm}$ \\
\hline$u_{m}=$ & 0.691 & $\mathrm{~mm}$ \\
\hline$u_{\text {infl }}=$ & 1.662 & $\mathrm{~mm}$ \\
\hline$u_{f}=$ & 1.475 & $\mathrm{~mm}$ \\
\hline \multicolumn{3}{|c|}{ Initial linear slope } \\
\hline Slope $_{\text {ini }}=$ & 3115.09 & $\mathrm{~N} / \mathrm{mm}$ \\
\hline \multicolumn{3}{|c|}{ Energy values } \\
\hline$E_{S P}=$ & 1.14 & J \\
\hline$E_{m}=$ & 0.44 & J \\
\hline $\mathrm{E}_{\mathrm{PL}}=$ & 0.29 & J \\
\hline
\end{tabular}




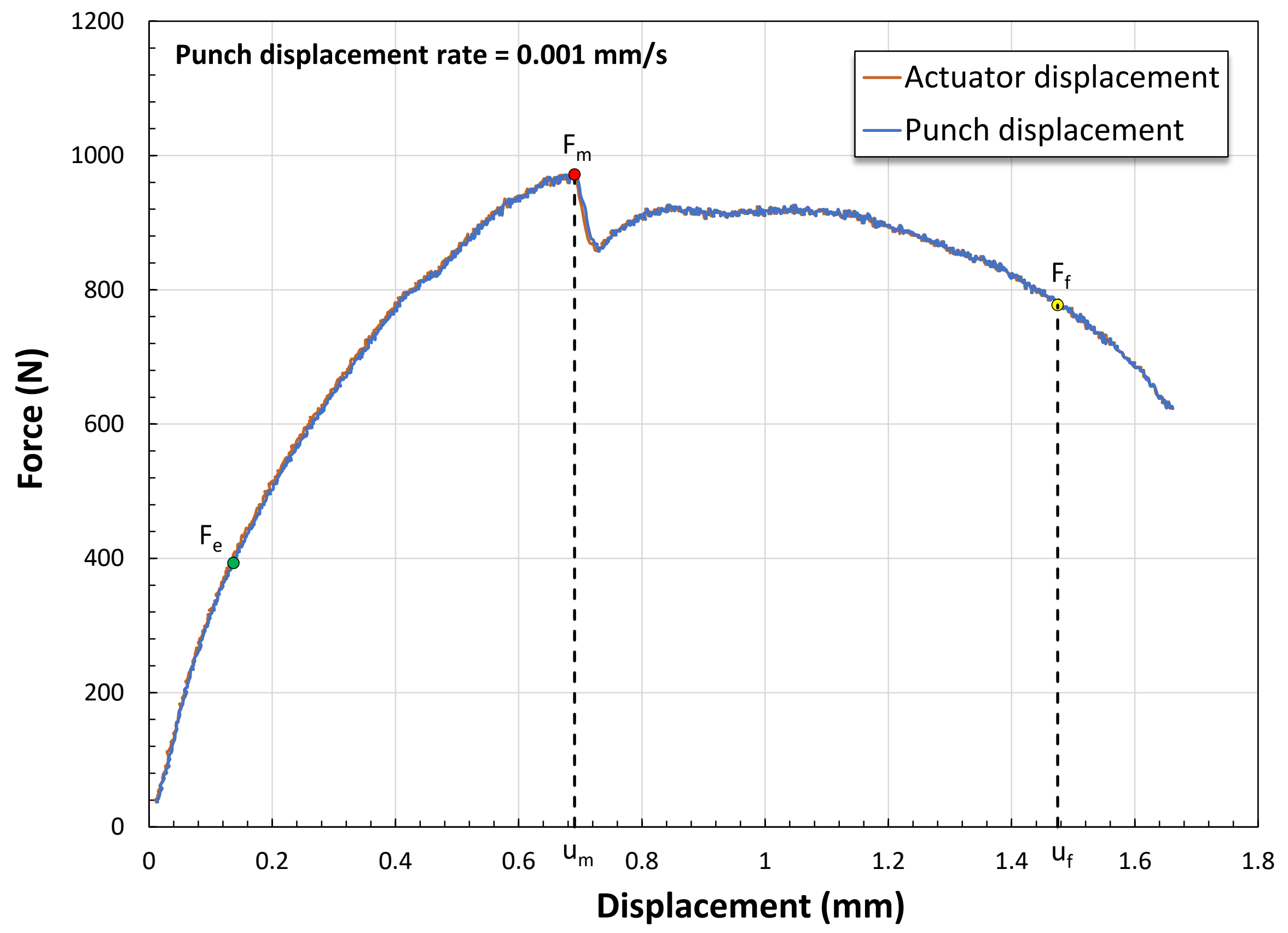




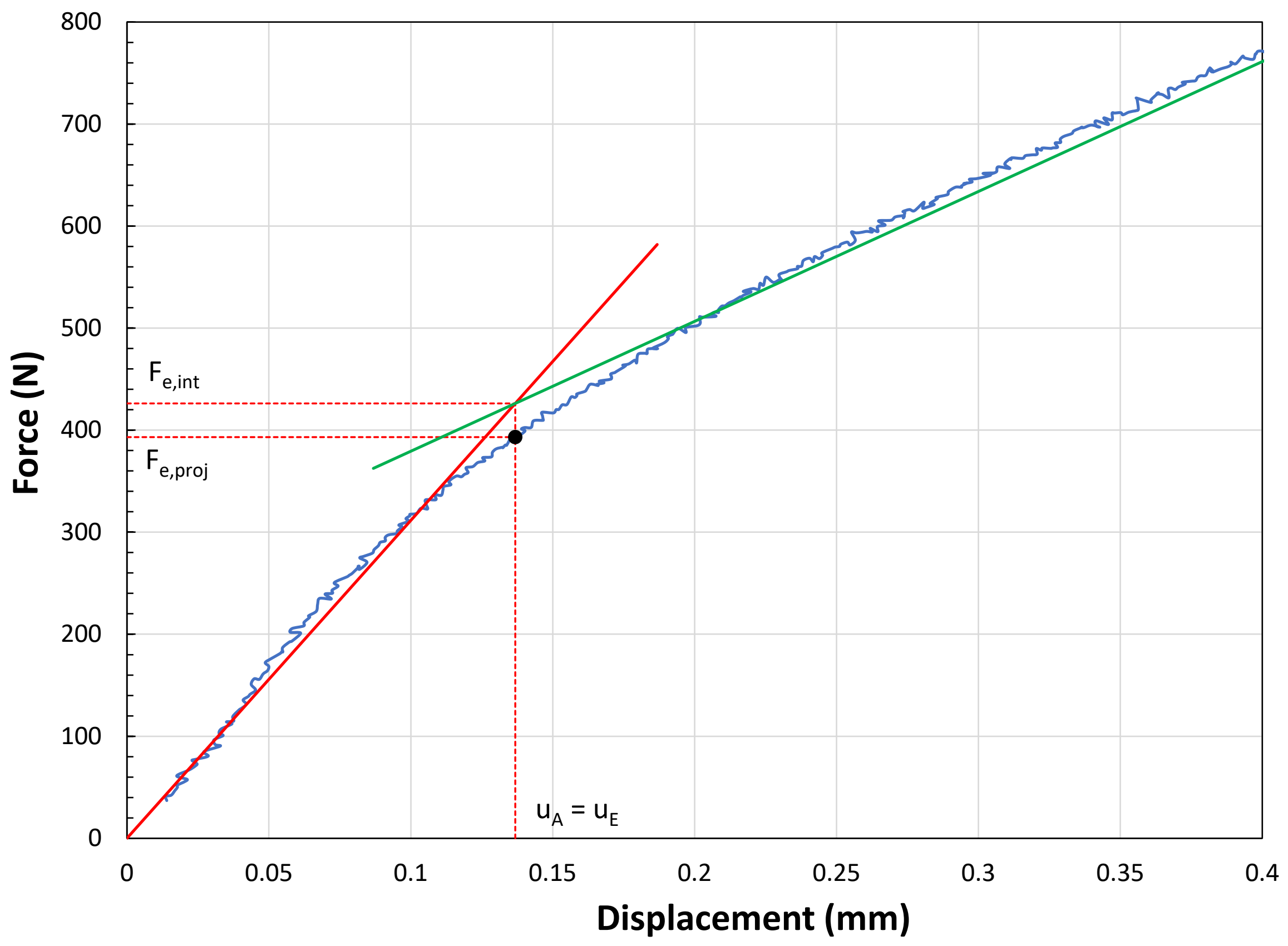


Specimen ID: 800HIP_1_N_a_SP10 (

Material: AM Ti-64

Test speed: $0.001428 \mathrm{~mm} / \mathrm{s}$

Max strain rate: $1.43 \mathrm{E}-03 \mathrm{~s}^{-1}$

\begin{tabular}{|c|c|c|}
\hline \multicolumn{3}{|c|}{ TEST RESULTS } \\
\hline \multicolumn{3}{|c|}{ Force values } \\
\hline$F_{e, \text { proj }}=$ & 348.2 & $\mathbf{N}$ \\
\hline$F_{e, \text { int }}=$ & 381.0 & $\mathbf{N}$ \\
\hline$F_{h 0 / 10, \text { off }}=$ & 484.1 & $\mathbf{N}$ \\
\hline$F_{0.1 \mathrm{~mm}, \text { off }}=$ & 630.0 & $\mathbf{N}$ \\
\hline$F_{0.1 \mathrm{~mm}}=$ & 314.7 & $\mathbf{N}$ \\
\hline$F_{0.48 \mathrm{~mm}}=$ & 854.8 & $\mathbf{N}$ \\
\hline $\mathrm{F}_{0.5 \mathrm{~mm}}=$ & 874.9 & $\mathbf{N}$ \\
\hline$F_{0.65 \mathrm{~mm}}=$ & 917.2 & $\mathbf{N}$ \\
\hline$F_{0.9 \mathrm{~mm}}=$ & 989.1 & $\mathbf{N}$ \\
\hline$F_{\text {ept }}=$ & 558.4 & $\mathbf{N}$ \\
\hline $\mathrm{F}_{\mathrm{e} 1.5}=$ & 589.4 & $\mathbf{N}$ \\
\hline$F_{m}=$ & 996.4 & $\mathbf{N}$ \\
\hline$F_{\text {infl }}=$ & 686.5 & $\mathbf{N}$ \\
\hline$F_{f}=$ & 797.1 & $\mathbf{N}$ \\
\hline \multicolumn{3}{|c|}{ Displacement values } \\
\hline $\mathrm{u}_{\mathrm{e}}=$ & 0.117 & $\mathrm{~mm}$ \\
\hline$u_{h 0 / 10, \text { off }}=$ & 0.193 & $\mathrm{~mm}$ \\
\hline$u_{0.1 \mathrm{~mm}, \text { off }}=$ & 0.294 & $\mathrm{~mm}$ \\
\hline$u_{e 1.5}=$ & 0.265 & $\mathrm{~mm}$ \\
\hline$u_{m}=$ & 0.900 & $\mathrm{~mm}$ \\
\hline$u_{\text {infl }}=$ & 1.416 & $\mathrm{~mm}$ \\
\hline$u_{f}=$ & 1.306 & $\mathrm{~mm}$ \\
\hline \multicolumn{3}{|c|}{ Initial linear slope } \\
\hline Slope $_{\text {ini }}=$ & 3258.38 & $\mathrm{~N} / \mathrm{mm}$ \\
\hline \multicolumn{3}{|c|}{ Energy values } \\
\hline$E_{S P}=$ & 1.02 & J \\
\hline$E_{m}=$ & 0.64 & J \\
\hline $\mathrm{E}_{\mathrm{PL}}=$ & 0.49 & J \\
\hline
\end{tabular}




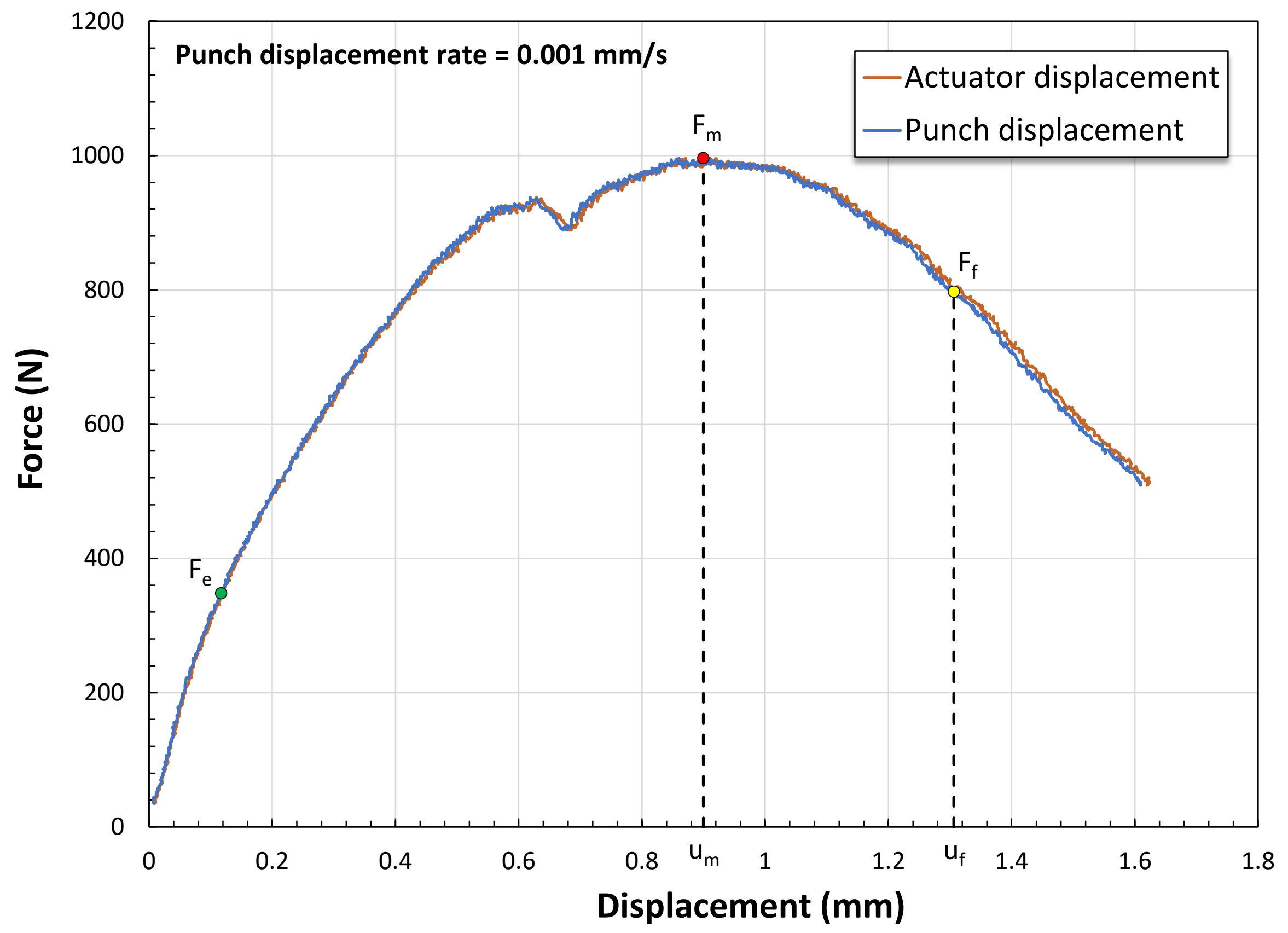




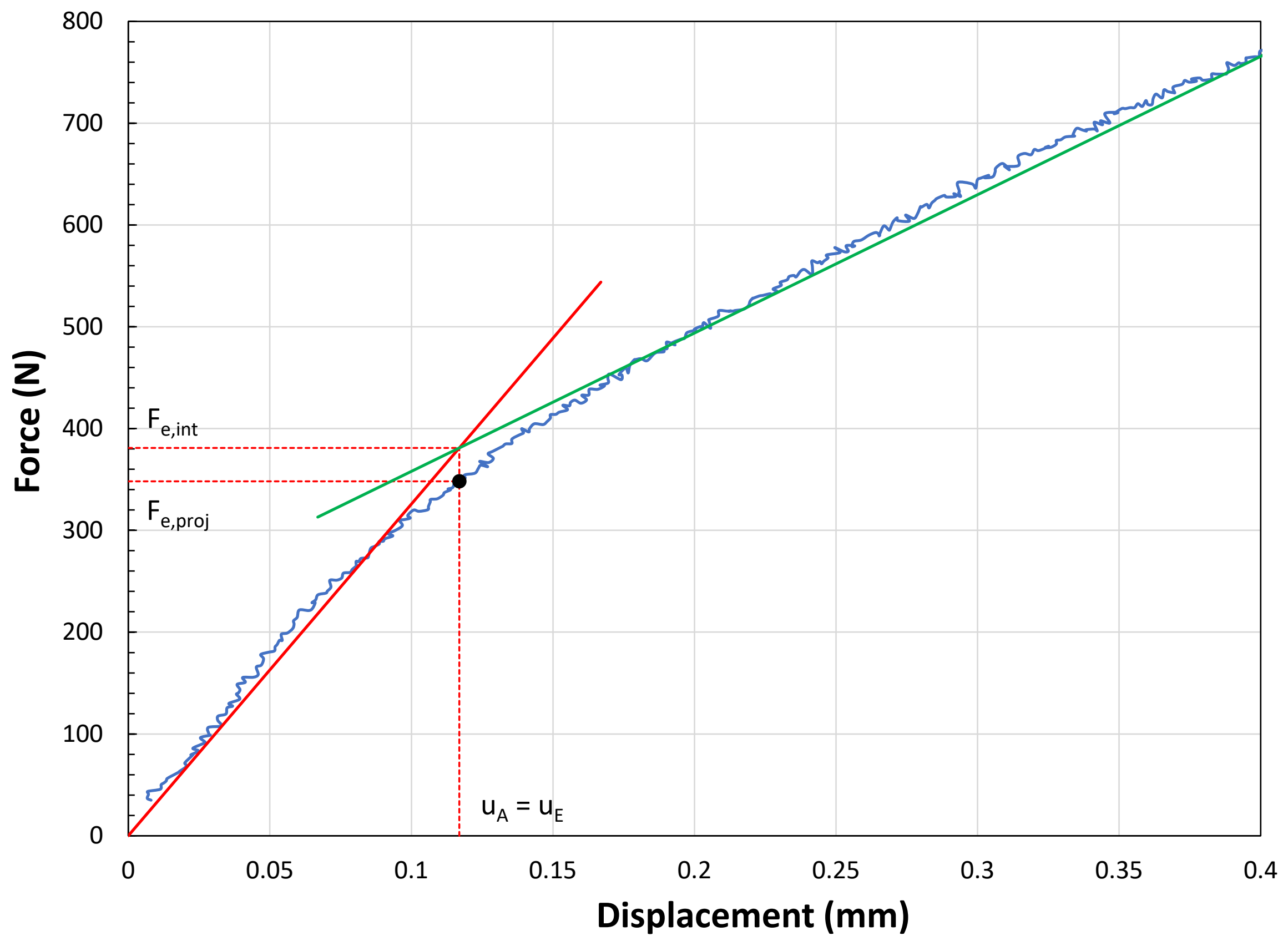




\section{ANNEX 8 \\ SP Test Results for \\ $900{ }^{\circ} \mathrm{C}$ HIP, scan length $=78 \mathrm{~mm}$, non-supported \\ ("rough" specimens)}


Specimen ID: 900HIP_1_N_a_SP2

Material: AM Ti-64

Test speed: $0.001017 \mathrm{~mm} / \mathrm{s}$

Max strain rate: $1.02 \mathrm{E}-03 \mathrm{~s}^{-1}$

\begin{tabular}{|c|c|c|}
\hline \multicolumn{3}{|c|}{ TEST RESULTS } \\
\hline \multicolumn{3}{|c|}{ Force values } \\
\hline$F_{e, p r o j}=$ & 401.3 & $\mathbf{N}$ \\
\hline$F_{e, \text { int }}=$ & 417.4 & $\mathbf{N}$ \\
\hline$F_{h 0 / 10, \text { off }}=$ & 583.9 & $\mathbf{N}$ \\
\hline$F_{0.1 \mathrm{~mm}, \mathrm{off}}=$ & 720.0 & $\mathbf{N}$ \\
\hline$F_{0.1 \mathrm{~mm}}=$ & 207.5 & $\mathbf{N}$ \\
\hline$F_{0.48 \mathrm{~mm}}=$ & 762.7 & $\mathbf{N}$ \\
\hline$F_{0.5 \mathrm{~mm}}=$ & 784.5 & $\mathbf{N}$ \\
\hline$F_{0.65 \mathrm{~mm}}=$ & 918.6 & $\mathbf{N}$ \\
\hline$F_{0.9 \mathrm{~mm}}=$ & 1071.1 & $\mathbf{N}$ \\
\hline $\mathbf{F}_{\mathrm{ept}}=$ & 1038.6 & $\mathbf{N}$ \\
\hline$F_{\mathrm{e} 1.5}=$ & 139.7 & $\mathbf{N}$ \\
\hline $\mathbf{F}_{\mathrm{m}}=$ & 1123.6 & $\mathbf{N}$ \\
\hline$F_{\text {infl }}=$ & 650.9 & $\mathbf{N}$ \\
\hline$F_{f}=$ & 898.9 & $\mathbf{N}$ \\
\hline \multicolumn{3}{|c|}{ Displacement values } \\
\hline $\mathrm{u}_{\mathrm{e}}=$ & 0.199 & $\mathrm{~mm}$ \\
\hline$u_{h 0 / 10, \text { off }}=$ & 0.328 & $\mathrm{~mm}$ \\
\hline$u_{0.1 \mathrm{~mm}, \mathrm{off}}=$ & 0.444 & $\mathrm{~mm}$ \\
\hline$u_{\mathrm{e} 1.5}=$ & 0.064 & $\mathrm{~mm}$ \\
\hline$u_{m}=$ & 1.091 & $\mathrm{~mm}$ \\
\hline$u_{\text {infl }}=$ & 1.748 & $\mathrm{~mm}$ \\
\hline $\mathbf{u}_{\mathrm{f}}=$ & 1.450 & $\mathrm{~mm}$ \\
\hline \multicolumn{3}{|c|}{ Initial linear slope } \\
\hline Slope $_{\text {ini }}=$ & 2099.36 & $\mathrm{~N} / \mathrm{mm}$ \\
\hline \multicolumn{3}{|c|}{ Energy values } \\
\hline$E_{S P}=$ & 1.18 & J \\
\hline$E_{m}=$ & 0.81 & J \\
\hline$E_{P L}=$ & 0.51 & $J$ \\
\hline
\end{tabular}




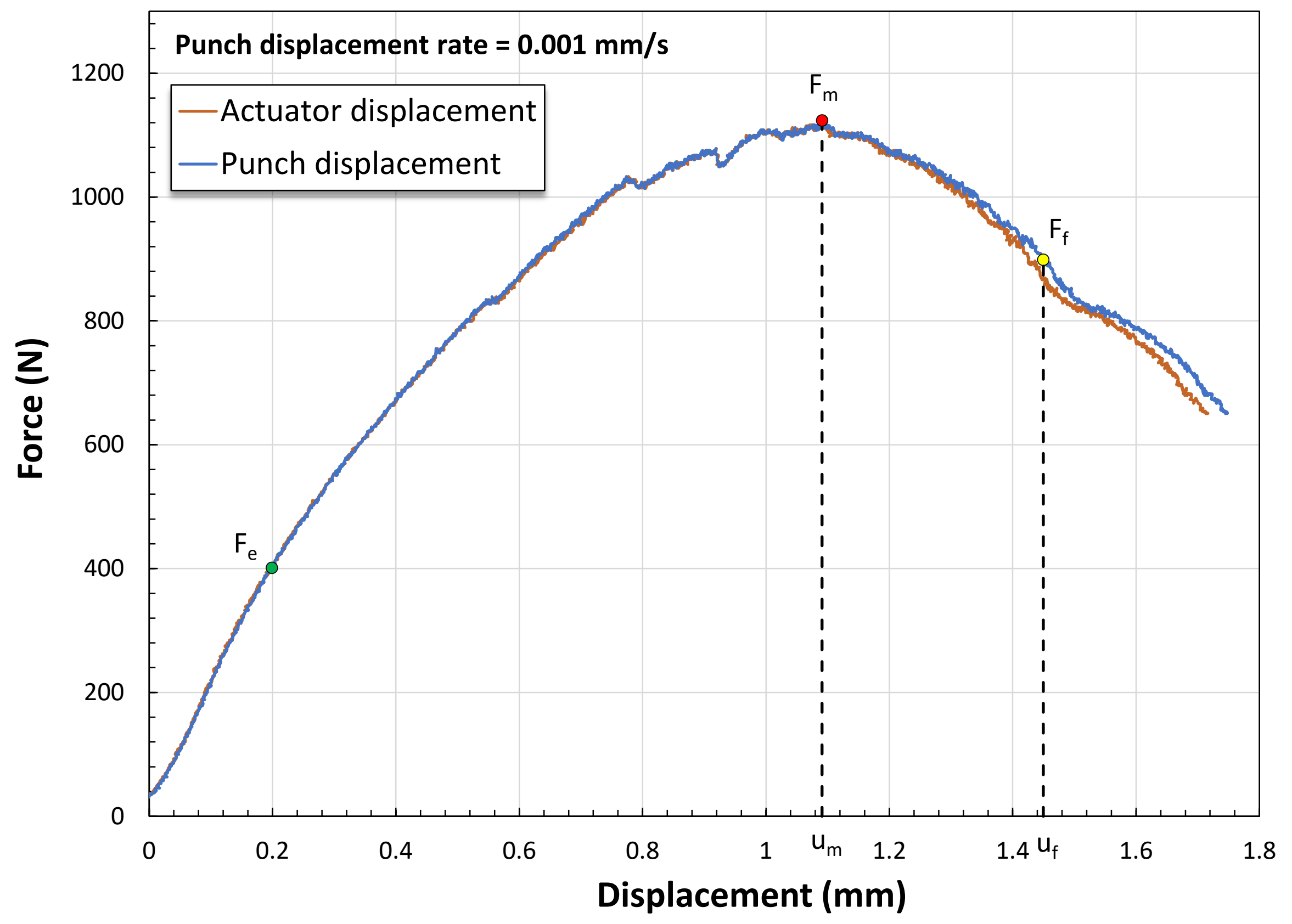




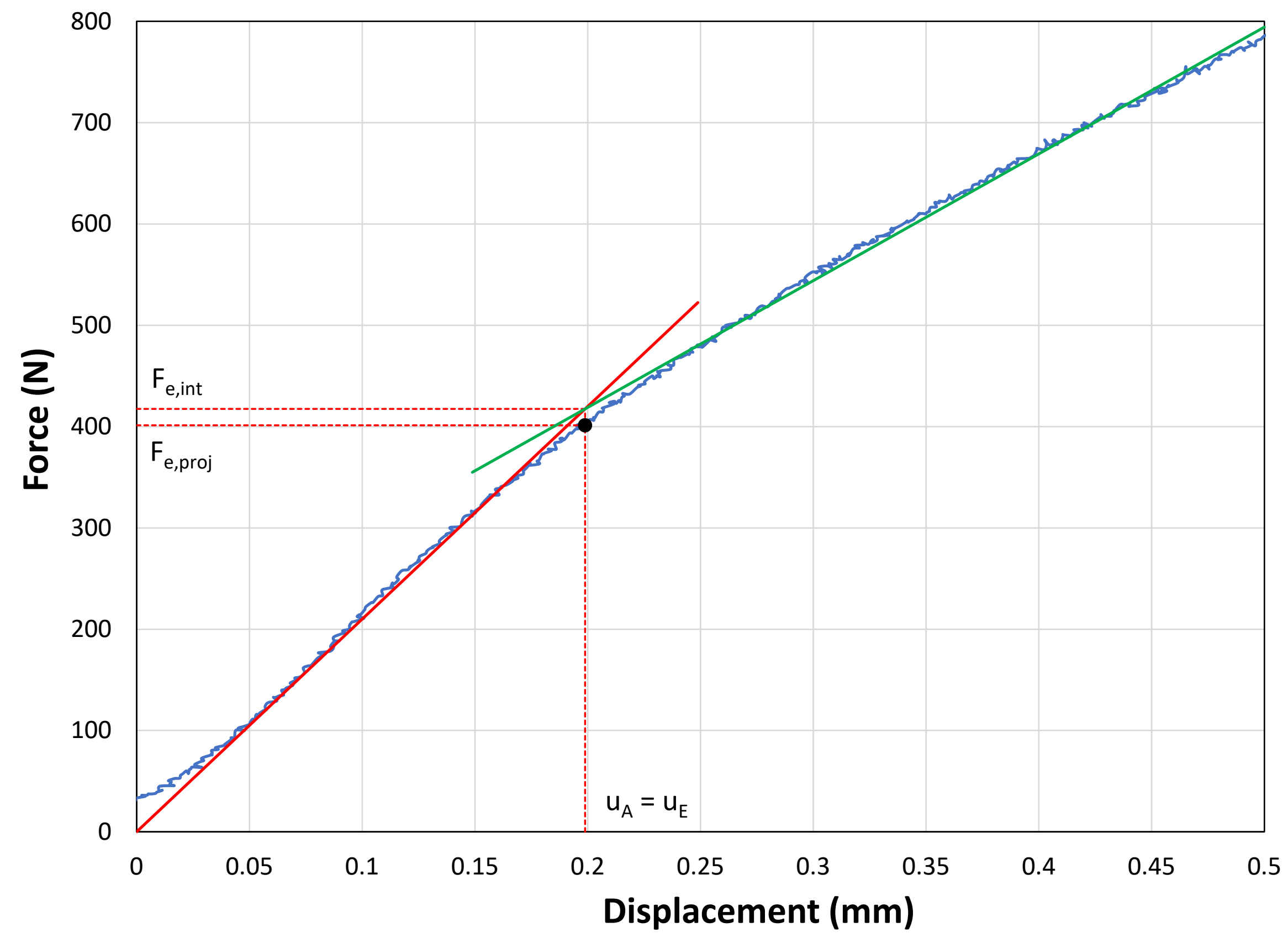


Specimen ID: 900HIP_1_N_a_SP3

Material: AM Ti-64

Test speed: $0.001014 \mathrm{~mm} / \mathrm{s}$

Max strain rate: $1.01 \mathrm{E}-03 \mathrm{~s}^{-1}$

\begin{tabular}{|c|c|c|}
\hline \multicolumn{3}{|c|}{ TEST RESULTS } \\
\hline \multicolumn{3}{|c|}{ Force values } \\
\hline$F_{e, p r o j}=$ & 415.5 & $\mathbf{N}$ \\
\hline$F_{e, \text { int }}=$ & 420.7 & $\mathbf{N}$ \\
\hline$F_{h 0 / 10, \text { off }}=$ & 609.6 & $\mathbf{N}$ \\
\hline$F_{0.1 \mathrm{~mm}, \mathrm{off}}=$ & 766.2 & $\mathbf{N}$ \\
\hline$F_{0.1 \mathrm{~mm}}=$ & 189.0 & $\mathbf{N}$ \\
\hline$F_{0.48 \mathrm{~mm}}=$ & 729.5 & $\mathbf{N}$ \\
\hline$F_{0.5 \mathrm{~mm}}=$ & 748.0 & $\mathbf{N}$ \\
\hline$F_{0.65 \mathrm{~mm}}=$ & 917.2 & $\mathbf{N}$ \\
\hline$F_{0.9 \mathrm{~mm}}=$ & 1037.6 & $\mathbf{N}$ \\
\hline$F_{\mathrm{ept}}=$ & 979.7 & $\mathbf{N}$ \\
\hline$F_{\mathrm{e} 1.5}=$ & 152.6 & $\mathbf{N}$ \\
\hline $\mathbf{F}_{\mathrm{m}}=$ & 1095.6 & $\mathbf{N}$ \\
\hline$F_{\text {infl }}=$ & 731.2 & $\mathbf{N}$ \\
\hline$F_{f}=$ & 876.5 & $\mathbf{N}$ \\
\hline \multicolumn{3}{|c|}{ Displacement values } \\
\hline $\mathrm{u}_{\mathrm{e}}=$ & 0.229 & $\mathrm{~mm}$ \\
\hline$u_{h 0 / 10, \text { off }}=$ & 0.383 & $\mathrm{~mm}$ \\
\hline$u_{0.1 \mathrm{~mm}, \mathrm{off}}=$ & 0.517 & $\mathrm{~mm}$ \\
\hline$u_{\mathrm{e} 1.5}=$ & 0.082 & $\mathrm{~mm}$ \\
\hline$u_{m}=$ & 1.094 & $\mathrm{~mm}$ \\
\hline$u_{\text {infl }}=$ & 0.483 & $\mathrm{~mm}$ \\
\hline$u_{f}=$ & 1.567 & $\mathrm{~mm}$ \\
\hline \multicolumn{3}{|c|}{ Initial linear slope } \\
\hline Slope $_{\text {ini }}=$ & 1839.49 & $\mathrm{~N} / \mathrm{mm}$ \\
\hline \multicolumn{3}{|c|}{ Energy values } \\
\hline$E_{S P}=$ & 1.28 & J \\
\hline$E_{m}=$ & 0.79 & $\mathbf{J}$ \\
\hline$E_{P L}=$ & 0.47 & $J$ \\
\hline
\end{tabular}




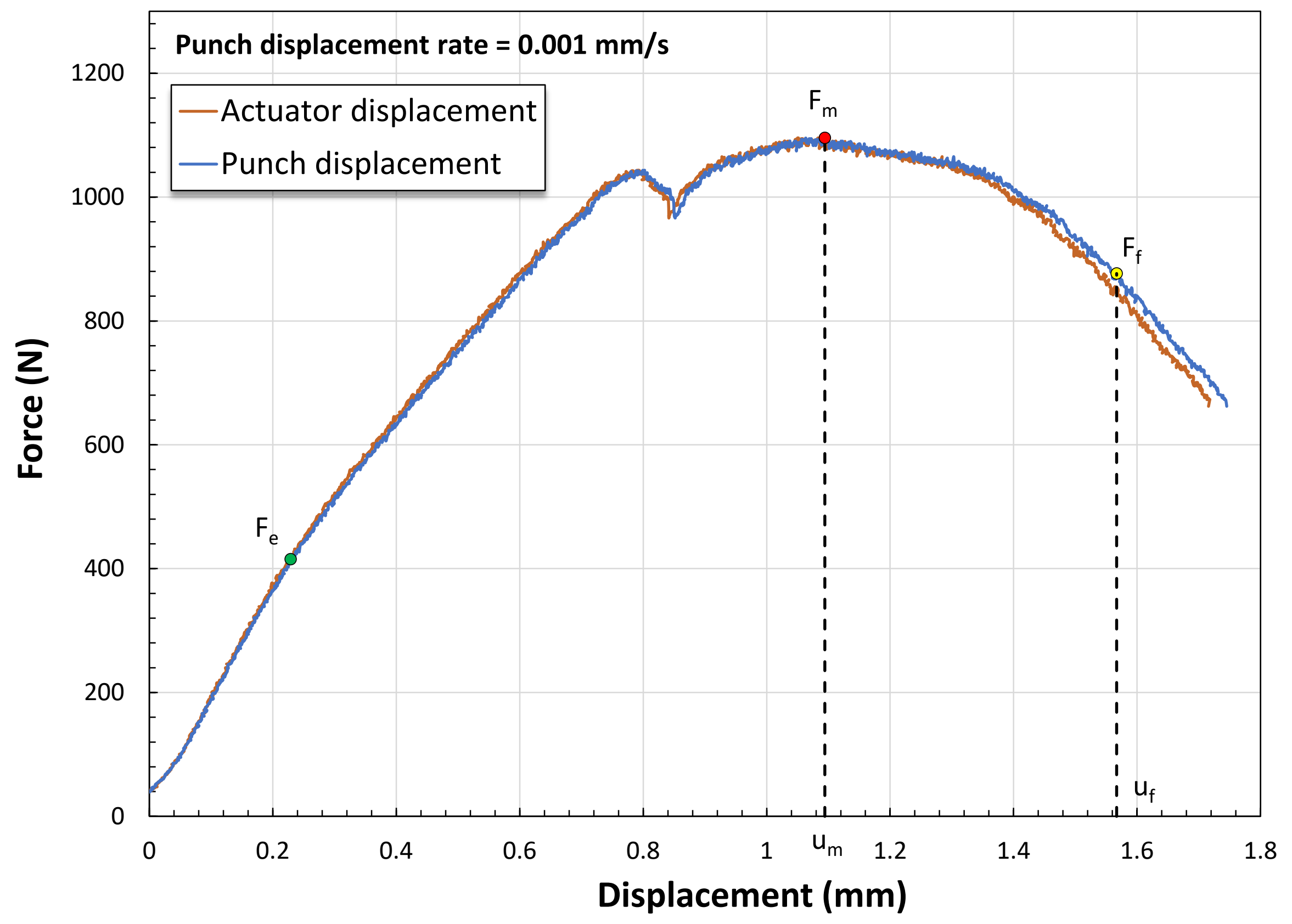




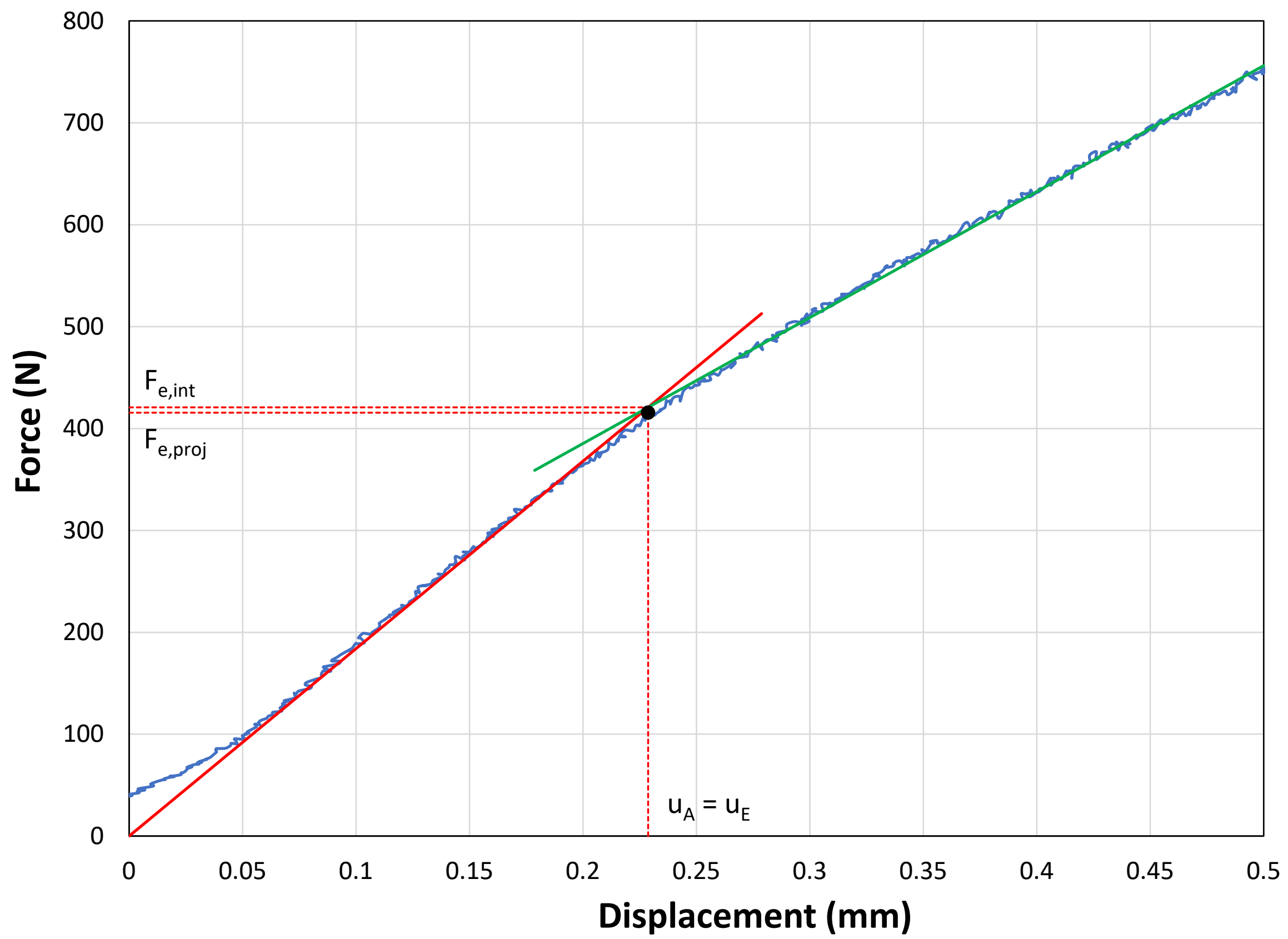


Specimen ID: 900HIP_1_N_a_SP4

Material: AM Ti-64

Test speed: $0.001005 \mathrm{~mm} / \mathrm{s}$

Max strain rate: $1.00 \mathrm{E}-03 \mathrm{~s}^{-1}$

\begin{tabular}{|c|c|c|}
\hline \multicolumn{3}{|c|}{ TEST RESULTS } \\
\hline \multicolumn{3}{|c|}{ Force values } \\
\hline$F_{e, p r o j}=$ & 345.3 & $\mathbf{N}$ \\
\hline$F_{e, \text { int }}=$ & 344.0 & $\mathbf{N}$ \\
\hline$F_{h 0 / 10, \text { off }}=$ & 1011.2 & $\mathbf{N}$ \\
\hline$F_{0.1 \mathrm{~mm}, \mathrm{off}}=$ & 1004.9 & $\mathbf{N}$ \\
\hline$F_{0.1 \mathrm{~mm}}=$ & 82.4 & $\mathbf{N}$ \\
\hline$F_{0.48 \mathrm{~mm}}=$ & 391.9 & $\mathbf{N}$ \\
\hline$F_{0.5 \mathrm{~mm}}=$ & 410.4 & $\mathbf{N}$ \\
\hline$F_{0.65 \mathrm{~mm}}=$ & 521.4 & $\mathbf{N}$ \\
\hline$F_{0.9 \mathrm{~mm}}=$ & 743.9 & $\mathbf{N}$ \\
\hline$F_{\mathrm{ept}}=$ & 193.3 & $\mathbf{N}$ \\
\hline$F_{\mathrm{e} 1.5}=$ & 144.1 & $\mathbf{N}$ \\
\hline $\mathbf{F}_{\mathrm{m}}=$ & 1022.4 & $\mathbf{N}$ \\
\hline$F_{\text {infl }}=$ & 5.0 & $\mathbf{N}$ \\
\hline$F_{f}=$ & 817.9 & $\mathbf{N}$ \\
\hline \multicolumn{3}{|c|}{ Displacement values } \\
\hline $\mathrm{u}_{\mathrm{e}}=$ & 0.417 & $\mathrm{~mm}$ \\
\hline$u_{h 0 / 10, \text { off }}=$ & 1.276 & $\mathrm{~mm}$ \\
\hline$u_{0.1 \mathrm{~mm}, \mathrm{off}}=$ & 1.320 & $\mathrm{~mm}$ \\
\hline$u_{\mathrm{e} 1.5}=$ & 0.176 & $\mathrm{~mm}$ \\
\hline$u_{m}=$ & 1.267 & $\mathrm{~mm}$ \\
\hline$u_{\text {infl }}=$ & -0.107 & $\mathrm{~mm}$ \\
\hline$u_{f}=$ & 1.653 & $\mathrm{~mm}$ \\
\hline \multicolumn{3}{|c|}{ Initial linear slope } \\
\hline Slope $_{\text {ini }}=$ & 825.63 & $\mathrm{~N} / \mathrm{mm}$ \\
\hline \multicolumn{3}{|c|}{ Energy values } \\
\hline$E_{S P}=$ & 1.02 & J \\
\hline$E_{m}=$ & 0.66 & $\mathbf{J}$ \\
\hline$E_{P L}=$ & 0.03 & J \\
\hline
\end{tabular}




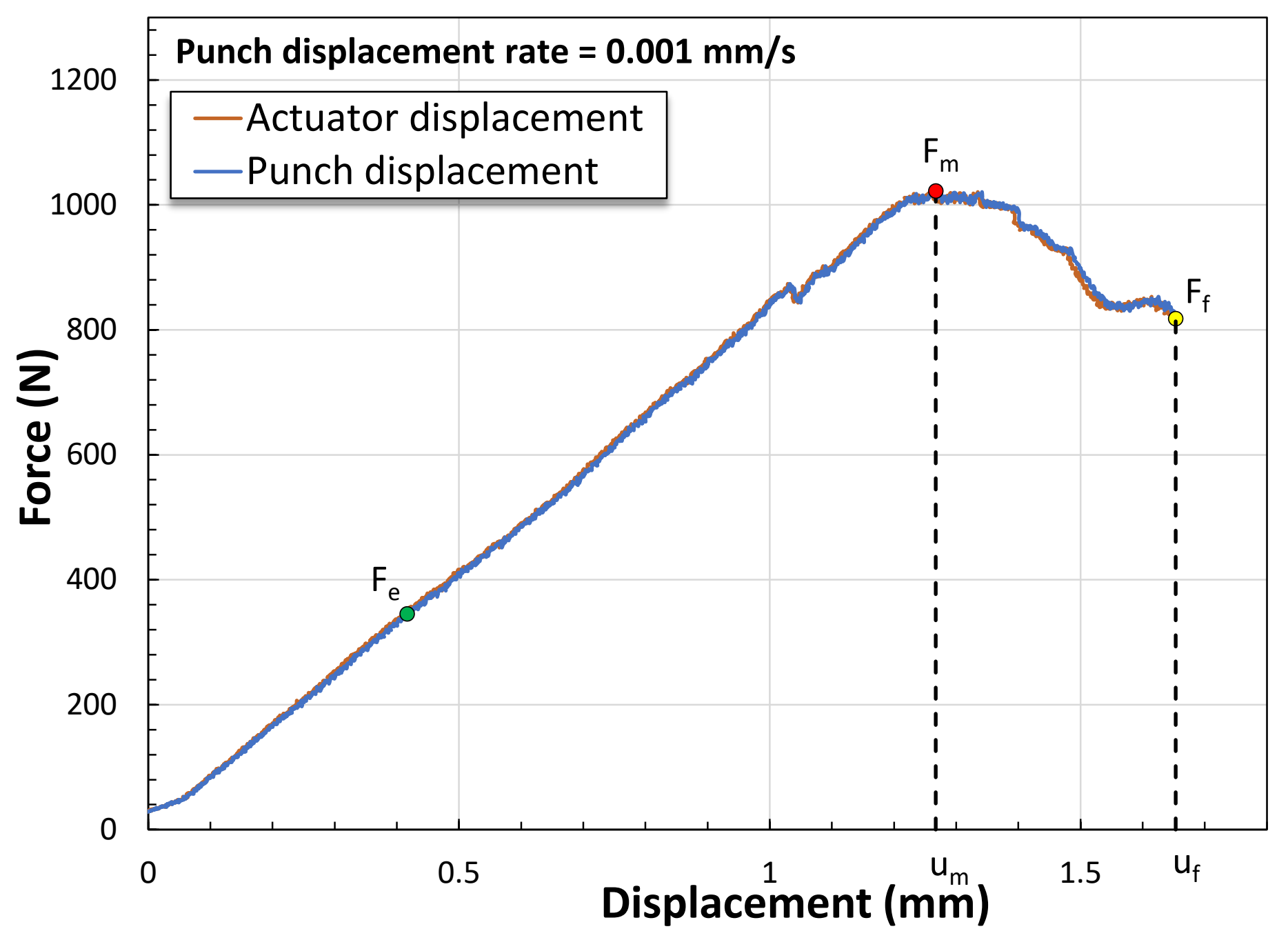




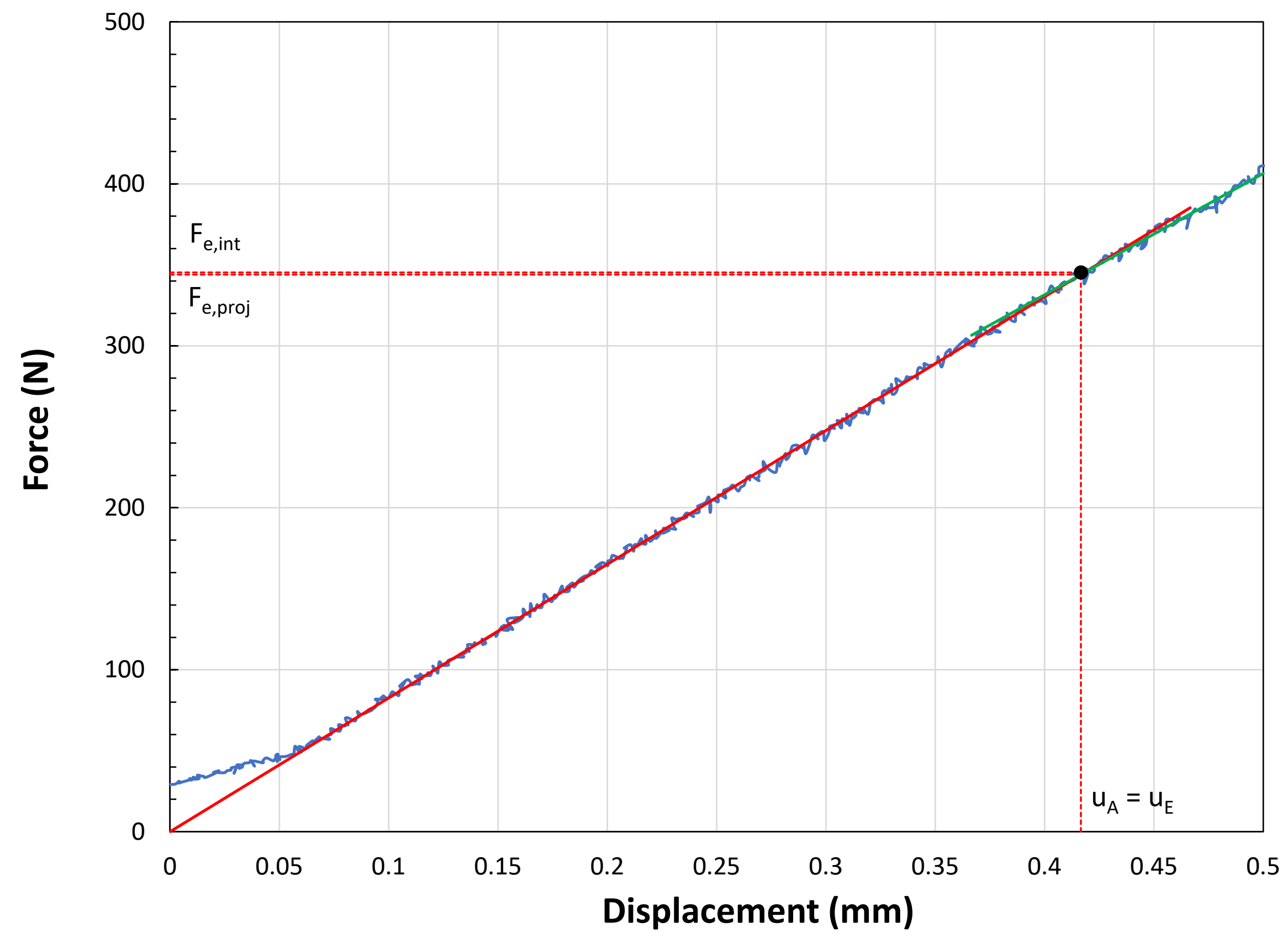


Specimen ID: 900HIP_1_N_a_SP5

Material: AM Ti-64

Test speed: $0.00101 \mathrm{~mm} / \mathrm{s}$

Max strain rate: $1.01 \mathrm{E}-03 \mathrm{~s}^{-1}$

\begin{tabular}{|c|c|c|}
\hline \multicolumn{3}{|c|}{ TEST RESULTS } \\
\hline \multicolumn{3}{|c|}{ Force values } \\
\hline$F_{e, p r o j}=$ & 342.7 & $\mathbf{N}$ \\
\hline$F_{e, \text { int }}=$ & 350.8 & $\mathbf{N}$ \\
\hline$F_{h 0 / 10, \text { off }}=$ & 539.8 & $\mathbf{N}$ \\
\hline$F_{0.1 \mathrm{~mm}, \mathrm{off}}=$ & 723.3 & $\mathbf{N}$ \\
\hline$F_{0.1 \mathrm{~mm}}=$ & 201.3 & $\mathbf{N}$ \\
\hline$F_{0.48 \mathrm{~mm}}=$ & 748.8 & $\mathbf{N}$ \\
\hline$F_{0.5 \mathrm{~mm}}=$ & 773.6 & $\mathbf{N}$ \\
\hline$F_{0.65 \mathrm{~mm}}=$ & 958.5 & $\mathbf{N}$ \\
\hline$F_{0.9 \mathrm{~mm}}=$ & 1047.8 & $\mathbf{N}$ \\
\hline $\mathbf{F}_{\mathrm{ept}}=$ & 97.4 & $\mathbf{N}$ \\
\hline$F_{\mathrm{e} 1.5}=$ & 128.8 & $\mathbf{N}$ \\
\hline $\mathbf{F}_{\mathrm{m}}=$ & 1085.0 & $\mathbf{N}$ \\
\hline$F_{\text {infl }}=$ & 577.3 & $\mathbf{N}$ \\
\hline$F_{f}=$ & 868.0 & $\mathbf{N}$ \\
\hline \multicolumn{3}{|c|}{ Displacement values } \\
\hline $\mathrm{u}_{\mathrm{e}}=$ & 0.177 & $\mathrm{~mm}$ \\
\hline$u_{h 0 / 10, \text { off }}=$ & 0.322 & $\mathrm{~mm}$ \\
\hline$u_{0.1 \mathrm{~mm}, \mathrm{off}}=$ & 0.465 & $\mathrm{~mm}$ \\
\hline$u_{\mathrm{e} 1.5}=$ & 0.064 & $\mathrm{~mm}$ \\
\hline$u_{m}=$ & 0.837 & $\mathrm{~mm}$ \\
\hline$u_{\text {infl }}=$ & 1.742 & $\mathrm{~mm}$ \\
\hline$u_{f}=$ & 1.463 & $\mathrm{~mm}$ \\
\hline \multicolumn{3}{|c|}{ Initial linear slope } \\
\hline Slope $_{\text {ini }}=$ & 1987.01 & $\mathrm{~N} / \mathrm{mm}$ \\
\hline \multicolumn{3}{|c|}{ Energy values } \\
\hline$E_{S P}=$ & 1.17 & J \\
\hline$E_{m}=$ & 0.54 & $\mathbf{J}$ \\
\hline$E_{P L}=$ & 0.24 & $\mathbf{J}$ \\
\hline
\end{tabular}




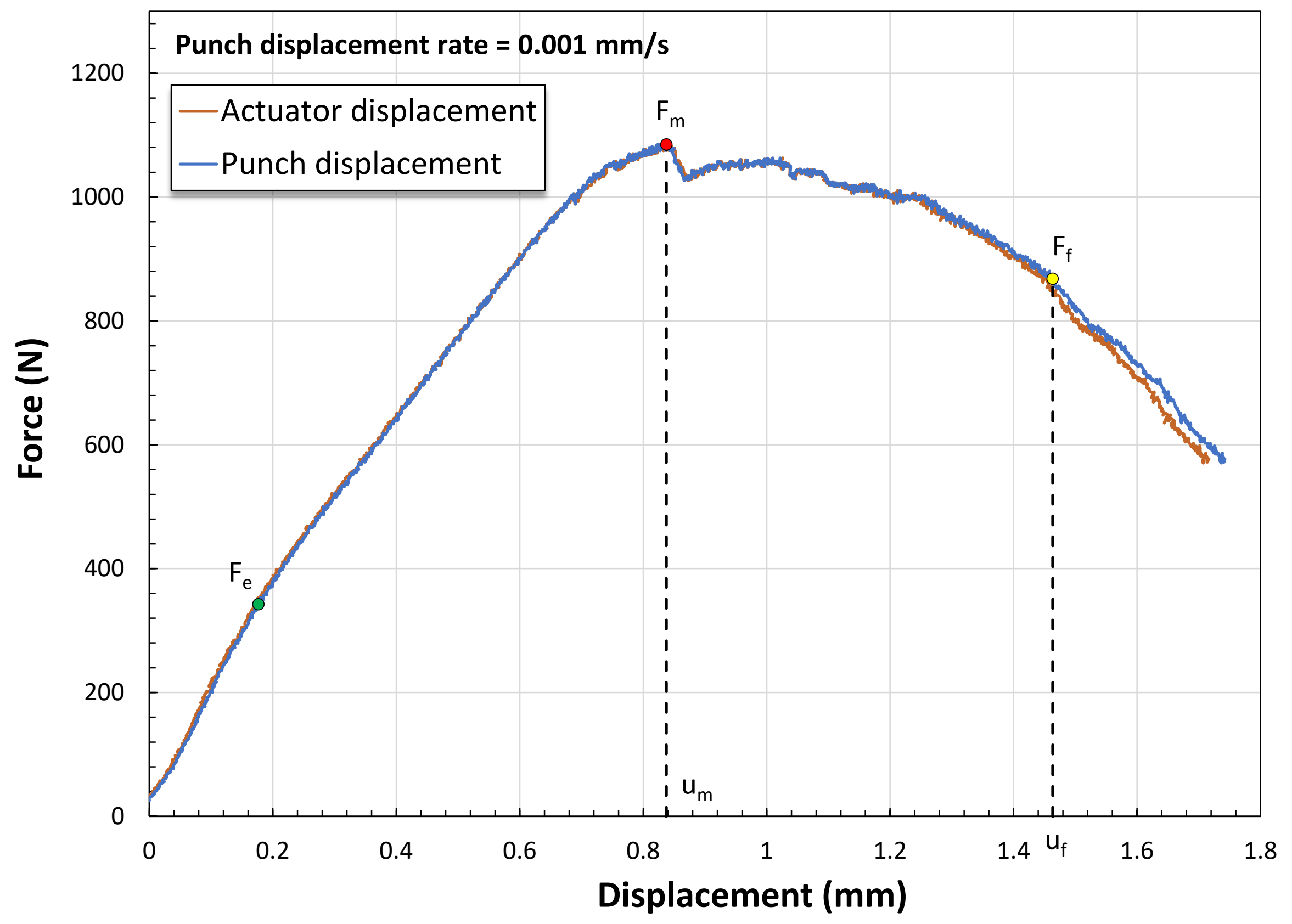




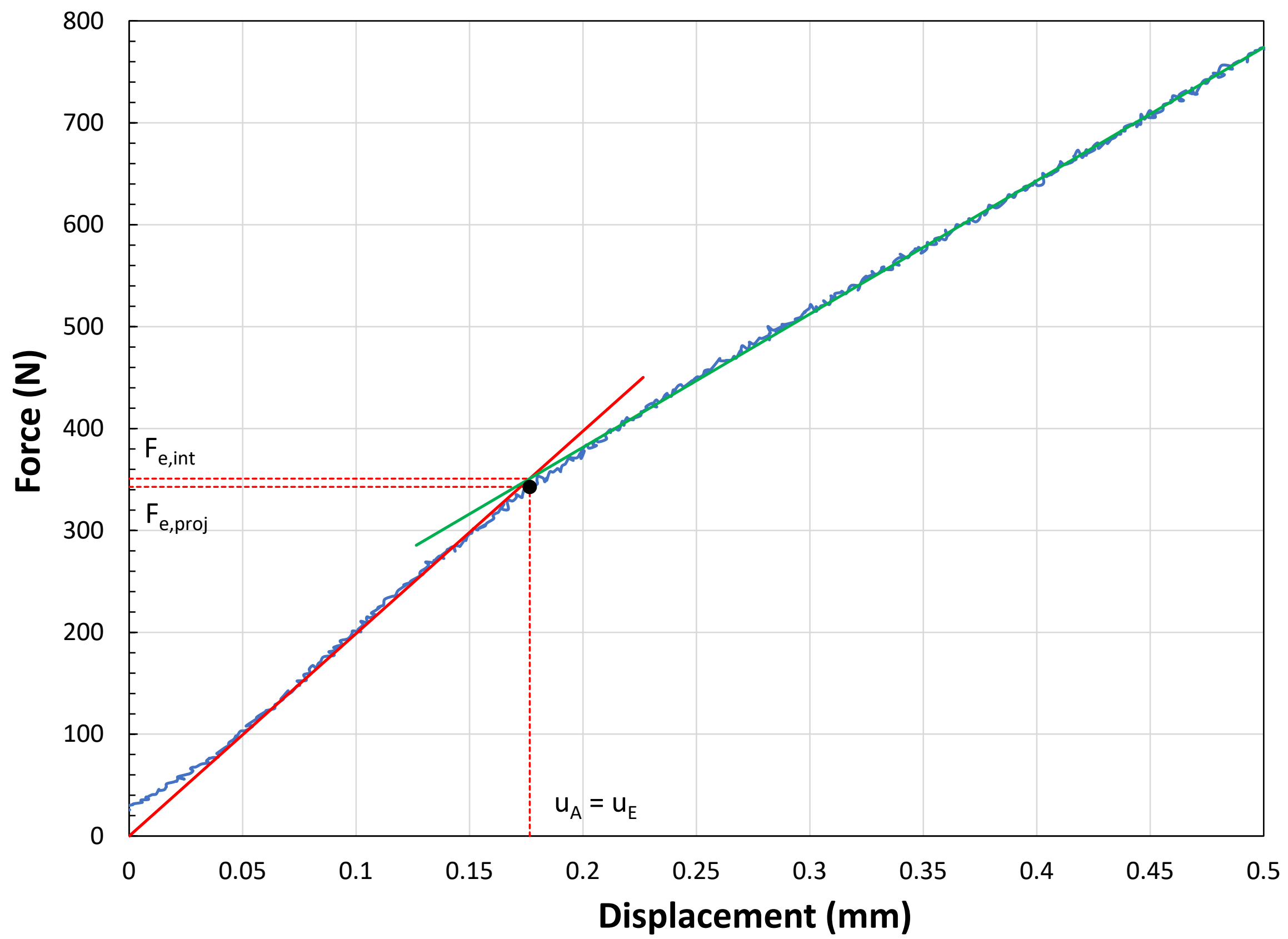


Specimen ID: 900HIP_1_N_a_SP7

Material: AM Ti-64

Test speed: $0.00102 \mathrm{~mm} / \mathrm{s}$

Max strain rate: $1.02 \mathrm{E}-03 \mathrm{~s}^{-1}$

\begin{tabular}{|c|c|c|}
\hline \multicolumn{3}{|c|}{ TEST RESULTS } \\
\hline \multicolumn{3}{|c|}{ Force values } \\
\hline$F_{e, p r o j}=$ & 447.6 & $\mathbf{N}$ \\
\hline $\mathbf{F}_{\mathrm{e}, \text { int }}=$ & 457.9 & $\mathbf{N}$ \\
\hline$F_{h 0 / 10, \text { off }}=$ & 662.2 & $\mathbf{N}$ \\
\hline$F_{0.1 \mathrm{~mm}, \text { off }}=$ & 792.1 & $\mathbf{N}$ \\
\hline$F_{0.1 \mathrm{~mm}}=$ & 211.6 & $\mathbf{N}$ \\
\hline$F_{0.48 \mathrm{~mm}}=$ & 791.3 & $\mathbf{N}$ \\
\hline $\mathrm{F}_{0.5 \mathrm{~mm}}=$ & 810.9 & $\mathbf{N}$ \\
\hline$F_{0.65 \mathrm{~mm}}=$ & 946.7 & $\mathbf{N}$ \\
\hline $\mathrm{F}_{0.9 \mathrm{~mm}}=$ & 1090.2 & $\mathbf{N}$ \\
\hline$F_{\text {ept }}=$ & 1017.1 & $\mathbf{N}$ \\
\hline$F_{\mathrm{e} 1.5}=$ & 134.1 & $\mathbf{N}$ \\
\hline$F_{m}=$ & 1112.3 & $\mathbf{N}$ \\
\hline$F_{\text {infl }}=$ & 515.1 & $\mathbf{N}$ \\
\hline$F_{f}=$ & 889.9 & $\mathbf{N}$ \\
\hline \multicolumn{3}{|c|}{ Displacement values } \\
\hline $\mathrm{u}_{\mathrm{e}}=$ & 0.223 & $\mathrm{~mm}$ \\
\hline$u_{h 0 / 10, \text { off }}=$ & 0.373 & $\mathrm{~mm}$ \\
\hline$u_{0.1 \mathrm{~mm}, \text { off }}=$ & 0.485 & $\mathrm{~mm}$ \\
\hline$u_{\mathrm{e} 1.5}=$ & 0.066 & $\mathrm{~mm}$ \\
\hline$u_{m}=$ & 0.870 & $\mathrm{~mm}$ \\
\hline$u_{i n f l}=$ & 0.268 & $\mathrm{~mm}$ \\
\hline$u_{f}=$ & 1.525 & $\mathrm{~mm}$ \\
\hline \multicolumn{3}{|c|}{ Initial linear slope } \\
\hline Slope $_{\text {ini }}=$ & 2057.23 & $\mathrm{~N} / \mathrm{mm}$ \\
\hline \multicolumn{3}{|c|}{ Energy values } \\
\hline$E_{S P}=$ & 1.25 & J \\
\hline$E_{m}=$ & 0.59 & J \\
\hline $\mathrm{E}_{\mathrm{PL}}=$ & 0.29 & J \\
\hline
\end{tabular}




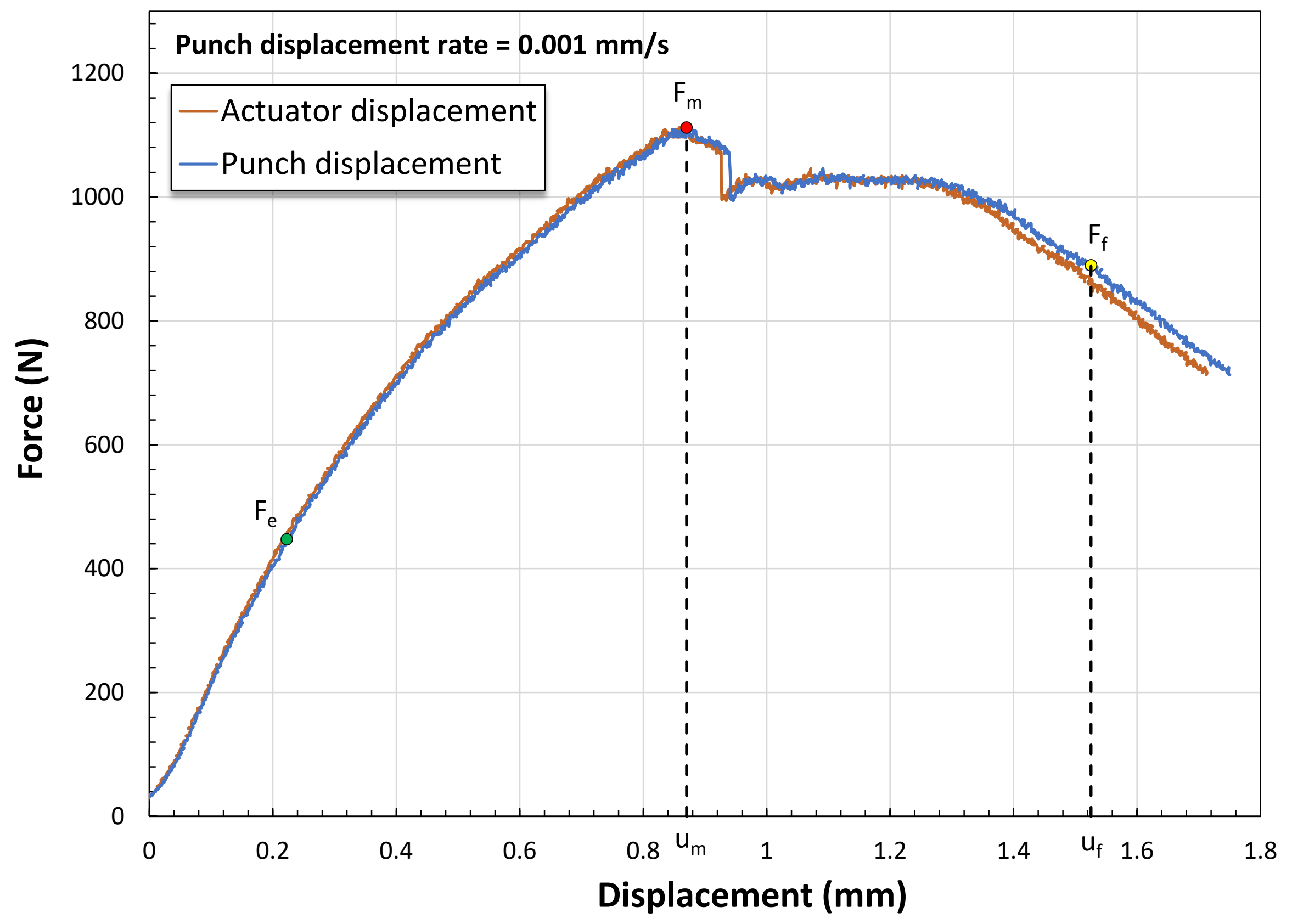




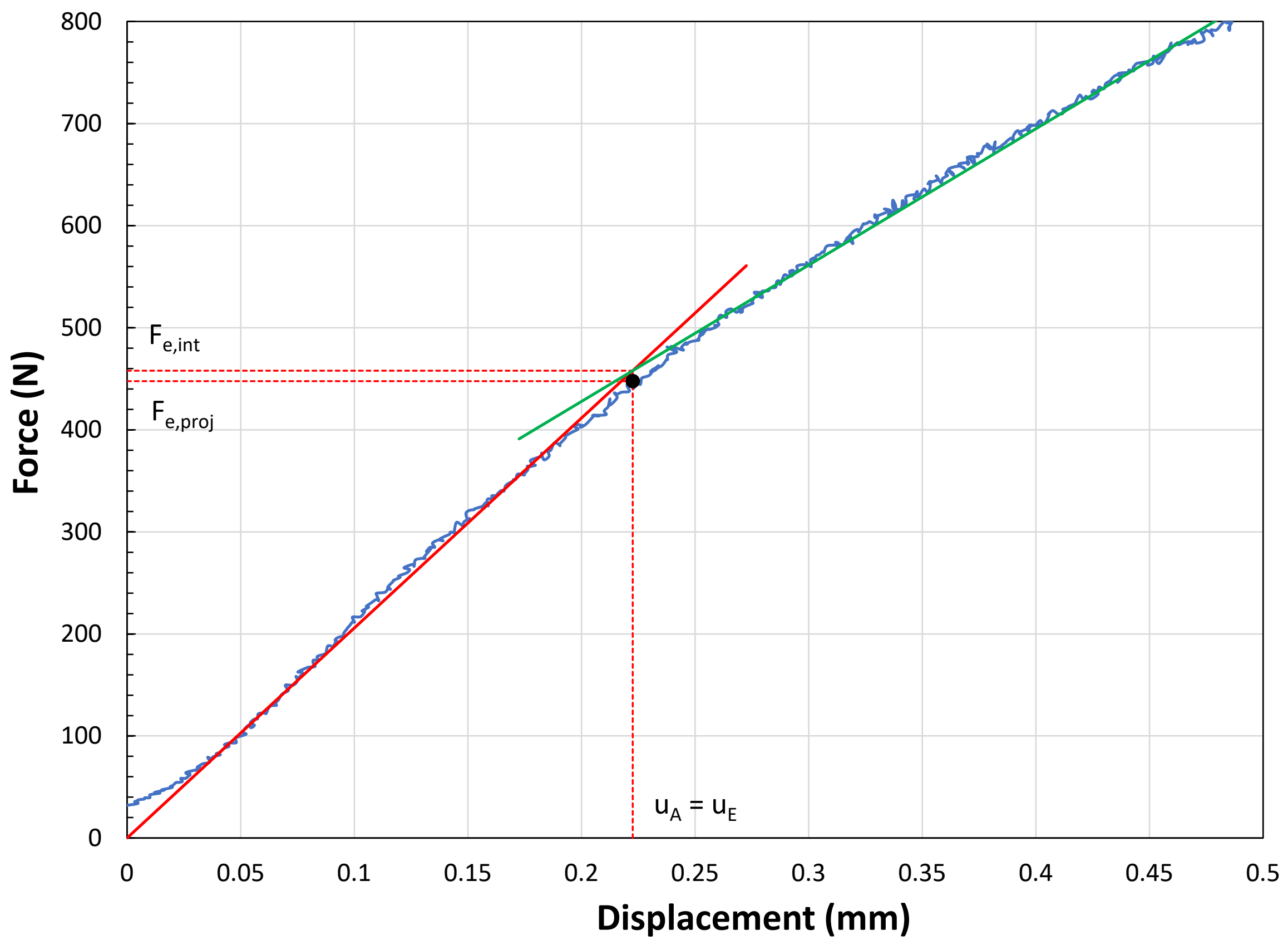


Specimen ID: 900HIP_1_N_a_SP8

Material: AM Ti-64

Test speed: $0.001006 \mathrm{~mm} / \mathrm{s}$

Max strain rate: $1.01 \mathrm{E}-03 \mathrm{~s}^{-1}$

\begin{tabular}{|c|c|c|}
\hline \multicolumn{3}{|c|}{ TEST RESULTS } \\
\hline \multicolumn{3}{|c|}{ Force values } \\
\hline$F_{e, p r o j}=$ & 358.3 & $\mathbf{N}$ \\
\hline$F_{e, \text { int }}=$ & 371.4 & $\mathbf{N}$ \\
\hline$F_{h 0 / 10, \text { off }}=$ & 595.4 & $\mathbf{N}$ \\
\hline$F_{0.1 \mathrm{~mm}, \mathrm{off}}=$ & 781.4 & $\mathbf{N}$ \\
\hline$F_{0.1 \mathrm{~mm}}=$ & 209.2 & $\mathbf{N}$ \\
\hline$F_{0.48 \mathrm{~mm}}=$ & 787.7 & $\mathbf{N}$ \\
\hline$F_{0.5 \mathrm{~mm}}=$ & 809.0 & $\mathbf{N}$ \\
\hline$F_{0.65 \mathrm{~mm}}=$ & 983.9 & $\mathbf{N}$ \\
\hline$F_{0.9 \mathrm{~mm}}=$ & 1042.8 & $\mathbf{N}$ \\
\hline $\mathbf{F}_{\mathrm{ept}}=$ & 1032.2 & $\mathbf{N}$ \\
\hline$F_{\mathrm{e} 1.5}=$ & 138.9 & $\mathbf{N}$ \\
\hline $\mathbf{F}_{\mathrm{m}}=$ & 1067.5 & $\mathbf{N}$ \\
\hline$F_{\text {infl }}=$ & 486.6 & $\mathbf{N}$ \\
\hline$F_{f}=$ & 854.0 & $\mathbf{N}$ \\
\hline \multicolumn{3}{|c|}{ Displacement values } \\
\hline$u_{e}=$ & 0.180 & $\mathrm{~mm}$ \\
\hline$u_{h 0 / 10, \text { off }}=$ & 0.339 & $\mathrm{~mm}$ \\
\hline$u_{0.1 \mathrm{~mm}, \mathrm{off}}=$ & 0.479 & $\mathrm{~mm}$ \\
\hline$u_{e 1.5}=$ & 0.066 & $\mathrm{~mm}$ \\
\hline$u_{m}=$ & 0.950 & $\mathrm{~mm}$ \\
\hline$u_{\text {infl }}=$ & 1.747 & $\mathrm{~mm}$ \\
\hline$u_{f}=$ & 1.337 & $\mathrm{~mm}$ \\
\hline \multicolumn{3}{|c|}{ Initial linear slope } \\
\hline Slope $_{\text {ini }}=$ & 2064.56 & $\mathrm{~N} / \mathrm{mm}$ \\
\hline \multicolumn{3}{|c|}{ Energy values } \\
\hline$E_{S P}=$ & 1.05 & J \\
\hline$E_{m}=$ & 0.67 & J \\
\hline$E_{P L}=$ & 0.39 & $\mathbf{J}$ \\
\hline
\end{tabular}




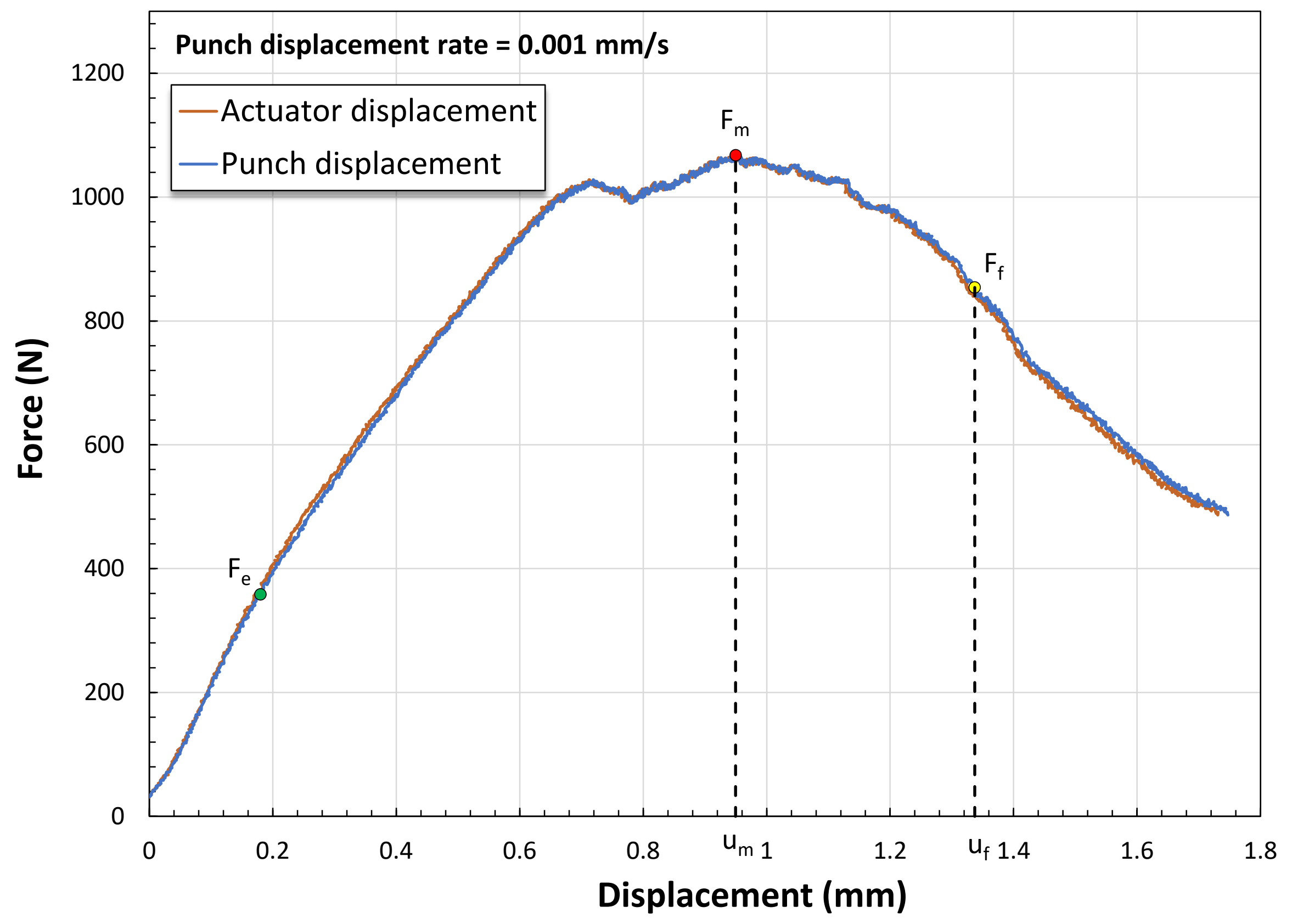




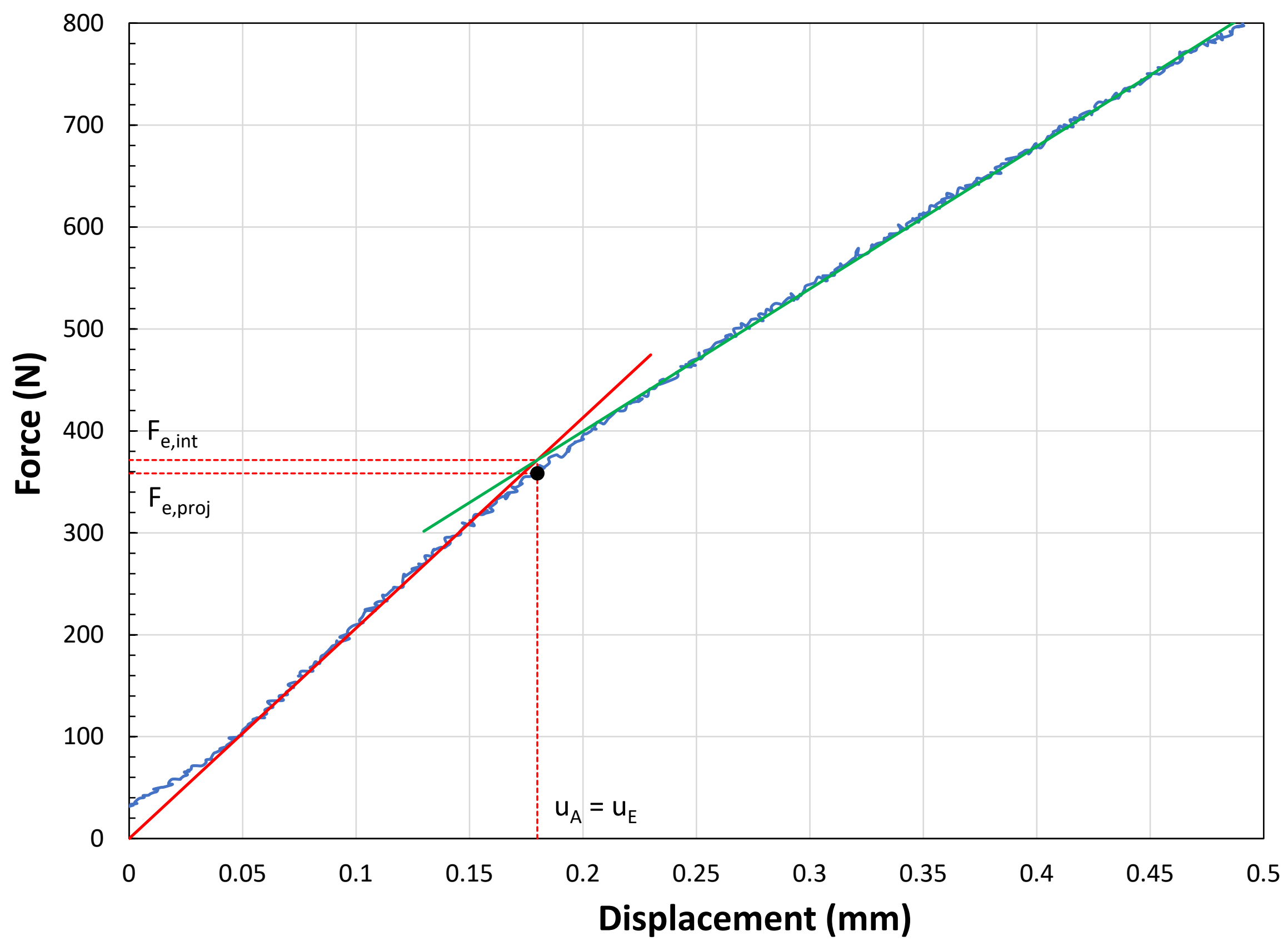




\section{ANNEX 9 \\ SP Test Results for \\ $900{ }^{\circ} \mathrm{C}$ HIP, scan length $=78 \mathrm{~mm}$, non-supported (polished specimens)}


Specimen ID: 900HIP_1_N_a_SP9 (p

Material: AM Ti-64

Test speed: $0.001428 \mathrm{~mm} / \mathrm{s}$

Max strain rate: $1.43 \mathrm{E}-03 \mathrm{~s}^{-1}$

\begin{tabular}{|c|c|c|}
\hline \multicolumn{3}{|c|}{ TEST RESULTS } \\
\hline \multicolumn{3}{|c|}{ Force values } \\
\hline$F_{e, p r o j}=$ & 414.0 & $\mathbf{N}$ \\
\hline $\mathbf{F}_{\mathrm{e}, \text { int }}=$ & 438.5 & $\mathbf{N}$ \\
\hline$F_{h 0 / 10, \text { off }}=$ & 568.5 & $\mathbf{N}$ \\
\hline$F_{0.1 \mathrm{~mm}, \text { off }}=$ & 715.2 & $\mathbf{N}$ \\
\hline$F_{0.1 \mathrm{~mm}}=$ & 301.3 & $\mathbf{N}$ \\
\hline$F_{0.48 \mathrm{~mm}}=$ & 877.3 & $\mathbf{N}$ \\
\hline $\mathrm{F}_{0.5 \mathrm{~mm}}=$ & 898.4 & $\mathbf{N}$ \\
\hline$F_{0.65 \mathrm{~mm}}=$ & 938.6 & $\mathbf{N}$ \\
\hline $\mathrm{F}_{0.9 \mathrm{~mm}}=$ & 1015.8 & $\mathbf{N}$ \\
\hline$F_{\text {ept }}=$ & 156.7 & $\mathbf{N}$ \\
\hline$F_{\mathrm{e} 1.5}=$ & 764.7 & $\mathbf{N}$ \\
\hline$F_{m}=$ & 1031.9 & $\mathbf{N}$ \\
\hline$F_{\text {infl }}=$ & 622.9 & $\mathbf{N}$ \\
\hline$F_{f}=$ & 825.5 & $\mathbf{N}$ \\
\hline \multicolumn{3}{|c|}{ Displacement values } \\
\hline $\mathrm{u}_{\mathrm{e}}=$ & 0.151 & $\mathrm{~mm}$ \\
\hline$u_{h 0 / 10, \text { off }}=$ & 0.241 & $\mathrm{~mm}$ \\
\hline$u_{0.1 \mathrm{~mm}, \text { off }}=$ & 0.347 & $\mathrm{~mm}$ \\
\hline$u_{\mathrm{e} 1.5}=$ & 0.391 & $\mathrm{~mm}$ \\
\hline$u_{m}=$ & 0.955 & $\mathrm{~mm}$ \\
\hline$u_{i n f l}=$ & 1.663 & $\mathrm{~mm}$ \\
\hline$u_{f}=$ & 1.448 & $\mathrm{~mm}$ \\
\hline \multicolumn{3}{|c|}{ Initial linear slope } \\
\hline Slope $_{\text {ini }}=$ & 2910.50 & $\mathrm{~N} / \mathrm{mm}$ \\
\hline \multicolumn{3}{|c|}{ Energy values } \\
\hline$E_{S P}=$ & 1.18 & J \\
\hline$E_{m}=$ & 0.71 & J \\
\hline $\mathrm{E}_{\mathrm{PL}}=$ & 0.53 & J \\
\hline
\end{tabular}




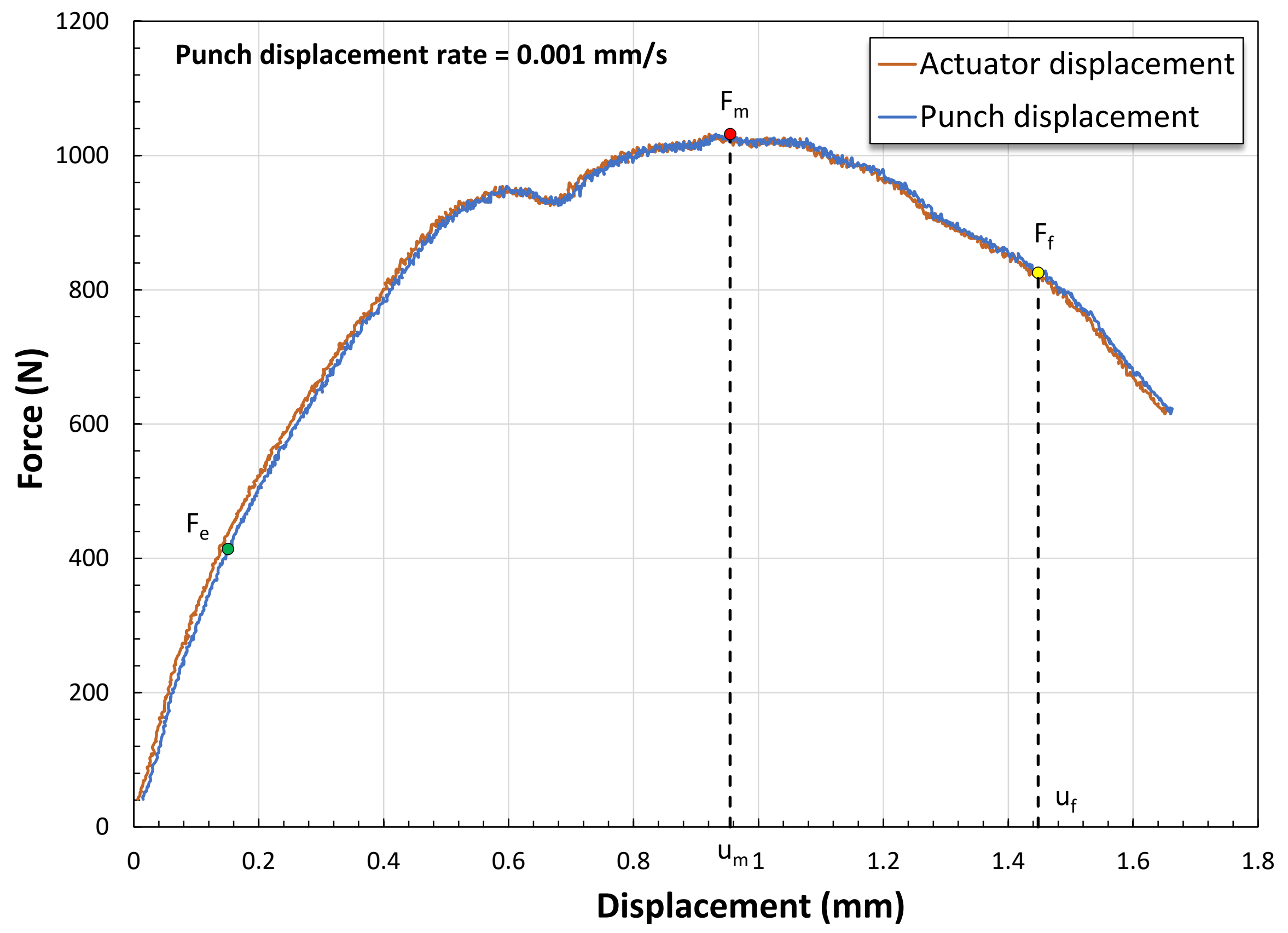




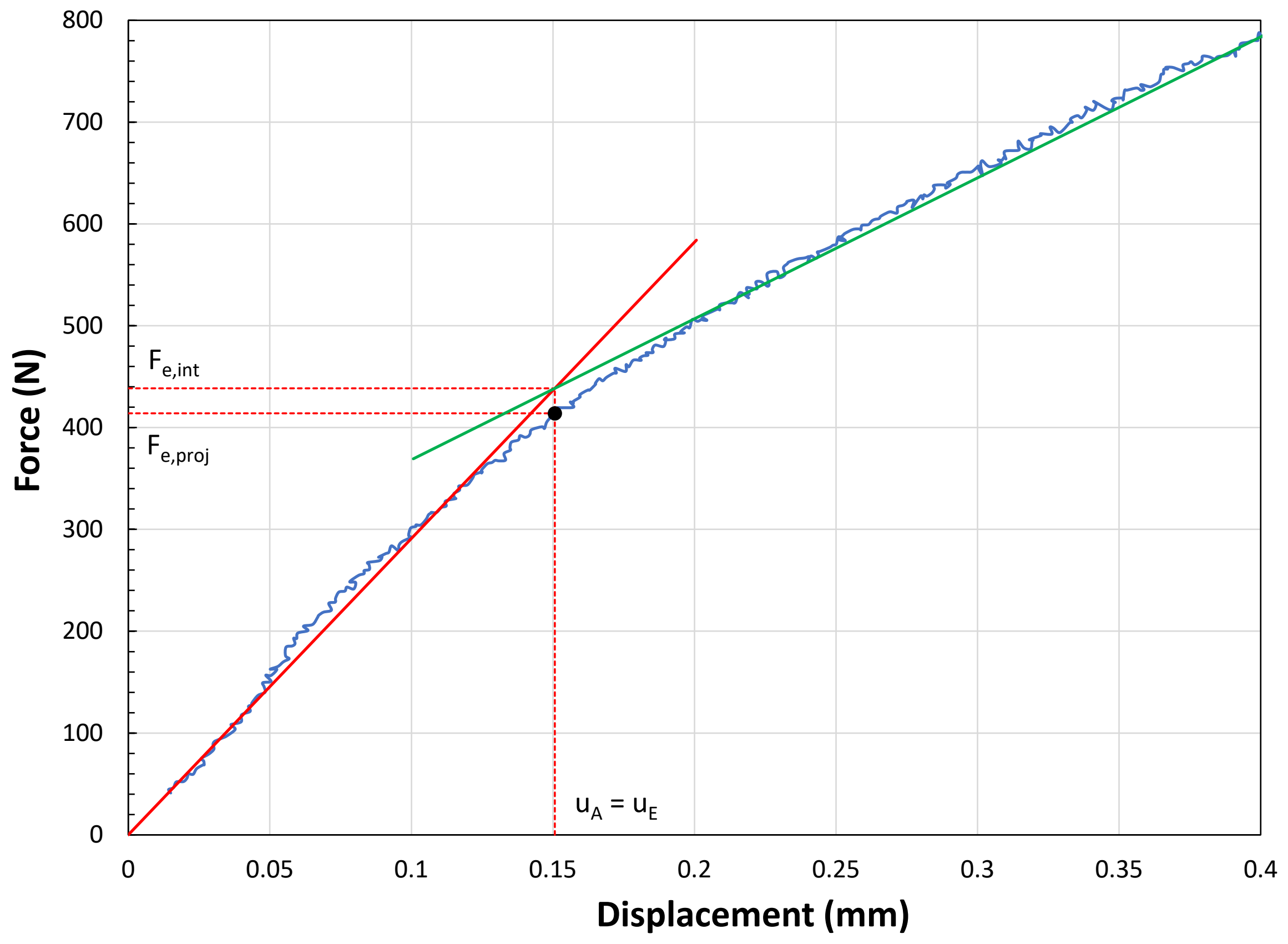


Specimen ID: 900HIP_1_N_a_SP10 (

Material: AM Ti-64

Test speed: $0.001438 \mathrm{~mm} / \mathrm{s}$

Max strain rate: $1.44 \mathrm{E}-03 \mathrm{~s}^{-1}$

\begin{tabular}{|c|c|c|}
\hline \multicolumn{3}{|c|}{ TEST RESULTS } \\
\hline \multicolumn{3}{|c|}{ Force values } \\
\hline$F_{e, p r o j}=$ & 357.8 & $\mathbf{N}$ \\
\hline $\mathbf{F}_{\mathrm{e}, \text { int }}=$ & 385.1 & $\mathbf{N}$ \\
\hline$F_{h 0 / 10, \text { off }}=$ & 496.9 & $\mathbf{N}$ \\
\hline$F_{0.1 \mathrm{~mm}, \text { off }}=$ & 656.5 & $\mathbf{N}$ \\
\hline$F_{0.1 \mathrm{~mm}}=$ & 311.3 & $\mathbf{N}$ \\
\hline$F_{0.48 \mathrm{~mm}}=$ & 869.2 & $\mathbf{N}$ \\
\hline $\mathrm{F}_{0.5 \mathrm{~mm}}=$ & 881.3 & $\mathbf{N}$ \\
\hline$F_{0.65 \mathrm{~mm}}=$ & 971.0 & $\mathbf{N}$ \\
\hline$F_{0.9 \mathrm{~mm}}=$ & 1074.2 & $\mathbf{N}$ \\
\hline$F_{\text {ept }}=$ & 676.9 & $\mathbf{N}$ \\
\hline$F_{\mathrm{e} 1.5}=$ & 714.7 & $\mathbf{N}$ \\
\hline$F_{m}=$ & 1081.8 & $\mathbf{N}$ \\
\hline$F_{\text {infl }}=$ & 573.1 & $\mathbf{N}$ \\
\hline$F_{f}=$ & 865.4 & $\mathbf{N}$ \\
\hline \multicolumn{3}{|c|}{ Displacement values } \\
\hline $\mathrm{u}_{\mathrm{e}}=$ & 0.121 & $\mathrm{~mm}$ \\
\hline$u_{h 0 / 10, \text { off }}=$ & 0.203 & $\mathrm{~mm}$ \\
\hline$u_{0.1 \mathrm{~mm}, \text { off }}=$ & 0.307 & $\mathrm{~mm}$ \\
\hline$u_{\mathrm{e} 1.5}=$ & 0.354 & $\mathrm{~mm}$ \\
\hline$u_{m}=$ & 0.891 & $\mathrm{~mm}$ \\
\hline$u_{i n f l}=$ & 1.676 & $\mathrm{~mm}$ \\
\hline$u_{f}=$ & 1.306 & $\mathrm{~mm}$ \\
\hline \multicolumn{3}{|c|}{ Initial linear slope } \\
\hline Slope $_{\text {ini }}=$ & 3178.87 & $\mathrm{~N} / \mathrm{mm}$ \\
\hline \multicolumn{3}{|c|}{ Energy values } \\
\hline$E_{S P}=$ & 1.07 & J \\
\hline$E_{m}=$ & 0.66 & J \\
\hline$E_{p L}=$ & 0.47 & J \\
\hline
\end{tabular}




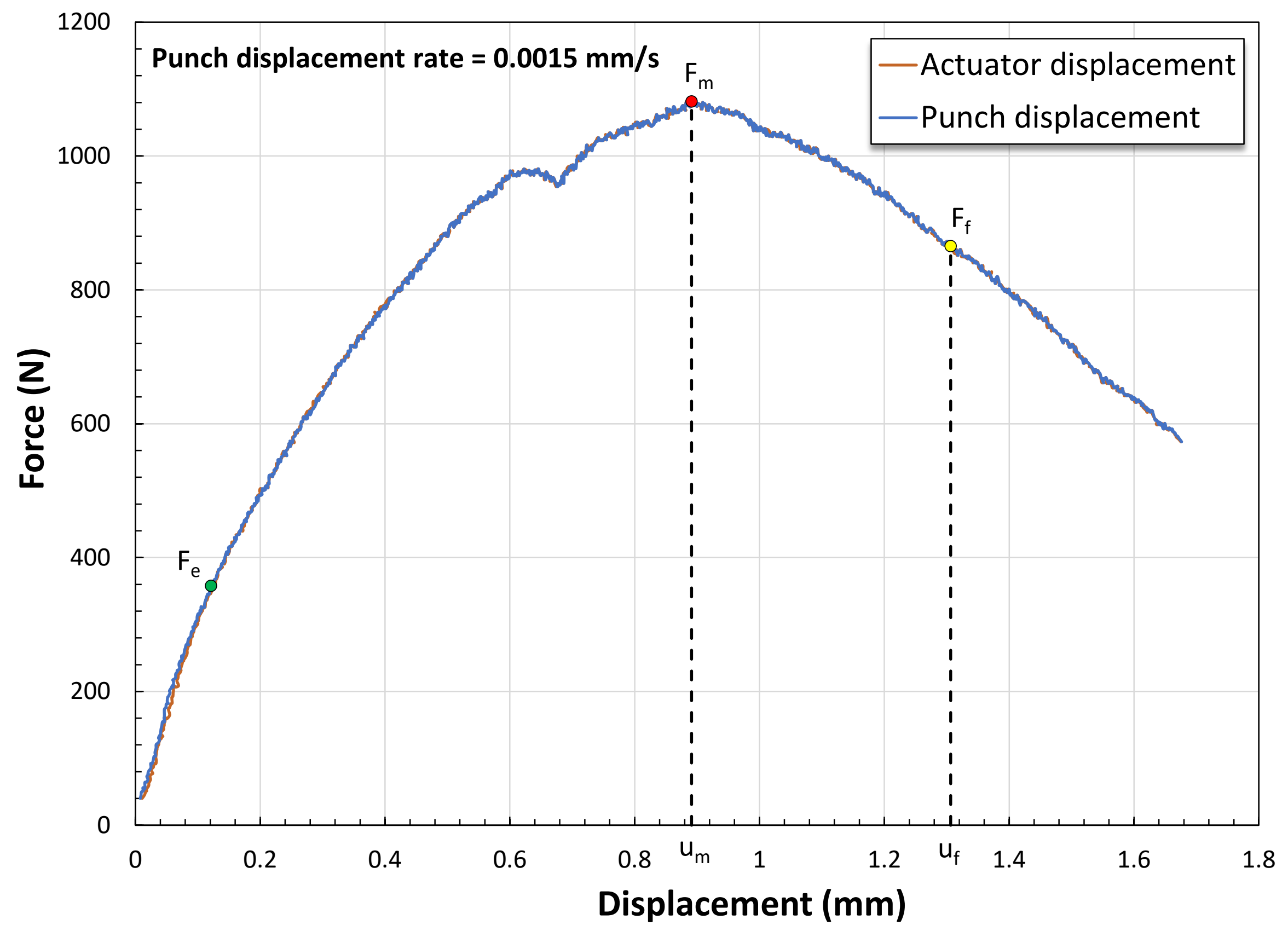




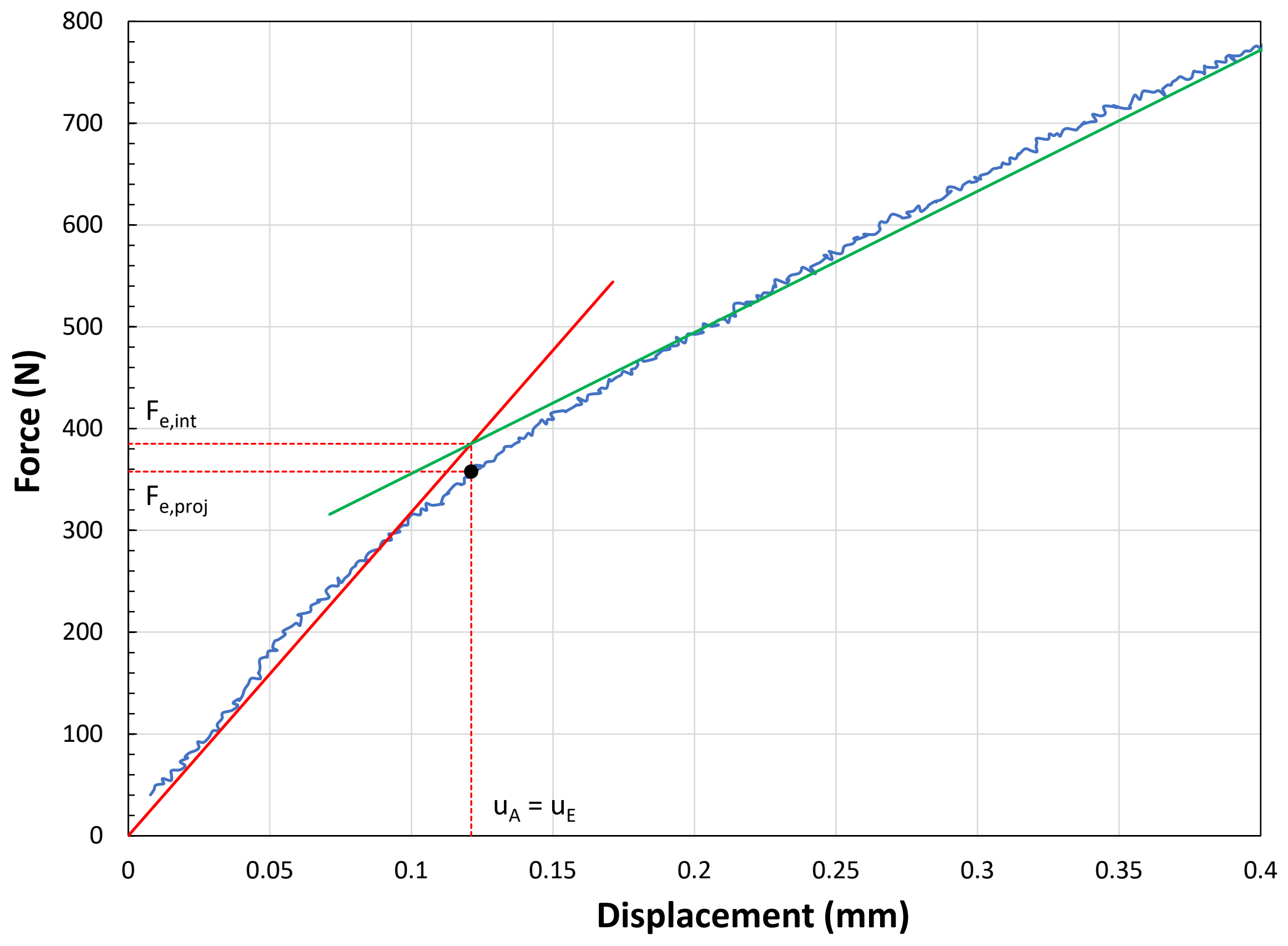




\section{ANNEX 10 \\ SP Test Results for \\ $900{ }^{\circ} \mathrm{C}$ HIP, scan length $=26 \mathrm{~mm}$, non-supported ("rough" specimens)}


Specimen ID: 900HIP_3_N_a_SP1

Material: AM Ti-64

Test speed: $0.001105 \mathrm{~mm} / \mathrm{s}$

Max strain rate: $1.10 \mathrm{E}-03 \mathrm{~s}^{-1}$

\begin{tabular}{|c|c|c|}
\hline \multicolumn{3}{|c|}{ TEST RESULTS } \\
\hline \multicolumn{3}{|c|}{ Force values } \\
\hline$F_{e, p r o j}=$ & 343.4 & $\mathbf{N}$ \\
\hline$F_{e, \text { int }}=$ & 356.2 & $\mathbf{N}$ \\
\hline$F_{h 0 / 10, \text { off }}=$ & 595.2 & $\mathbf{N}$ \\
\hline$F_{0.1 \mathrm{~mm}, \mathrm{off}}=$ & 759.3 & $\mathbf{N}$ \\
\hline$F_{0.1 \mathrm{~mm}}=$ & 176.4 & $\mathbf{N}$ \\
\hline$F_{0.48 \mathrm{~mm}}=$ & 682.5 & $\mathbf{N}$ \\
\hline$F_{0.5 \mathrm{~mm}}=$ & 710.3 & $\mathbf{N}$ \\
\hline$F_{0.65 \mathrm{~mm}}=$ & 880.1 & $\mathbf{N}$ \\
\hline$F_{0.9 \mathrm{~mm}}=$ & 1035.2 & $\mathbf{N}$ \\
\hline$F_{\mathrm{ept}}=$ & 1102.4 & $\mathbf{N}$ \\
\hline$F_{\mathrm{e} 1.5}=$ & 77.5 & $\mathbf{N}$ \\
\hline $\mathbf{F}_{\mathrm{m}}=$ & 1121.8 & $\mathbf{N}$ \\
\hline$F_{\text {infl }}=$ & 584.1 & $\mathbf{N}$ \\
\hline$F_{f}=$ & 897.5 & $\mathbf{N}$ \\
\hline \multicolumn{3}{|c|}{ Displacement values } \\
\hline $\mathrm{u}_{\mathrm{e}}=$ & 0.211 & $\mathrm{~mm}$ \\
\hline$u_{h 0 / 10, \text { off }}=$ & 0.403 & $\mathrm{~mm}$ \\
\hline$u_{0.1 \mathrm{~mm}, \mathrm{off}}=$ & 0.552 & $\mathrm{~mm}$ \\
\hline$u_{\mathrm{e} 1.5}=$ & 0.042 & $\mathrm{~mm}$ \\
\hline$u_{m}=$ & 1.201 & $\mathrm{~mm}$ \\
\hline$u_{\text {infl }}=$ & 0.389 & $\mathrm{~mm}$ \\
\hline $\mathbf{u}_{\mathrm{f}}=$ & 1.491 & $\mathrm{~mm}$ \\
\hline \multicolumn{3}{|c|}{ Initial linear slope } \\
\hline Slope $_{\text {ini }}=$ & 1685.89 & $\mathrm{~N} / \mathrm{mm}$ \\
\hline \multicolumn{3}{|c|}{ Energy values } \\
\hline$E_{S P}=$ & 1.18 & J \\
\hline$E_{m}=$ & 0.89 & $\mathbf{J}$ \\
\hline$E_{P L}=$ & 0.51 & $\mathbf{J}$ \\
\hline
\end{tabular}




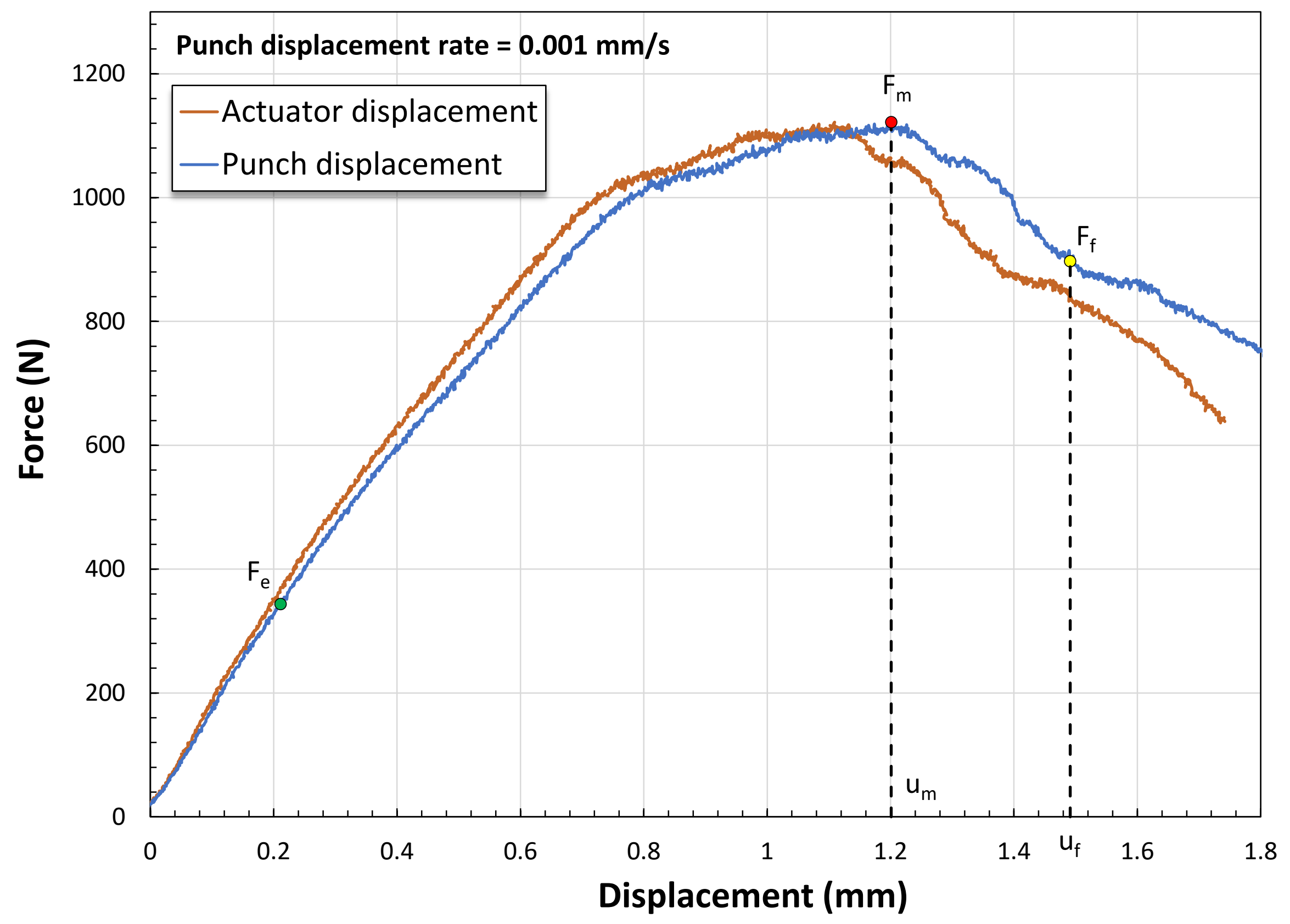




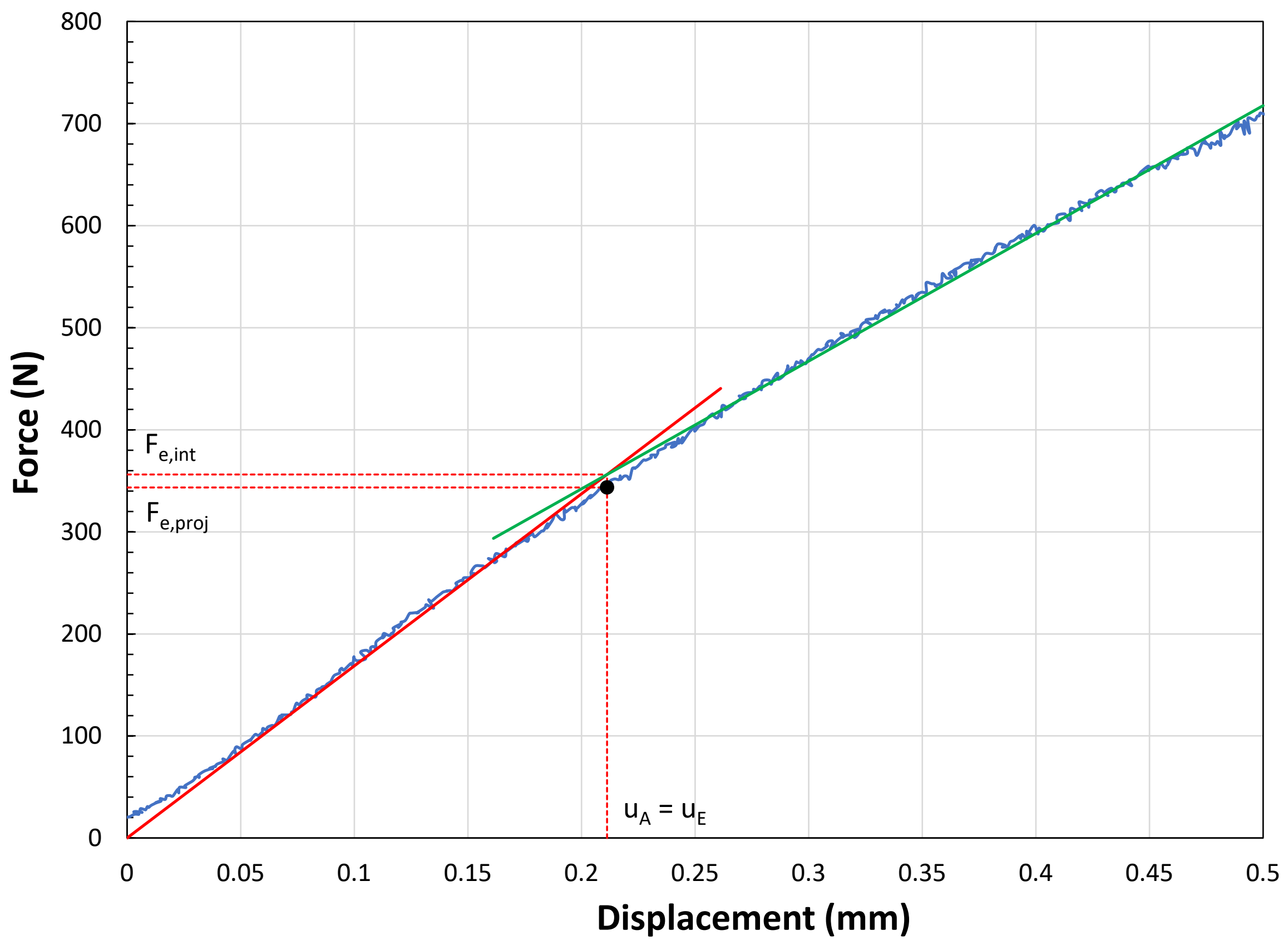


Specimen ID: 900HIP_3_N_a_SP2

Material: AM Ti-64

Test speed: $0.001171 \mathrm{~mm} / \mathrm{s}$

Max strain rate: $1.17 \mathrm{E}-03 \mathrm{~s}^{-1}$

\begin{tabular}{|c|c|c|}
\hline \multicolumn{3}{|c|}{ TEST RESULTS } \\
\hline \multicolumn{3}{|c|}{ Force values } \\
\hline$F_{e, p r o j}=$ & 412.2 & $\mathbf{N}$ \\
\hline$F_{e, \text { int }}=$ & 420.5 & $\mathbf{N}$ \\
\hline$F_{h 0 / 10, \text { off }}=$ & 668.2 & $\mathbf{N}$ \\
\hline$F_{0.1 \mathrm{~mm}, \mathrm{off}}=$ & 920.9 & $\mathbf{N}$ \\
\hline$F_{0.1 \mathrm{~mm}}=$ & 146.5 & $\mathbf{N}$ \\
\hline$F_{0.48 \mathrm{~mm}}=$ & 641.9 & $\mathbf{N}$ \\
\hline$F_{0.5 \mathrm{~mm}}=$ & 668.0 & $\mathbf{N}$ \\
\hline$F_{0.65 \mathrm{~mm}}=$ & 844.5 & $\mathbf{N}$ \\
\hline$F_{0.9 \mathrm{~mm}}=$ & 1062.3 & $\mathbf{N}$ \\
\hline$F_{\text {ept }}=$ & \#DIV/0! & $\mathbf{N}$ \\
\hline$F_{\mathrm{e} 1.5}=$ & 82.3 & $\mathbf{N}$ \\
\hline$F_{m}=$ & 1085.3 & $\mathbf{N}$ \\
\hline$F_{\text {infl }}=$ & \#DIV/0! & $\mathbf{N}$ \\
\hline$F_{f}=$ & 868.2 & $\mathbf{N}$ \\
\hline \multicolumn{3}{|c|}{ Displacement values } \\
\hline$u_{e}=$ & 0.284 & $\mathrm{~mm}$ \\
\hline$u_{h 0 / 10, \text { off }}=$ & 0.501 & $\mathrm{~mm}$ \\
\hline $\mathrm{u}_{0.1 \mathrm{~mm}, \mathrm{off}}=$ & 0.721 & $\mathrm{~mm}$ \\
\hline$u_{e 1.5}=$ & 0.057 & $\mathrm{~mm}$ \\
\hline$u_{m}=$ & 0.924 & $\mathrm{~mm}$ \\
\hline$u_{\text {infl }}=$ & \#DIV/0! & $\mathrm{mm}$ \\
\hline$u_{f}=$ & 1.799 & $\mathrm{~mm}$ \\
\hline \multicolumn{3}{|c|}{ Initial linear slope } \\
\hline Slope $_{\text {ini }}=$ & 1482.61 & $\mathrm{~N} / \mathrm{mm}$ \\
\hline \multicolumn{3}{|c|}{ Energy values } \\
\hline$E_{S P}=$ & 1.44 & J \\
\hline $\mathbf{E}_{m}=$ & 0.56 & J \\
\hline$E_{P L}=$ & 0.16 & J \\
\hline
\end{tabular}




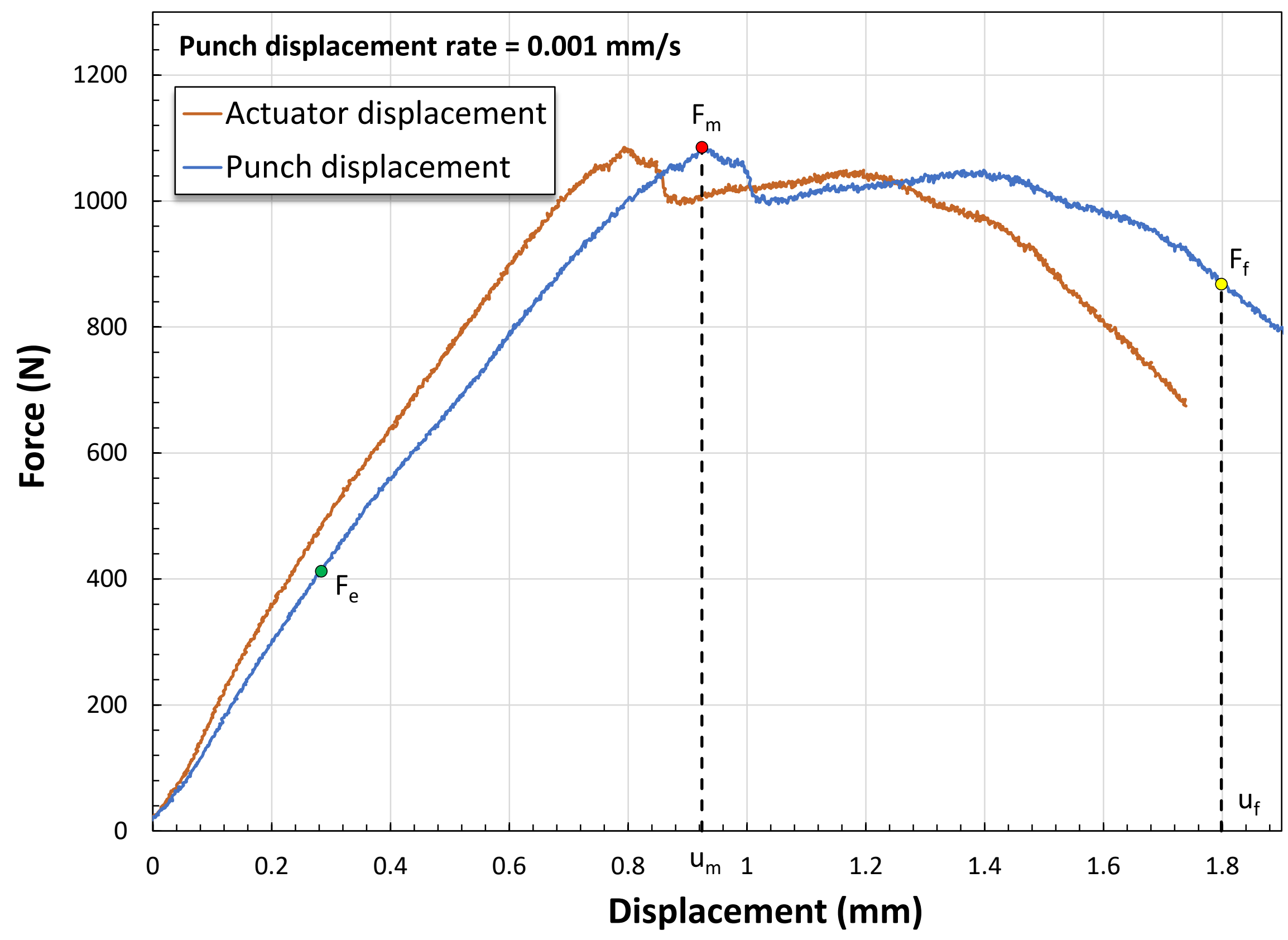




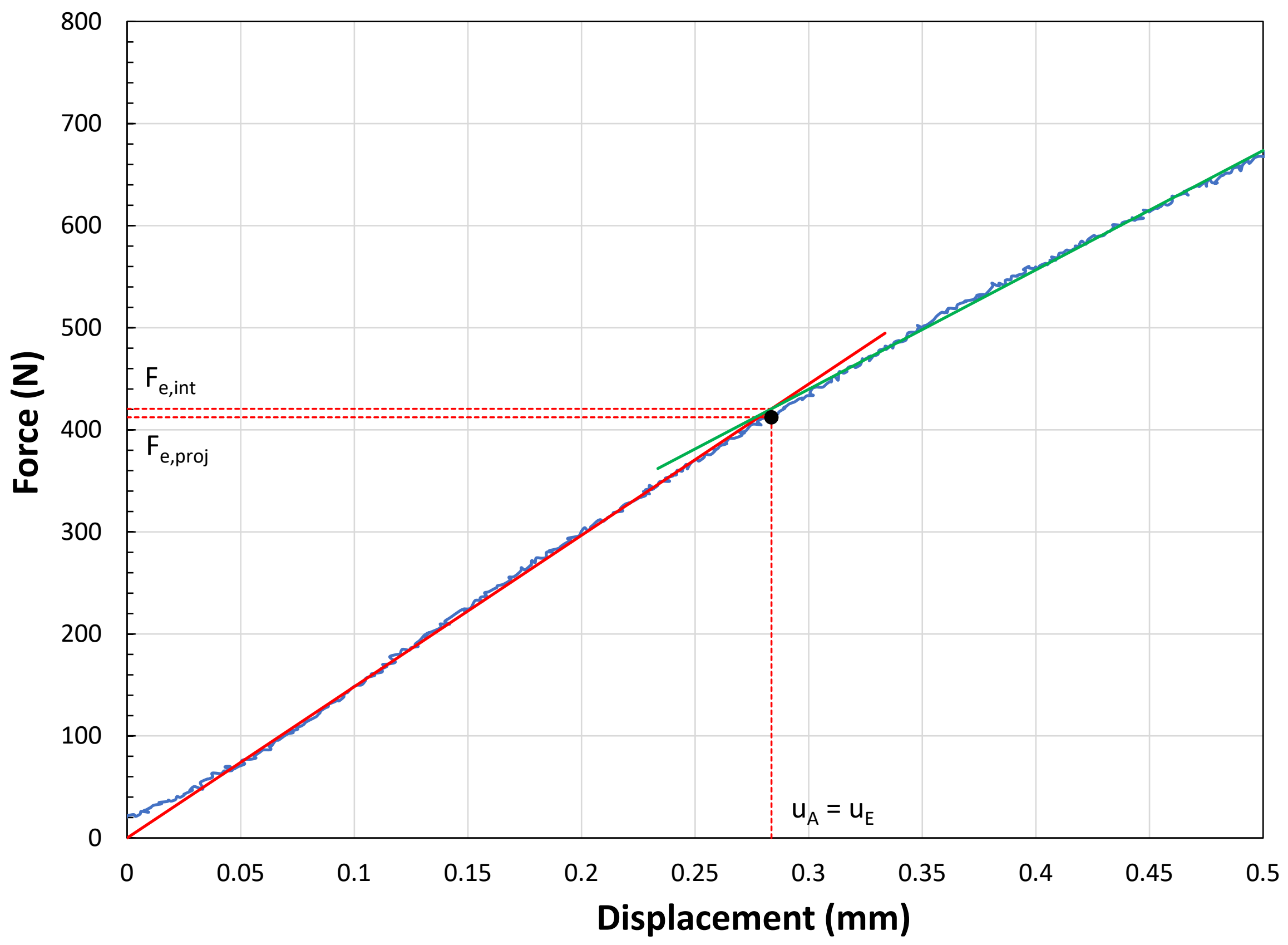


Specimen ID: 900HIP_3_N_a_SP3

Material: AM Ti-64

Test speed: $0.001142 \mathrm{~mm} / \mathrm{s}$

Max strain rate: $1.14 \mathrm{E}-03 \mathrm{~s}^{-1}$

\begin{tabular}{|c|c|c|}
\hline \multicolumn{3}{|c|}{ TEST RESULTS } \\
\hline \multicolumn{3}{|c|}{ Force values } \\
\hline$F_{e, p r o j}=$ & 371.8 & $\mathbf{N}$ \\
\hline $\mathbf{F}_{\mathrm{e}, \mathrm{int}}=$ & 383.3 & $\mathbf{N}$ \\
\hline$F_{h 0 / 10, \text { off }}=$ & 584.3 & $\mathbf{N}$ \\
\hline$F_{0.1 \mathrm{~mm}, \mathrm{off}}=$ & 743.4 & $\mathbf{N}$ \\
\hline$F_{0.1 \mathrm{~mm}}=$ & 157.2 & $\mathbf{N}$ \\
\hline$F_{0.48 \mathrm{~mm}}=$ & 663.6 & $\mathbf{N}$ \\
\hline$F_{0.5 \mathrm{~mm}}=$ & 691.1 & $\mathbf{N}$ \\
\hline$F_{0.65 \mathrm{~mm}}=$ & 838.0 & $\mathbf{N}$ \\
\hline$F_{0.9 \mathrm{~mm}}=$ & 1046.7 & $\mathbf{N}$ \\
\hline$F_{\mathrm{ept}}=$ & 392.2 & $\mathbf{N}$ \\
\hline$F_{\mathrm{e} 1.5}=$ & 131.6 & $\mathbf{N}$ \\
\hline $\mathbf{F}_{\mathrm{m}}=$ & 1097.0 & $\mathbf{N}$ \\
\hline$F_{\text {infl }}=$ & 1083.1 & $\mathbf{N}$ \\
\hline$F_{f}=$ & 877.6 & $\mathbf{N}$ \\
\hline \multicolumn{3}{|c|}{ Displacement values } \\
\hline $\mathrm{u}_{\mathrm{e}}=$ & 0.237 & $\mathrm{~mm}$ \\
\hline$u_{h 0 / 10, \text { off }}=$ & 0.412 & $\mathrm{~mm}$ \\
\hline$u_{0.1 \mathrm{~mm}, \mathrm{off}}=$ & 0.561 & $\mathrm{~mm}$ \\
\hline$u_{\mathrm{e} 1.5}=$ & 0.082 & $\mathrm{~mm}$ \\
\hline$u_{m}=$ & 1.213 & $\mathrm{~mm}$ \\
\hline$u_{\text {infl }}=$ & 1.131 & $\mathrm{~mm}$ \\
\hline$u_{f}=$ & 1.726 & $\mathrm{~mm}$ \\
\hline \multicolumn{3}{|c|}{ Initial linear slope } \\
\hline Slope $_{\text {ini }}=$ & 1615.91 & $\mathrm{~N} / \mathrm{mm}$ \\
\hline \multicolumn{3}{|c|}{ Energy values } \\
\hline$E_{S P}=$ & 1.39 & J \\
\hline$E_{m}=$ & 0.88 & J \\
\hline$E_{P L}=$ & 0.50 & $\mathbf{J}$ \\
\hline
\end{tabular}




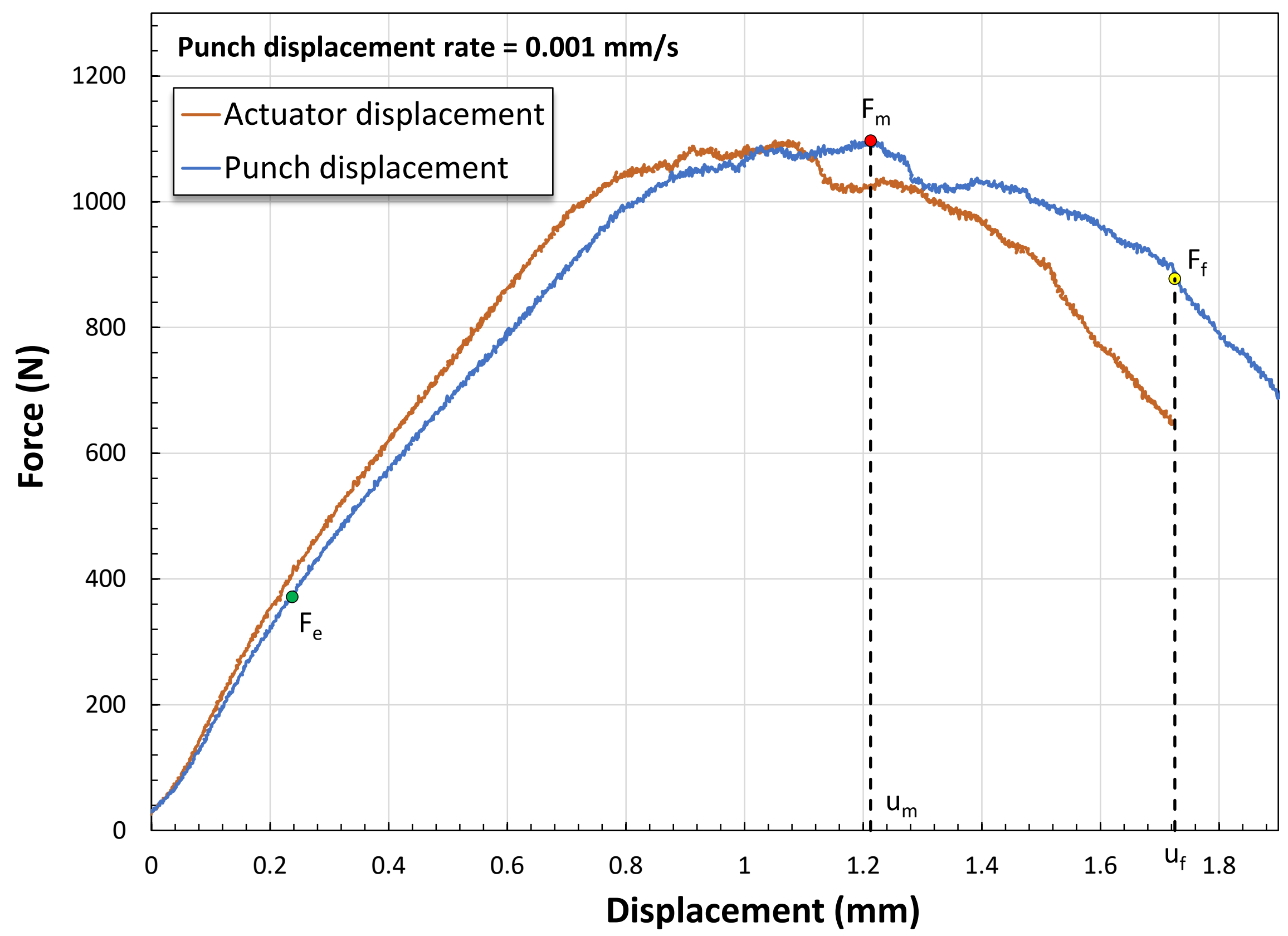




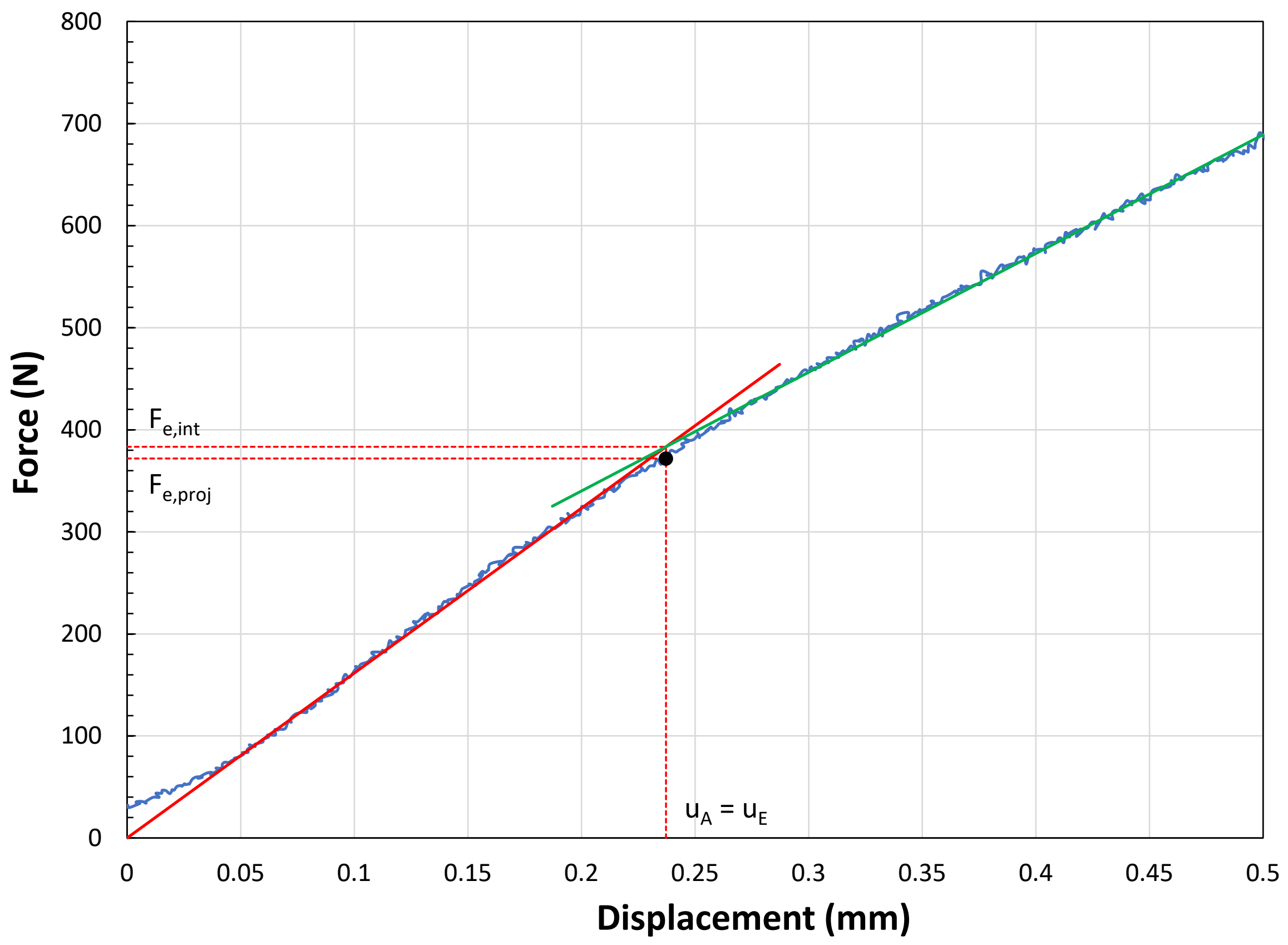


Specimen ID: 900HIP_3_N_a_SP4

Material: AM Ti-64

Test speed: $0.001164 \mathrm{~mm} / \mathrm{s}$

Max strain rate: $1.16 \mathrm{E}-03 \mathrm{~s}^{-1}$

\begin{tabular}{|c|c|c|}
\hline \multicolumn{3}{|c|}{ TEST RESULTS } \\
\hline \multicolumn{3}{|c|}{ Force values } \\
\hline$F_{e, p r o j}=$ & 369.8 & $\mathbf{N}$ \\
\hline$F_{e, \text { int }}=$ & 379.0 & $\mathbf{N}$ \\
\hline$F_{h 0 / 10, \text { off }}=$ & 580.9 & $\mathbf{N}$ \\
\hline$F_{0.1 \mathrm{~mm}, \mathrm{off}}=$ & 724.9 & $\mathbf{N}$ \\
\hline$F_{0.1 \mathrm{~mm}}=$ & 187.2 & $\mathbf{N}$ \\
\hline$F_{0.48 \mathrm{~mm}}=$ & 704.5 & $\mathbf{N}$ \\
\hline$F_{0.5 \mathrm{~mm}}=$ & 724.9 & $\mathbf{N}$ \\
\hline$F_{0.65 \mathrm{~mm}}=$ & 860.5 & $\mathbf{N}$ \\
\hline$F_{0.9 \mathrm{~mm}}=$ & 1049.7 & $\mathbf{N}$ \\
\hline$F_{\mathrm{ept}}=$ & 60.7 & $\mathbf{N}$ \\
\hline$F_{e 1.5}=$ & 210.0 & $\mathbf{N}$ \\
\hline $\mathbf{F}_{\mathrm{m}}=$ & 1094.2 & $\mathbf{N}$ \\
\hline$F_{\text {infl }}=$ & 688.8 & $\mathbf{N}$ \\
\hline$F_{f}=$ & 875.4 & $\mathbf{N}$ \\
\hline \multicolumn{3}{|c|}{ Displacement values } \\
\hline $\mathrm{u}_{\mathrm{e}}=$ & 0.208 & $\mathrm{~mm}$ \\
\hline$u_{h 0 / 10, \text { off }}=$ & 0.369 & $\mathrm{~mm}$ \\
\hline$u_{0.1 \mathrm{~mm}, \mathrm{off}}=$ & 0.498 & $\mathrm{~mm}$ \\
\hline$u_{\mathrm{e} 1.5}=$ & 0.114 & $\mathrm{~mm}$ \\
\hline$u_{m}=$ & 1.238 & $\mathrm{~mm}$ \\
\hline$u_{\text {infl }}=$ & 2.005 & $\mathrm{~mm}$ \\
\hline $\mathbf{u}_{\mathrm{f}}=$ & 1.800 & $\mathrm{~mm}$ \\
\hline \multicolumn{3}{|c|}{ Initial linear slope } \\
\hline Slope $_{\text {ini }}=$ & 1821.89 & $\mathrm{~N} / \mathrm{mm}$ \\
\hline \multicolumn{3}{|c|}{ Energy values } \\
\hline$E_{S P}=$ & 1.49 & J \\
\hline$E_{m}=$ & 0.92 & $\mathbf{J}$ \\
\hline$E_{P L}=$ & 0.60 & $\mathbf{J}$ \\
\hline
\end{tabular}




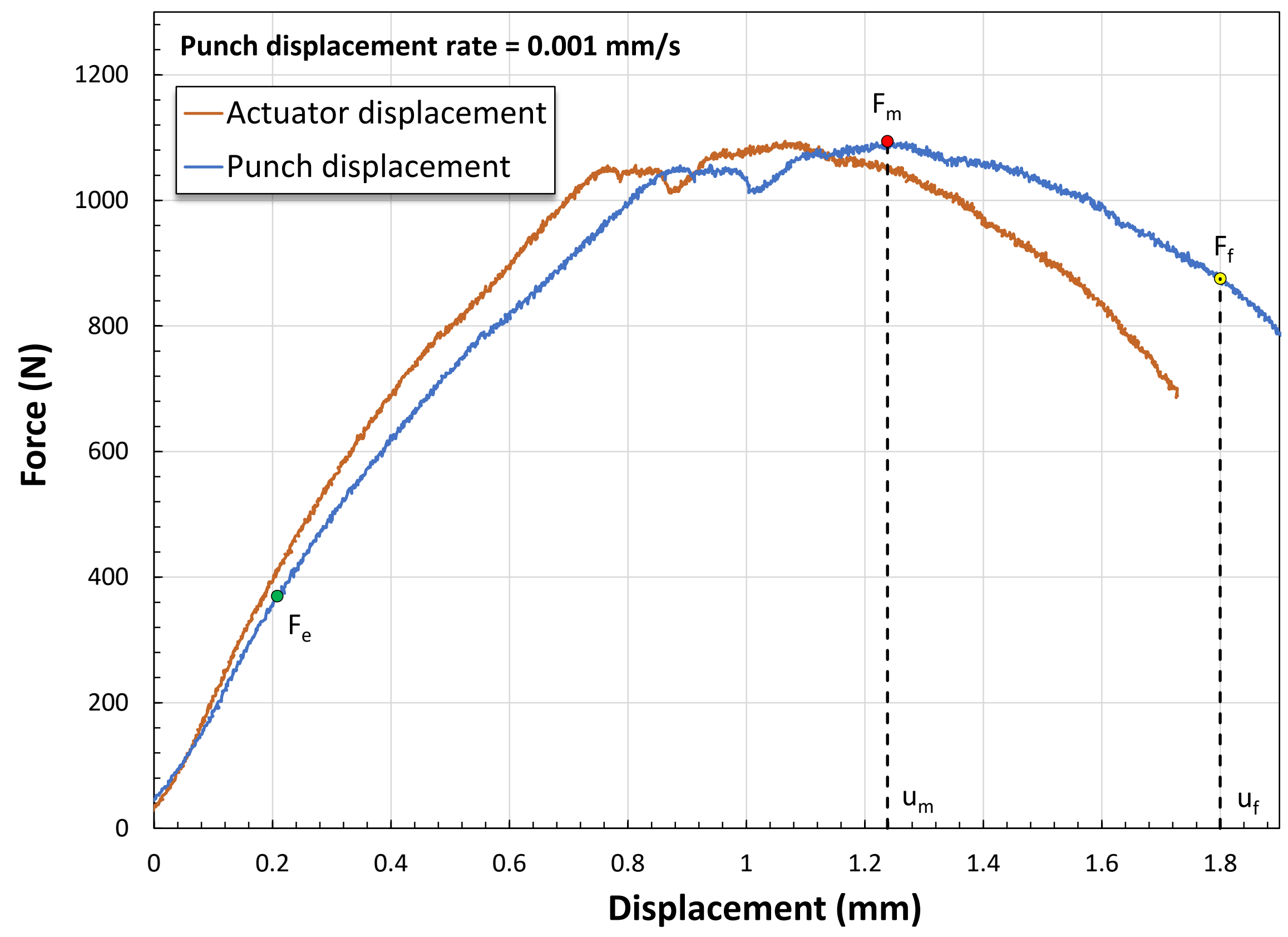




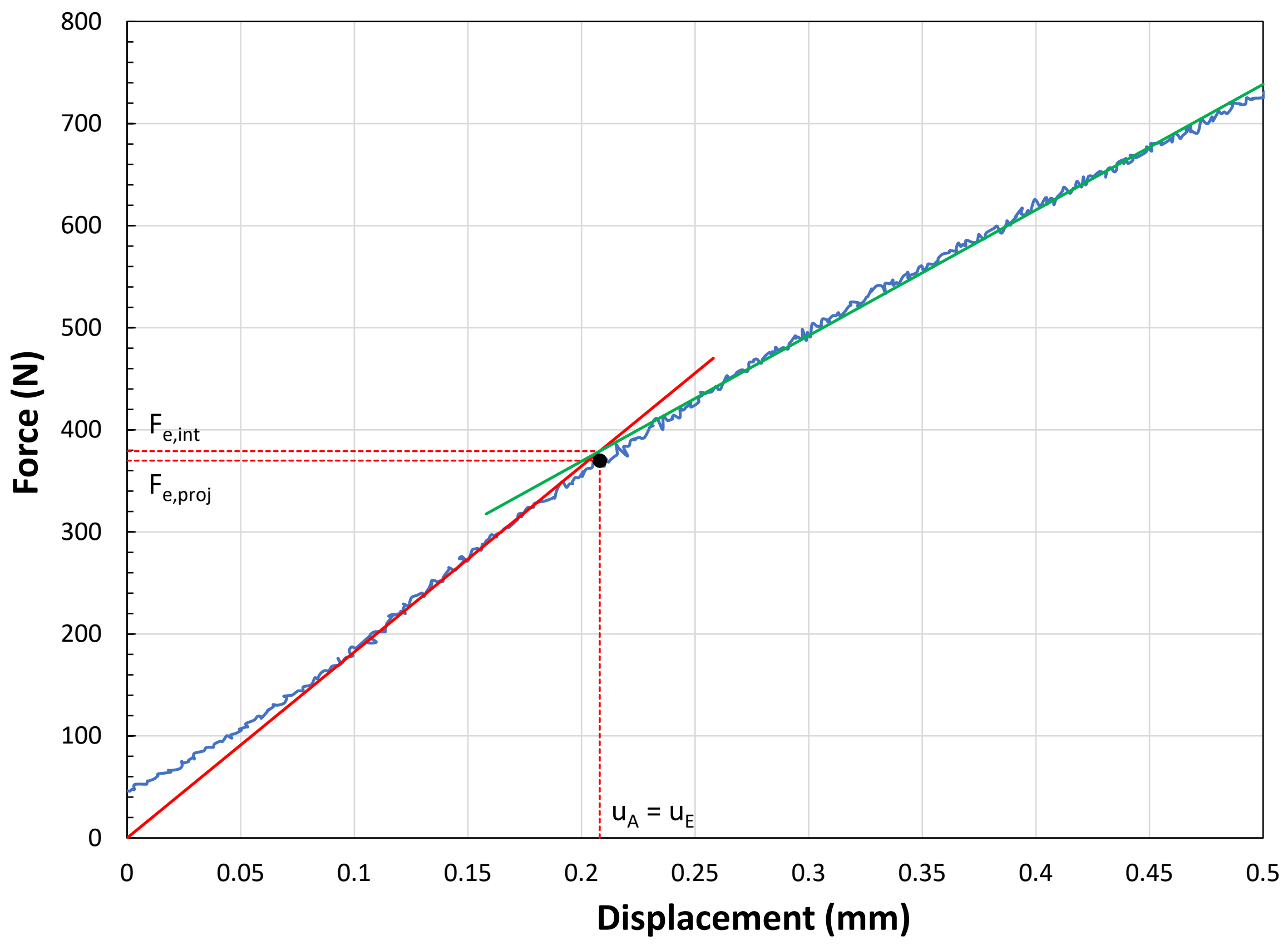


Specimen ID: 900HIP_3_N_a_SP5

Material: AM Ti-64

Test speed: $0.001171 \mathrm{~mm} / \mathrm{s}$

Max strain rate: $1.17 \mathrm{E}-03 \mathrm{~s}^{-1}$

\begin{tabular}{|c|c|c|}
\hline \multicolumn{3}{|c|}{ TEST RESULTS } \\
\hline \multicolumn{3}{|c|}{ Force values } \\
\hline$F_{e, p r o j}=$ & 381.6 & $\mathbf{N}$ \\
\hline$F_{e, \text { int }}=$ & 390.5 & $\mathbf{N}$ \\
\hline$F_{h 0 / 10, \text { off }}=$ & 573.8 & $\mathbf{N}$ \\
\hline$F_{0.1 \mathrm{~mm}, \mathrm{off}}=$ & 715.8 & $\mathbf{N}$ \\
\hline$F_{0.1 \mathrm{~mm}}=$ & 162.4 & $\mathbf{N}$ \\
\hline$F_{0.48 \mathrm{~mm}}=$ & 662.9 & $\mathbf{N}$ \\
\hline$F_{0.5 \mathrm{~mm}}=$ & 679.5 & $\mathbf{N}$ \\
\hline$F_{0.65 \mathrm{~mm}}=$ & 828.5 & $\mathbf{N}$ \\
\hline$F_{0.9 \mathrm{~mm}}=$ & 1026.5 & $\mathbf{N}$ \\
\hline $\mathbf{F}_{\mathrm{ept}}=$ & 1027.3 & $\mathbf{N}$ \\
\hline$F_{\mathrm{e} 1.5}=$ & 131.3 & $\mathbf{N}$ \\
\hline $\mathbf{F}_{\mathrm{m}}=$ & 1071.5 & $\mathbf{N}$ \\
\hline$F_{\text {infl }}=$ & 46.1 & $\mathbf{N}$ \\
\hline$F_{f}=$ & 857.2 & $\mathbf{N}$ \\
\hline \multicolumn{3}{|c|}{ Displacement values } \\
\hline $\mathrm{u}_{\mathrm{e}}=$ & 0.238 & $\mathrm{~mm}$ \\
\hline$u_{h 0 / 10, \text { off }}=$ & 0.400 & $\mathrm{~mm}$ \\
\hline$u_{0.1 \mathrm{~mm}, \mathrm{off}}=$ & 0.536 & $\mathrm{~mm}$ \\
\hline$u_{\mathrm{e} 1.5}=$ & 0.078 & $\mathrm{~mm}$ \\
\hline$u_{m}=$ & 1.146 & $\mathrm{~mm}$ \\
\hline$u_{\text {infl }}=$ & 0.014 & $\mathrm{~mm}$ \\
\hline$u_{f}=$ & 1.733 & $\mathrm{~mm}$ \\
\hline \multicolumn{3}{|c|}{ Initial linear slope } \\
\hline Slope $_{\text {ini }}=$ & 1641.54 & $\mathrm{~N} / \mathrm{mm}$ \\
\hline \multicolumn{3}{|c|}{ Energy values } \\
\hline$E_{S P}=$ & 1.38 & J \\
\hline$E_{m}=$ & 0.80 & J \\
\hline$E_{P L}=$ & 0.45 & $\mathbf{J}$ \\
\hline
\end{tabular}




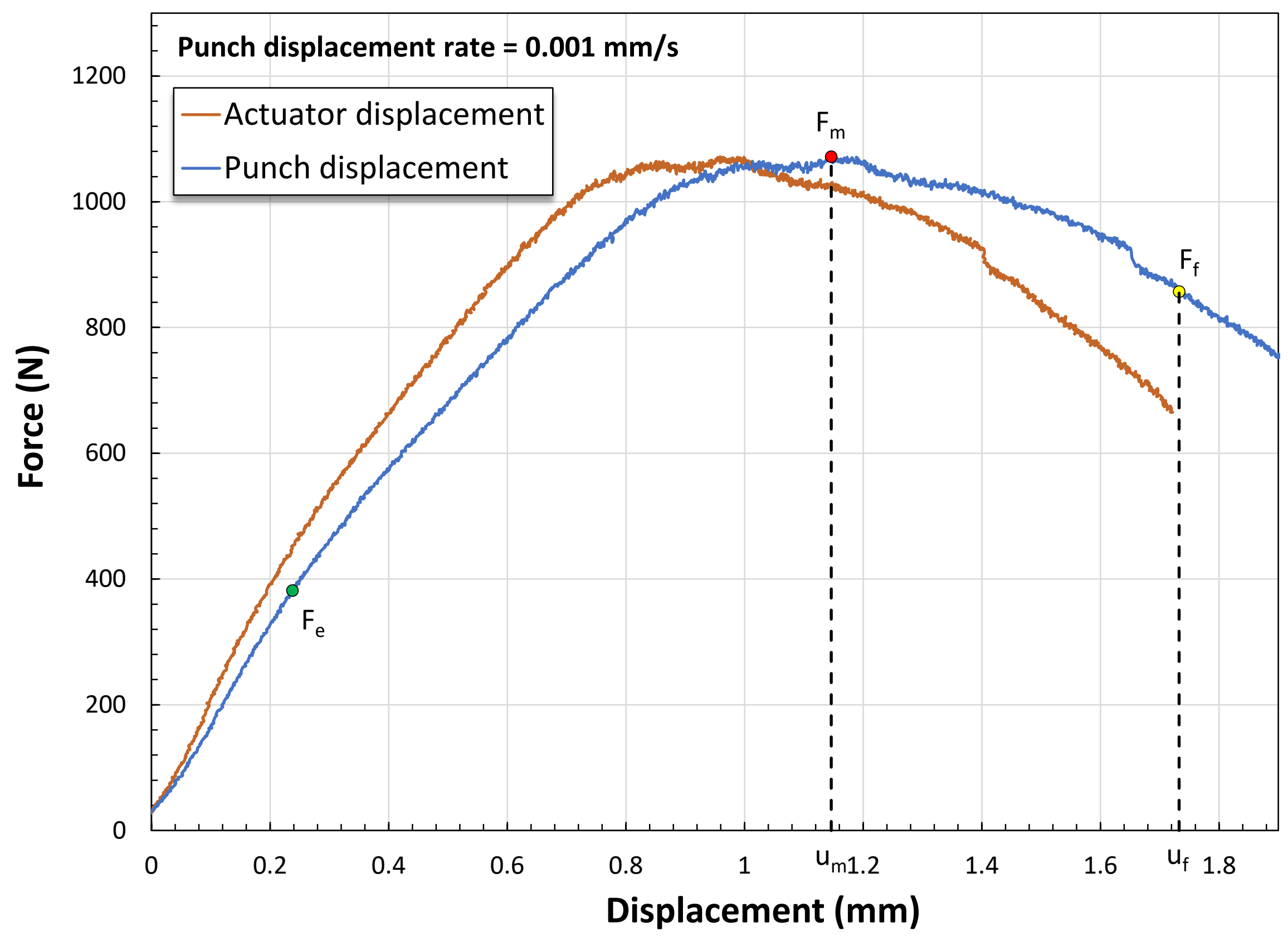




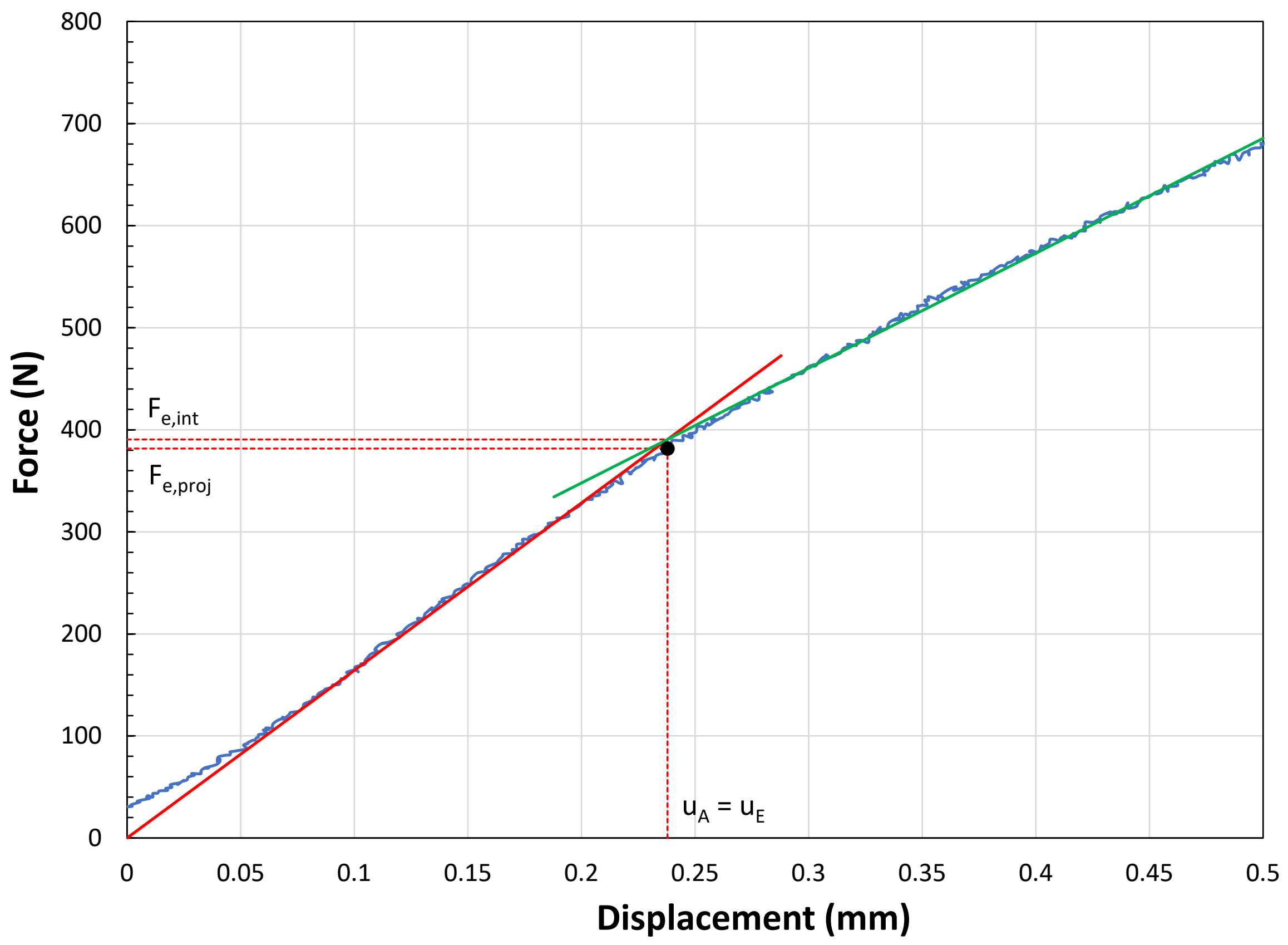


Specimen ID: 900HIP_3_N_a_SP6

Material: AM Ti-64

Test speed: $0.001153 \mathrm{~mm} / \mathrm{s}$

Max strain rate: $1.15 \mathrm{E}-03 \mathrm{~s}^{-1}$

\begin{tabular}{|c|c|c|}
\hline \multicolumn{3}{|c|}{ TEST RESULTS } \\
\hline \multicolumn{3}{|c|}{ Force values } \\
\hline$F_{e, p r o j}=$ & 410.2 & $\mathbf{N}$ \\
\hline$F_{e, \text { int }}=$ & 423.9 & $\mathbf{N}$ \\
\hline$F_{h 0 / 10, \text { off }}=$ & 590.8 & $\mathbf{N}$ \\
\hline$F_{0.1 \mathrm{~mm}, \mathrm{off}}=$ & 712.0 & $\mathbf{N}$ \\
\hline$F_{0.1 \mathrm{~mm}}=$ & 200.5 & $\mathbf{N}$ \\
\hline$F_{0.48 \mathrm{~mm}}=$ & 728.9 & $\mathbf{N}$ \\
\hline$F_{0.5 \mathrm{~mm}}=$ & 753.7 & $\mathbf{N}$ \\
\hline$F_{0.65 \mathrm{~mm}}=$ & 902.6 & $\mathbf{N}$ \\
\hline$F_{0.9 m m}=$ & 1027.1 & $\mathbf{N}$ \\
\hline$F_{\mathrm{ept}}=$ & 847.9 & $\mathbf{N}$ \\
\hline$F_{\mathrm{e} 1.5}=$ & 807.9 & $\mathbf{N}$ \\
\hline $\mathbf{F}_{\mathrm{m}}=$ & 1057.0 & $\mathbf{N}$ \\
\hline$F_{\text {infl }}=$ & 632.8 & $\mathbf{N}$ \\
\hline$F_{f}=$ & 845.6 & $\mathbf{N}$ \\
\hline \multicolumn{3}{|c|}{ Displacement values } \\
\hline $\mathrm{u}_{\mathrm{e}}=$ & 0.217 & $\mathrm{~mm}$ \\
\hline$u_{h 0 / 10, \text { off }}=$ & 0.353 & $\mathrm{~mm}$ \\
\hline$u_{0.1 \mathrm{~mm}, \mathrm{off}}=$ & 0.465 & $\mathrm{~mm}$ \\
\hline$u_{\mathrm{e} 1.5}=$ & 0.559 & $\mathrm{~mm}$ \\
\hline$u_{m}=$ & 0.948 & $\mathrm{~mm}$ \\
\hline$u_{\text {infl }}=$ & 1.994 & $\mathrm{~mm}$ \\
\hline$u_{f}=$ & 1.708 & $\mathrm{~mm}$ \\
\hline \multicolumn{3}{|c|}{ Initial linear slope } \\
\hline Slope $_{\text {ini }}=$ & 1952.20 & $\mathrm{~N} / \mathrm{mm}$ \\
\hline \multicolumn{3}{|c|}{ Energy values } \\
\hline$E_{S P}=$ & 1.38 & J \\
\hline$E_{m}=$ & 0.63 & $\mathbf{J}$ \\
\hline$E_{P L}=$ & 0.34 & $\mathbf{J}$ \\
\hline
\end{tabular}




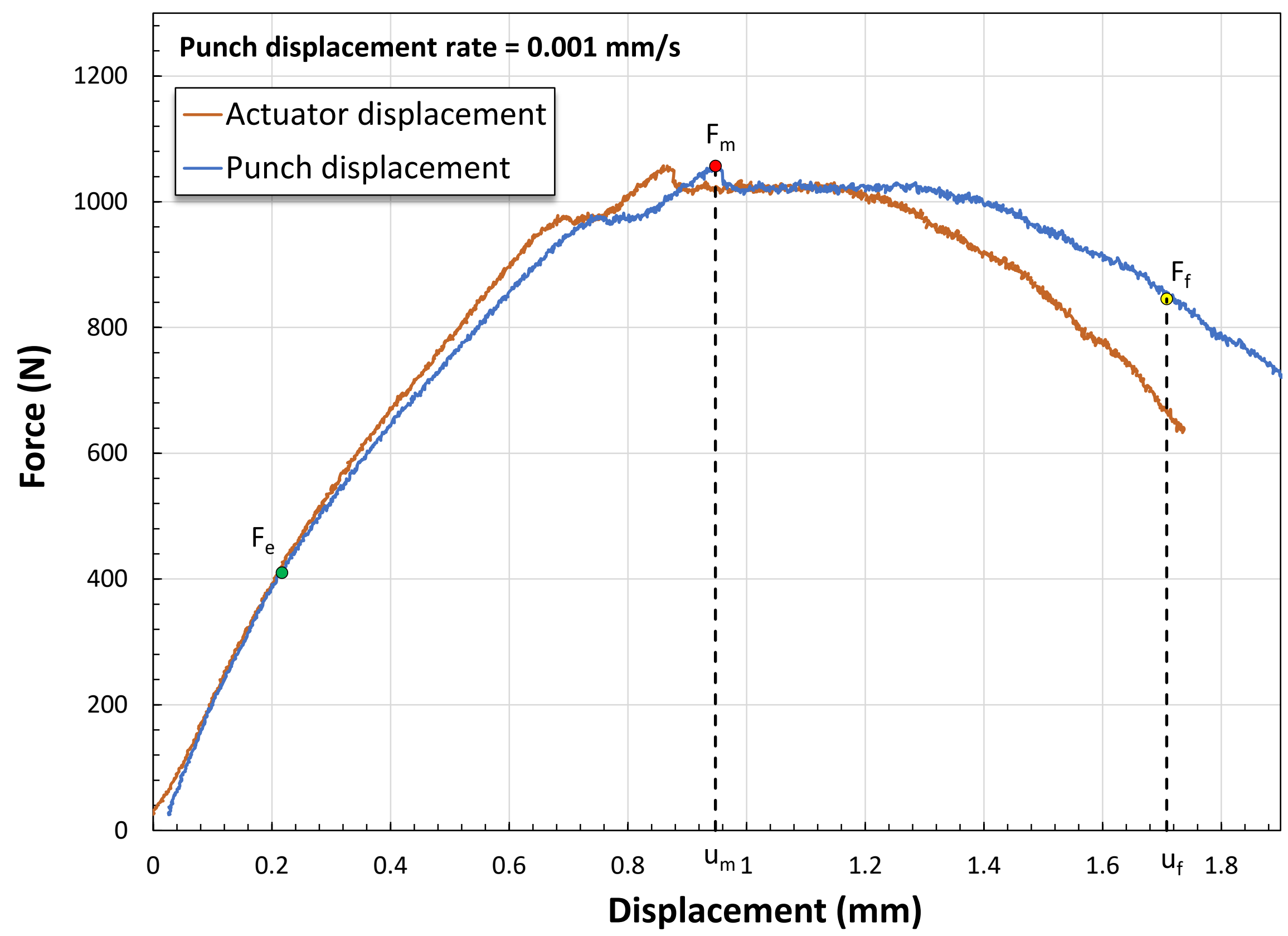




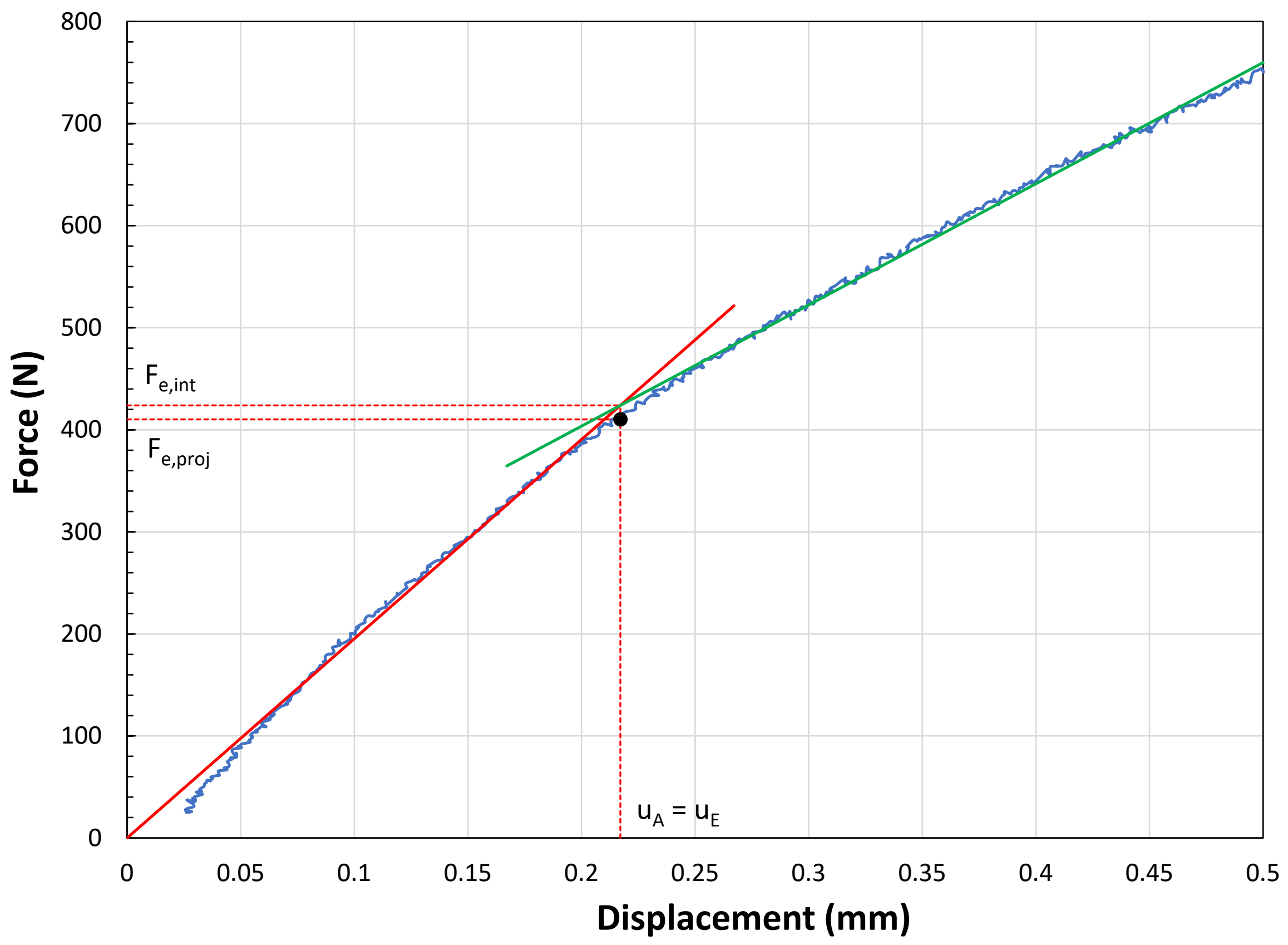




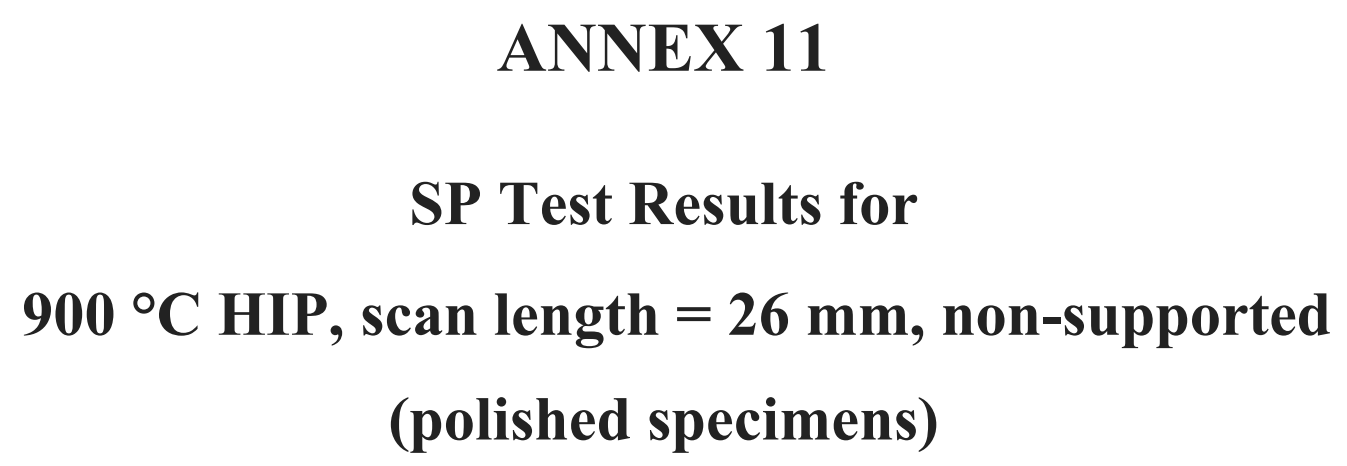


Small Punch Test Results

Specimen ID: 900HIP_3_N_a_SP7 (pc

Material: AM Ti-64

Test speed: $0.001441 \mathrm{~mm} / \mathrm{s}$

Max strain rate: $1.44 \mathrm{E}-03 \mathrm{~s}^{-1}$

\begin{tabular}{|c|c|c|}
\hline \multicolumn{3}{|c|}{ TEST RESULTS } \\
\hline \multicolumn{3}{|c|}{ Force values } \\
\hline$F_{e, p r o j}=$ & 373.5 & $\mathbf{N}$ \\
\hline$F_{e, \text { int }}=$ & 397.2 & $\mathbf{N}$ \\
\hline$F_{h 0 / 10, \text { off }}=$ & 522.2 & $\mathbf{N}$ \\
\hline$F_{0.1 \mathrm{~mm}, \mathrm{off}}=$ & 653.3 & $\mathbf{N}$ \\
\hline$F_{0.1 \mathrm{~mm}}=$ & 309.9 & $\mathbf{N}$ \\
\hline$F_{0.48 \mathrm{~mm}}=$ & 870.1 & $\mathbf{N}$ \\
\hline$F_{0.5 \mathrm{~mm}}=$ & 882.4 & $\mathbf{N}$ \\
\hline$F_{0.65 \mathrm{~mm}}=$ & 965.2 & $\mathbf{N}$ \\
\hline$F_{0.9 \mathrm{~mm}}=$ & 918.4 & $\mathbf{N}$ \\
\hline$F_{\text {ept }}=$ & 831.9 & $\mathbf{N}$ \\
\hline$F_{\mathrm{e} 1.5}=$ & 600.9 & $\mathbf{N}$ \\
\hline$F_{m}=$ & 992.2 & $\mathbf{N}$ \\
\hline$F_{\text {infl }}=$ & 593.6 & $\mathbf{N}$ \\
\hline$F_{f}=$ & 793.7 & $\mathbf{N}$ \\
\hline \multicolumn{3}{|c|}{ Displacement values } \\
\hline$u_{e}=$ & 0.124 & $\mathrm{~mm}$ \\
\hline$u_{h 0 / 10, \text { off }}=$ & 0.209 & $\mathrm{~mm}$ \\
\hline $\mathrm{u}_{0.1 \mathrm{~mm}, \mathrm{off}}=$ & 0.305 & $\mathrm{~mm}$ \\
\hline$u_{e 1.5}=$ & 0.269 & $\mathrm{~mm}$ \\
\hline$u_{m}=$ & 0.618 & $\mathrm{~mm}$ \\
\hline$u_{\text {infl }}=$ & 1.667 & $\mathrm{~mm}$ \\
\hline$u_{f}=$ & 1.369 & $\mathrm{~mm}$ \\
\hline \multicolumn{3}{|c|}{ Initial linear slope } \\
\hline Slope $_{\text {ini }}=$ & 3201.67 & $\mathrm{~N} / \mathrm{mm}$ \\
\hline \multicolumn{3}{|c|}{ Energy values } \\
\hline$E_{S P}=$ & 1.05 & J \\
\hline$E_{m}=$ & 0.38 & J \\
\hline$E_{P L}=$ & 0.23 & J \\
\hline
\end{tabular}




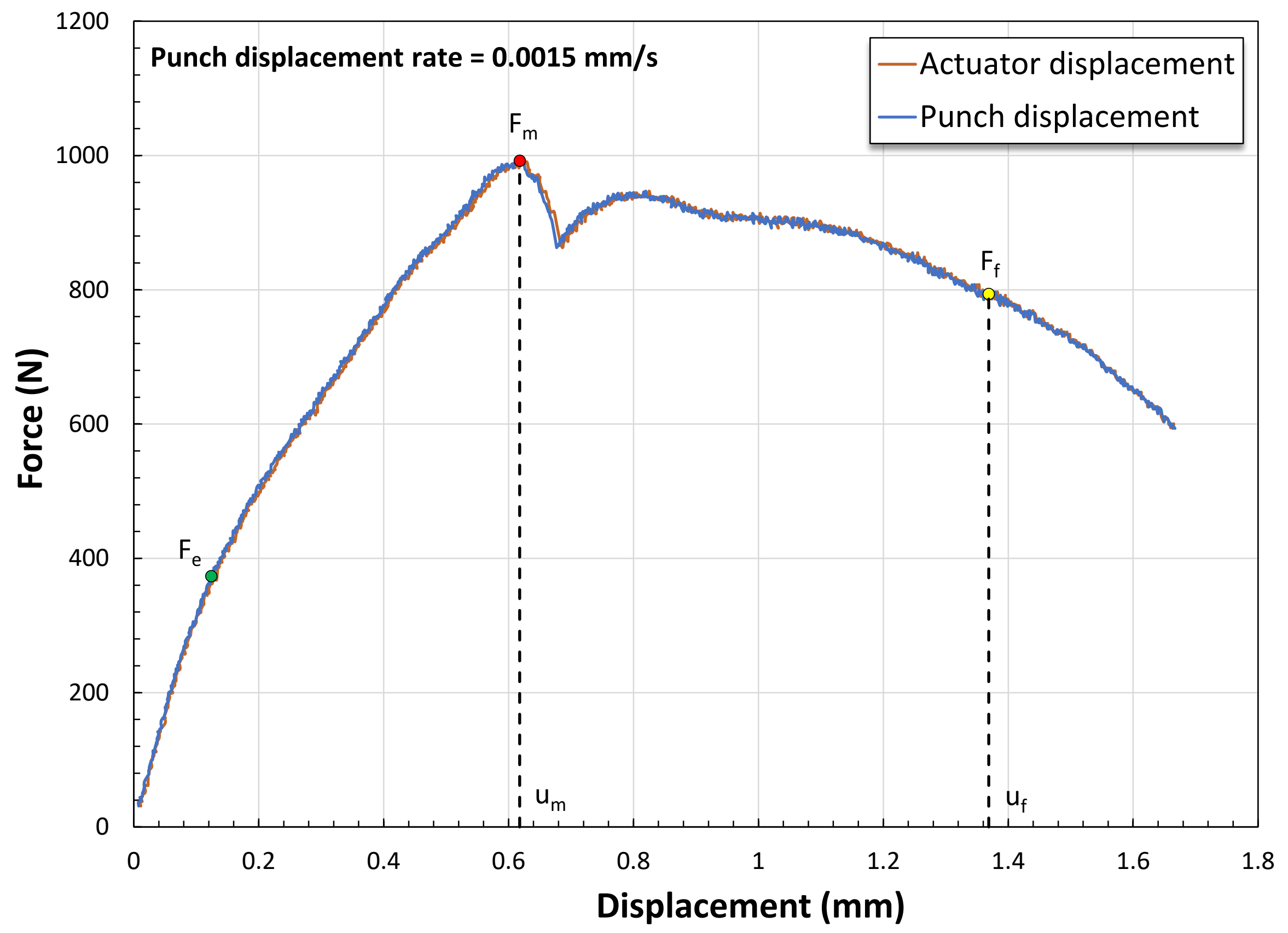




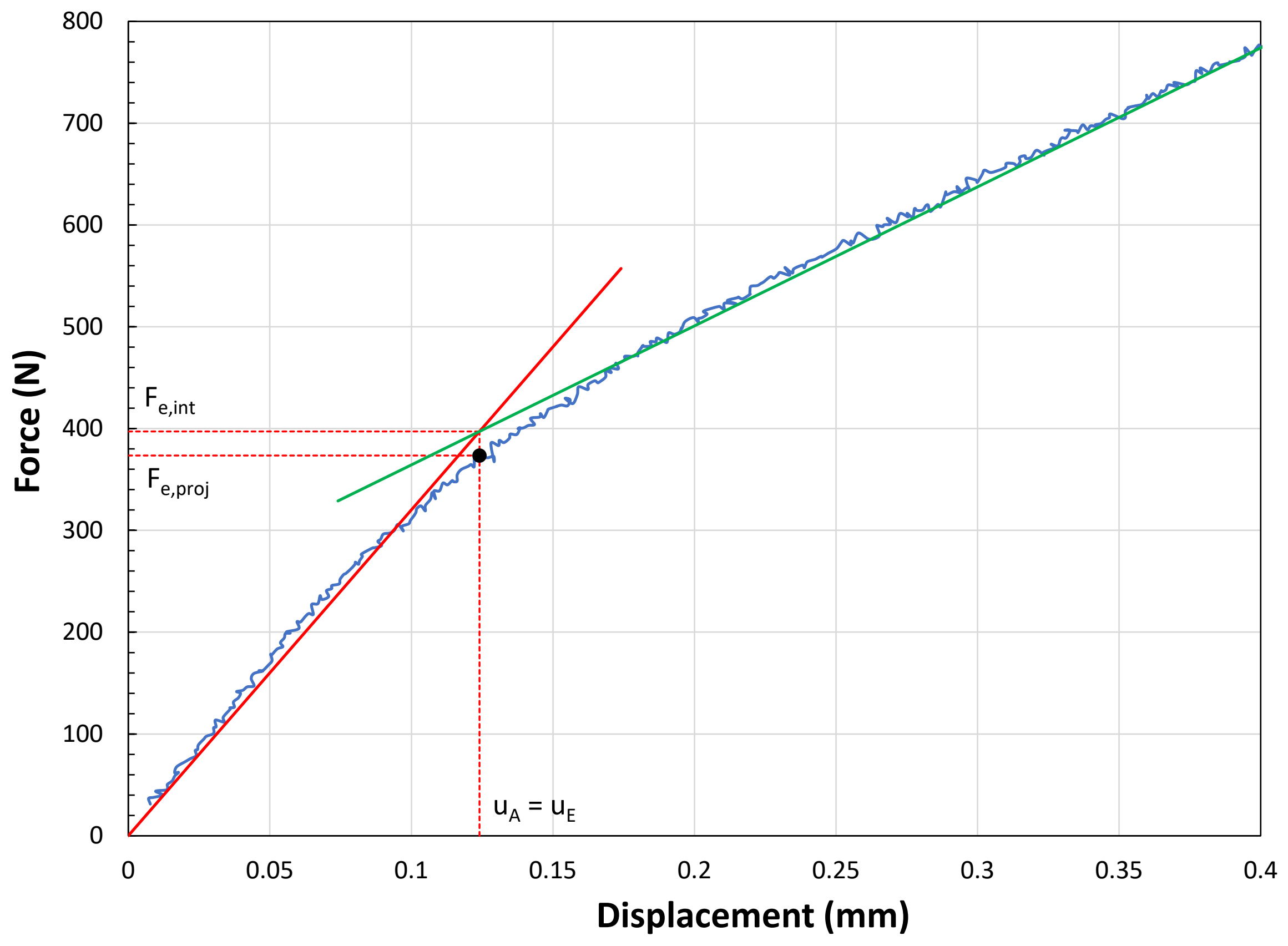


Small Punch Test Results

Specimen ID: 900HIP_3_N_a_SP8 (pc

Material: AM Ti-64

Test speed: $0.001426 \mathrm{~mm} / \mathrm{s}$

Max strain rate: $1.43 \mathrm{E}-03 \mathrm{~s}^{-1}$

\begin{tabular}{|c|c|c|}
\hline \multicolumn{3}{|c|}{ TEST RESULTS } \\
\hline \multicolumn{3}{|c|}{ Force values } \\
\hline$F_{e, p r o j}=$ & 372.2 & $\mathbf{N}$ \\
\hline$F_{e, \text { int }}=$ & 402.0 & $\mathbf{N}$ \\
\hline$F_{h 0 / 10, \text { off }}=$ & 515.6 & $\mathbf{N}$ \\
\hline$F_{0.1 \mathrm{~mm}, \mathrm{off}}=$ & 660.5 & $\mathbf{N}$ \\
\hline$F_{0.1 \mathrm{~mm}}=$ & 324.6 & $\mathbf{N}$ \\
\hline$F_{0.48 \mathrm{~mm}}=$ & 882.8 & $\mathbf{N}$ \\
\hline$F_{0.5 \mathrm{~mm}}=$ & 902.5 & $\mathbf{N}$ \\
\hline$F_{0.65 \mathrm{~mm}}=$ & 1026.6 & $\mathbf{N}$ \\
\hline$F_{0.9 \mathrm{~mm}}=$ & 1079.7 & $\mathbf{N}$ \\
\hline$F_{\text {ept }}=$ & 369.2 & $\mathbf{N}$ \\
\hline$F_{\mathrm{e} 1.5}=$ & 735.9 & $\mathbf{N}$ \\
\hline$F_{m}=$ & 1091.8 & $\mathbf{N}$ \\
\hline$F_{\text {infl }}=$ & 559.0 & $\mathbf{N}$ \\
\hline$F_{f}=$ & 873.4 & $\mathbf{N}$ \\
\hline \multicolumn{3}{|c|}{ Displacement values } \\
\hline $\mathrm{u}_{\mathrm{e}}=$ & 0.122 & $\mathrm{~mm}$ \\
\hline$u_{h 0 / 10, \text { off }}=$ & 0.203 & $\mathrm{~mm}$ \\
\hline$u_{0.1 \mathrm{~mm}, \mathrm{off}}=$ & 0.302 & $\mathrm{~mm}$ \\
\hline $\mathrm{u}_{\mathrm{e} 1.5}=$ & 0.357 & $\mathrm{~mm}$ \\
\hline $\mathbf{u}_{\mathrm{m}}=$ & 0.832 & $\mathrm{~mm}$ \\
\hline$u_{\text {infl }}=$ & 1.487 & $\mathrm{~mm}$ \\
\hline$u_{f}=$ & 1.273 & $\mathrm{~mm}$ \\
\hline \multicolumn{3}{|c|}{ Initial linear slope } \\
\hline Slope $_{\text {ini }}=$ & 3288.00 & $\mathrm{~N} / \mathrm{mm}$ \\
\hline \multicolumn{3}{|c|}{ Energy values } \\
\hline$E_{S P}=$ & 1.06 & J \\
\hline$E_{m}=$ & 0.62 & J \\
\hline$E_{P L}=$ & 0.43 & J \\
\hline
\end{tabular}




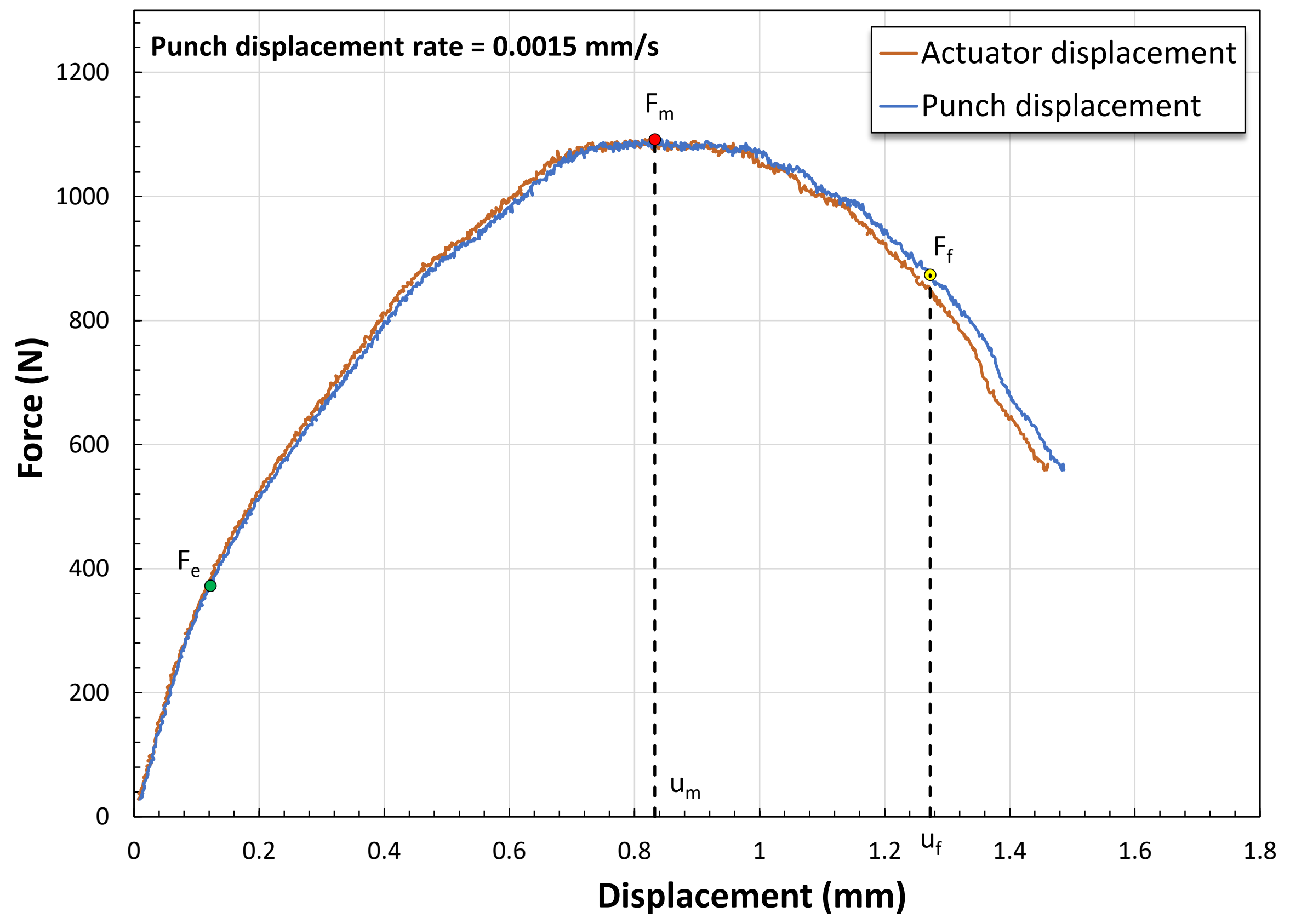




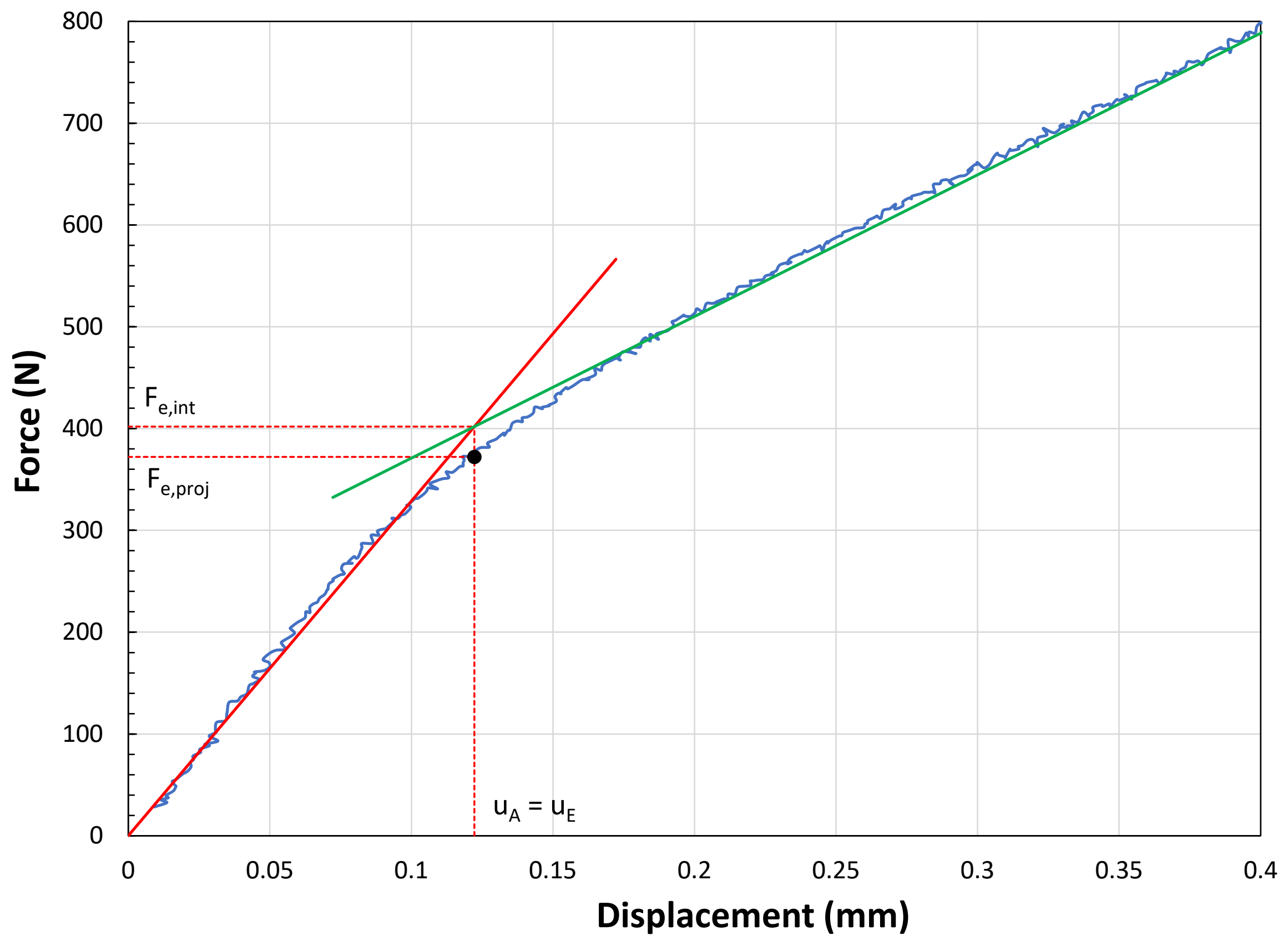


Small Punch Test Results

Specimen ID: 900HIP_3_N_a_SP9 (pc

Material: AM Ti-64

Test speed: $0.001459 \mathrm{~mm} / \mathrm{s}$

Max strain rate: $1.46 \mathrm{E}-03 \mathrm{~s}^{-1}$

\begin{tabular}{|c|c|c|}
\hline \multicolumn{3}{|c|}{ TEST RESULTS } \\
\hline \multicolumn{3}{|c|}{ Force values } \\
\hline$F_{e, \text { proj }}=$ & 373.8 & $\mathbf{N}$ \\
\hline$F_{e, \text { int }}=$ & 406.2 & $\mathbf{N}$ \\
\hline$F_{h 0 / 10, \text { off }}=$ & 531.7 & $\mathbf{N}$ \\
\hline$F_{0.1 \mathrm{~mm}, \mathrm{off}}=$ & 690.7 & $\mathbf{N}$ \\
\hline$F_{0.1 \mathrm{~mm}}=$ & 300.6 & $\mathbf{N}$ \\
\hline$F_{0.48 \mathrm{~mm}}=$ & 882.8 & $\mathbf{N}$ \\
\hline$F_{0.5 \mathrm{~mm}}=$ & 915.7 & $\mathbf{N}$ \\
\hline$F_{0.65 \mathrm{~mm}}=$ & 961.6 & $\mathbf{N}$ \\
\hline$F_{0.9 \mathrm{~mm}}=$ & 969.8 & $\mathbf{N}$ \\
\hline$F_{e p t}=$ & 721.9 & $\mathbf{N}$ \\
\hline$F_{e 1.5}=$ & 746.2 & $\mathbf{N}$ \\
\hline$F_{m}=$ & 995.9 & $\mathbf{N}$ \\
\hline$F_{\text {infl }}=$ & 509.4 & $\mathbf{N}$ \\
\hline$F_{f}=$ & 796.7 & $\mathbf{N}$ \\
\hline \multicolumn{3}{|c|}{ Displacement values } \\
\hline$u_{e}=$ & 0.136 & $\mathrm{~mm}$ \\
\hline$u_{h 0 / 10, \text { off }}=$ & 0.223 & $\mathrm{~mm}$ \\
\hline$u_{0.1 \mathrm{~mm}, \mathrm{off}}=$ & 0.333 & $\mathrm{~mm}$ \\
\hline $\mathrm{u}_{\mathrm{e} 1.5}=$ & 0.380 & $\mathrm{~mm}$ \\
\hline$u_{m}=$ & 0.558 & $\mathrm{~mm}$ \\
\hline$u_{\text {infl }}=$ & 1.648 & $\mathrm{~mm}$ \\
\hline$u_{f}=$ & 1.323 & $\mathrm{~mm}$ \\
\hline \multicolumn{3}{|c|}{ Initial linear slope } \\
\hline Slope $_{\text {ini }}=$ & 2989.78 & $\mathrm{~N} / \mathrm{mm}$ \\
\hline \multicolumn{3}{|c|}{ Energy values } \\
\hline$E_{S P}=$ & 1.04 & J \\
\hline$E_{m}=$ & 0.32 & J \\
\hline $\mathrm{E}_{\mathrm{PL}}=$ & 0.16 & J \\
\hline
\end{tabular}




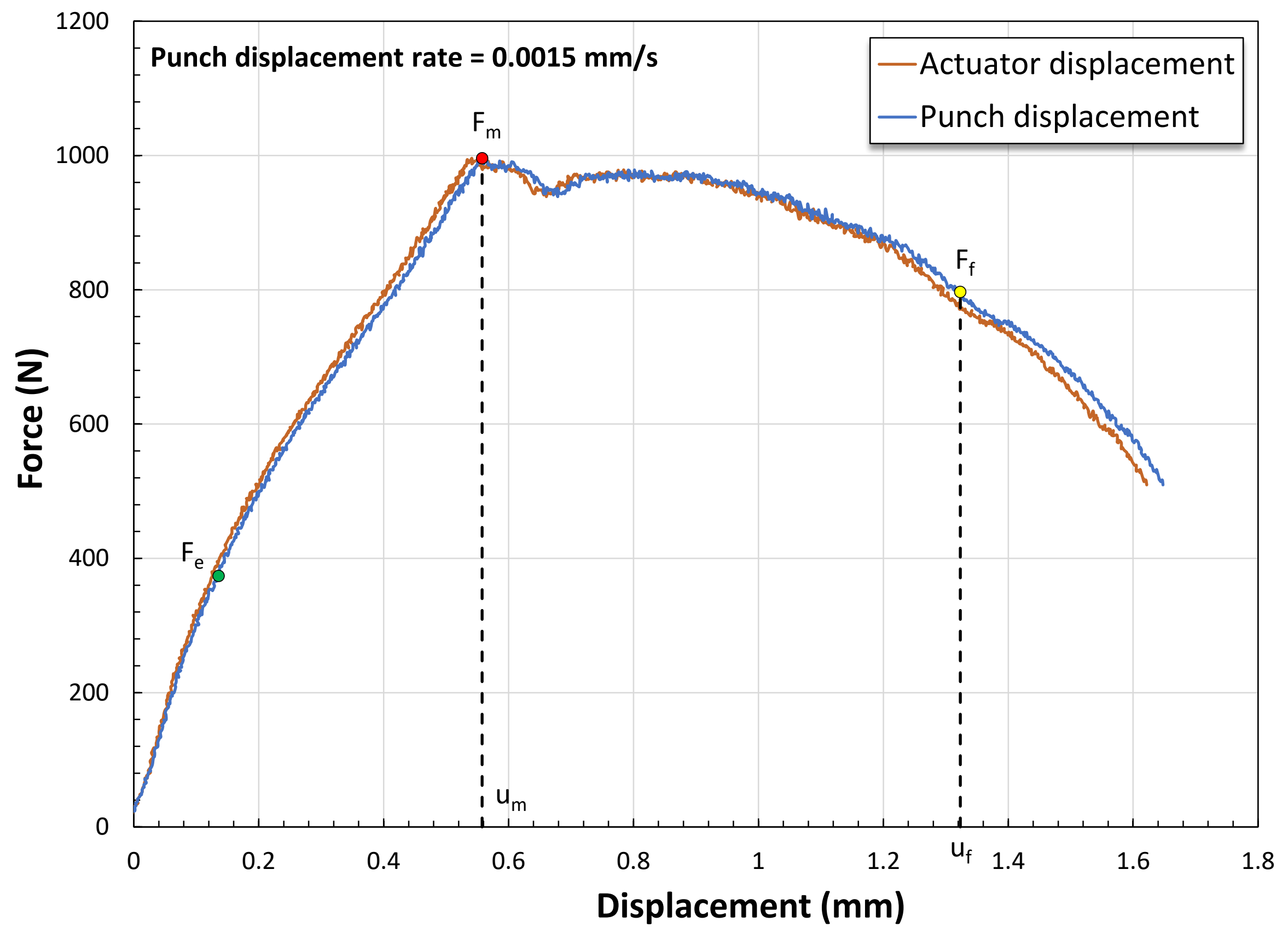




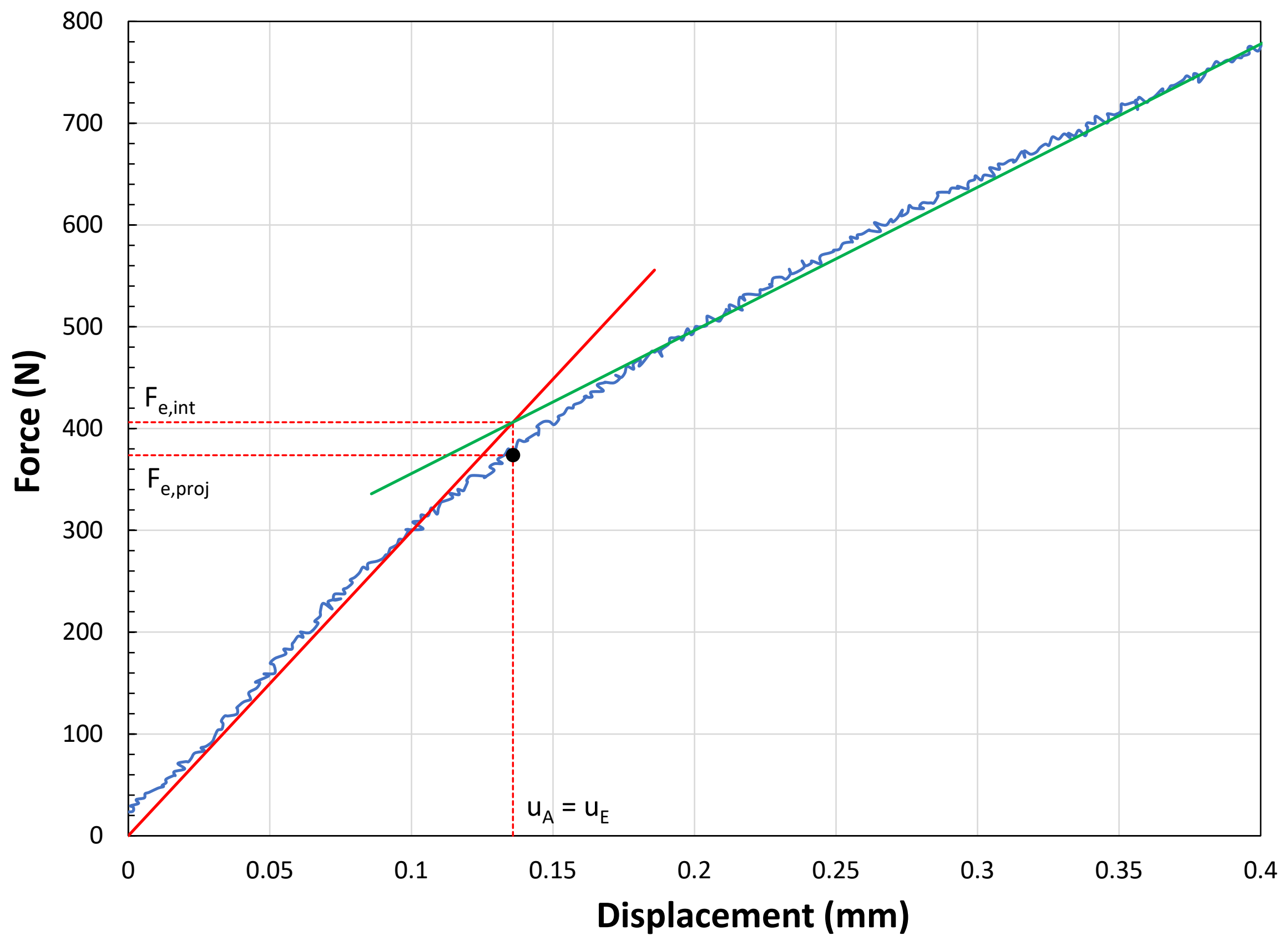


Small Punch Test Results

Specimen ID: 900HIP_3_N_a_SP10 (

Material: AM Ti-64

Test speed: $0.001444 \mathrm{~mm} / \mathrm{s}$

Max strain rate: $1.44 \mathrm{E}-03 \mathrm{~s}^{-1}$

\begin{tabular}{|c|c|c|}
\hline \multicolumn{3}{|c|}{ TEST RESULTS } \\
\hline \multicolumn{3}{|c|}{ Force values } \\
\hline$F_{e, \text { proj }}=$ & 434.7 & $\mathbf{N}$ \\
\hline$F_{e, \text { int }}=$ & 467.2 & $\mathbf{N}$ \\
\hline$F_{h 0 / 10, \text { off }}=$ & 595.3 & $\mathbf{N}$ \\
\hline$F_{0.1 \mathrm{~mm}, \mathrm{off}}=$ & 748.0 & $\mathbf{N}$ \\
\hline$F_{0.1 \mathrm{~mm}}=$ & 293.0 & $\mathbf{N}$ \\
\hline$F_{0.48 \mathrm{~mm}}=$ & 872.8 & $\mathbf{N}$ \\
\hline$F_{0.5 \mathrm{~mm}}=$ & 878.4 & $\mathbf{N}$ \\
\hline$F_{0.65 \mathrm{~mm}}=$ & 921.6 & $\mathbf{N}$ \\
\hline$F_{0.9 \mathrm{~mm}}=$ & 1033.5 & $\mathbf{N}$ \\
\hline$F_{e p t}=$ & 884.7 & $\mathbf{N}$ \\
\hline$F_{\text {e1.5 }}=$ & 858.4 & $\mathbf{N}$ \\
\hline$F_{m}=$ & 1040.8 & $\mathbf{N}$ \\
\hline$F_{\text {infl }}=$ & 967.1 & $\mathbf{N}$ \\
\hline$F_{f}=$ & 832.7 & $\mathbf{N}$ \\
\hline \multicolumn{3}{|c|}{ Displacement values } \\
\hline$u_{e}=$ & 0.164 & $\mathrm{~mm}$ \\
\hline$u_{h 0 / 10, \text { off }}=$ & 0.254 & $\mathrm{~mm}$ \\
\hline$u_{0.1 \mathrm{~mm}, \mathrm{off}}=$ & 0.364 & $\mathrm{~mm}$ \\
\hline $\mathrm{u}_{\mathrm{e} 1.5}=$ & 0.457 & $\mathrm{~mm}$ \\
\hline$u_{m}=$ & 0.899 & $\mathrm{~mm}$ \\
\hline$u_{\text {infl }}=$ & 0.707 & $\mathrm{~mm}$ \\
\hline$u_{f}=$ & 1.356 & $\mathrm{~mm}$ \\
\hline \multicolumn{3}{|c|}{ Initial linear slope } \\
\hline Slope $_{\text {ini }}=$ & 2849.28 & $\mathrm{~N} / \mathrm{mm}$ \\
\hline \multicolumn{3}{|c|}{ Energy values } \\
\hline$E_{S P}=$ & 1.09 & J \\
\hline$E_{m}=$ & 0.65 & J \\
\hline $\mathrm{E}_{\mathrm{PL}}=$ & 0.46 & J \\
\hline
\end{tabular}




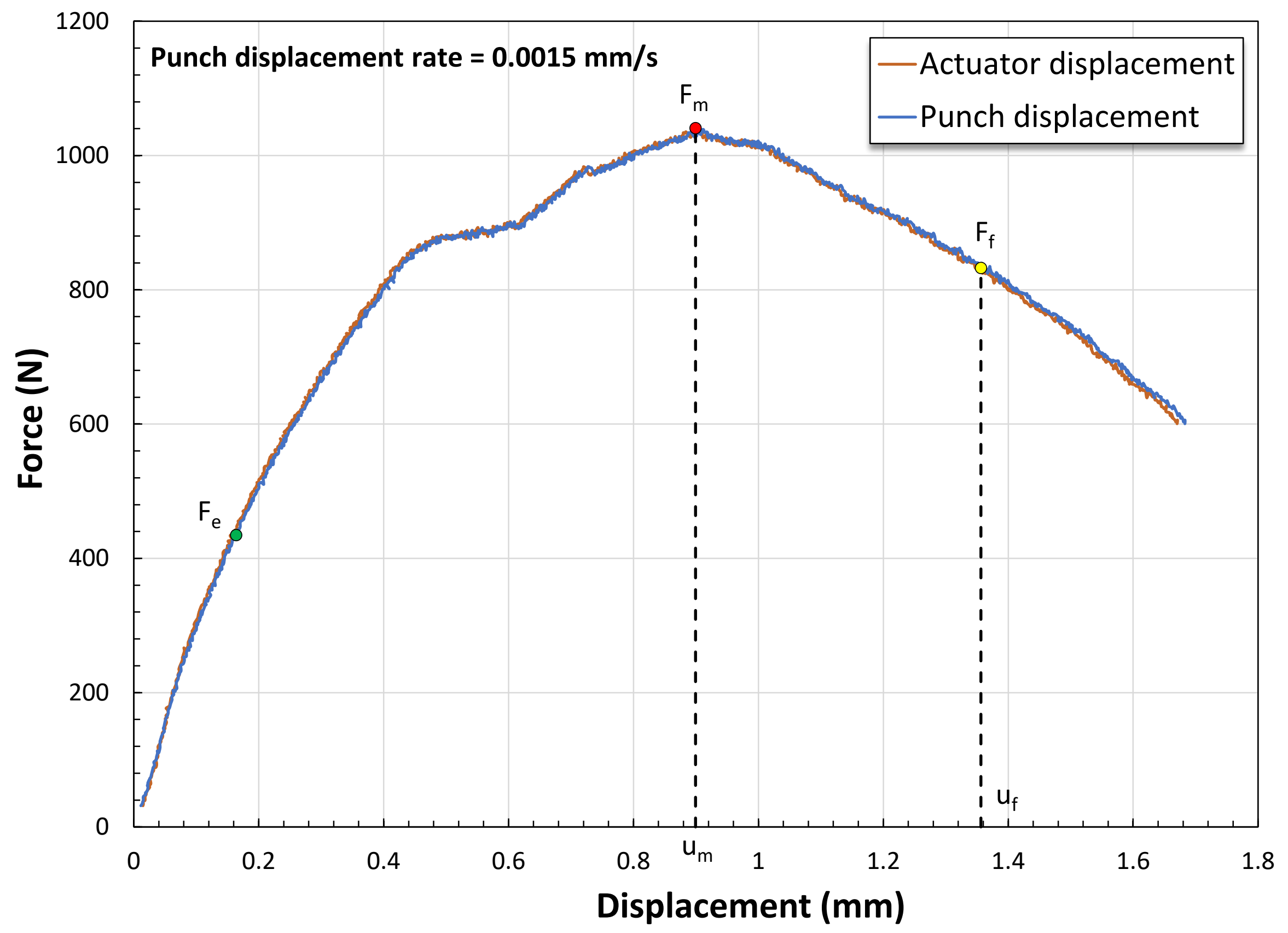




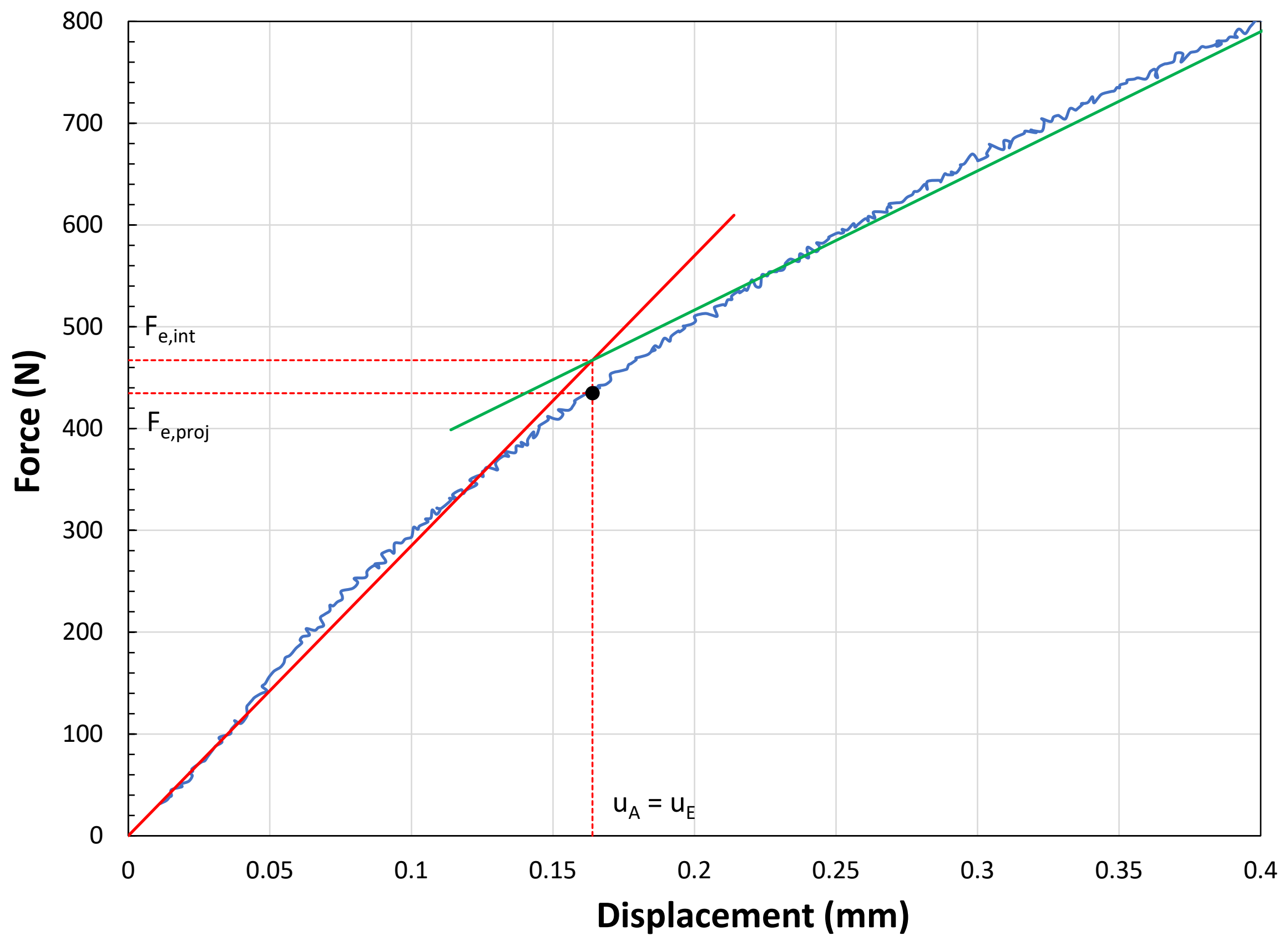




\section{ANNEX 12 \\ SP Test Results for \\ $1050{ }^{\circ} \mathrm{C}+800{ }^{\circ} \mathrm{C} \mathrm{HIP}$, scan length $=78 \mathrm{~mm}$, non-supported ("rough" specimens)}


Small Punch Test Results

Specimen ID: SuperB_1_N_a_SP1

Material: AM Ti-64

Test speed: $0.001043 \mathrm{~mm} / \mathrm{s}$

Max strain rate: $1.04 \mathrm{E}-03 \mathrm{~s}^{-1}$

\begin{tabular}{|c|c|c|}
\hline \multicolumn{3}{|c|}{ TEST RESULTS } \\
\hline \multicolumn{3}{|c|}{ Force values } \\
\hline$F_{e, \text { proj }}=$ & 407.3 & $\mathbf{N}$ \\
\hline$F_{e, \text { int }}=$ & 420.1 & $\mathbf{N}$ \\
\hline$F_{h 0 / 10, \text { off }}=$ & 576.8 & $\mathbf{N}$ \\
\hline$F_{0.1 \mathrm{~mm}, \mathrm{off}}=$ & 703.8 & $\mathbf{N}$ \\
\hline$F_{0.1 \mathrm{~mm}}=$ & 218.3 & $\mathbf{N}$ \\
\hline$F_{0.48 \mathrm{~mm}}=$ & 768.0 & $\mathbf{N}$ \\
\hline$F_{0.5 \mathrm{~mm}}=$ & 791.8 & $\mathbf{N}$ \\
\hline$F_{0.65 \mathrm{~mm}}=$ & 932.3 & $\mathbf{N}$ \\
\hline$F_{0.9 \mathrm{~mm}}=$ & 1126.7 & $\mathbf{N}$ \\
\hline$F_{e p t}=$ & 1179.1 & $\mathbf{N}$ \\
\hline$F_{\text {e1.5 }}=$ & 231.7 & $\mathbf{N}$ \\
\hline$F_{m}=$ & 1192.9 & $\mathbf{N}$ \\
\hline$F_{\text {infl }}=$ & 702.3 & $\mathbf{N}$ \\
\hline$F_{f}=$ & 954.3 & $\mathbf{N}$ \\
\hline \multicolumn{3}{|c|}{ Displacement values } \\
\hline$u_{e}=$ & 0.196 & $\mathrm{~mm}$ \\
\hline$u_{h 0 / 10, \text { off }}=$ & 0.320 & $\mathrm{~mm}$ \\
\hline$u_{0.1 \mathrm{~mm}, \mathrm{off}}=$ & 0.430 & $\mathrm{~mm}$ \\
\hline $\mathrm{u}_{\mathrm{e} 1.5}=$ & 0.104 & $\mathrm{~mm}$ \\
\hline$u_{m}=$ & 1.066 & $\mathrm{~mm}$ \\
\hline$u_{\text {infl }}=$ & 1.760 & $\mathrm{~mm}$ \\
\hline$u_{f}=$ & 1.498 & $\mathrm{~mm}$ \\
\hline \multicolumn{3}{|c|}{ Initial linear slope } \\
\hline Slope $_{\text {ini }}=$ & 2139.70 & $\mathrm{~N} / \mathrm{mm}$ \\
\hline \multicolumn{3}{|c|}{ Energy values } \\
\hline$E_{S P}=$ & 1.29 & J \\
\hline$E_{m}=$ & 0.81 & J \\
\hline $\mathrm{E}_{\mathrm{PL}}=$ & 0.48 & J \\
\hline
\end{tabular}




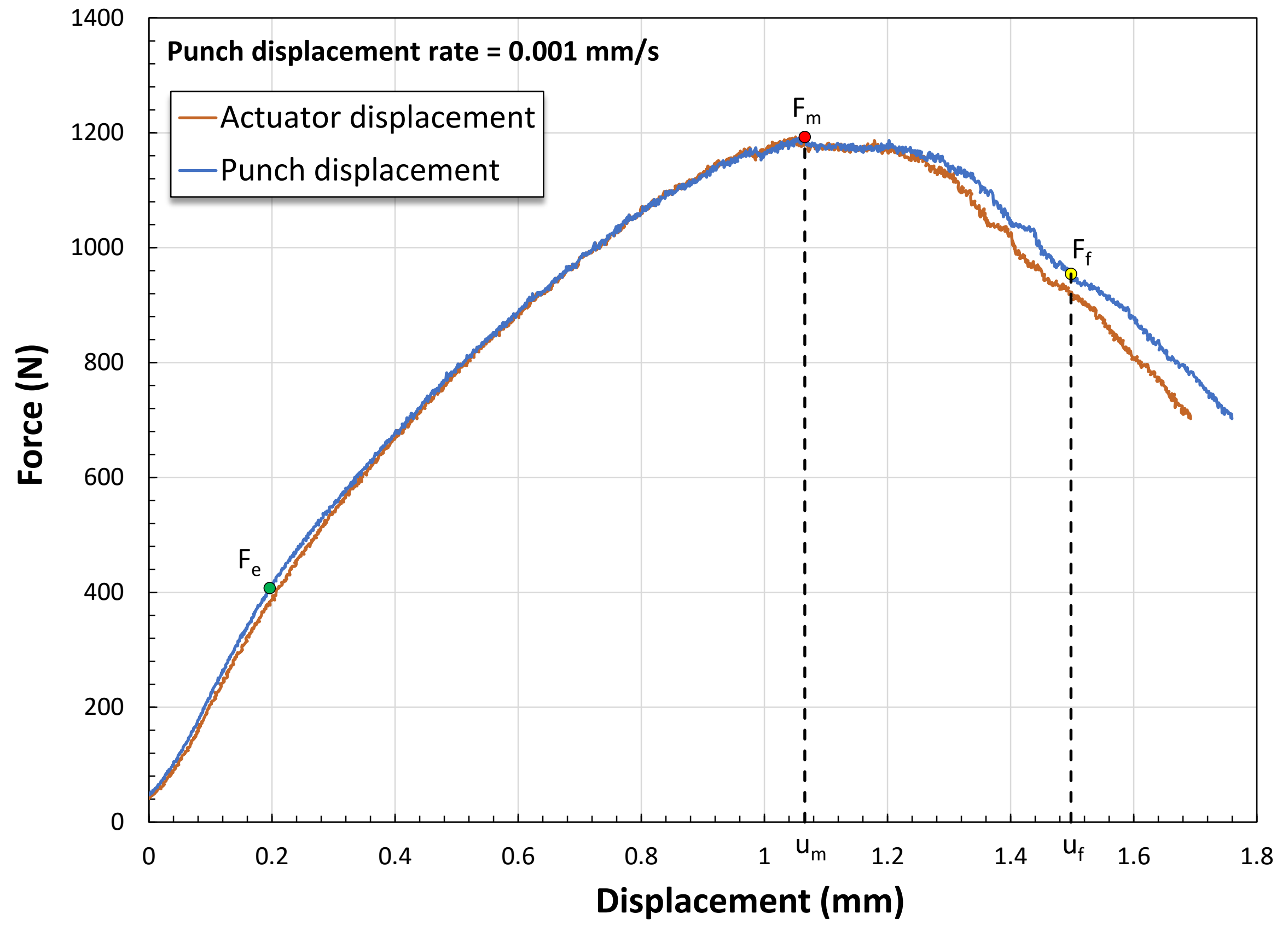




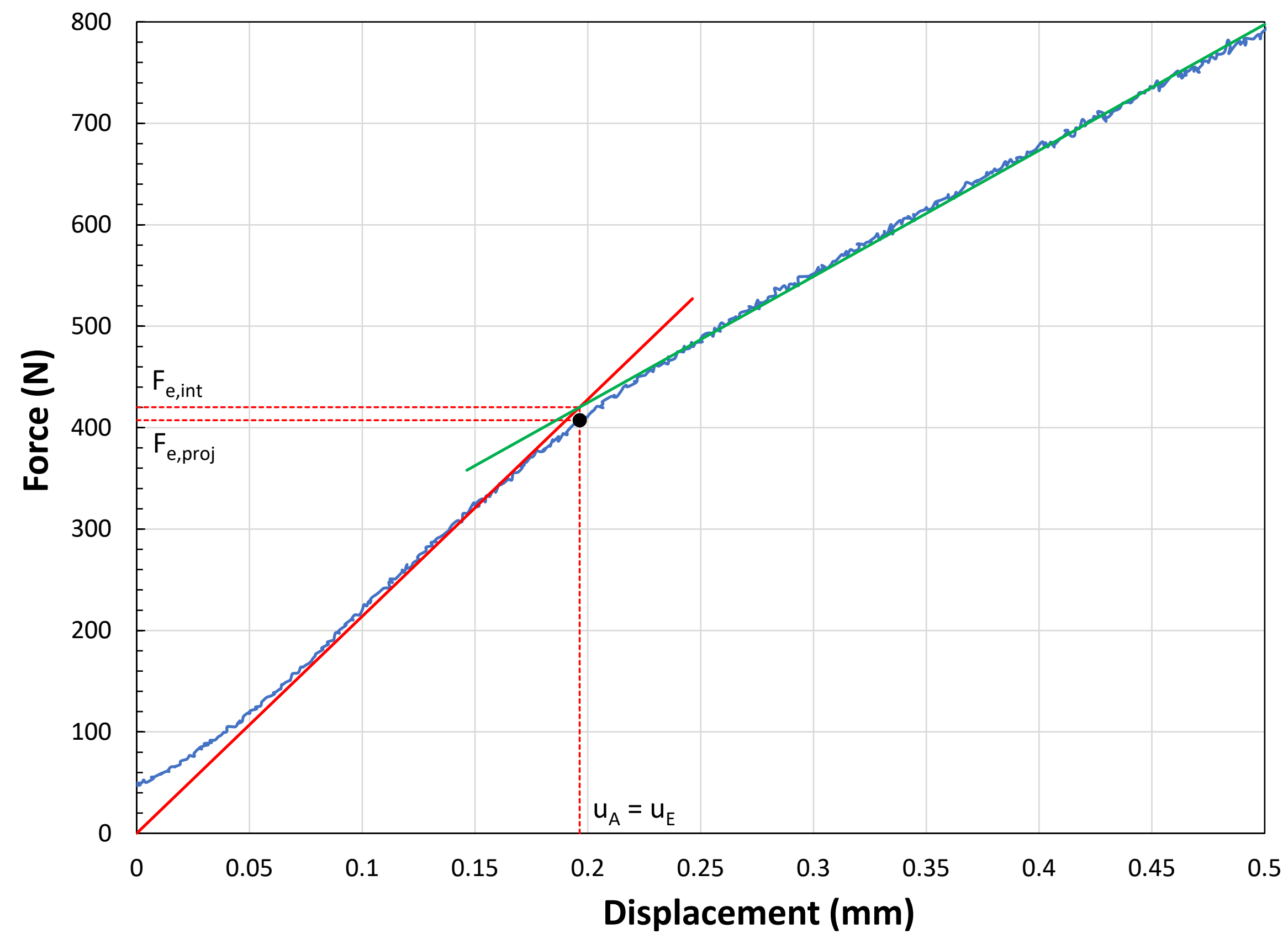


Small Punch Test Results

Specimen ID: SuperB_1_N_a_SP2

Material: AM Ti-64

Test speed: $0.001067 \mathrm{~mm} / \mathrm{s}$

Max strain rate: $1.07 \mathrm{E}-03 \mathrm{~s}^{-1}$

\begin{tabular}{|c|c|c|}
\hline \multicolumn{3}{|c|}{ TEST RESULTS } \\
\hline \multicolumn{3}{|c|}{ Force values } \\
\hline$F_{e, \text { proj }}=$ & 365.1 & $\mathbf{N}$ \\
\hline$F_{e, \text { int }}=$ & 377.5 & $\mathbf{N}$ \\
\hline$F_{h 0 / 10, \text { off }}=$ & 570.1 & $\mathbf{N}$ \\
\hline$F_{0.1 \mathrm{~mm}, \mathrm{off}}=$ & 732.7 & $\mathbf{N}$ \\
\hline$F_{0.1 \mathrm{~mm}}=$ & 207.9 & $\mathbf{N}$ \\
\hline$F_{0.48 \mathrm{~mm}}=$ & 748.0 & $\mathbf{N}$ \\
\hline$F_{0.5 \mathrm{~mm}}=$ & 779.8 & $\mathbf{N}$ \\
\hline$F_{0.65 \mathrm{~mm}}=$ & 931.9 & $\mathbf{N}$ \\
\hline$F_{0.9 \mathrm{~mm}}=$ & 1138.0 & $\mathbf{N}$ \\
\hline$F_{e p t}=$ & 1127.1 & $\mathbf{N}$ \\
\hline$F_{e 1.5}=$ & 146.1 & $\mathbf{N}$ \\
\hline$F_{m}=$ & 1194.5 & $\mathbf{N}$ \\
\hline$F_{\text {infl }}=$ & 1189.5 & $\mathbf{N}$ \\
\hline$F_{f}=$ & 955.6 & $\mathbf{N}$ \\
\hline \multicolumn{3}{|c|}{ Displacement values } \\
\hline$u_{e}=$ & 0.188 & $\mathrm{~mm}$ \\
\hline$u_{h 0 / 10, \text { off }}=$ & 0.335 & $\mathrm{~mm}$ \\
\hline$u_{0.1 \mathrm{~mm}, \mathrm{off}}=$ & 0.465 & $\mathrm{~mm}$ \\
\hline $\mathrm{u}_{\mathrm{e} 1.5}=$ & 0.072 & $\mathrm{~mm}$ \\
\hline$u_{m}=$ & 1.071 & $\mathrm{~mm}$ \\
\hline$u_{\text {infl }}=$ & 1.077 & $\mathrm{~mm}$ \\
\hline$u_{f}=$ & 1.458 & $\mathrm{~mm}$ \\
\hline \multicolumn{3}{|c|}{ Initial linear slope } \\
\hline Slope $_{\text {ini }}=$ & 2011.63 & $\mathrm{~N} / \mathrm{mm}$ \\
\hline \multicolumn{3}{|c|}{ Energy values } \\
\hline$E_{S P}=$ & 1.23 & J \\
\hline$E_{m}=$ & 0.80 & J \\
\hline $\mathrm{E}_{\mathrm{PL}}=$ & 0.45 & J \\
\hline
\end{tabular}




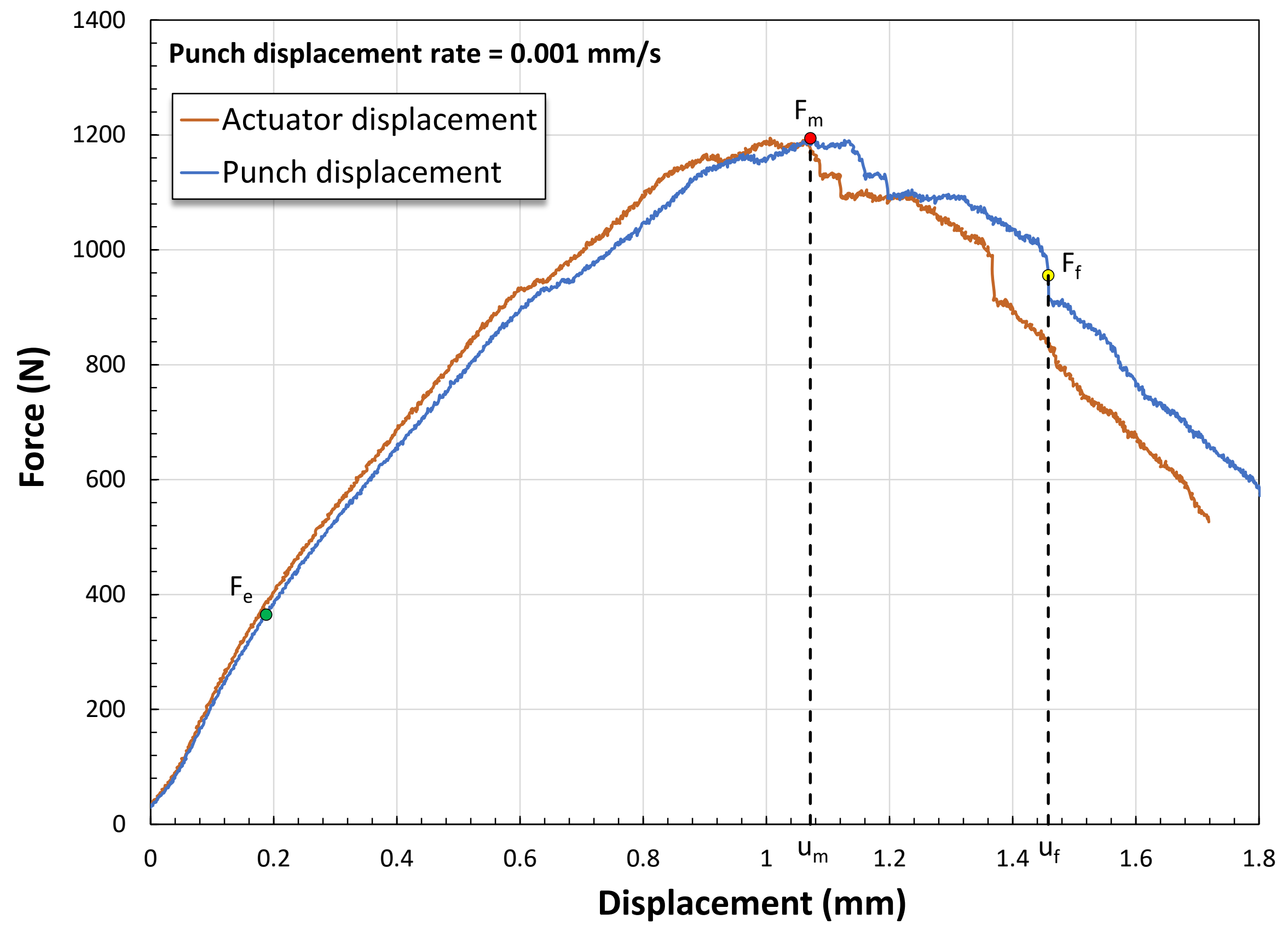




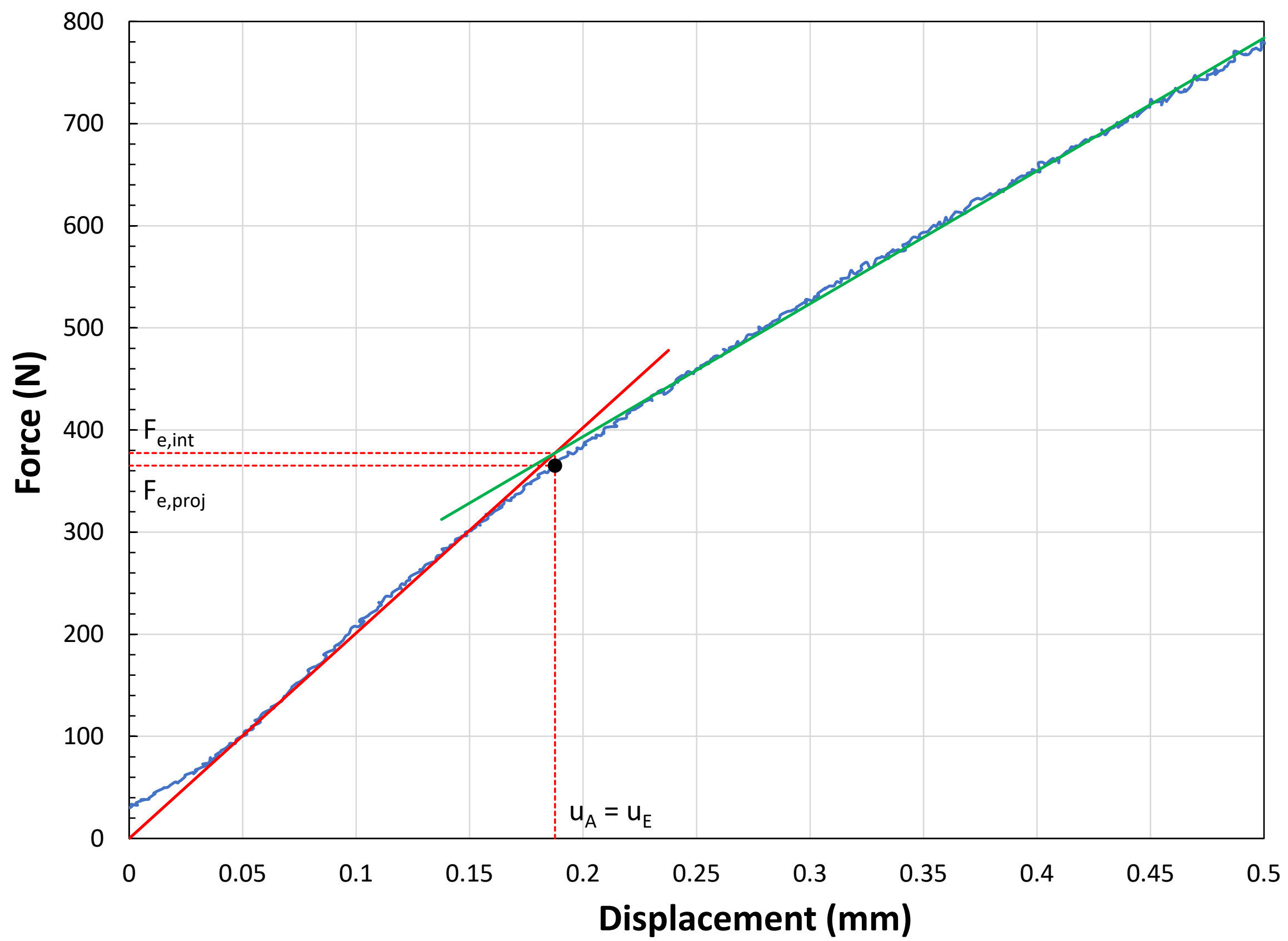


Small Punch Test Results

Specimen ID: SuperB_1_N_a_SP4

Material: AM Ti-64

Test speed: $0.00107 \mathrm{~mm} / \mathrm{s}$

Max strain rate: $1.07 \mathrm{E}-03 \mathrm{~s}^{-1}$

\begin{tabular}{|c|c|c|}
\hline \multicolumn{3}{|c|}{ TEST RESULTS } \\
\hline \multicolumn{3}{|c|}{ Force values } \\
\hline$F_{e, \text { proj }}=$ & 456.7 & $\mathbf{N}$ \\
\hline$F_{e, \text { int }}=$ & 465.1 & $\mathbf{N}$ \\
\hline$F_{h 0 / 10, \text { off }}=$ & 646.7 & $\mathbf{N}$ \\
\hline$F_{0.1 \mathrm{~mm}, \mathrm{off}}=$ & 771.7 & $\mathbf{N}$ \\
\hline$F_{0.1 \mathrm{~mm}}=$ & 182.3 & $\mathbf{N}$ \\
\hline$F_{0.48 \mathrm{~mm}}=$ & 731.7 & $\mathbf{N}$ \\
\hline$F_{0.5 \mathrm{~mm}}=$ & 753.7 & $\mathbf{N}$ \\
\hline$F_{0.65 \mathrm{~mm}}=$ & 910.0 & $\mathbf{N}$ \\
\hline$F_{0.9 \mathrm{~mm}}=$ & 1138.2 & $\mathbf{N}$ \\
\hline$F_{e p t}=$ & 868.4 & $\mathbf{N}$ \\
\hline$F_{\text {e1.5 }}=$ & 173.5 & $\mathbf{N}$ \\
\hline$F_{m}=$ & 1230.4 & $\mathbf{N}$ \\
\hline$F_{\text {infl }}=$ & 714.9 & $\mathbf{N}$ \\
\hline$F_{f}=$ & 984.3 & $\mathbf{N}$ \\
\hline \multicolumn{3}{|c|}{ Displacement values } \\
\hline$u_{e}=$ & 0.252 & $\mathrm{~mm}$ \\
\hline$u_{h 0 / 10, \text { off }}=$ & 0.401 & $\mathrm{~mm}$ \\
\hline$u_{0.1 \mathrm{~mm}, \mathrm{off}}=$ & 0.519 & $\mathrm{~mm}$ \\
\hline $\mathrm{u}_{\mathrm{e} 1.5}=$ & 0.096 & $\mathrm{~mm}$ \\
\hline$u_{m}=$ & 1.065 & $\mathrm{~mm}$ \\
\hline$u_{\text {infl }}=$ & 1.831 & $\mathrm{~mm}$ \\
\hline$u_{f}=$ & 1.560 & $\mathrm{~mm}$ \\
\hline \multicolumn{3}{|c|}{ Initial linear slope } \\
\hline Slope $_{\text {ini }}=$ & 1844.34 & $\mathrm{~N} / \mathrm{mm}$ \\
\hline \multicolumn{3}{|c|}{ Energy values } \\
\hline$E_{S P}=$ & 1.34 & J \\
\hline$E_{m}=$ & 0.79 & J \\
\hline $\mathrm{E}_{\mathrm{PL}}=$ & 0.38 & J \\
\hline
\end{tabular}




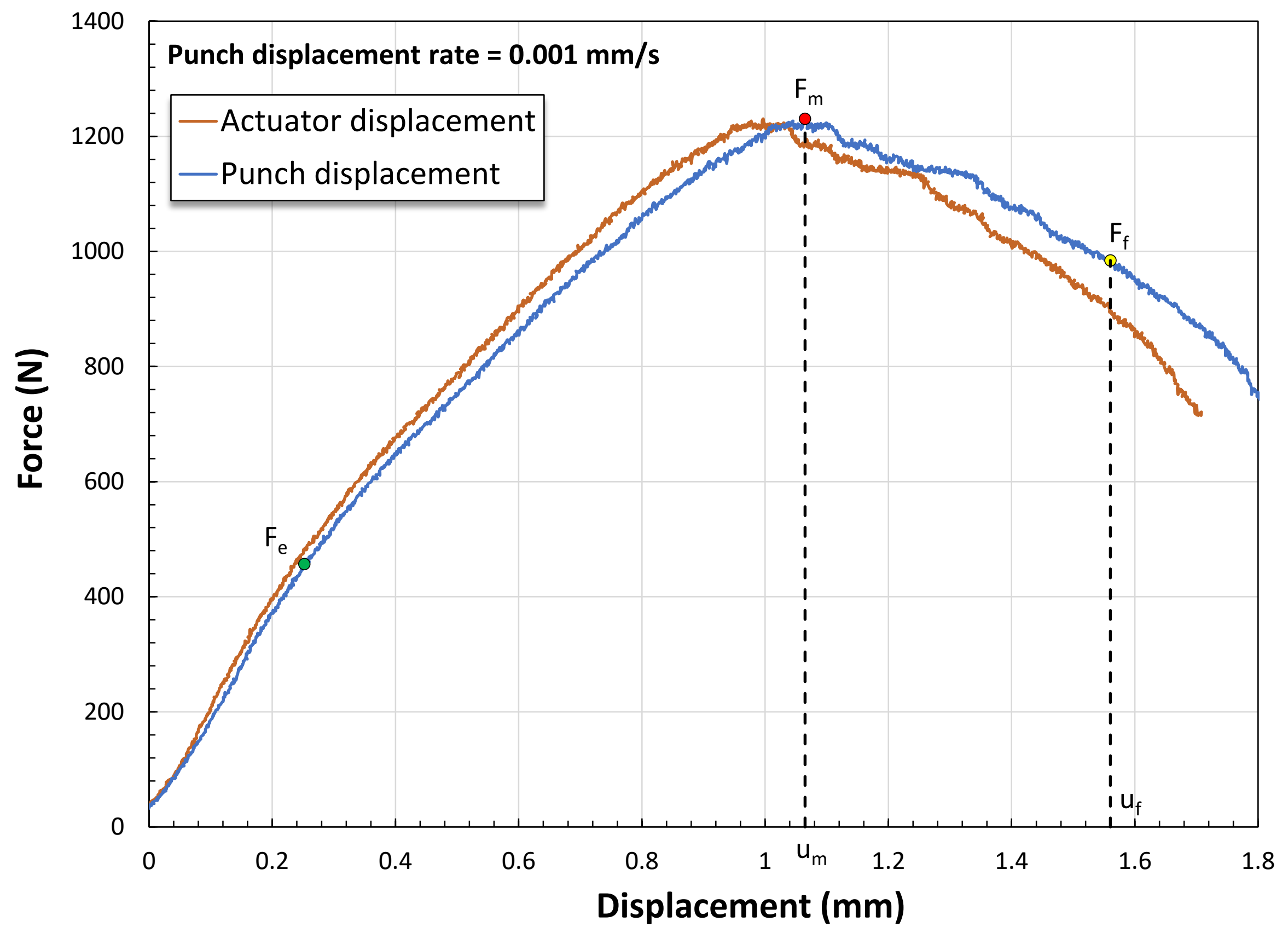




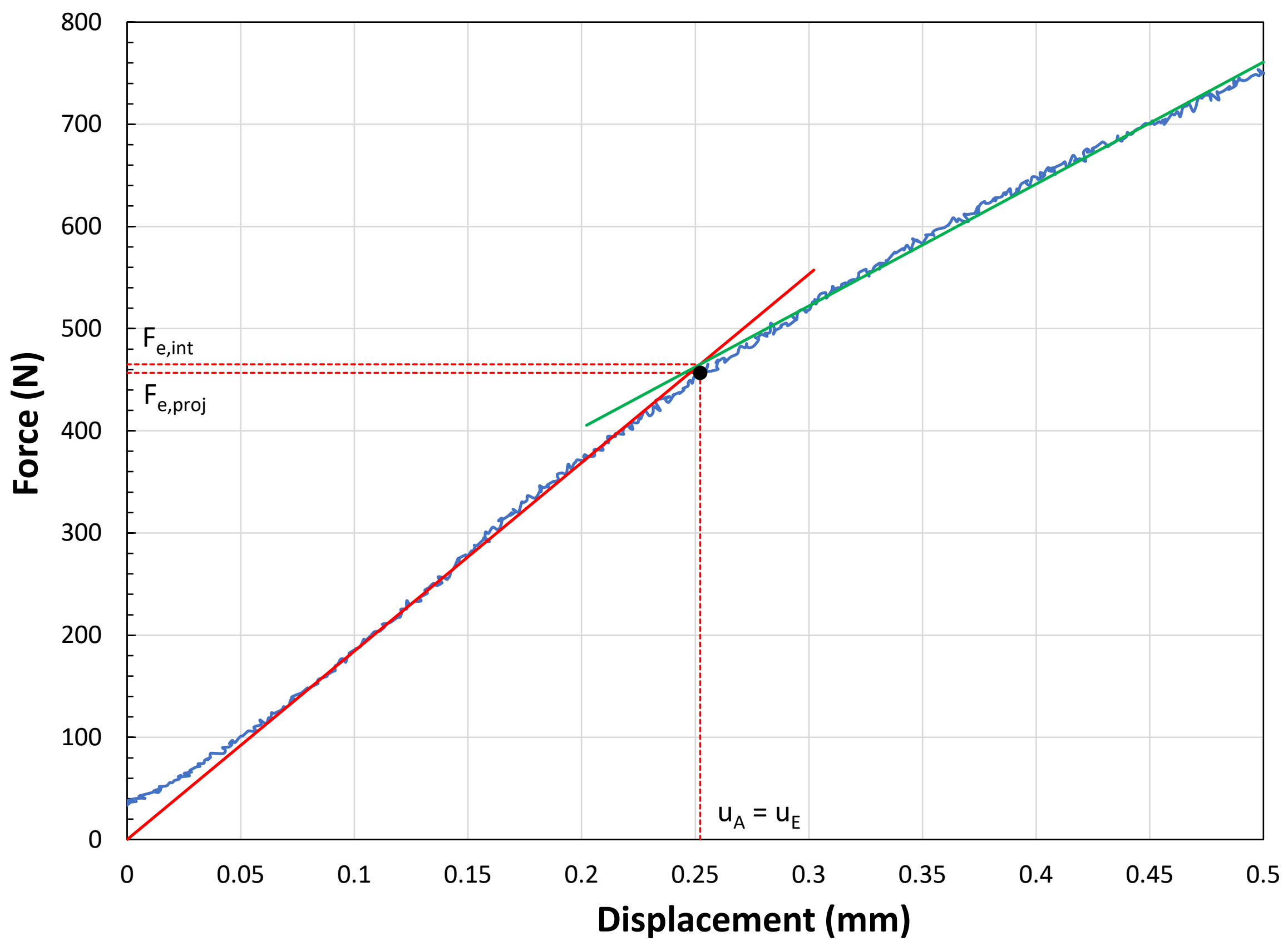


Small Punch Test Results

Specimen ID: SuperB_1_N_a_SP5

Material: AM Ti-64

Test speed: $0.001076 \mathrm{~mm} / \mathrm{s}$

Max strain rate: $1.08 \mathrm{E}-03 \mathrm{~s}^{-1}$

\begin{tabular}{|c|c|c|}
\hline \multicolumn{3}{|c|}{ TEST RESULTS } \\
\hline \multicolumn{3}{|c|}{ Force values } \\
\hline$F_{e, \text { proj }}=$ & 475.7 & $\mathbf{N}$ \\
\hline$F_{e, \text { int }}=$ & 485.1 & $\mathbf{N}$ \\
\hline$F_{h 0 / 10, \text { off }}=$ & 644.0 & $\mathbf{N}$ \\
\hline$F_{0.1 \mathrm{~mm}, \mathrm{off}}=$ & 765.3 & $\mathbf{N}$ \\
\hline$F_{0.1 \mathrm{~mm}}=$ & 196.2 & $\mathbf{N}$ \\
\hline$F_{0.48 \mathrm{~mm}}=$ & 748.9 & $\mathbf{N}$ \\
\hline$F_{0.5 \mathrm{~mm}}=$ & 772.8 & $\mathbf{N}$ \\
\hline$F_{0.65 \mathrm{~mm}}=$ & 928.4 & $\mathbf{N}$ \\
\hline$F_{0.9 \mathrm{~mm}}=$ & 1162.1 & $\mathbf{N}$ \\
\hline$F_{e p t}=$ & 539.2 & $\mathbf{N}$ \\
\hline$F_{\text {e1.5 }}=$ & 126.7 & $\mathbf{N}$ \\
\hline$F_{m}=$ & 1225.3 & $\mathbf{N}$ \\
\hline$F_{\text {infl }}=$ & 61.4 & $\mathbf{N}$ \\
\hline$F_{f}=$ & 980.2 & $\mathbf{N}$ \\
\hline \multicolumn{3}{|c|}{ Displacement values } \\
\hline$u_{e}=$ & 0.251 & $\mathrm{~mm}$ \\
\hline$u_{h 0 / 10, \text { off }}=$ & 0.385 & $\mathrm{~mm}$ \\
\hline$u_{0.1 \mathrm{~mm}, \mathrm{off}}=$ & 0.496 & $\mathrm{~mm}$ \\
\hline $\mathrm{u}_{\mathrm{e} 1.5}=$ & 0.066 & $\mathrm{~mm}$ \\
\hline$u_{m}=$ & 1.005 & $\mathrm{~mm}$ \\
\hline$u_{\text {infl }}=$ & 0.027 & $\mathrm{~mm}$ \\
\hline$u_{f}=$ & 1.524 & $\mathrm{~mm}$ \\
\hline \multicolumn{3}{|c|}{ Initial linear slope } \\
\hline Slope $_{\text {ini }}=$ & 1930.46 & $\mathrm{~N} / \mathrm{mm}$ \\
\hline \multicolumn{3}{|c|}{ Energy values } \\
\hline$E_{S P}=$ & 1.32 & J \\
\hline$E_{m}=$ & 0.74 & J \\
\hline $\mathrm{E}_{\mathrm{PL}}=$ & 0.35 & J \\
\hline
\end{tabular}




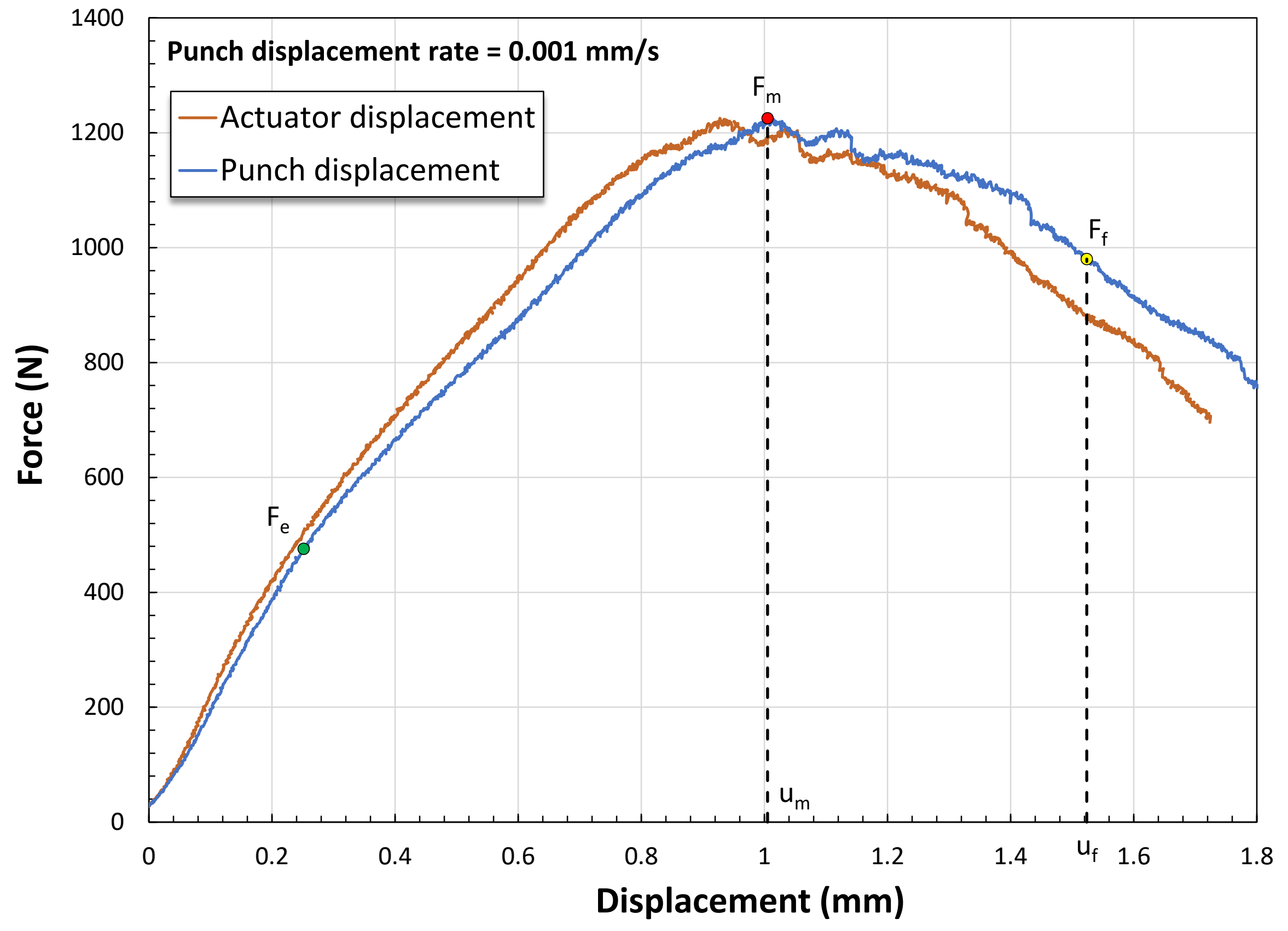




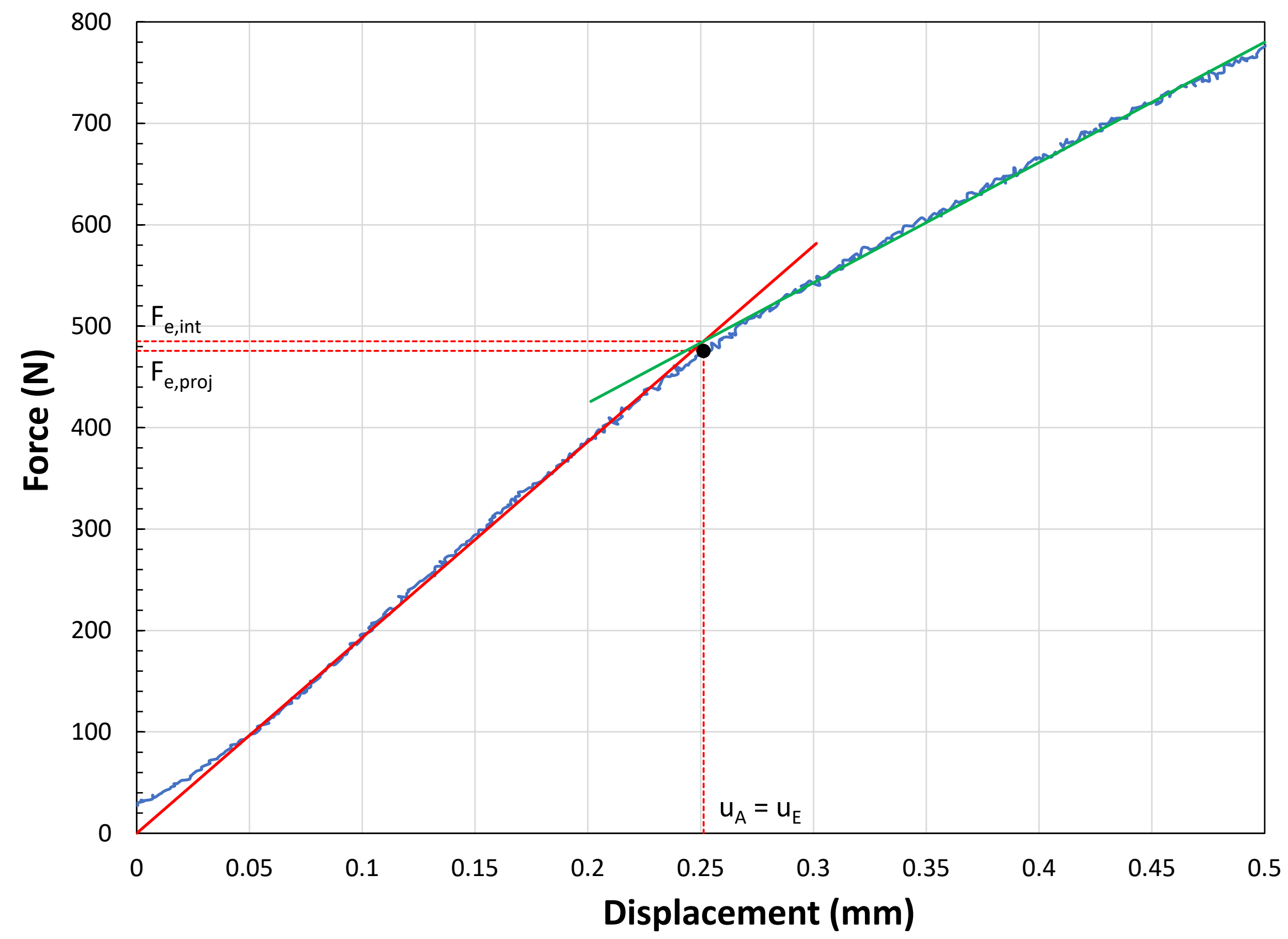


Small Punch Test Results

Specimen ID: SuperB_1_N_a_SP6

Material: AM Ti-64

Test speed: $0.001113 \mathrm{~mm} / \mathrm{s}$

Max strain rate: $1.11 \mathrm{E}-03 \mathrm{~s}^{-1}$

\begin{tabular}{|c|c|c|}
\hline \multicolumn{3}{|c|}{ TEST RESULTS } \\
\hline \multicolumn{3}{|c|}{ Force values } \\
\hline$F_{e, \text { proj }}=$ & 414.5 & $\mathbf{N}$ \\
\hline$F_{e, \text { int }}=$ & 430.1 & $\mathbf{N}$ \\
\hline$F_{h 0 / 10, \text { off }}=$ & 594.2 & $\mathbf{N}$ \\
\hline$F_{0.1 \mathrm{~mm}, \mathrm{off}}=$ & 713.8 & $\mathbf{N}$ \\
\hline$F_{0.1 \mathrm{~mm}}=$ & 193.2 & $\mathbf{N}$ \\
\hline$F_{0.48 \mathrm{~mm}}=$ & 724.3 & $\mathbf{N}$ \\
\hline$F_{0.5 \mathrm{~mm}}=$ & 747.6 & $\mathbf{N}$ \\
\hline$F_{0.65 \mathrm{~mm}}=$ & 901.9 & $\mathbf{N}$ \\
\hline$F_{0.9 \mathrm{~mm}}=$ & 1106.7 & $\mathbf{N}$ \\
\hline$F_{e p t}=$ & 431.9 & $\mathbf{N}$ \\
\hline$F_{\text {e1.5 }}=$ & 143.3 & $\mathbf{N}$ \\
\hline$F_{m}=$ & 1156.0 & $\mathbf{N}$ \\
\hline$F_{\text {infl }}=$ & 901.5 & $\mathbf{N}$ \\
\hline$F_{f}=$ & 924.8 & $\mathbf{N}$ \\
\hline \multicolumn{3}{|c|}{ Displacement values } \\
\hline$u_{e}=$ & 0.224 & $\mathrm{~mm}$ \\
\hline$u_{h 0 / 10, \text { off }}=$ & 0.360 & $\mathrm{~mm}$ \\
\hline$u_{0.1 \mathrm{~mm}, \mathrm{off}}=$ & 0.472 & $\mathrm{~mm}$ \\
\hline $\mathrm{u}_{\mathrm{e} 1.5}=$ & 0.075 & $\mathrm{~mm}$ \\
\hline$u_{m}=$ & 1.002 & $\mathrm{~mm}$ \\
\hline$u_{\text {infl }}=$ & 0.649 & $\mathrm{~mm}$ \\
\hline$u_{f}=$ & 1.628 & $\mathrm{~mm}$ \\
\hline \multicolumn{3}{|c|}{ Initial linear slope } \\
\hline Slope $_{\text {ini }}=$ & 1918.27 & $\mathrm{~N} / \mathrm{mm}$ \\
\hline \multicolumn{3}{|c|}{ Energy values } \\
\hline$E_{S P}=$ & 1.36 & J \\
\hline$E_{m}=$ & 0.71 & J \\
\hline $\mathrm{E}_{\mathrm{PL}}=$ & 0.36 & J \\
\hline
\end{tabular}




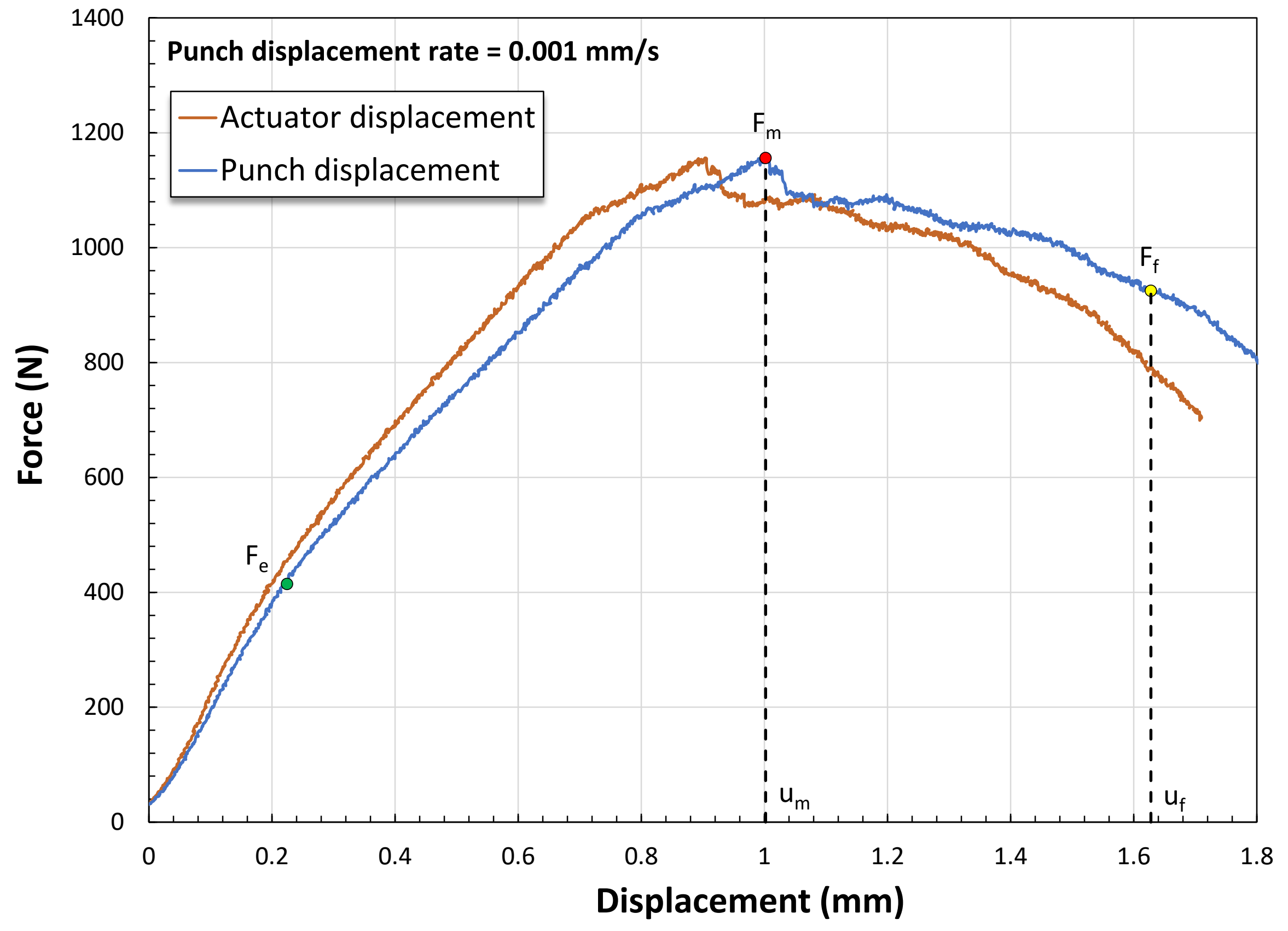




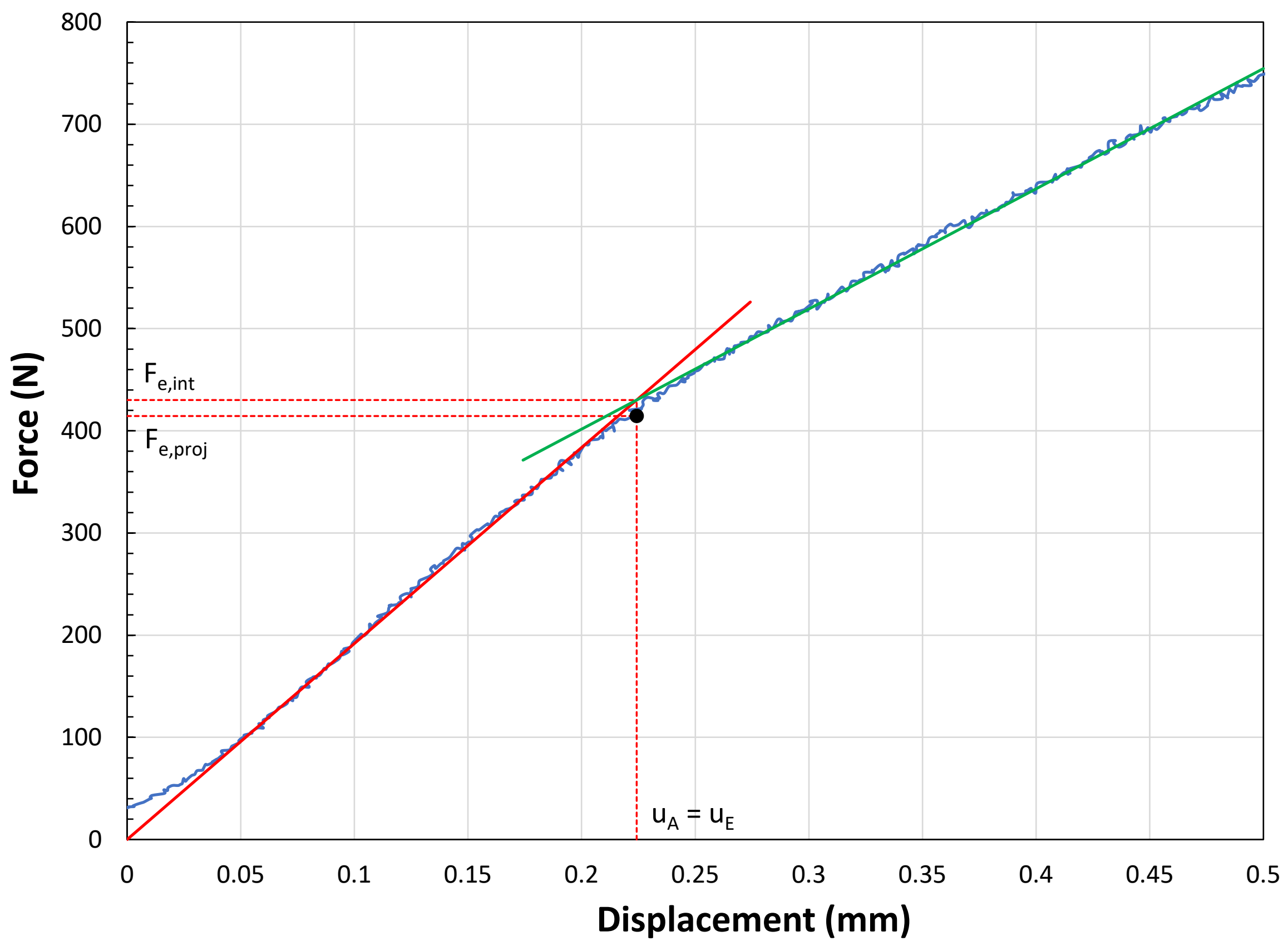


Small Punch Test Results

Specimen ID: SuperB_1_N_a_SP7

Material: AM Ti-64

Test speed: $0.001064 \mathrm{~mm} / \mathrm{s}$

Max strain rate: $1.06 \mathrm{E}-03 \mathrm{~s}^{-1}$

\begin{tabular}{|c|c|c|}
\hline \multicolumn{3}{|c|}{ TEST RESULTS } \\
\hline \multicolumn{3}{|c|}{ Force values } \\
\hline$F_{e, \text { proj }}=$ & 380.2 & $\mathbf{N}$ \\
\hline$F_{e, \text { int }}=$ & 393.6 & $\mathbf{N}$ \\
\hline$F_{h 0 / 10, \text { off }}=$ & 604.0 & $\mathbf{N}$ \\
\hline$F_{0.1 \mathrm{~mm}, \mathrm{off}}=$ & 742.3 & $\mathbf{N}$ \\
\hline$F_{0.1 \mathrm{~mm}}=$ & 191.4 & $\mathbf{N}$ \\
\hline$F_{0.48 \mathrm{~mm}}=$ & 732.4 & $\mathbf{N}$ \\
\hline$F_{0.5 \mathrm{~mm}}=$ & 752.2 & $\mathbf{N}$ \\
\hline$F_{0.65 \mathrm{~mm}}=$ & 912.0 & $\mathbf{N}$ \\
\hline$F_{0.9 \mathrm{~mm}}=$ & 1122.9 & $\mathbf{N}$ \\
\hline$F_{e p t}=$ & 1096.6 & $\mathbf{N}$ \\
\hline$F_{e 1.5}=$ & 155.8 & $\mathbf{N}$ \\
\hline$F_{m}=$ & 1196.6 & $\mathbf{N}$ \\
\hline$F_{\text {infl }}=$ & 678.8 & $\mathbf{N}$ \\
\hline$F_{f}=$ & 957.2 & $\mathbf{N}$ \\
\hline \multicolumn{3}{|c|}{ Displacement values } \\
\hline$u_{e}=$ & 0.210 & $\mathrm{~mm}$ \\
\hline$u_{h 0 / 10, \text { off }}=$ & 0.372 & $\mathrm{~mm}$ \\
\hline$u_{0.1 \mathrm{~mm}, \mathrm{off}}=$ & 0.496 & $\mathrm{~mm}$ \\
\hline $\mathrm{u}_{\mathrm{e} 1.5}=$ & 0.082 & $\mathrm{~mm}$ \\
\hline$u_{m}=$ & 1.023 & $\mathrm{~mm}$ \\
\hline$u_{\text {infl }}=$ & 1.843 & $\mathrm{~mm}$ \\
\hline$u_{f}=$ & 1.412 & $\mathrm{~mm}$ \\
\hline \multicolumn{3}{|c|}{ Initial linear slope } \\
\hline Slope $_{\text {ini }}=$ & 1874.92 & $\mathrm{~N} / \mathrm{mm}$ \\
\hline \multicolumn{3}{|c|}{ Energy values } \\
\hline$E_{S P}=$ & 1.14 & J \\
\hline$E_{m}=$ & 0.73 & J \\
\hline $\mathrm{E}_{\mathrm{PL}}=$ & 0.35 & J \\
\hline
\end{tabular}




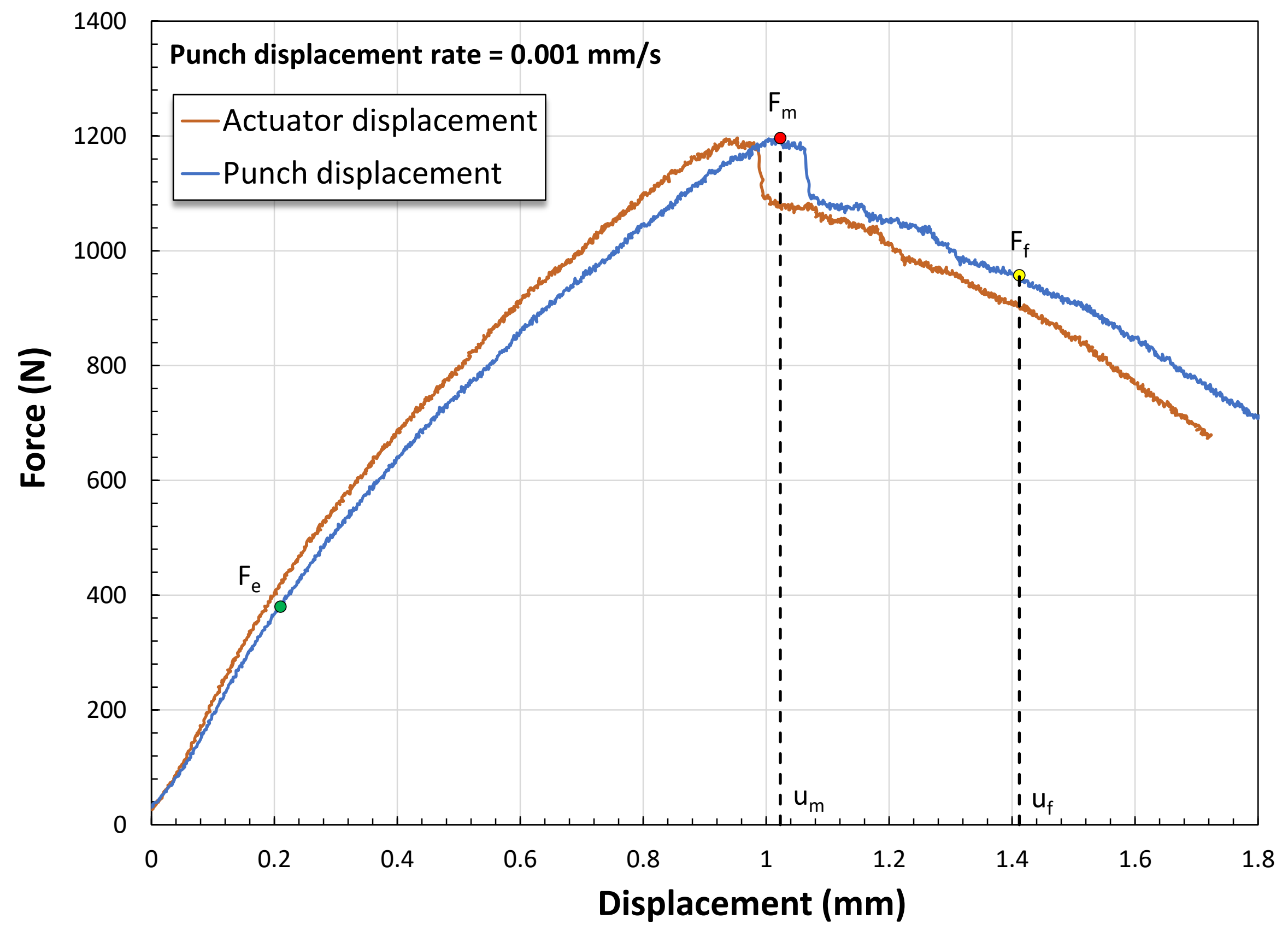




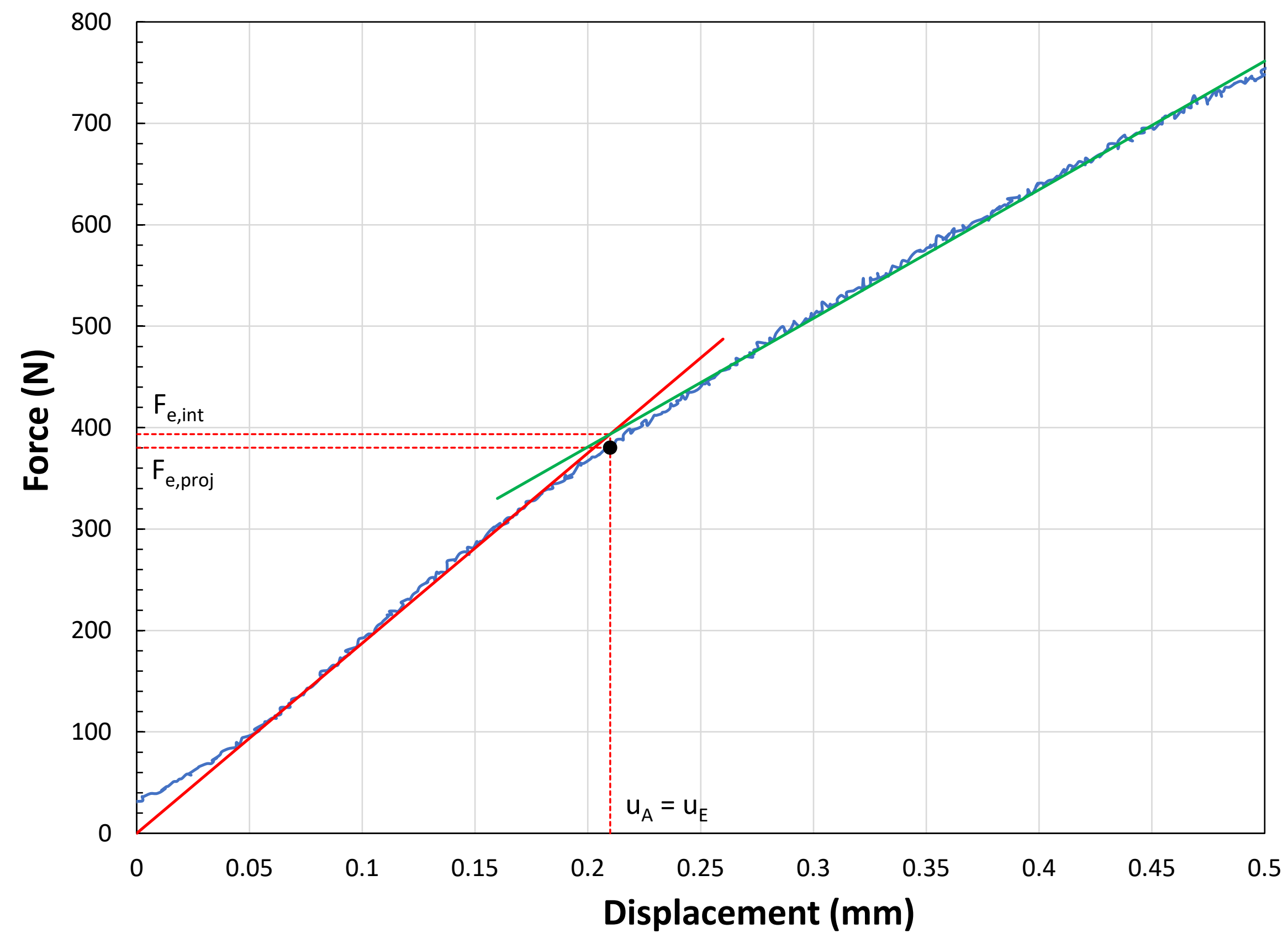




$$
\begin{gathered}
\text { ANNEX } 13 \\
\text { SP Test Results for } \\
1050^{\circ} \mathrm{C}+800^{\circ} \mathrm{C} \mathrm{HIP,} \text {, scan length }=78 \mathrm{~mm} \text {, non-supported } \\
\text { (polished specimens) }
\end{gathered}
$$


Small Punch Test Results

Specimen ID: 900HIP_3_N_a_SP7 (pc

Material: AM Ti-64

Test speed: $0.001441 \mathrm{~mm} / \mathrm{s}$

Max strain rate: $1.44 \mathrm{E}-03 \mathrm{~s}^{-1}$

\begin{tabular}{|c|c|c|}
\hline \multicolumn{3}{|c|}{ TEST RESULTS } \\
\hline \multicolumn{3}{|c|}{ Force values } \\
\hline$F_{e, p r o j}=$ & 373.5 & $\mathbf{N}$ \\
\hline$F_{e, \text { int }}=$ & 397.2 & $\mathbf{N}$ \\
\hline$F_{h 0 / 10, \text { off }}=$ & 522.2 & $\mathbf{N}$ \\
\hline$F_{0.1 \mathrm{~mm}, \mathrm{off}}=$ & 653.3 & $\mathbf{N}$ \\
\hline$F_{0.1 \mathrm{~mm}}=$ & 309.9 & $\mathbf{N}$ \\
\hline$F_{0.48 \mathrm{~mm}}=$ & 870.1 & $\mathbf{N}$ \\
\hline$F_{0.5 \mathrm{~mm}}=$ & 882.4 & $\mathbf{N}$ \\
\hline$F_{0.65 \mathrm{~mm}}=$ & 965.2 & $\mathbf{N}$ \\
\hline$F_{0.9 \mathrm{~mm}}=$ & 918.4 & $\mathbf{N}$ \\
\hline$F_{\text {ept }}=$ & 831.9 & $\mathbf{N}$ \\
\hline$F_{\mathrm{e} 1.5}=$ & 600.9 & $\mathbf{N}$ \\
\hline$F_{m}=$ & 992.2 & $\mathbf{N}$ \\
\hline$F_{\text {infl }}=$ & 593.6 & $\mathbf{N}$ \\
\hline$F_{f}=$ & 793.7 & $\mathbf{N}$ \\
\hline \multicolumn{3}{|c|}{ Displacement values } \\
\hline$u_{e}=$ & 0.124 & $\mathrm{~mm}$ \\
\hline$u_{h 0 / 10, \text { off }}=$ & 0.209 & $\mathrm{~mm}$ \\
\hline $\mathrm{u}_{0.1 \mathrm{~mm}, \mathrm{off}}=$ & 0.305 & $\mathrm{~mm}$ \\
\hline$u_{e 1.5}=$ & 0.269 & $\mathrm{~mm}$ \\
\hline$u_{m}=$ & 0.618 & $\mathrm{~mm}$ \\
\hline$u_{\text {infl }}=$ & 1.667 & $\mathrm{~mm}$ \\
\hline$u_{f}=$ & 1.369 & $\mathrm{~mm}$ \\
\hline \multicolumn{3}{|c|}{ Initial linear slope } \\
\hline Slope $_{\text {ini }}=$ & 3201.67 & $\mathrm{~N} / \mathrm{mm}$ \\
\hline \multicolumn{3}{|c|}{ Energy values } \\
\hline$E_{S P}=$ & 1.05 & J \\
\hline$E_{m}=$ & 0.38 & J \\
\hline$E_{P L}=$ & 0.23 & J \\
\hline
\end{tabular}




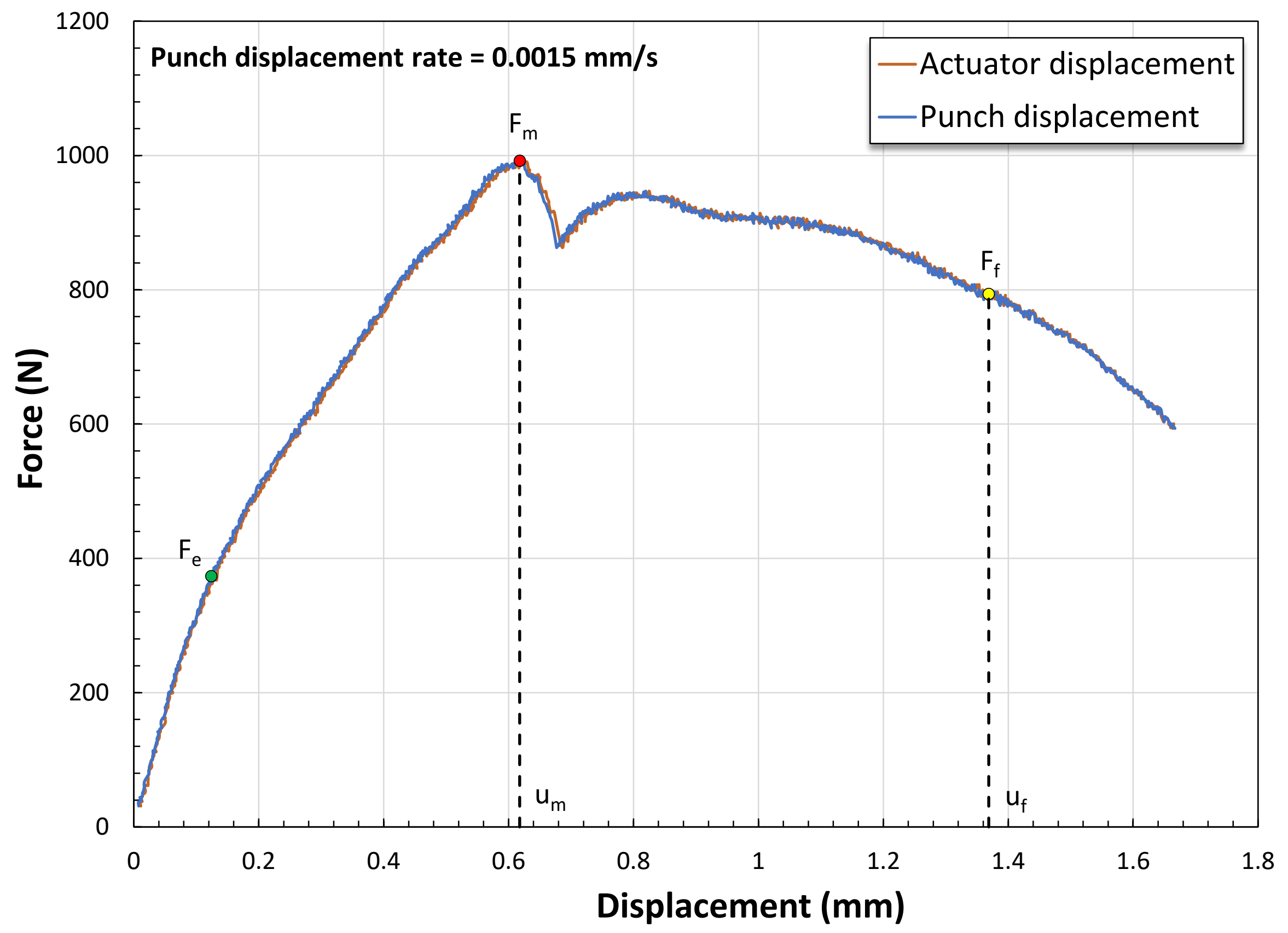




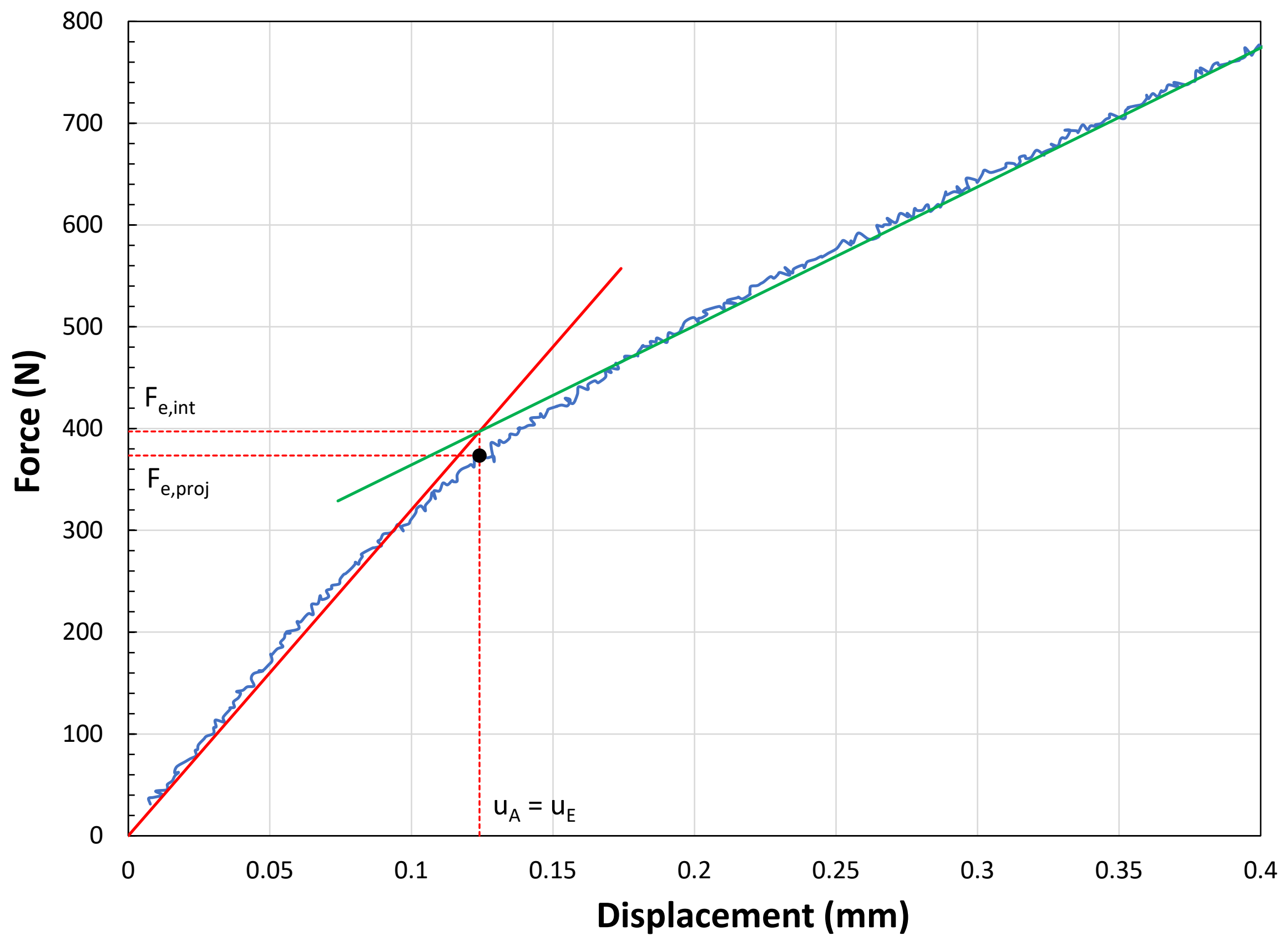


Small Punch Test Results

Specimen ID: 900HIP_3_N_a_SP8 (pc

Material: AM Ti-64

Test speed: $0.001426 \mathrm{~mm} / \mathrm{s}$

Max strain rate: $1.43 \mathrm{E}-03 \mathrm{~s}^{-1}$

\begin{tabular}{|c|c|c|}
\hline \multicolumn{3}{|c|}{ TEST RESULTS } \\
\hline \multicolumn{3}{|c|}{ Force values } \\
\hline$F_{e, p r o j}=$ & 372.2 & $\mathbf{N}$ \\
\hline$F_{e, \text { int }}=$ & 402.0 & $\mathbf{N}$ \\
\hline$F_{h 0 / 10, \text { off }}=$ & 515.6 & $\mathbf{N}$ \\
\hline$F_{0.1 \mathrm{~mm}, \mathrm{off}}=$ & 660.5 & $\mathbf{N}$ \\
\hline$F_{0.1 \mathrm{~mm}}=$ & 324.6 & $\mathbf{N}$ \\
\hline$F_{0.48 \mathrm{~mm}}=$ & 882.8 & $\mathbf{N}$ \\
\hline$F_{0.5 \mathrm{~mm}}=$ & 902.5 & $\mathbf{N}$ \\
\hline$F_{0.65 \mathrm{~mm}}=$ & 1026.6 & $\mathbf{N}$ \\
\hline$F_{0.9 \mathrm{~mm}}=$ & 1079.7 & $\mathbf{N}$ \\
\hline$F_{\text {ept }}=$ & 369.2 & $\mathbf{N}$ \\
\hline$F_{\mathrm{e} 1.5}=$ & 735.9 & $\mathbf{N}$ \\
\hline$F_{m}=$ & 1091.8 & $\mathbf{N}$ \\
\hline$F_{\text {infl }}=$ & 559.0 & $\mathbf{N}$ \\
\hline$F_{f}=$ & 873.4 & $\mathbf{N}$ \\
\hline \multicolumn{3}{|c|}{ Displacement values } \\
\hline $\mathrm{u}_{\mathrm{e}}=$ & 0.122 & $\mathrm{~mm}$ \\
\hline$u_{h 0 / 10, \text { off }}=$ & 0.203 & $\mathrm{~mm}$ \\
\hline$u_{0.1 \mathrm{~mm}, \mathrm{off}}=$ & 0.302 & $\mathrm{~mm}$ \\
\hline $\mathrm{u}_{\mathrm{e} 1.5}=$ & 0.357 & $\mathrm{~mm}$ \\
\hline $\mathbf{u}_{\mathrm{m}}=$ & 0.832 & $\mathrm{~mm}$ \\
\hline$u_{\text {infl }}=$ & 1.487 & $\mathrm{~mm}$ \\
\hline$u_{f}=$ & 1.273 & $\mathrm{~mm}$ \\
\hline \multicolumn{3}{|c|}{ Initial linear slope } \\
\hline Slope $_{\text {ini }}=$ & 3288.00 & $\mathrm{~N} / \mathrm{mm}$ \\
\hline \multicolumn{3}{|c|}{ Energy values } \\
\hline$E_{S P}=$ & 1.06 & J \\
\hline$E_{m}=$ & 0.62 & J \\
\hline$E_{P L}=$ & 0.43 & J \\
\hline
\end{tabular}




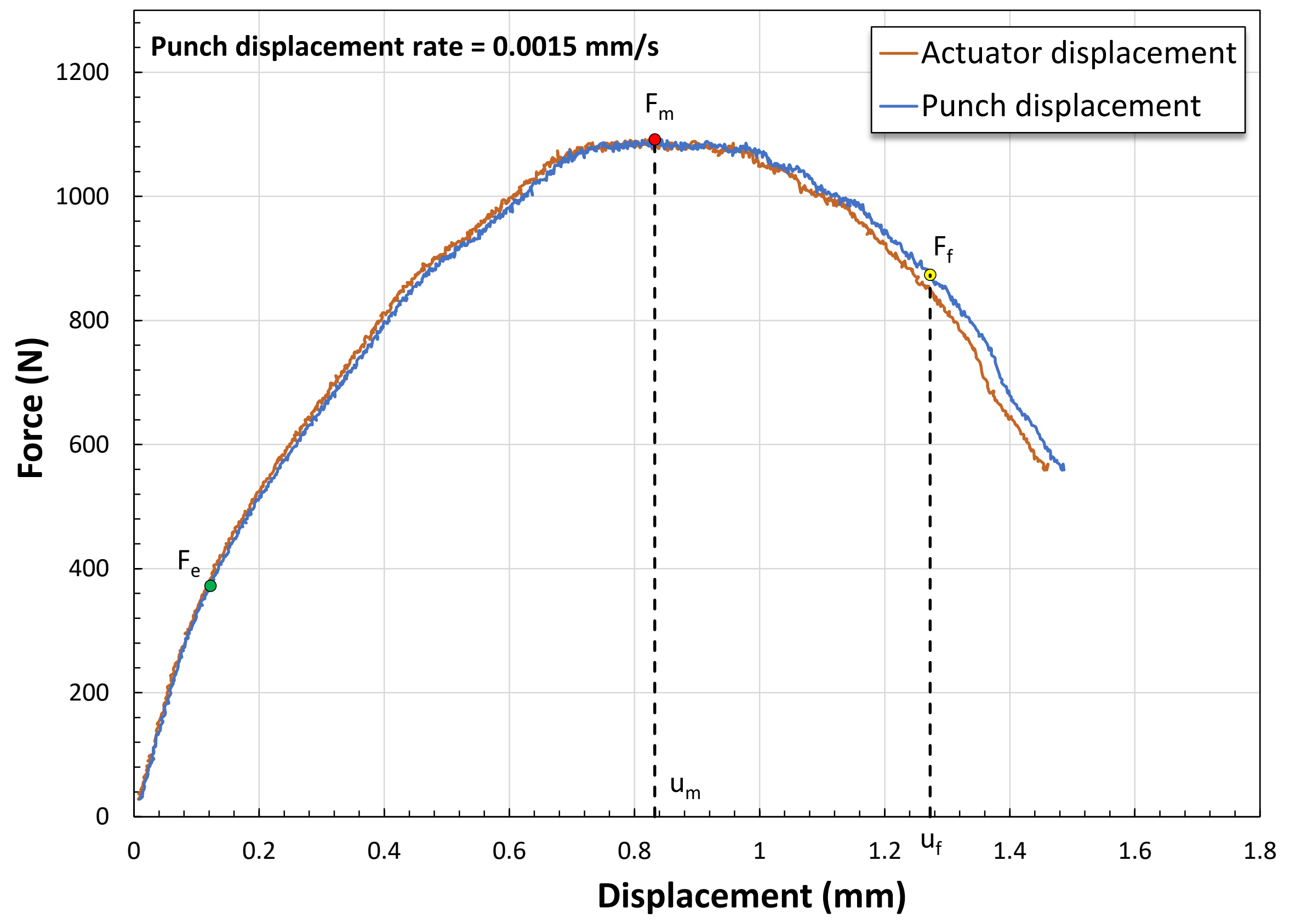




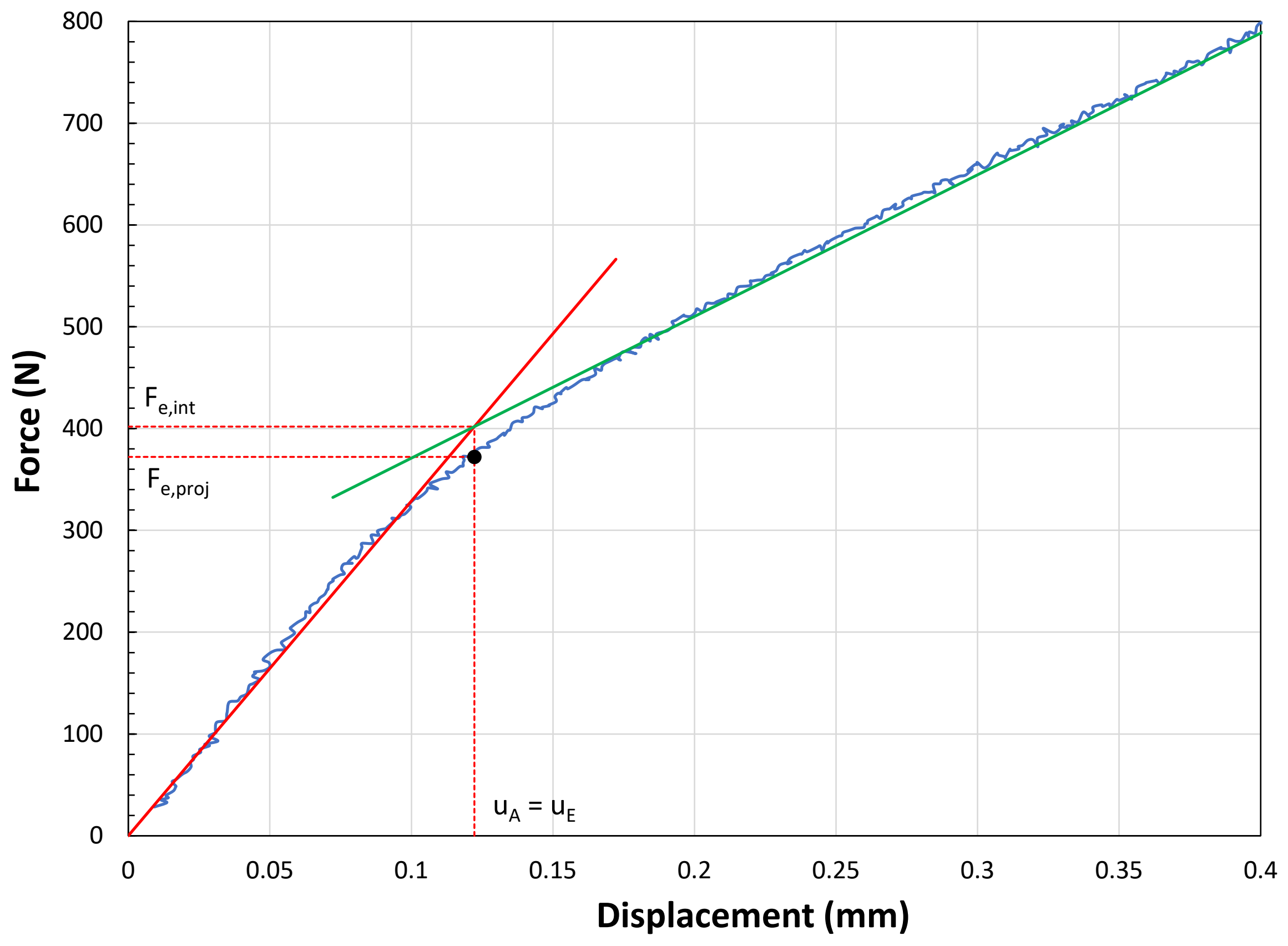


Small Punch Test Results

Specimen ID: 900HIP_3_N_a_SP9 (pc

Material: AM Ti-64

Test speed: $0.001459 \mathrm{~mm} / \mathrm{s}$

Max strain rate: $1.46 \mathrm{E}-03 \mathrm{~s}^{-1}$

\begin{tabular}{|c|c|c|}
\hline \multicolumn{3}{|c|}{ TEST RESULTS } \\
\hline \multicolumn{3}{|c|}{ Force values } \\
\hline$F_{e, \text { proj }}=$ & 373.8 & $\mathbf{N}$ \\
\hline$F_{e, \text { int }}=$ & 406.2 & $\mathbf{N}$ \\
\hline$F_{h 0 / 10, \text { off }}=$ & 531.7 & $\mathbf{N}$ \\
\hline$F_{0.1 \mathrm{~mm}, \mathrm{off}}=$ & 690.7 & $\mathbf{N}$ \\
\hline$F_{0.1 \mathrm{~mm}}=$ & 300.6 & $\mathbf{N}$ \\
\hline$F_{0.48 \mathrm{~mm}}=$ & 882.8 & $\mathbf{N}$ \\
\hline$F_{0.5 \mathrm{~mm}}=$ & 915.7 & $\mathbf{N}$ \\
\hline$F_{0.65 \mathrm{~mm}}=$ & 961.6 & $\mathbf{N}$ \\
\hline$F_{0.9 \mathrm{~mm}}=$ & 969.8 & $\mathbf{N}$ \\
\hline$F_{e p t}=$ & 721.9 & $\mathbf{N}$ \\
\hline$F_{e 1.5}=$ & 746.2 & $\mathbf{N}$ \\
\hline$F_{m}=$ & 995.9 & $\mathbf{N}$ \\
\hline$F_{\text {infl }}=$ & 509.4 & $\mathbf{N}$ \\
\hline$F_{f}=$ & 796.7 & $\mathbf{N}$ \\
\hline \multicolumn{3}{|c|}{ Displacement values } \\
\hline$u_{e}=$ & 0.136 & $\mathrm{~mm}$ \\
\hline$u_{h 0 / 10, \text { off }}=$ & 0.223 & $\mathrm{~mm}$ \\
\hline$u_{0.1 \mathrm{~mm}, \mathrm{off}}=$ & 0.333 & $\mathrm{~mm}$ \\
\hline $\mathrm{u}_{\mathrm{e} 1.5}=$ & 0.380 & $\mathrm{~mm}$ \\
\hline$u_{m}=$ & 0.558 & $\mathrm{~mm}$ \\
\hline$u_{\text {infl }}=$ & 1.648 & $\mathrm{~mm}$ \\
\hline$u_{f}=$ & 1.323 & $\mathrm{~mm}$ \\
\hline \multicolumn{3}{|c|}{ Initial linear slope } \\
\hline Slope $_{\text {ini }}=$ & 2989.78 & $\mathrm{~N} / \mathrm{mm}$ \\
\hline \multicolumn{3}{|c|}{ Energy values } \\
\hline$E_{S P}=$ & 1.04 & J \\
\hline$E_{m}=$ & 0.32 & J \\
\hline $\mathrm{E}_{\mathrm{PL}}=$ & 0.16 & J \\
\hline
\end{tabular}




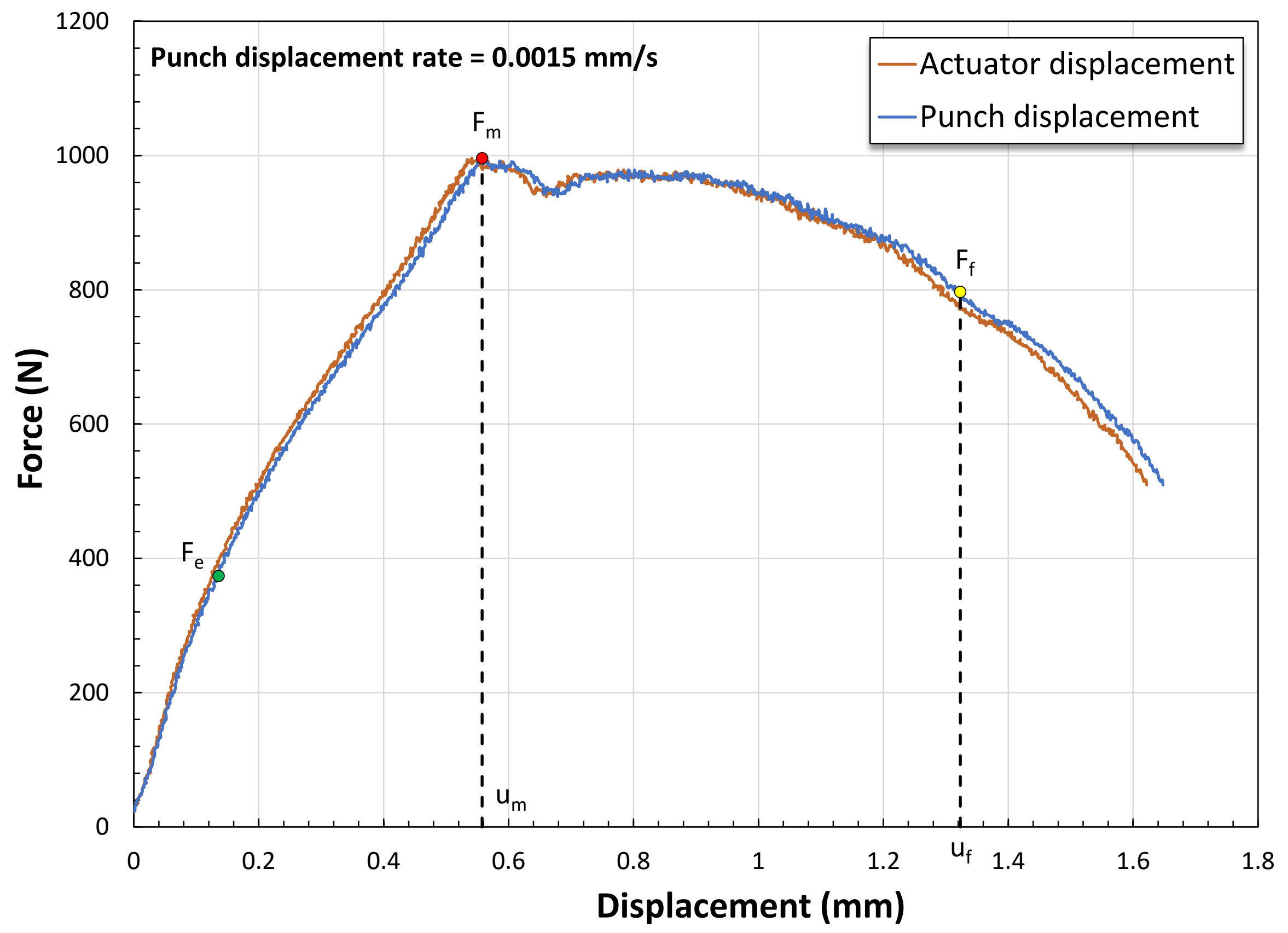




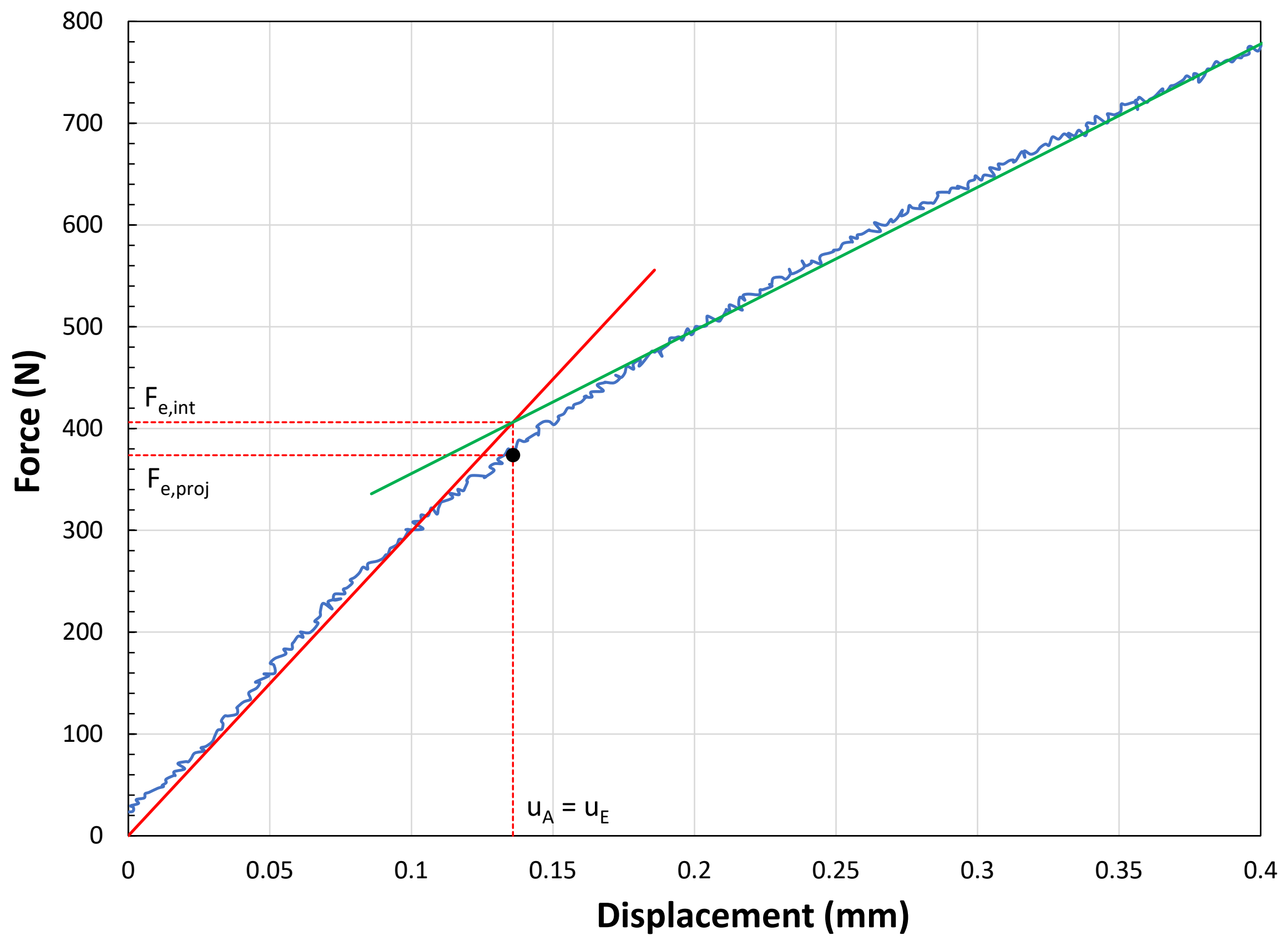


Small Punch Test Results

Specimen ID: 900HIP_3_N_a_SP10 (

Material: AM Ti-64

Test speed: $0.001444 \mathrm{~mm} / \mathrm{s}$

Max strain rate: $1.44 \mathrm{E}-03 \mathrm{~s}^{-1}$

\begin{tabular}{|c|c|c|}
\hline \multicolumn{3}{|c|}{ TEST RESULTS } \\
\hline \multicolumn{3}{|c|}{ Force values } \\
\hline$F_{e, \text { proj }}=$ & 434.7 & $\mathbf{N}$ \\
\hline$F_{e, \text { int }}=$ & 467.2 & $\mathbf{N}$ \\
\hline$F_{h 0 / 10, \text { off }}=$ & 595.3 & $\mathbf{N}$ \\
\hline$F_{0.1 \mathrm{~mm}, \mathrm{off}}=$ & 748.0 & $\mathbf{N}$ \\
\hline$F_{0.1 \mathrm{~mm}}=$ & 293.0 & $\mathbf{N}$ \\
\hline$F_{0.48 \mathrm{~mm}}=$ & 872.8 & $\mathbf{N}$ \\
\hline$F_{0.5 \mathrm{~mm}}=$ & 878.4 & $\mathbf{N}$ \\
\hline$F_{0.65 \mathrm{~mm}}=$ & 921.6 & $\mathbf{N}$ \\
\hline$F_{0.9 \mathrm{~mm}}=$ & 1033.5 & $\mathbf{N}$ \\
\hline$F_{e p t}=$ & 884.7 & $\mathbf{N}$ \\
\hline$F_{\text {e1.5 }}=$ & 858.4 & $\mathbf{N}$ \\
\hline$F_{m}=$ & 1040.8 & $\mathbf{N}$ \\
\hline$F_{\text {infl }}=$ & 967.1 & $\mathbf{N}$ \\
\hline$F_{f}=$ & 832.7 & $\mathbf{N}$ \\
\hline \multicolumn{3}{|c|}{ Displacement values } \\
\hline$u_{e}=$ & 0.164 & $\mathrm{~mm}$ \\
\hline$u_{h 0 / 10, \text { off }}=$ & 0.254 & $\mathrm{~mm}$ \\
\hline$u_{0.1 \mathrm{~mm}, \mathrm{off}}=$ & 0.364 & $\mathrm{~mm}$ \\
\hline $\mathrm{u}_{\mathrm{e} 1.5}=$ & 0.457 & $\mathrm{~mm}$ \\
\hline$u_{m}=$ & 0.899 & $\mathrm{~mm}$ \\
\hline$u_{\text {infl }}=$ & 0.707 & $\mathrm{~mm}$ \\
\hline$u_{f}=$ & 1.356 & $\mathrm{~mm}$ \\
\hline \multicolumn{3}{|c|}{ Initial linear slope } \\
\hline Slope $_{\text {ini }}=$ & 2849.28 & $\mathrm{~N} / \mathrm{mm}$ \\
\hline \multicolumn{3}{|c|}{ Energy values } \\
\hline$E_{S P}=$ & 1.09 & J \\
\hline$E_{m}=$ & 0.65 & J \\
\hline $\mathrm{E}_{\mathrm{PL}}=$ & 0.46 & J \\
\hline
\end{tabular}




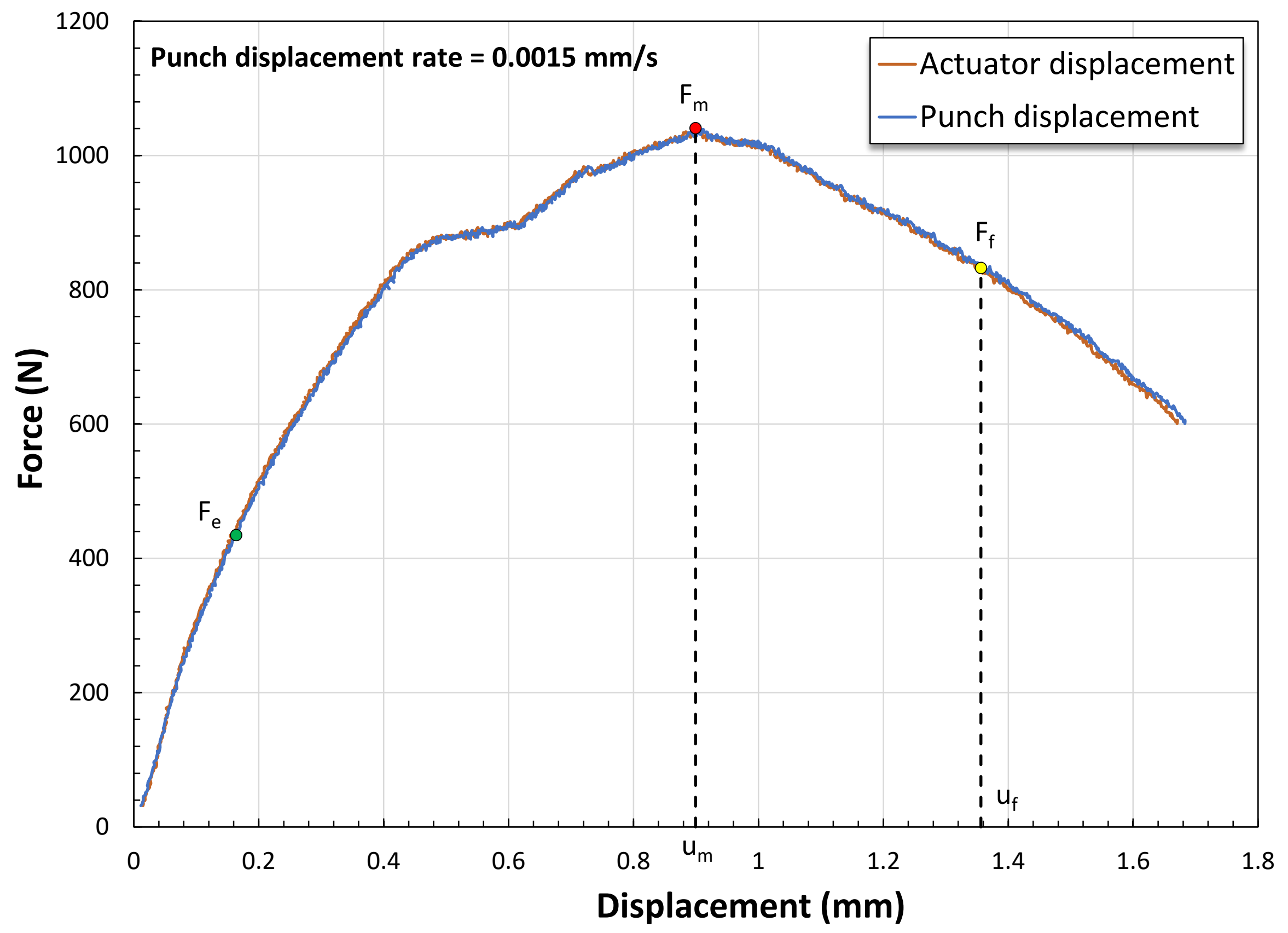




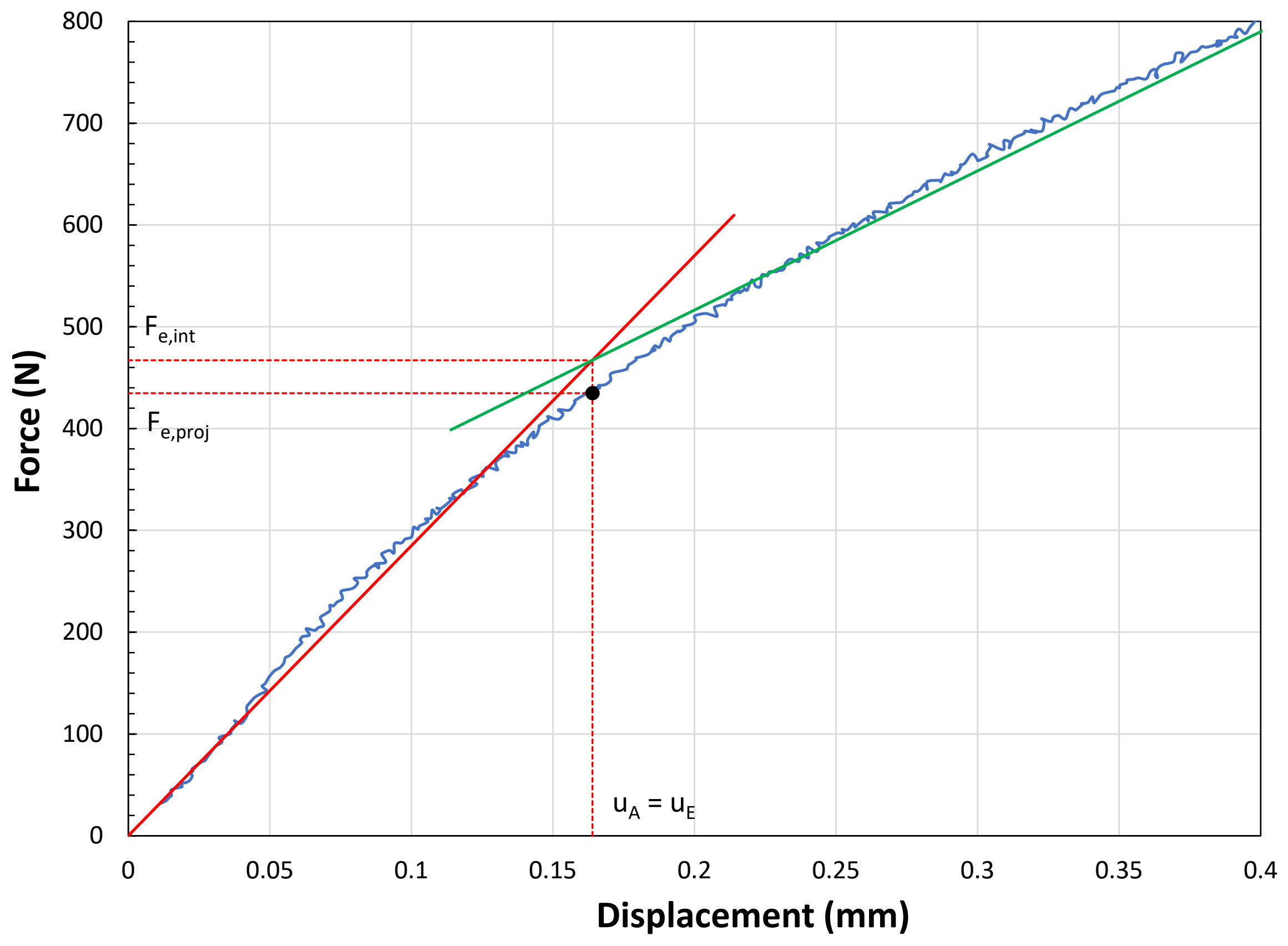




\section{ANNEX 14 \\ Correlations obtained for AM Ti64 \\ between yield strength and SP parameters}




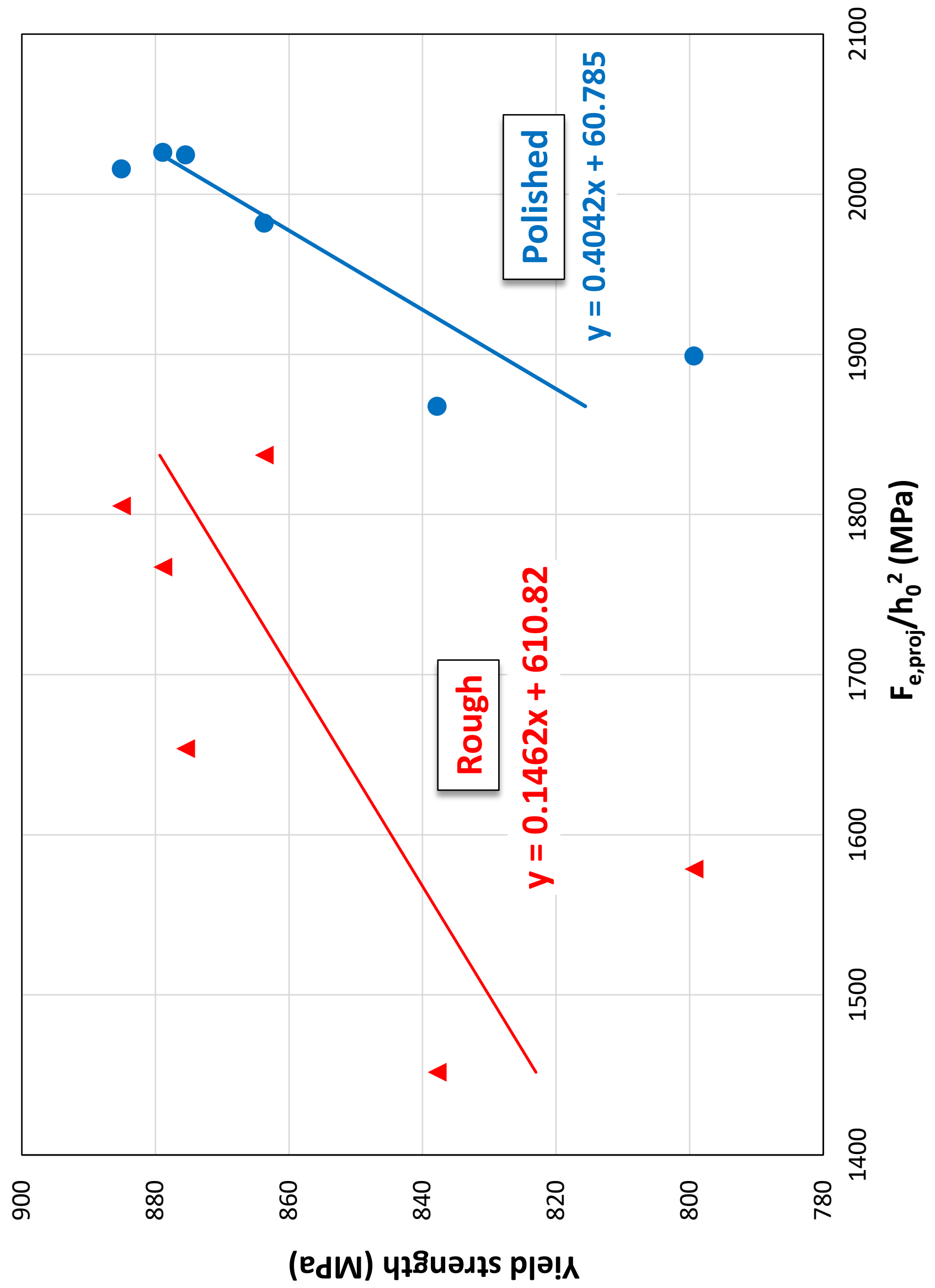




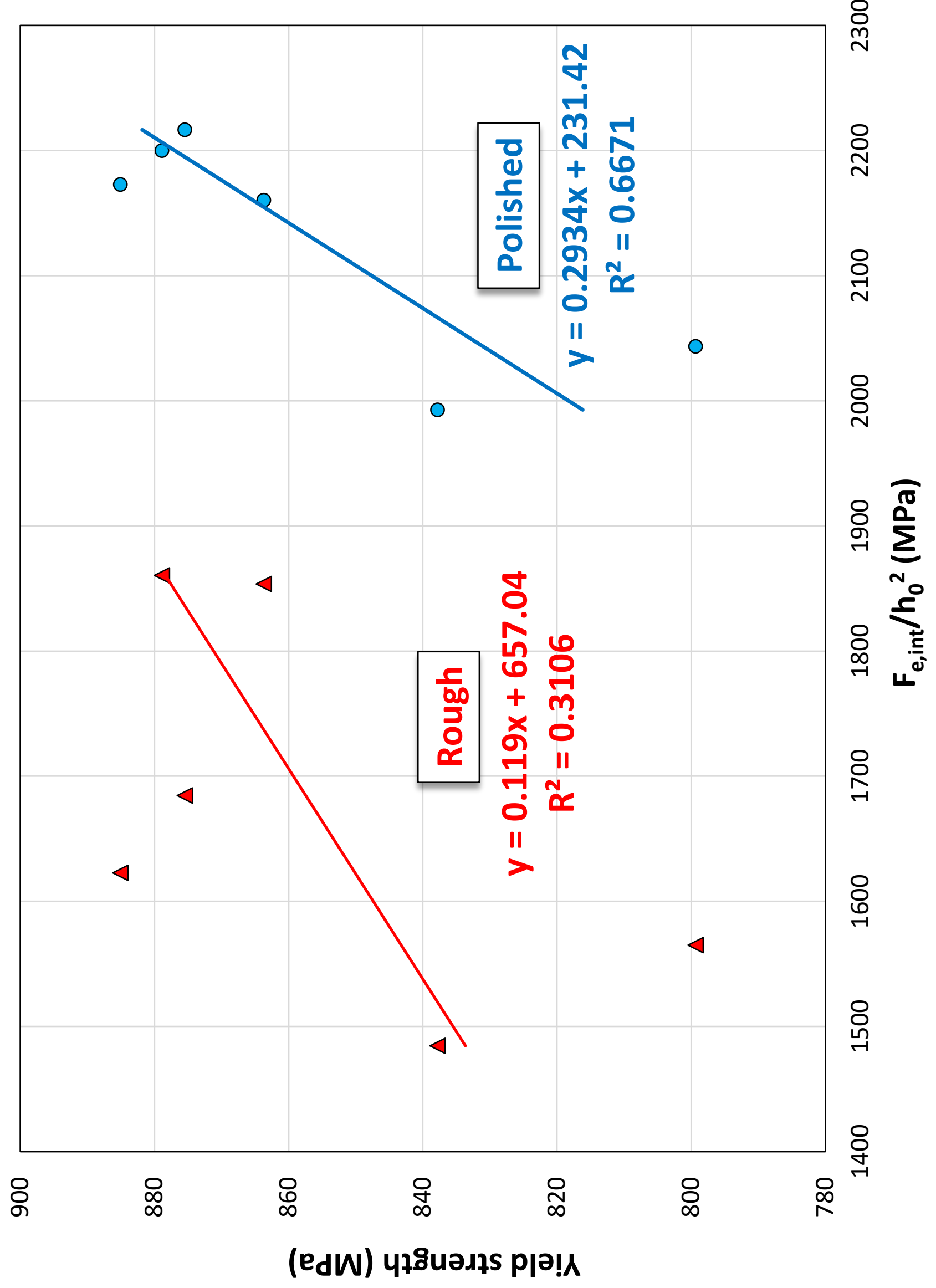




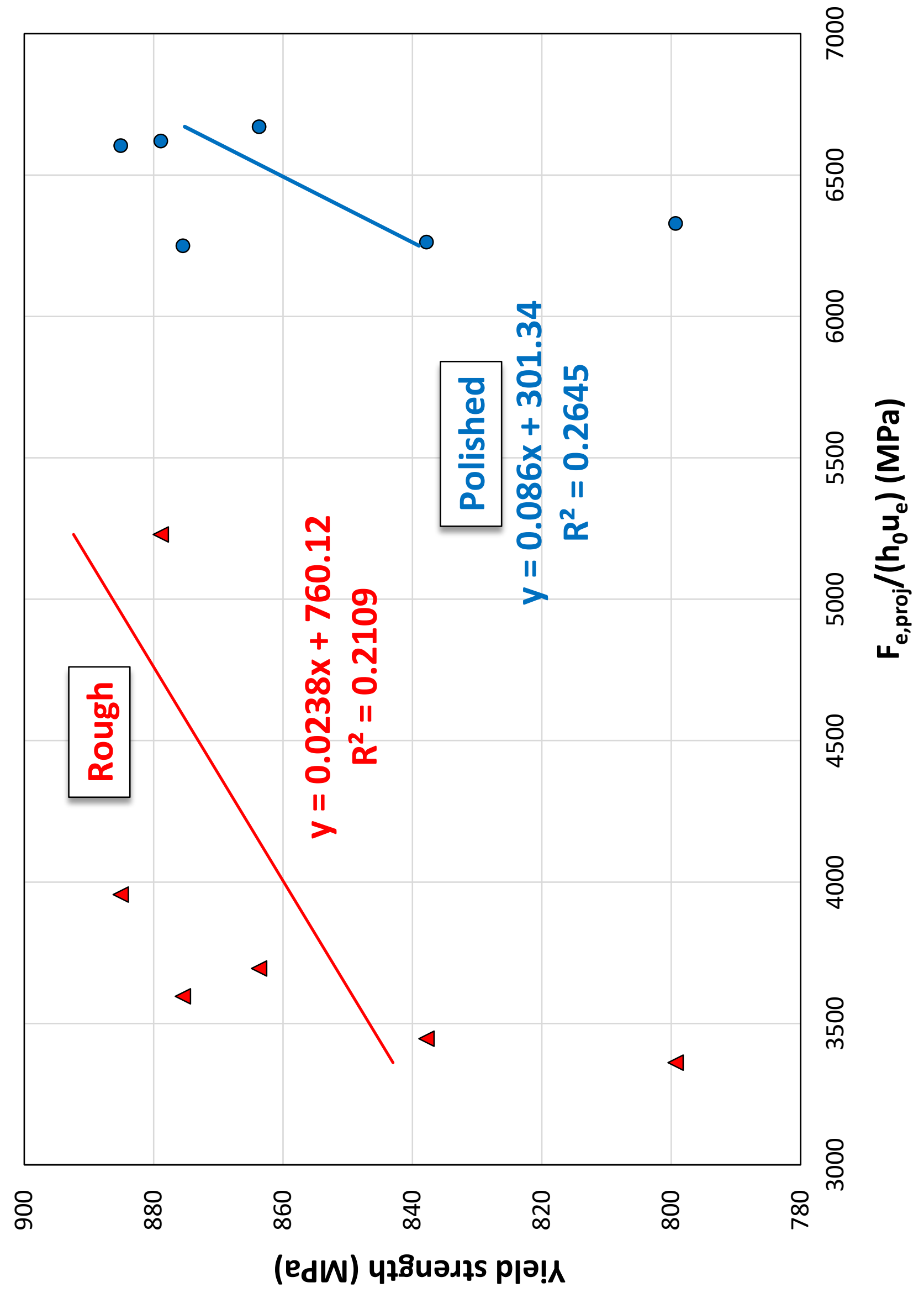




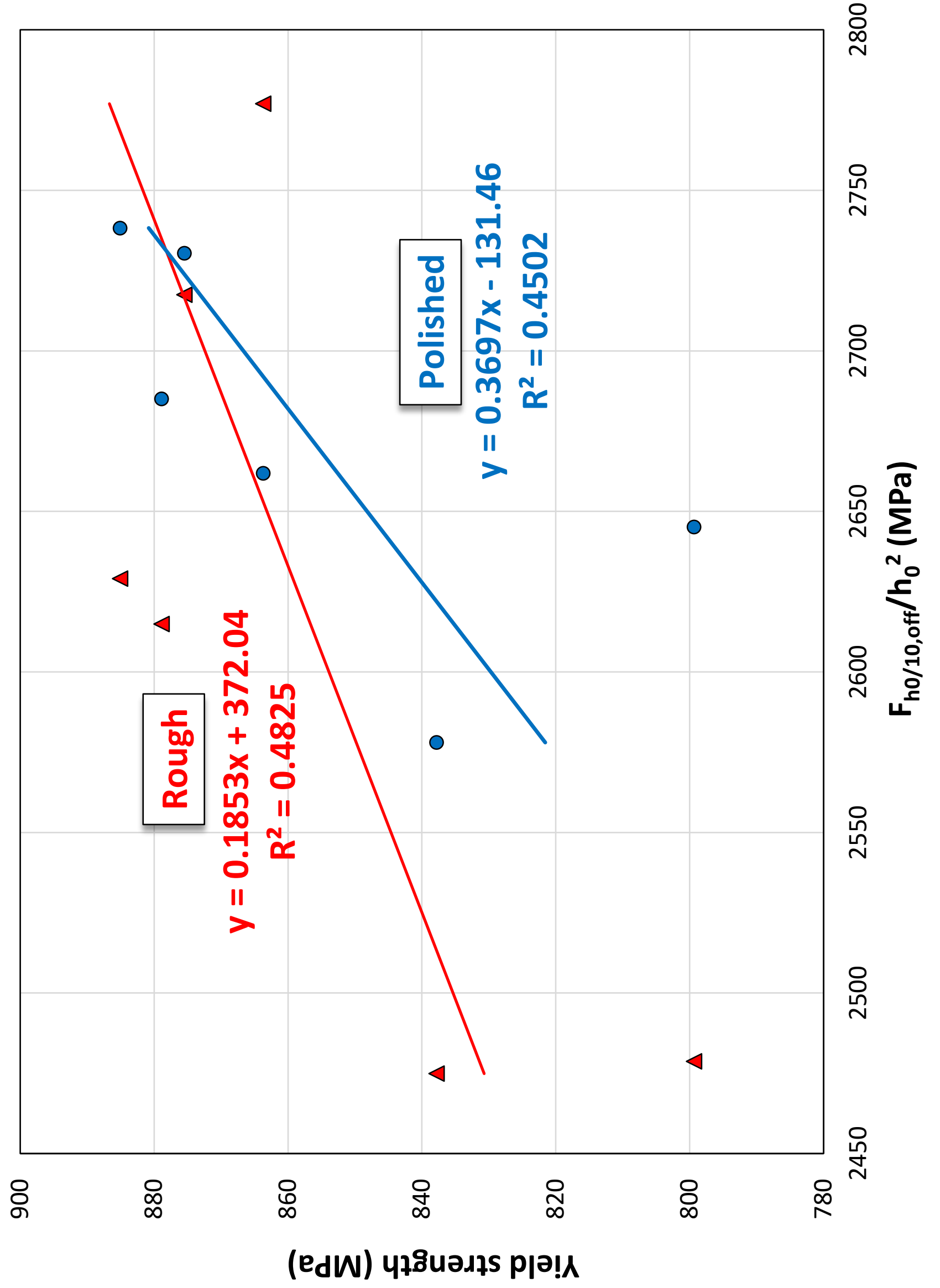




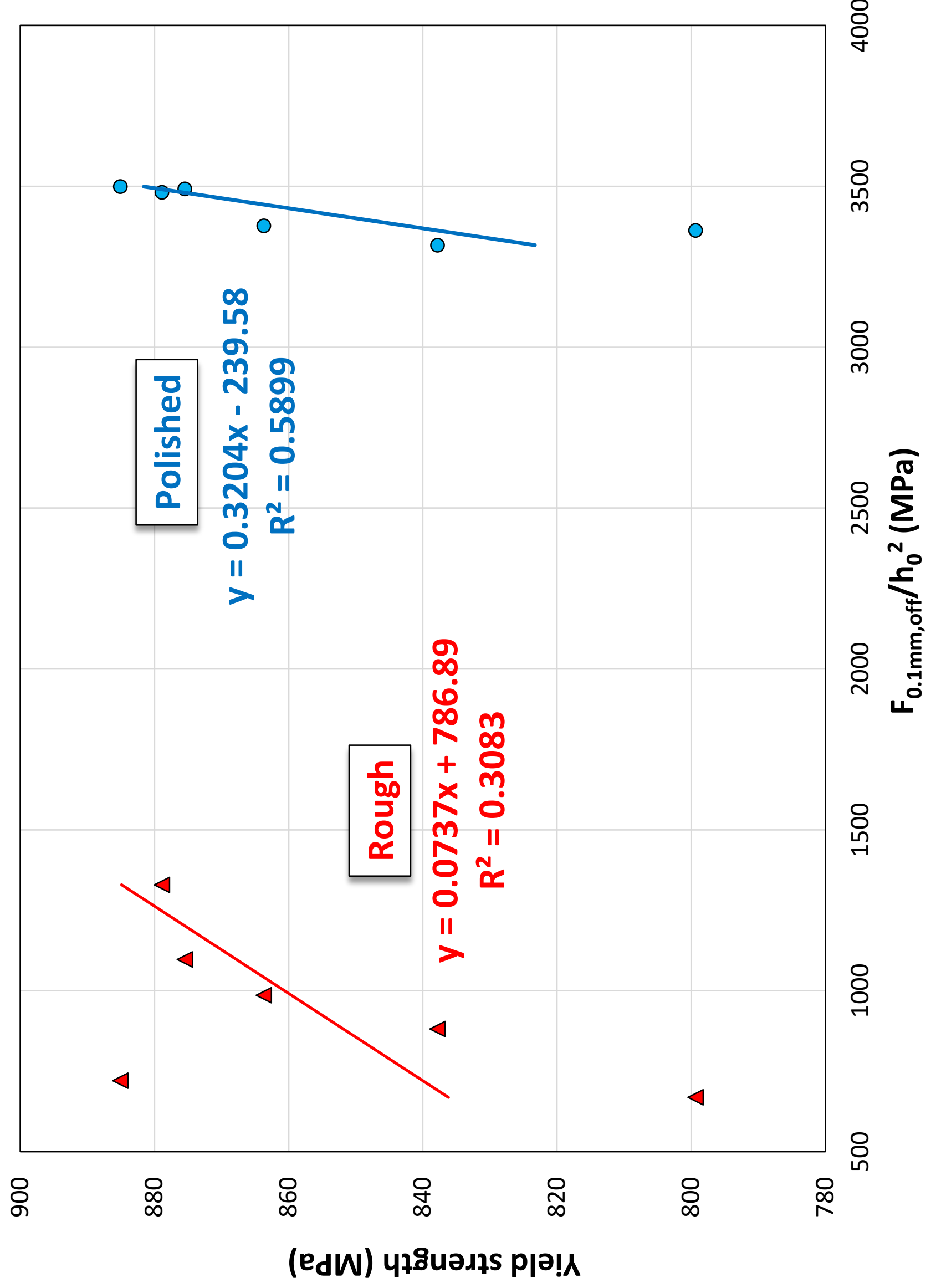




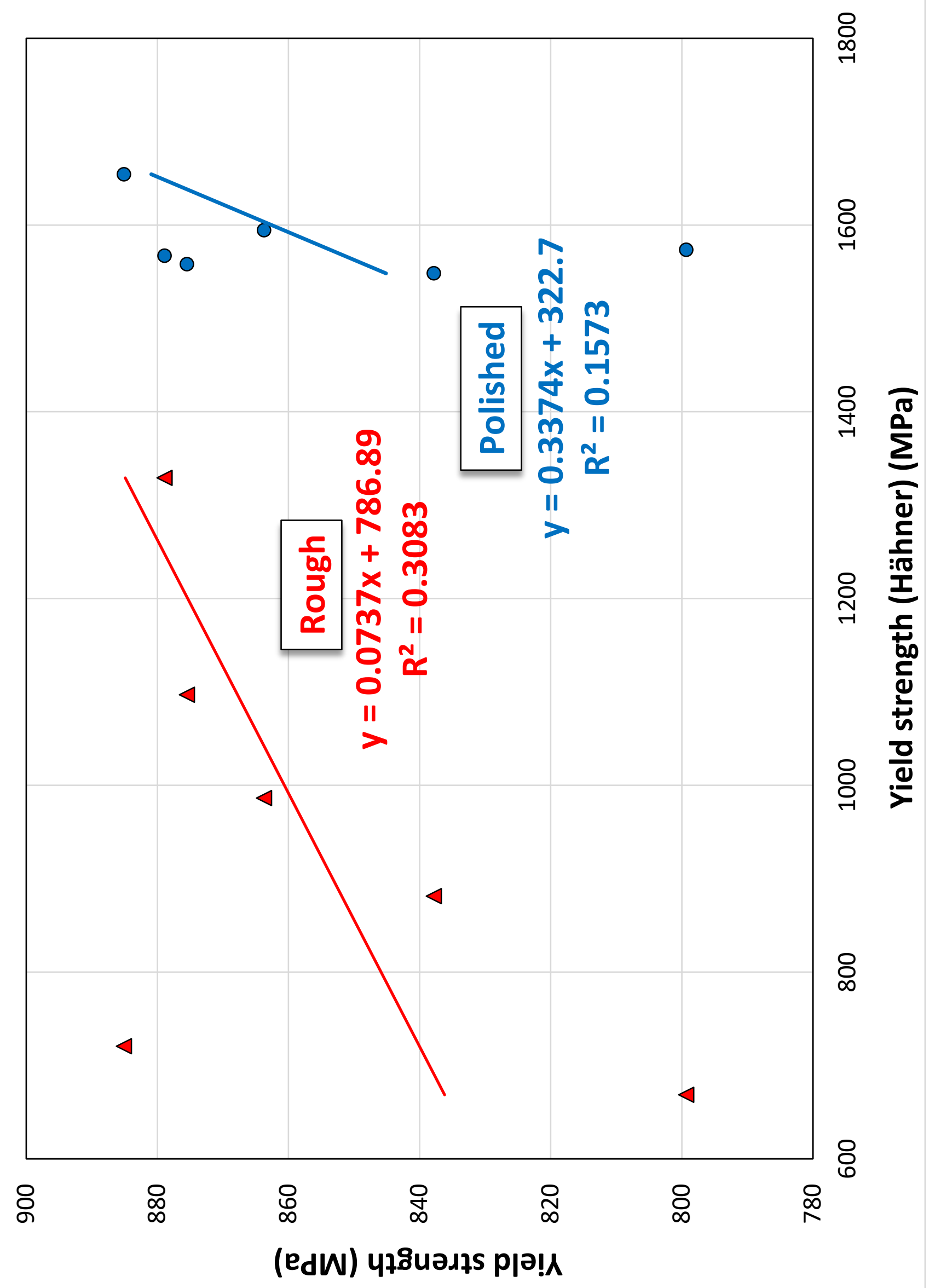




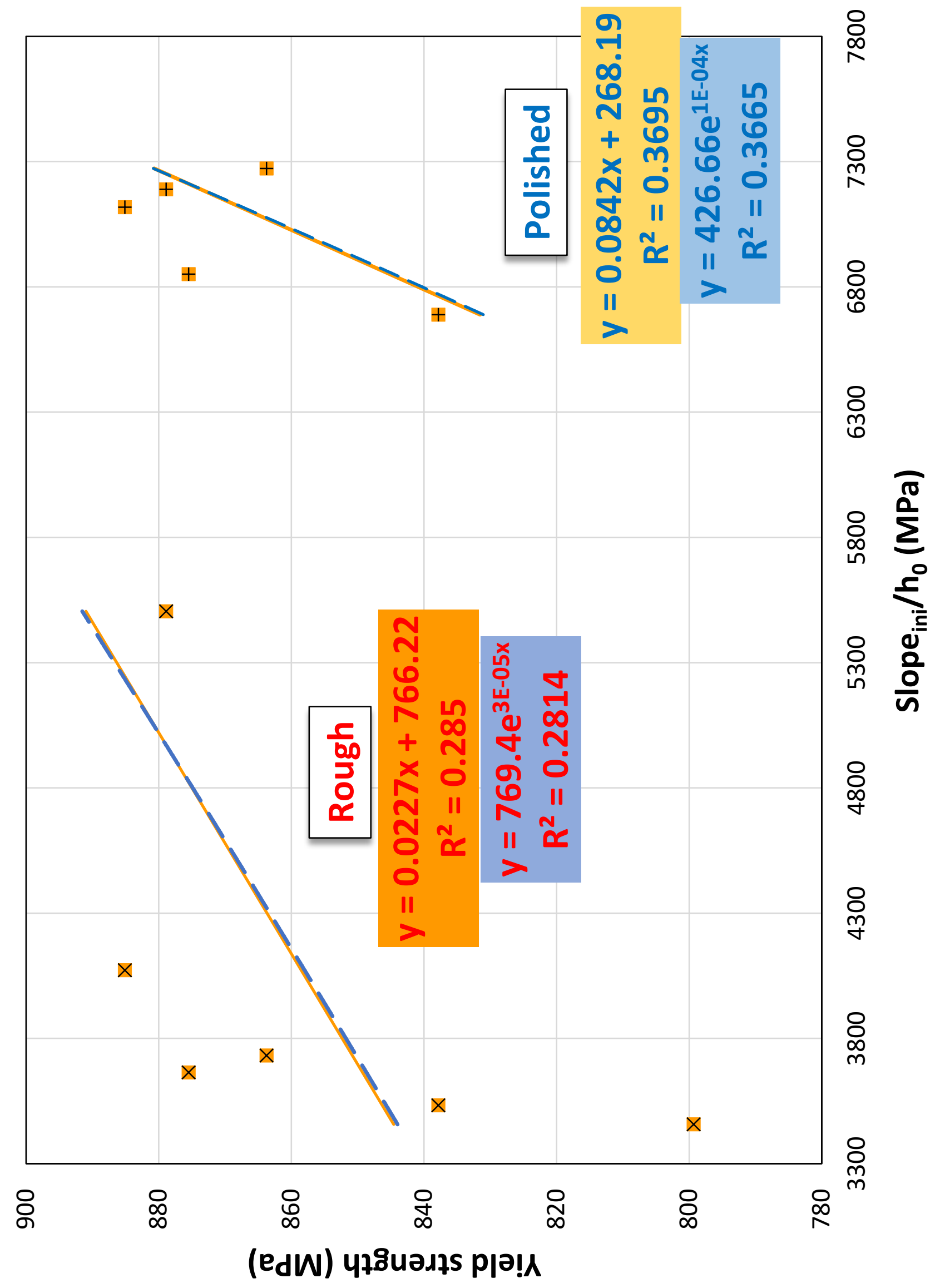




\section{ANNEX 15 \\ Correlations obtained for AM Ti64 between ultimate tensile strength and SP parameters}




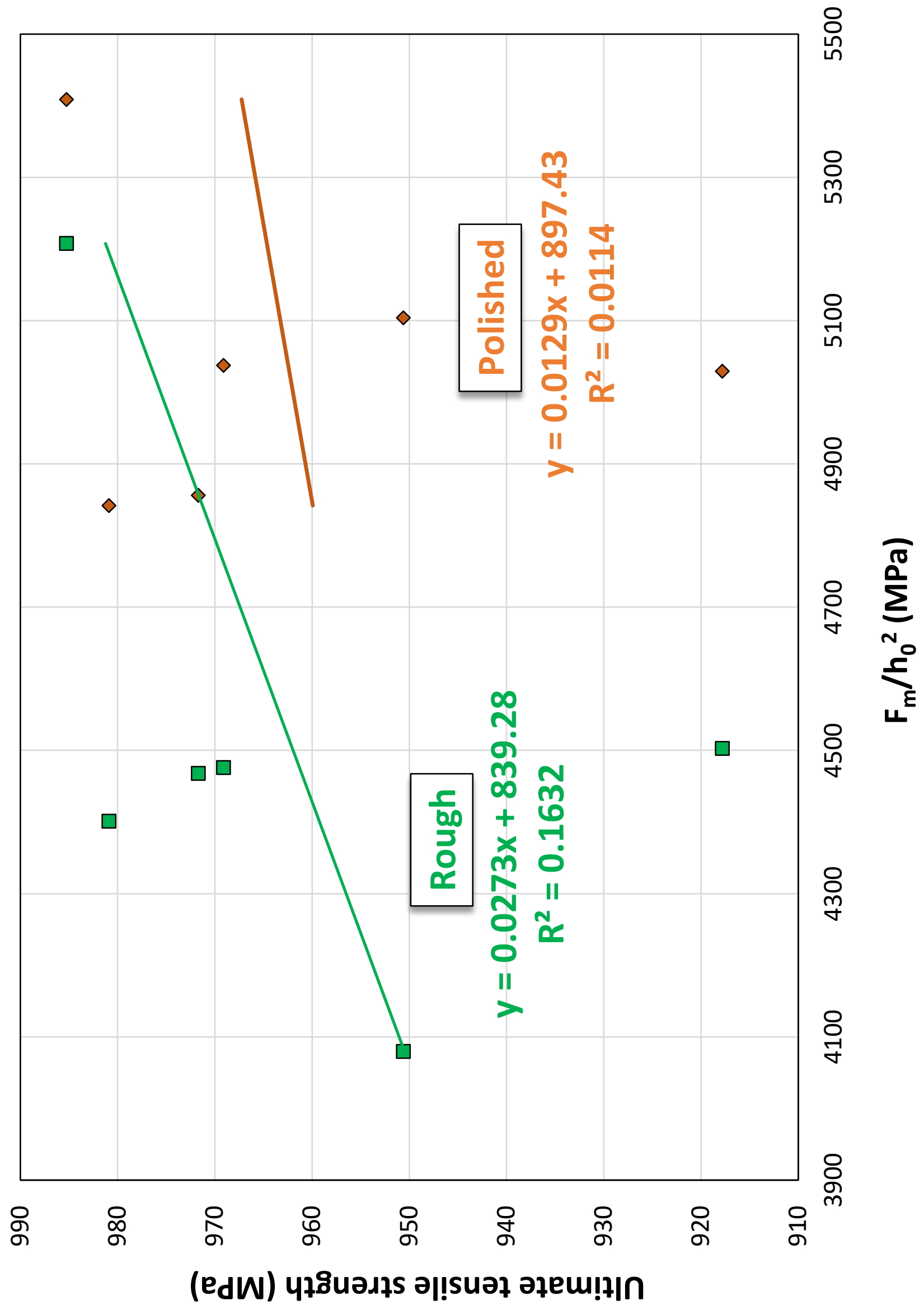




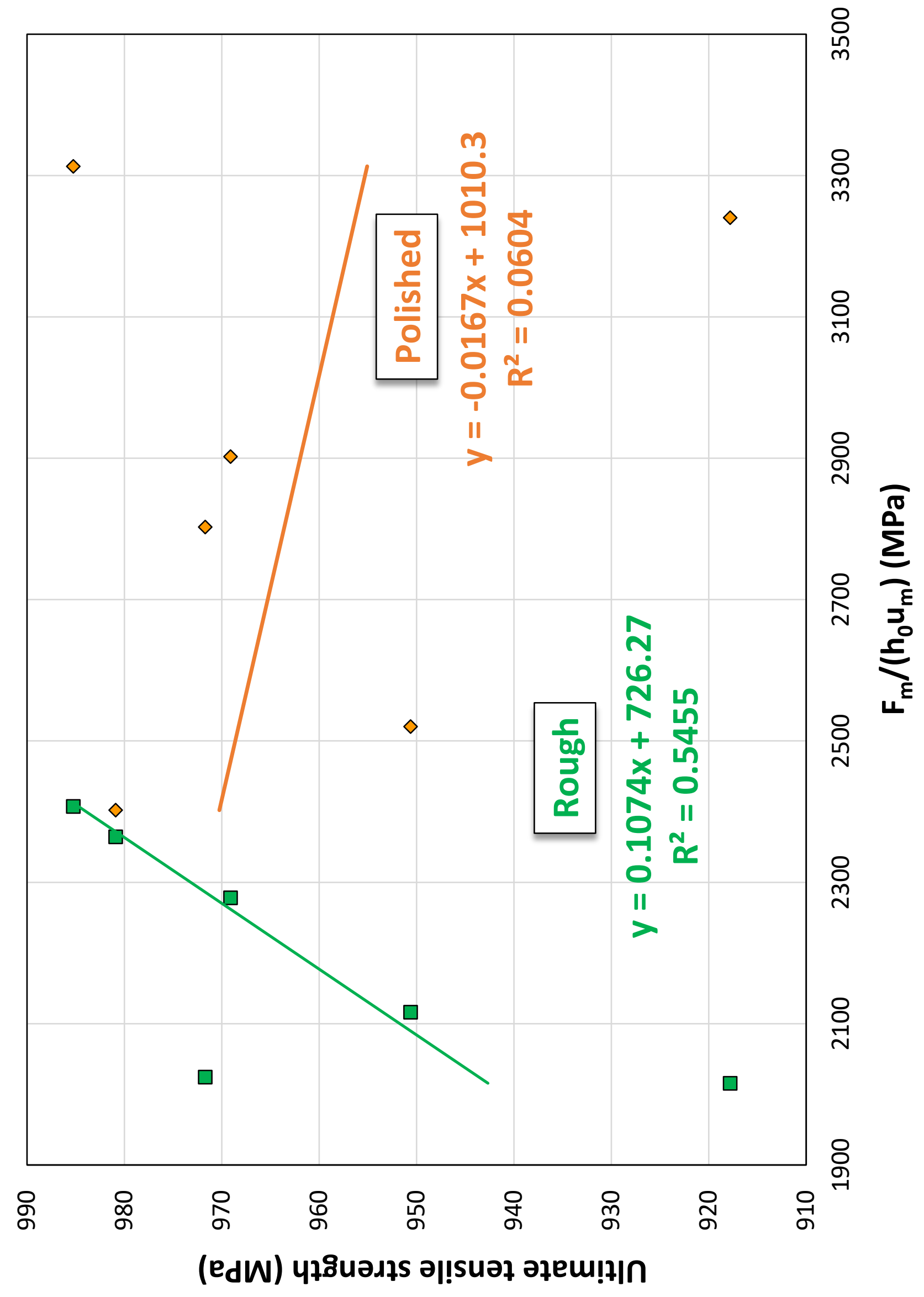




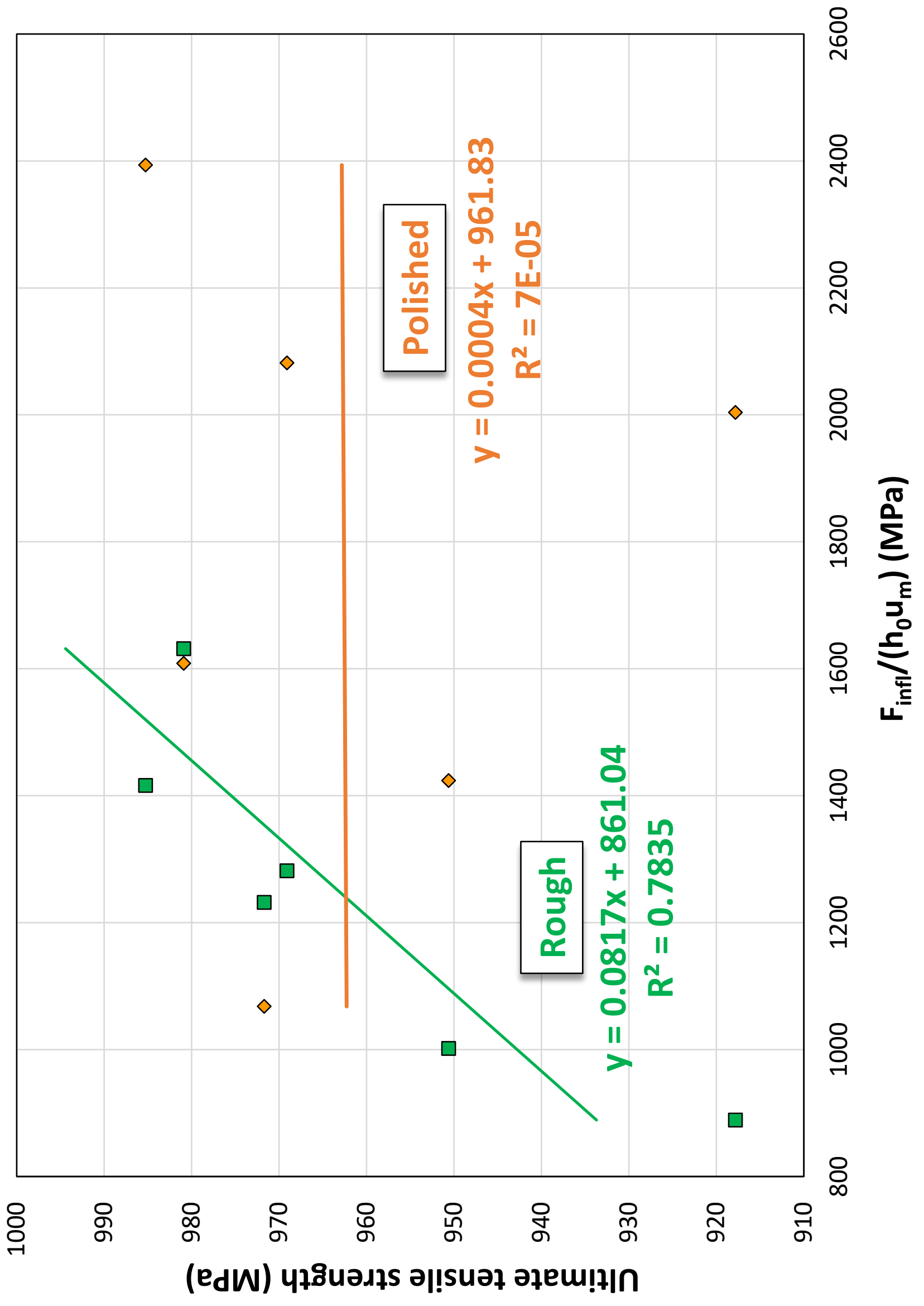




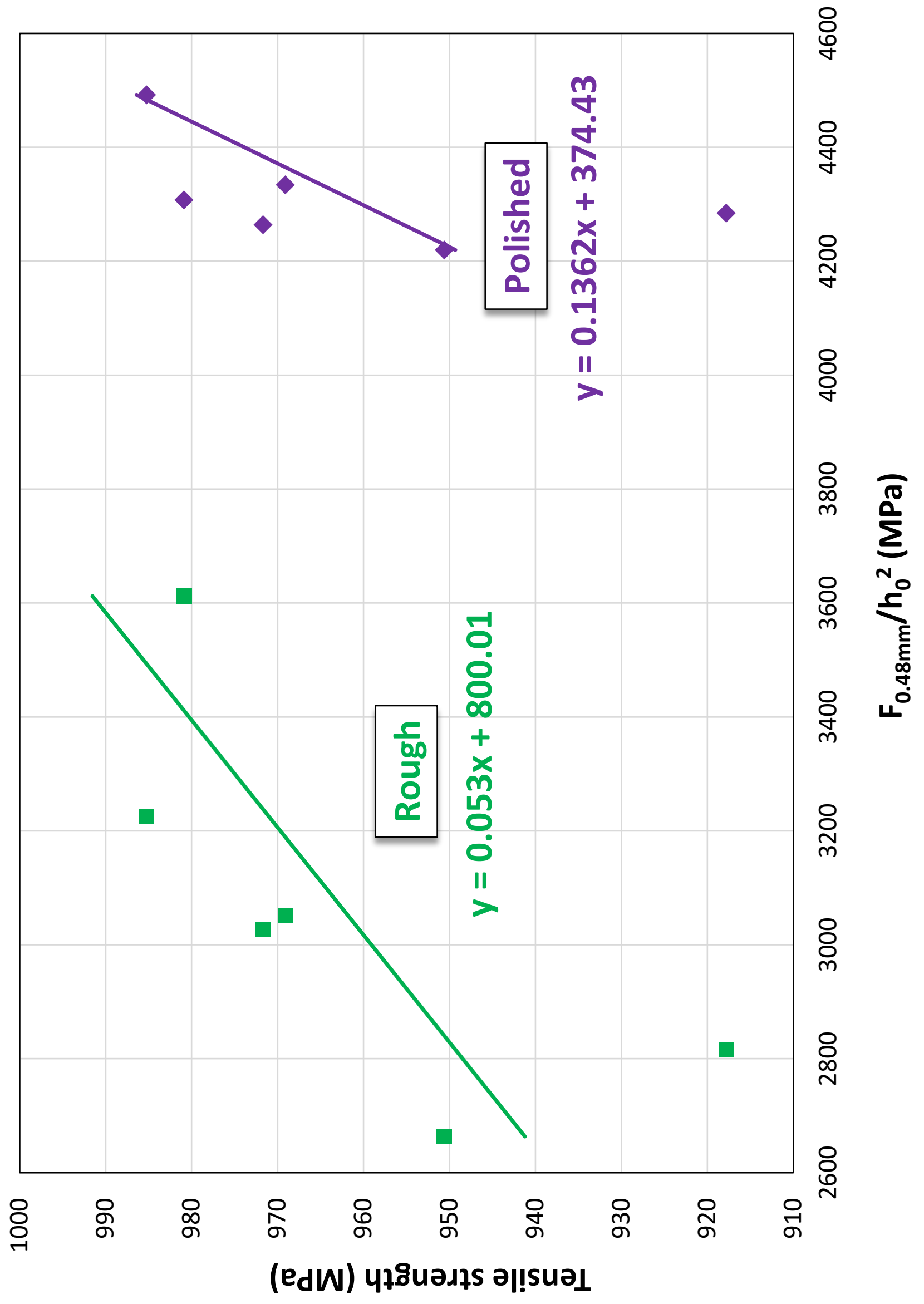




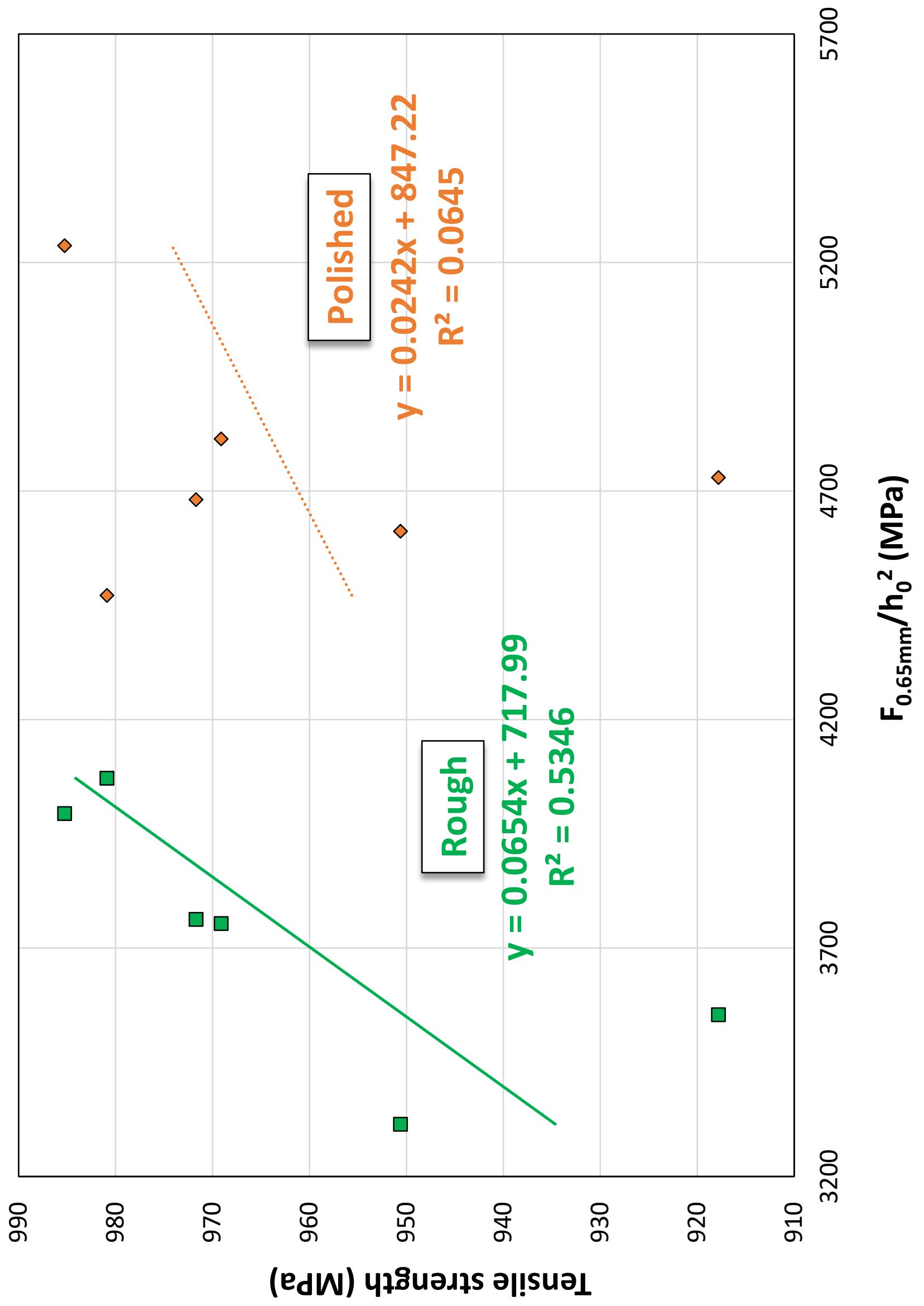




\section{ANNEX 16 \\ Correlations obtained for AM Ti64 \\ between total/uniform elongation and SP parameters}




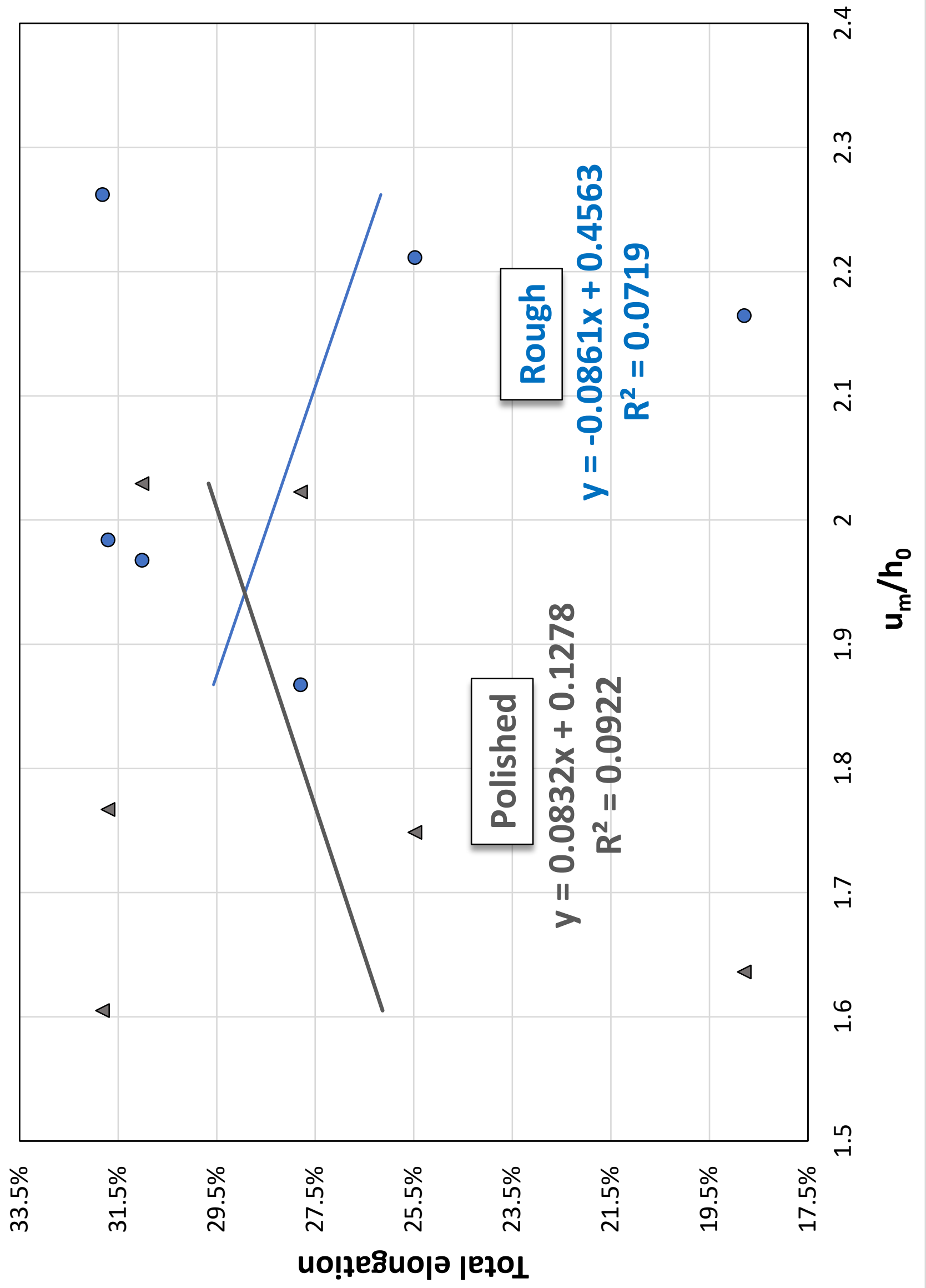




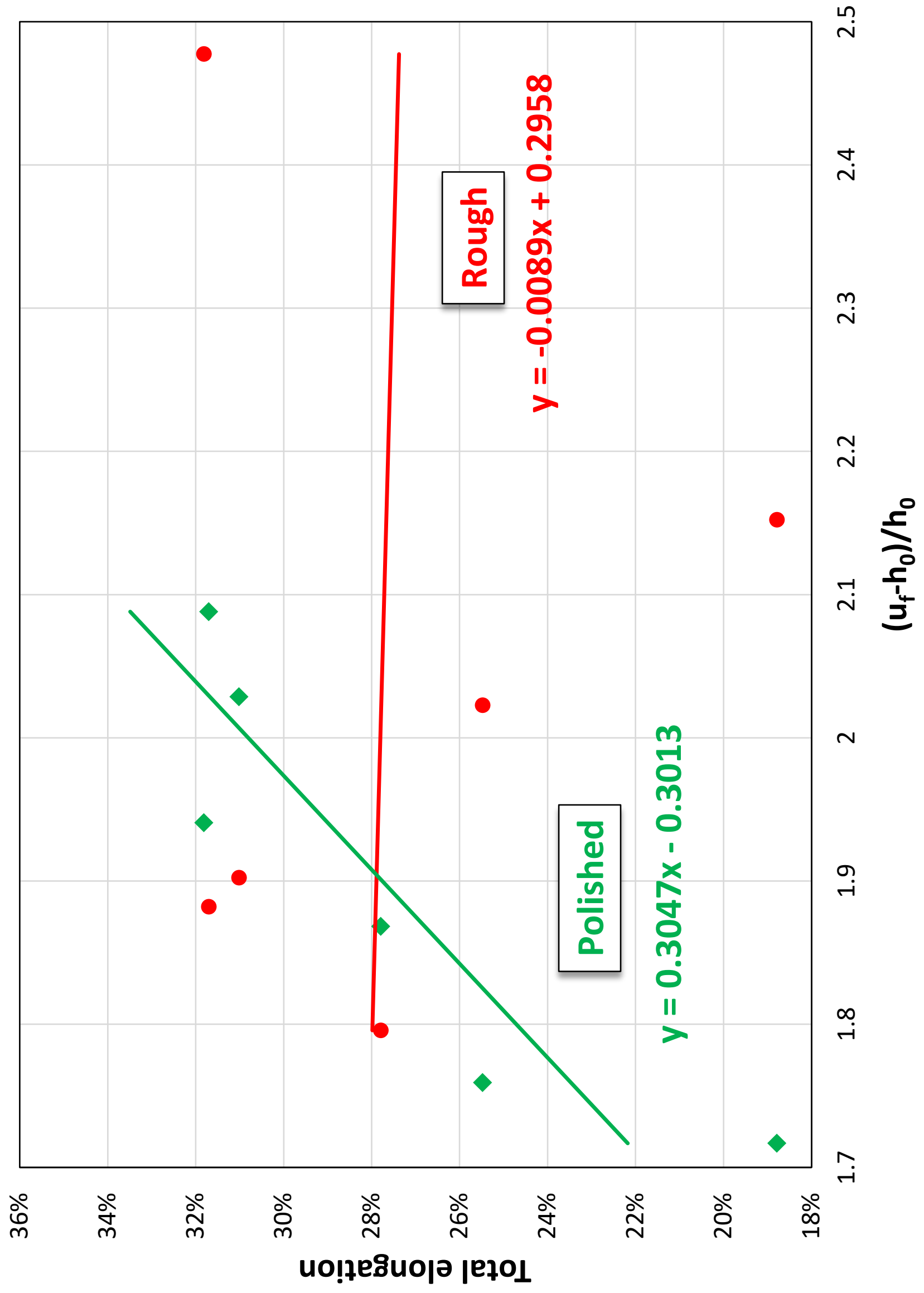




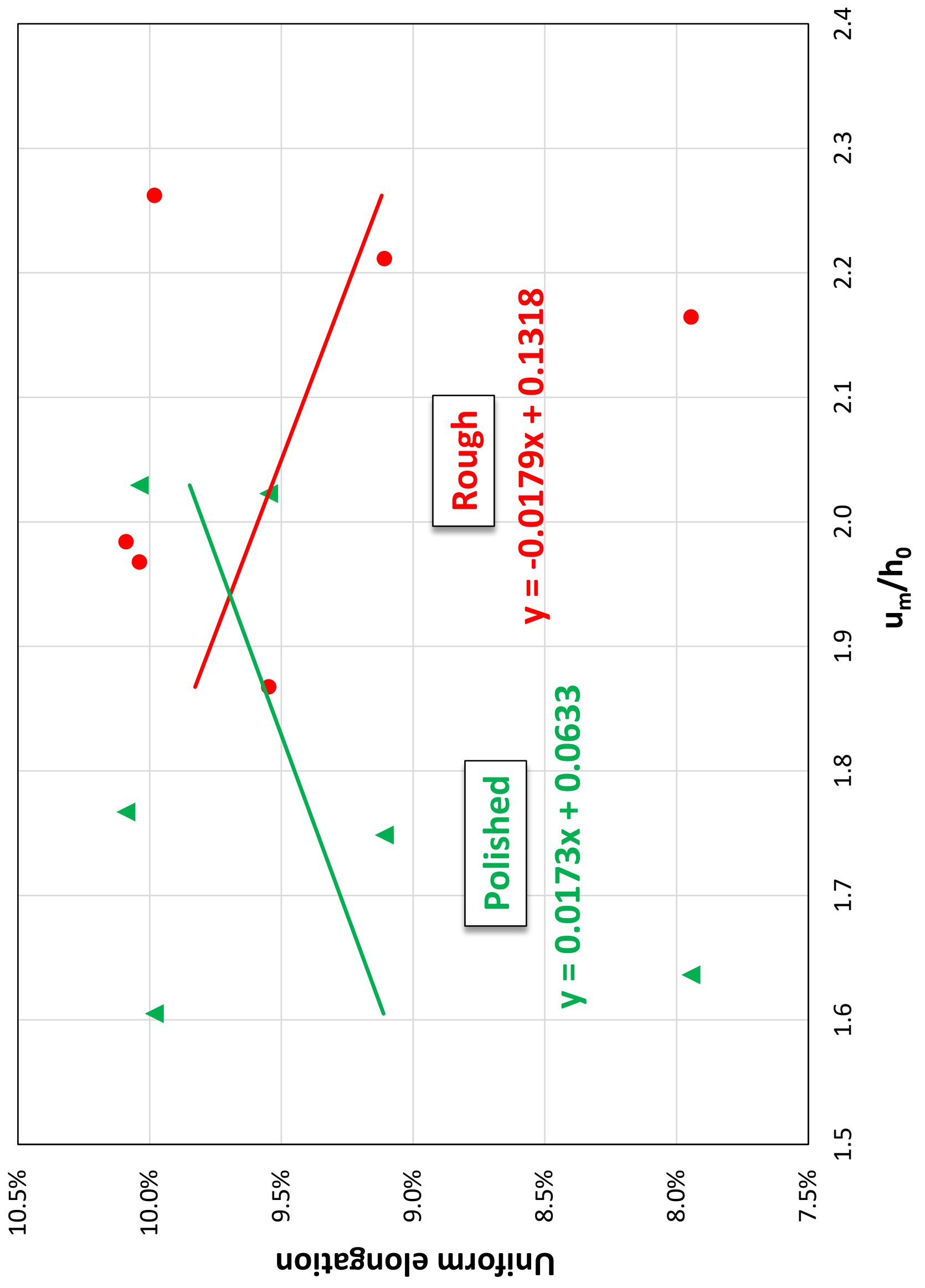

